\title{
Towards diagnostic and therapeutic integration of advanced MR imaging in thoracic and neuro-oncology
}

Citation for published version (APA):

Peerlings, J. (2019). Towards diagnostic and therapeutic integration of advanced MR imaging in thoracic and neuro-oncology. [Doctoral Thesis, Maastricht University]. Ipskamp Printing BV. https://doi.org/10.26481/dis.20190418jp

Document status and date:

Published: 01/01/2019

DOI:

10.26481/dis.20190418jp

Document Version:

Publisher's PDF, also known as Version of record

\section{Please check the document version of this publication:}

- A submitted manuscript is the version of the article upon submission and before peer-review. There can be important differences between the submitted version and the official published version of record.

People interested in the research are advised to contact the author for the final version of the publication, or visit the DOI to the publisher's website.

- The final author version and the galley proof are versions of the publication after peer review.

- The final published version features the final layout of the paper including the volume, issue and page numbers.

Link to publication

\footnotetext{
General rights rights.

- You may freely distribute the URL identifying the publication in the public portal. please follow below link for the End User Agreement:

www.umlib.nl/taverne-license

Take down policy

If you believe that this document breaches copyright please contact us at:

repository@maastrichtuniversity.nl

providing details and we will investigate your claim.
}

Copyright and moral rights for the publications made accessible in the public portal are retained by the authors and/or other copyright owners and it is a condition of accessing publications that users recognise and abide by the legal requirements associated with these

- Users may download and print one copy of any publication from the public portal for the purpose of private study or research.

- You may not further distribute the material or use it for any profit-making activity or commercial gain

If the publication is distributed under the terms of Article $25 \mathrm{fa}$ of the Dutch Copyright Act, indicated by the "Taverne" license above, 


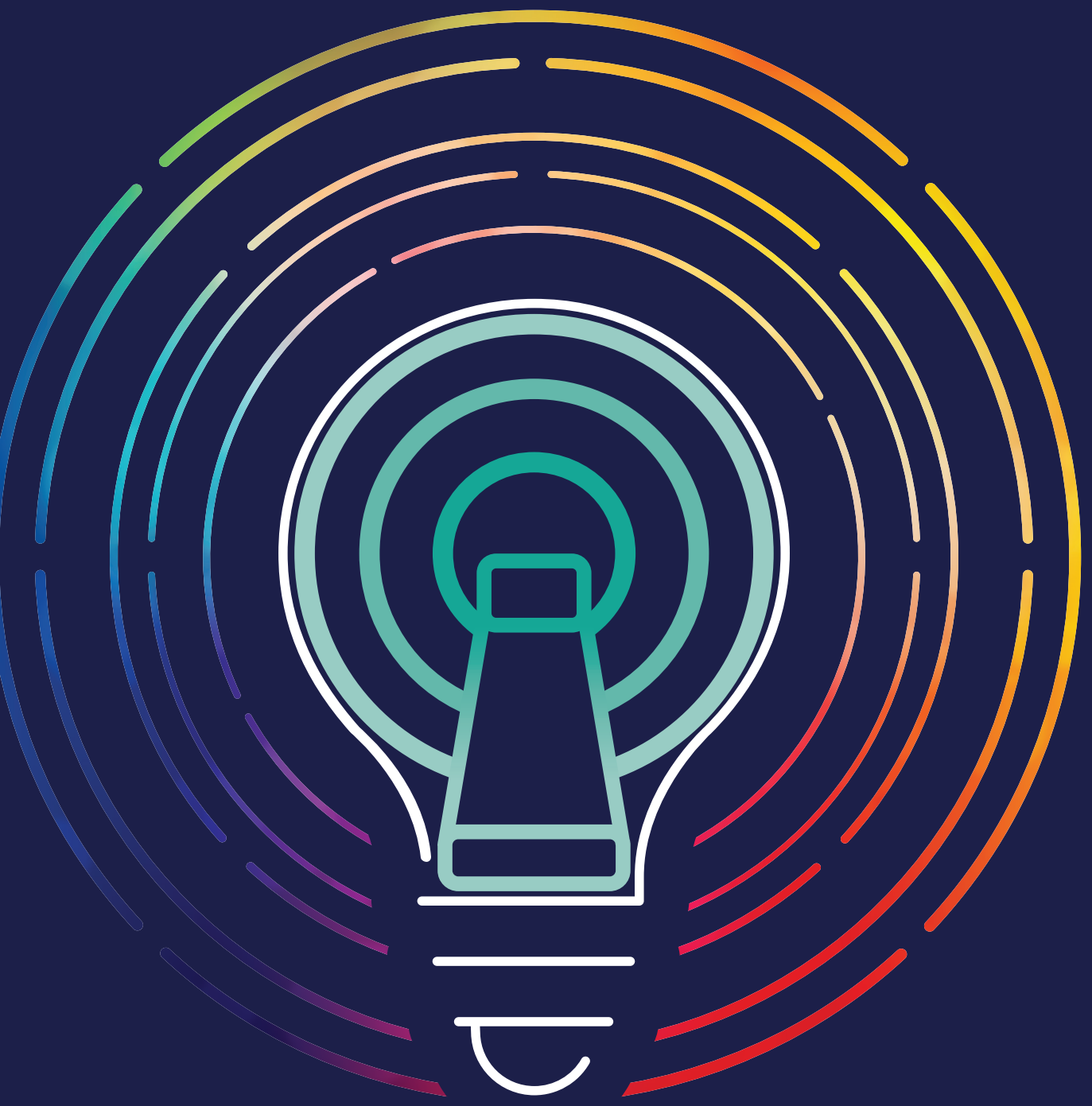

Towards diagnostic and therapeutic integration of advanced IIRR imaging in thoracic and neuro-oncology

\section{Jurgen Peerlings}





\section{Towards diagnostic and}

therapeutic integration of advanced MR imaging in thoracic and neuro-oncology

- by Jurgen Peerlings 


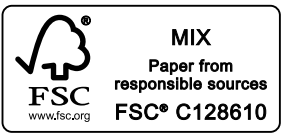

\section{Production}

Cover: [ don't eat the cat ]

Printing: Ipskamp Printing, Enschede

ISBN: 978-94-028-1451-4

(C) Copyright J. Peerlings, Maastricht 2019

All rights are reserved. No part of this thesis publication may be reproduced or transmitted in any form or by any means, without prior written permission of the holder of the copyrights 


\title{
Towards diagnostic and therapeutic integration of advanced MR imaging in thoracic and neuro-oncology
}

\author{
Proefschrift
}

ter verkrijging van de graad van doctor aan de Universiteit Maastricht, op gezag van de Rector Magnificus Prof. dr. Rianne M. Letschert, volgens het besluit van het College van Decanen,

in het openbaar te verdedigen

op donderdag 18 april 2019 om 16:00 uur

door

Jurgen Peerlings 


\section{Promotoren}

Prof. dr. P. Lambin

Prof. dr. F.M. Mottaghy

\section{Copromotor}

Dr. ir. A. L. Hoffmann

\section{Beoordelingscommissie}

Prof. dr. ir. W. H. Backes (Voorzitter)

Prof. dr. D. de Ruysscher

Prof. dr. A-M. C. Dingemans

Prof. dr. D. Thorwarth (Eberhard Karls Universiteit, Tübingen, Duitsland)

Dr. W. Vogel (Nederlands Kanker Instituut, Amsterdam, Nederland) 


\section{CONTENT}

\section{Introduction}

Chapter 1 Introduction and Outline of the thesis

PART I: Anatomical MR imaging and qualitative image analysis

Chapter 2 Thoracic imaging: Meta-analysis on determining the regional lymph node status in NSCLC using MRI

Chapter 3 Performing clinical ${ }^{18}$ F-FDG-PET/MR imaging of the mediastinum using a dedicated, patient friendly protocol

Chapter 4 Integrating 7 Tesla anatomical MRI in neuro-oncology and image-guided radiotherapy

Chapter 5 Characterizing geometrical accuracy in clinically optimised 7T and 3T magnetic resonance images for high-precision radiation treatment of brain tumours

PART II: Functional (PET-)MR imaging and quantitative image analysis

Chapter 6 Hypoxia and hypoxia response-associated molecular markers in oesophageal cancer: a systematic review

Chapter $7{ }^{18} \mathrm{~F}-\mathrm{HX} 4-\mathrm{PET} / \mathrm{MRI}$ in oesophageal cancer: assessing 153 tumour hypoxia and early treatment response

Chapter 8 Stability of radiomics features in apparent diffusion 163 coefficient maps from a multi-centre test-retest trial

\section{Discussion}




\section{Addendum}

$\begin{array}{ll}\text { Summary } & 225\end{array}$

Samenvatting (Dutch summary) 233

$\begin{array}{ll}\text { Valorisation } & 241\end{array}$

Dankwoord (Acknowledgements) 249

Curriculum Vitae 257 


\section{List of Abbreviations}

\begin{tabular}{|c|c|}
\hline $\mathrm{AC}$ & Adenocarcinoma \\
\hline $\mathrm{ADC}$ & Apparent diffusion coefficient \\
\hline ARCON & Accelerated radiotherapy with carbogen breathing and nicotinamide \\
\hline AUC & Area under the curve \\
\hline B0 & Static magnetic field strength \\
\hline $\mathrm{BH}$ & Breath-hold \\
\hline BTV & Biological target volume \\
\hline CA IX & Carbonic Anhydrase \\
\hline CCC & Concordance correlation coefficient \\
\hline CCRT & Concurrent chemoradiotherapy \\
\hline CI & Confidence interval \\
\hline $\mathrm{CoV}$ & Coefficient of variance \\
\hline CR & Contrast ratio \\
\hline CT & Computed tomography \\
\hline CTV & Clinical tumour volume \\
\hline Cu-ATSM & Copper-62 labelled diacetyl-bis (N4-methylthiosemicarbazone) \\
\hline DFS & Disease-free survival \\
\hline DSV & Diameter of a spherical volume \\
\hline DW & Diffusion-weighted \\
\hline$D_{x y z}$ & 3D displacement vector \\
\hline EC & Oesophageal cancer \\
\hline ECG & Electrocardiogram \\
\hline ESCC & Oesophageal squamous cell carcinomas \\
\hline${ }^{18} \mathrm{~F}-\mathrm{FAZA}$ & ${ }^{18}$ F-Fluoroazomycin Arabinoside \\
\hline${ }^{18} \mathrm{~F}-\mathrm{FDG}$ & ${ }^{18}$ F-Fluorodeoxyglucose \\
\hline${ }^{18} \mathrm{~F}-\mathrm{FETA}$ & ${ }^{18}$ F-Fluoroetanidazole \\
\hline${ }^{18} \mathrm{~F}-\mathrm{FETNIM}$ & ${ }^{18}$ F-Fluoroerythronitroimidazole \\
\hline${ }^{18} \mathrm{~F}-\mathrm{FMISO}$ & ${ }^{18}$ F-Fluoromisonidazole \\
\hline${ }^{18} \mathrm{~F}-\mathrm{HX} 4$ & ${ }^{18}$ F-Flortanidazole \\
\hline FB & Free-breathing \\
\hline FFE & Fast field gradient echo \\
\hline FID & Free induced decay \\
\hline FLAIR & Fluid attenuation inversion recovery \\
\hline FOV & Field-of-view \\
\hline GBM & Glioblastoma \\
\hline GD & Geometrical distortion \\
\hline GLCM & Grey-level co-occurrence \\
\hline GLDZM & Grey-level distance-zone \\
\hline GLM & Grey-level matrix \\
\hline GLRLM & Grey-level run-length \\
\hline GLSZM & Grey-level size-zone \\
\hline GLUT-1 & Glucose-transporter-1 \\
\hline GRE & Multi-echo gradient echo \\
\hline GRE & Multi-echo gradient echo \\
\hline GTV & Gross tumour volume \\
\hline HAP & Hypoxia-activated prodrug \\
\hline HASTE & Half Fourier Acquisition Single Shot Turbo Spin Echo \\
\hline HIF & Hypoxia-inducible factor \\
\hline HRE & Hypoxia response element \\
\hline hsROC & Hierarchical summary Receiver Operating Characteristic \\
\hline
\end{tabular}


$\mathrm{IH}$

INV

IQ

LC

LN

LocInt

LPR

LR

MAD

MESH

MP2RAGE

MRI

Mxy

$\mathrm{Mz}$

NGLDM

NGTDM

NSCLC

OE-MRI

OP-OSEM3D

OS

PDT

PET

pO2

POI

PTV

rBW

rDOR

RF

ROS

RTP

SAR

SD

SI

SNR

SPACE

SPAIR

STIR-TSE

SUV

$\mathrm{T}$

TBR

TE

TR

3D-TFE

TSE

UHF

VEGF

VIBE

VISTA

VOI
Intensity histogram

Inversion times

Image quality

Local control

Lymph node

Local intensity

Lesion-phantom ratio

Left-right

Mean absolute deviation

Medical Subject Headings

Magnetization-prepared rapid gradient-echo

Magnetic resonance imaging

Longitudinal magnetization

Transverse magnetization

Neighbouring grey-level dependence

Neighbourhood grey-tone difference matrix

Non-small cell lung cancer

Oxygen-enhanced magnetic resonance imaging

3D-iterative ordinary Poisson ordered-subsets expectation maximization

Overall survival

Photodynamic therapy

Positron-emission tomography

Intravascular oxygen partial pressure

Point-of-interest

Planned target volume

Read-out bandwidth

Relative diagnostic odds ratio

Radiofrequency

Reactive oxygen species

Radiation treatment planning

Specific absorption rate

Standard deviation

Signal intensity

Signal-to-noise ratio

Sampling perfection with application optimized contrasts using different flip

angle evolution

Spectral adiabatic inversion recovery

Short inversion-time inversion-recovery turbo spin-echo

Standardized-uptake values

Tesla

Tumour-to-blood ratio

Echo time

Repetition time

Turbo field echo

Turbo Spin-Echo

Ultra-high field

Vascular endothelial growth factor

Volumetric Interpolated 3D Gradient Echo

Volume isotropic Turbo spin echo

Volumes-of-interest 


\section{Chapter 1}

General introduction

\&

Outline of the thesis 


\section{CANCER AS A DISEASE}

\section{CANCER BY NUMBERS}

Globally, cancer is considered as the second most common cause of disease-related deaths [1]. Over 14.1 million people were affected by cancer in 2012, leading to 8.2 million deaths worldwide [2]. Lung cancer remains the most prevalent type of cancer in both incidence (1.8 million) and mortality (1.6 million) [2]. Alarmingly, the 5 -year overall survival rate drops dramatically from $90-95 \%$ for patients with earlystage cancer to $20 \%$ for patients with late-stage non-small cell lung cancer (NSCLC) [3]. This large difference in survival rates stresses the need for early detection and treatment, as early-stage cancer is more likely to respond to effective curative treatment than late-stage cancer [1].

Although occurring less frequently, oesophageal cancer (EC) and brain cancer (i.e., 16.940 and 21.014 new cancer patients in 2017, respectively) are of interest for this thesis [4,5]. EC can be considered as one of the most lethal forms of cancer in which only $7 \%$ of all patients will not die from the disease and merely $20 \%$ will survive 5 years after initial diagnosis [6,7]. For brain cancer, on the other hand, the 5 -year survival rate is $35 \%$ [5]. However, additional disease-related deaths can arise from secondary brain metastases originating from e.g., primary lung cancer. The global impact of thoracic and brain cancer demonstrates the need for improvements in early diagnostics and cancer treatment, and will be ventured in this thesis using non-invasive imaging techniques and image analyses.

\section{HALLMARKS OF CANCER}

Cancer originates after multiple genetic mutations (i.e., oncogenic events) resulting in altered cell function and abnormal cell reproduction. Eventually, unregulated growth and uncontrollable spread of cells in the human body results in the formation of tumour masses [8]. However, cancer as a disease is more complex than masses of proliferating cancer cells. Tumours are considered to be heterogenic, being composed of multiple types of oncogenic cells, and to have a tumour-specific microenvironment that both play an important role in cancer development [9]. During cancer development, cancer cells attain the ability to maintain continuous growth, infiltrate other tissue, and survive anti-cancer defence mechanisms of normal cells. The first two characteristics are accomplished by self-sufficiency in growthstimulating signals, insensitivity to growth suppressions, sustained angiogenesis, and active invasion and metastasis [10]. To assure survival, tumour cells counter telomere-erosion, undergo reprogramming of energy metabolism, and acquire resistance to apoptosis and elimination by the immune system. The underlying mechanisms of acquiring these hallmarks are enabled by genomic instability and manifestation of tumour-promoting inflammation [9]. Despite the diversity and complexity of cancer cells, it remains challenging to differentiate cancer cells from 
normal cells and detect early development of the disease in an infant stage. The first step towards curative treatment and patient survival is to improve the ability to accurately detect cancer as soon as possible.

\section{MEDICAL IMAGING FOR CANCER DIAGNOSIS AND ANTI-CANCER TREATMENT}

Medical imaging has established a central role in modern cancer management, ranging from initial staging and therapy planning towards response monitoring and follow-up. Cancer screening and initial tumour staging are one of the most important prognostic factors as diagnostic assessment heavily influences treatment decision-making for each patient. Selection of the most suitable treatment option is different for each patient and depends on the type of cancer, extent of the disease, operability, and patient-related factors. Currently, anti-cancer treatment regimens balance the use of three main treatment options: surgery, radiation therapy, and/or chemotherapy including immunomodulating therapy. In radiation therapy, both anatomical and functional imaging is used as a tool to identify tumour characteristics and differentiate radiation target volumes and healthy tissues to be spared. During and after treatment, imaging is used to evaluate therapeutic changes (e.g., effect and potential complications) and adapt treatment if necessary.

Anti-cancer treatment of lung, oesophageal, and brain cancer is challenging. For NSCLC and EC, mis-diagnoses of tumour stage and tumour mobility are detrimental for patient outcome and require imaging with high soft-tissue contrast and spatial resolution for early staging and treatment monitoring. For brain cancer, tumours are difficult to access (e.g., resectability, impermeable blood-brain barrier) and surrounded by delicate healthy tissue that, when damaged, could result in neuro-cognitive decay. It is therefore essential that anatomical images visualise brain cancer in great detail, differentiate healthy and tumour tissue, and allow for accurate target volume definition.

\section{CANCER IMAGING MODALITIES}

\section{CT}

Computed tomography (CT) imaging is able to visualise cellular malformities due to differences in X-ray absorption properties of tissues, related to electron density (i.e., Hounsfield units). Tissues attenuate ionising radiation differently, creating a radiographic image with anatomical information. CT imaging can be considered as the workhorse for volumetric imaging in oncology used for diagnostic staging, tumour segmentation and radiation therapy dose calculations. A disadvantage of CT imaging is the limited soft-tissue contrast and the need to use ionising radiation (i.e. expose the patient to imaging dose). 


\section{MRI}

In 1977, Damadian et al. pioneered in magnetic resonance (MR) imaging by acquiring the first MR image of a living human's thorax, based on the discoveries of Drs. Lauterbur and Mansfield. Generally, MR images are created by exploiting the nuclear spin properties of hydrogen-atoms (Fig. 1.1) [11]. When a body is placed in a static magnetic field $\left(\mathrm{B}_{0}\right)$, tissue becomes magnetized and nuclear spins align with Bo. To create an MR signal, the spin alignment is distorted by applying an excitation radio-frequency pulse at the Larmor resonance frequency $\left(\omega_{0}=-\gamma B_{0}\right)$. The nuclear spins will absorb this energy and transition to a higher energy state. Next, longitudinal spin-lattice relaxation and transversal spin-spin relaxation processes occur naturally for spins to return to the equilibrium state. By doing so, excited molecules transmit energy in the radio-frequency range, creating detectable MRsignals. In addition, switched magnetic gradient fields are applied in each orthogonal direction to spatially encode the MR signal and create anatomical $2 \mathrm{D}$ or $3 \mathrm{D}$ images. The time needed for relaxation is tissue-dependent and is labelled as $\mathrm{T}_{1}$ and $\mathrm{T}_{2}$-relaxation time, respectively. By selecting a specific echo time (TE) and a repetition time (TR) of MR pulse sequences, an image becomes predominantly $\mathrm{T}_{1}$ or $\mathrm{T}_{2}$-weighted and contrast within the MR image is created [12-15].

a.

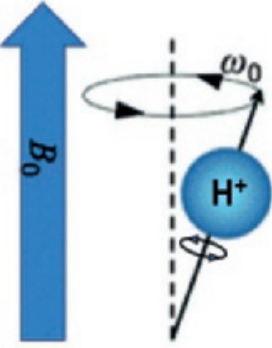

b.

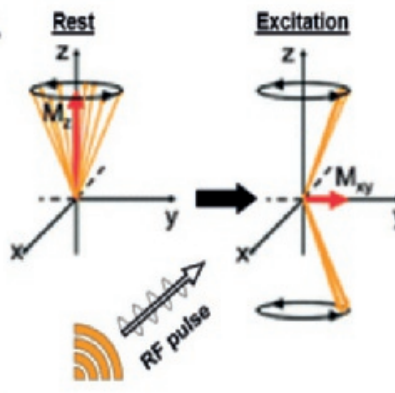

c.

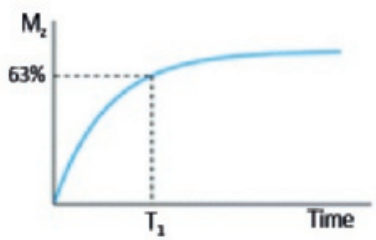

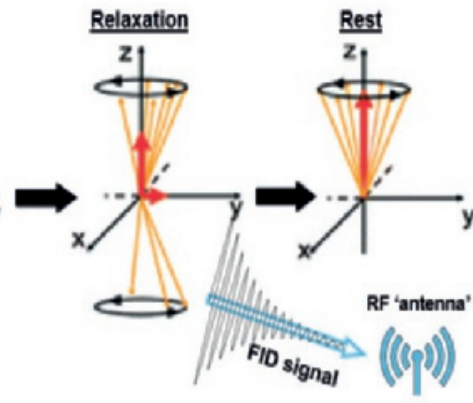

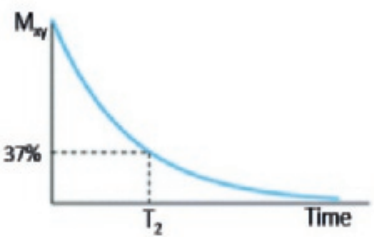

Fig. 1.1: Principles of MR imaging - (a.) In a static magnetic field $B_{0}$, the hydrogen nuclear spins align with (parallel) or against (antiparallel) the external magnetic field. (b.) Excitation by a pulse of resonant radio-frequency (RF) results in decrease in longitudinal magnetization $(\mathrm{Mz})$ and generation of transverse magnetization (Mxy). Relaxation occurs naturally and, the nuclear spins return to their initial state by transmitting free-induced decay (FID) signal that can be detected by an RF coil or 'antenna'. (c.) T1 is the time required for longitudinal magnetization to recover to $63 \%$ of its equilibrium, and T2 is the time required for transverse magnetization to drop to $37 \%$ of its initial magnitude.

[Adapted from: Nohyun Lee and Taeghwan Hyeon -Chem. Soc. Rev., 2012,41, 2575-2589 [11]] 
Currently, anatomical and functional MR images are being used to evaluate various tumour types throughout the entire human body with high soft-tissue contrast and spatial resolution. This thesis focuses on the clinical implementation of advanced MRI in thoracic and neuro-oncology, and studies its role in radiology and radiation oncology for improving patient care.

\section{MR IMAGING IN THORACIC ONCOLOGY}

The thorax has long been regarded as a 'black hole' in MR imaging. Hence, MRI is currently not being used in standard clinical practice to image lung, oesophageal, or mediastinal malignancies in thoracic oncology [16]. Acquiring high-quality MR images of thoracic tissues is extremely challenging due to the intrinsic properties of lung and mediastinal tissues, and respiratory and cardiac motion. Related to the alveolar architecture, tissue density of healthy lungs is about a tenfold lower (i.e., $0.1 \mathrm{~g} / \mathrm{cm}^{3}$ ) than in other tissues and since the ${ }^{1} \mathrm{H}$-MRI signal is directly proportional to proton-density, the signal-to-noise ratio (SNR) is relatively low [17]. Furthermore, MR-related artefacts are prominent in the thoracic region. At air-tissue interfaces, large differences in magnetic susceptibility between paramagnetic air and diamagnetic tissue often result in image-distorting artefacts. Additional distortion of thoracic MR images might be caused by motion artefacts related to respiration and cardiac pulsations. The predisposition to low SNR and imaging artefacts hinders accurate diagnostic interpretation and has limited the integration of thoracic MRI in clinical practice. However, innovations in MR imaging hardware and software (e.g. parallel imaging, faster sequences, triggering-techniques, motion correction) as well as improvements in receiver-coil sensitivity have enabled modern MRI to potentially overcome these obstacles and attain clinical advances in thoracic oncology.

Besides technological challenges, however, practical challenges remain to be conquered to integrate thoracic MRI in clinical practice $[18,19]$. The main motivation of this thesis is to overcome these obstacles with clinically available MR tools, acquire high-quality thoracic MR images suitable for diagnostic use in radiology and therapeutic use in radiation oncology, and gain insights to improve its clinical implementation.

\section{MR IMAGING IN NEURO-ONCOLOGY}

Although MRI is still in its infancy in thoracic oncology, MRI is considered as the standard clinical imaging modality in neurology and neuro-oncology. Currently for radiation therapy planning (RTP) of brain tumours, 1.5 Tesla (T) or 3T MR images are used to delineate the tumour volume after co-registering them with CT images. The latter is needed for dose calculation that is based on electron density information, which is lacking in the former. However, current clinical MRI is limited in visualising detailed neurologic malformations such as intracerebral tumour spread and is unable to adequately visualise intracerebral tumour spread [20]. 
Hence, there is a need for MR images with even higher SNR and spatial resolution up to the sub-micrometer scale [21-23]. By increasing the magnetic field strength an increase in SNR, T2*-weighted contrast, and spatial resolution can be achieved, enabling the visualisation of small lesions, basal ganglia and tumour angiogenesis [24-26].

In the past decade, systems for ultra-high field (UHF-)MR imaging at magnetic field strengths above $3 \mathrm{~T}$ have become available. It is expected that UHFMR images could improve the accuracy and precision of target volume definition by depicting the infiltrative nature of glioblastoma along the white matter tracts (Fig. 1.2) and by visualising microvasculature (diameter $\sim 100 \mu \mathrm{m}$ ) outside the contrast-enhanced tumour [20,27-29]. However, the clinical implementation of UHFMRI in neuro-oncology is not straightforward. Imaging protocols and patient handling require optimisation as image artefacts (i.e., geometrical distortions, increased tissue susceptibility and chemical shift effects) and patient-discomfort (e.g., nausea, vertigo, peripheral nerve stimulation, and thermal responses related to $\mathrm{RF}$ excitation pulses) might impede its use in clinical practice. In addition, concerns regarding the geometrical integrity have compromised the integration of UHF-MRI into radiation therapy planning. For high-precision radiation dose delivery, the spatial accuracy of anatomical brain images needs to be within $2 \mathrm{~mm}$ and $1 \mathrm{~mm}$ for conventional and stereotactic radiotherapy, respectively [30,31]. Hence, the accuracy of hardware-related geometrical distortion in clinically optimised 7T MR scan protocols needs to be assessed before UHF-MR images can be used for highprecision radiation therapy planning.
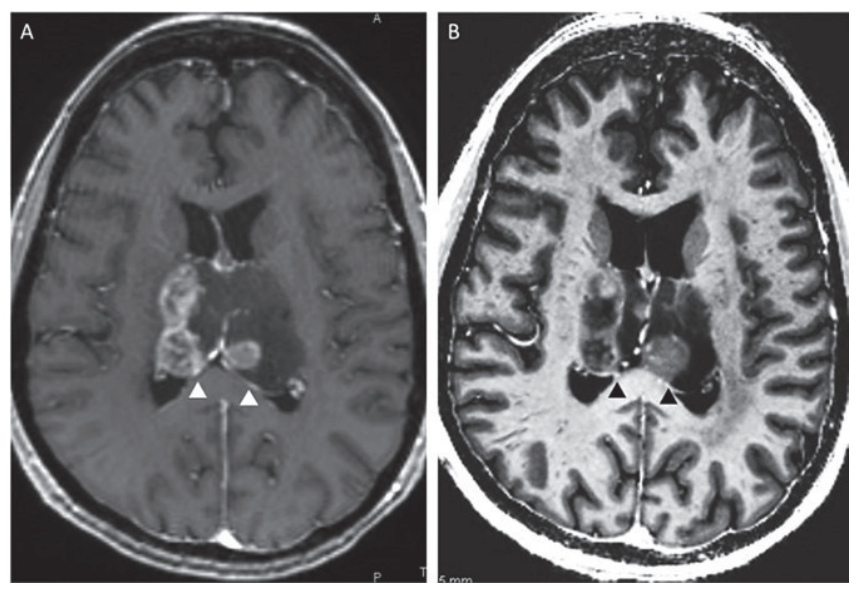

Fig. 1.2: (a.) $3 \mathrm{~T}$ axial post-gadolinium T1-weighted image shows an infiltrative glioblastoma involving both thalami (white triangles). (b.) 7T axial post-gadolinium MP2RAGE image shows improved detailed margins of the tumour involving of the thalami (black triangles) and infiltration of glioblastoma across the hemispheres.

[Rights acquired from EC Obusez -NeuroImage 168 (2018) 459-476 [32]] 


\section{PET}

Positron emission tomography imaging is able to visualise the glucose metabolism of cells using the radioactively labelled glucose analogue, fluorodeoxyglucose $\left({ }^{18} \mathrm{~F}\right.$ FDG). In general, active cells with a high turnover rate present an overall high metabolic activity. The same holds true for rapidly growing cancer cells but these cancer cells also upregulate glucose membrane transporters and downregulate hexokinase. This means that, when injected, more ${ }^{18} \mathrm{~F}-\mathrm{FDG}$-tracer will accumulate in cancer cells and will be retained longer in solid tumour than in normal tissues. PET imaging can exploit this biological phenomenon to visualise tumours by detecting two annihilation photons of $511 \mathrm{keV}$ associated with radioactive decay of ${ }^{18} \mathrm{~F}-\mathrm{FDG}$ and positron-electron annihilation events.

\section{Multi-modality imaging}

Although PET-scanners can sensitively detect cells with tracer build-up, detailed anatomical structures are not easily detectable in PET-images of low spatial resolution (i.e., 3-4 mm). Multi-modal imaging systems have been developed that combine the single PET-modality with either CT- or MRI-modalities. The resulting hybrid imaging systems (i.e., PET/CT and PET/MR, respectively) enable clinicians to pin-point the origin of the low-resolution PET-signal on anatomical CT or MRimages and correlate ${ }^{18} \mathrm{~F}-\mathrm{FDG}$-avidity to an underlying pathology. By doing so, multi-modal imaging plays an important role in modern cancer management through advances in diagnostic staging, treatment-decision-making, radiation therapy planning, and treatment response monitoring.

\section{HYBRID PET/CT IMAGING}

Over the years, ${ }^{18} \mathrm{~F}-\mathrm{FDG}-\mathrm{PET} / \mathrm{CT}$ imaging has become well integrated in clinical practice and has proven its diagnostic and therapeutic value in thoracic oncology. However, the limited spatial resolution of PET/CT imaging may induce false anatomic allocation or cause the reader to miss small lymph nodes that harbour microscopic metastases, leading to false-negative results [33-36]. Furthermore, uptake of ${ }^{18} \mathrm{~F}-\mathrm{FDG}$-tracer is not tumour-specific and also accumulates in metabolically active cells and in tissues with hyperplasia or inflammation. This makes that PET imaging cannot differentiate between tumour cells and other cells with accumulated ${ }^{18} \mathrm{~F}$-FDG-tracer, potentially resulting in false-positive findings $[37,38]$. In oncology, misdiagnoses could translate to over- and under-treatment, resulting in excess side-effects and possible regional recurrence, respectively. Nevertheless, ${ }^{18}$ F-FDG-PET/CT imaging is considered as the clinical standard for diagnostic staging and RTP in thoracic oncology, and is therefore considered as the clinical benchmark $[3,39,40]$. The work in this thesis evaluates the value of advanced MRI for thoracic oncology in the context of PET/CT imaging as the clinical benchmark. 


\section{HYBRID PET/MR IMAGING}

Approximately 5 year ago, the first fully-integrated whole-body PET/MR system has been developed to allow for simultaneous PET and MR image acquisition (Fig. 1.2) [41]. This novel hybrid system opened up numerous possibilities for anatomical and functional imaging, by using various PET-tracers and MR sequences. Although highly anticipated, the role of PET/MR imaging in oncology is still under investigation and key clinical applications remain to be defined [42-44]. In patients with mediastinal malignancies, initial studies have reported non-inferiority to ${ }^{18} \mathrm{~F}$ FDG-PET/CT imaging for diagnostic staging and treatment-decision making [45-49]. These studies indicated the necessity to optimise imaging protocols to acquire highquality images within the shortest time possible. By optimising MR sequences, a trade-off between SNR, spatial resolution, and acquisition time can be established. However, high-quality images should not be distorted by artefacts. Methods to reduce motion-artefacts in PET/MR images involve the use of ECG-triggering and image-acquisition during breath-hold.

So far, effects of these methods on the trade-off between image quality, diagnostic accuracy, and patient compliance to scanning instructions has not been assessed in mediastinal imaging and will therefore be studied in this thesis. In addition, the clinical gain of region-specific PET/MR imaging will be evaluated in patients with EC or NSCLC. In EC, detailed visualisation of oesophageal wall thickening on MRI could benefit accurate tumour staging as this is determined on the extent of tumour invasion into the oesophageal wall layers. This work is expected to bring us closer to define key applications for clinical PET/MR imaging in oncology and to validate dedicated, high-quality and patient-friendly PET/MR protocols as an alternative to PET/CT imaging in the thoracic region. 


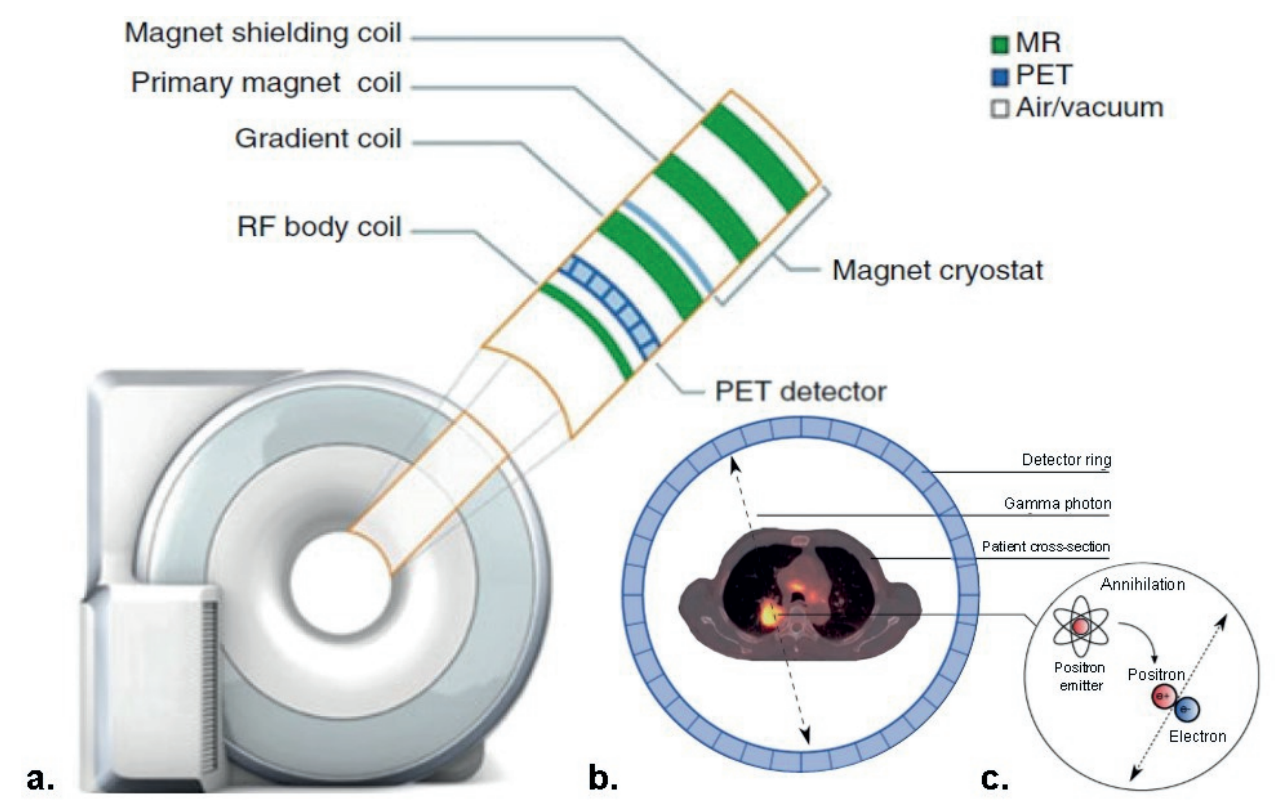

Fig. 1.3: (a.) Schematic drawing of an integrated PET/MR system (Biograph mMR, Siemens Healthineers, DE) showing the integration of PET detectors (Blue) within an MR system infrastructure (Green). (b.) MR-compatible PET detector ring surrounding a patient with lung cancer. (c.) Annihilation event between positron and electron, emitting detectable gamma photons that can be reconstructed within the image.

\section{FUNCTIONAL IMAGING IN RADIATION THERAPY}

The goal of curative radiation therapy is to deliver a therapeutic (homogeneous) radiation dose of ionising radiation to a target volume while sparing surrounding normal tissue and sensitive organs-at-risk as much as possible. However, tumours contain heterogenic sub-volumes that may present different radiosensitivity and response to radiation therapy. Functional imaging using dedicated PET and MR imaging biomarkers has the potential to determine tumour phenotype and reveal the radiobiological nature of patient-specific malignancies. Information regarding tumour aggressiveness and radioresistant sub-volumes can be defined as 'biological target volumes' (BTV) for radiotherapy and could help select the most beneficial treatment-regime for an individual patient. By using state-of-the art radiation dose delivery techniques the BTV can be incorporated into personalised treatment planning in which heterogenous dose distributions are sculpted to deliver an increased radiation "boost" dose to the BTV sub-volumes [50].

In this thesis, the quantitative assessment of imaging biomarkers for both tumour hypoxia and hyper-cellularity are addressed using a dedicated PET-tracer and diffusion-weighted MR imaging (DWI), respectively. Alternative PET-tracers 
and MRI techniques (e.g., perfusion MRI, MR angiography, MR spectroscopy) for functional imaging are beyond the scope of this thesis.

\section{HYPOXIA IMAGING}

Hypoxia is a state in which tissues are insufficiently oxygenated. It occurs when oxygen demand (i.e., metabolic activity) exceeds oxygen supply (i.e., perfusion or diffusion). In cancerous tissue, the rapid and uncontrolled cell division causes tumours to outgrow their blood supply and become out of the reach of diffusing oxygen-molecules (i.e., 100-180 $\mu \mathrm{m}$ ). Oxygen starvation in this region cannot be resolved by physiological homeostasis, resulting in chronic hypoxia [51,52].

For effective radiation treatment, oxygen is needed to induce irreversible DNA-damage. Consequently, badly oxygenated cells generally express a 2-3 times higher resistance to (chemo-)radiotherapy than cells with normal levels of oxygen [53,54]. Assessment of hypoxia is therefore used as a prognostic marker for patient outcome and could be targeted as BTV to counteract treatment-resistance $[55,56]$. However, accurate detection and detailed visualisation of tumour hypoxia is needed to specifically target hypoxic cells [53]. Although several techniques are known, no single modality is approved for assessing hypoxic status of tumours in clinical practice [57].

An interesting new PET-tracer for hypoxia, Fluor-labelled flortanidazole $\left({ }^{18} \mathrm{~F}-\right.$ HX4), was developed with better pharmacokinetic properties compared to other 2nitroimidazole-based PET-tracers. The tracer diffuses passively into cells and is specifically trapped in hypoxic cells after irreversible electron reduction. By using this tracer for ${ }^{18} \mathrm{~F}-\mathrm{HX} 4-\mathrm{PET} / \mathrm{MR}$ imaging, hypoxic regions can be detected, correlated with anatomical images with high soft-tissue resolution in the mediastinal region, and targeted for patient-specific treatment options and early treatment response assessment [58-61]. In addition, tracers beyond ${ }^{18} \mathrm{~F}-\mathrm{FDG}$ have the potential to define novel key applications of PET/MR imaging in oncology and personalized cancer care [62]. For efficient PET/MR imaging protocols, however, truly synergic information must be acquired from both PET and MR imaging to maximise the clinical value of PET/MR for anatomical and functional imaging. The work in this thesis therefore investigates the combined use of ${ }^{18} \mathrm{~F}-\mathrm{HX} 4$ PET imaging, anatomical and functional MR imaging (i.e., DWI) for efficient assessment of tumour heterogeneity in EC patients.

\section{HYPER-CELLULARITY IMAGING}

DWI is an MR-method that detects the free random motion of extracellular water molecules (i.e., diffusion). For DWI, typically two bipolar diffusion gradient pulses with a selected strength, duration and time interval (i.e., diffusion-sensitizing $b$ value) are added to conventional MR-sequences. Molecules that diffuse in the time between pulses are affected differently by the second pulse and acquire a phase shift that ultimately results in diffusion-specific MR signal loss. Static molecules are 
unaffected by diffusion gradients and contrast in DWI is created. When motion is restricted by cellular barriers or macromolecules in tissue, the MR signal is attenuated less and DWI can be used as an indirect imaging biomarker for hypercellularity. By compiling DWI with different $b$-values, apparent diffusion coefficients (ADC) maps can be generated and the DWI signal can be quantified.

Clinically, quantitative ADC maps are being used to determine tumour malignancy and assess early treatment response as molecular changes are arguably presented sooner than anatomical changes related to tumour remission [63]. Nevertheless, thoracic DWI remains challenging in oncology due to motion-related artefacts and low SNR [64-66]. Potentially, DWI would be valuable for RTP by differentiating metastatic lymph nodes and defining the BTV for elective nodal irradiation that demonstrate reduced oesophageal toxicity and regional failure in lung cancer patients $[67,68]$.

Complementary information regarding tumour heterogeneity can be gained from computational image-based analyses (i.e., radiomics) by extracting quantitative phenotypic features (i.e., shape, texture, and signal intensity) $[69,70]$. However, the lack of standardized DWI protocols does not permit the translation of ADC-based radiomics information into clinical practice across multiple centres [71,72]. Moreover, there is a realistic risk of overfitting when creating prediction models where the number of radiomics features exceeds the number of patients studied. Reducing the number of unstable features would substantially improve the reliability of radiomics analyses and the correlation with underlying pathology and tumour biology [73]. To reach the goal of clinical implementation of reliable radiomics analyses, features need to be selected that are stable across multiple centres and are unaffected by different sources of data variability (i.e., tumour type, MR system, magnetic field strength) when applying standardized protocols. The work in this thesis aims to establish a method to evaluate the stability of radiomics features extracted from ADC maps based on standardised test-retest images, facilitating future clinical implementation.

\section{OBJECTIVES}

The goal of the work described in this thesis is to investigate the clinical potential to integrate advanced MRI techniques (i.e., UHF-MRI and PET/MR) and innovative MR image analysis methods (i.e., MR radiomics) into oncological radiology and radiation therapy in order to improve the diagnostic quality and therapeutic use of anatomical and functional MR imaging, in particular for thoracic and neurooncology.

Anatomical MRI has the potential to become as important as ${ }^{18} \mathrm{~F}-\mathrm{FDG}-\mathrm{PET} / \mathrm{CT}$ imaging in diagnostic staging, treatment decision-making, RTP, and treatment 
response monitoring in clinical oncology. However, acquiring high-quality MR images of thoracic tissues (i.e., lung, oesophagus, mediastinal structures) is challenging due to the low SNR and proneness to motion artefacts. Despite technological advances in coil sensitivity, acquisition speed, and motion correction strategies, the role of MR imaging in thoracic oncology relative to ${ }^{18} \mathrm{~F}-\mathrm{FDG}-\mathrm{PET} / \mathrm{CT}$ imaging has not been established yet.

The first objective of this work was therefore to assess the diagnostic quality of thoracic MRI and PET/MRI and compare it against ${ }^{18} \mathrm{~F}-\mathrm{FDG}-\mathrm{PET} / \mathrm{CT}$ imaging. To this end, the following hypotheses were tested:

H1. Detection and differentiation of (non-)metastatic hilar and mediastinal lymph nodes in NSCLC patients can be accurately realized by advanced thoracic MR imaging methods.

H2. A mediastinal-specific ${ }^{18 F-F D G-P E T / M R ~ s c a n ~ p r o t o c o l, ~ g e n e r a t i n g ~ h i g h-~}$ quality MR-images within the shortest possible acquisition time, provides a diagnostic performance similar to ${ }^{18} \mathrm{~F}-\mathrm{FDG}-\mathrm{PET} / \mathrm{CT}$ imaging in patients with NSCLC and EC.

Through advances in anatomical MRI the question arises whether MR images could be incorporated into radiation treatment planning. For this purpose, inherent geometric distortion of MR imaging could be a limiting factor. Especially for UHFMRI visualisation of brain tumours and microvasculature, the spatial accuracy could be a major concern and needs to be evaluated before UHF-MRI can be clinically applied. Furthermore, the UHF-MRI scan protocol needs to be evaluated and optimised in terms of image quality and patient tolerability (e.g., dizziness and thermal responses to high-energy MR pulses).

The second objective of this work was therefore to assess the clinical potential of 7T MRI for high-precision radiation therapy of brain tumours. More specifically, the following hypothesis were investigated:

H3. 7T MRI protocols can be optimised towards therapeutic image quality and tolerability in patients with brain tumours.

H4. System- and object-related geometrical distortion in 7T MRI meets the clinical acceptance criteria to be incorporated into radiation therapy planning in neuro-oncology.

Functional imaging using a dedicated hypoxia PET-tracer and DWI, has the potential to provide additional quantitative information regarding tumour phenotypes that affect radio-sensitivity and radiation treatment outcome. This work investigates the clinical potential of PET/MR imaging for patients with EC by using the ${ }^{18} \mathrm{~F}-\mathrm{HX} 4$ tracer to assess tumour heterogeneity. Furthermore, this work aims to assess the treatment response by correlating changes in anatomy, hypoxia, and 
ADC-values mid-therapy. Finally, the potential of ADC-based radiomics analysis to extract stable phenotypic features across multiple clinical centres is investigated.

The third objective of this work was therefore to assess the value of hypoxia PET/MR-imaging and quantitative radiomics analysis. To this end, the following hypotheses were tested:

H5. ${ }^{18} \mathrm{~F}-\mathrm{HX} 4-\mathrm{PET} / \mathrm{MR}$ imaging can visualise hypoxic regions and assess tumour heterogeneity in patients with EC.

H6. ADC-based radiomics analysis can extract stable phenotypic features from standardized DWI in a multi-institutional setting using different MRI systems.

\section{OUTLINE OF THE THESIS}

This thesis is divided into two parts that investigate the clinical applicability of advanced MRI techniques and MR image analysis methods for anatomical and functional imaging in chapters 2-5 and chapters 6-8, respectively (Fig. 1.4).

\section{Part I. Anatomical MR imaging and qualitative image analysis}

In this part anatomical MR imaging and qualitative image analysis for diagnostic evaluation and reliable RTP is addressed. In more detail, hypothesis 1 is tested in Chapter 2. This chapter comprises a meta-analysis summarizing existing evidence on the diagnostic quality of thoracic MRI in determining lymph node status in patients with NSCLC. Following, in Chapter 3, ${ }^{18} \mathrm{~F}-\mathrm{FDG}-\mathrm{PET} / \mathrm{MR}$ imaging protocols for different MR pulse sequences are developed to test hypothesis 2 and investigate the effect of ECG-triggering and breath-hold acquisition on diagnostic MR image quality and patient compliance in volunteers and patients with NSCLC and EC. Next, hypothesis 3 and 4 are tested in chapter 4 and chapter 5, respectively. Chapter 4 presents a preparatory study to investigate the technical feasibility of incorporating anatomical UHF-MR images of patients with glioblastoma into neurosurgical navigation and radiation therapy planning by qualitatively evaluating the clinical image quality and the geometrical reliability. Chapter 5 investigates the geometrical accuracy of clinically optimised 3T and 7T MR images to be incorporated into high-precision radiation therapy planning of patients with brain tumours.

\section{Part II. Functional (PET-)MR imaging and quantitative image analysis}

This part focuses on functional (PET-)MR imaging and quantitative image interpretation for the definition of a BTV in radiation therapy. Chapter 6 systematically reviews the clinically available hypoxia-associated molecular response biomarkers in EC and aims to assess the prognostic role of hypoxia imaging 
in radiation treatment outcome and efficacy. In Chapter 7, hypothesis 5 is tested. Here, the feasibility of visualising tumour hypoxia in patients with EC is assessed by using ${ }^{18} \mathrm{~F}-\mathrm{HX} 4-\mathrm{PET} / \mathrm{MR}$ imaging and our previously optimised PET/MR protocol for mediastinal imaging. Furthermore, the effectiveness of chemo-radiotherapy was assessed mid-treatment by correlating changes in hypoxic fraction, tumour volume, and ADC values. Finally, hypothesis 6 and the feasibility of DWI for quantitative radiomics analysis is addressed in Chapter 8 by analysing the test-retest repeatability of ADC-extracted features in a multi-centric study.

A general discussion is presented in Chapter 9. This discussion comprises a recapitulation of the advances in knowledge this work has produced, as well as a critical reflection of aspects that need further investigation before the proposed MRI techniques and MR image analysis methods can be integrated into the clinical practice of oncologic radiology and radiation therapy.

The addendum provides a summary (in English and Dutch), valorisation section, acknowledgments, CV, and a list of publications.

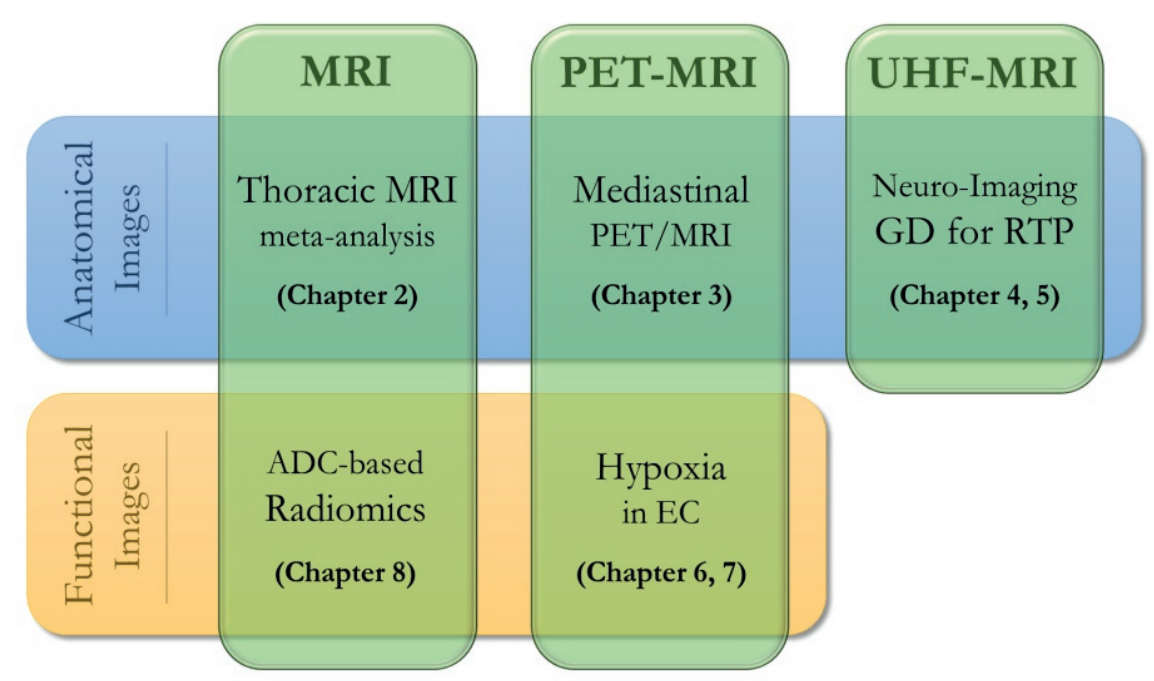

Fig 1.4: Schematic outline of the thesis showing the inter-relationship the between the various research topics. 


\section{REFERENCES}

1. Reboux G. 2018 Cancer. <http://www.who.int/news-room/fact-sheets/detail/cancer>.

2. Ferlay J, Soerjomataram I, Ervik M, Dikshit R, Eser S, Mathers C, Rebelo M, Parkin DM, Forman D, Bray F. 2013 24/04/2014. GLOBOCAN 2012 v1.0, Cancer Incidence and Mortality Worldwide: IARC CancerBase No. 11 [Internet]. International Agency for Research on Cancer <http://globocan.iarc.fr/>. Accessed 2014 24/04/2014.

3. Landelijke Werkgroep Longtumoren. 2014 01/03/2014. Niet-kleincellig longcarcinoom: Landelijke richtlijn. In Oncoline richtlijnen oncologische zorg. 2.0. Integraal Kankercentrum Nederland $<$ http://oncoline.nl/niet-kleincellig-longcarcinoom>. Accessed 2014 01/03/2014.

4. Siegel RL, Miller KD, Jemal A. Cancer statistics, 2016. CA Cancer J Clin 2016;66(1):7-30.

5. American Cancer Society. Cancer facts \& figures 2018. Atlanta: American Cancer Society 2018.

6. Ping W, Sun W, Zu Y, Chen W, Fu X. Clinicopathological and prognostic significance of hypoxia-inducible factor-1alpha in esophageal squamous cell carcinoma: a meta-analysis. Tumour Biol 2014;35(5):4401-9.

7. van Hagen P, Hulshof M, van Lanschot J, Steyerberg EW, Henegouwen MIV, Wijnhoven BPL, Richel DJ, Nieuwenhuijzen GaP, group C. Preoperative chemoradiotherapy for esophageal or junctional cancer. N Engl J Med 2012;366:2074-2084.

8. Vogelstein B, Papadopoulos N, Velculescu VE, Zhou S, Diaz LA, Jr., Kinzler KW. Cancer genome landscapes. Science 2013;339(6127):1546-58.

9. Hanahan D, Weinberg RA. Hallmarks of cancer: the next generation. Cell 2011;144(5):646-74.

10. Hanahan D, Weinberg RA. The hallmarks of cancer. Cell 2000;100(1):57-70.

11. Lee N, Hyeon T. Designed synthesis of uniformly sized iron oxide nanoparticles for efficient magnetic resonance imaging contrast agents. Chem Soc Rev 2012;41(7):2575-89.

12. Haacke EM, Brown RW, Thompson MR, Venkatesan R. Magnetic resonance imaging: physical principles and sequence design. Wiley-Liss New York:; 1999.

13. McRobbie DW, Moore EA, Graves MJ, Prins MH. MRI From Picture to Proton. Cambridge, UK: Cambridge University Press; 2006.

14. Schild HH. MRI Made Easy:(--well Almost). Berlex Laboratories; 1992.

15. Bernstein MA, King KF, Zhou XJ. Handbook of MRI pulse sequences. Elsevier; 2004.

16. Hochhegger B, Marchiori E, Sedlaczek O, Irion K, Heussel CP, Ley S, Ley-Zaporozhan J, Soares Souza A, Jr., Kauczor HU. MRI in lung cancer: a pictorial essay. Br J Radiol 2011;84(1003):661-8.

17. Wild JM, Marshall H, Bock M, Schad LR, Jakob PM, Puderbach M, Molinari F, Van Beek EJ, Biederer J. MRI of the lung (1/3): methods. Insights Imaging 2012;3(4):345-53.

18. Biederer J, Mirsadraee S, Beer M, Molinari F, Hintze C, Bauman G, Both M, Van Beek EJ, Wild J, Puderbach M. MRI of the lung (3/3)-current applications and future perspectives. Insights Imaging 2012;3(4):373-86.

19. Puderbach M, Hintze C, Ley S, Eichinger M, Kauczor HU, Biederer J. MR imaging of the chest: a practical approach at 1.5T. Eur J Radiol 2007;64(3):345-55.

20. Claes A, Idema AJ, Wesseling P. Diffuse glioma growth: a guerilla war. Acta Neuropathol 2007;114(5):44358.

21. Kraff O, Fischer A, Nagel AM, Monninghoff C, Ladd ME. MRI at 7 Tesla and above: demonstrated and potential capabilities. J Magn Reson Imaging 2015;41(1):13-33.

22. Barrett TF, Sarkiss CA, Dyvorne HA, Lee J, Balchandani P, Shrivastava RK. Application of Ultrahigh Field Magnetic Resonance Imaging in the Treatment of Brain Tumors: A Meta-Analysis. World Neurosurg 2016;86:450-65.

23. Trattnig S, Springer E, Bogner W, Hangel G, Strasser B, Dymerska B, Cardoso PL, Robinson SD. Key clinical benefits of neuroimaging at 7T. Neuroimage 2016.

24. Christoforidis GA, Grecula JC, Newton HB, Kangarlu A, Abduljalil AM, Schmalbrock P, Chakeres DW. Visualization of microvascularity in glioblastoma multiforme with 8-T high-spatial-resolution MR imaging. AJNR Am J Neuroradiol 2002;23(9):1553-6.

25. Lau JC, Khan AR, Zeng TY, MacDougall KW, Parrent AG, Peters TM. Quantification of local geometric distortion in structural magnetic resonance images: Application to ultra-high fields. Neuroimage 2017.

26. Trattnig S, Bogner W, Gruber S, Szomolanyi P, Juras V, Robinson S, Zbyn S, Haneder S. Clinical applications at ultrahigh field (7 T). Where does it make the difference? NMR Biomed 2016;29(9):1316-34.

27. Moenninghoff C, Maderwald S, Theysohn JM, Kraff O, Ladd ME, El Hindy N, van de Nes J, Forsting M, Wanke I. Imaging of adult astrocytic brain tumours with 7 T MRI: preliminary results. Eur Radiol 
2010;20(3):704-13.

28. Christoforidis GA, Yang M, Abduljalil A, Chaudhury AR, Newton HB, McGregor JM, Epstein CR, Yuh WT, Watson S, Robitaille PM. "Tumoral pseudoblush" identified within gliomas at high-spatial-resolution ultrahigh-field-strength gradient-echo MR imaging corresponds to microvascularity at stereotactic biopsy. Radiology 2012;264(1):210-7.

29. Lupo JM, Li Y, Hess CP, Nelson SJ. Advances in ultra-high field MRI for the clinical management of patients with brain tumors. Curr Opin Neurol 2011;24(6):605-15.

30. Weygand J, Fuller CD, Ibbott GS, Mohamed AS, Ding Y, Yang J, Hwang KP, Wang J. Spatial Precision in Magnetic Resonance Imaging-Guided Radiation Therapy: The Role of Geometric Distortion. Int J Radiat Oncol Biol Phys 2016;95(4):1304-16.

31. Schmidt MA, Wells EJ, Davison K, Riddell AM, Welsh L, Saran F. Stereotactic radiosurgery planning of vestibular schwannomas: Is MRI at 3 Tesla geometrically accurate? Med Phys 2017;44(2):375-381.

32. Obusez EC, Lowe M, Oh SH, Wang I, Jennifer B, Ruggieri P, Hill V, Lockwood D, Emch T, Moon D and others. 7T MR of intracranial pathology: Preliminary observations and comparisons to 3T and 1.5T. Neuroimage 2018;168:459-476.

33. Rosenbaum SJ, Lind T, Antoch G, Bockisch A. False-positive FDG PET uptake-the role of PET/CT. Eur Radiol 2006;16(5):1054-65.

34. Roberts PF, Follette DM, von Haag D, Park JA, Valk PE, Pounds TR, Hopkins DM. Factors Associated With False-Positive Staging of Lung Cancer by Positron Emission Tomography. Ann Thorac Surg 2000;70:7.

35. Wunderbaldinger P. Problems and prospects of modern lymph node imaging. Eur J Radiol 2006;58(3):32537.

36. Yeh DW, Lee KS, Han J, Yi CA, Lee HY, Chung MJ, Kim TS. Mediastinal nodes in patients with non-small cell lung cancer: MRI findings with PET/CT and pathologic correlation. AJR Am J Roentgenol 2009;193(3):813-21.

37. Liewald F. How Useful is Positron Emission Tomography for Lymphnode Staging in Non-Small-Cell Lung Cancer? 2000.

38. Lv Y, Yuan D, Wang K, Miao X, Qian Q, Wei S, Zhu X, Song Y. Diagnostic Performance of Integrated Positron Emission Tomography/Computed Tomography for Mediastinal Lymph Node Staging in Nonsmall Cell Lung Cancer: A Bivariate Systematic Review and Meta-Analysis. J Thorac Oncol 2011;6(8):9.

39. Vansteenkiste J, De Ruysscher D, Eberhardt WE, Lim E, Senan S, Felip E, Peters S, Group EGW. Early and locally advanced non-small-cell lung cancer (NSCLC): ESMO Clinical Practice Guidelines for diagnosis, treatment and follow-up. Ann Oncol 2013;24 Suppl 6:vi89-98.

40. Lordick F, Mariette C, Haustermans K, Obermannova R, Arnold D. Oesophageal cancer: ESMO Clinical Practice Guidelines for diagnosis, treatment and follow-up. Ann Oncol 2016;27(suppl 5):v50-v57.

41. Rausch I, Quick HH, Cal-Gonzalez J, Sattler B, Boellaard R, Beyer T. Technical and instrumentational foundations of PET/MRI. Eur J Radiol 2017;94:A3-a13.

42. Bailey DL, Pichler BJ, Guckel B, Antoch G, Barthel H, Bhujwalla ZM, Biskup S, Biswal S, Bitzer M, Boellaard R and others. Combined PET/MRI: Global Warming-Summary Report of the 6th International Workshop on PET/MRI, March 27-29, 2017, Tubingen, Germany. Mol Imaging Biol 2018;20(1):4-20.

43. Ohno Y, Koyama H, Lee HY, Yoshikawa T, Sugimura K. Magnetic Resonance Imaging (MRI) and Positron Emission Tomography (PET)/MRI for Lung Cancer Staging. J Thorac Imaging 2016;31(4):215-27.

44. Sotoudeh H, Sharma A, Fowler KJ, McConathy J, Dehdashti F. Clinical application of PET/MRI in oncology. J Magn Reson Imaging 2016.

45. Schaarschmidt BM, Grueneisen J, Metzenmacher M, Gomez B, Gauler T, Roesel C, Heusch P, Ruhlmann V, Umutlu L, Antoch G and others. Thoracic staging with 18F-FDG PET/MR in non-small cell lung cancer - does it change therapeutic decisions in comparison to 18F-FDG PET/CT? Eur Radiol 2017;27(2):681-688.

46. Huellner MW, de Galiza Barbosa F, Husmann L, Pietsch CM, Mader CE, Burger IA, Stolzmann P, Delso G, Frauenfelder T, von Schulthess GK and others. TNM Staging of Non-Small Cell Lung Cancer: Comparison of PET/MR and PET/CT. J Nucl Med 2016;57(1):21-6.

47. Heusch P, Nensa F, Schaarschmidt B, Sivanesapillai R, Beiderwellen K, Gomez B, Kohler J, Reis H, Ruhlmann V, Buchbender C. Diagnostic accuracy of whole-body PET/MRI and whole-body PET/CT for TNM staging in oncology. Eur J Nucl Med Mol Imaging 2015;42(1):42-8.

48. Rauscher I, Eiber M, Furst S, Souvatzoglou M, Nekolla SG, Ziegler SI, Rummeny EJ, Schwaiger M, Beer AJ. PET/MR imaging in the detection and characterization of pulmonary lesions: technical and diagnostic 
evaluation in comparison to PET/CT. J Nucl Med 2014;55(5):724-9.

49. Lee G, I H, Kim SJ, Jeong YJ, Kim IJ, Pak K, Park DY, Kim GH. Clinical implication of PET/MR imaging in preoperative esophageal cancer staging: comparison with PET/CT, endoscopic ultrasonography, and CT. J Nucl Med 2014;55(8):1242-7.

50. Ling CC, Humm J, Larson S, Amols H, Fuks Z, Leibel S, Koutcher JA. Towards multidimensional radiotherapy (MD-CRT): biological imaging and biological conformality. Int J Radiat Oncol Biol Phys 2000;47(3):551-60.

51. Brown JM, Giaccia AJ. The unique physiology of solid tumors: opportunities (and problems) for cancer therapy. Cancer Res 1998;58(7):1408-16.

52. Thomlinson R, Gray L. The histological structure of some human lung cancers and the possible implications for radiotherapy. British journal of cancer 1955;9(4):539.

53. Horsman MR, Mortensen LS, Petersen JB, Busk M, Overgaard J. Imaging hypoxia to improve radiotherapy outcome. Nat Rev Clin Oncol 2012;9(12):674-87.

54. Gray LH, Conger AD, Ebert M, Hornsey S, Scott OC. The concentration of oxygen dissolved in tissues at the time of irradiation as a factor in radiotherapy. Br J Radiol 1953;26(312):638-48.

55. Kelada OJ, Carlson DJ. Molecular imaging of tumor hypoxia with positron emission tomography. Radiat Res 2014;181(4):335-49.

56. Horsman MR, Overgaard J. The impact of hypoxia and its modification of the outcome of radiotherapy. J Radiat Res 2016;57 Suppl 1:i90-i98.

57. Walsh JC, Lebedev A, Aten E, Madsen K, Marciano L, Kolb HC. The clinical importance of assessing tumor hypoxia: relationship of tumor hypoxia to prognosis and therapeutic opportunities. Antioxid Redox Signal 2014;21(10):1516-54.

58. Wack LJ, Monnich D, van Elmpt W, Zegers CM, Troost EG, Zips D, Thorwarth D. Comparison of [18F]FMISO, [18F]-FAZA and [18F]-HX4 for PET imaging of hypoxia--a simulation study. Acta Oncol 2015;54(9):1370-7.

59. Zegers CM, Hoebers FJ, van Elmpt W, Bons JA, Ollers MC, Troost EG, Eekers D, Balmaekers L, ArtsPechtold M, Mottaghy FM and others. Evaluation of tumour hypoxia during radiotherapy using [18F]HX4 PET imaging and blood biomarkers in patients with head and neck cancer. Eur J Nucl Med Mol Imaging 2016;43(12):2139-2146.

60. van Elmpt W, Zegers CM, Reymen B, Even AJ, Dingemans AM, Oellers M, Wildberger JE, Mottaghy FM, Das M, Troost EG and others. Multiparametric imaging of patient and tumour heterogeneity in non-smallcell lung cancer: quantification of tumour hypoxia, metabolism and perfusion. Eur J Nucl Med Mol Imaging 2016;43(2):240-8.

61. Klaassen R, Bennink RJ, van Tienhoven G, Bijlsma MF, Besselink MGH, Henegouwen MIV, Wilmink JW, Nederveen AJ, Windhorst AD, Hulshof $\mathrm{M}$ and others. Feasibility and repeatability of PET with the hypoxia tracer F-18 HX4 in oesophageal and pancreatic cancer. Radiotherapy and Oncology 2015;116(1):94-99.

62. De Ruysscher D, Haustermans K, Thorwarth D. FDG and Beyond. Recent Results Cancer Res 2016;198:163-73.

63. Koh DM, Collins DJ. Diffusion-weighted MRI in the body: applications and challenges in oncology. AJR Am J Roentgenol 2007;188(6):1622-35.

64. Koh DM, Lee JM, Bittencourt LK, Blackledge M, Collins DJ. Body Diffusion-weighted MR Imaging in Oncology: Imaging at 3 T. Magn Reson Imaging Clin N Am 2016;24(1):31-44.

65. Koh DM, Blackledge M, Padhani AR, Takahara T, Kwee TC, Leach MO, Collins DJ. Whole-body diffusionweighted MRI: tips, tricks, and pitfalls. AJR Am J Roentgenol 2012;199(2):252-62.

66. Taouli B, Beer AJ, Chenevert T, Collins D, Lehman C, Matos C, Padhani AR, Rosenkrantz AB, ShuklaDave A, Sigmund E and others. Diffusion-weighted imaging outside the brain: Consensus statement from an ISMRM-sponsored workshop. J Magn Reson Imaging 2016;44(3):521-40.

67. De Ruysscher D, Wanders S, van Haren E, Hochstenbag M, Geeraedts W, Utama I, Simons J, Dohmen J, Rhami A, Buell U and others. Selective mediastinal node irradiation based on FDG-PET scan data in patients with non-small-cell lung cancer: a prospective clinical study. Int J Radiat Oncol Biol Phys 2005;62(4):988-94.

68. Van De Voorde L, Larue RT, Pijls M, Buijsen J, Troost EG, Berbee M, Sosef M, van Elmpt W, Schraepen $\mathrm{MC}$, Vanneste B and others. A qualitative synthesis of the evidence behind elective lymph node irradiation in oesophageal cancer. Radiother Oncol 2014;113(2):166-74. 
69. Aerts HJ, Velazquez ER, Leijenaar RT, Parmar C, Grossmann P, Carvalho S, Bussink J, Monshouwer R, Haibe-Kains B, Rietveld D and others. Decoding tumour phenotype by noninvasive imaging using a quantitative radiomics approach. Nat Commun 2014;5:4006.

70. Lambin P, Rios-Velazquez E, Leijenaar R, Carvalho S, van Stiphout RG, Granton P, Zegers CM, Gillies R, Boellard R, Dekker A and others. Radiomics: extracting more information from medical images using advanced feature analysis. Eur J Cancer 2012;48(4):441-6.

71. Barnes A, Alonzi R, Blackledge M, Charles-Edwards G, Collins DJ, Cook G, Coutts G, Goh V, Graves M, Kelly $\mathrm{C}$ and others. UK quantitative WB-DWI technical workgroup: consensus meeting recommendations on optimisation, quality control, processing and analysis of quantitative whole-body diffusion-weighted imaging for cancer. Br J Radiol 2018;91(1081):20170577.

72. deSouza NM, Winfield JM, Waterton JC, Weller A, Papoutsaki MV, Doran SJ, Collins DJ, Fournier L, Sullivan D, Chenevert $\mathrm{T}$ and others. Implementing diffusion-weighted MRI for body imaging in prospective multicentre trials: current considerations and future perspectives. Eur Radiol 2017.

73. Lambin P, Leijenaar RTH, Deist TM, Peerlings J, de Jong EEC, van Timmeren J, Sanduleanu S, Larue R, Even AJG, Jochems A and others. Radiomics: the bridge between medical imaging and personalized medicine. Nat Rev Clin Oncol 2017;14(12):749-762. 


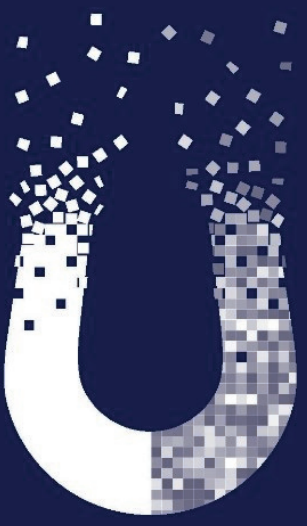




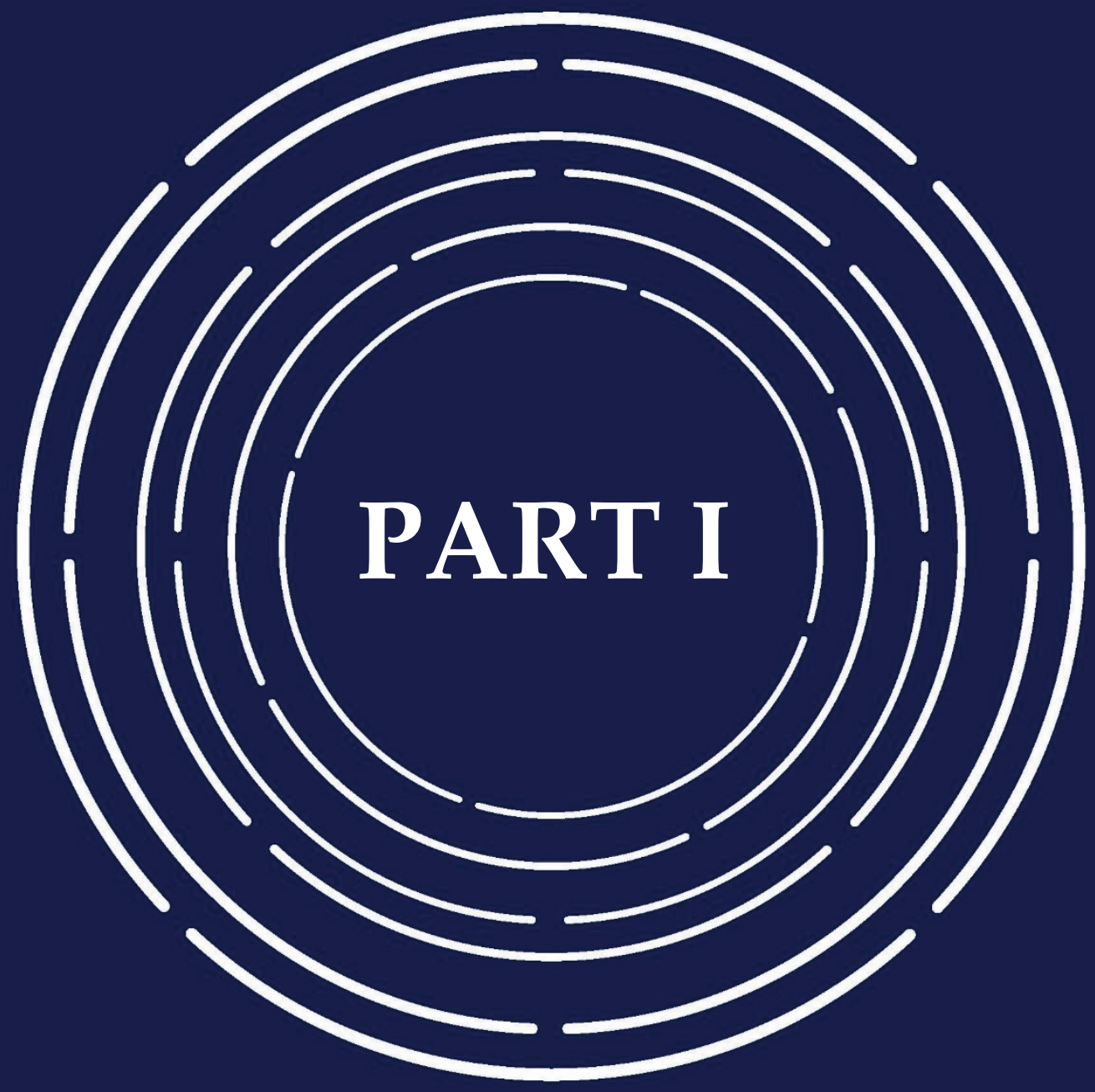

Anatomical MR imaging and qualitative image analysis 


\section{Chapter}

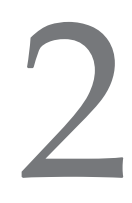

\section{Thoracic imaging:}

Meta-analysis on determining the regional lymph node status in NSCLC using MRI

Authors:

J. Peerlings, E.G.C. Troost, P.J. Nelemans, D.C.P. Cobben, J.C.J. de Boer,A.L. Hoffmann, R.G.H. Beets-Tan

Adapted from:

The diagnostic value of MRI in determining the lymph node status of patients with non-small cell lung cancer: a meta-analysis Radiology, 2016; 281(1):86-98 DOI: $10.1148 /$ radiol.2016151631 


\section{ABSTRACT}

\section{PURPOSE}

The purpose of this meta-analysis is to summarize existing evidence of thoracic MRI in determining the nodal status of NSCLC and aims to elucidate its diagnostic value on a per-patient basis (e.g., in treatment decision making) and a per-nodal basis (e.g., in target volume delineation for radiotherapy) with cytology and/or histology as reference standard.

\section{METHODS}

A systematic literature search for original diagnostic studies was performed in PubMed, Web-of-Science, Embase, and MEDLINE. The methodological quality was evaluated using QUADAS-2. Hierarchical summary Receiver Operating Characteristic (hsROC)curves were generated to estimate the diagnostic performance of MRI. Subgroupanalyses, expressed as relative diagnostic odds ratio (rDOR), were performed to evaluate whether publication year, methodological quality, and/or method of evaluation [qualitative (i.e., lesion size/morphology) vs. quantitative (e.g., ADC-values in diffusionweighted images)] affected the diagnostic performance.

\section{RESULTS}

Twelve of 2551 initially identified studies are included in this metaanalysis (1122 patients, $4302 \mathrm{LN}$ ). On a per-patient basis, the pooled estimates of MRI [95\% CI] for sensitivity, specificity, and DOR-values were 0.87[0.78-0.92], 0.88[0.77-0.94], and 48.1 [23.4-98.9], respectively. On a per-nodal basis, the respective measures were 0.88 [0.78-0.94], 0.95 [0.87-0.98], and 129.5 [49.3-340.0]. Subgroup-analyses suggest greater diagnostic performance of quantitative evaluation on both a per-patient and per-nodal basis (rDOR=2.76 [0.83-9.10], $P=0.09$ and $\mathrm{rDOR}=7.25$ [1.75-30.09], $P=0.01)$.

\section{CONCLUSION}

This meta-analysis demonstrates high diagnostic performance of MRI in staging hilar/mediastinal LN in NSCLC on both per-patient and pernodal basis. 


\section{INTRODUCTION}

With an incidence of roughly 1.8 million cases in 2012, lung cancer has become the most prevalent cancer-type worldwide [1]. More alarmingly, lung cancer has the highest mortality rate of all solid tumors with cause-specific 5-year survival rates ranging from $90-95 \%$ (stage I) to $20 \%$ (stage III) [2]. Therapeutic outcome depends on tumor type [i.e., small cell or non-small cell lung cancer (NSCLC)], nodal involvement, and the presence of distant metastases [3]. In particular, accurate nodal staging is critical for reliable treatment decision-making on a per-patient basis [4]. In fact, the general treatment strategy in NSCLC patients without distant metastases (cM0) is defined by their clinical lymph node stage ( $\mathrm{cN} 0 / 1$ versus $\mathrm{cN} 2 / 3)$ determining the choice for surgical resection or (chemo-)radiotherapy [5].

Currently, the reference standard for detecting metastatic hilar/mediastinal lymph nodes is cytological or histological examination by means of endo-bronchial/esophageal ultrasound guided transbronchial fine-needle aspiration (EBUS/EUSTBNA), or mediastinoscopy $[2,6]$. Nonetheless, this invasive procedure remains subject to sampling errors ( $7 \%$ and $11 \%$ false-negative rates, respectively) and induces patient discomfort [7-9]. The most frequently investigated imaging method for non-invasive nodal staging is positron emission tomography using the radioactive tracer ${ }^{18} \mathrm{~F}$-fluorodeoxyglucose combined with computed tomography $\left({ }^{18} \mathrm{~F}-\mathrm{FDG}-\mathrm{PET} / \mathrm{CT}\right)$. However, ${ }^{18} \mathrm{~F}-\mathrm{FDG}-\mathrm{PET} / \mathrm{CT}$ is associated with many falsepositive and false-negative findings, limiting its diagnostic capabilities [10,11]. Confounding effects of hyperplasia, sarcoidosis, and inflammatory lymph nodes may result in false-positive readings, whereas the limited spatial resolution of ${ }^{18}$ F-FDG-PET/CT may induce false anatomical allocation or miss small lymph nodes harboring microscopic metastases leading to false-negatives [12-17]. A high ${ }^{18} \mathrm{~F}-\mathrm{FDG}$-signal of lymph nodes should therefore not be interpreted as proof of malignancy, and confirmation by cytology and/or histology is still required $[2,18,19]$.

Magnetic resonance imaging (MRI) provides detailed anatomical information with a higher spatial and temporal resolution than ${ }^{18} \mathrm{~F}-\mathrm{FDG}$-PET/CT [20]. However, MRI of the lung and mediastinal structures is quite challenging due to the intrinsic properties of the surrounding tissues. The alveolar architecture of the lung, the related magnetic susceptibility effects, as well as motion from breathing and cardiac pulsation results in a low signal to noise ratio (SNR) and limits overall image quality [21-23]. Modern MRI has recently shown potential to overcome these hurdles through technical breakthroughs such as fast imaging sequences, parallel acquisition and triggering techniques [22-24]. These advances enable MRI to visualize even small cancerous lesions $[25,26]$. We therefore hypothesize that detection and differentiation of (non-)metastatic hilar and mediastinal lymph nodes may be realized by an imaging modality other than ${ }^{18} \mathrm{~F}-\mathrm{FDG}-\mathrm{PET} / \mathrm{CT}$. 
The purpose of this meta-analysis is to summarize the existing evidence of thoracic MRI in determining the nodal status of NSCLC and aims to elucidate its diagnostic value on a per-patient basis (e.g., in treatment decision making) and a per-nodal basis (e.g., in target volume delineation for radiotherapy) with cytology and/or histology as reference standard.

\section{MATERIAL \& METHODS}

\section{SEARCH STRATEGY}

To develop the research question and identify the scope of the review, a structured approach was followed to identify the patient population, interventions, comparators, outcomes and study design (PICOS criteria). The research question was formulated as: What is the diagnostic accuracy of MRI in detecting regional lymph nodes metastases in patients with NSCLC, when compared with cytology or histology [27-29]?

Based on this research question, a comprehensive literature search was conducted to identify relevant studies published in PubMed (National Center for Biotechnology Information, NCBI), MEDLINE (U.S. National Library of Medicine, using OvidSP), Web of Science (Thomson Reuters), Cochrane Library (Cochrane collaboration, using Wiley online library), and/or in the Excerpta Medica database (Embase, using OvidSP). For MEDLINE, Web of Science, and Embase a free-text construction of the search string was made with the following terms: magnetic resonance imaging AND non-small cell lung cancer AND regional metastatic lymph nodes AND lung. Additionally, all known synonyms, spelling, and associated terms (e.g., diffusion weighted imaging, hilar and mediastinal lymphadenopathy, adenocarcinoma, squamous cell carcinoma, and large cell carcinoma) were incorporated into the search string. Although 'bronchioloalveolar carcinomas' (currently referred as adenocarcinomas with lepidic growth-pattern) is thought to be a premalignant form of NSCLC, we excluded related studies because this cancer type does not develop lymph node metastases. In addition, nodules, thymus carcinomas, and mesotheliomas were excluded using the Boolean NOT-operator. Searches in PubMed and Cochrane Library databases were conducted with following Medical Subject Headings (MeSH terms): magnetic resonance imaging AND non-small cell lung cancer AND (lymph node OR lymphatic metastases). Several restrictions were used to limit the number of hits. A paper was only retrieved when written in English or Dutch, and published within the period 2000-2014.

\section{STUDY SELECTION}

To be included for review, full-text content had to be accessible online or as a printed copy, and the study had to meet pre-specified PICOS-criteria [30]: (1) the patient population consisted of human adults that were affected by or suspected of having NSCLC with regional nodal metastasis; (2) the index test comprised of MRI for 
detecting and differentiating (non-)metastatic hilar/mediastinal lymph nodes; (3) for comparison, diagnostic results from EBUS/EUS-TBNA and/or mediastinoscopy were available as reference standard; (4) study outcome described the diagnostic performance of MRI and absolute numbers of true-positive, true-negative, falsepositive, and false-negative test results were reported or could be derived; (5) studies had to describe original results, prospectively or retrospectively, on both per-patient based and per-nodal based analyses.

The described search strategy was applied weekly, with the last update being performed on June $16^{\text {th }}, 2014$. To complete the systematic search, the reference lists of included papers and relevant reviews were manually cross-checked (i.e., citation tracking) to find potential studies fitting the inclusion criteria. Next, duplicate findings were manually discarded to ensure that no data overlap was used. Publications using the same patient cohort were included only once by selecting the one with the largest sample size. If studies presented results from both qualitative and quantitative analyses, the results with the highest diagnostic performance was selected for further analyses.

\section{DATA EXTRACTION AND PROCESSING}

The extracted data contained information regarding patient characteristics, study design, and features of the index and reference test. We recorded publication year, the number of patients or lymph nodes evaluated by the reference standard, whether MRI scans were evaluated in a qualitative (i.e., diagnoses based on visual inspection of lesion size and morphology for quantitative (i.e., diagnoses based on ADC-values in diffusion-weighted image or lesion-to-phantom ratio (LPR) calculated by normalizing signal intensities of MR images with a phantom placed next to the patient) manner, and what MR scanning sequence was primarily used [31,32]. Outcome parameters were either extracted from the original study or derived from sensitivity and specificity, in combination with prevalence. Either way, the diagnostic performance of MRI in discriminating metastatic from non-metastatic lymph nodes ( $\mathrm{cN} 0$ vs. cN1-3) was extracted and manually verified by recalculating the absolute values from the raw data, and used to construct a $2 \times 2$ contingency table. Furthermore, we recorded whether these results originated from per-patient or pernodal based analyses.

\section{DATA QUALITY ASSESSMENT}

We used the standard QUADAS-2 checklist and signaling questions (Bristol University, Bristol, UK) to evaluate the methodological quality of the studies included $[33,34]$. The QUADAS-2 tool consists of 4 key domains that discuss patient selection, index test, reference standard, and flow of patients through the study and timing of the index tests and reference standard (flow and timing). Each domain was scored in terms of risk of bias and concerns regarding applicability to the research 
question. With this checklist, the quality of each diagnostic study was individually evaluated and used for further subgroup-analyses.

A schematic overview of the pre-specified research protocol is documented in a web-appendix (online only) [35]. Two reviewers (J.P., PhD candidate, 2 years' experience in clinical research related to thoracic radiology and radiotherapy, and E.T., radiation oncologist and professor of image-guided high precision radiation therapy, 7 years' experience in radiation oncology of thoracic tumors) performed each step of this protocol independently and attempted to reach consensus in cases of disagreement. Moreover, the corresponding authors of ambiguous studies were contacted for more information. If discrepancy remained, a third party (R.B.T., radiologist and professor, more than 20 years' experience in oncological MRI, and A.H., medical physicist and senior scientist, 10 years' experience in medical physics and image-guided radiation therapy) was consulted to adjudicate.

\section{DATA ANALYSIS}

The reference standard was considered positive when EBUS/EUS-TBNA and/or mediastinoscopy indicated the presence of lymphatic metastasis regardless of the location of the lymph node (i.e., $\mathrm{cN} 1, \mathrm{cN} 2$, or $\mathrm{cN} 3$ ). Likewise, a positive index test was based on MRI findings and the resulting qualitative or quantitative diagnosis $[31,32,36,37]$.

Results from individual diagnostic studies were summarized using Rutter \& Gatsonis' hierarchical summary Receiver Operating Characteristic (hsROC) model, which weights both within- and between-study variability, and allows flexibility in the estimation of summary statistics [38-39]. The model provides an hsROC curve illustrating which sensitivity can be achieved at specific levels of specificity with $95 \%$ CI (and visa versa), with the optimal trade-off located at the point closest to the top left-hand corner of the graph [40]. Diagnostic performance is expressed by diagnostic odds ratios (DOR) which combine sensitivity and specificity into one single measure of discriminative ability of a diagnostic test. Heterogeneity in test accuracy between studies was explored using the inconsistency index $\left(\mathrm{I}^{2}\right)$ and further subgroup analyses. $I^{2}$-values $>50 \%$ indicated substantial heterogeneity in the diagnostic parameters across studies [41]. Publication bias was assessed using a Deeks' funnel plot asymmetry test. Slope coefficients with a P-value $\leq 0.10$ were considered to show statistically significant asymmetry [42].

Subgroup analyses were separately performed according to publication year (i.e., until 2008 vs. after 2008, for this is the median publication year), method of diagnostic assessment (i.e., qualitative vs. quantitative), and QUADAS-2 quality scoring per domain to explore the relationship between these study characteristics and pooled estimates of diagnostic accuracy. Next, differences in diagnostic 
performance between subgroups were tested for statistical significance and expressed as relative DORs (rDOR) with 95\% confidence interval (CI) [43-45].

Statistical meta-analyses were performed with Stata/SE statistical software version 12.0 (StataCorp LP, College Station, TX), using the Metandi- and Midas-modules [46,47]. Meta-regression analyses were performed with Meta-DiSc 1.4 (Ramón y Cajal Hospital, Madrid, Spain) using the DerSimonian-Laird method (randomeffects model) weighted for study sample size [45]. P-values $\leq 0.05$ were considered to indicate statistically significant results for all analyses, except for the Deeks' funnel plot asymmetry test.

\section{RESULTS}

\section{LITERATURE SEARCH}

Initially, 2551 records were identified throughout all databases (609 Web of Science, 55 PubMed, 870 MEDLINE, 1017 Embase). The Cochrane Library was also searched but no eligible papers could be retrieved. This number was narrowed down using inclusion and exclusion criteria (Fig. 2.1).
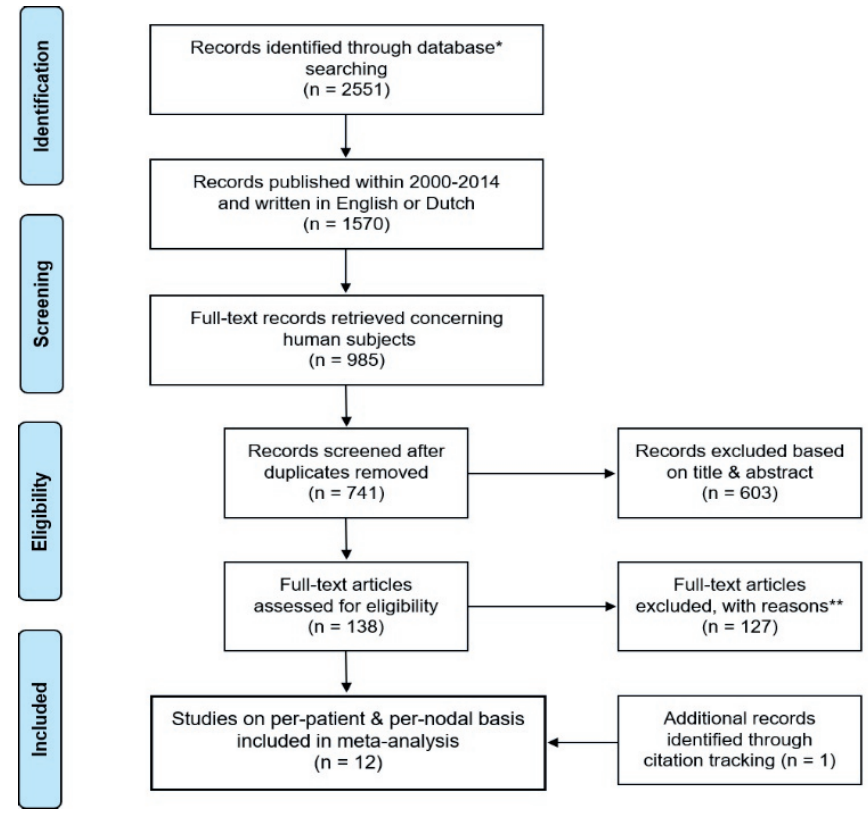

Figure 2.1: Flow of information through the different phases of systematic review towards a meta-analysis

${ }^{*} \mid$ Web of Science $(\mathrm{n}=609)$, PubMed $(\mathrm{n}=55)$, MEDLINE $(\mathrm{n}=870)$, Embase $(\mathrm{n}=1017)$

$\left.\right|^{* *} \mid$ No accessible full text $(n=2)$, Overlapping dataset $(n=2)$, Unable to construct $2 \times 2$ table $(n=3)$, Studies involving no NSCLC patients $(n=7)$, studies not using MRI of the thorax $(n=10)$, studies using PET/MRI $(n=4)$, studies assessing extra-thoracic regions/distant metastases $(n=4)$, studies not assessing mediastinal/hilar lymph nodes $(n=8)$, Non-diagnostic studies $(n=77)$, Unfit reference standard $(\mathrm{n}=8)$, Only abstract was written in English $(\mathrm{n}=2)$. 
After screening title and abstract, we excluded irrelevant records and rejected all reviews, meta-analyses, comments, supplements, conference abstracts, symposiums reports, essays, guidelines, and letters to the editor. A total of 138 full-text articles were retrieved to assess whether the studies met the pre-defined inclusion criteria. Two out of 138 studies were excluded because the full-text content could not be accessed or provided by the corresponding author. Furthermore, studies with overlapping datasets $(\mathrm{n}=2)$, studies that did not allow a $2 \times 2$ diagnostic accuracy assessment $(\mathrm{n}=3)$, and studies not meeting our pre-specified inclusion criteria $(n=120)$ were excluded (Fig. 2.1). One additional record was identified through citation tracking. Ultimately, a total of 12 eligible studies (1122 pathologically confirmed patients and 4302 lymph nodes) were included to be reviewed and considered for further analyses [31,32,48-57].

\section{DATA EXTRACTION AND QUALITY ASSESSMENT}

Relevant study characteristics and the obtained MR sequences are summarized in Table 2.1. While qualitative assessment was based on visual inspection of lesion size and morphology on anatomical images, studies in the quantitative subgroup used either cardiac- and/or respiratory-triggered conventional or black-blood short inversion-time inversion-recovery turbo spin-echo (STIR-TSE) imaging, or diffusion-weighted imaging (DWI) to examine the clinical utility of MRI in defining the nodal status. STIR-TSE images were analyzed quantitatively by normalizing signal intensities from detected lymph nodes with those from a phantom filled with either $0.9 \%$ saline or $2 \%$ copper sulfate. The resulting LPR shows a direct relationship with the likelihood of a metastatic lymph node [31,32,50,51,54,55]. Alternatively, DWI images were evaluated quantitatively by calculating apparent diffusing coefficient (ADC) values for each lymph node. In a prospective study with histopathologic validation, $\mathrm{ADC}$-values of metastatic lymph nodes were reported to be significantly lower than the ADC-values of lymph nodes without metastasis $[52,55]$.

The distribution of QUADAS-2 scores of methodological quality of every included study (i.e., risk of bias and concerns regarding applicability) is presented in Figure 2.2. The majority of studies are assessed as having low risk of bias and minimal concerns regarding applicability. Common weaknesses relate to uncertainties regarding the blinding of the reference test from MRI results, and to the poorly documented time interval between cytological/histological examination and MRI. Both the slope and symmetry of Deeks' funnel plot as well as the nonsignificant result of the asymmetry test indicate a low likelihood of publication bias for MRI-studies on both per-patient $(P=0.86)$ and per-nodal basis ( $P=0.74)$ (Fig. A2.1). 


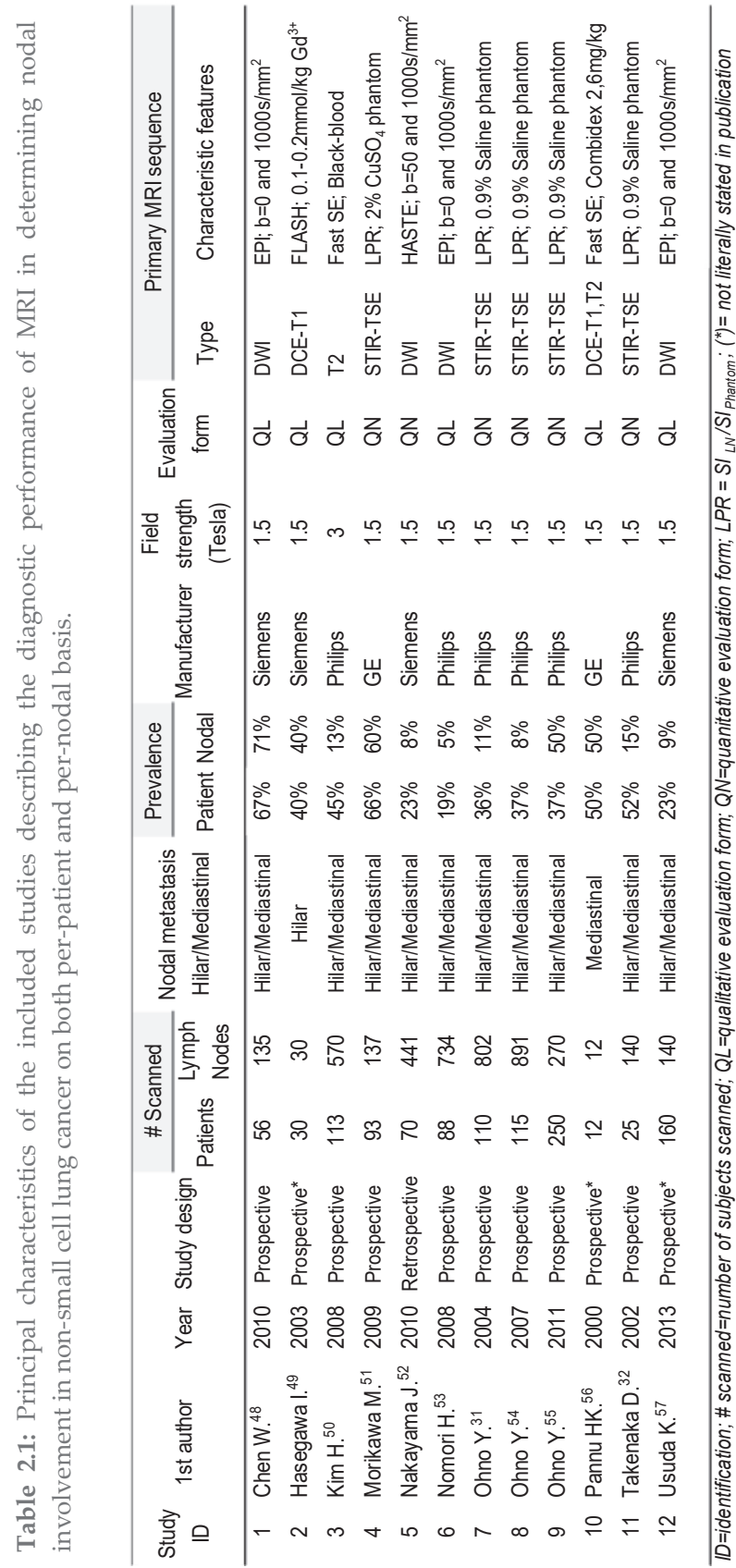




\section{DATA ANALYSis}

The observed sensitivity and specificity values from individual studies are presented in Forest plots, along with 95\% CI and a summary estimate for MRI's diagnostic performance (Fig. 2.3). Per-patient based results reveal pooled estimates of 0.87 [0.78-0.92] for sensitivity and 0.88 [0.77-0.94] for specificity (Fig. 2.3A). Results on a per-nodal basis show pooled estimates of 0.88 [0.78-0.94] and 0.95 [0.87-0.98], respectively (Fig. 2.3B). In addition, these plots indicate that heterogeneity was high with $\mathrm{I}^{2}$-values exceeding $50 \%$ for sensitivity and specificity on a per-patient basis and on a per-nodal basis.

Resulting from the hsROC model, the discriminative power of a diagnostic test is expressed as summary DORs of 48.1 [23.4-98.9] and 129.5 [49.3-340.0] for per-patient based analyses and per-nodal based analyses, respectively (Fig. 2.4A and 2.4B). As a reference, DOR-values of 10, 50, 100, and 200 are equivalent to area under the curve (AUC)-values of $0.83,0.94,0.96$, and 0.98 , respectively, for symmetric hsROC curves [58].
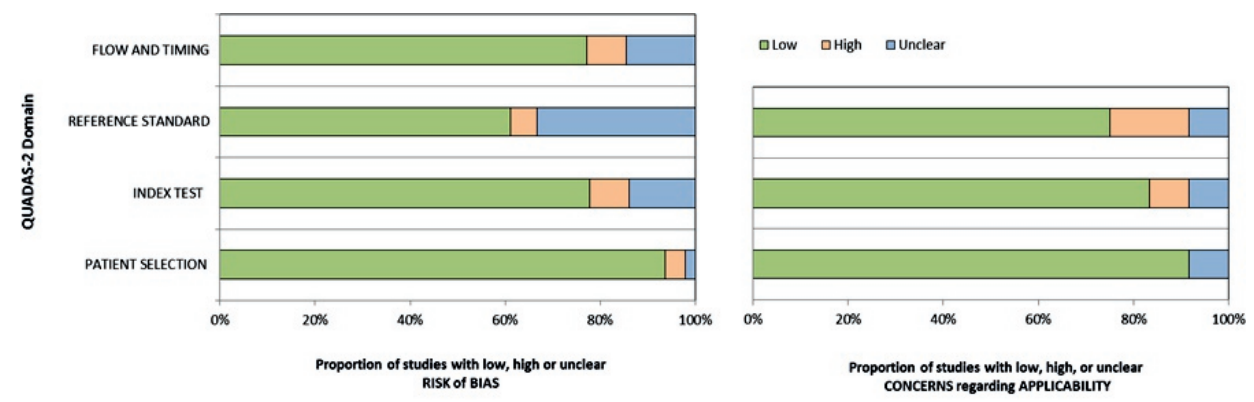

Figure 2.2: Stacked bar chart of QUADAS-2 scores of methodological study quality expressed as a percent of studies meeting each criterion to give a quick overview of the study quality within the whole meta-analysis. For each quality domain, the proportion of included studies that suggest low, high, or unclear risk of bias and/or concerns regarding applicability are respectively displayed in green, orange, and blue.

Six studies using quantitative diagnostic assessment of MRI-images were compared with six studies using qualitative assessment. Subgroup analyses on a per-patient basis reported summary DORs of 61.3 [36.2-103.7] and 27.4 [7.83-95.9], respectively, but the rDOR of 2.76 [0.83-9.10] was not statistically significant $(P=0.09)$. However, for per-nodal based analyses, the difference between quantitative (DOR=254.6 [69.2937.4]) and qualitative evaluation ( $\mathrm{DOR}=53.3$ [14.9-190.2]) proved to be statistically significant with an rDOR of 7.25 [1.75-30.09] $(P=0.01)$. 

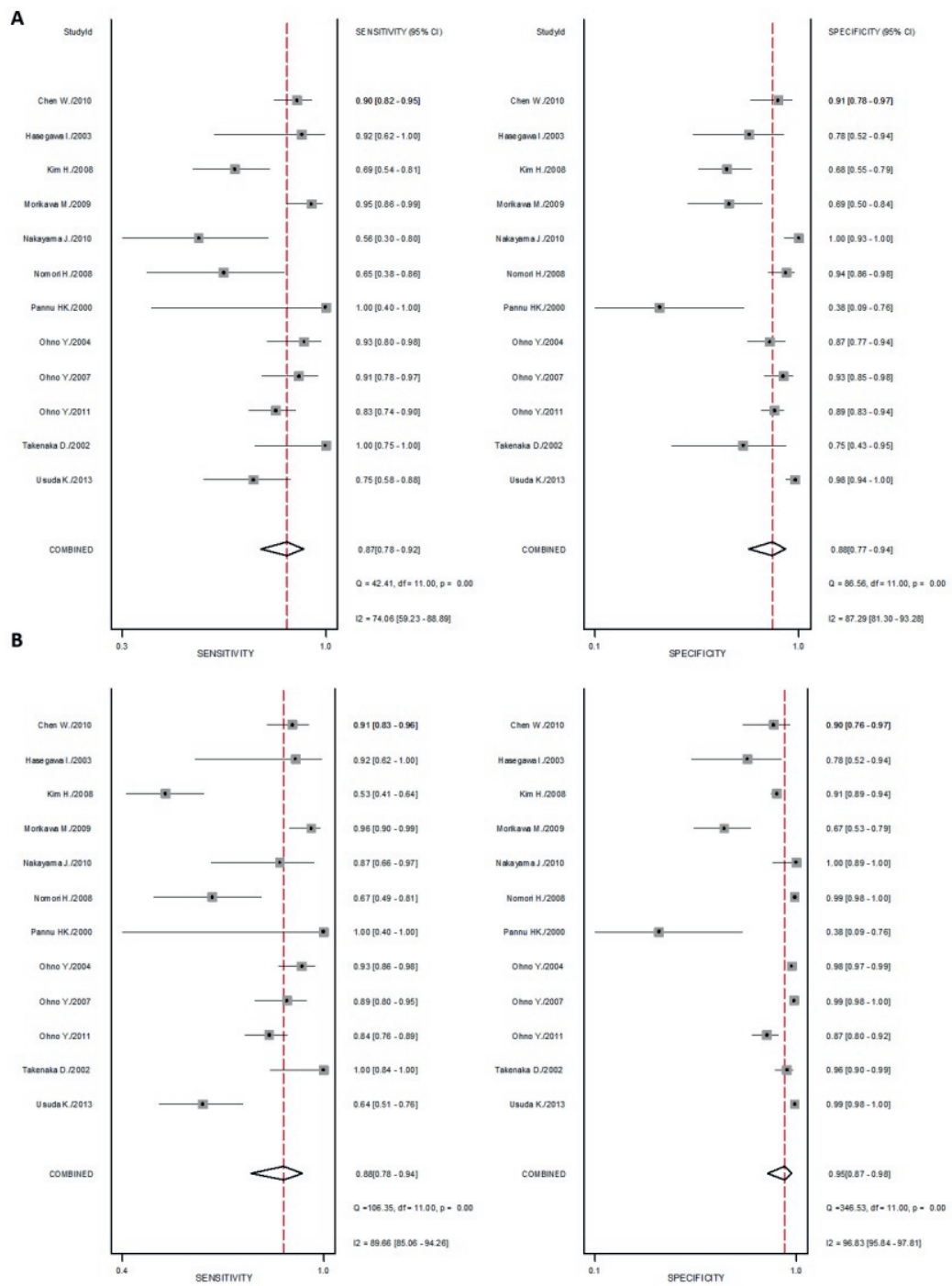

Figure 2.3: Forest plots displaying diagnostic performance estimates (i.e., sensitivity and specificity, dotted squares) of each study on a per-patient basis (a) and on a per-nodal basis (b) with $95 \%$ confidence interval (horizontal lines). The diamond at the bottom and the vertical dotted line describe pooled summary estimates of sensitivity and specificity. Heterogeneity statistics $\left(\mathrm{I}^{2}\right.$,

Q) for sensitivity and specificity are also shown.

Subgroup analyses according to publication year indicated non-significant differences between publications until 2008 and thereafter for per-patient based studies (34.8 [10.8-111.8] versus 79.2 [32.2-195.3], $P=0.25$ ) and per-nodal based studies (155.9 [31.8-763.8] versus 82.2 [33.6-200.9], $P=0.40$ ). An overview of these results is provided in Table 2.2. The third subgroup analysis assessing differences in methodological quality per QUADAS-2 domain yielded inconsistent results due to the small number of studies within most subgroups and therefore are not shown. 
A

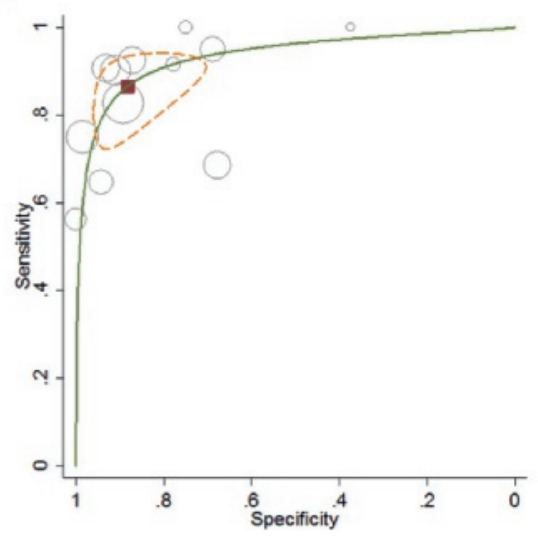

B

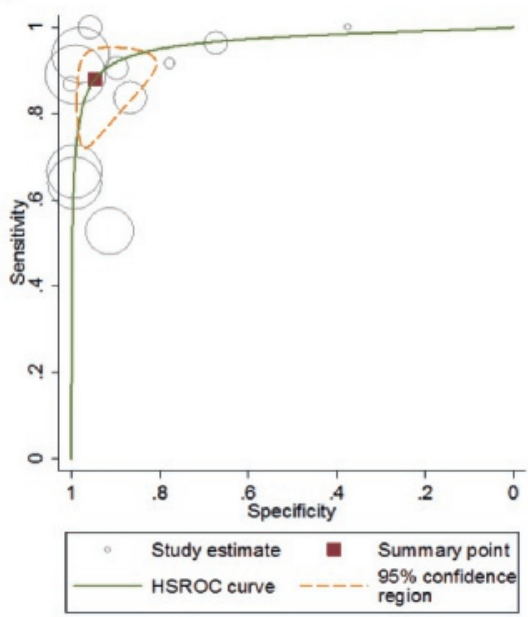

Figure 2.4: Hierarchical summary receiver operating curves (hsROC) for the diagnostic performance of MRI on per-patient basis (a) per-nodal basis (b) in determining the lymph node status of non-small cell lung cancer patients. These hsROC curves illustrate which sensitivity can be achieved at specific levels of specificity, with the optimal trade-off between sensitivity and specificity located at the top left-hand corner.

Table 2.2: Summary estimates diagnostic performance of MRI, in detecting and differentiating (non-)metastatic regional lymph in patients with non-small cell lung cancer in respect to cytology and/or histology, derived from all studies and subgroups of studies performed on per-patient and on per-nodal basis. Sensitivity and specificity are expressed in percentages (\%) and accompanied with 95\% confidence intervals (square brackets).

\begin{tabular}{lclllll}
\hline Per-Patient Basis & $\mathrm{N}_{\text {studies }}$ & Se $(\%)$ & Spec (\%) & DOR & rDOR & P-value \\
\hline Overall & 12 & $86.5[77.6-92.3]$ & $88.2[77.3-94.3]$ & $48.1[23.4-98.9]$ & 1 & \\
Year & 12 & & & & & \\
$\quad \leq 2008$ & 7 & $88.4[73.4-95.5]$ & $82.0[67.8-90.8]$ & $34.8[10.8-111.8]$ & $2.17[0.52-9.04]$ & 0.25 \\
$\quad>2008$ & 5 & $83.0[69.2-91.4]$ & $94.2[79.3-98.6]$ & $79.2[32.2-195.3]$ & & \\
Assessment & 12 & & & & & \\
$\quad$ Qualitative & 6 & $81.2[68.6-89.6]$ & $86.4[65.7-95.4]$ & $27.4[7.83-95.9]$ & $2.76[0.83-9.10]$ & 0.09 \\
$\quad$ Quantitative & 6 & $88.4[76.4-94.7]$ & $88.9[76.6-95.2]$ & $61.3[36.2-103.7]$ & & \\
\hline Per-Nodal Basis & $\mathrm{N}_{\text {studies }}$ & Se (\%) & Spec (\%) & DOR & rDOR & P-value \\
\hline Overall & 12 & $87.9[78.2-93.7]$ & $94.7[86.9-98.0]$ & $129.5[49.3-340.0]$ & 1 & \\
Year & 12 & & & & & \\
$\quad \leq 2008$ & 7 & $89.5[70.3-96.8]$ & $94.8[83.3-98.5]$ & $155.9[31.8-763.8]$ & $2.18[0.30-15.65]$ & 0.40 \\
$\quad>$ 2008 & 5 & $85.6[73.4-92.7]$ & $93.3[78.1-98.2]$ & $82.2[33.6-200.9]$ & & \\
Assessment & 12 & & & & & \\
$\quad$ Qualitative & 6 & $80.3[59.7-91.8]$ & $92.9[73.6-98.4]$ & $53.3[14.9-190.2]$ & $7.25[1.75-30.09]$ & 0.01 \\
$\quad$ Quantitative & 6 & $91.7[85.7-95.3]$ & $95.9[86.8-98.8]$ & $254.6[69.2-937.4]$ & & \\
\hline
\end{tabular}




\section{DISCUSSION}

This meta-analysis demonstrates high pooled sensitivities and specificities for the detection of hilar/mediastinal lymph node metastases with MRI in NSCLC, both on a per-patient and per-nodal basis. Subgroup analyses revealed that studies using quantitative diagnostic assessment of metastatic regional lymph nodes had a significantly higher diagnostic accuracy on a per-nodal basis than studies using qualitative assessment.

A high diagnostic accuracy is essential for reliable cN-staging, determining resectability, and associated treatment decision-making (i.e., surgical resection vs. (chemo-)radiotherapy) on a per-patient basis $[4,59,60]$. These decisions are currently based on EBUS/EUS-TBNA and/or mediastinoscopy and additional 18F-FDGPET/CT. However, the diagnostic power of ${ }^{18} \mathrm{~F}-\mathrm{FDG}$-PET/CT only is hampered by false-positive and false-negative readings $[10,61,62]$. The question is whether MRI, which in literature has shown potential for mediastinal nodal staging could overcome the limitations of ${ }^{18} \mathrm{~F}-\mathrm{FDG}-\mathrm{PET} / \mathrm{CT}$ and further improves the performance of clinical nodal staging $[15,63,64]$. Ideally, one would perform a meta-analysis based on studies that directly compared both MRI and ${ }^{18} \mathrm{~F}-\mathrm{FDG}-\mathrm{PET} / \mathrm{CT}$ in the same study population, with cytological/histological examination as reference standard. As such studies are thus far lacking, we compared the overall diagnostic performance of MRI from our meta-analysis to four recently published meta-analyses assessing the diagnostic performance of ${ }^{18} \mathrm{~F}-\mathrm{FDG}$-PET/CT in N-staging patients with NSCLC in respect to cytology/histology (Table 2.3).

Table 2.3: Summary estimates of diagnostic performance of ${ }^{18} \mathrm{~F}-\mathrm{FDG}-\mathrm{PET} / \mathrm{CT}$ in respect to cytology and/or histology, derived from 4 published meta-analyses, compared to the diagnostic power of MRI for defining metastatic regional lymph nodes on per-patient and per-nodal basis in patients with NSCLC, as stated in present meta-analysis. Sensitivity and specificity are expressed in percentages (\%) and accompanied with 95\% confidence intervals (square brackets).

\begin{tabular}{|c|c|c|c|c|c|c|c|}
\hline \multirow[t]{2}{*}{ Meta-analyses } & \multirow[b]{2}{*}{ \#Studies } & \multirow[b]{2}{*}{ \#Patients } & \multirow[b]{2}{*}{ \#Nodes } & \multicolumn{2}{|c|}{ PET/CT per-patient basis } & \multicolumn{2}{|c|}{ PET/CT per-nodal basis } \\
\hline & & & & Se $(\%)$ & Spec (\%) & Se (\%) & Spec (\%) \\
\hline $\mathrm{Wu}(2013)^{10}$ & 36 & 3248 & 5383 & $72[65-78]^{*}$ & $91[86-94]^{*}$ & $78[64-87]$ & $90[84-94]$ \\
\hline $\operatorname{Lv}(2012)^{18}$ & 14 & 2550 & 8188 & $76[65-84]$ & $88[82-92]$ & $65[62-68]$ & 95 [94-95] \\
\hline Zhao (2012) & 20 & 3028 & - & $72[68-75]$ & $90[88-91]$ & $61[58-64]$ & $93[92-93]$ \\
\hline $\mathrm{Wu}(2012)^{66}$ & 19 & 2845 & - & $75[68-81]$ & $89[85-91]$ & - & - \\
\hline \multirow{3}{*}{$\begin{array}{l}\text { Presented } \\
\text { meta-analysis }\end{array}$} & & & & \multicolumn{2}{|c|}{ MRI per-patient basis } & \multicolumn{2}{|c|}{ MRI per-nodal basis } \\
\hline & \#Studies & \#Patients & \#Nodes & Se $(\%)$ & Spec (\%) & Se $(\%)$ & Spec (\%) \\
\hline & 12 & 1122 & 4302 & $86.5[77.6-92.3]$ & 88.2 [77.3-94.3] & 87.9 [78.2-93.7] & 94.7 [86.9-98.0] \\
\hline
\end{tabular}

|*| Mediastinal lymph nodes only 
These meta-analyses revealed sensitivity- and specificity-values on per-patient basis ranging from 0.72-0.76 and 0.89-0.91, respectively, and from 0.61-0.78 and 0.90-0.95, respectively, on per-nodal basis $[10,18,65,66]$. Relative to our findings, the results of ${ }^{18} \mathrm{~F}-\mathrm{FDG}-\mathrm{PET} / \mathrm{CT}$ meta-analyses indicate that on a per-patient basis, MRI is able to achieve an overall sensitivity that is at least as high as ${ }^{18} \mathrm{~F}-\mathrm{FDG}-\mathrm{PET} / \mathrm{CT}$ at equivalent specificity values. Moreover, the overall diagnostic performance of MRI on a pernodal basis is substantially higher than ${ }^{18} \mathrm{~F}-\mathrm{FDG}-\mathrm{PET} / \mathrm{CT}$ with superior sensitivity values at comparable specificity values. The high diagnostic performance of MRI could possibly be due to its versatile contrast determinants. Anatomical MRI can produce images with superior soft-tissue contrast compared to CT. Physiological MRI can assess functional tissue parameters (e.g., molecular diffusion, and water content and mobility), whereas PET-imaging provide information on molecular, cell receptor, and metabolic status of affected tissue [25].

We further investigated the impact of method of diagnostic assessment on the overall diagnostic performance of MRI through rDOR-calculations. The results suggest that quantitative assessment is associated with higher discriminatory power than qualitative assessment, with a statistically significant difference in analyses on a per-nodal basis. It should be noted that four studies, quantitatively evaluating STIR-TSE images through LPR calculations, originated from the same research group pioneering in quantitative detection and differentiation of (non-)metastatic lymph nodes in patients with NSCLC $[31,32,54,55]$. Nevertheless, results from this meta-analysis suggests that an objective quantitative parameter (i.e., not based on human expertise) serves as a better measurement than size and morphology as criteria for detection of metastatic hilar and/or mediastinal lymph nodes [63,67-69]. Our finding is in line with the main conclusion of the six individual studies included for subgroup-analysis [31,32,51,54,55].

This meta-analysis investigated the overall diagnostic value of MRI in detecting metastatic hilar/mediastinal lymph nodes in NSCLC patients on a per-patient and a per-nodal basis, in reference to cytology and/or histology. Related meta-analyses either studied different anatomical locations or mixed cancer types (e.g., pulmonary nodules, distant metastases, small cell lung cancer), and/or confined themselves to the diagnostic capabilities of DWI or contrast-enhanced MRI [3,7,66,70-72]. Our study does not discriminate articles based on MRI-methods used and is unique in presenting per-patient based analyses parallel to per-nodal based analyses.

Though quantitative MRI demonstrates a high diagnostic performance for nodal staging in our review, literature shows conflicting results with some authors reporting that quantitative evaluation may hold several pitfalls. Pitfalls such as DWI misinterpretation, multi-vendor/-center variability, and standardization-issues (e.g., contrast dose, specific acquisition parameters, and post-processing analysis) need to be into account during quantitative image assessment [73,74]. Nevertheless, it can 
be hypothesized that accurate quantitative individual lymph node evaluation could be of potential value for user-independent target volume delineation during pernodal based radiation therapy planning [63,75]. In 2005, De Ruysscher et al. showed that selective nodal irradiation of ${ }^{18}$ F-FDG-PET-positive, CT-enlarged and/or pathological confirmed metastatic lymph nodes of NSCLC patients resulted in a low regional failure and reduced esophageal toxicity in the era of 3-dimensional conformal radiation therapy [76]. It is envisioned that MRI could possibly reduce detrimental treatment effects currently caused by false-positive (i.e., over-treatment causing excess side-effects) and false-negative ${ }^{18} \mathrm{~F}-\mathrm{FDG}$-PET/CT readings (i.e., under-treatment leading to regional recurrence). After its potential diagnostic value, compared to or along with ${ }^{18} \mathrm{~F}-\mathrm{FDG}-\mathrm{PET} / \mathrm{CT}$, is proven in future studies, MRI-based quantitative nodal staging-techniques may be implemented in radiation therapy planning, allowing radiation-oncologists to evaluate each lymph node at highspatial resolution facilitating selective nodal irradiation [76].

Before MRI can be incorporated in routine clinical practice for detecting and differentiating regional lymph nodes, the safety of this approach has to be evaluated in a prospective clinical trial. Furthermore, possible geometrical distortions and standardization-issues could prove to be problematic for radiotherapy planning and should first be thoroughly investigated before being implemented in radiation oncology [77,78]. Moreover, new lymph node specific MR contrast agents, such as Gadofosveset (Ablavar®; Lantheus Medical Imaging, MA), may hold additional benefits to further improve the diagnostic performance of MRI [79-81]. Additionally, MRI does not expose patients and medical personnel to irradiation, is less invasive and less expensive than ${ }^{18} \mathrm{~F}-\mathrm{FDG}-\mathrm{PET} / \mathrm{CT}[82]$.

Although the meta-analysis adheres to the PRISMA statement, it has a number of limitations $[27,28]$. First, the search strategy was restricted to articles published between 2000-2014, since studies performed before 2000 were deemed technologically irrelevant. Within this period, MRI has undergone a massive technologic evolution with faster imaging sequences, cardiac and respiratory triggering, enabling a good correlation with cytology/histology at a higher spatial and temporal resolution [64,83]. To investigate the relationship of an additional technological progress within this period on the overall pooled estimates and thus to overcome this shortcoming, we performed subgroup analyses according to publication year. However, the non-significant results suggest that publication year had no or little impact on the overall diagnostic performance. Second, the exact location of metastatic lymph nodes in relation to the primary tumor was not taken into account in (most of) the publications serving as basis for this meta-analysis, hence, we were not able to differentiate the $\mathrm{cN}$-stages (cN1-3). Instead, we focused on the ability of MRI to discriminate metastatic from non-metastatic lymph nodes (cN0 versus cN1-3), as this was the aim of our study. Third, the reference standard of this study, EBUS/EUS-TBNA and/or mediastinoscopy, is not infallible with 
sensitivity-values ranging from $93-96 \%$ and $82-95 \%$, respectively, at $100 \%$ specificity $[9,84,85]$. Fourth, there was substantial heterogeneity between study results. Subgroup analyses indicated that part of the variation in study results can be explained by method of diagnostic assessment (i.e., qualitative vs. quantitative). Differences in methodological quality according to QUADAS-2 could also be a source of heterogeneity, but comparison of the effects of risk of bias per domain (high vs. unclear vs. low) on diagnostic performance, yielded inconsistent results due to the small number of studies within most subgroups. In addition, we investigated the effect of studies with low methodological quality (e.g., high likelihood of bias) on overall diagnostic performance of MRI on per-patient and pernodal basis. We found that the study by Pannu et al. affects both the results of perpatient and per-nodal based analyses [56]. Nevertheless, the inclusion of this pioneering study did not alter the main conclusion of our meta-analysis.

Finally, only a relatively small number of studies met our inclusion criteria, limiting the power to retrieve statistically significant differences between the subgroups of studies and to estimate the diagnostic performance of mediastinal MRI more precisely. Thoracic MRI remains an innovative imaging technique but is rapidly gaining more interest and is increasingly being used for, e.g., studying lung cancer lymph node metastases.

The results of this meta-analysis suggest that MRI could be of additional value in the clinical diagnosis and selective treatment of regional lymph node metastasis in NSCLC on both per-patient and per-nodal basis. However, further validation of mediastinal MRI in relation to ${ }^{18} \mathrm{~F}$-FDG-PET/CT and cytology/histology is needed in prospective settings to elucidate its value in diagnosis and treatment of NSCLC. 


\section{SUMMARY STATEMENT}

This meta-analysis demonstrates high pooled sensitivities and specificities for the detection of hilar/mediastinal lymph node metastases with MRI in NSCLC, both on a per-patient and per-nodal basis.

\section{SUPPLEMENTARY DATA}
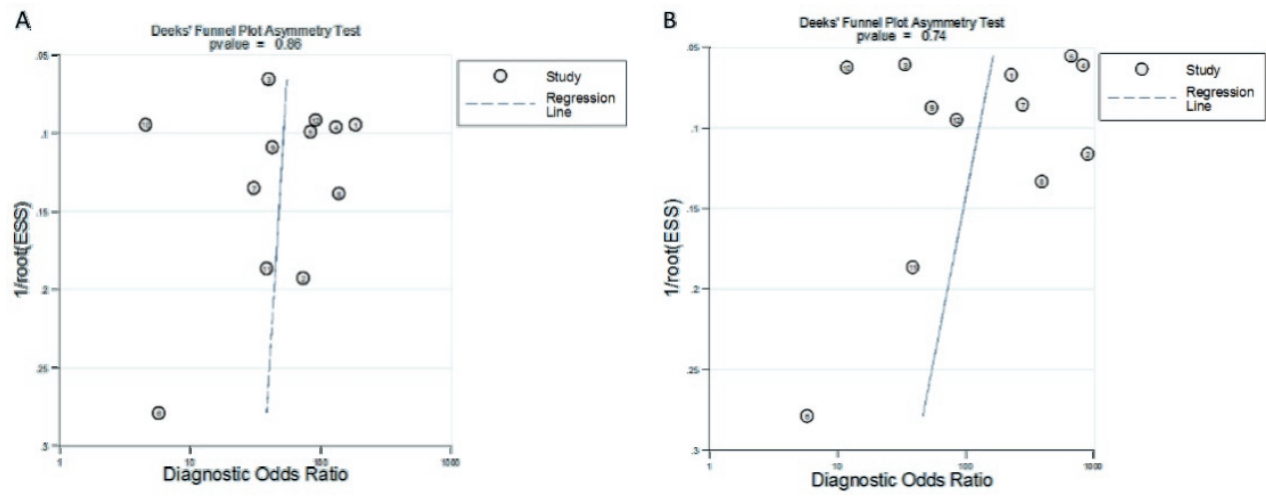

Figure A2.1: Deeks' funnel plot asymmetry test, as determined by linear regression of inverse root of effective sample size (ESS) plotted against diagnostic odds ratio (DOR) on per-patient basis (A) and on per-nodal basis (B). The non-significant regression coefficients indicate funnel plot symmetry $(\mathrm{P} \leq 0.10)$ (online only). 


\section{REFERENCES}

1. Ferlay J, Soerjomataram I, Ervik M, et al. GLOBOCAN 2012 v1.0, Cancer Incidence and Mortality Worldwide: IARC CancerBase No. 11. Available from URL: http://globocan.iarc.fr/ [accessed 24/04/2014].

2. Landelijke Werkgroep Longtumoren. Oncoline: Niet-kleincellig longcarcinoom: Landelijke richtlijn. Edition 2.2. Integraal Kankercentrum Nederland. Available from URL: http://oncoline.nl/niet-kleincelliglongcarcinoom [updated 10/07/2015, accessed 14/07/2015].

3. Chen L, Zhang J, Bao J, et al. Meta-analysis of diffusion-weighted MRI in the differential diagnosis of lung lesions. J Magn Reson Imaging. 2013;37: 1351-1358.

4. Tsim S, O'Dowd CA, Milroy R, Davidson S. Staging of non-small cell lung cancer (NSCLC): a review. Respir Med. 2010;104: 1767-1774.

5. Shankar LK, Sullivan DC. Functional imaging in lung cancer. J Clin Oncol. 2005;23: 3203-3211.

6. Vansteenkiste J, De Ruysscher D, Eberhardt WE, et al. Early and locally advanced non-small-cell lung cancer (NSCLC): ESMO Clinical Practice Guidelines for diagnosis, treatment and follow-up. Ann Oncol. 2013;24 Suppl 6: vi89-98.

7. Wu LM, Xu JR, Liu MJ, et al. Value of magnetic resonance imaging for nodal staging in patients with head and neck squamous cell carcinoma: a meta-analysis. Acad Radiol. 2012;19: 331-340.

8. Abdel Razek AA, Elkammary S, Elmorsy AS, Elshafey M, Elhadedy T. Characterization of mediastinal lymphadenopathy with diffusion-weighted imaging. Magn Reson Imaging. 2011;29: 167-172.

9. Walker CM, Chung JH, Abbott GF, et al. Mediastinal lymph node staging: from noninvasive to surgical. AJR Am J Roentgenol. 2012;199: W54-64.

10. Wu Y, Li P, Zhang H, et al. Diagnostic value of fluorine 18 fluorodeoxyglucose positron emission tomography/computed tomography for the detection of metastases in non-small-cell lung cancer patients. Int J Cancer. 2013;132: E37-47.

11. Devaraj A, Cook GJ, Hansell DM. PET/CT in non-small cell lung cancer staging-promises and problems. Clin Radiol. 2007;62: 97-108.

12. Rosenbaum SJ, Lind T, Antoch G, Bockisch A. False-positive FDG PET uptake-the role of PET/CT. Eur Radiol. 2006;16: 1054-1065.

13. Roberts PF, Follette DM, von Haag D, et al. Factors Associated With False-Positive Staging of Lung Cancer by Positron Emission Tomography. Ann Thorac Surg. 2000;70: 7.

14. Pauls S, Schmidt SA, Juchems MS, et al. Diffusion-weighted MR imaging in comparison to integrated [(1)(8)F]-FDG PET/CT for N-staging in patients with lung cancer. Eur J Radiol. 2012;81: 178-182.

15. Yeh DW, Lee KS, Han J, et al. Mediastinal nodes in patients with non-small cell lung cancer: MRI findings with PET/CT and pathologic correlation. AJR Am J Roentgenol. 2009;193: 813-821.

16. Luciani A, Itti E, Rahmouni A, Meignan M, Clement O. Lymph node imaging: basic principles. Eur J Radiol. 2006;58: 338-344.

17. Wunderbaldinger P. Problems and prospects of modern lymph node imaging. Eur J Radiol. 2006;58: 325337.

18. Lv Y, Yuan D, Wang K, et al. Diagnostic Performance of Integrated Positron Emission Tomography/Computed Tomography for Mediastinal Lymph Node Staging in Non-small Cell Lung Cancer: A Bivariate Systematic Review and Meta-Analysis. J Thorac Oncol. 2011;6: 9.

19. Liewald F, Große S, Storck M, et al. How Useful is Positron Emission Tomography for Lymphnode Staging in Non-Small-Cell Lung Cancer? Thorac Cardiov Surg. 2000;48: 4.

20. Schmidt GP, Baur-Melnyk A, Herzog P, et al. High-Resolution Whole-Body Magnetic Resonance Imaging Tumor Staging With the Use of Parallel Imaging Versus Dual-Modality Positron Emission TomographyComputed Tomography: Experience on a 32-Channel System. Invest Radiol. 2005;40: 743-753.

21. Wild JM, Marshall H, Bock M, et al. MRI of the lung (1/3): methods. Insights Imaging. 2012;3: 345-353.

22. Biederer J, Beer M, Hirsch W, et al. MRI of the lung (2/3). Why ... when ... how? Insights Imaging. 2012;3: 355-371.

23. Puderbach M, Hintze C, Ley S, Eichinger M, Kauczor HU, Biederer J. MR imaging of the chest: a practical approach at 1.5T. Eur J Radiol. 2007;64: 345-355.

24. Kauczor HU, Plathow C. Imaging tumour motion for radiotherapy planning using MRI. Cancer Imaging. 2006;6: S140-144. 
25. Regier M, Derlin T, Schwarz D, et al. Diffusion weighted MRI and 18F-FDG PET/CT in non-small cell lung cancer (NSCLC): does the apparent diffusion coefficient (ADC) correlate with tracer uptake (SUV)? Eur J Radiol. 2012;81: 2913-2918.

26. Weick S, Breuer FA, Ehses P, et al. DC-gated high resolution three-dimensional lung imaging during freebreathing. J Magn Reson Imaging. 2013;37: 727-732.

27. Liberati A, Altman DG, Tetslaff J, et al. The PRISMA Statement for Reporting Systematic Reviews and Meta-Analyses of Studies That Evaluate Health Care Interventions Explanation and Elaboration. Ann Intern Med. 2009;151.

28. Moher D, Liberati A, Tetzlaff J, Altman DG. Preferred Reporting Items for Systematic Reviews and MetaAnalyses: The PRISMA Statement. Ann Intern Med. 2009;151: 261-269.

29. O'Connor D, Green S, Higgins JP. Chapter 5: Defining the review question and developing criteria for including studies. In: Higgins JPT, Green S (editors), Cochrane Handbook of Systematic Reviews of Intervention. Version 5.1.0 (updated March 2011). The Cochrane Collaboration, 2011. Available from www.cochrane-handbook.org.

30. Bossuyt PM, Leeflang MMG. Chapter 6: Developing Criteria for Including Studies. In: Cochrane Handbook for Systematic Reviews of Diagnostic Test Accuracy Version 0.4 [updated September 2008]. The Cochrane Collaboration, 2008.

31. Ohno Y, Hatabu H, Takenaka D, et al. Metastases in Mediastinal and Hilar Lymph Nodes in Patients with Non-Small Cell Lung Cancer: Quantitative and Qualitative Assessment with STIR Turbo Spin-Echo MR imaging. Radiology. 2004;231: 8.

32. Takenaka D, Ohno Y, Hatabu H, et al. Differentiation of metastatic versus non-metastatic mediastinal lymph nodes in patients with non-small cell lung cancer using respiratorytriggered short inversion time inversion recovery (STIR) turbo spinecho MR imaging. Eur J Radiol. 2002;44: 9.

33. Whiting PF, Rutjes AWS, Westwood ME, et al. QUADAS-2: A Revised Tool for the Quality Assessment of Diagnostic Accuracy Studies. Ann Intern Med. 2011;155: 529-536.

34. Reitsma JB, Rutjes AWS, Whiting P, Vlassov VV, Leeflang MMG, Deeks JJ. Chapter 9: Assessing methodological quality. In: Deeks JJ, Bossuyt PM, Gatsonis C (editors), Cochrane Handbook for Systematic Reviews of Diagnostic Test Accuracy Version 1.0.0. The Cochrane Collaboration, 2009. Available from: http://srdta.cochrane.org/.

35. Deeks JJ, Wisniewski S, Davenport C. Chapter 4: Guide to the contents of a Cochrane Diagnostic Test Accuracy Protocol. In: Deeks JJ, Bossuyt PM, Gatsonis C (editors), Cochrane Handbook for Systematic Reviews of Diagnostic Test Accuracy Version 1.0.0. The Cochrane Collaboration, 2013. Available from: http://srdta.cochrane.org/.

36. Gloria C, Li Q, Xu L, Zhang W. Differentiation of diffusion coefficients to distinguish malignant and benign tumor. J Xray Sci Technol. 2010;18: 235-249.

37. Koh DM, Collins DJ. Diffusion-weighted MRI in the body: applications and challenges in oncology. AJR Am J Roentgenol. 2007;188: 1622-1635.

38. Rutter CM, Gatsonis CA. A hierarchical regression approach to meta-analysis of diagnostic test accuracy evaluations. Stat Med. 2001;20: 2865-2884.

39. Harbord RM, Whiting P, Sterne JA, et al. An empirical comparison of methods for meta-analysis of diagnostic accuracy showed hierarchical models are necessary. J Clin Epidemiol. 2008;61: 1095-1103.

40. Macaskill P, Gatsonis CA, Deeks JJ, Harbord RM, Y. T. Chapter 10: Analysing and Presenting Results. In: Deeks JJ, Bossuyt PM, Gatsonis C (editors), Cochrane Handbook for Systematic Reviews of Diagnostic Test Accuracy Version 1.0. The Cochrane Collaboration, 2010. Available from: http://srdta.cochrane.org/.

41. Thompson SG, Higgins JP. How should meta-regression analyses be undertaken and interpreted? Stat Med. 2002;21: 1559-1573.

42. Deeks JJ, Macaskill P, Irwig L. The performance of tests of publication bias and other sample size effects in systematic reviews of diagnostic test accuracy was assessed. J Clin Epidemiol. 2005;58: 882-893.

43. Lijmer JG, Bossuyt PM, Heisterkamp SH. Exploring sources of heterogeneity in systematic reviews of diagnostic tests. Stat Med. 2002;21: 1525-1537.

44. Bossuyt PM, Davenport C, Deeks JJ, Hyde C, Leeflang MMG, Scholten R. Chapter 11: Interpreting results and drawing conclusions. In: Deeks JJ, Bossuyt PM, Gatsonis C (editors), Cochrane Handbook for Systematic Reviews of Diagnostic Test Accuracy: The Cochrane Collaboration, 2013. 
45. Zamora J, Abraira V, Muriel A, Khan K, Coomarasamy A. Meta-DiSc: a software for meta-analysis of test accuracy data. BMC Med Res Methodol. 2006;6: 31.

46. Harbord RM, Whiting P. Metandi: Meta-analysis of diagnostic accuracy using hierarchical logistic regression. The Stata Journal. 2009;9: 211-229.

47. Dwamena BA, Sylvester R, Carlos RC. Midas: A program for Meta-analytical Integration of Diagnostic Accuracy Studies in Stata. Ann Arbor. 2007;MI: Division of Nuclear Medicine, Department of Radiology, University of Michigan Medical School.

48. Chen W, Jian W, Li HT, et al. Whole-body diffusion-weighted imaging vs. FDG-PET for the detection of non-small-cell lung cancer. How do they measure up? Magn Reson Imaging. 2010;28: 613-620.

49. Hasegawa I, Eguchi K, Kohda E, et al. Pulmonary hilar lymph nodes in lung cancer: assessment with 3Ddynamic contrast-enhanced MR imaging. Eur J Radiol. 2003;45: 129-134.

50. Kim HY, Yi CA, Lee KS, et al. Nodal Metastasis in Non-Small Cell Lung Cancer: Accuracy of 3.0-T MR Imaging. Radiology. 2008;246: 596-604.

51. Morikawa M, Demura Y, Ishizaki T, et al. The effectiveness of 18F-FDG PET/CT combined with STIR MRI for diagnosing nodal involvement in the thorax. J Nucl Med. 2009;50: 81-87.

52. Nakayama J, Miyasaka K, Omatsu T, et al. Metastases in Mediastinal and Hilar Lymph Nodes in Patients With Non-Small Cell Lung Cancer: Quantitative Assessment With Diffusion-Weighted Magnetic Resonance Imaging and Apperent Diffusion Coefficient. J Comput Assist Tomogr. 2010;34: 8.

53. Nomori H, Mori T, Ikeda K, et al. Diffusion-weighted magnetic resonance imaging can be used in place of positron emission tomography for $\mathrm{N}$ staging of non-small cell lung cancer with fewer false-positive results. J Thorac Cardiovasc Surg. 2008;135: 816-822.

54. Ohno Y, Koyama H, Nogami M, et al. STIR turbo SE MR imaging vs. coregistered FDG-PET/CT: quantitative and qualitative assessment of N-stage in non-small-cell lung cancer patients. J Magn Reson Imaging. 2007;26: 1071-1080.

55. Ohno Y, Koyama H, Yoshikawa T, et al. N stage Disease in Patients with Non-Small Cell Lung Cancer: Efficacy of Quantitative and Qualitative Assessment with STIR Turbo Spin-Echo Imaging, Diffusionweighted MR Imaging, and Fluorodeoxyglucose PET/CT. Radiology. 2011;261: 605-615.

56. Pannu H, Wang K, Borman TL, Bluemke DA. MR Imaging of Mediastinal Lymph Nodes: Evaluation Using a Superparamagnetic Contrast Agent. J Magn Reson Imaging. 2000;12: 899-904.

57. Usuda K, Sagawa M, Motono N, et al. Advantages of diffusion-weighted imaging over positron emission tomography-computed tomography in assessment of hilar and mediastinal lymph node in lung cancer. Ann Surg Oncol. 2013;20: 1676-1683.

58. Walter SD. Properties of the summary receiver operating characteristic (SROC) curve for diagnostic test data. Stat Med. 2002;21: 1237-1256.

59. Nair A, Klusmann MJ, Jogeesvaran KH, Grubnic S, Green S, Vlahos I. Revisions to the TNM Staging of Non-Small Cell Lung Cancer: Rationale, Clinicoradiologic Implications, and Persistent Limitations. Radiographics. 2011;31: 215-238.

60. Imai K, Minamiya Y, Saito H, et al. Diagnostic imaging in the preoperative management of lung cancer. Surg Today. 2014;44: 1197-1206.

61. Sommer G, Wiese M, Winter L, et al. Preoperative staging of non-small-cell lung cancer: comparison of whole-body diffusion-weighted magnetic resonance imaging and 18F-fluorodeoxyglucose-positron emission tomography/computed tomography. Eur Radiol. 2012;22: 2859-2867.

62. Wynants J, Stroobants S, Dooms C, Vansteenkiste J. Staging of lung cancer. Radiol Clin North Am. 2007;45: 609-625.

63. Kim HS, Lee KS, Ohno Y, van Beek EJ, Biederer J. PET/CT versus MRI for diagnosis, staging, and followup of lung cancer. J Magn Reson Imaging. 2014.

64. Ohno Y. New applications of magnetic resonance imaging for thoracic oncology. Semin Respir Crit Care Med. 2014;35: 27-40.

65. Zhao L, He ZY, Zhong XN, Cui ML. (18)FDG-PET/CT for detection of mediastinal nodal metastasis in non-small cell lung cancer: a meta-analysis. Surg Oncol. 2012;21: 230-236.

66. Wu LM, Xu JR, Gu HY, et al. Preoperative mediastinal and hilar nodal staging with diffusion-weighted magnetic resonance imaging and fluorodeoxyglucose positron emission tomography/computed tomography in patients with non-small-cell lung cancer: which is better? J Surg Res. 2012;178: 304-314. 
67. Webb WR, Sarin M, Zerhouni EA, Heelan RT, Glazer GM, Gatsonis C. Interobserver variability in CT and MR staging of lung cancer. J Comput Assist Tomogr. 1993;17: 841-846.

68. Koyama H, Ohno Y, Kono A, et al. Quantitative and qualitative assessment of non-contrast-enhanced pulmonary MR imaging for management of pulmonary nodules in 161 subjects. Eur Radiol. 2008;18: 21202131.

69. Koyama H, Ohno Y, Aoyama N, et al. Comparison of STIR turbo SE imaging and diffusion-weighted imaging of the lung: capability for detection and subtype classification of pulmonary adenocarcinomas. Eur Radiol. 2010;20: 790-800.

70. Klerkx WM, Bax L, Veldhuis WB, et al. Detection of lymph node metastases by gadolinium-enhanced magnetic resonance imaging: systematic review and meta-analysis. J Natl Cancer Inst. 2010;102: 244-253.

71. Li B, Li Q, Chen C, Guan Y, Liu S. A systematic review and meta-analysis of the accuracy of diffusionweighted MRI in the detection of malignant pulmonary nodules and masses. Acad Radiol. 2014;21: 21-29.

72. Will O, Purkayastha S, Chan C, et al. Diagnostic precision of nanoparticle-enhanced MRI for lymph-node metastases: a meta-analysis. The Lancet Oncology. 2006;7: 52-60.

73. Kurland BF, Gerstner ER, Mountz JM, et al. Promise and pitfalls of quantitative imaging in oncology clinical trials. Magn Reson Imaging. 2012;30: 1301-1312.

74. Koh DM, Blackledge M, Padhani AR, et al. Whole-body diffusion-weighted MRI: tips, tricks, and pitfalls. AJR Am J Roentgenol. 2012;199: 252-262.

75. De Leyn P, Lardinois D, Van Schil PE, et al. ESTS guidelines for preoperative lymph node staging for nonsmall cell lung cancer. Eur J Cardiothorac Surg. 2007;32: 1-8.

76. De Ruysscher D, Wanders S, van Haren E, et al. Selective mediastinal node irradiation based on FDG-PET scan data in patients with non-small-cell lung cancer: a prospective clinical study. Int J Radiat Oncol Biol Phys. 2005;62: 988-994.

77. Koch N, Liu HH, Olsson LE, Jackson EF. Assessment of geometrical accuracy of magnetic resonance images for radiation therapy of lung cancers. J Appl Clin Med Phys. 2003;4: 352-364.

78. Blackall JM, Ahmad S, Miquel ME, McClelland JR, Landau DB, Hawkes DJ. MRI-based measurements of respiratory motion variability and assessment of imaging strategies for radiotherapy planning. Phys Med Biol. 2006;51: 4147-4169.

79. Lambregts DM, Beets GL, Maas M, et al. Accuracy of gadofosveset-enhanced MRI for nodal staging and restaging in rectal cancer. Ann Surg. 2011;253: 539-545.

80. Schipper R, Smidt ML, van Roozendaal LM, et al. Noninvasive Nodal Staging in Patients With Breast Cancer Using Gadofosveset-Enhanced Magnetic Resonance Imaging: A Feasibility Study. Invest Radiol. 2013;48: 134-139.

81. Yamashita T, Takahara T, Kwee TC, et al. Diffusion magnetic resonance imaging with gadofosveset trisodium as a negative contrast agent for lymph node metastases assessment. Jpn J Radiol. 2011;29: 2532.

82. Plathow C, Walz M, Lichy MP, et al. [Cost considerations for whole-body MRI and PET/CT as part of oncologic staging]. Der Radiologe 2008; 48(4): 384-396.

83. Kauczor HU, Ley S. Thoracic Magnetic Resonance Imaging 1985 to 2010. J Thorac Imaging. 2010;25: 34-38.

84. Herth FJ. Nonsurgical staging of the mediastinum: EBUS and EUS. Semin Respir Crit Care Med. 2011;32: 62-68.

85. Zhang R, Ying K, Shi L, Zhang L, Zhou L. Combined endobronchial and endoscopic ultrasound-guided fine needle aspiration for mediastinal lymph node staging of lung cancer: a meta-analysis. Eur J Cancer. 2013;49: 1860-1867. 


\section{Chapter 3}

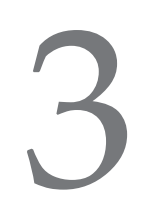

\section{Performing clinical ${ }^{18}$ F-FDG-PET/MR imaging of the mediastinum using a dedicated, patient friendly protocol}

Authors:

J. Peerlings, L. Paulis, C. Mitea, F. C. Bakers, M. Berbée, R. Wierts, S. Vöö, J. E. Wildberger, A. L. Hoffmann, P. Lambin, F. M. Mottaghy

Adapted from:

Nuclear Medicine Communications Article in review 


\section{ABSTRACT}

\section{OBJECTIVE}

To construct a mediastinal-specific ${ }^{18} \mathrm{~F}-\mathrm{FDG}-\mathrm{PET} / \mathrm{MR}$ protocol containing high-quality MR-images with minimal acquisition-time, and comparable diagnostic performance to ${ }^{18} \mathrm{~F}-\mathrm{FDG}-\mathrm{PET} / \mathrm{CT}$.

\section{METHODS}

Fifteen healthy subjects and ten patients with mediastinal malignancies (8 non-small cell lung cancer, 2 oesophageal cancer) received ${ }^{18} \mathrm{~F}-\mathrm{FDG}-$ $\mathrm{PET} / \mathrm{MR}$ imaging immediately after ${ }^{18} \mathrm{~F}-\mathrm{FDG}-\mathrm{PET} / \mathrm{CT}$. Sequences volume interpolated breath-hold examination (T1-VIBE) and Half-Fourier acquisition single-shot turbo spin echo (T2-HASTE) were optimized by varying the parameters: breath-hold (BH, end-expiration), fat suppression (SPAIR), and electrocardiogram-triggering (ECG, in end-diastole). Image quality (IQ) of each sequence-variation was qualitatively scored by medical experts and quantitatively assessed by calculating signal-to-noise ratios (SNR), contrast relative to muscletissue (CR), standardized-uptake-values (SUVs), and tumour-to-blood ratios (TBRs). Differences in CR determined contrast between adjacent tissues and tumour visibility. Patient comfort was evaluated on patients' experience. Diagnostic accuracy of ${ }^{18} \mathrm{~F}$-FDG-PET/MRI was compared to ${ }^{18} \mathrm{~F}-\mathrm{FDG}-\mathrm{PET} / \mathrm{CT}$, with reference to clinical reports and histo-/cytopathological analyses.

\section{RESULTS}

Quantitative analysis showed that ECG-triggering T1-VIBE images presented highest SNR $(\mathrm{p}<0.01)$ and largest differences in $\mathrm{CR}$ between mediastinal soft-tissues, regardless of breath-hold or free-breathing acquisition. Qualitative IQ of ECG-triggered T1-VIBE scans in BH were scored qualitatively highest with good reader agreement $(\kappa=0.62)$. Quantitative and qualitative IQ of T2-HASTE was not significantly affected by breath-hold acquisition ( $p>0.9)$. Qualitative IQ of both T1VIBE and T2-HASTE deteriorated after SPAIR fat-suppression. Patients could maintain breath-hold and reported no discomfort. Diagnostic performance of ${ }^{18} \mathrm{~F}$-FDG-PET/MR was not significantly different from ${ }^{18} \mathrm{~F}-\mathrm{FDG}-\mathrm{PET} / \mathrm{CT}$ with comparable staging, SUVs, and TBRs.

\section{CONCLUSION}

ECG-triggered T1-VIBE sequences acquired during short, multiple breath-holds are recommended for mediastinal imaging using ${ }^{18} \mathrm{~F}-\mathrm{FDG}$ PET/MR. With dedicated protocols, ${ }^{18}$ F-FDG-PET/MRI could be implemented in thoracic oncology and aid in diagnostic evaluation, tailored treatment decision-making and personalized patientcare. 


\section{INTRODUCTION}

Hybrid imaging, combining positron emission tomography (PET) with either computed tomography (i.e., PET/CT) or magnetic resonance imaging (i.e., PET/MRI), has been implemented in a variety of oncological management steps [1,2]. In thoracic oncological imaging, PET/CT is considered to be the current clinical standard. Nevertheless, an additional MR scan is often needed to clarify equivocal findings, detect tumour infiltration, and differentiate metastatic lymph nodes (LN) [3-5]. It was generally anticipated that with the development of integrated PET/MR systems, the diagnostic accuracy would improve for these situations. However, a clear clinical improvement of whole-body ${ }^{18} \mathrm{~F}-\mathrm{FDG}-\mathrm{PET} / \mathrm{MR}$ over ${ }^{18} \mathrm{~F}-\mathrm{FDG}$-PET/CT has yet to be determined in oncology [6]. Potentially, an alternative approach using region-specific instead of whole-body ${ }^{18} \mathrm{~F}-\mathrm{FDG}-\mathrm{PET} / \mathrm{MRI}$-protocols could lead to more insight in the efficient application and further integration of PET/MR in thoracic oncological imaging. Here, PET-images could be used as the leading diagnostic tool by detecting FDG-avid lesions with high sensitivity, while MRimages of FDG-positive regions would be better suited as the matching tool to determine the underlying pathological correlate (e.g., tumour infiltration, mediastinal LN involvement, inflammation).

For an efficient patient workflow, PET/MR protocol development rests on three fundamental pillars: (1) producing clinically valuable images with high image quality (IQ) and diagnostic accuracy, (2) implementing time-efficient protocols by simultaneous acquisition of PET and MR images, (3) efficient acquisition of complementary, non-redundant data [7]. For successful mediastinal-specific protocols, also MR-artefacts related to respiratory and cardiac motion need to be minimized using e.g., cardiac triggering and breath-hold (BH) techniques, while preserving patient comfort. However, patients with non-small cell lung cancer (NSCLC) often present dyspnoea and may thus find it problematic to maintain a $\mathrm{BH}$ for a certain period of time. Therefore, MRI sequences have to be tailored to this specific patient population.

The goal of this study was to obtain a mediastinal-specific clinical ${ }^{18 F-F D G-P E T / M R ~}$ protocol containing dedicated MRI-sequences able to produce high-quality images with optimal patient compliance and diagnostic performance comparable to ${ }^{18}$ F-FDG-PET/CT. The different T1-/T2-sequences will not be compared with each other. 


\section{MATERIAL \& METHODS}

\section{STUDY POPULATION}

Between June 2016 and February 2017, 25 participants were included for PET/MR imaging after approval by the local Ethics Review Committee (METC 16-4-036). Fifteen adult, healthy volunteers were included to optimize clinical MRI pulsesequences for imaging mediastinal structures without acquiring PET-images. Next, 10 patients suspected of having mediastinal malignancies (NSCLC $n=8$, oesophageal cancer; EC $n=2$ ) were included to validate the optimized protocol. All patients received a conventional ${ }^{18} \mathrm{~F}-\mathrm{FDG}$-PET/CT scan for primary staging, immediately followed by a dedicated ${ }^{18}$ F-FDG-PET/MRI scan.

\section{IMAGE ACQUISITION}

${ }^{18}$ F-FDG-PET/CT imaging

Whole-body PET images were obtained on a Gemini TF 64 scanner (Philips Healthcare, NL) using the default manufacturer's time-of-flight reconstruction algorithm (BLOB-OS-TF). The injected dose of ${ }^{18} \mathrm{~F}-\mathrm{FDG}$ was $2 \mathrm{MBq} / \mathrm{kg}(162 \pm 34 \mathrm{MBq})$ and administered after patients fasted for four hours. CT images of $4 \mathrm{~mm}$ thickness were acquired at $120 \mathrm{kV}$ and $30 \mathrm{mAs}$.

\section{${ }^{18}$ F-FDG-PET/MR imaging}

Mediastinal PET/MRI was performed on a 3-Tesla Magnetom Biograph mMR scanner using two body coils. ${ }^{18 F-F D G-P E T ~ d a t a ~ w a s ~ a c q u i r e d ~ i n ~ l i s t-m o d e ~ f o r ~}$ $15 \mathrm{~min}$ at one bed-position. PET-images were reconstructed using a 3D-iterative ordinary Poisson ordered-subsets expectation maximization (OP-OSEM3D) reconstruction algorithm (3 iterations, 21 subsets, $4 \mathrm{~mm}$ Gaussian smoothing) and fused with MR-images using Syngo.via software (Siemens Healthcare, GER).

The dedicated PET/MR-protocol was centred over the carina and included a standard localizer sequence, followed by a 3D T1-DIXON-VIBE sequence for MRbased attenuation correction. Based on literature and preliminary evaluation of clinically available sequences, two sequences were selected for further optimisation for tumour imaging: 1) T1-VIBE (T1-weighted Volumetric Interpolated 3D Gradient Echo) and 2) T2-HASTE (T2-weighted Half Fourier Acquisition Single Shot Turbo Spin Echo) (Supplementary Table S3.1) [8-10]. Sequences were optimised to acquire transversal images of high spatial resolution (in-plane resolution $<1.6 \mathrm{~mm}$ for LN detection) within a reasonable acquisition time [11].

\section{Variations of mediastinal-specific MR scanning}

Images were acquired using all combinations of MR settings: (1) free-breathing (FB) or BH acquisition, (2) with or without electrocardiogram (ECG)-triggering, (3) with or without fat suppression. 
Breath-hold scans were performed in multiple sessions of 4 times 5.7s for T1-VIBE and 7 times 8.1s for T2-HASTE. ECG-triggering was used to synchronize MR signal acquisition to the cardiac cycle by real-time R-wave detection. However, ECGtriggering is only effective when images were acquired during the diastolic phase with minimal pulsatile motion. Effective ECG-triggering in diastole was not possible within the desired acquisition time for T2-HASTE and fat-suppressed T1-VIBE using spectral adiabatic inversion recovery (SPAIR), and thus has not been evaluated for these sequence-variants.

\section{Data AnAlysis}

\section{Quantitative image analysis}

Volumes-of-interest (VOIs) were established by delineating mediastinal structures (i.e., adipose tissue, muscles, bone, oesophagus, lung, and liver) and tumorous lesions. All VOIs had the same size of $1.6 \mathrm{~cm}^{3}$ and were placed over 3 slices around the level of the carina. To quantify IQ, signal-to-noise ratio (SNR) and contrast ratio relative to muscle (CR) were calculated using Equations 1 and 2, respectively.

$$
\begin{aligned}
& \text { (1) } \quad S N R=\frac{S I_{\text {Tissue }}}{0.655 * S D_{\text {Noise }}} \\
& \text { (2) } \quad C R=\frac{S I_{\text {Tissue }}}{S I_{\text {Muscle }}}
\end{aligned}
$$

SITissue and SIMuscle represented the measured signal intensities of delineated tissues and muscle, respectively [12]. The Rayleigh-distribution in noise outside the body was accounted for in the standard deviation (SDNoise) [12]. Tissue visibility and contrast between adjacent tissues was quantified by differences in $\mathrm{CR}$ of aforementioned tissues $(\triangle \mathrm{CR})$

On ${ }^{18}$ F-FDG-PET/MR images, FDG-avid lesions were manually delineated and maximal and mean standard uptake values (SUVs) were obtained. Tumour-to-blood ratios (TBRs) were calculated from ${ }^{18}$ F-FDG-activity of delineated lesions and aortic blood.

\section{Qualitative image analysis}

Two clinical experts in thoracic oncological imaging (radiologist F.B, and radiationoncologist M.B.) independently rated the MR image quality using a 5-point scoring scale (Supplementary Table S3.2). A score of 1 represented poor IQ, not suitable for clinical use, while a score of 5 represented high IQ. 


\section{CLINICAL VALIDATION}

\section{Patient compliance and comfort}

Patient compliance was based on the ability to follow and maintain BH instructions at end-expiration and the ability to remain lying still during scanning. Comfort during scanning was evaluated by enquiring the emotional status of all participants (feelings of stress, claustrophobia, physical discomfort from laying still or acoustic noise) and their perception of scan-time (BH duration, resting periods between $\mathrm{BHs,}$ and total scan duration).

\section{Diagnostic performance}

While blinded, independent reviewers diagnostically evaluated all ${ }^{18} \mathrm{~F}-\mathrm{FDG}$-PET/MRI and 18F-FDG-PET/CT images according to the AJCC/UICC TNM-system with corresponding stage grouping ( $7^{\text {th }}$ edition). M-stage was evaluated on whole-body PET/CT and on thoracic PET/MR images. Clinical reports with diagnoses based on PET/CT and histo-/cytopathologic analyses after mediastinoscopy or ultrasoundguided transbronchial needle aspiration were obtained as part of standard clinical care and served as the golden standard for further reference. All PET/MR image analyses were performed using OsiriX software (Pixmeo SARL, SUI).

\section{STATISTICAL ANALYSIS}

Firstly, for protocol-optimisation statistical differences between volunteer and patient data in SNR of normal tissue was tested using a paired Student T-test. Populations were pooled when no significant differences were apparent. Secondly, differences in SNR and CR between sequence-variations were tested for statistical significance using 2-way ANOVA with Tukey correction, respectively. Thirdly, differences in CR between tumour/LN and adjacent tissue were statistically tested per sequence-variation using 2-way ANOVA with Tukey correction to quantify contrast between tissues, and tumour visibility, relative to muscle-tissue. Finally, reader agreement in qualitative image assessment was analysed using weighted Kappa reliability statistics $(\kappa<0.2=$ slight agreement, $\quad \kappa=0.2-0.4=$ fair, $\quad \kappa=0.41$ $0.6=$ moderate, $\kappa=0.6-0.8=$ good, $\kappa>0.8=$ excellent).

For clinical validation in patients, we first tested the hypothesis that there is no statistically significant difference in SUV Mean, SUV $\mathrm{Max}_{\text {, }}$ and TBR acquired on PET/MRI and PET/CT using a paired Student $T$-test. The diagnostic performances of PET/MR and PET/CT (i.e., TNM staging) were compared in terms of sensitivity and specificity using a McNemar test. All statistical analyses were performed using GraphPad Prism version 6.01 (GraphPad, USA). P-values $<0.05$ were considered statistically significant. 


\section{RESULTS}

\section{MR-PROTOCOL OPTIMIZATION FOR MEDIASTINAL IMAGING}

\section{Quantitative image analyses}

Figure 3.1 present SNRs of various tissues for each sequence-variation of T1-VIBE and T2-HASTE. For T1-VIBE and T2-HASTE, SNRs of healthy subjects and patients were not significantly different $(\mathrm{p}=0.52, \mathrm{p}=0.065)$. In the pooled population of volunteer and patient data, there were no statistically significant differences between T1-VIBE sequences acquired in BH and FB ( $p>0.05)$. However, the addition of ECG-triggering to either T1-VIBE BH or FB sequence-variations resulted in significantly greater SNR for all soft-tissue structures of the mediastinum $(p \leq 0.001)$ But did not significantly affect SNR of bone structures ( $\mathrm{p}=0.76, \mathrm{p}=0.73$ ) and lung tissue ( $p \geq 0.99, p=0.998$ ), respectively (Fig. 3.1a). The addition of SPAIR to T1-VIBE $\mathrm{BH}$ or T1-VIBE FB did not result in statistically significant differences in SNR ( $\mathrm{p}>0.1)$. In tumorous lesions, SNR was only significantly different between T1-VIBE BH with and without ECG-triggering $(\mathrm{p}=0.030)$. In FDG-avid LN, no significant difference was found between T1-VIBE sequence-variants ( $p>0.05)$.

In T2-HASTE images, SNR was unaffected by breath-hold compared to free breathing for all tissues ( $p>0.97$ ). The addition of SPAIR fat-saturation to T2-HASTE $\mathrm{BH}$ and T2-HASTE FB significantly reduced quantitative SNR in adipose tissue $(\mathrm{p} \leq 0.0001, \mathrm{p} \leq 0.0001)$ and bone $(\mathrm{p}=0.0236, \mathrm{p}=0.0197)$, but remained unaffected for all other tissues

Contrast within images indicated the ability to differentiate various tissues from SIMuscle (Fig. 3.2). CR-values were not significantly different between T1-VIBE and between T2-HASTE sequence-variants ( $>0.9$ ), except between sequences acquired with and without SPAIR fat-saturation $(p<0.0001)$. However, within T1-VIBE sequence-variants contrast between adjacent tissues $(\triangle C R)$ was significant between oesophagus, lung, and fat ( $\mathrm{x} \leq 0.0001)$, between lung, bone, and liver $(\mathrm{p} \leq 0.0001)$, and between tumour and fat $(\mathrm{p} \leq 0.0001)$. In ECG-triggered T1-VIBE sequences, $\triangle \mathrm{CR}$ was also significant between oesophagus and bone $(\mathrm{p}<0.05)$, and between tumour and lung $(\mathrm{p}<0.05)$. The addition of SPAIR did not result in additional significant results of $\triangle \mathrm{CR}$. For T2-HASTE BH and T2-HASTE FB, $\triangle \mathrm{CR}$ was significant between lung, oesophagus ( $\mathrm{p}=0.013, \mathrm{p}=0.008)$, and bone $(\mathrm{p}=0.0008, \mathrm{p}=0.0012)$ and between fat and all other tissues $(p \leq 0.0001)$. When fat was suppressed by SPAIR, no significant differences in contrast were observed. In addition, differences in SNR and CR can be appreciated and the visibility of mediastinal tissues and cancerous lesions can be deduced for each T1-VIBE sequence-variant (Fig. 3.2). 

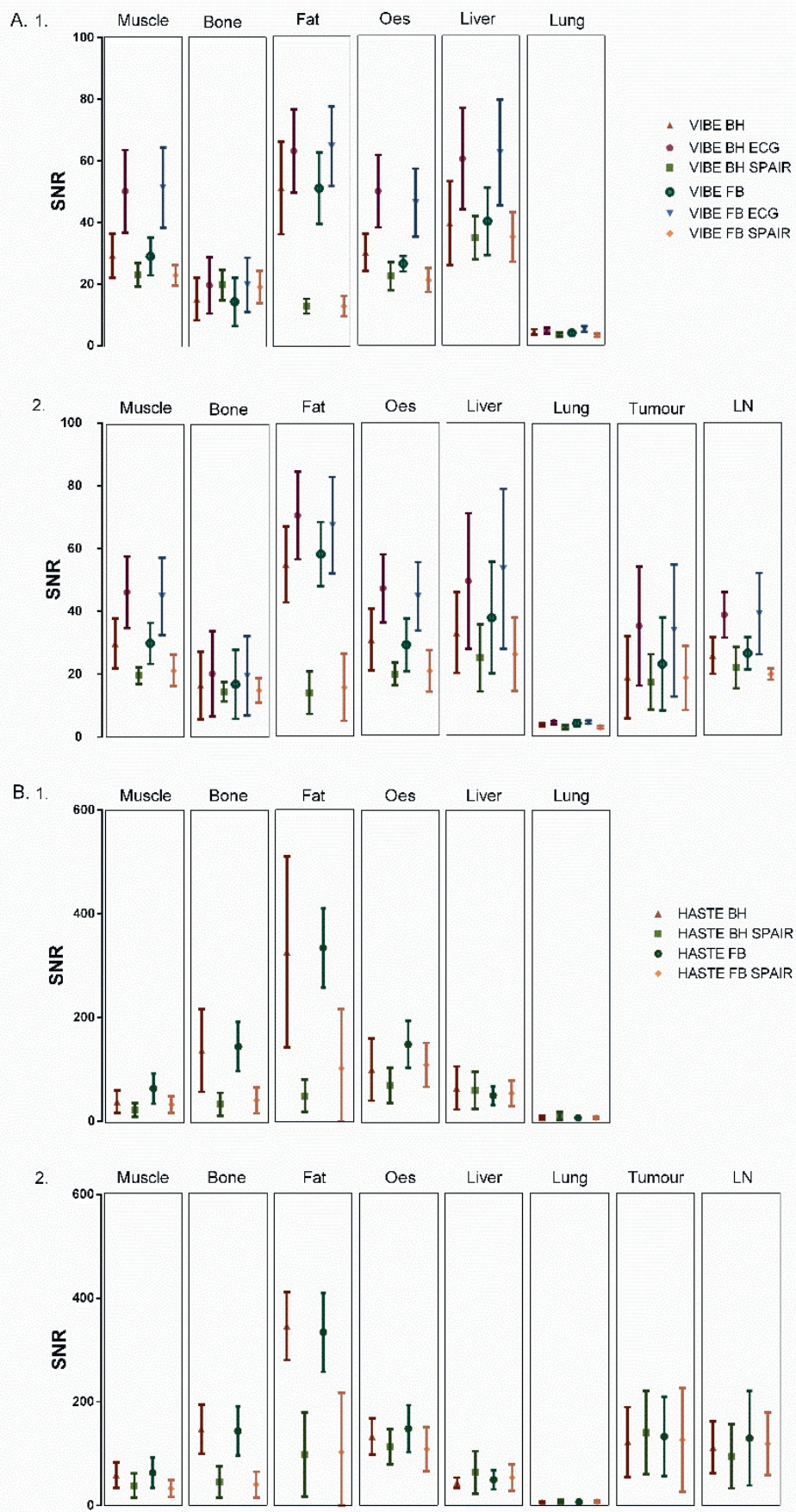

Figure 3.1: Quantitative analyses of T1-VIBE (a) and T2-HASTE (b) image quality acquired in healthy volunteers (1) and cancer patients (2) by assessment of signal-to-noise ratio (SNR) per tissue-type over different sequences. VOIs were drawn over target regions and backgroundregions located outside the body 
Tumour visibility was highest in ECG-triggered T1-VIBE sequences acquired in $\mathrm{BH}$ ( $S N R=62.6, C R=0.86)$, followed closely by T1-VIBE ECG FB (SNR=59.2, CR=0.83). However, overall IQ and visibility of all mediastinal structures is determined by the balance of SNR and $\triangle \mathrm{CR}$, which is demonstrated by the spread between data-points and the slope of the plots in figure 3.2. Visibility of all mediastinal structures was lower in T1-VIBE sequences acquired without ECG-triggering and further decreased in fat-saturated sequences, which presented more clustering of data-points and a lower slope. For T2-HASTE sequences, an acceptable distribution of SNR-CR data points was noted (Supplementary Figure S3.1). However, no differences between sequence-variants were evident.

\section{Qualitative image analyses}

T1-VIBE and T2-HASTE image quality was scored with good inter-reader agreement ( $\kappa=0.617, \kappa=0.625$ respectively) (Table 3.1, Fig. 3.3). For T1-VIBE, qualitative IQ was scored highest for images acquired during $\mathrm{BH}$ and with ECG-triggering, and contained a homogeneous distribution of SI within VOIs and visually less noise. T1VIBE BH ECG showed clear organ-edge detection with low incidences of artefacts at the mediastinum. In free-breathing ECG-triggered T1-VIBE images more motion artefacts were present at the mediastinum, leading to qualitatively moderate IQ.

Generally, the IQ of T2-HASTE images was scored lower than T1-VIBE images. Moderate IQ was registered for both T2-HASTE BH and FB sequencevariants with moderate contouring of mediastinal structures. However, a notable increase in IQ of T2-HASTE was perceived towards abdominal regions (e.g., gastroesophageal junction, liver).

For both T1-VIBE and T2-HASTE, SPAIR adequately suppressed fat near the mediastinum. However, the addition of SPAIR visually increased image noise and led to poor IQ, making it difficult to detect and differentiate mediastinal structures. 

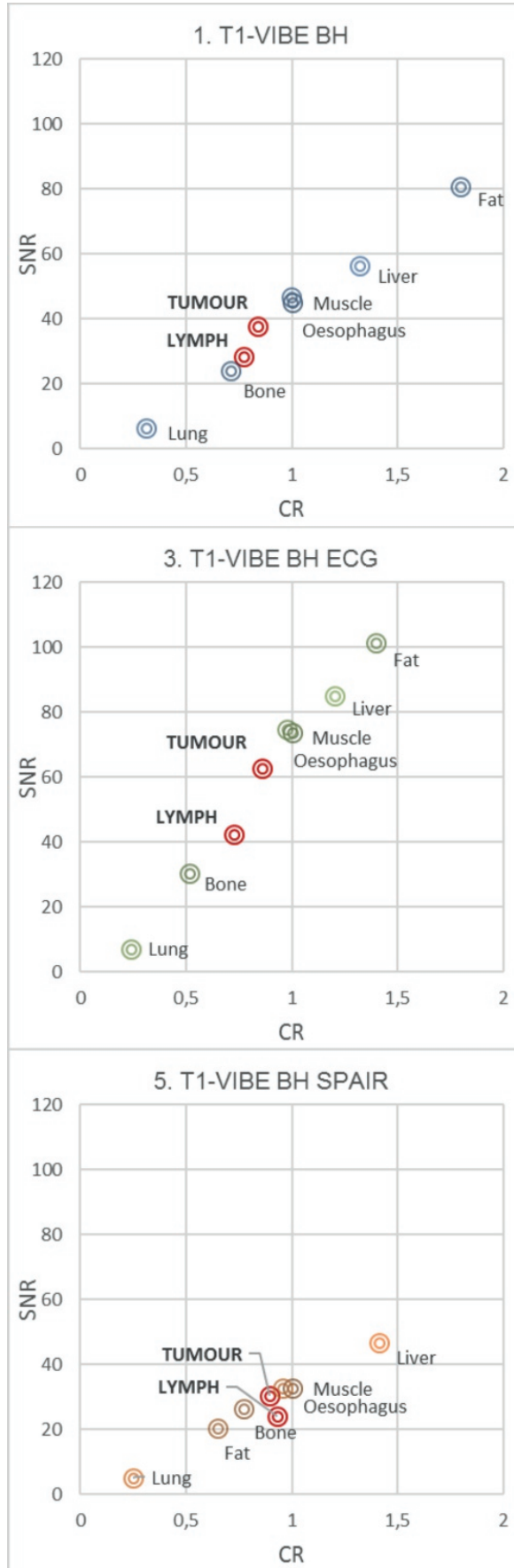

2. T1-VIBE FB

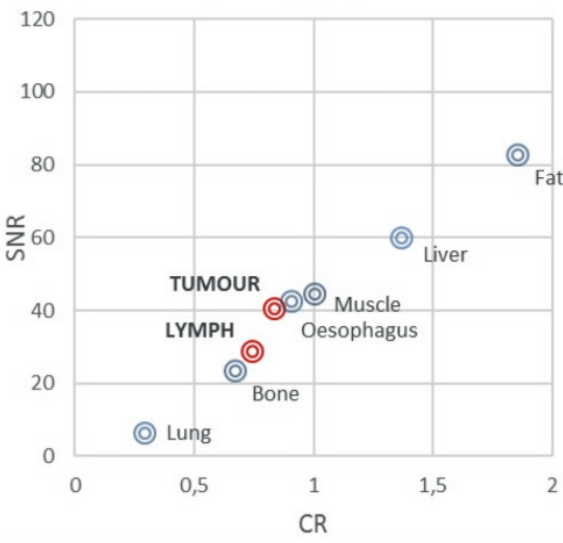

4. T1-VIBE FB ECG

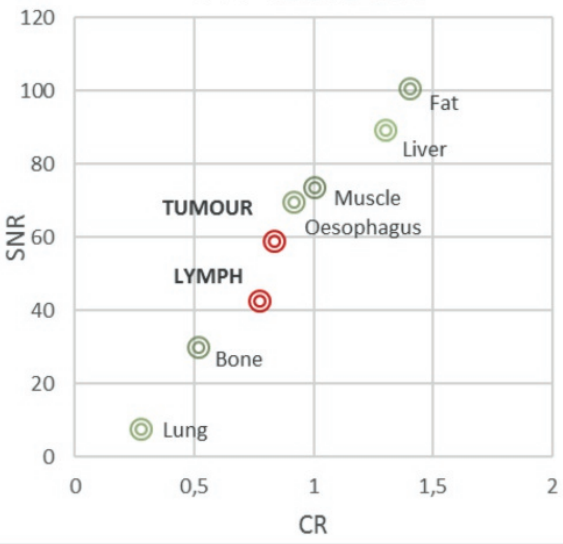

6. T1-VIBE FB SPAIR

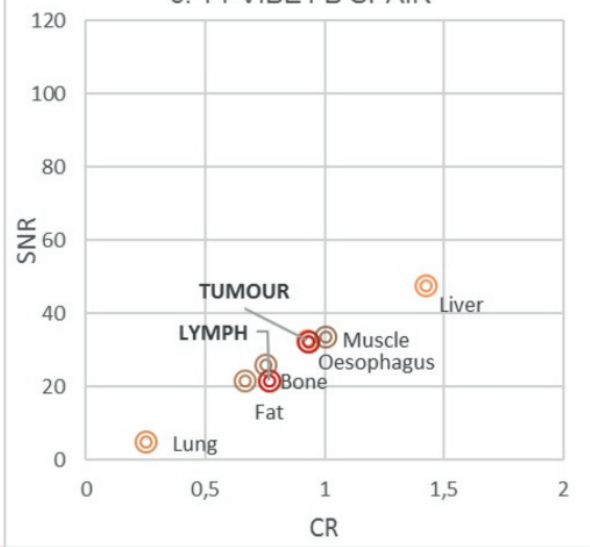

Figure 3.2: Quantitative analyses of T1-VIBE image quality acquired in cancer patients $\left(n=10^{* *}\right)$ by assessment of signal-to-noise ratio (SNR) and contrast ratio (CR) per sequence-variant over different tissues. VOIs were drawn over target regions and background-regions located outside the body ${ }^{* *} 8$ primary tumours, $5 \mathrm{LN}$ metastases) 
A.

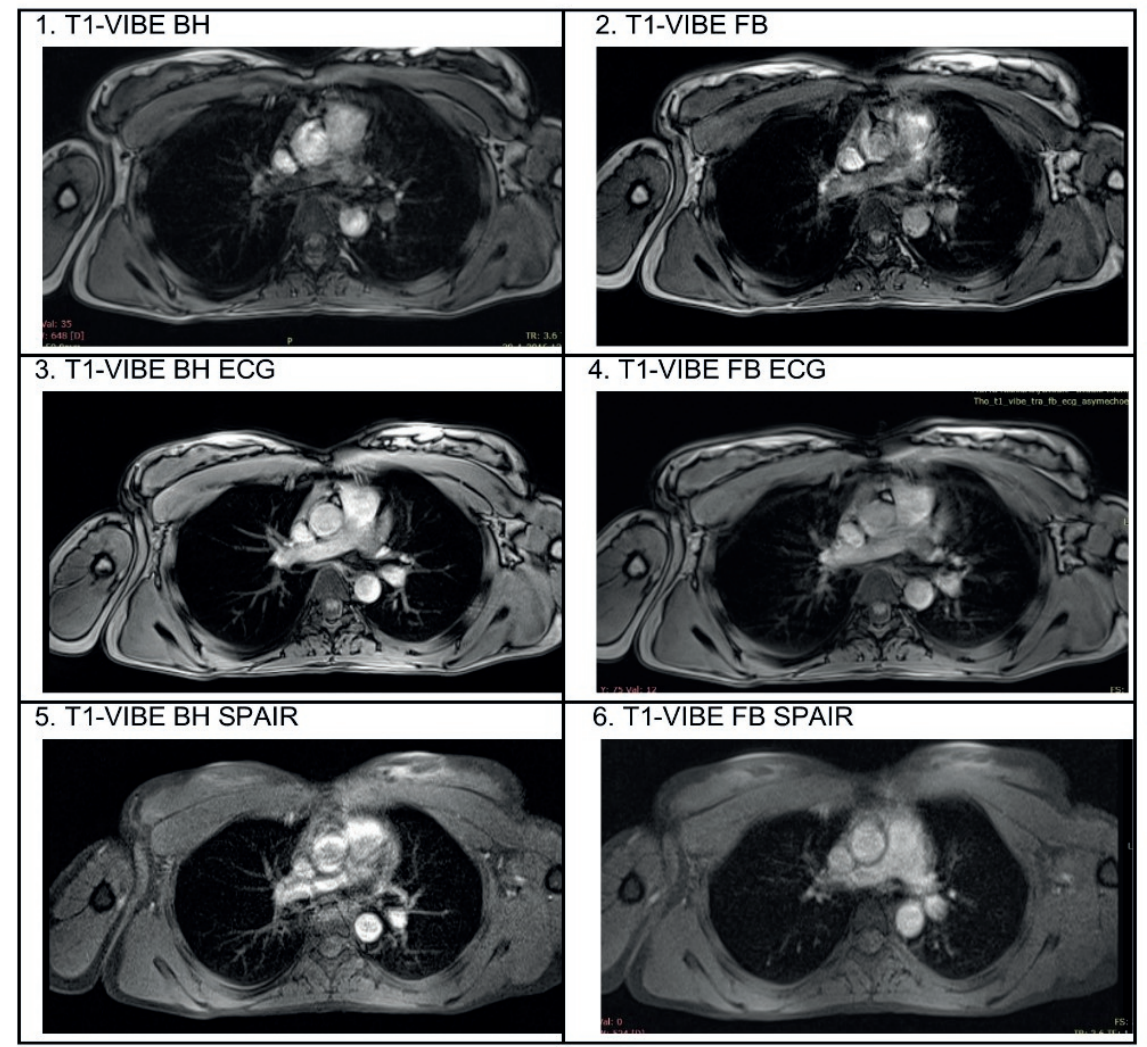

B.

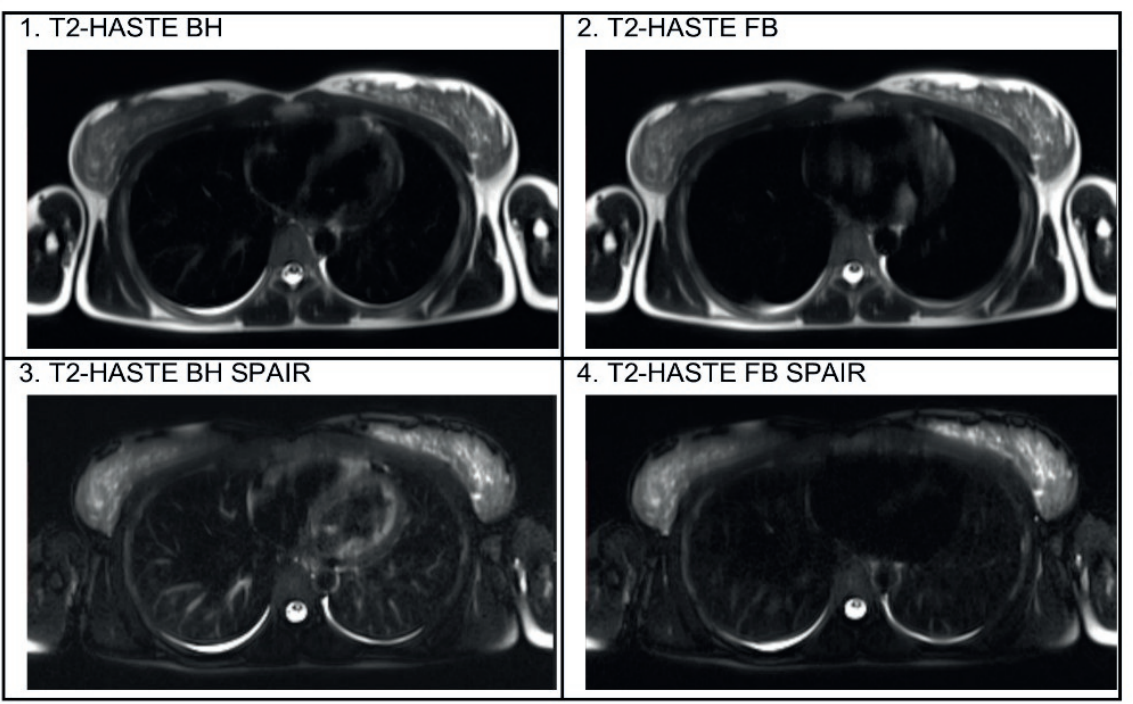

Figure 3.3: T1-VIBE (a) and T2-HASTE (b) images acquired in healthy volunteers at varying patient-specific parameters (in breath hold (BH), in free-breathing (FB), and with/without ECGtriggering, or SPAIR fat saturation 
Table 3.1: Qualitative assessment of MR image quality assessed in healthy volunteers and patients using a 5-point scoring scale (score $1=$ low IQ, score 5= high IQ)

\begin{tabular}{lccc}
\hline & \multicolumn{3}{c}{ Qualitative 5-point scoring } \\
\cline { 2 - 4 } Sequence & $\mathrm{R} 1$ & $\mathrm{R} 2$ & $\kappa$ \\
\hline T1-VIBE & & & 0.617 \\
BH & $2 \pm 0.5$ & $3 \pm 0.4$ & \\
BH-ECG & $4 \pm 0.5$ & $4 \pm 0.3$ & \\
BH-SPAIR & $2 \pm 0.5$ & $2 \pm 0.5$ & \\
FB & $2 \pm 0.5$ & $3 \pm 0.5$ & \\
FB-ECG & $3 \pm 0.0$ & $3 \pm 0.3$ & \\
FB-SPAIR & $2 \pm 0.6$ & $2 \pm 0.5$ & \\
T2-HASTE & & & 0.625 \\
BH & $3 \pm 0.5$ & $3 \pm 0.3$ & \\
BH-SPAIR & $2 \pm 0.3$ & $2 \pm 0.3$ & \\
FB & $2 \pm 0.6$ & $3 \pm 0.5$ & \\
FB-SPAIR & $2 \pm 0.4$ & $2 \pm 0.3$ & \\
\hline
\end{tabular}

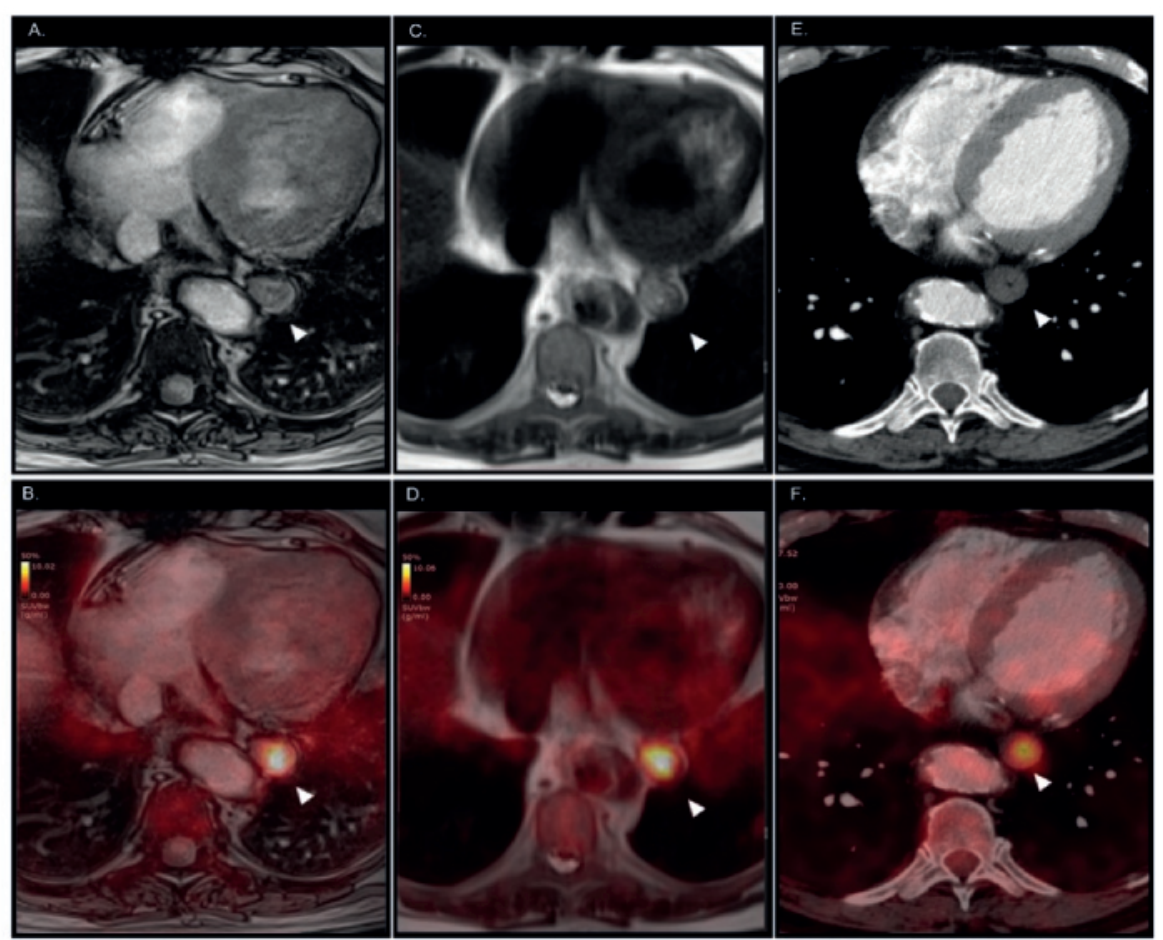

Figure 3.4: ${ }^{18} \mathrm{~F}-\mathrm{FDG}-\mathrm{PET} / \mathrm{MR}(\mathrm{a}-\mathrm{d})$ and ${ }^{18} \mathrm{~F}-\mathrm{FDG}-\mathrm{PET} / \mathrm{CT}(\mathrm{e}, \mathrm{f})$ images of patient 1 (Male, 68y) with oesophageal cancer (white triangle). Fused images $(c, d, f)$ are respectively compiled of T1-VIBE BH ECG (a), T2-HASTE BH (c), and thoracic CT (e) 


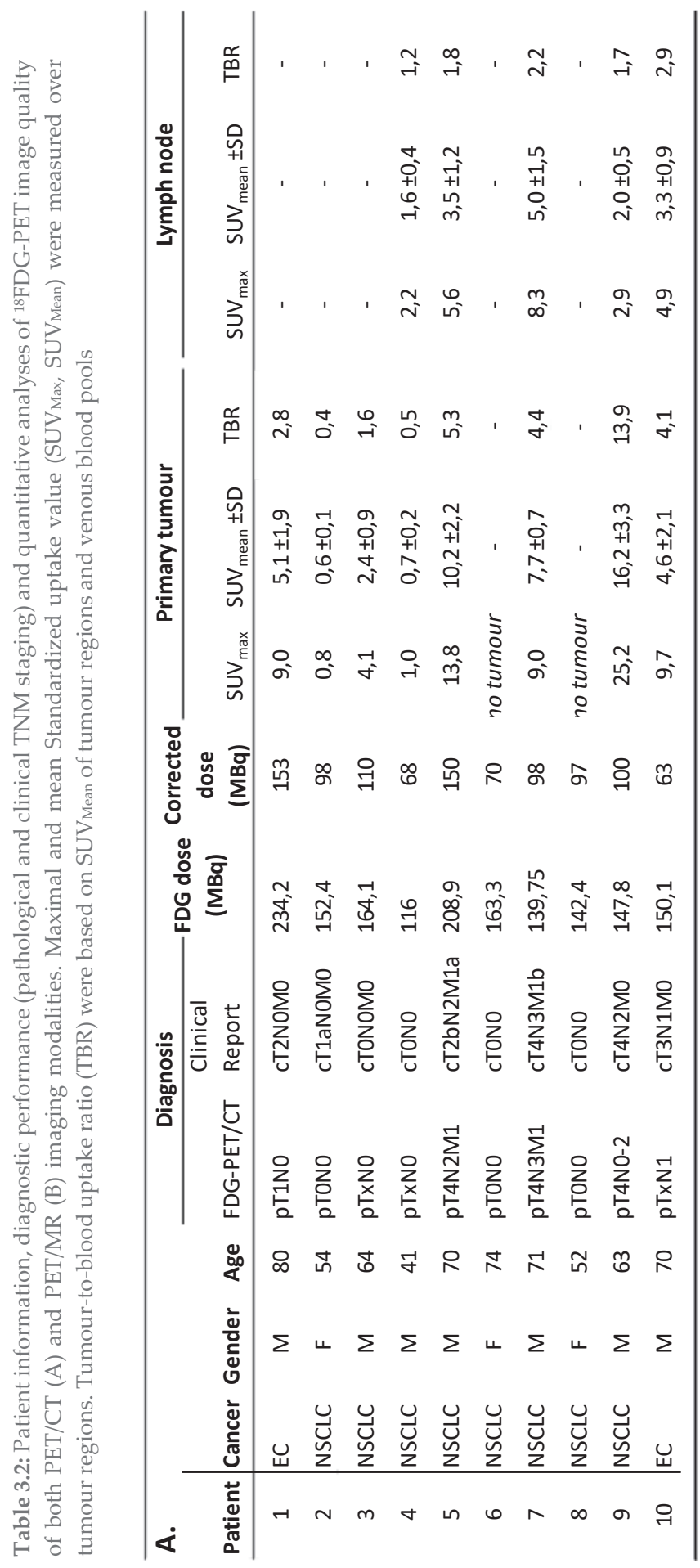




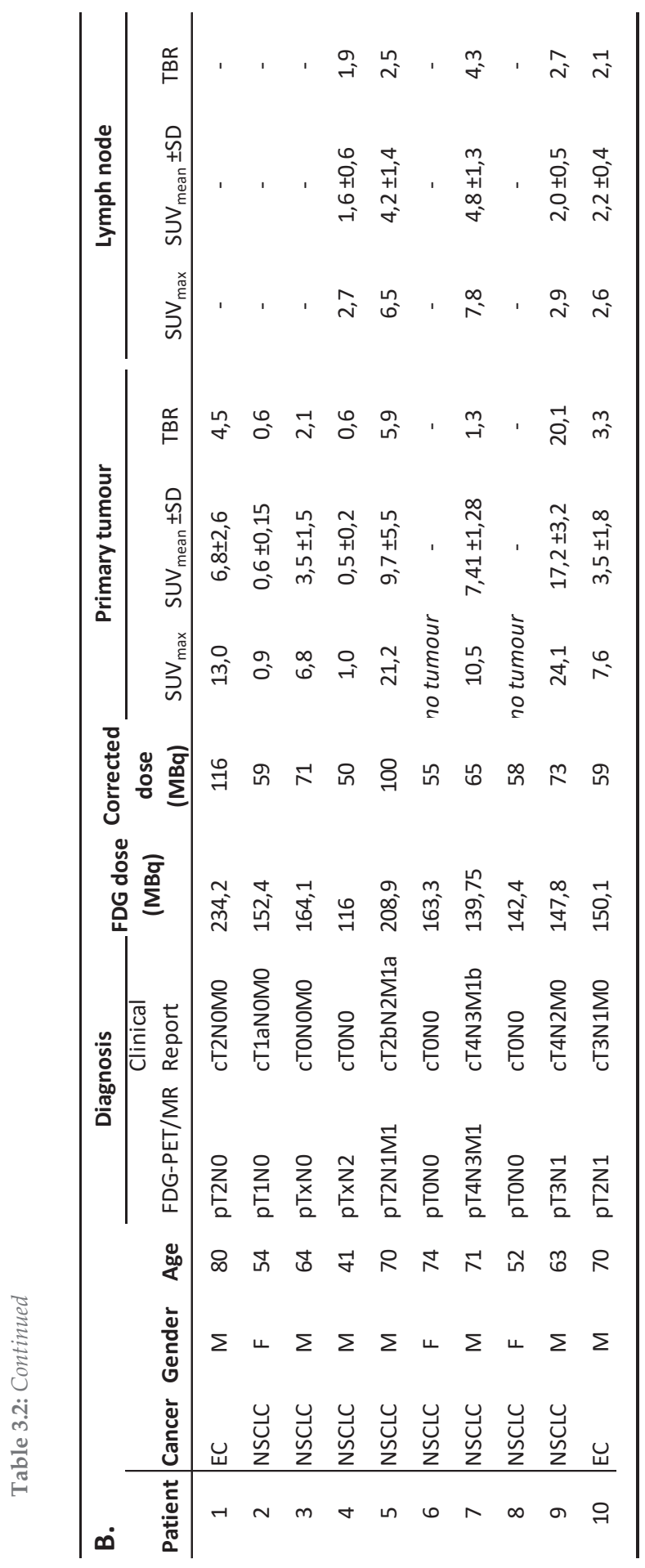




\section{CLINICAL VALIDATION}

Patient compliance and comfort

All included participants successfully underwent PET/MRI and were able to maintain BHs at end-expiration for all sequences (Table 3.2). However, patients with mediastinal malignancies had more trouble complying to scanning instructions and reported that the repetition frequency of multiple $\mathrm{BHs}$ made it increasingly difficult to consistently maintain $\mathrm{BH}$. The $\mathrm{BH}$-duration of $8.7 \mathrm{~s}$ during T2-HASTE image acquisition was perceived as nearing the patients' limits. Longer BHs, more $\mathrm{BH}$ repetitions, or the use of single $\mathrm{BH}$ schemes are therefore unlikely to be successful in patients with mediastinal malignancies. After optimisation, the total scan duration was 15-20 minutes and no excessive physical or psychological discomfort was reported.

\section{PET-image quality}

The radioactive dose at the start of ${ }^{18} \mathrm{~F}-\mathrm{FDG}-\mathrm{PET} / \mathrm{CT}$ was $105 \pm 33 \mathrm{MBq}$ at $65 \pm 10 \mathrm{~min}$ post-injection and $71 \pm 21 \mathrm{MBq}$ at the start of ${ }^{18} \mathrm{~F}-\mathrm{FDG}-\mathrm{PET} / \mathrm{MR}(127 \pm 19 \mathrm{~min}$ postinjection). No significant differences were found between modalities in SUVMean and SUVMax for FDG-avid tumours $(p=0.736, p=0.177$ ) and suspicious LNs ( $p=0.184$, $\mathrm{p}=0.535)$, respectively. Likewise, TBR-values of PET/MR were not significantly different from PET/CT for FDG-avid tumours $(p=0.082)$ and suspicious LNs $(\mathrm{p}=0.125)$. Subjective assessment of PET-images indicated clinically-acceptable IQ and excellent alignment with anatomical substrate on MRI and CT, respectively. Generally, lesions could be well differentiated with slightly blurred lesion edges. In patients 3 and 4, considerable blurring resulted in uncertain definition of FDGavidity and lesion differentiation.

\section{Diagnostic performance}

No significant differences in sensitivity and specificity on defining T-stage $(\mathrm{p}>0.99)$ or N-stage $(p>0.99)$ was presented between PET/MR and PET/CT, compared with histo-/cytopathological analyses. However, T-status was more often over-staged on ${ }^{18}$ F-FDG-PET/CT (3/10 patients) and N-status was more frequently under-staged on ${ }^{18}$ F-FDG-PET/MR (2/5 patients) (Table 3.2, Fig. 3.4, Fig. 3.5). In patient 5 and 7, Mstatus were accurately evaluated on both whole-body ${ }^{18} \mathrm{~F}-\mathrm{FDG}-\mathrm{PET} / \mathrm{CT}$ and thorax ${ }^{18}$ F-FDG-PET/MR images. 

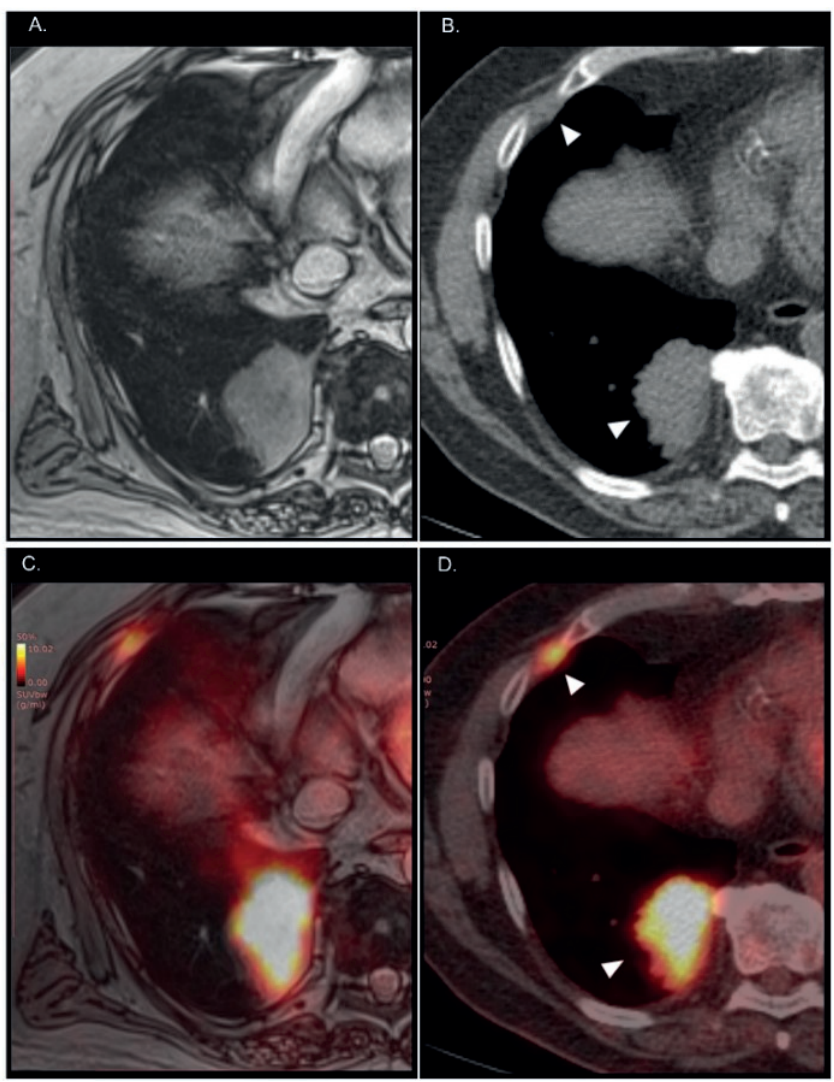

Figure 3.5: ${ }^{18} \mathrm{~F}-\mathrm{FDG}-\mathrm{PET} / \mathrm{MR}(\mathrm{a}, \mathrm{c})$ and ${ }^{18} \mathrm{~F}-\mathrm{FDG}-\mathrm{PET} / \mathrm{CT}(\mathrm{b}, \mathrm{d})$ images of patient 5 (Male, 70y) with NSCLC and thoracic metastases (white triangles). Tumour infiltration was dismissed on ECGtriggered T1-VIBE images acquired in breath-hold(a)

\section{DISCUSSION}

In this study, we developed a clinically-manageable, region-specific PET/MR protocol with high patient compliance and stable pulse sequences that consistently produce images of high IQ and diagnostic accuracy, within a reasonable acquisition time.

Based on qualitative and quantitative image analyses, T1-VIBE and T2HASTE were adequate sequences for mediastinal MR imaging and tumour visualisation. For breath-hold or free-breathing T1-VIBE, no significant differences were found in SNR and CR between images acquired with or without SPAIR. Although SPAIR performed well at fat saturation in all sequences, organ-edges became blurred which made visually differentiating mediastinal structures more difficult. In contrast, the addition of ECG-triggering to either BH- or FB-variants resulted in a significant increase in quantitative IQ, indicating that ECG-triggering had the most impact on IQ by reducing the effect of cardiac and aortic motion during 
scanning. As such, ECG-triggered T1-VIBE presented high contrast between adjacent tissues and ease of differentiating tumours and metastatic LNs, relative to muscle. The application of ECG-triggered T1-VIBE could be especially useful in assessing mediastinal tumours with infiltrates into the pericardium or large vessels [11]. However, ECG-triggering might be inefficient in patients with arrhythmia or complex QRS-patterns.

Qualitative assessment of ECG-triggered T1-VIBE indicated a slight preference for $\mathrm{BH}$-sequences as fewer MR-artefacts were present at the mediastinum. By maintaining $\mathrm{BH}$ at end-expiration, mean diaphragm motion is more stable $(0.15 \mathrm{~mm} / \mathrm{s})$ compared to end-inspiration $\mathrm{BH}(0.1-7.9 \mathrm{~mm} / \mathrm{s})$ [13]. This recommendation is in contrast to the suggested protocol of Biederer et al. that contain end-inspiration $\mathrm{BH}$ instructions of 20s [11,14]. However, ample time between $\mathrm{BH}$-intervals is needed to avoid fatigue and failure to maintain reproducible BHs. Therefore, short, multiple $\mathrm{BHs}$ are preferred without tiring the patient. When a patient is unfit to perform these BHs, a free-breathing ECGtriggered T1-VIBE could still produce adequate images for clinical use. In this, however, motion artefacts would appear more frequent. Alternatively, respiratory motion could be handled by automated respiratory triggering or gated acquisition using a navigator sequence to monitor the diaphragm's position [15-17]. However, these techniques are not flawless in compensating motion artefacts and will extend the total scan duration [11]. In literature, T1-VIBE has been shown to be robust in the presence of cardiac pulsation, able to identify small pulmonary nodules, and has been promoted for thoracic imaging $[8,10,18]$. For T2-HASTE, quantitative and qualitative IQ was unaffected by acquisition in BH or FB. However, lengthy BHdurations were not patient friendly and prolonged acquisition-time. T2-HASTE acquisition in FB might therefore be preferred in clinical practice. Previous studies recommended applying fat saturation in contrast-enhanced MRI to differentiate mediastinal LNs from the surrounding fat $[10,11,19]$. However, in this study no contrast agent was used and the addition of SPAIR did not significantly affect SNR and contrast between tumours, LN, and surrounding tissue. Nevertheless, unenhanced T2-HASTE sequences have been reported to sensitively detect pleural effusion and intra-atelectatic tumour growth $[9,20,21]$.

For successful clinical imaging, PET/MR protocols need to be developed for timeefficient, region-specific acquisition of high-quality images [7,22]. Efficient PET/MR protocols could be expanded with diffusion-weighted (DW) MRI, steady-state free precession or inversion recovery sequences that have proven to be clinically useful in EC and NSCLC $[10,23,24]$. However, several studies suggest an overlap in metabolic activity and tumour-cellularity as assessed by ${ }^{18} \mathrm{~F}-\mathrm{FDG}$-PET and DW-MRI, respectively $[25,26]$. Acquisition of time-consuming DW-MRI is only worthwhile when truly synergistic information could be gained for either diagnostics or treatment-decision making $[6,27]$. Therefore, efficient PET/MR protocols may need 
to combine anatomical and functional MRI with PET-imaging that uses radioactive tracers beyond ${ }^{18} \mathrm{~F}-\mathrm{FDG}$, such as ${ }^{18} \mathrm{~F}-\mathrm{HX} 4$ (i.e., hypoxia imaging), but reserve ${ }^{18} \mathrm{~F}-\mathrm{FDG}$ (i.e., glucose metabolism) for PET/CT that is already well-integrated in clinical practice and holds great diagnostic value. In radiation therapy, PET/MR images could be valuable in precisely differentiating malignant lesions from organs-at-risk, defining the metabolic phenotype of mediastinal tumours, and defining treatment strategies. The search for key applications of PET/MR needs to be further stimulated to work towards personalized patient care [28-31].

In this study, the diagnostic performance of ${ }^{18} \mathrm{~F}-\mathrm{FDG}-\mathrm{PET} / \mathrm{MR}$ was not inferior to ${ }^{18}$ F-FDG-PET/CT in defining tumour or LN status. SUVMean and SUVMax were not significantly different between ${ }^{18}$ F-FDG-PET/MR and ${ }^{18} \mathrm{~F}-\mathrm{FDG}-\mathrm{PET} / \mathrm{CT}$. In T-staging, pleural infiltration of NSCLC (Fig. 3.5) and thickening of oesophageal wall layers in EC (Fig. 3.4a-d) could be better detected on high-resolution MR images, resulting in less over-staging compared to ${ }^{18}$ F-FDG-PET/CT (Fig. 3.4e-f) [3,15,32]. In N-staging, contradicting results of diagnostic performance have been reported in singleinjection ${ }^{18} \mathrm{~F}-\mathrm{FDG}$-PET/CT versus ${ }^{18} \mathrm{~F}-\mathrm{FDG}$-PET/MR studies. In EC, Lee et al. (2014) showed that PET/MR presented the highest diagnostic performance for T-staging, followed by EUS, PET/CT and CT [32]. In agreement to our study, however, these authors demonstrated no significant differences in diagnostic accuracy for diagnosing nodal metastasis. In NSCLC, no clear (dis)advantage of ${ }^{18} \mathrm{~F}-\mathrm{FDG}-$ PET/MR over ${ }^{18} \mathrm{~F}-\mathrm{FDG}-\mathrm{PET} / \mathrm{CT}$ is determined in TNM-staging or therapeutic decision-making [33-35]. Heusch et al. showed no difference in T-staging or Nstaging NSCLC with sensitivity, specificity, and accuracy $(\mathrm{p}=0.48)$. However, fewer over/under-staging (9\%) were discovered using ${ }^{18} \mathrm{~F}-\mathrm{FDG}-\mathrm{PET} / \mathrm{MR}$, which concurred to our findings [36]. In our study, ${ }^{18}$ F-FDG-PET/MR produces clinically acceptable images from simultaneously acquired PET and MR-data of similar value as the clinically standard ${ }^{18} \mathrm{~F}-\mathrm{FDG}$-PET/CT [34,36,37]. In addition, ${ }^{18}$ F-FDG-PET/MR images could be clinically valuable by detecting tumour infiltration in adjacent organs. However, the impact of mediastinal-specific ${ }^{18} \mathrm{~F}-\mathrm{FDG}-\mathrm{PET} / \mathrm{MRI}$ on TNM-staging needs to be investigated further in large-scale studies.

In this study, the diagnostic performance of ${ }^{18} \mathrm{~F}-\mathrm{FDG}-\mathrm{PET} / \mathrm{MR}$ was not inferior to ${ }^{18} \mathrm{~F}-\mathrm{FDG}-\mathrm{PET} / \mathrm{CT}$ in defining tumour or LN status. SUVMean and SUVMax were not significantly different between ${ }^{18}$ F-FDG-PET/MR and ${ }^{18} \mathrm{~F}-\mathrm{FDG}-\mathrm{PET} / \mathrm{CT}$. In T-staging, pleural infiltration of NSCLC (Fig. 3.5) and thickening of oesophageal wall layers in EC (Fig. 3.4a-d) could be better detected on high-resolution MR images, resulting in less over-staging compared to ${ }^{18}$ F-FDG-PET/CT (Fig. 3.4e--f) [3,15,32]. In N-staging, contradicting results of diagnostic performance have been reported in singleinjection ${ }^{18}$ F-FDG-PET/CT versus ${ }^{18}$ F-FDG-PET/MR studies. In EC, Lee et al. (2014) showed that PET/MR presented the highest diagnostic performance for T-staging, followed by EUS, PET/CT and CT [32]. In agreement to our study, however, these 
authors demonstrated no significant differences in diagnostic accuracy for diagnosing nodal metastasis. In NSCLC, no clear (dis)advantage of ${ }^{18} \mathrm{~F}-\mathrm{FDG}$ PET/MR over ${ }^{18} \mathrm{~F}-\mathrm{FDG}-\mathrm{PET} / \mathrm{CT}$ is determined in TNM-staging or therapeutic decision-making [33-35]. Heusch et al. showed no difference in T-staging or Nstaging NSCLC with sensitivity, specificity, and accuracy $(\mathrm{p}=0.48)$. However, fewer over/under-staging $(9 \%)$ were discovered using ${ }^{18 F-F D G-P E T / M R, ~ w h i c h ~ c o n c u r r e d ~}$ to our findings [36]. In our study, ${ }^{18}$ F-FDG-PET/MR produces clinically acceptable images from simultaneously acquired PET and MR-data of similar value as the clinically standard ${ }^{18} \mathrm{~F}-\mathrm{FDG}-\mathrm{PET} / \mathrm{CT}[34,36,37]$. In addition, ${ }^{18} \mathrm{~F}-\mathrm{FDG}-\mathrm{PET} / \mathrm{MR}$ images could be clinically valuable by detecting tumour infiltration in adjacent organs. However, the impact of mediastinal-specific ${ }^{18} \mathrm{~F}-\mathrm{FDG}$-PET/MRI on TNM-staging needs to be investigated further in large-scale studies.

This study is limited in patient population and in the diversity of MR-sequences tested. We selected conventional, clinical T1- and T2-weighted sequences and did not alter sequence-parameter settings while testing aforementioned sequencevariables. In some patients, the gastroesophageal junction was not depicted due to the limited field-of-view (FOV) of T1-VIBE. For future depiction of EC, cranio-caudal FOV-settings need to be adjusted to extent from commonly affected cervical to celiac LN-stations in EC. Alternative sequences to T1-VIBE (radial-VIBE, CAIPIRINHAVIBE, contrast-enhanced VIBE) have been previously studied and could potentially improve mediastinal imaging [38]. Radial-VIBE is less susceptible to respiratorymotion artefacts and showed high overall IQ in paediatric abdominal MRI [39]. However, this experimental sequence is prone to streaking artefacts and not standardly-available on PET/MR-systems [40]. CAIPIRINHA-VIBE has been shown to produce abdominal and thoracic images with higher SNR and spatial resolution compared to T1-VIBE by improving parallel imaging and reducing aliasing artefacts [41].

\section{CONCLUSION}

A region-specific PET/MRI protocol, including T1-VIBE ECG and T2-HASTE, can be used for dedicated mediastinal examinations and produce images with acceptable quality that is both patient-friendly and clinically valuable. Tumour-specific protocols using PET-tracers beyond ${ }^{18} \mathrm{~F}-\mathrm{FDG}$ and dedicated MRI sequences will become valuable treatment decision tools in thoracic oncology in the near future. 


\section{REFERENCES}

1. Singnurkar A, Poon R, Metser U. Comparison of 18F-FDG-PET/CT and 18F-FDG-PET/MR imaging in oncology: a systematic review. Annals of nuclear medicine 2017; 31(5): 366-78.

2. Rosenkrantz AB, Friedman K, Chandarana H, et al. Current Status of Hybrid PET/MRI in Oncologic Imaging. AJR American journal of roentgenology 2016; 206(1): 162-72.

3. Schwenzer NF, Schraml C, Muller M, et al. Pulmonary lesion assessment: comparison of whole-body hybrid MR/PET and PET/CT imaging--pilot study. Radiology 2012; 264(2): 551-8.

4. Reck M, Popat S, Reinmuth N, De Ruysscher D, Kerr KM, Peters S. Metastatic non-small-cell lung cancer (NSCLC): ESMO Clinical Practice Guidelines for diagnosis, treatment and follow-up. Annals of oncology : official journal of the European Society for Medical Oncology 2014; 25 Suppl 3: iii27-39.

5. Lordick F, Mariette C, Haustermans K, Obermannova R, Arnold D. Oesophageal cancer: ESMO Clinical Practice Guidelines for diagnosis, treatment and follow-up. Annals of oncology : official journal of the European Society for Medical Oncology 2016; 27(suppl 5): v50-v7.

6. Bailey DL, Pichler BJ, Guckel B, et al. Combined PET/MRI: from Status Quo to Status Go. Summary Report of the Fifth International Workshop on PET/MR Imaging; February 15-19, 2016; Tubingen, Germany. Molecular imaging and biology : MIB : the official publication of the Academy of Molecular Imaging 2016; 18(5): 637-50.

7. von Schulthess GK, Veit-Haibach P. Workflow Considerations in PET/MR Imaging. Journal of nuclear medicine : official publication, Society of Nuclear Medicine 2014; 55(Supplement 2): 19S-24S.

8. Biederer J, Hintze C, Fabel M. MRI of pulmonary nodules: technique and diagnostic value. Cancer imaging : the official publication of the International Cancer Imaging Society 2008; 8: 125-30.

9. Henzler T, Dietrich O, Krissak R, et al. Half-Fourier-acquisition single-shot turbo spin-echo (HASTE) MRI of the lung at 3 Tesla using parallel imaging with 32-receiver channel technology. Journal of magnetic resonance imaging : JMRI 2009; 30(3): 541-6.

10. Kumar S, Liney G, Rai R, Holloway L, Moses D, Vinod SK. Magnetic resonance imaging in lung: a review of its potential for radiotherapy. The British journal of radiology 2016; 89(1060): 20150431.

11. Biederer J. General Requirements of MRI of the Lung and Suggested Standard Protocol. In: Kauczor HU, ed. MRI of the Lung Medical Radiology. Berlin, Heidelberg: Springer; 2009.

12. Potchen EJ, Siebert JE, Haacke EM, Gottschalk A. Signal-to-noise, contrast-to-noise, and resolution. In: Patterson AS, ed. Magnetic Resonance Angiography: Concepts \& Applications. St. Louis, Missouri, USA: Mosby; 1993.

13. Holland AE, Goldfarb JW, et al. Diaphragmatic and cardiac motion during suspended breathing: preliminary experience and implications for breath-hold MR imaging. Radiology 1998; 209(2): 483-9.

14. Biederer J, Beer M, Hirsch W, et al. MRI of the lung (2/3). Why ... when ... how? Insights into imaging 2012; 3(4): 355-71.

15. van Rossum PS, van Lier AL, Lips IM, et al. Imaging of oesophageal cancer with FDG-PET/CT and MRI. Clin Radiol 2015; 70(1): 81-95.

16. Lever FM, Lips IM, Crijns SP, et al. Quantification of esophageal tumor motion on cine-magnetic resonance imaging. International journal of radiation oncology, biology, physics 2014; 88(2): 419-24.

17. Dutta J, Huang C, Li Q, El Fakhri G. Pulmonary imaging using respiratory motion compensated simultaneous PET/MR. Medical physics 2015; 42(7): 4227-40.

18. Biederer J, Mirsadraee S, Beer M, et al. MRI of the lung (3/3)-current applications and future perspectives. Insights into imaging 2012; 3(4): 373-86.

19. Biederer J, Ohno Y, Hatabu H, et al. Screening for lung cancer: Does MRI have a role? European journal of radiology 2017; 86: 353-60.

20. Ishii S, Hara T, Nanbu T, et al. Optimized workflow and imaging protocols for whole-body oncologic PET/MRI. Japanese journal of radiology 2016; 34(11): 754-62.

21. Schaarschmidt BM, Grueneisen J, Heusch P, et al. Oncological whole-body staging in integrated (18)FFDG PET/MR: Value of different MR sequences for simultaneous PET and MR reading. European journal of radiology 2015; 84(7): 1285-92.

22. Kuperman V. Magnetic Resonance Imaging: Physical Principles and Applications. University of Chicago, Illinois: Academic press; 2000.

23. van Rossum PS, van Lier AL, van Vulpen M, et al. Diffusion-weighted magnetic resonance imaging for the prediction of pathologic response to neoadjuvant chemoradiotherapy in esophageal cancer. Radiotherapy and oncology : journal of the European Society for Therapeutic Radiology and Oncology 
2015; 115(2): 163-70.

24. Alper F, Turkyilmaz A, Kurtcan S, et al. Effectiveness of the STIR turbo spin-echo sequence MR imaging in evaluation of lymphadenopathy in esophageal cancer. European journal of radiology 2011; 80(3): 6258.

25. Schaarschmidt BM, Buchbender C, Nensa F, et al. Correlation of the apparent diffusion coefficient (ADC) with the standardized uptake value (SUV) in lymph node metastases of non-small cell lung cancer (NSCLC) patients using hybrid 18F-FDG PET/MRI. PLoS One 2015; 10(1): e0116277.

26. Heusch P, Buchbender C, Kohler J, et al. Correlation of the apparent diffusion coefficient (ADC) with the standardized uptake value (SUV) in hybrid 18F-FDG PET/MRI in non-small cell lung cancer (NSCLC) lesions: initial results. RoFo : Fortschritte auf dem Gebiete der Rontgenstrahlen und der Nuklearmedizin 2013; 185(11): 1056-62.

27. Taouli B, Beer AJ, Chenevert T, et al. Diffusion-weighted imaging outside the brain: Consensus statement from an ISMRM-sponsored workshop. Journal of magnetic resonance imaging : JMRI 2016.

28. Karki K, Saraiya S, Hugo GD, et al. Variabilities of Magnetic Resonance Imaging-, Computed Tomography-, and Positron Emission Tomography-Computed Tomography-Based Tumor and Lymph Node Delineations for Lung Cancer Radiation Therapy Planning. International journal of radiation oncology, biology, physics 2017; 99(1): 80-9.

29. Paulus DH, Oehmigen M, Gruneisen J, Umutlu L, Quick HH. Whole-body hybrid imaging concept for the integration of PET/MR into radiation therapy treatment planning. Physics in medicine and biology 2016; 61(9): 3504-20.

30. Thorwarth D, Leibfarth S, Mönnich D. Potential role of PET/MRI in radiotherapy treatment planning. Clinical and Translational Imaging 2013; 1(1): 45-51.

31. Bailey DL, Pichler BJ, Guckel B, et al. Combined PET/MRI: Global Warming-Summary Report of the 6th International Workshop on PET/MRI, March 27-29, 2017, Tubingen, Germany. Molecular imaging and biology : MIB : the official publication of the Academy of Molecular Imaging 2018; 20(1): 4-20.

32. Lee G, I H, Kim SJ, et al. Clinical implication of PET/MR imaging in preoperative esophageal cancer staging: comparison with PET/CT, endoscopic ultrasonography, and CT. Journal of nuclear medicine : official publication, Society of Nuclear Medicine 2014; 55(8): 1242-7.

33. Schaarschmidt BM, Grueneisen J, Metzenmacher M, et al. Thoracic staging with F-FDG PET/MR in nonsmall cell lung cancer - does it change therapeutic decisions in comparison to F-FDG PET/CT? European radiology 2016.

34. Rauscher I, Eiber M, Furst S, et al. PET/MR imaging in the detection and characterization of pulmonary lesions: technical and diagnostic evaluation in comparison to PET/CT. Journal of nuclear medicine : official publication, Society of Nuclear Medicine 2014; 55(5): 724-9.

35. Huellner MW, de Galiza Barbosa F, Husmann L, et al. TNM Staging of Non-Small Cell Lung Cancer: Comparison of PET/MR and PET/CT. Journal of nuclear medicine : official publication, Society of Nuclear Medicine 2016; 57(1): 21-6.

36. Heusch P, Buchbender C, Kohler J, et al. Thoracic staging in lung cancer: prospective comparison of $18 \mathrm{~F}-$ FDG PET/MR imaging and 18F-FDG PET/CT. Journal of nuclear medicine : official publication, Society of Nuclear Medicine 2014; 55(3): 373-8.

37. Spick C, Herrmann K, Czernin J. 18F-FDG PET/CT and PET/MRI Perform Equally Well in Cancer: Evidence from Studies on More Than 2,300 Patients. Journal of nuclear medicine : official publication, Society of Nuclear Medicine 2016; 57(3): 420-30.

38. Qu J, Zhang H, Wang Z, et al. Comparison between free-breathing radial VIBE on 3-T MRI and endoscopic ultrasound for preoperative $\mathrm{T}$ staging of resectable oesophageal cancer, with histopathological correlation. European radiology 2017.

39. Shin HJ, Kim MJ, Lee MJ, Kim HG. Comparison of image quality between conventional VIBE and radial VIBE in free-breathing paediatric abdominal MRI. Clin Radiol 2016; 71(10): 1044-9.

40. Bamrungchart S, Tantaway EM, Midia EC, et al. Free breathing three-dimensional gradient echosequence with radial data sampling (radial 3D-GRE) examination of the pancreas: Comparison with standard 3D-GRE volumetric interpolated breathhold examination (VIBE). Journal of magnetic resonance imaging : JMRI 2013; 38(6): 1572-7.

41. Dewes P, Frellesen C, Al-Butmeh F, et al. Comparative evaluation of non-contrast CAIPIRINHA-VIBE 3T-MRI and multidetector CT for detection of pulmonary nodules: In vivo evaluation of diagnostic accuracy and image quality. European journal of radiology 2016; 85(1): 193-8. 


\section{SUPPLEMENTARY DATA:}
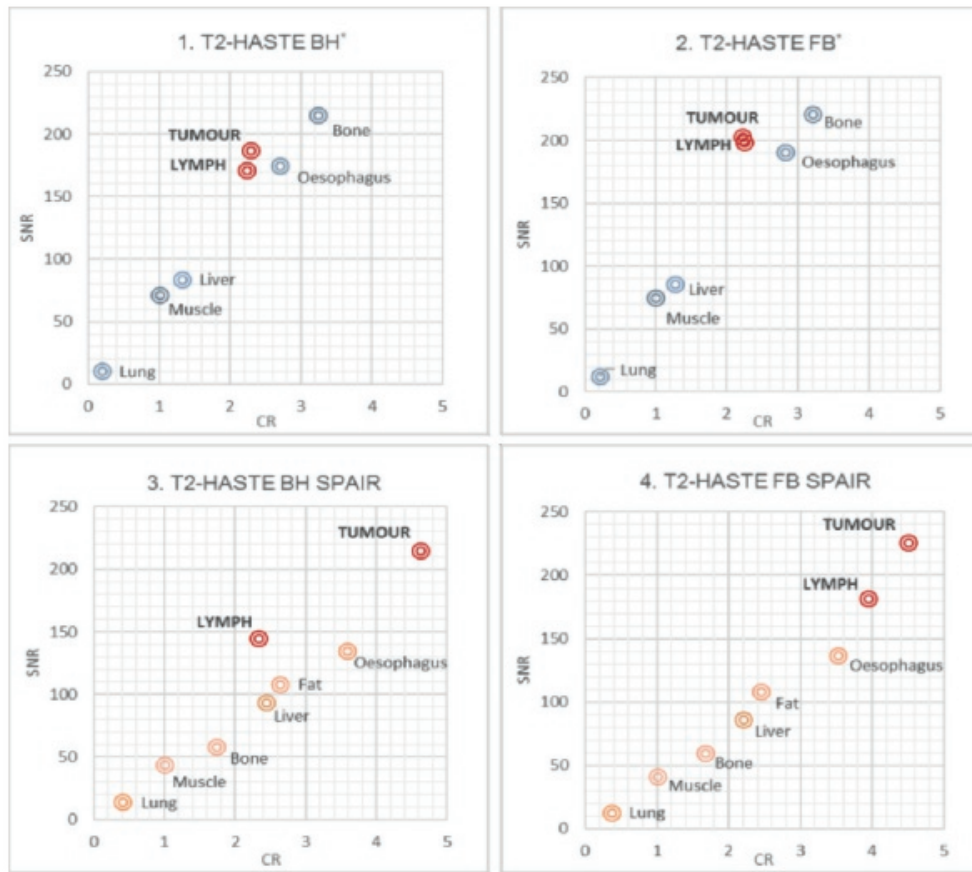

Figure S3.1: Quantitative analyses of T2-HASTE image quality acquired in cancer patients $\left(n=10^{* *}\right)$ by assessment of signal-to-noise ratio (SNR) and contrast ratio (CR). VOIs were drawn over target regions and background-regions located outside the body.

(** 8 primary tumours, 5 LN metastases). $\left(^{*}\right)$ SNR/CR for VOI(fat) is off-scale in T2-HASTE BH (512.1/8.4) an T2-HASTE FB (701.5/10.6)

Table S3.1: Details of (PET)/MR imaging sequences. (*) TA measured during single breath-hold

\begin{tabular}{l|llll}
\hline Parameter & Localizer & $\begin{array}{l}\text { T1-DIXON } \\
\text { VIBE }\end{array}$ & T1-VIBE & T2-HASTE \\
\hline Sequence type & SE & GE & GE & SE \\
Acquisition & 2D & 3D & 3D & $2 \mathrm{D}$ \\
Image plane & All & Coronal & Axial & Axial \\
TR $(\mathrm{ms})$ & 1000 & 3.6 & 3.64 & 1500 \\
TE $(\mathrm{ms})$ & 84 & 2.46 & 1.23 & 113 \\
Flip angle $\left({ }^{\circ}\right)$ & 143 & 10 & 9 & 90 \\
Number of slices & $3 /$ plane & 128 & 48 & 48 \\
Slice thickness $(\mathrm{mm})$ & 6 & 3.12 & 4 & 5.5 \\
Distance factor & $150 \%$ & $20 \%$ & $0 \%$ & $0 \%$ \\
Field of view & $430 \times 430$ & $329 \times 500$ & $400 \times 262$ & $450 \times 450$ \\
Matrix (reconstructed) & $256 \times 179$ & $192 \times 120$ & $189 \times 384$ & $259 \times 320$ \\
Resolution (mm) & $2.4 \times 1.7 \times 6$ & $4.1 \times 2.6 \times 3.1$ & $1.4 \times 1.0 \times 4$ & $1.7 \times 1.4 \times 5.5$ \\
Bandwidth $($ Hz/pixel) & 476 & 965 & 592 & 710 \\
Echo train length & 256 & 1 & 1 & 256 \\
Parallel imaging & GRAPPA 2 & GRAPPA 2 & GRAPPA 2 & GRAPPA 2 \\
TA (s) & 9.1 & 19 & $5.7(\times 4)^{*}$ & $8.1(\times 7)^{*}$ \\
\hline
\end{tabular}




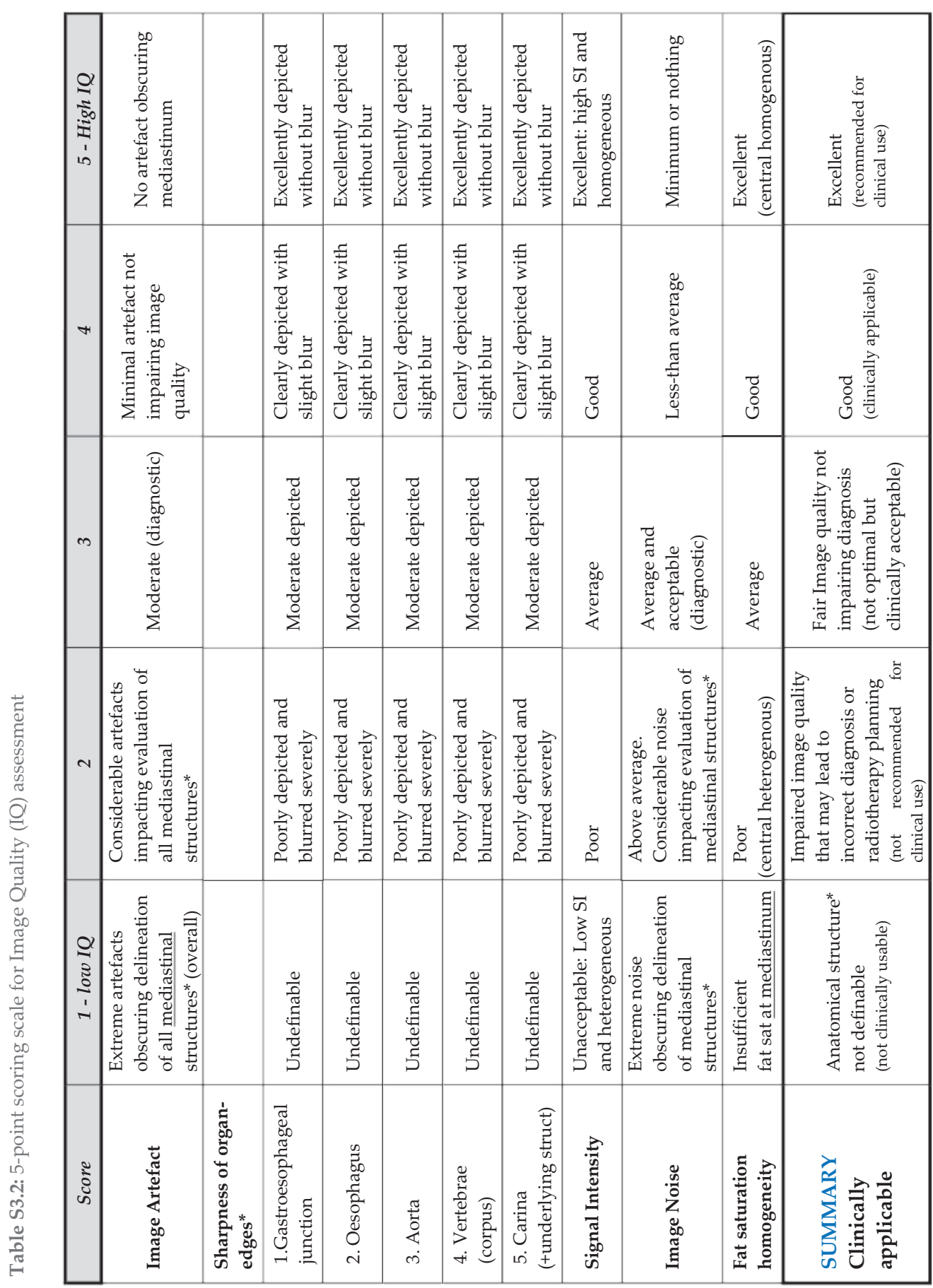




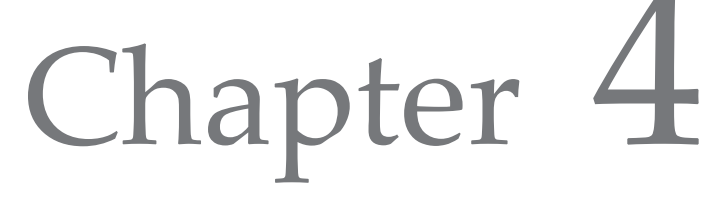

\section{Integrating 7 Tesla anatomical MRI in neuro- oncology and image-guided radiotherapy}

Authors:

I. Compter*, J. Peerlings*, D.B.P. Eekers, A. A. Postma, D. Ivanov, C. J. Wiggins, P. Kubben, B. Küsters, P. Wesseling, L. Ackermans, O. E.M.G. Schijns, P.Lambin, A. L. Hoffmann * equal contribution

Adapted from:

Technical feasibility of integrating 7 Tesla anatomical MRI in image-guided radiotherapy of glioblastoma: a preparatory study Magnetic Resonance Materials in Physics, Biology, Medicine (MAGMA). 2016; 29 (3):591-603 DOI: 10.1007/s10334-016-0534-7 


\section{ABSTRACT}

\section{OBJECTIVE}

7 Tesla (T) MRI has recently shown great potential for high-resolution soft-tissue neuroimaging and visualization of microvascularization in glioblastoma (GBM). We have designed a clinical trial to explore the value of 7T MRI for radiation treatment of GBM. For this clinical study we performed a preparatory study to investigate the technical feasibility of incorporating 7T MR images into the neurosurgical navigation and radiotherapy treatment planning (RTP) systems by qualitative and quantitative assessment of the image quality.

\section{MATERIALS \& METHODS}

The MR images were acquired with a Siemens Magnetom 7T wholebody scanner and a Nova Medical 32-channel head coil. 7T MRI pulse sequences included MP2RAGE, T2-SPACE, SPACE-FLAIR and GRE. A pilot study with 3 healthy volunteers and an anthropomorphic $3 \mathrm{D}$ phantom was used to assess image quality and geometrical image accuracy.

\section{RESULTS}

The MRI scans were well tolerated by the volunteers. Susceptibility artefacts were observed in both the cortex and subcortical white matter at close proximity to air-tissue interfaces. Regional loss of signal and contrast could be minimized by the use of dielectric pads. Image transfer and processing did not degrade image quality. The system-related spatial uncertainty of geometrical distortion-corrected MP2RAGE pulse sequences is $\leq 2 \mathrm{~mm}$.

\section{CONCLUSION}

Integration of high-quality and geometrically-reliable 7T MR images into neurosurgical navigation and RTP software is technically feasible and safe. 


\section{INTRODUCTION}

Glioblastoma (GBM) is the most common type of primary brain tumour having a peak incidence in the $6^{\text {th }}$ and $7^{\text {th }}$ decade. Patients with a GBM have an extremely poor prognosis with a median overall survival of 14.6 months [1]. The main goals of surgery are verification of histological diagnosis and reduction of any mass effect. A complete resection is nearly impossible because of the infiltrative nature of this disease and spread along white matter tracts [2]. Therefore, patients up to 70 years old with a good performance status are treated with adjuvant radiotherapy in combination with temozolomide to delay local tumour recurrence and increase overall survival. Radiotherapy usually consists of conventionally fractionated regimens, delivering a dose of 59.4-60 Gy in 6-7 weeks. Computed tomography (CT) images ( $1 \mathrm{~mm}$ slices) are commonly used for radiation therapy planning (RTP) because of the excellent spatial quality (i.e., no geometrical distortions) and the electron density information that is required for accurate dose calculations. In current RTP practice for GBM, contrast-enhanced (CE) CT images are co-registered with 1.5 or 3 Tesla (T) magnetic resonance (MR) images because the latter offer superior soft-tissue contrast over CT images. Subsequently, a gross tumour volume (GTV) is delineated based on the resection cavity and any residual disease visible on CE T1-weighted MRI scans. According to European guidelines a 2-3 cm isotropic margin is added to the GTV to encompass any non-enhancing tumour tissue and to establish the clinical target volume (CTV) [3]. This margin is based on the fact that over $80 \%$ of recurrences occur within $2 \mathrm{~cm}$ of the GTV [4,5]. However, the isotropic margin does not take into account spatially varying tumour-growth dynamics in different brain tissues and tumour spread along white matter tracts, which evidently results in needlessly damaging healthy cells while leaving viable malignant cells outside the CTV [6]. A planning target volume (PTV) margin is added to account for systematic and random errors such as setup errors, inter- and intrafraction motion, but also uncertainty in image registration and delineation. Current clinically available MRI techniques are unable to adequately visualize tumour spread throughout the brain. Ultra-high field (UHF) $(>3$ T) MRI might be able to overcome these limitations, because the increased signal-to-noise ratio (SNR) and susceptibility effects which allow for an increased spatial resolution and better contrast in comparison to clinically-used 1.5T and 3T MRI. However, the potential of UHF MRI for RTP of GBM has not been investigated so far.

With regard to neurosurgical planning, UHF MRI may benefit image-guided biopsies through identification of increased vascularity suggestive for tumour grade. Endothelial proliferation and increased microvascularization are key features for the diagnosis of GBM according to the WHO criteria [7]. An increase in susceptibility effects, as seen with UHF MRI in comparison to lower field strengths, results in novel contrast mechanisms on quantitative $\mathrm{T}_{2}{ }^{*}$-weighted images by which 
microvascularization with a vessel diameter as small as $100 \mu \mathrm{m}$ can be visualized [8-11].

We therefore hypothesize that 7T MRI allows for better delineation of the GTV of GBM due to improved visualization of microvascularization. We have designed a future clinical study to investigate this, which includes a 7T-image guided biopsy and RTP (clinicaltrials.gov NCT 02062372). In this paper we describe the technical aspects of the preparatory work for this clinical trial. If 7T MR images are to be used for neurosurgical and RTP purposes, a high spatial reliability is required. Although 7T MRI may hold promise to be included into neurosurgical navigation and RTP, it also presents technical challenges such as inhomogeneity of the transmit B 1 field, an increased specific absorption rate (SAR) and geometrical distortions caused by increased static magnetic field $\left(\mathrm{B}_{0}\right)$ inhomogeneity, tissue susceptibility differences and chemical shift effects [12-14]. In order to measure the geometric accuracy, a phantom study was conducted to quantify system-related geometrical image distortions. Furthermore, a pilot study with healthy volunteers was conducted to investigate whether the 7T MRI images meet the requirements for clinical application in RTP, such as visualization of brain anatomy structures on different sequences, differences in scanning times and patient tolerability of the scan. In this paper we report on the challenges and pitfalls we encountered in preparation of our clinical study, and present solutions we developed to quantify the system-related geometrical image distortions, optimize 7T MRI scanning protocols as well as the image transfer and processing workflow.

\section{MATERIAL \& METHODS}

\section{PILOT STUDY}

A pilot study with healthy volunteers was conducted to assess the image quality in terms of field inhomogeneity and susceptibility artefacts and to optimize the pulse sequences and scanning protocols for RTP purposes. Prior to the 7T MRI scan all volunteers received detailed information regarding the purpose of the study and possible temporary sensory side effects due to the applied magnetic field. Written informed consent was obtained prior to participation. A total of three volunteers were recruited: two females ( 26 and 37 years old) and one male (29 years old). Subjects filled out a safety questionnaire prior to the scan regarding e.g. medication, claustrophobia and metallic objects, and were instructed not to move during the scanning procedure. They were positioned in a head-first supine position and dielectric pads were fixed on both sides of the subject's head next to the temporal lobes after which the head coil was placed. The dielectric pads contained a $25 \%$ suspension of barium titanate in deuterated water and were used to locally increase 
the transmit $\mathrm{B}_{1}^{+}$field to improve its homogeneity across the brain [15]. Cushions were placed under the knees to provide extra comfort.

\section{PHANTOM STUDY}

Geometrical distortion is a recognized problem in anatomical MRI sometimes resulting in pixel shifts of several millimetres, which is detrimental for application of MRI in image-guided interventions in neurosurgery and radiotherapy. Geometric inaccuracies originate from system (i.e., Bo-inhomogeneity, gradient field nonlinearity) or object (i.e., chemical shift, susceptibility effects) related causes, and can to some extent be corrected for by manufacturer-developed distortion correction methods and shimming procedures. As magnetic field inhomogeneity, chemical shift and susceptibility are proportional to $\mathrm{B}_{0}$, the spatial inaccuracy increases with higher magnetic field strength. Hence, estimation of the geometrical distortion is essential before 7T MRI can be integrated into image-guided interventions.

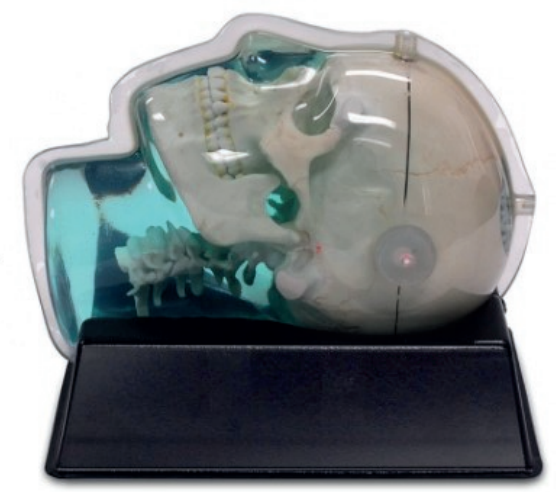

Fig. 4.1 Anthropomorphic skull phantom CIRS 603A. Computerized Imaging Reference Systems, Inc (CIRS), viewed on 22 November 2015

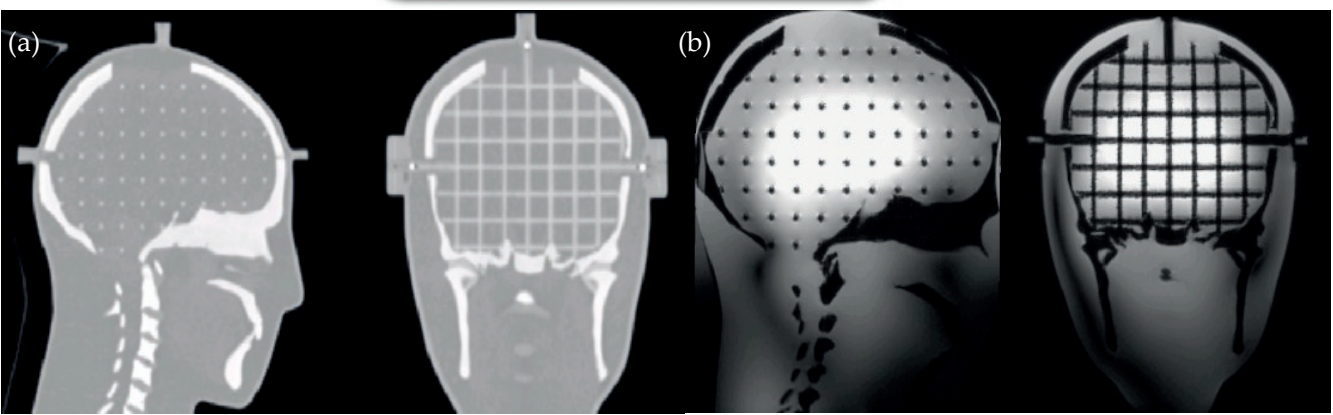

Fig. 4.2 Sagittal and coronal slice of the CIRS 603A phantom on CT (a), and 7T MRI (b) scan

For the assessment of system- and sequence-related geometrical image distortions, we used a dedicated 3D anthropomorphic skull phantom (CIRS Model 603A, Computerized Imaging Reference Systems, Inc., Norfolk, Virginia, United States; Figure 4.1 and $4.2 \mathrm{a} \& 2 \mathrm{~b}$ ). This phantom is made up of a plastic-bone tissue substitute 
and soft-tissue equivalent material consisting of a water-based polyacrylamide (Zerdine ${ }^{\circledR}$, CIRS). The entire phantom is encased in a clear vacuum-formed plastic shell to protect the gel from desiccation. The cranial portion of the skull volume is filled with an orthogonal 3D grid of $3 \mathrm{~mm}$ diameter rods (reinforced Nylon) spaced $15 \mathrm{~mm}$ apart. The maximum $3 \mathrm{D}$ grid-size is $15 \times 12 \times 13.5 \mathrm{~cm}^{3}(\mathrm{AP} \times \mathrm{LR} \times \mathrm{SI})$, resulting in 436 measurable grid-intersection points. Since Nylon shows magnetic susceptibility properties similar to water (i.e., difference in susceptibility $<3 \mathrm{ppm}$ ), the phantom rods are expected not to induce artefacts and image abnormalities in either spin-echo or gradient-echo sequences [16]. The phantom was placed in an ABS vacuum-formed cradle to ensure reproducible placement within the scanner. Both $\mathrm{CT}$ and 7T MRI images were acquired in order to compare the geometrical distortions of both imaging modalities.

\section{IMAGE ACQUISITION}

For the phantom study, CT images (SOMATOM Sensation 10, Siemens Healthcare, Erlangen, Germany) were acquired at MAASTRO CLINIC with a $1 \mathrm{~mm}$ slice thickness, 306 slices, $50 \times 50 \mathrm{~cm}^{2}$ field of view, $140 \mathrm{kV}, 400 \mathrm{mAs}$.

For the pilot and phantom study, MR images were acquired at the Scannexus facility with a $7 \mathrm{~T}$ whole-body scanner (Magnetom 7T, Siemens Healthcare, Erlangen, Germany) in combination with a 32-channel head coil (Nova Medical, Wilmington, United States). Pulse sequences included multi-echo gradient echo (GRE), magnetization-prepared rapid gradient-echo (MP2RAGE) [17], T2- sampling perfection with application optimized contrasts using different flip angle evolution (T2-SPACE), and sampling perfection with application optimized contrasts using different flip angle evolution FLAIR (SPACE-FLAIR) (Table 4.1).

Table 4.1 Scan parameters per 7T MRI pulse sequence

\begin{tabular}{lllll}
\hline & MP2RAGE & SPACE & $\begin{array}{l}\text { SPACE } \\
\text { FLAIR }\end{array}$ & GRE \\
& & & 8000 & 33 \\
\hline Repetition time TR (ms) & 5000 & 4000 & 302 & 2.5 \\
Echo time TE (ms) & 2.5 & 283 & nvt \\
Inversion time (ms) & TI1 900 & nvt & 2330 & \\
& TI2 27500 & & & $160 \times 223$ \\
Field of View (mm) & $223 \times 223$ & $192 \times 192$ & $193 \times 206$ & 11 \\
Nominal flip angle ( $\left.{ }^{\circ}\right)$ & $5 \& 3$ & variable & variable & $320 \times 320$ \\
Acquisition matrix (pixel) & $320 \times 320$ & $320 \times 320$ & $256 \times 240$ & 290 \\
Bandwidth (Hz/pixel) & 248 & 372 & 383 & 208 \\
Slices (n) & 240 & 288 & 208 & 0.7 \\
Slice thickness (mm) & 0.7 & 0.6 & 0.8 & 8.33 \\
Acquisition time (min) & 8.02 & 7.50 & 10.58 & \\
\hline
\end{tabular}




\section{T MRI SEQUENCE SELECTION AND OPTIMIZATION}

Sequences were selected to highlight differences in tissue contrast [18] and visualize microvascularization [10,9]. A standard method for obtaining $\mathrm{T}_{1}$-contrast is the MPRAGE (magnetization-prepared rapid gradient-echo) sequence, which provides good grey-white matter contrast [19]. Due to inhomogeneities in both the transmit and receive radiofrequency (RF) fields, a newer variant (MP2RAGE) was used which generates two different images at different inversion times and allows for selfcorrection of the bias fields was used [20].

The MP2RAGE sequence with optimized TR-FOCI inversion pulse was chosen for $\mathrm{T}_{1}$-weighted imaging instead of the MPRAGE because receive bias field could be corrected online (Figure 4.3a) [21-24]. In particular, the $\mathrm{T}_{1}$-weighted images were obtained as the ratio of the two volumes acquired at different inversion times (INV1 and INV2), which minimizes the effect of $\mathrm{B}_{1}$-variations through space. A quantitative $\mathrm{T}_{1}$-map was calculated online by linear interpolation of the INV1 and INV2 images [17]. An optimized four-echo GRE was used instead of the typical single-echo GRE with a long echo time in order to correct for $\mathrm{B}_{1}$-inhomogeneities and obtain quantitative $\mathrm{T}_{2}{ }^{*}$ images (Figure $4.3 \mathrm{~b}$ ).
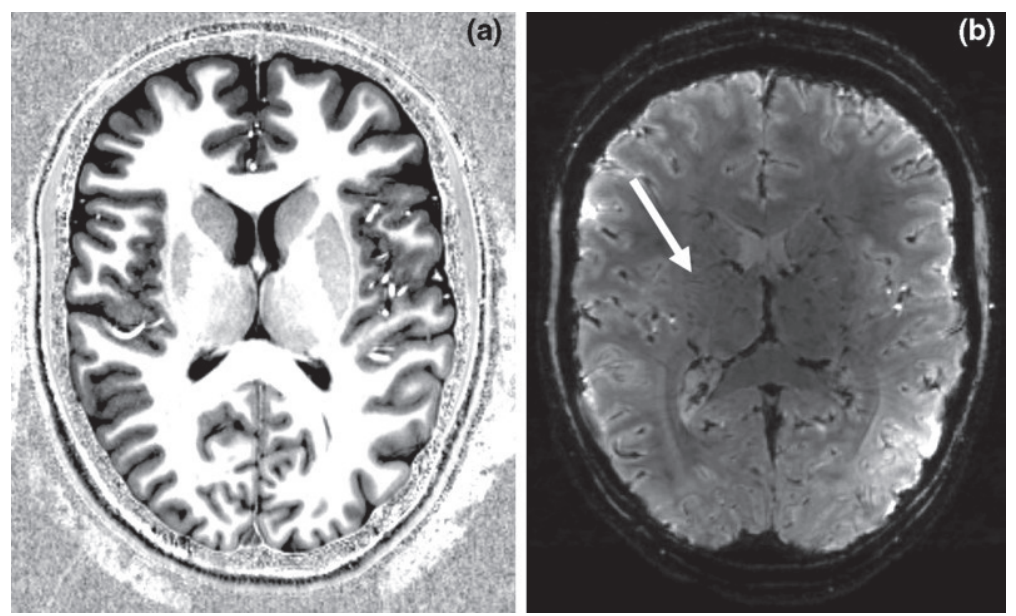

Fig. 4.3 Axial slices of 7T MRI T1-weighted image from MP2RAGE (b) and T2*-weighted image from GRE (a) of a healthy volunteer. White arrow exemplifies the fine vascularisation detail that is observed

T2-weighted imaging using standard Turbo Spin Echo sequences becomes increasingly difficult at UHF due to relatively high SAR induced by multiple 180 degree refocussing pulses [25]. For this reason, a technique involving the use of a tailored RF flip angle train was used (T2-SPACE) to obtain $\mathrm{T}_{2}$-like contrast [26-28]. This same technique was also used as the basis for a FLAIR-type contrast (SPACE- 
FLAIR), where an inversion pulse, with inversion time of $2330 \mathrm{~ms}$, was added to null fluid signals. For both $\mathrm{T}_{2}$-weighted sequences, a Works-In-Progress package [WIP 692] from Siemens was used and parameters were optimised to obtain excellent contrast, with reference to standard clinical imaging. All images were reconstructed with and without manufacturer-developed correction procedures to restore gradient uniformity.

\section{IMAGE TRANSFER AND PROCESSING WORKFLOW}

Since MAASTRO CLINIC and Scannexus each operate independently from the Maastricht University Medical Centre (MUMC), a dedicated inter-institutional imaging workflow was developed to be able to collaboratively share the 7T MR images acquired at Scannexus with the department of Neurosurgery at MUMC and Radiation Oncology department at MAASTRO CLINIC. Our solution comprised a cloud-based medical image sharing environment (Quentry, version 3.0, Brainlab AG, Feldkirchen, Germany) to upload the 7T MR images into the neurosurgical cranial navigation software (iPlan Net, version 3.6, Brainlab AG, Feldkirchen, Germany) that will be used to obtain the biopsy specimen for the future clinical study. The MiTools software package was used to generate quantitative $\mathrm{T}_{2}{ }^{*}$-maps from the multi-echo GRE images (http://od1n.sourceforge.net/). The 7T MRI scans were imported directly as DICOM files into the radiation treatment planning system (Eclipse version 11, Varian, Palo Alto, United States of America) from the Scannexus facility.

\section{IMAGE QUALITY ASSESSMENT}

\section{Qualitative image analysis}

During a multi-disciplinary session, a physicist, neuro-radiologist and radiation oncologist visually verified that the MR image quality was warranted after acquisition, transfer and processing of the images. Image quality was assessed by evaluating the images of all three volunteers on the MR scanner console and within the neurosurgical navigation and RTP software. The MP2RAGE, T2-SPACE and SPACE-FLAIR sequences were visually evaluated by an experienced neuroradiologist for SNR, visualisation of the cerebral lobes, depiction of grey-white matter, basal ganglia, ventricles and CSF around the brain. Each of these factors was classified on a scale of $1-4$ (1. Excellent, 2. Good, 3. Marginal, but still diagnostic, 4. Non-diagnostic). The GRE images were not assessed as these images were postprocessed in order to obtain quantitative $\mathrm{T}_{2}{ }^{*}$-images.

\section{Quantitative image analysis - geometrical image distortion}

To assess the geometrical reliability of the 7T MR images two clinically-relevant pulse-sequences (GRE and MP2RAGE) were used to acquire images of the phantom, both with and without automatic geometric distortion correction. Unfortunately, it 
was found that the distortion correction processing was not compatible with the multi-echo data, causing severe artefacts. For this reason, only the data from the MP2RAGE is presented. We aim to resolve this incompatibility in future clinical studies. The GRE and MP2RAGE sequences were selected to assess sequence-related geometrical image distortions as they will be primarily used for GTV delineation in the clinical study.

Next, the 3D coordinates of all 436 grid-intersection points were acquired after manual processing of the respective CT and MR images in Eclipse. The coordinates of the reference points were deduced from the phantom's known geometry.

Two methods were used to measure the geometrical distortion at different levels of sophistication. The first method was used to assess the overall image distortion using an in-house developed MATLAB script (R2014b, MathWorks Inc, Natick, USA). All unique pairs of grid-intersection point coordinates were generated from which subsequently the Euclidean distance was determined, and absolute differences in distance were calculated between the reference (i.e., phantom-based) and measured (i.e., image-based) distances. In a second method, Euclidean distances were measured between a fixed reference point located at the magnetic field isocenter, and all other grid-intersection points in the image, and compared to corresponding distances within the reference frame. Hence, a measure of the geometrical dispersion relative to the magnetic field isocenter was obtained. Both methods were applied to the CT and MRI scans. The overall geometric distortion was quantified by the global mean absolute deviation (MAD global) and its standard deviation (SD), while geometrical dispersion relative to the isocenter was quantified by local mean absolute deviation (MADlocal) and its SD:

$$
\begin{aligned}
& \text { a. } M A D_{\text {global }}=\frac{1}{i} \sum_{i}\left|D_{\text {ref }}-D_{m}\right|_{i} \\
& \text { b. } M A D_{\text {local }}=\frac{1}{i} \sum_{i}\left|D_{\text {ref }}-D_{\text {isoc }}\right|_{i}
\end{aligned}
$$

where $D_{\text {ref }}$ represents the Euclidian distance between reference points, $D_{m}$ is the measured distance between image-based grid points, and $D_{i s o c}$ is the measured distance between the magnetic field isocenter and surrounding grid points. For each measurement within a cluster of unique distances $i$, the MAD is then calculated as the average of the absolute difference in Euclidian distances. Given the MAD and its standard deviation $(\mathrm{SD})$, a $95 \%$ confidence interval $(\Delta)$ was calculated as:

$$
\Delta= \pm(M A D+2 S D)
$$

Based on this confidence interval, a maximum acceptable tolerance level of $2.0 \mathrm{~mm}$ was defined. When MAD $>1.0 \mathrm{~mm}$ and $|\Delta|>2.0 \mathrm{~mm}$, the level of geometric deviation between the measured and reference dimensions were considered unacceptable. 


\section{RESULTS}

\section{IMAGE ACQUISITION}

The duration of the scanning sessions for the volunteers was 50 minutes. All three volunteers reported slight vestibular effects while being moved in and out of the magnetic field. One of the volunteers reported twitching of the nose tip during the scanning procedure. No other clinically relevant sensory effects were reported. The duration of the scanning session for the phantom was up to 150 minutes as it was scanned with the same sequences as the volunteers in three orthogonal planes (axial, sagittal, coronal).

\section{IMAGE QUALITY}

\section{Qualitative image analysis}

Images acquired with MP2RAGE (Figure 4.3a), T2-SPACE and SPACE-FLAIR sequences all showed good to excellent SNR and visualization of the frontal and parietal lobes. Furthermore, there was a good to excellent reproduction of the ventricles and CSF surrounding the brain. All sequences showed marginal to good SNR and visualization of the cerebellum. The reproduction of CSF around the cerebellum was good, except for on T2-SPACE where it was judged to be nondiagnostic to marginal. All sequences demonstrated non-diagnostic to marginal image quality for SNR, visualization of the cerebral lobes, depiction of grey-white matter and ventricles and depiction of CSF around the brain at the frontobasal and temporal lobes. This decrease in image quality was primarily caused by a decrease in signal and susceptibility artefacts near the skull base (Figure 4.4). The reproduction of the basal ganglia was good to excellent on T2-SPACE and MP2RAGE, respectively, but was considered marginal in one of the volunteers on SPACE-FLAIR due to flow-artefacts. The visualization of the cerebral vessels was good to excellent on both T2-SPACE and MP2RAGE and considered marginal to good on the SPACE-FLAIR. There was a signal inhomogeneity present in the T2SPACE and SPACE- FLAIR images in both the medial-lateral and anterior-posterior direction. Moreover, the T2-SPACE showed a signal drop at both the skull base and the temporal lobes. Ghosting artefacts were anteriorly and posteriorly present in the T2-SPACE and SPACE-FLAIR. These may be the result of the relatively high amount of image acceleration used (2x2 GRAPPA) and it may be possible to reduce these artefacts through enhanced image reconstruction. In addition, flow-artefacts were observed near major intra-cranial vessels such as the carotid and basillary arteries. Image transfer and processing did not visually degrade the image quality. 


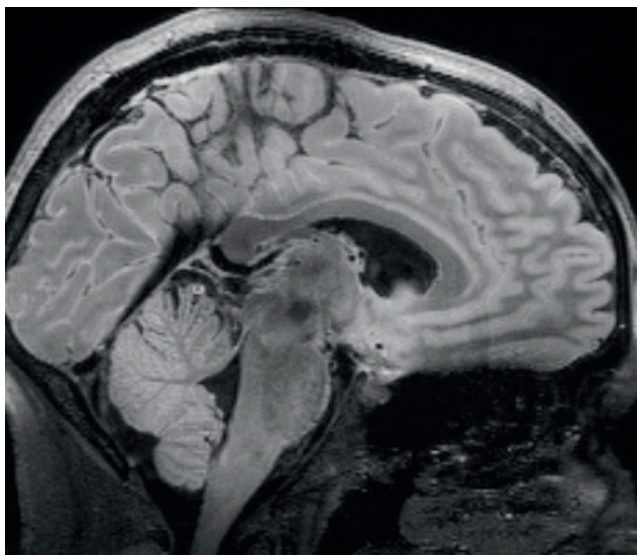

Fig. 4.4 Sagittal 7T MRI SPACE-FLAIR image of a healthy volunteer, showing increased geometrical distortion near the skull base, the ventral part of the temporal cortices, and the orbito-frontal cortex

Quantitative image analysis - geometrical image distortion

\section{GLOBAL DISTORTION METHOD}

Based on the 436 reference points of the 3D grid, 95266 unique distances between grid-intersection points were calculated and paired into 100 unique distance clusters, ranging from 15 to $164.32 \mathrm{~mm}$. The corresponding image-based distances were paired into the same clusters for calculating MADglobal.
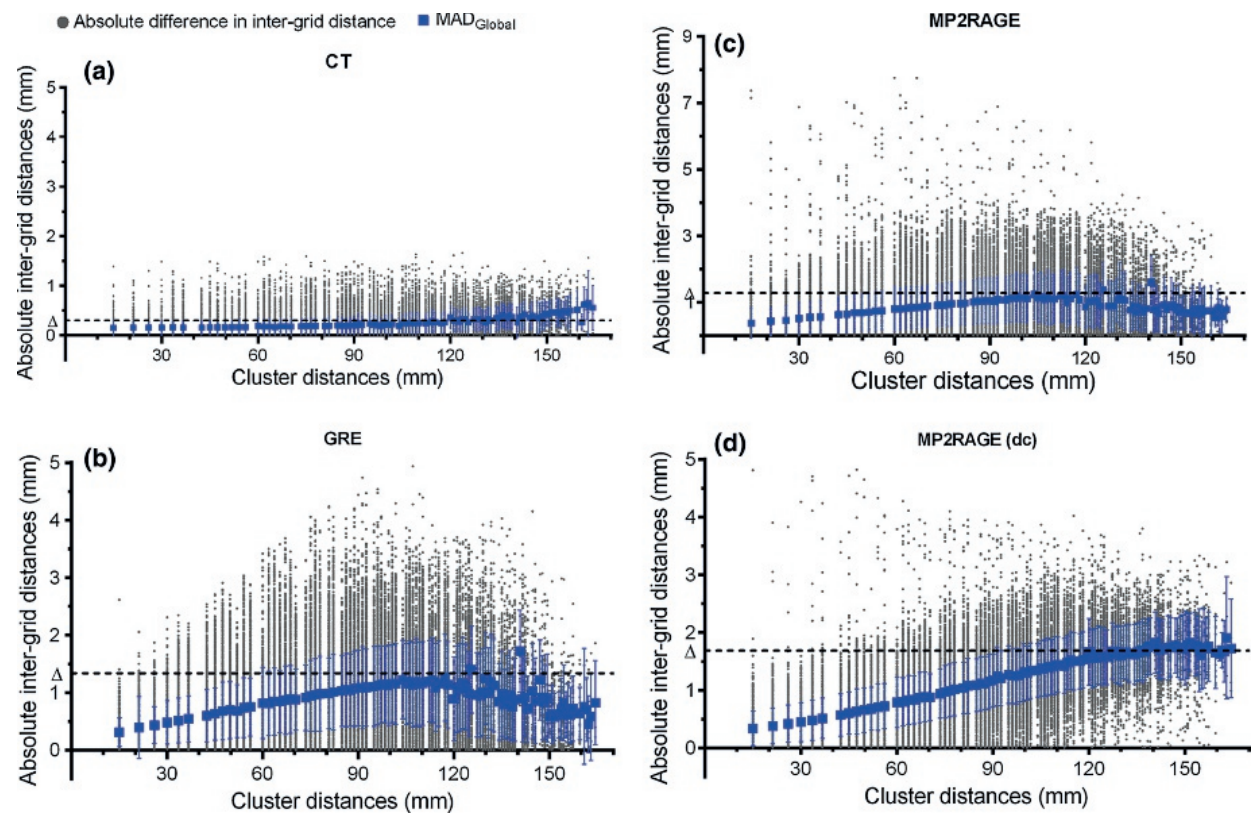

Fig. 4.5 Absolute inter-grid distances (grey dot) measured within CT (a), distortion-uncorrected GRE images (b), distortion-uncorrected MP2RAGE images (c), and distortion-corrected MP2RAGE (d) relative to the possible cluster distances in $\mathrm{mm}$. The overall geometric distortion was quantified by MADGlobal (blue square) and its standard deviation. The $95 \%$ confidence interval $(\Delta)$ is shown as the dotted line 
Figure 4.5 presents the absolute difference between phantom- and image-based distances within each cluster, as well as the MADglobal of that cluster. The global geometric distortion within the MR-images is more pronounced than in CT images. However, $\mathrm{CT}$ is not completely free of distortion errors with average MAD global of $0.20 \mathrm{~mm}$ (SD $\pm 0.05 \mathrm{~mm}$ ) (Fig. 4.5a). The GRE and MP2RAGE sequences present MADglobal ranges of $0.31-1.35 \mathrm{~mm}$ and $0.38-1.62 \mathrm{~mm}$, respectively in distortionuncorrected images (Fig. 4.5b, 4.5c). Furthermore, the measured differences were less consistent in MP2RAGE images with various outliers above the $95 \%$ confidence interval $(\Delta=1.29 \mathrm{~mm})$, mostly at small intergrid distances. In the distortioncorrected MP2RAGE images, relatively similar patterns of MAD global could be noted (Fig. 4.5d) with values ranging from $0.34-1.91 \mathrm{~mm}(\Delta=1.68 \mathrm{~mm})$. Table 4.3 provides a clear overview of MAD ranges, average MAD and MAD confidence intervals per selected image.

Table 4.3 Geometric distortion measures for 7T MRI

\begin{tabular}{lcccccccc}
\hline & & \multicolumn{3}{c}{ MAD global $(\mathbf{m m})$} & & \multicolumn{3}{c}{ MADLocal $(\mathrm{mm})$} \\
\cline { 3 - 5 } \cline { 8 - 9 } Sequence & DC & Range & Mean \pm SD & $\Delta$ & & Range & Mean \pm SD & $\Delta$ \\
\hline GRE & No & $0.31-1.35$ & $0.88 \pm 0.22$ & 1.33 & & $0.28-1.31$ & $0.65 \pm 0.17$ & 0.99 \\
MP2RAGE & No & $0.38-1.62$ & $0.88 \pm 0.21$ & 1.29 & & $0.26-1.46$ & $0.64 \pm 0.20$ & 1.04 \\
MP2RAGE & 3D & $0.34-1.91$ & $0.98 \pm 0.35$ & 1.68 & & $0.22-1.82$ & $0.85 \pm 0.36$ & 1.56 \\
\hline
\end{tabular}

\section{LOCAL DISTORTION METHOD}

Figure 4.6 shows the geometrical dispersion relative to the isocenter (MADlocal) and illustrates modality-and sequence-dependent distortion effects and the quality of the MR-distortion correction methods. In CT-images, MADlocal ranges from 0.06-0.27 $\mathrm{mm}$ indicating small spatial deviations. In the distortion-uncorrected 7T MR images, MADlocal ranges from $0.28-1.31 \mathrm{~mm}(\Delta=0.99 \mathrm{~mm})$ in GRE images and $0.26-1.46 \mathrm{~mm}$ $(\Delta=1.04 \mathrm{~mm})$ in MP2RAGE images. In the distortion-corrected MR-images, MADlocal ranges from $0.22-1.82 \mathrm{~mm}(\Delta=1.56 \mathrm{~mm})$ in MP2RAGE images. The value for MADlocal is observed with increasing distance from the magnetic field isocenter, with a maximum of $1.82 \mathrm{~mm}$ near the edges of the phantom. At equal distance from the isocenter, geometric distortion was found to be anisotropic. For distortion-corrected MP2RAGE images major spatial displacement could be noted in the superiorinferior (SI) direction (e.g., $0.82 \mathrm{~mm}$ at $45 \mathrm{~mm}$ isocenter distance). 

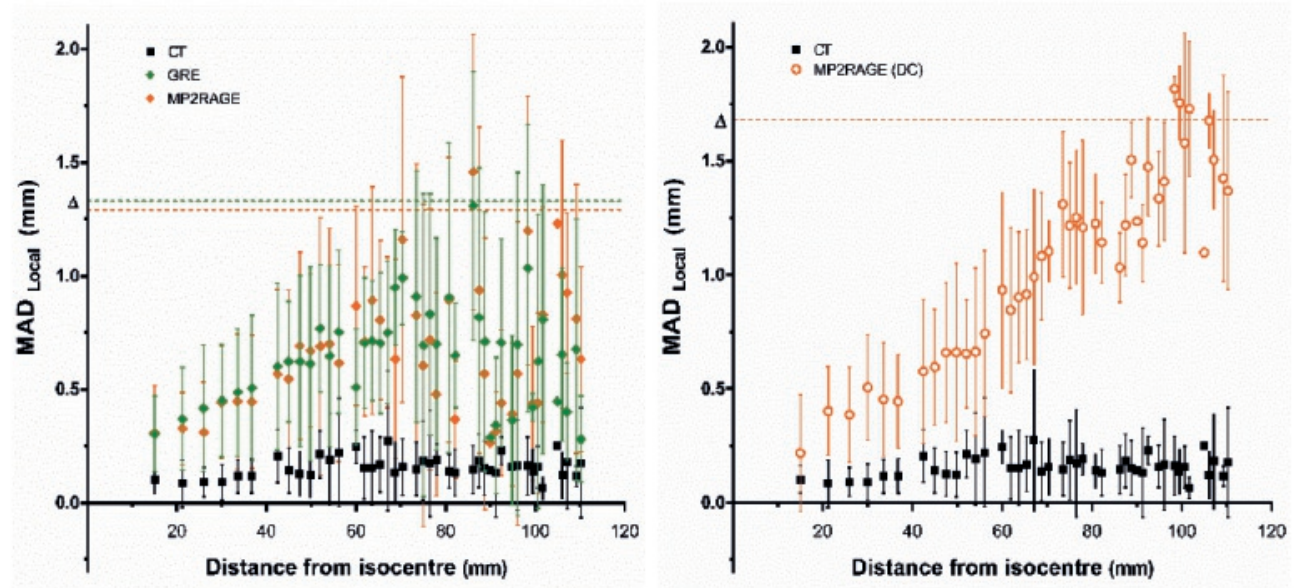

Fig. 4.6 Geometric dispersion relative to the magnetic field isocenter, quantified by MADlocal and its standard deviation within CT (black square) and distortion-uncorrected 7T MR images (a) acquired with GRE (green diamond) and MP2RAGE (orange diamond) pulse sequences. Measured MADlocal values for CT and distortion-corrected 7T MR images (b) acquired with MP2RAGE (orange circle) pulse sequence are shown relative to the distance.

Based on the average MAD-values for CT and 7T MRI, both modalities present tolerable levels of geometrical image distortion with average $\mathrm{MAD} \leq 1.0 \mathrm{~mm}$ and $\Delta$ $\leq 2.0 \mathrm{~mm}$ (Table 4.2 and 4.3). However, MAD global and MADlocal of larger cluster distances often exceed this tolerance limit. For corrected MP2RAGE images, $\mathrm{MAD}_{\text {global }}$ was $>1.0 \mathrm{~mm}$ for intergrid distances $>77.9 \mathrm{~mm}$. MAD local measurements in distortion-corrected MP2RAGE images was $<1.0 \mathrm{~mm}$ up until $68.7 \mathrm{~mm}$ from the isocenter.

Table 4.2 Geometric distortion measures for CT

\begin{tabular}{lccccccc}
\hline & \multicolumn{3}{c}{ MAD $_{\text {global }}(\mathrm{mm})$} & & \multicolumn{3}{c}{ MAD Local $(\mathrm{mm})$} \\
\cline { 2 - 3 } & Range & Mean \pm SD & $\Delta$ & & Range & Mean \pm SD & $\Delta$ \\
\hline CT & $0.14-0.64$ & $0.20 \pm 0.05$ & 0.30 & & $0.06-0.27$ & $0.16 \pm 0.04$ & 0.24 \\
\hline
\end{tabular}

\section{DISCUSSION}

This paper reports on the preparatory work for our clinical study to integrate 7T MR images into neurosurgical navigation and RTP systems. It includes a pilot study with healthy volunteers and a phantom study. The volunteers in our pilot study reported limited side effects of the 7T MRI. Similar to other studies on UHF MRI, mainly transient vestibular effects were noted [29,30]. In our study these effects were only present during a change in table position. The cause of transient vertigo and 
dizziness is not yet fully understood, but magnetic stimulation of the ear's labyrinth has been suggested [31].

There are currently still a few shortcomings in image quality that need to be resolved before clinical implementation of UHF MRI. Although image quality in our study was good to excellent in the frontal and parietal lobes, all sequences demonstrated a non-diagnostic to marginal image quality for all evaluated parameters at the frontobasal and temporal lobes. This is a well-known issue for UHF-MRI and is generally caused by a decrease in signal and susceptibility artefacts near the skull base. As a consequence, patients with tumours near the skull base will be excluded from our clinical trial.

Many researchers have been working on improving the inversion contrast in MPRAGE, particularly trying to reduce the sensitivity to off-resonance and RF inhomogeneity effects [19]. RF transmit technology is a very active research field within UHF MRI, and better transmitter designs may become available. The application of dielectric pads improves the transmit field homogeneity, which in our case ensured obtaining sufficient signal from the temporal regions [15,32]. Concurrently, the application of dielectric pads also affects the receive $\mathrm{B}_{1}$ field and can lead to signal increases in their immediate vicinity. Image intensity inhomogeneities of this kind are also typical for multi-channel RF receive coils. They can be circumvented by image post-processing techniques such as division by an image with the appropriate weighting or by model fitting of at least two images differing by a single parameter [24]. The latter approach is utilized when generating the $\mathrm{T}_{2}{ }^{*}$ maps from the multi-echo GRE data and $\mathrm{T}_{1}$ maps from the MP2RAGE data, whereas the former approach is used to obtain the MP2RAGE T1-weighted images. It is worth mentioning that the inversion pulse of the MP2RAGE sequence has been specifically designed to deliver homogeneous $\mathrm{T}_{1}$-contrast across the whole brain despite $\mathrm{B}_{1}$-inhomogeneities [22]. Yet further gains may be possible through the use of parallel transmit (pTx) technology, where 8 or 16 separate elements may be combined to produce a more uniform or targeted excitation. However, the use of such systems involves significant additional experimental setup (e.g., more complicated RF coils and pre-scans to determine per-subject transmit patterns, etc.), which may limit the applicability to patients. Additionally, SAR considerations are significantly more complicated in a pTx setup, as different excitation patterns will produce different SAR values within the subject. While considerable progress has been made, there is still significant work required to implement this in clinical studies.

The phantom we used provides a realistic anthropomorphic scenario for CT and MRI to assess spatial distortion in 7T MRI for clinical use. Although there is no standard procedure for assessing spatial distortion in 7T MRI, AAPM and the 
Institute of Physics and Engineering in Medicine recommend to measure the distance between two selected points in the MR-image and to calculate the deviation from the known distance $[33,34]$. Based on these recommendations we assessed the geometrical image distortion using two different methods, MAD global and MADlocal. We selected two variable points in the image for MAD global assessment and selected one variable and one fixed point in the image (e.g., magnetic field isocenter) for MADlocal assessment [35,36].

The assessment of geometrical distortion in our study is similar to the study by Cho et al. investigating the visualisation of targets for deep brain stimulation in Parkinson disease [13]. However, in the present study an updated version of the CIRS phantom was used. Furthermore, we did not consider the CT as a reference standard and therefore did not co-register the CT to the MRI scan. Instead the known geometry of the phantom was considered the reference.

Although no geometrical distortion was expected, we do report minimal distortion from the CT images. This is probably caused by measurement uncertainties from manually assessing the grid coordinates and was not found to be correlated to inter-grid distances or distances from the isocenter. For MRI however, overall geometric distortion increases exponentially with increasing distances between grid-intersection points. In uncorrected GRE and MP2RAGE images, a plateau-stage starts to manifest at $58 \mathrm{~mm}$ from the isocenter. Presumably, this plateau-stage could be attributed to non-linearity of the gradient fields. Near the edges of the image, the assumed linear relationship between spin position and precession frequency in the local field is false, leading to a compression of the image. In distortion-corrected images, gradient uniformity was restored and as a result, the image was decompressed to its correct dimensions. Consequently, MP2RAGE sequences show a more linear increase of spatial deviation and higher MADlocal maxima are measured (Table 4.3). The larger range of MAD-values is related to the increase in measurable distance between grid-intersection points and the residual system-related geometrical distortion factors, such as eddy currents and Bo inhomogeneity, which were not corrected for. Others have used a similar method as applied in this study to estimate the reliability of stereotactic coordinates on $1.5 \mathrm{~T}$ and 3T MR images [37]. They reported a range of mean errors of $0.30-1.20 \mathrm{~mm}$ and $0.43-1.78 \mathrm{~mm}$ in $\mathrm{T}_{1} \mathrm{w}$-images at $1.5 \mathrm{~T}$ and $3 \mathrm{~T}$, respectively. For $\mathrm{T}_{2} \mathrm{~W}$-images, they have shown ranges of $0.29-0.58 \mathrm{~mm}$ and $0.31-1.85 \mathrm{~mm}$, respectively.

Geometric distortion can occur to different extents depending on the imaging sequence parameter settings [38]. Differences in MADlocal between GRE and MP2RAGE could be attributed to differences in read-out bandwidth (rBW). With a lower $\mathrm{rBW}$, more geometric distortion could be noted in the frequency-encoding or read-out direction. For MP2RAGE, a low rBW was used $(250 \mathrm{~Hz} /$ pixel $)$ for measurements in the SI read-out direction, while the read-out direction for GRE 
images was AP. To minimise this type of geometric distortion, a larger rBW could be selected, however this in turn implies a loss in SNR. Increasing slice thickness could compensate for this loss in SNR, but is undesirable in clinical practise as resolution decreases. Based on Figure 4.5 and 4.6, we conclude that geometric distortion is anisotropic. When measuring local displacement at equal distances from the magnetic field isocenter, the geometric distortion correlates with the frequency-encoding direction. However, the exact quantification of geometric distortion anisotropy was not investigated and will be analysed in future work. When interpreting MADglobal and MADlocal, it is important to note that the phantom used in this study describes the dimensions of a realistic human head. This implies that larger measurable distances from the isocenter could be measured in AP and SI direction than are possible in LR direction, leading to a possible misinterpretation of MAD global and MADlocal and their respective SD.

Geometrical distortion, image registration, and delineation inaccuracies should all be taken into account while establishing the PTV margin for the clinical application of the 7T MRI [39]. Although a larger PTV margin might be less relevant for RTP in GBM in which an isotropic CTV margin of $2-3 \mathrm{~cm}$ is added. An increase in PTV margin is highly detrimental for stereotactic radiosurgery, in which a very high radiation dose is given to a small volume of the brain. Manufacturer's distortion correction routines (i.e., gradient non-linearity correction and automated shimming) were applied to decrease geometric inaccuracies. However, Wang et al. showed that despite these measures, distortion can still be significantly present in UHF MR images [40]. As confirmed by our measurements (Fig. 4.5), the spatial deformation in geometrically corrected MR images is non-negligible for RTP purposes, especially for tumours located far from the magnetic field isocenter. This finding corresponds to the results obtained by other studies using a different phantom $[12,13]$. Current use of MR images in radiotherapy planning requires co-registration to CT images as information of electron density is needed for dose calculation. Although CT shows negligible geometric distortion, co-registration of CT and MR images introduces registration errors [41]. MRI-only based treatment planning could provide an alternative to errors introduced through registration, but requires thorough investigation before clinical implementation [42].

Any technical and logistic challenges we encountered in the image transfer and processing workflow were primarily caused by the different image acquisition and processing platforms used at the different institutions and departments involved in this study, e.g. 7T MRI scanner at Scannexus, neurosurgical and RTP software. Not all image data formats (e.g. NIfTi, xBrain) were compatible with these software systems. Fortunately, we could solve these problems by converting all image datasets into DICOM format and by using a cloud-based environment to collaboratively share the images. However, transfer and conversion of images could 
carry an inherent risk of data degradation and should be checked prior to clinical use.

The present study has several limitations and some factors that need to be considered when attempting to setup a similar study. First, the number of volunteers included in our pilot study was small. Moreover, sequence optimization was limited by the increase in SAR and scan duration and leaves room for future improvement. Second, unlike a human head, the anthropomorphic skull phantom did not contain any air cavities (i.e., sinuses) and only allowed assessment of the system-related geometrical distortion. Third, we only investigated geometrical distortion of 7T MRI in comparison to $\mathrm{CT}$ and did not yet evaluate lower field strength MR images. Geometrical distortion in relation to 1.5T and 3T field strengths will be addressed in a future study. Fourth, other hardware related geometrical distortions such as eddy currents and $\mathrm{B}_{0}$ inhomogeneity are not corrected and remain present in the images. In general, $B_{0}$ inhomogeneity could be resolved with shimming and field map correction, although these corrections are mostly effective in EPI sequences [43,44]. Finally, the distortion corrected GRE images could not be assessed for geometrical distortion due to severe artefacts, therefore a comparison between distortion corrected pulse sequences was not possible.

Several investigators suggested the presence of microvasculature on UHF MRI may aid in improved identification of WHO tumour grade in GBM as compared to 3T MRI $[8,10]$. So far, these findings have not resulted in a systematic investigation of the potential value of 7T MRI for improved neurosurgical navigation and target volume definition in RTP. Therefore, we aim to demonstrate the potential benefit of UHF MRI in identifying tumour infiltration for the delineation of GBM in our upcoming clinical study. This study is expected to open for inclusion in the first quarter of 2016. During the preparatory phase of the current study image quality has significantly improved. However, further improvement of the image quality by reducing susceptibility artefacts in the frontobasal region and in the temporal lobe is required before clinical implementation.

\section{CONCLUSION}

Although the integration of high quality and geometrically reliable 7T anatomical MR images into neurosurgical navigation and RTP software is technically feasible and safe for the system and pulse sequences studied, image quality needs further improvement before it can be integrated into the clinical workflow. System-related geometrical distortions of the 7T sequences studied are clinically acceptable for image co-registration with $\mathrm{CT}$ prior to radiotherapy and for direct use in neurosurgical procedures. 


\section{Acknowledgments}

This research was partially supported by the Brains Unlimited Pioneer Fund of the Limburg University Fund/SWOL. We would like to thank B.G. Baumert MD, PhD, MBA for her support with the study concept. Furthermore, we would like to thank the Support Team at Scannexus, Margo van de Wetering and Esther Steijvers, for their support in the acquisition and optimization of the 7T MRI sequences.

\section{REFERENCES}

1. Stupp R, Hegi ME, Mason WP, van den Bent MJ, Taphoorn MJ, Janzer RC, Ludwin SK, Allgeier A, Fisher B, Belanger K, Hau P, Brandes AA, Gijtenbeek J, Marosi C, Vecht CJ, Mokhtari K, Wesseling P, Villa S, Eisenhauer E, Gorlia T, Weller M, Lacombe D, Cairncross JG, Mirimanoff RO (2009) Effects of radiotherapy with concomitant and adjuvant temozolomide versus radiotherapy alone on survival in glioblastoma in a randomised phase III study: 5-year analysis of the EORTC-NCIC trial. Lancet Oncol 10 (5):459-466. doi:S1470-2045(09)70025-7

2. Claes A, Idema AJ, Wesseling P (2007) Diffuse glioma growth: a guerilla war. Acta neuropathol. 114 (5):443-458. doi:10.1007/s00401-007-0293-7

3. Weller M, van den Bent M, Hopkins K, Tonn JC, Stupp R, Falini A, Cohen-Jonathan-Moyal E, Frappaz D, Henriksson R, Balana C, Chinot O, Ram Z, Reifenberger G, Soffietti R, Wick W (2014) EANO guideline for the diagnosis and treatment of anaplastic gliomas and glioblastoma. Lancet Oncol 15 (9):e395-403. doi:10.1016/s1470-2045(14)70011-7

4. Chang EL, Akyurek S, Avalos T, Rebueno N, Spicer C, Garcia J, Famiglietti R, Allen PK, Chao KS, Mahajan A, Woo SY, Maor MH (2007) Evaluation of peritumoral edema in the delineation of radiotherapy clinical target volumes for glioblastoma. Int J Radiat Oncol Biol Phys 68 (1):144-150. doi:S0360-3016(06)03649-2

5. Oppitz U, Maessen D, Zunterer H, Richter S, Flentje M (1999) 3D-recurrence-patterns of glioblastomas after CT-planned postoperative irradiation. Radiother Oncol 53 (1):53-57. doi:S0167814099001176

6. Kuroiwa T, Ueki M, Chen Q, Suemasu H, Taniguchi I, Okeda R (1994) Biomechanical characteristics of brain edema: the difference between vasogenic-type and cytotoxic-type edema. Acta Neurochir Suppl (Wien) 60:158-161

7. Louis DN, International Agency for Research on Cancer, World Health Organization (2007) WHO classification of tumours of the central nervous system. WHO series on histological and genetic typing of human tumours ; 4th ed., 1st v, 4th edn. International Agency for Research on Cancer, 2007., Lyon. doi:9283224302

8. Moenninghoff C, Maderwald S, Theysohn JM, Kraff O, Ladd ME, El Hindy N, van de Nes J, Forsting M, Wanke I (2010) Imaging of adult astrocytic brain tumours with 7 T MRI: preliminary results. Eur Radiol 20 (3):704-713. doi:10.1007/s00330-009-1592-2

9. Christoforidis GA, Grecula JC, Newton HB, Kangarlu A, Abduljalil AM, Schmalbrock P, Chakeres DW (2002) Visualization of microvascularity in glioblastoma multiforme with 8-T high-spatial-resolution MR imaging. AJNR Am J Neuroradiol 23 (9):1553-1556

10. Christoforidis GA, Yang M, Abduljalil A, Chaudhury AR, Newton HB, McGregor JM, Epstein CR, Yuh WT, Watson S, Robitaille PM (2012) "Tumoral pseudoblush" identified within gliomas at high-spatialresolution ultrahigh-field-strength gradient-echo MR imaging corresponds to microvascularity at stereotactic biopsy. Radiology 264 (1):210-217. doi:radiol.12110799

11. Lupo JM, Li Y, Hess CP, Nelson SJ (2011) Advances in ultra-high field MRI for the clinical management of patients with brain tumors. Curr Opin Neurol 24 (6):605-615. doi:10.1097/WCO.0b013e32834cd495

12. Dammann P, Kraff O, Wrede KH, Ozkan N, Orzada S, Mueller OM, Sandalcioglu IE, Sure U, Gizewski ER, Ladd ME, Gasser T (2011) Evaluation of hardware-related geometrical distortion in structural MRI at 7 Tesla for image-guided applications in neurosurgery. Acad Radiol 18 (7):910-916. doi:S10766332(11)00127-9

13. Cho ZH, Min HK, Oh SH, Han JY, Park CW, Chi JG, Kim YB, Paek SH, Lozano AM, Lee KH (2010) Direct visualization of deep brain stimulation targets in Parkinson disease with the use of 7-tesla magnetic resonance imaging. J Neurosurg 113 (3):639-647. doi:10.3171/2010.3.jns091385 
14. Duchin Y, Abosch A, Yacoub E, Sapiro G, Harel N (2012) Feasibility of using ultra-high field (7 T) MRI for clinical surgical targeting. PLoS One 7 (5):e37328. doi:10.1371/journal.pone.0037328

15. Teeuwisse WM, Brink WM, Haines KN, Webb AG (2012) Simulations of high permittivity materials for $7 \mathrm{~T}$ neuroimaging and evaluation of a new barium titanate-based dielectric. Magn. reson. med. 67 (4):912-918. doi:10.1002/mrm.24176

16. Schenck JF (1996) The role of magnetic susceptibility in magnetic resonance imaging: MRI magnetic compatibility of the first and second kinds. Med. phys. 23 (6):815-850

17. Marques JP, Kober T, Krueger G, van der Zwaag W, Van de Moortele PF, Gruetter R (2010) MP2RAGE, a self bias-field corrected sequence for improved segmentation and T1-mapping at high field. Neuroimage 49 (2):1271-1281. doi:10.1016/j.neuroimage.2009.10.002

18. Mugler JP, 3rd, Brookeman JR (1990) Three-dimensional magnetization-prepared rapid gradient-echo imaging (3D MP RAGE). Magn. reson. med. 15 (1):152-157

19. Wrede KH, Johst S, Dammann P, Umutlu L, Schlamann MU, Sandalcioglu IE, Sure U, Ladd ME, Maderwald S (2012) Caudal image contrast inversion in MPRAGE at 7 Tesla: problem and solution. Acad Radiol 19 (2):172-178. doi:10.1016/j.acra.2011.10.004

20. Marques JP, Gruetter R (2013) New developments and applications of the MP2RAGE sequence--focusing the contrast and high spatial resolution R1 mapping. PLoS One 8 (7):e69294. doi:10.1371/journal.pone.0069294

21. O'Brien KR, Kober T, Hagmann P, Maeder P, Marques J, Lazeyras F, Krueger G, Roche A (2014) Robust T1-weighted structural brain imaging and morphometry at 7T using MP2RAGE. PLoS One 9 (6):e99676. doi:10.1371/journal.pone.0099676

22. Hurley AC, Al-Radaideh A, Bai L, Aickelin U, Coxon R, Glover P, Gowland PA (2010) Tailored RF pulse for magnetization inversion at ultrahigh field. Magn. reson. med. 63 (1):51-58. doi:10.1002/mrm.22167

23. Okubo G, Okada T, Yamamoto A, Kanagaki M, Fushimi Y, Okada T, Murata K, Togashi K (2016) MP2RAGE for deep gray matter measurement of the brain: A comparative study with MPRAGE. J Magn Reson Imaging 43 (1):55-62. doi:10.1002/jmri.24960

24. Van de Moortele PF, Auerbach EJ, Olman C, Yacoub E, Ugurbil K, Moeller S (2009) T1 weighted brain images at 7 Tesla unbiased for Proton Density, T2* contrast and RF coil receive B1 sensitivity with simultaneous vessel visualization. Neuroimage 46 (2):432-446. doi:10.1016/j.neuroimage.2009.02.009

25. Hennig J, Nauerth A, Friedburg H (1986) RARE imaging: a fast imaging method for clinical MR. Magn. reson. med. 3 (6):823-833

26. Mugler John P. KRB, Brookeman James R. (2000) Three-dimensional T2-weighted imaging of the brain using very long spin-echo trains. In: Proceedings of the 8th Scientific Meeting of the International Society for Magn. reson. med., Denver, Colorado, p 687

27. Mugler John P. WLL, Brookeman James R. (2001) T2-weighted 3D spin-echo train imaging of the brain at 3 Tesla: reduced power deposition using low flip-angle refocusing RF pulses. In: Proceedings of the 9th Scientific Meeting of the International Society for Magn. reson. med., Glasgow, Scotland, UK, p438

28. Busse RF, Hariharan H, Vu A, Brittain JH (2006) Fast spin echo sequences with very long echo trains: design of variable refocusing flip angle schedules and generation of clinical T2 contrast. Magn. reson. med. 55 (5):1030-1037. doi:10.1002/mrm.20863

29. Schaap K, Christopher-de Vries Y, Mason CK, de Vocht F, Portengen L, Kromhout H (2014) Occupational exposure of healthcare and research staff to static magnetic stray fields from 1.5-7 Tesla MRI scanners is associated with reporting of transient symptoms. Occup. Environ. Med. 71 (6):423-429. doi:10.1136/oemed-2013-101890

30. Heilmaier C, Theysohn JM, Maderwald S, Kraff O, Ladd ME, Ladd SC (2011) A large-scale study on subjective perception of discomfort during 7 and $1.5 \mathrm{~T}$ MRI examinations. Bioelectromagnetics 32 (8):610-619. doi:10.1002/bem.20680

31. Ward BK, Roberts DC, Della Santina CC, Carey JP, Zee DS (2015) Vestibular stimulation by magnetic fields. Annals of the New York Academy of Sciences 1343:69-79. doi:10.1111/nyas.12702

32. Brink WM, van der Jagt AM, Versluis MJ, Verbist BM, Webb AG (2014) High permittivity dielectric pads improve high spatial resolution magnetic resonance imaging of the inner ear at 7 T. Invest Radiol 49 (5):271-277. doi:10.1097/rli.0000000000000026

33. Price RR, Axel L, Morgan T, Newman R, Perman W, Schneiders N, Selikson M, Wood M, Thomas SR 
(1990) Quality assurance methods and phantoms for magnetic resonance imaging: report of AAPM nuclear magnetic resonance Task Group No. 1. Med. phys. 17 (2):287-295

34. Lerski RA, Schad LR (1998) The use of reticulated foam in texture test objects for magnetic resonance imaging. Magn Reson Imaging 16 (9):1139-1144

35. Wang D, Doddrell DM, Cowin G (2004) A novel phantom and method for comprehensive 3-dimensional measurement and correction of geometric distortion in magnetic resonance imaging. Magn Reson Imaging 22 (4):529-542. doi:10.1016/j.mri.2004.01.008

36. Roue A, Ferreira IH, Van Dam J, Svensson H, Venselaar JL (2006) The EQUAL-ESTRO audit on geometric reconstruction techniques in brachytherapy. Radiother Oncol 78 (1):78-83. doi:10.1016/j.radonc.2005.12.004

37. Kim HY, Lee SI, Jin SJ, Jin SC, Kim JS, Jeon KD (2014) Reliability of stereotactic coordinates of 1.5-tesla and 3-tesla MRI in radiosurgery and functional neurosurgery. J Korean Neurosurg Soc. 55 (3):136-141. doi:10.3340/jkns.2014.55.3.136

38. Walker A, Liney G, Metcalfe P, Holloway L (2014) MRI distortion: considerations for MRI based radiotherapy treatment planning. Australas Phys Eng Sci Med. 37 (1):103-113. doi:10.1007/s13246-0140252-2

39. van Herk M (2004) Errors and margins in radiotherapy. Semin Radiat Oncol. 14 (1):52-64. doi:10.1053/j.semradonc.2003.10.003

40. Wang D, Strugnell W, Cowin G, Doddrell DM, Slaughter R (2004) Geometric distortion in clinical MRI systems Part II: correction using a 3D phantom. Magn Reson Imaging 22 (9):1223-1232. doi:10.1016/j.mri.2004.08.014

41. Baldwin LN, Wachowicz K, Thomas SD, Rivest R, Fallone BG (2007) Characterization, prediction, and correction of geometric distortion in 3 T MR images. Med. phys. 34 (2):388-399

42. Schmidt MA, Payne GS (2015) Radiotherapy planning using MRI. Phys Med Biol. 60 (22):R323-361. doi:10.1088/0031-9155/60/22/r323

43. Chang HC, Chuang TC, Lin YR, Wang FN, Huang TY, Chung HW (2013) Correction of geometric distortion in Propeller echo planar imaging using a modified reversed gradient approach. Quantitative imaging in medicine and surgery 3 (2):73-81. doi:10.3978/j.issn.2223-4292.2013.03.05

44. Wang FN, Huang TY, Lin FH, Chuang TC, Chen NK, Chung HW, Chen CY, Kwong KK (2005) PROPELLER EPI: an MRI technique suitable for diffusion tensor imaging at high field strength with reduced geometric distortions. Magn Reson Med. 54 (5):1232-1240. doi:10.1002/mrm.20677 

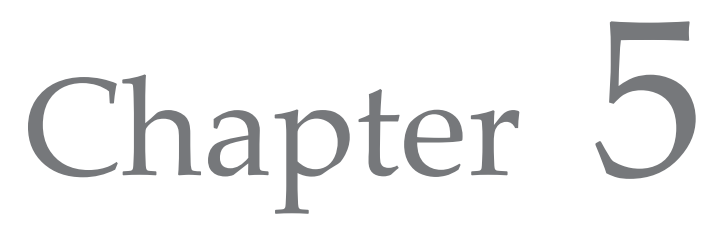

Characterizing geometrical accuracy in clinically optimised 7T and 3T MRI for high-precision radiation treatment of brain tumours

Authors:

J. Peerlings, I. Compter, F. Janssen, C. Wiggins, A. Postma, F. Mottaghy, P. Lambin, A. L. Hoffmann

Adapted from:

Physics \& Imaging in Radiation Oncology 2019, Jan; (9):35-42 DOI: $10.1016 /$ j.phro.2018.12.001 


\section{ABSTRACT}

\section{PURPOSE}

To assess whether the spatial accuracy in 7-Tesla (7T) MR images, previously optimised for anatomical imaging, is sufficient for clinicallyacceptable radiation treatment planning (RTP) in neuro-oncology with respect to standard clinical imaging-modalities.

\section{MATERIALS \& METHODS}

System- and phantom-related geometrical distortion (GD) were quantified on clinically-relevant MR-sequences at 7T and 3T, and on CTimages using a dedicated anthropomorphic head-phantom incorporating a 3D grid-structure, creating 436 points-of-interest. Global GD was assessed by mean absolute deviation (MADGlobal). Local GD relative to the magnetic isocentre was assessed by MADLocal. Using 3D displacement-vectors of individual points-of-interest, GD maps were created. For clinically acceptable radiotherapy, 7T-images need to meet the criteria for accurate dose delivery $(\mathrm{GD}<1 \mathrm{~mm})$ and present comparable GD as tolerated in clinically-standard 3T-MR/CT-based RTP.

\section{RESULTS}

MADGlobal in 7T- and 3T-images ranged from $0.3-2.2 \mathrm{~mm}$ and $0.2-0.8$ $\mathrm{mm}$, respectively. MADLocal increased with increasing distance from the isocentre, showed an anisotropic distribution, and was significantly larger in 7T MR-sequences (MADLocal=0.2-1.2 $\mathrm{mm}$ ) than in 3T $($ MAD Local $=0.1-0.7 \mathrm{~mm}) \quad(\mathrm{p}<0.05)$. Significant differences in GD were detected between $7 \mathrm{~T}$-images $(\mathrm{p}<0.001)$. However, maximum MADLocal remained $\leq 1 \mathrm{~mm}$ within $68.7 \mathrm{~mm}$ diameter spherical volume. No significant differences in GD were found between 7T- and 3T-protocols near the isocentre.

\section{CONCLUSION}

System- and phantom-related GD remained $\leq 1 \mathrm{~mm}$ in central brain regions, suggesting that $7 \mathrm{~T}$ MR-images could be implemented in radiotherapy with clinically-acceptable spatial accuracy and equally tolerated GD as in 3T-MR/CT-based RTP. For peripheral regions, GD should be incorporated in safety margins for treatment uncertainties. Moreover, the effects of sequence-related factors on GD needs further investigation to obtain RTP-specific MR protocols. 


\section{INTRODUCTION}

In radiation treatment planning (RTP) of brain tumours, magnetic resonance imaging (MRI) at 1.5 or 3 Tesla (T) is currently being used as the standard anatomical imaging modality owing to its superior soft-tissue contrast compared to computer tomography (CT) [1]. Generally, after co-registration of MR and CT images, the former is used for target volume definition and the latter is used for dose calculation, as tissue electron density information is missing in MR-images. However, the current clinical MR-techniques are limited in depicting detailed neurologic malformations such as intracerebral tumour spread [2]. With the prospect of clinically-certified ultra-high field (UHF-)MRI systems ( $\geq 7 \mathrm{~T})$, images with higher signal-to-noise ratio (SNR), higher spatial resolution, and novel contrast mechanisms such as quantitative susceptibility-weighted images will become available, enabling the visualisation of small lesions, basal ganglia, and tumour angiogenesis [3-5]. The improved ability in detecting microvasculature (diameter $\sim 100 \mu \mathrm{m}$ ) could play a decisive role in staging primary brain tumours such as glioblastoma (GBM), as tumour angiogenesis is directly associated with tumour grade, and could aid in determining relevant location of dedifferentiated cells for image-guided biopsies [6, 7]. In addition, 7T-MRI could aid target volume definition in RTP by visualising microvasculature outside the contrast-enhanced tumour and infiltration of GBM cells along white matter tracts [7-9]. During follow-up scans, 7TMRI has been shown to reveal radiation-induced microbleeds, indicating potential neurocognitive decline and the need to adapt RTP $[10,11]$.

However, concerns regarding geometrical distortion (GD) with increasing static magnetic field strength $\left(\mathrm{B}_{0}\right)$ have compromised the integration of UHF-MRI into radiation treatment planning. System-related GD are mainly induced by imperfections in hardware (Bo-inhomogeneity, gradient field nonlinearity, and eddy currents), applied gradient pulse scheme and parameter settings (sequencedependent GD), while phantom-related GD (chemical shift and susceptibility differences) are related to the object's shape placed in the MR-system [12, 13]. To be applicable for high-precision RTP, the spatial accuracy of anatomical images needs to be within $2 \mathrm{~mm}$ for non-stereotactic radiotherapy and within $1 \mathrm{~mm}$ for stereotactic radiotherapy, as is the case for 3T-MRI [13-18]. In fact, profound GD may have significant dosimetric impact in radiotherapy as the precision of beam targeting and dose calculations could be affected [19, 20]. For high-precision dose delivery techniques that apply steep 3D dose gradients (intracranial stereotactic radiotherapy or radiosurgery), spatial inaccuracies of $>1 \mathrm{~mm}$ could result in clinicallyunacceptable dose variations of more than $15 \%$ that could lead to underdosage of target volume and/or overdosage of surrounding tissue [20, 21]. Ultimately, inaccuracy of beam targeting and dose calculation could lead to a different clinical outcome and local tumour control [13, 18]. For instance, techniques that apply 
extremely steep dose gradients, such as stereotactic radiotherapy and radiosurgery, are susceptible to significant dosimetric effects (5-32\% underdosage) caused by even small GD of $1.3 \mathrm{~mm}[21,22]$. The question therefore arises whether our clinical 7TMRI protocol, optimised for anatomical imaging, can produce images with acceptable spatial accuracy needed for reliable high-precision radiation treatment of brain tumours. Previously several investigators have evaluated geometrical distortion on either CT, 3T- or 7T-MRI, demonstrating a maximum GD of up to several $\mathrm{mm}$ in large cylindrical or cubical phantoms [14, 23-27].

This study aims to investigate the clinical applicability of anatomical 7T-MRI in comparison to 3T-MRI and CT for reliable high-precision radiotherapy by evaluating the magnitude and location of hardware-related GD. We hypothesise that: 1) acceptable submillimetre spatial accuracy could be achieved in our 7T-MRI protocol and no significant differences in GD are present between different 7Tsequences; 2) the tolerated level of GD in 7T-MRI is not significantly different from system-related GD in a predefined 3T-MRI protocol, clinically used for RTP. To test these hypotheses on both global level (GD throughout the entire volume-of-interest (VOI)) and on local level (GD relative to the distance from the magnetic isocentre), we performed quantitative image analyses using a dedicated anthropomorphic head-phantom in a clinically-realistic set-up. Hence, different sequence parameter settings and distortion correction methods were not explored in this study.

\section{MATERIAL \& METHODS}

\section{PHANTOM}

A dedicated anthropomorphic head-phantom (CIRS Model 603A, Computerized Imaging Reference Systems Inc., USA) was used to evaluate system-related GD in 7T-MRI, 3T-MRI, and CT-images (Fig. S5.1). The anatomical shape demanded similar procedures used for clinically imaging patients (FOV setting, shimming, robust positioning inside the scanner and head-coil). Furthermore, the centre of the phantom was aligned with the magnetic isocentre.

The phantom was composed of a plastic-based bone substitute and soft-tissue equivalent fluid consisting of a water-based polyacrylamide with added $\mathrm{NiCl}_{2}$ (Zerdine $\left.{ }^{\circledR}, \mathrm{CIRS}\right)$. Inside the phantom, 3-mm thick nylon rods formed a 3D grid with $15 \mathrm{~mm}$ spacing $( \pm 0.05 \mathrm{~mm}$ manufacturing tolerance), defining a VOI of $15 \times 12 \times 13.5 \mathrm{~cm}^{3}$ (anterior-posterior $(\mathrm{AP}) \times$ left-right $(\mathrm{LR}) \times$ superior-inferior $(\mathrm{SI})$ ). At grid-intersections, 437 measurable points-of-interest (POIs) were identified. Nylon shows similar magnetic susceptibility properties to water $(\chi<3 \mathrm{ppm})$ and was assumed not to induce such susceptibility artefacts in spin-echo or gradient-echo sequences [24, 28]. 


\section{IMAGE ACQUISITION}

MR images were acquired on a 7T-MR system (Magnetom 7T, Siemens, DEU) and on a 3T-MR system (Achieva 3T, Philips, NL) using 32-channel head coils from Nova Medical inc. (1Tx/32Rx, Wilmington, USA) and Invivo Corp. (Florida, USA), respectively. The 7T-MR system was equipped with Siemens' SC72-gradient system $(80 \mathrm{mT} / \mathrm{m}$ peak gradient amplitude, $200 \mathrm{~T} / \mathrm{m} / \mathrm{s}$ maximum slew rate). The 3T-MR system was fitted with Philips' Dual Quasar gradient system $(80 \mathrm{mT} / \mathrm{m}$ peak gradient amplitude, $100 \mathrm{mT} / \mathrm{m} / \mathrm{s}$ maximum slew rate). All MR sequences were previously optimised to acquire anatomical images of high image-quality within an acceptable scan duration, and were not altered for this study [9]. The selected 7TMR sequences included magnetization-prepared rapid gradient-echo (MP2RAGE), T2-sampling perfection with application optimized contrasts using different flip angle evolution (T2-SPACE), T2-SPACE with fluid attenuation inversion recovery (T2-SPACE FLAIR), and multi-echo gradient-echo (T1-GRE) [9]. Sequences with equivalent pulse schemes were selected from a predefined clinical 3T-MR protocol used in RTP and included turbo field echo (3D TFE), volume isotropic turbo spinecho (T2-VISTA) with and without FLAIR, and fast field gradient echo (T1-FFE), respectively (Table S5.1). Vendor-installed 3D distortion correction methods were applied to restore gradient non-linearity and 3D volume-based second order shimming procedures were applied to improve Bo-homogeneity [14, 29, 30].

CT images were acquired (SOMATOM Sensation 10, Siemens, Germany) with a slice thickness of $1 \mathrm{~mm}, 306$ slices, $50 \times 50 \mathrm{~cm}^{2} \mathrm{FOV}, 140 \mathrm{kV}$, and $400 \mathrm{mAs}$.

\section{METHODS OF ANALYSES}

The known 3D coordinates of all POIs were defined as reference dataset and manually reconstructed in MRI and CT images using Eclipse treatment planning system (Varian Medical Systems, USA). The X-, Y-, and Z-axes defined the LR-, SI-, and AP-directions in both datasets, respectively.

Three methods were used to assess GD at different levels of sophistication using in-house developed MATLAB software (R2014b, MathWorks Inc, USA) (Fig. 5.1). Firstly, mean absolute deviation (MAD) was computed to assess GD on a global level (MADGlobal) throughout the entire VOI, independently of location within the phantom. Secondly, MAD was calculated to assess GD on a local level (MAD Local) relative to the distance from the magnetic isocentre. MAD was calculated according to:

$$
M A D(i)=\frac{1}{N(i)} \sum_{j=1}^{N(i)}\left|D_{i}(j)-\delta_{i}\right|
$$

where $i=1 \ldots M$ is an index into set $\Delta=\left\{\delta_{1}, \delta_{2}, \ldots, \delta_{M}\right\}$ of $M$ unique distances, $D_{i}(j)$ is the measured Euclidean distance for the $j$-th unique pair of POIs having an Euclidean distance $\delta_{i}$ in the reference dataset, and $N(i)$ represents the number of 
unique pairs of POIs. For MADGlobal, $\Delta$ contained all unique distances that could be observed within the entire phantom. For MADLocal, $\Delta$ contained only unique distances that originate from the POI nearest the magnetic isocentre. The mean MAD was averaged over all $M$ unique distances in $\Delta$.

The third method of analyses pin-pointed GD by calculating 3D displacement of each individual POI by computing the Euclidean distance directly between the POI-coordinates in the image $\left(X_{\mathrm{m}}, Y_{\mathrm{m}}, Z_{\mathrm{m}}\right)$ and the coordinates in the reference datasets $\left(X_{\text {ref, }} Y_{\text {ref, }} Z_{\text {ref }}\right)$ :

$$
D_{x y z}=\sqrt{\left(X_{m}-X_{\text {ref }}\right)^{2}+\left(Y_{m}-Y_{\text {ref }}\right)^{2}+\left(Z_{m}-Z_{\text {ref }}\right)^{2}}
$$

The resulting 3D displacement vector $\left(D_{\mathrm{xyz}}\right)$ indicated relative GD of each individual POI, independently of all other POIs.

The three methods of analyses provided complementary information on the geometrical quality of the images. The MADGlobal quantified the level of GD throughout the entire VOI, thereby ignoring information on the absolute and relative locations of the POIs. The MADLocal retained spatial information by quantifying the GD with respect to the diameter of a spherical volume (DSV) at the magnetic isocentre. To quantify spatial integrity of an entire image, $95 \%$ confidence interval $\left(\left|\mathrm{Cl}_{95}\right|\right)$ was calculated from MAD and standard deviation (SD) as:

$$
\left|C I_{95}\right|=M A D+2 S D
$$

Based on this interval, a maximum acceptability-level of $1 \mathrm{~mm}$ was defined. The level of GD was considered acceptable when MADLocal $\leq 1 \mathrm{~mm}$ (at a specific DSV) and $\left|\mathrm{Cl}_{95}\right| \leq 1 \mathrm{~mm}$.

\section{Statistical Analyses}

To assess differences in sequence-dependent GD, MADGlobal and MADLocal were analysed between 7T-MRI sequences and between 3T-MRI sequences using a Kruskal-Wallis test. To assess differences in tolerated GD-levels, MADGlobal and MADLocal were analysed between equivalent sequence pulse schemes in 7T- and 3TMRI using a multiple student $\mathrm{t}$-test with Bonferonni-Sidak correction. For MADLocal, DSV at which statistical significance in MADLocal between sequences occurred, was identified by a separate Kruskal-Wallis test for each set of unique distances. All statistical analyses were performed using GraphPad Prism v6.01 (GraphPad, USA). $P$-values $<0.05$ were considered to indicate statistical significance. 
1a. Measured dataset: image-based

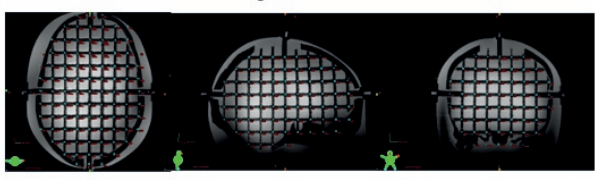

2. Mean Absolute Deviation (MAD)

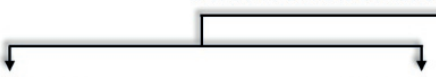

2a. Global MAD *

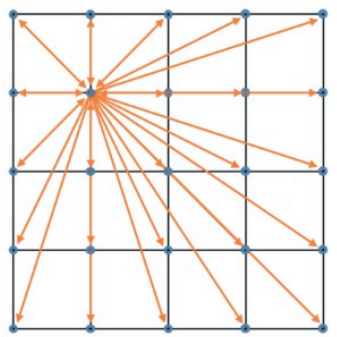

2b. Local MAD

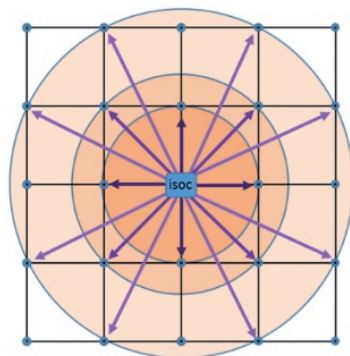

1b. Reference data: phantom-based
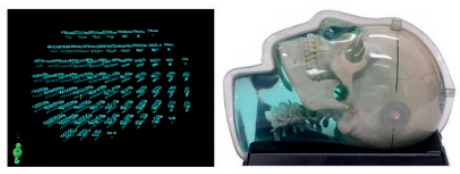

3. Relative deviation

3a. Displacement vector

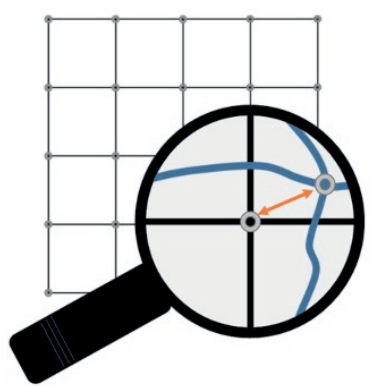

Figure 5.1: Overview of 3D data acquisition and analyses. XYZ-coordinates are determined on CT and MR images (1a.), and based on product characteristics of the CIRS' phantom model 603A (1b.). Global MAD (2a.) is based on distances with 2 variable grid-intersection points (*example given for 1 intersection but applies for all points). Local MAD (2b.) is based on distances between magnetic field isocenter and 1 variable grid-intersection points. Displacements vectors (3a.) are determined between the measured and reference coordinates of each individual data-point and indicate the relative $3 \mathrm{D}$ geometrical distortion

\section{RESULTS}

\section{GLOBAL GD}

In total, 95.266 pairs of distances were identified and binned into 100 unique distances, ranging from $15 \mathrm{~mm}$ to $164.3 \mathrm{~mm}$. In CT images, MADGlobal ranged from 0.1-0.6 mm with mean MADGlobal of $0.3 \pm 0.1 \mathrm{~mm}$. For MR images, MADGlobal ranged from 0.3-2.2 $\mathrm{mm}$ and $0.2-0.8 \mathrm{~mm}$ in 7T- and 3T-MRI, respectively (Table 5.1, Fig.5.2). Based on $\left|\mathrm{CI}_{95}\right|$, only 3T-MR sequences presented GD $<1 \mathrm{~mm}$ throughout the entire image. No statistical significance in MADGlobal were found between 7T-MR images $(\mathrm{P}=0.38)$ or 3T-MR images $(\mathrm{P}=0.13)$. However, significant differences in MADGlobal were detected between all equivalent sequences on $7 \mathrm{~T}$ and $3 \mathrm{~T}$ MR-system $(\mathrm{P}<0.001)$. 

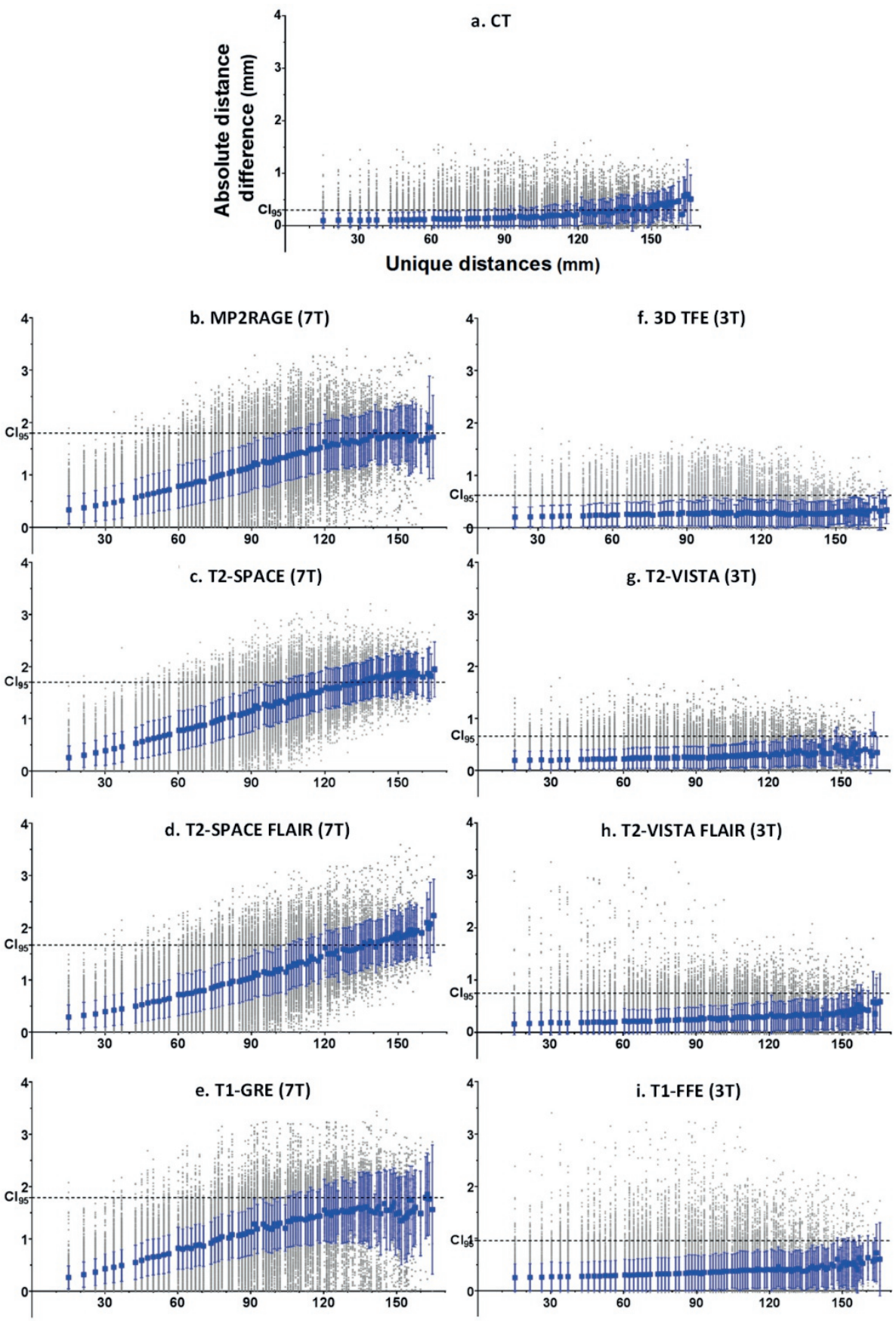

Figure 5.2: Absolute differences in Euclidian distance between the measured and reference dataset (grey dot) relative to the unique distances found in the reference dataset, observed within CT (a), MP2RAGE (b), T2-SPACE (c), T2-SPACE FLAIR (d), T1-GRE (e), 3D TFE (f), T2-TSE (g), T2-TSE FLAIR (h), T1-FFE (i). The overall geometric distortion was quantified by MADGlobal $( \pm \mathrm{SD})$ (blue square). The $95 \%$ confidence interval (C195) is shown as the dotted horizontal line 

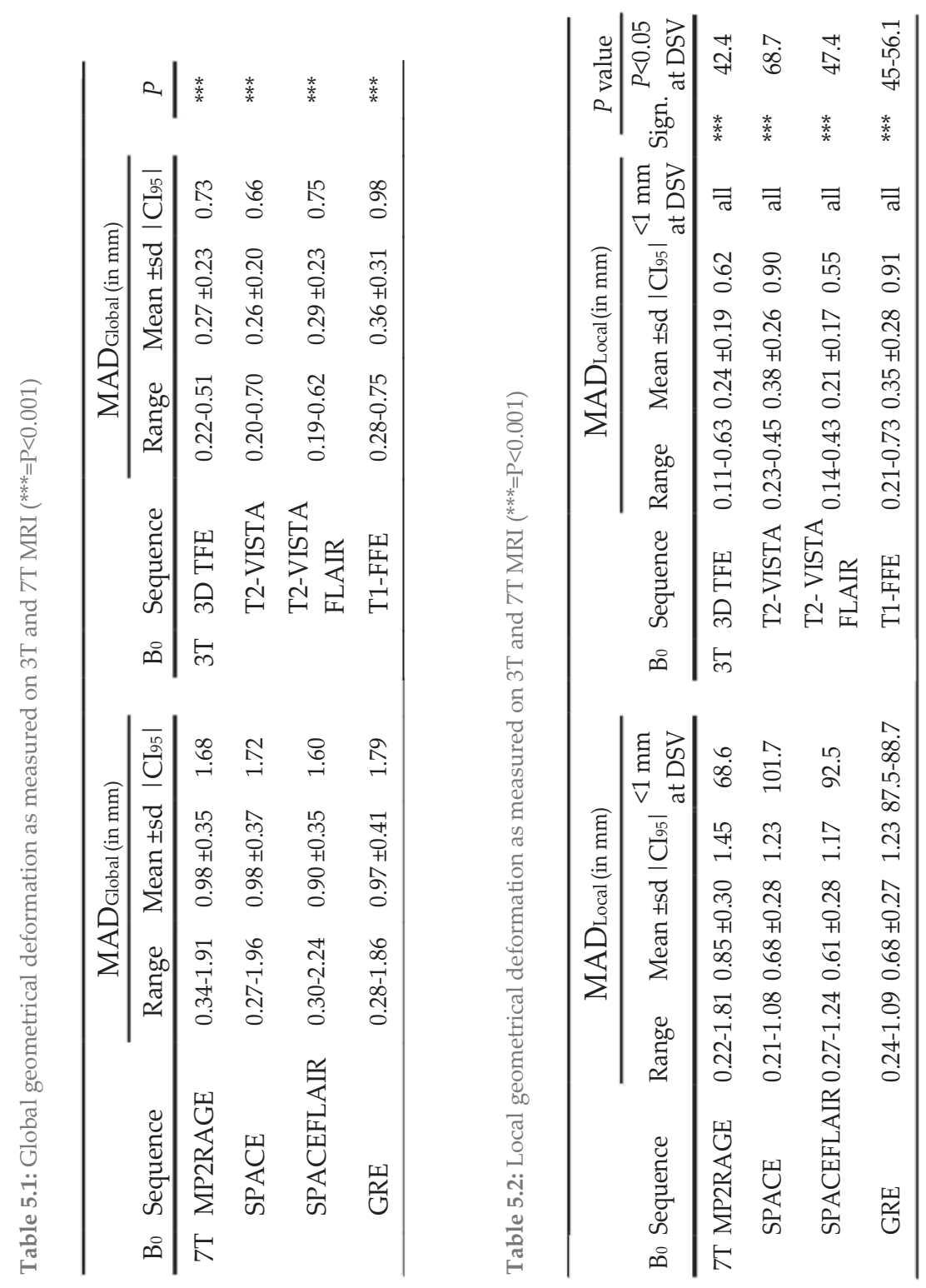


\section{LOCAL GD}

In CT images, MADLocal ranged from 0.1-0.3 mm with mean MADLocal of $0.2 \pm 0.04 \mathrm{~mm}$ (Fig. 5.3). All MR images acquired at 7T presented a steady increase in MAD Local with increasing distance from the magnetic isocentre. MADLocal ranged from $0.2-1.8 \mathrm{~mm}$ for 7T-MRI and from 0.1-0.7 mm for 3T-MRI. Each 7T-MR sequence remained below the clinical tolerance-level of $1 \mathrm{~mm}$ up until a defined DSV (Table 5.2). For T1-GRE, MADLocal exceeded $1 \mathrm{~mm}$ only at DSV of 87.5-88.7 $\mathrm{mm}$.

For all 3T-MR sequences, MADLocal remained below $1 \mathrm{~mm}$ throughout the whole VOI. MADLocal of MP2RAGE was statistically greater than all other 7T-MR sequences $(\mathrm{P}<0.001$, Fig. S5.2). No significant difference in MAD Local was found between T2-SPACE, T2-SPACE FLAIR ( $\mathrm{P}=0.064)$, and T1-GRE ( $\mathrm{P}=0.093)$, respectively. Within the 3T-dataset, MADLocal of 3D TFE was significantly different from T2-VISTA $(\mathrm{P}<0.001)$, T2-VISTA FLAIR $(\mathrm{P}=0.046)$, and T1-FFE $(\mathrm{P}<0.001)$. T2VISTA FLAIR presented significantly different MADLocal from T1-FFE $(\mathrm{P}<0.001)$. MADLocal was not significantly different between T1-FFE and T2-VISTA ( $\mathrm{P}=0.51)$, and the T2-VISTA and T2-VISTA FLAIR sequences $(\mathrm{P}=0.061)$. However, all sequences meet the criteria for GD and seem to have found an equilibrium with requirements for anatomical imaging.

Statistical analyses of MADLocal between equivalent 7T and 3T-sequences indicated significant differences for all sequences $(\mathrm{P}<0.001)$. However, no significant difference in MADLocal was found between equivalent sequences when the DSV was considered (Table 5.2).

\section{D DISPLACEMENT}

The magnitude of each displacement vector is presented in a 2D mesh-plot in axial $(\mathrm{XZ})$, coronal $(\mathrm{XY})$, and sagittal $(\mathrm{YZ})$ planes, intersecting the magnetic isocentre. The worst GD was presented in MP2RAGE and 3D TFE (Fig. 5.4). Additionally, anisotropic distributions of GD were observed along the phase-encoding direction and frequency-encoding direction. Particularly the anterior-superior section was affected by spatial deformation and maximal GD was observed in the right-superior section of the anterior region (frontal lobe). Distortion maps of all sequences are presented in Figures S5.3-5. 

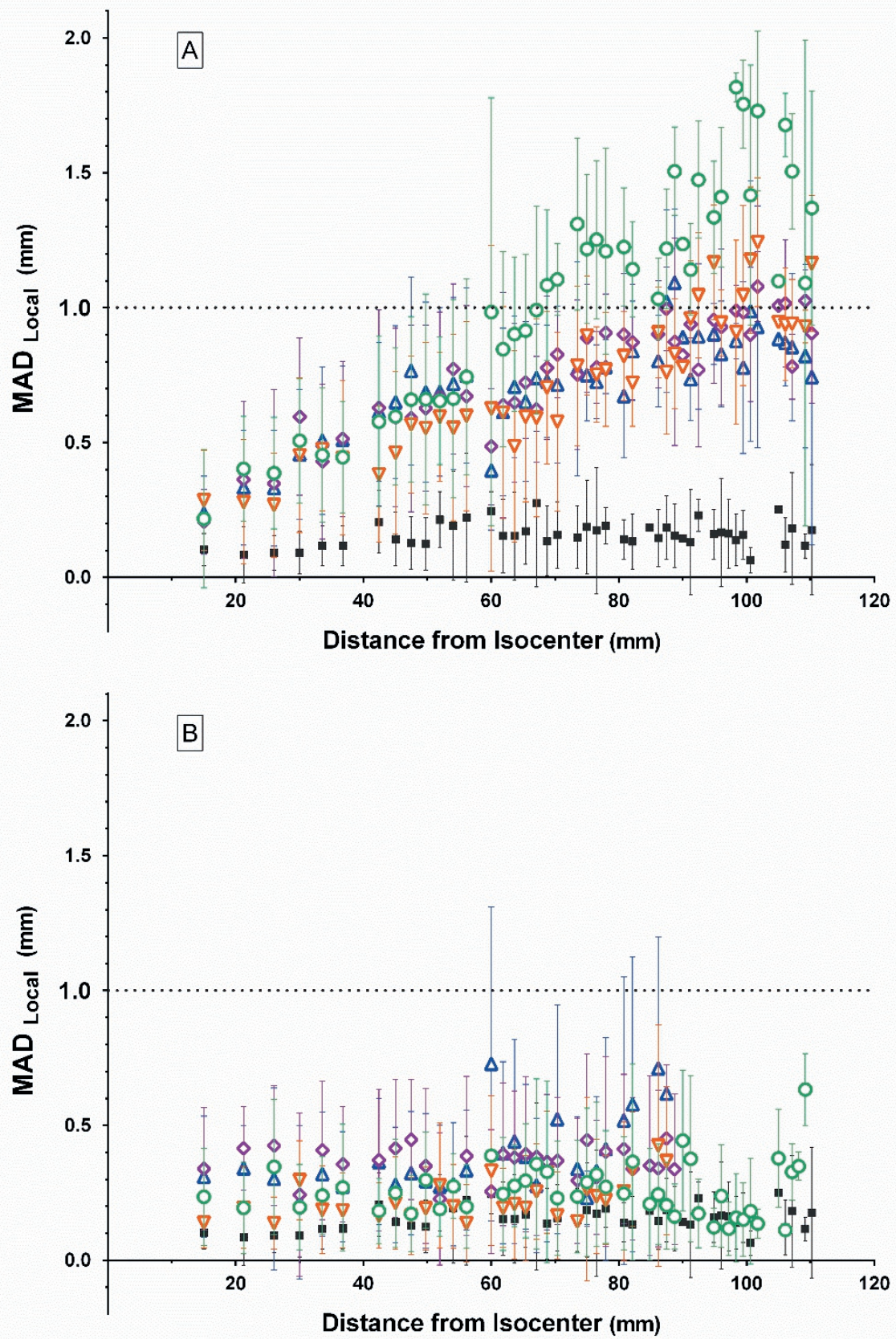

Figure 5.3: MADLocal values $( \pm \mathrm{SD})$ relative to the distance from the magnetic isocenter at $7 \mathrm{~T}(\mathrm{a})$ and 3T (b) MRI, both relative to CT (black square). Presented 7T MR sequence include MP2RAGE (green circle), T2-SPACE (purple diamond), T2-SPACE FLAIR (orange downward triangle), and T1-GRE (blue upward triangle). The same colour- and shape-code was used for the equivalent 3T sequences, 3D TFE, T2-TFE, T2-TSE FLAIR, and T1-FFE, respectively. The dotted horizontal line represents the $1 \mathrm{~mm}$-acceptability level required for spatially reliable RTP 
MP2RAGE (7T)

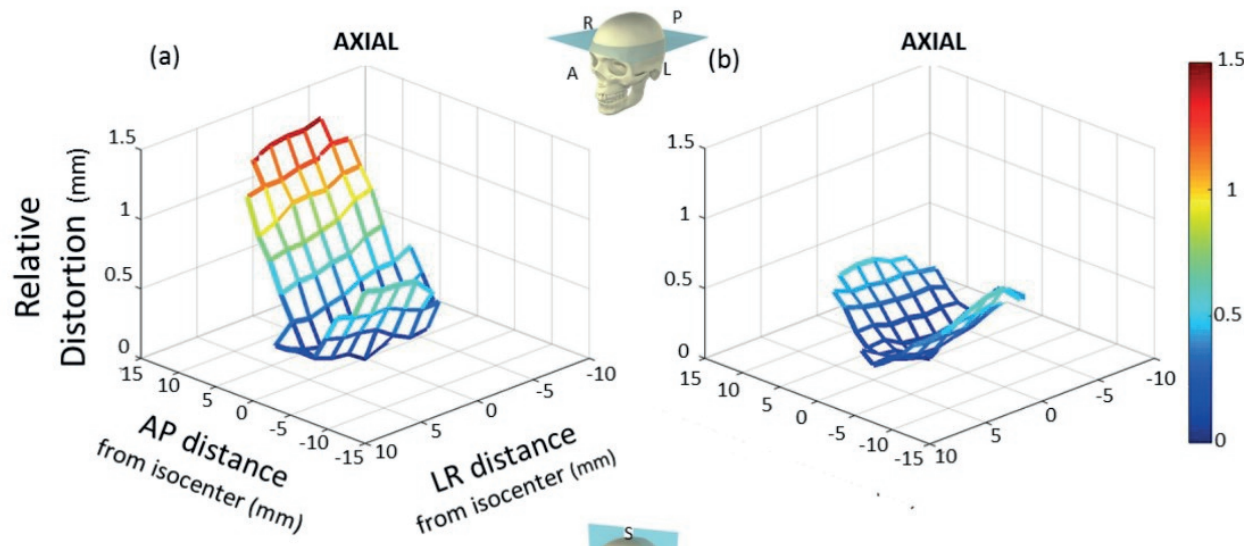

(c)

CORONAL

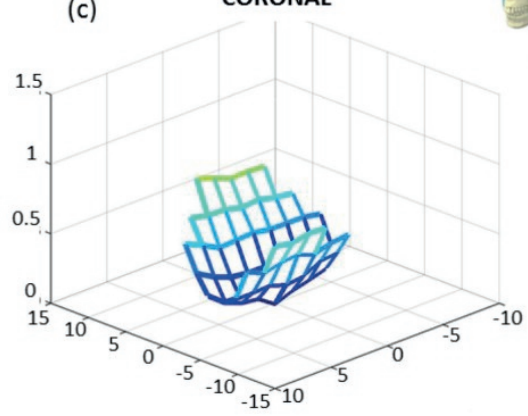

3D TFE (3T)

AXIAL
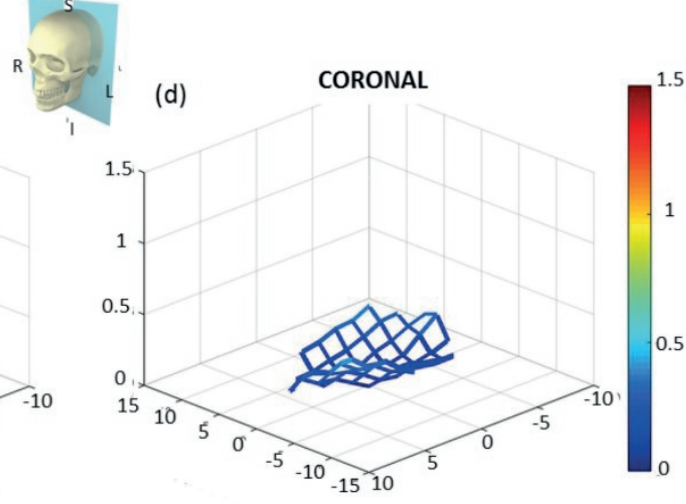
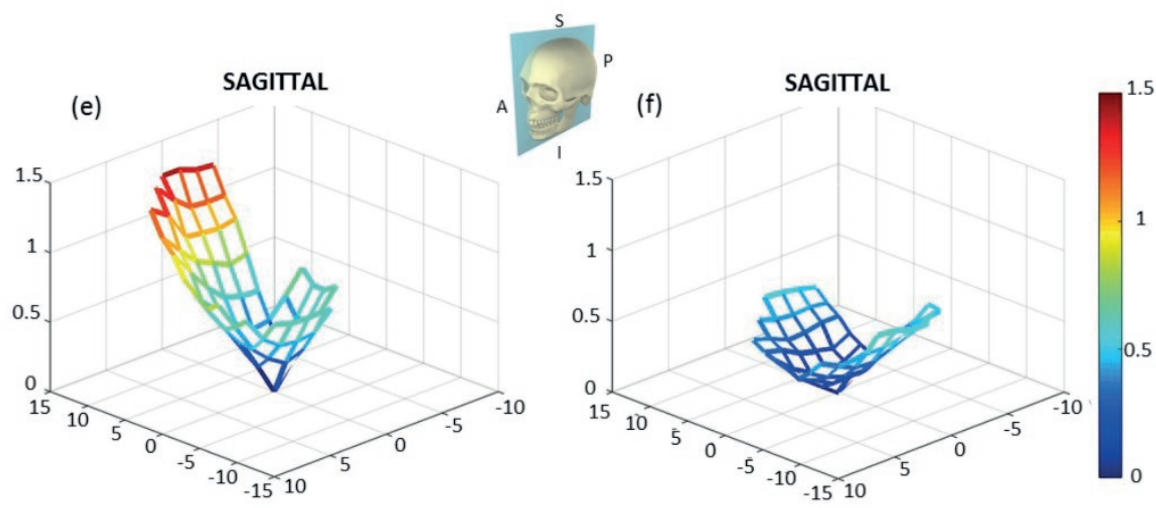

Figure 5.4: Distortion maps of MP2RAGE on 7T MRI (a) and 3D TFE on 3T MRI (b) measured in the axial plane nearest the magnetic isocenter $(\mathrm{Y}=0)$, in the coronal plane $(\mathrm{Z}=0)$, and in the sagittal plane $(X=0)$ 


\section{DISCUSSION}

This study reports both system- and phantom-related GD on clinically-relevant MRsequences at 7T-MRI for neurological imaging and allows direct comparison to GD on both 3T-MRI and CT-images. System-related GD was present in all 3T- and 7TMRI and increased with increasing distance from the magnetic isocentre, even though vendor-provided correction methods and $2^{\text {nd }}$ order $3 \mathrm{D}$ shimming were applied. However, GD of $\leq 1 \mathrm{~mm}$ could be assured within a sequence-dependent DSV near the magnetic isocentre.

While UHF-MRI promises clinical gain in terms of spatial resolution, SNR, contrast, and delineation of pathology, implementation of 7T-MRI in RTP requires mm-scale spatial accuracy for reliable, high-precision radiotherapy. This geometrical requirement was met by all 3T-MR sequences throughout the entire VOI while the magnitude of GD in 7T-MRI depended on the distance from the magnetic isocentre and applied sequence. GD $\leq 1 \mathrm{~mm}$ was apparent in 7T-MRI within a sequencespecific DSV. Moreover, no significant differences in system-related GD were found between 3T- and 7T-images near the magnetic isocentre. This implies that these 7TMRI with clinically-acceptable/tolerable levels of spatial uncertainty could be applied for high-precision RTP in central brain regions, under the assumption that tissue-related GD is negligible. In addition, it has previously been shown that GD in 7T-MRI did not affect targeting of basal ganglia and subthalamic nuclei in deepbrain stimulation $[23,31]$.

Some sequences are more prone to GD than others as gradient pulse scheme and parameter settings affect Bo-homogeneity and eddy currents [25, 29, 32]. Our MP2RAGE sequence, which acquires 2 gradient-echo images at different inversion times, presented the largest GD at 7T (and smallest DSV where GD $<1 \mathrm{~mm}$ ) [33]. Nevertheless, even in this worst-case scenario, system-related GD at 7T was below the clinically acceptable limit of $\leq 1 \mathrm{~mm}$ within $68.7 \mathrm{~mm}$ DSV. Presumably, GD in MP2RAGE could be related to the combination of different sequence-parameter settings (receiver bandwidth (rBW), FOV, and matrix size) [13, 14, 25, 29, 31, 32, 34]. The effect of varying rBW on GD and SNR has extensively been studied by Walker et al. (2014) in 4 different MR-systems [31]. For instance, increasing rBW would reduce GD, chemical shift, and susceptibility artefacts but would also reduce SNR. Limiting FOV-settings (i.e., fewer acquisition lines in k-space) could subside the impact of time-dependent off-resonance effects, reducing GD [34, 35]. However, this could also influence spatial resolution, SNR, and induce potential fold-over artefacts.

Residual GD in phase-encoding direction is expected to be related to gradient non-uniform and could be adequately reduced by vendor-installed distortion correction methods $[14,18,25,26]$. The technique behind this correction is similar 
for all main MR-manufacturers and achieve comparable and reproducible results for 3T- and 7T-MRI [4, 14, 15, 18, 31]. Nonetheless, some residual system-dependent GD was detected in this study despite applying correction methods and 3D shimming. It is therefore important to note that vendor-provided distortion correction is not flawless, uncorrected Bo-inhomogeneity and eddy currents could still induce GD, and gradient coils are designed with a tolerated performance variation [24, 36]. For example, general gradient errors of body gradient coils should be $>2 \%$ of the gradient strength over a $40 \mathrm{~cm}$ DSV [37, 38].

Our findings of GD corresponded well with literature. At 3T, Stanescu et al. (2010) reported maximal overall GD of $0.6 \mathrm{~mm}$ in 3D TFE sequences and Schmidt et al. (2016) have shown global GD of less than $0.5 \mathrm{~mm}$ in an IR-sequence (rBW= 890 $\mathrm{Hz} /$ pixel, resolution $1 \mathrm{~mm}^{3}$ ) [14, 39]. However, some studies report GD of several centimetres [25-27]. These findings, however, occurred at the edge of the scanner's FOV on a large cubical phantom that was placed in a body-coil. In contrast to this, we used a dedicated anthropomorphic head phantom to mimic a clinically realistic scan-procedure with standard radiofrequency-coils, shimming, distortion correction, and FOV settings ( $\leq 256 \mathrm{~mm}$ ).

Similar to our approach and FOV setting, Dammann et al. (2011) investigated hardware-related GD in 7T-MRI using a cylindrical phantom (190 POIs) and presented local GD smaller than $1 \mathrm{~mm}$ at $<80 \mathrm{~mm}$ DSV, except in T2-TSE (rBW= 350 $\mathrm{Hz} /$ pixel, resolution $0.6 \mathrm{~mm}^{2}$ ) that showed maximal local GD of $1.6 \mathrm{~mm}$ [26]. Presumably, this sequence was affected by intravoxel dephasing, image blurring and inaccurate measurements at the phantom's edge [26]. This could also could explain our finding of $1.2 \mathrm{~mm}$ local GD at $80 \mathrm{~mm}$ DSV. Differences in rBW-setting could explain GD differences found in MPRAGE (rBW= $600 \mathrm{~Hz} /$ pixel, resolution 1 $\mathrm{mm}^{3}$ ) and MP2RAGE $\left(\mathrm{rBW}=248 \mathrm{~Hz} /\right.$ pixel, $\left.0.7 \mathrm{~mm}^{3}\right)$. Recently, Lau et al. (2017) demonstrated differences in local GD between 3T and 7T larger than $1 \mathrm{~mm}$ at $80 \mathrm{~mm}$ DSV for T1-MPRAGE (rBW= $195 \mathrm{~Hz} /$ pixel, $1 \mathrm{~mm}^{3}$ ) [4]. In our study, MP2RAGE showed differences in MADLocal between $3 \mathrm{~T}$ and $7 \mathrm{~T}$ to be below $1 \mathrm{~mm}$ up for a $\leq 87.5$ mm DSV. Distortion maps of MP2RAGE indicated an anisotropic distribution of GD along the phase-encoding (AP) and frequency-encoding (SI) direction (Fig. 5.4). In a comparable 7T phantom study by Cho et al. (2010), coronal distortion maps showed $0.4 \mathrm{~mm}$ GD along the frequency-encoding direction and $0.8 \mathrm{~mm}$ along the phaseencoding direction [22].

We observed GD in CT-images with MADGlobal ranging from $0.1-0.6 \mathrm{~mm}$ and MADLocal ranging from $0.1-0.3 \mathrm{~mm}$. These findings were unanticipated as it is generally recognized that CT-images are distortion-free. Other studies have merged MRI with CT, ignoring this innate GD and measured fundamental errors. Artefacts in CT-images could originate from incorrectly reported scanner table-speed or 
shearing distortion (incorrectly reported gantry-tilt or table-bending). In this study, GD in CT-images could represent partial volume effects and variations in manual reconstruction leading to slightly different xyz-coordinates measurements of POIs (experimental inaccuracies).

In this study, we limited ourselves to evaluating system-related GD and not tissuerelated GD. Relative to system-related GD, tissue-induced GD is rather small in 3Tsequences that are currently being used for RTP of brain tumours, but it is nevertheless not negligible [16]. Simulations on 3T-MRI suggest that susceptibilityinduced GD were on average $0.6 \mathrm{~mm}$ at cranial air-cavities [40]. This has recently been confirmed by Schmidt et al. (2016), who specified susceptibility-related GD of $0.4 \mathrm{~mm}$ in the naso-oropharyngeal cavities and around the internal ear canal using a T1-weighted sequence $\left(\mathrm{rBW}=890 \mathrm{~Hz} /\right.$ pixel, resolution $\left.1 \mathrm{~mm}^{3}\right)$ [14]. It remains to be determined what the impact of tissue-related GD is for 7T-MRI. Susceptibility artefacts are proportional to the magnetic field strength and TE but could be reduced by increasing $\mathrm{rBW}$ [12]. Regardless, patient-specific correction methods are required [16, 37, 40]. Recently, Rai et al. (2018) developed an 3D printed human skull with sinuses and mastoid air cells, water-based brain tissue and eyes and surrogate cortical bone [41]. Such realistic anatomical skulls could be used to further assess tissue-induced GD in simulated air cavities, bony anatomy, and soft tissue. Since system-related GD could be further reduced by appropriate setting of MR-sequence parameters, research has been performed to systematically assess the impact of e.g., $\mathrm{rBW}, \mathrm{FOV}$, matrix size, scanning direction, and various (post-)processing correction algorithms [16, 18, 27, 31, 36]. However, 7T-MR protocols need to be optimised specifically for each clinical application and the trade-off between anatomical imagequality (spatial resolution, SNR, visibility of pathology) and image integrity (spatial accuracy) needs to be made, while respecting specific absorption rate restriction [29, 32]. For example, high-precision RTP relies heavily on high geometrical accuracy but could compensate on image-resolution, while in radiology larger GD could be tolerated.

To apply 7T-images in radiotherapy, GD should be $\leq 1 \mathrm{~mm}$ to ensure reliable RTP and dose delivery with local dose variations less than $5-10 \%$ [21]. This strict criterion was met by all the clinically relevant sequences we tested on 3T- and 7TMRI up until 92.5-101.7 mm DSV around the magnetic isocentre, except for the MP2RAGE sequence. Delineations of brain lesions and organs-at-risk in the frontal or occipital lobe on 7T-MRI can be significantly influenced by GD as the spatial uncertainty exceeds $1 \mathrm{~mm}$ in these regions. Even though systematic and random treatment uncertainties are foreseen in a planning treatment volume (PTV), the degree and location of GD is rarely taken into account during radiation dose planning. This study has shown that system-related GD should be incorporated into the PTV-margin to achieve a high level of dose delivery accuracy for intracranial 
(stereotactic) radiotherapy. This can be accomplished by incorporating the GD present at the tumour location within a margin recipe for dose delivery as suggested by Van Herk et al. (2004) and Seravelli et al. (2015) [42, 43]. We therefore recommend applying larger tumour site-specific PTV-margins in regions where spatial integrity of 7T-MRI could not be warranted within $1 \mathrm{~mm}$. Apart from adjustment of PTVmargins, it is advised to find a trade-off between image quality and image distortion, and apply MR-imaging protocols that are dedicated for RTP purposes.

In conclusion, GD of $\leq 1 \mathrm{~mm}$ could be assured within a sequence-dependent DSV near the magnetic isocentre, implying that this 7T-MRI protocol could be applied with clinically acceptable/tolerable levels of spatial uncertainty for high-precision RTP in central brain regions, under the assumption that tissue-related GD can be ignored. For peripheral regions, 7T-MR protocol for RTP should incorporate GD in tumour-site specific PTV-margins for treatment uncertainties. Nevertheless, dedicated MR-protocols are aspired for application in radiation oncology and further optimisation of sequence parameter settings and GD reduction methods are needed. 


\section{REFERENCES}

1. Niyazi M, Brada M, Chalmers AJ, Combs SE, Erridge SC, Fiorentino A et al. ESTRO-ACROP guideline "target delineation of glioblastomas". Radiother Oncol. 2016;118(1):35-42. doi:10.1016/j.radonc.2015.12.003.

2. Claes A, Idema AJ, Wesseling P. Diffuse glioma growth: a guerilla war. Acta Neuropathol. 2007;114(5):443-58. doi:10.1007/s00401-007-0293-7.

3. Barrett TF, Sarkiss CA, Dyvorne HA, Lee J, Balchandani P, Shrivastava RK. Application of Ultrahigh Field Magnetic Resonance Imaging in the Treatment of Brain Tumors: A Meta-Analysis. World Neurosurg. 2016;86:450-65. doi:10.1016/j.wneu.2015.09.048.

4. Lau JC, Khan AR, Zeng TY, MacDougall KW, Parrent AG, Peters TM. Quantification of local geometric distortion in structural magnetic resonance images: Application to ultra-high fields. Neuroimage. 2017. doi:10.1016/j.neuroimage.2016.12.066.

5. Trattnig S, Bogner W, Gruber S, Szomolanyi P, Juras V, Robinson S et al. Clinical applications at ultrahigh field $(7 \mathrm{~T})$. Where does it make the difference? NMR Biomed. 2016;29(9):1316-34. doi:10.1002/nbm.3272.

6. Trattnig S, Springer E, Bogner W, Hangel G, Strasser B, Dymerska B et al. Key clinical benefits of neuroimaging at 7T. Neuroimage. 2016. doi:10.1016/j.neuroimage.2016.11.031.

7. Christoforidis GA, Yang M, Abduljalil A, Chaudhury AR, Newton HB, McGregor JM et al. "Tumoral pseudoblush" identified within gliomas at high-spatial-resolution ultrahigh-field-strength gradientecho MR imaging corresponds to microvascularity at stereotactic biopsy. Radiology. 2012;264(1):210-7. doi:radiol.12110799 [pii]10.1148/radiol..

8. Moenninghoff C, Maderwald S, Theysohn JM, Kraff O, Ladd ME, El Hindy N et al. Imaging of adult astrocytic brain tumours with 7T MRI: preliminary results. Eur Radiol. 2010;20(3):704-13. doi:10.1007/s0033000915922.

9. Compter I, Peerlings J, Eekers DB, Postma AA, Ivanov D, Wiggins CJ et al. Technical feasibility of integrating $7 \mathrm{~T}$ anatomical MRI in image-guided radiotherapy of glioblastoma: a preparatory study. MAGMA. 2016;29(3):591-603. doi:10.1007/s10334-016-0534-7.

10. Bian W, Hess CP, Chang SM, Nelson SJ, Lupo JM. Susceptibility-weighted MR imaging of radiation therapy-induced cerebral microbleeds in patients with glioma: a comparison between 3T and 7T. Neuroradiology. 2014;56(2):91-6. doi:10.1007/s00234-013-1297-8.

11. Lupo JM, Chuang CF, Chang SM, Barani IJ, Jimenez B, Hess CP et al. 7-Tesla susceptibility-weighted imaging to assess the effects of radiotherapy on normal-appearing brain in patients with glioma. Int J Radiat Oncol Biol Phys. 2012;82(3):e493-500. doi:S0360-3016(11)02787-8 [pii] 10.1016/j.ijrobp.2011.05.046.

12. Dietrich $\mathrm{O}$, Reiser MF, Schoenberg SO. Artifacts in 3-T MRI: physical background and reduction strategies. Eur J Radiol. 2008;65(1):29-35. doi:10.1016/j.ejrad.2007.11.005.

13. Weygand J, Fuller CD, Ibbott GS, Mohamed AS, Ding Y, Yang J et al. Spatial Precision in Magnetic Resonance Imaging-Guided Radiation Therapy: The Role of Geometric Distortion. Int J Radiat Oncol Biol Phys. 2016;95(4):1304-16. doi:10.1016/j.ijrobp.2016.02.059.

14. Schmidt MA, Wells EJ, Davison K, Riddell AM, Welsh L, Saran F. Stereotactic Radiosurgery Planning of Vestibular Schwannomas: Is MRI at 3 Tesla Geometrically Accurate? Medical physics. 2016. doi:10.1002/mp.12068.

15. Torfeh T, Hammoud R, Perkins G, McGarry M, Aouadi S, Celik A et al. Characterization of 3D geometric distortion of magnetic resonance imaging scanners commissioned for radiation therapy planning. Magn Reson Imaging. 2016;34(5):645-53. doi:10.1016/j.mri.2016.01.001.

16. Wang H, Balter J, Cao Y. Patient-induced susceptibility effect on geometric distortion of clinical brain MRI for radiation treatment planning on a 3T scanner. Phys Med Biol. 2013;58(3):465-77. doi:10.1088/0031-9155/58/3/465.

17. Klein EE, Hanley J, Bayouth J, Yin FF, Simon W, Dresser S et al. Task Group 142 report: quality assurance of medical accelerators. Medical physics. 2009;36(9):4197-212. doi:10.1118/1.3190392. 
18. Duchin Y, Abosch A, Yacoub E, Sapiro G, Harel N. Feasibility of using ultra-high field (7 T) MRI for clinical surgical targeting. PLoS One. 2012;7(5):e37328. doi:10.1371/journal.pone.0037328.

19. Jursinic PA, Rickert K, Gennarelli TA, Schultz CJ. Effect of image uncertainty on the dosimetry of trigeminal neuralgia irradiation. International Journal of Radiation Oncology Biology Physics. 2005;62(5):1559-67. doi:10.1016/j.ijrobp.2005.01.059.

20. Karaiskos P, Moutsatsos A, Pappas E, Georgiou E, Roussakis A, Torrens M et al. A simple and efficient methodology to improve geometric accuracy in gamma knife radiation surgery: implementation in multiple brain metastases. Int J Radiat Oncol Biol Phys. 2014;90(5):1234-41. doi:10.1016/j.ijrobp.2014.08.349.

21. Seibert TM, White NS, Kim GY, Moiseenko V, McDonald CR, Farid N et al. Distortion inherent to magnetic resonance imaging can lead to geometric miss in radiosurgery planning. Pract Radiat Oncol. 2016;6(6):e319-e28. doi:10.1016/j.prro.2016.05.008.

22. Cho ZH, Min HK, Oh SH, Han JY, Park CW, Chi JG et al. Direct visualization of deep brain stimulation targets in Parkinson disease with the use of 7-tesla magnetic resonance imaging. J Neurosurg. 2010;113(3):639-47. doi:10.3171/2010.3.jns091385.

23. Wang D, Doddrell DM. Geometric Distortion in Structural Magnetic Resonance Imaging. Current Medical Imaging Reviews. 2005;1(1):49-60.

24. O'Callaghan J, Wells J, Richardson S, Holmes H, Yu Y, Walker-Samuel S et al. Is your system calibrated? MRI gradient system calibration for pre-clinical, high-resolution imaging. PLoS One. 2014;9(5):e96568. doi:10.1371/journal.pone.0096568.

25. Baldwin LN, Wachowicz K, Thomas SD, Rivest R, Fallone BG. Characterization, prediction, and correction of geometric distortion in 3 T MR images. Medical physics. 2007;34(2):388-99.

26. Dammann P, Kraff O, Wrede KH, Ozkan N, Orzada S, Mueller OM et al. Evaluation of hardware-related geometrical distortion in structural MRI at 7 Tesla for image-guided applications in neurosurgery. Acad Radiol. 2011;18(7):910-6. doi:10.1016/j.acra.2011.02.011.

27. Doran SJ, Charles-Edwards L, Reinsberg SA, Leach MO. A complete distortion correction for MR images: I. Gradient warp correction. Phys Med Biol. 2005;50(7):1343-61. doi:10.1088/0031-9155/50/7/001.

28. Schenck JF. The role of magnetic susceptibility in magnetic resonance imaging: MRI magnetic compatibility of the first and second kinds. Medical physics. 1996;23(6):815-50.

29. Liney GP, Moerland MA. Magnetic resonance imaging acquisition techniques for radiotherapy planning. Semin Radiat Oncol. 2014;24(3):160-8. doi:10.1016/j.semradonc.2014.02.014.

30. Teeuwisse WM, Brink WM, Haines KN, Webb AG. Simulations of high permittivity materials for $7 \mathrm{~T}$ neuroimaging and evaluation of a new barium titanate-based dielectric. Magn Reson Med. 2012;67(4):912-8. doi:10.1002/mrm.24176.

31. Walker A, Liney G, Metcalfe P, Holloway L. MRI distortion: considerations for MRI based radiotherapy treatment planning. Australas Phys Eng Sci Med. 2014;37(1):103-13. doi:10.1007/s13246-014-0252-2.

32. Kraff O, Fischer A, Nagel AM, Monninghoff C, Ladd ME. MRI at 7 Tesla and above: demonstrated and potential capabilities. J Magn Reson Imaging. 2015;41(1):13-33. doi:10.1002/jmri.24573.

33. Marques JP, Gruetter R. New Developments and Applications of the MP2RAGE Sequence - Focusing the Contrast and High Spatial Resolution R-1 Mapping. Plos One. 2013;8(7):11. doi:10.1371/journal.pone.0069294.

34. Hong C, Lee DH, Han BS. Characteristics of geometric distortion correction with increasing field-ofview in open-configuration MRI. Magn Reson Imaging. 2014;32(6):786-90. doi:10.1016/j.mri.2014.02.007.

35. Hashemi RH, Bradley Jr WG, Lisanti CJ. MRI: the Basics. 3rd ed. Philadelphia: Lippincott Williams and Wilkins; 2010.

36. Wang D, Strugnell W, Cowin G, Doddrell DM, Slaughter R. Geometric distortion in clinical MRI systems Part II: correction using a 3D phantom. Magn Reson Imaging. 2004;22(9):1223-32. doi:10.1016/j.mri.2004.08.014.

37. Schmidt MA, Payne GS. Radiotherapy planning using MRI. Phys Med Biol. 2015;60(22):R323-61. doi:10.1088/0031-9155/60/22/r323. 
38. Jezzard P. The physical basis of spatial distortion in magnetic resonance images. In: Bankman IN, editor. Handbook of medical image processing and analysis. 2nd ed. Amsterdam: Elsevier/Academic Press; 2009. p. 499-514.

39. Stanescu T, Jans HS, Wachowicz K, Fallone BG. Investigation of a 3D system distortion correction method for MR images. Journal of Applied Clinical Medical Physics. 2010;11(1):200-16.

40. Stanescu T, Wachowicz K, Jaffray DA. Characterization of tissue magnetic susceptibility-induced distortions for MRIgRT. Medical physics. 2012;39(12):7185-93. doi:10.1118/1.4764481.

41. Rai R, Manton D, Jameson MG, Josan S, Barton MB, Holloway LC et al. 3D printed phantoms mimicking cortical bone for the assessment of ultrashort echo time magnetic resonance imaging. Medical physics. 2018;45(2):758-66. doi:10.1002/mp.12727.

42. van Herk M. Errors and margins in radiotherapy. Semin Radiat Oncol. 2004;14(1):52-64. doi:10.1053/j.semradonc.2003.10.003.

43. Seravalli E, van Haaren PM, van der Toorn PP, Hurkmans CW. A comprehensive evaluation of treatment accuracy, including end-to-end tests and clinical data, applied to intracranial stereotactic radiotherapy. Radiother Oncol. 2015;116(1):131-8. doi:10.1016/j.radonc.2015.06.004 


\section{SUPPLEMENTARY MATERIAL}

Table S5.1: Scan parameters of the 7T- and 3T-MR sequences investigated in this study. All sequences were acquired in 3D.

\begin{tabular}{|c|c|c|c|c|}
\hline 7T MRI sequences & MP2RAGE & T2-SPACE & $\begin{array}{l}\text { T2-SPACE } \\
\text { FLAIR }\end{array}$ & T1-GRE \\
\hline Imaging plane & Sag & Sag & Sag & Tra \\
\hline Phase encode direction & $\mathrm{AP}$ & $\mathrm{AP}$ & $\mathrm{AP}$ & RL \\
\hline Repetition time TR (ms) & 5000 & 4000 & 8000 & 33 \\
\hline Echo time TE (ms) & 2.5 & 283 & 302 & 2.5 \\
\hline Inversion time (ms) & $\begin{array}{l}\text { TI1 } 900 \\
\text { TI2 } 2750\end{array}$ & N/A & 2330 & N/A \\
\hline Turbo factor & 1 & 164 & 126 & N/A \\
\hline Field of View (mm) & $223 \times 223 \times 240$ & $192 \times 192 \times 288$ & $193 \times 206 \times 208$ & $160 \times 223 \times 208$ \\
\hline Acquisition matrix (voxel) & $320 \times 320 \times 340$ & $320 \times 320 \times 480$ & $240 \times 256 \times 260$ & $320 \times 320 \times 300$ \\
\hline In-plane resolution (mm/voxel) & $0.7 \times 0.7 \times 0.7$ & $0.6 \times 0.6 \times 0.6$ & $0.8 \times 0.8 \times 0.8$ & $0.5 \times 0.7 \times 0.7$ \\
\hline Nominal flip angle $\left(^{\circ}\right)$ & $5 \& 3$ & variable & variable & 11 \\
\hline Bandwidth (Hz/pixel) & 248 & 372 & 383 & 290 \\
\hline Acceleration factor (iPAT) & 3 & 4 & 4 & 2 \\
\hline Acquisition time (minutes) & 08:02 & 07:50 & $10: 58$ & 08:33 \\
\hline 3T MRI sequences & 3D TFE & T2-VISTA & $\begin{array}{l}\text { T2-VISTA } \\
\text { FLAIR }\end{array}$ & T1-FFE \\
\hline Imaging plane & Tra & Sag & Sag & Tra \\
\hline Phase encoding direction & $\mathrm{AP}$ & $\mathrm{AP}$ & $\mathrm{AP}$ & RL \\
\hline Repetition time TR (ms) & 8.1 & 2500 & 8000 & 11 \\
\hline Echo time TE (ms) & 3.7 & 243 & 331.5 & 4.6 \\
\hline Inversion time (ms) & 800 & N/A & 2400 & $\mathrm{~N} / \mathrm{A}$ \\
\hline Turbo factor & 188 & 133 & 110 & N/A \\
\hline Field of View (mm) & $256 \times 256 \times 180$ & $256 \times 256 \times 180$ & $256 \times 256 \times 180$ & $256 \times 192 \times 180$ \\
\hline Acquisition matrix (voxel) & $256 \times 256 \times 180$ & $256 \times 256 \times 180$ & $228 \times 226 \times 150$ & $256 \times 256 \times 180$ \\
\hline In-plane resolution (mm/voxel) & $1 \times 1 \times 1$ & $1 \times 1 \times 1$ & $1.1 \times 1.1 \times 1.2$ & $1 \times 0.75 \times 1$ \\
\hline Nominal flip angle $\left(^{\circ}\right)$ & 8 & 90 & variable & 8 \\
\hline Bandwidth (Hz/pixel) & 191 & 1005 & 502 & 288 \\
\hline Acceleration factor (SENSE) & 1 & 2 & 2 & 1 \\
\hline Acquisition time (minutes) & $06: 00$ & $05: 42$ & $08: 24$ & $06: 21$ \\
\hline
\end{tabular}




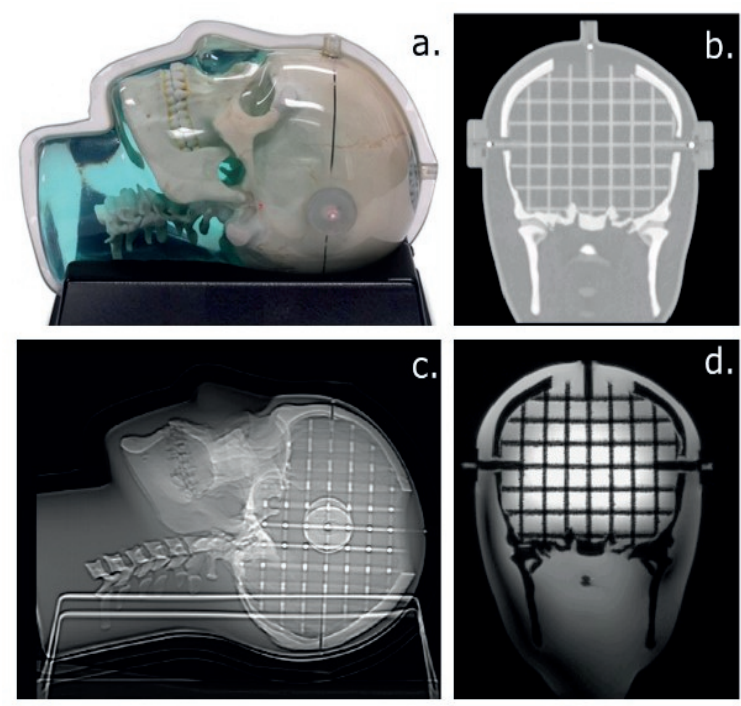

Figure S5.1: Anthropomorphic skull phantom CIRS 603A (a) contains a 3D rigid grid structure and is compatible for $\mathrm{CT}(\mathrm{b}, \mathrm{c})$ and MR imaging $(\mathrm{d})$. Source: Computerized Imaging Reference Systems, Inc (CIRS)

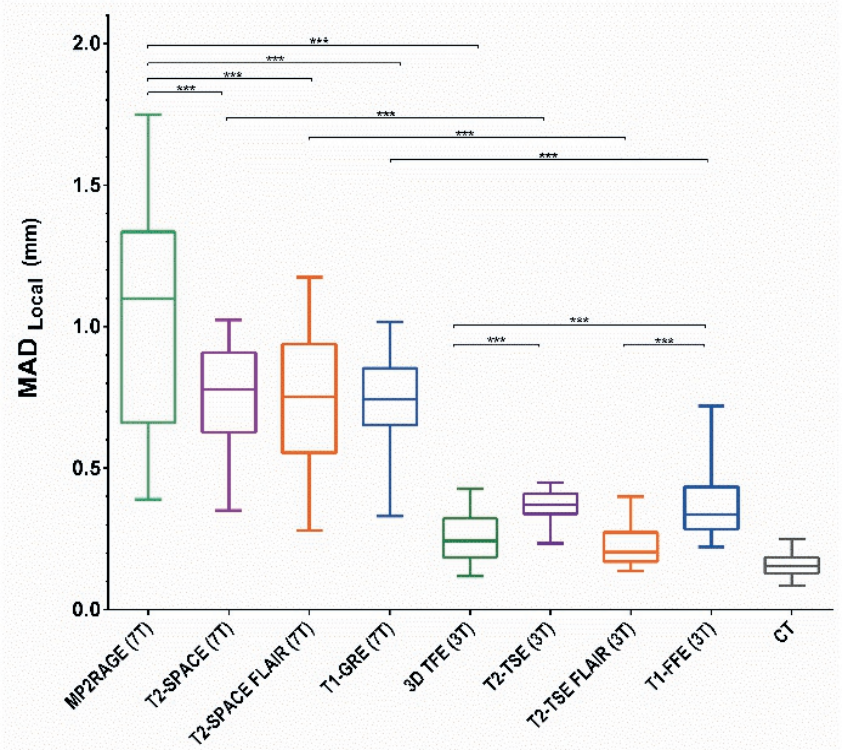

Figure S5.2: Boxplot of MADLocal values with 5-95 percentile whiskers for 7T pulse sequences, equivalent $3 \mathrm{~T}$ sequences pulse schemes, and CT. Statistically significant results within and between MR datasets are indicated by asterisks $(* * *=\mathrm{P}<0.001)$ 
T2-SPACE (7T)

T2- VISTA (3T)

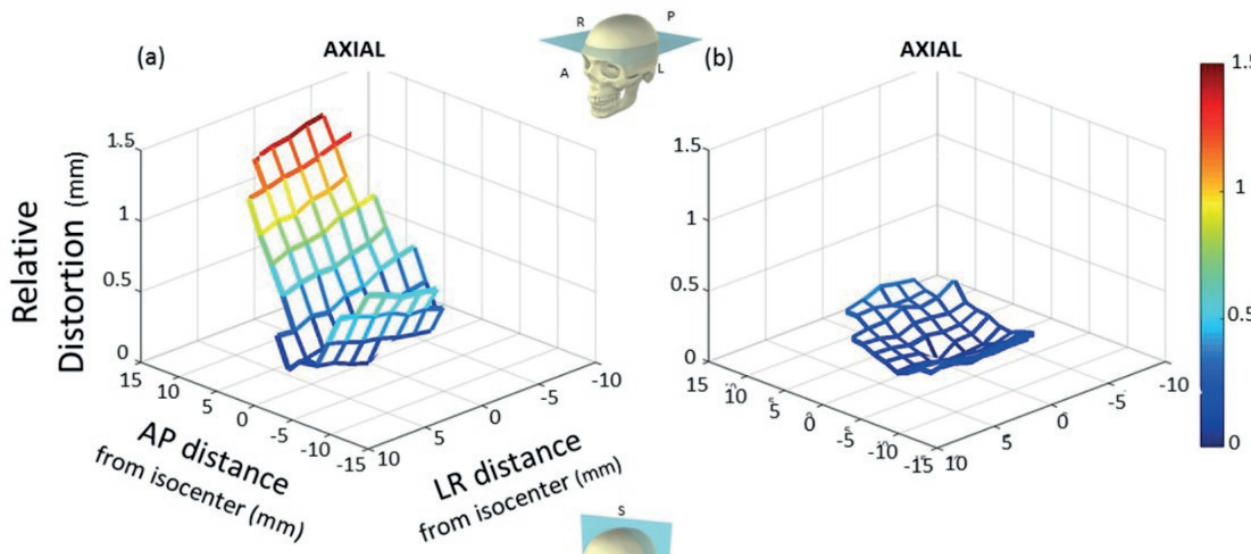

(c)

CORONAL

(d)
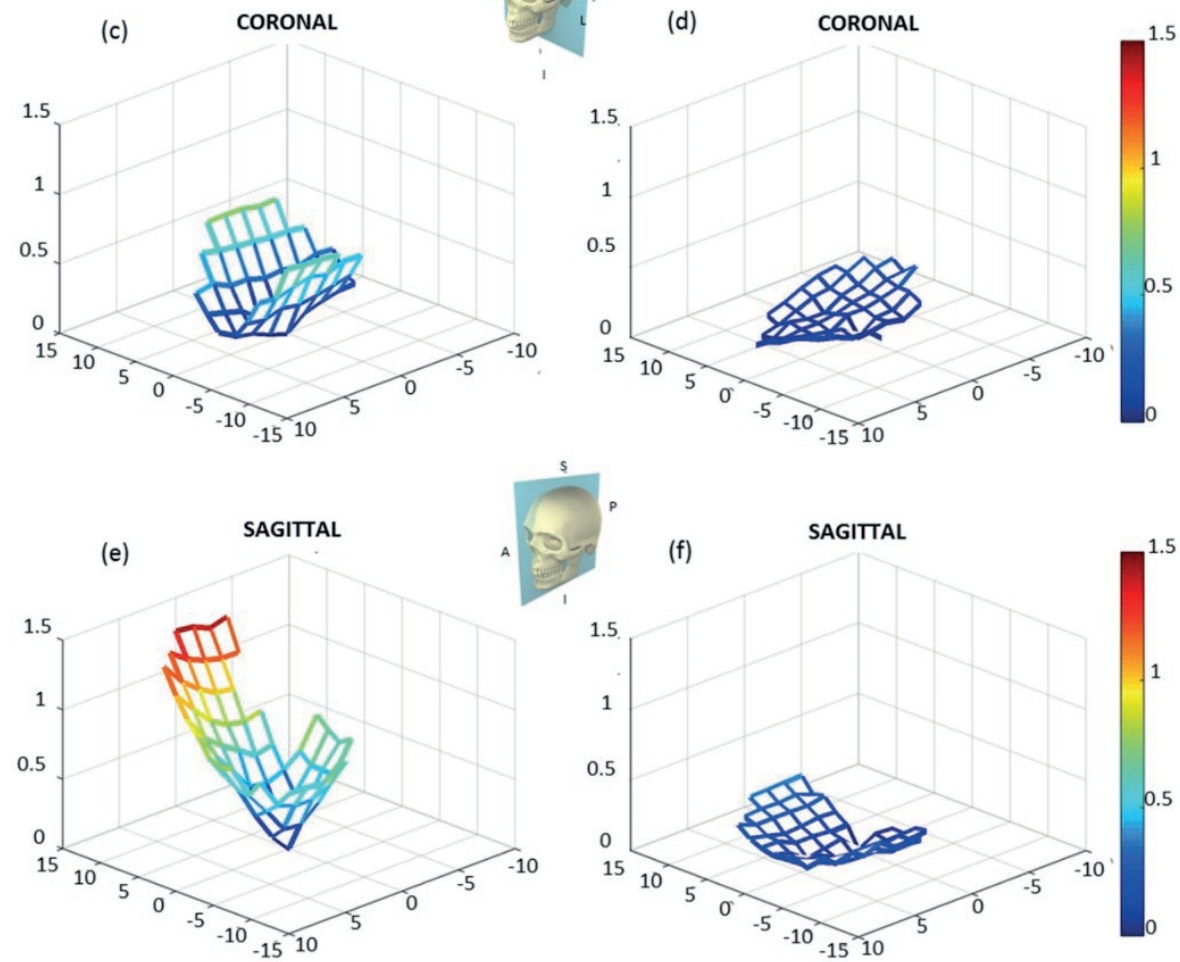

Figure S5.3: Distortion maps of T2-SPACE on 7T MRI (a) and T2-VISTA on 3T MRI (b) measured in the axial plane nearest the magnetic isocenter $(\mathrm{Y}=0)$, in the coronal plane $(\mathrm{Z}=0)$, and in the sagittal plane $(X=0)$ 
T2-SPACE FLAIR (7T)

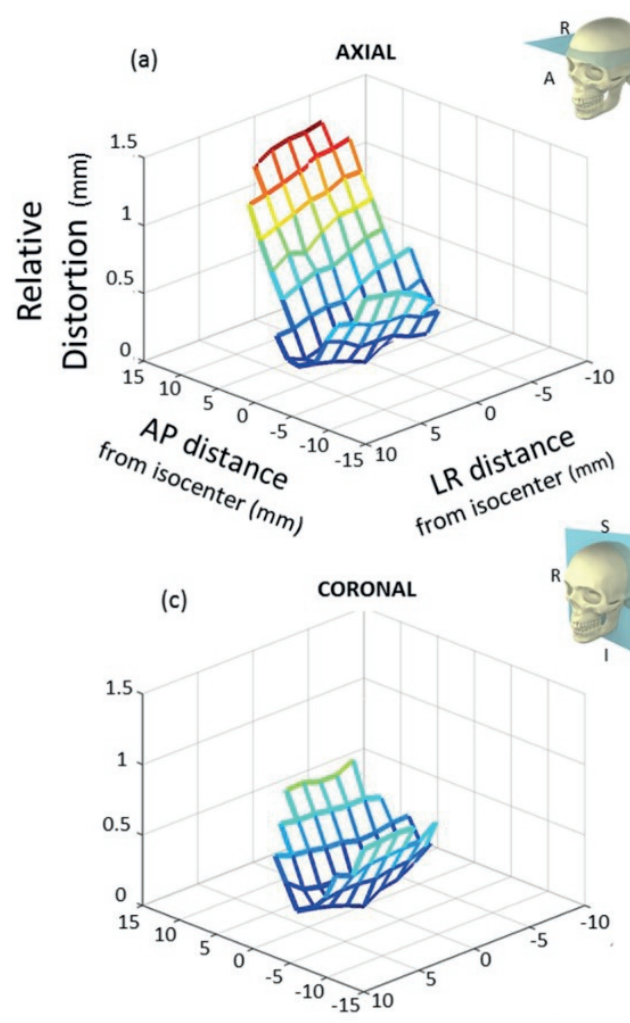

T2- VISTA FLAIR (3T)

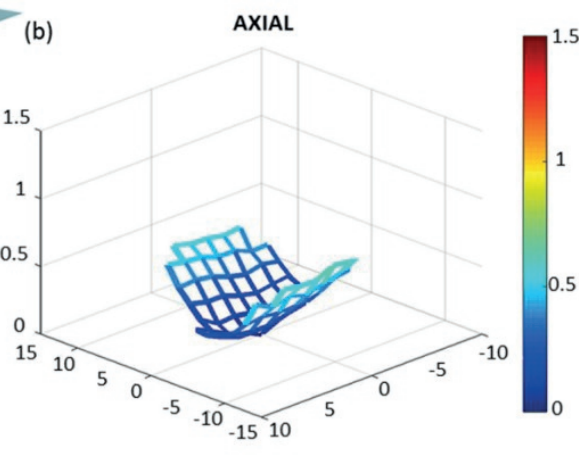

(d)

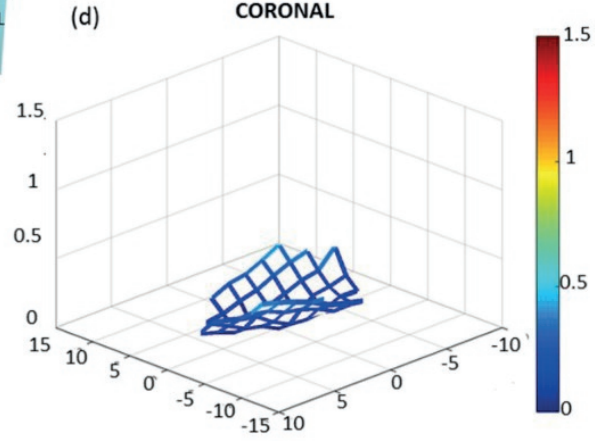

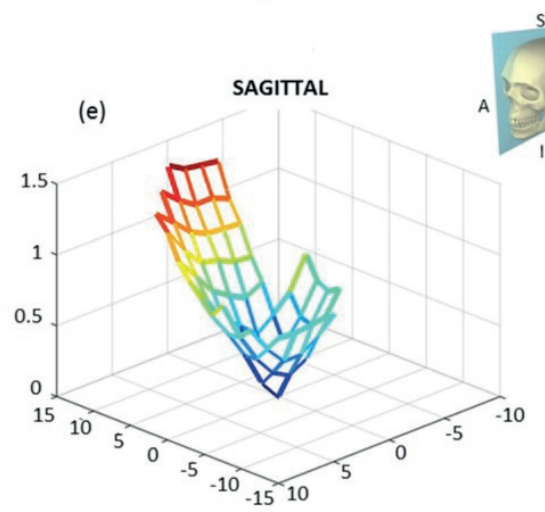

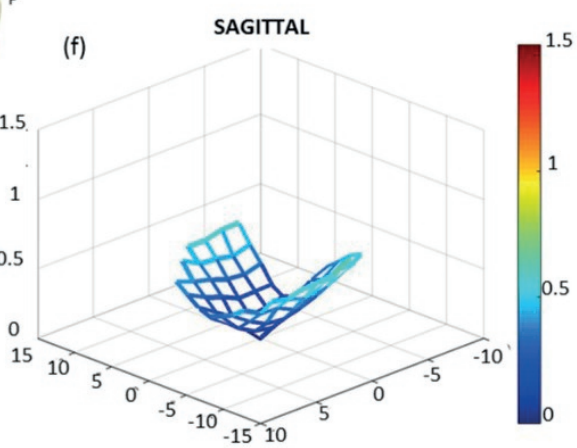

Figure S5.4: Distortion maps of T2-SPACE FLAIR on 7T MRI (a) and T2-VISTA FLAIR on 3T MRI (b) measured in the axial plane nearest the magnetic isocenter $(Y=0)$, in the coronal plane $(\mathrm{Z}=0)$, and in the sagittal plane $(X=0)$ 
T1-GRE (7T)

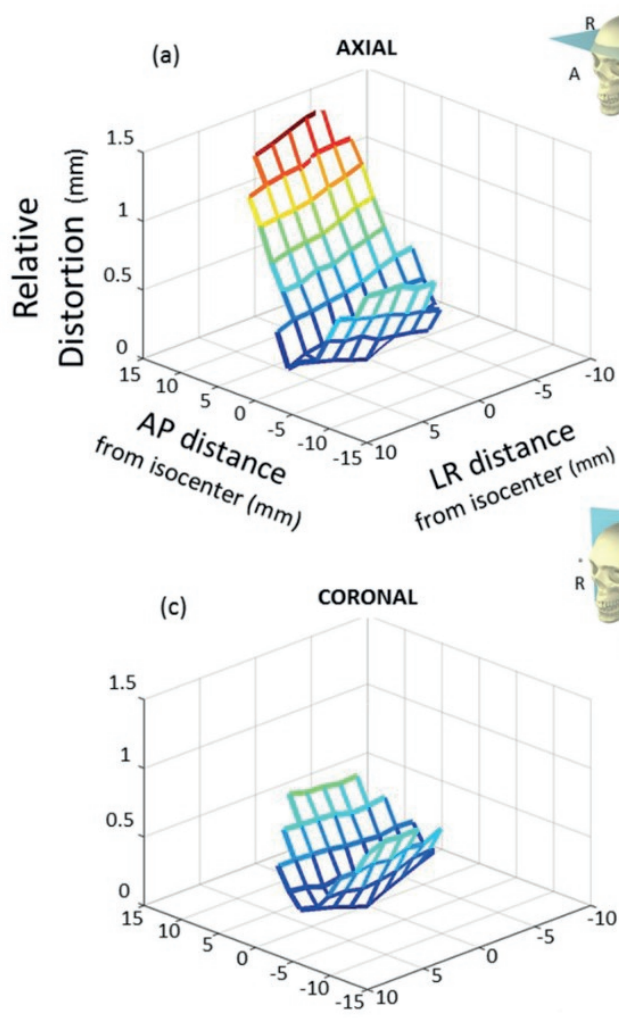

T1- FFE (3T)

(b)

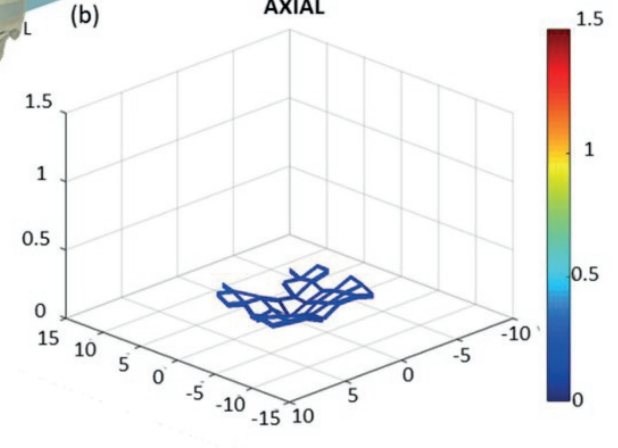

(e)
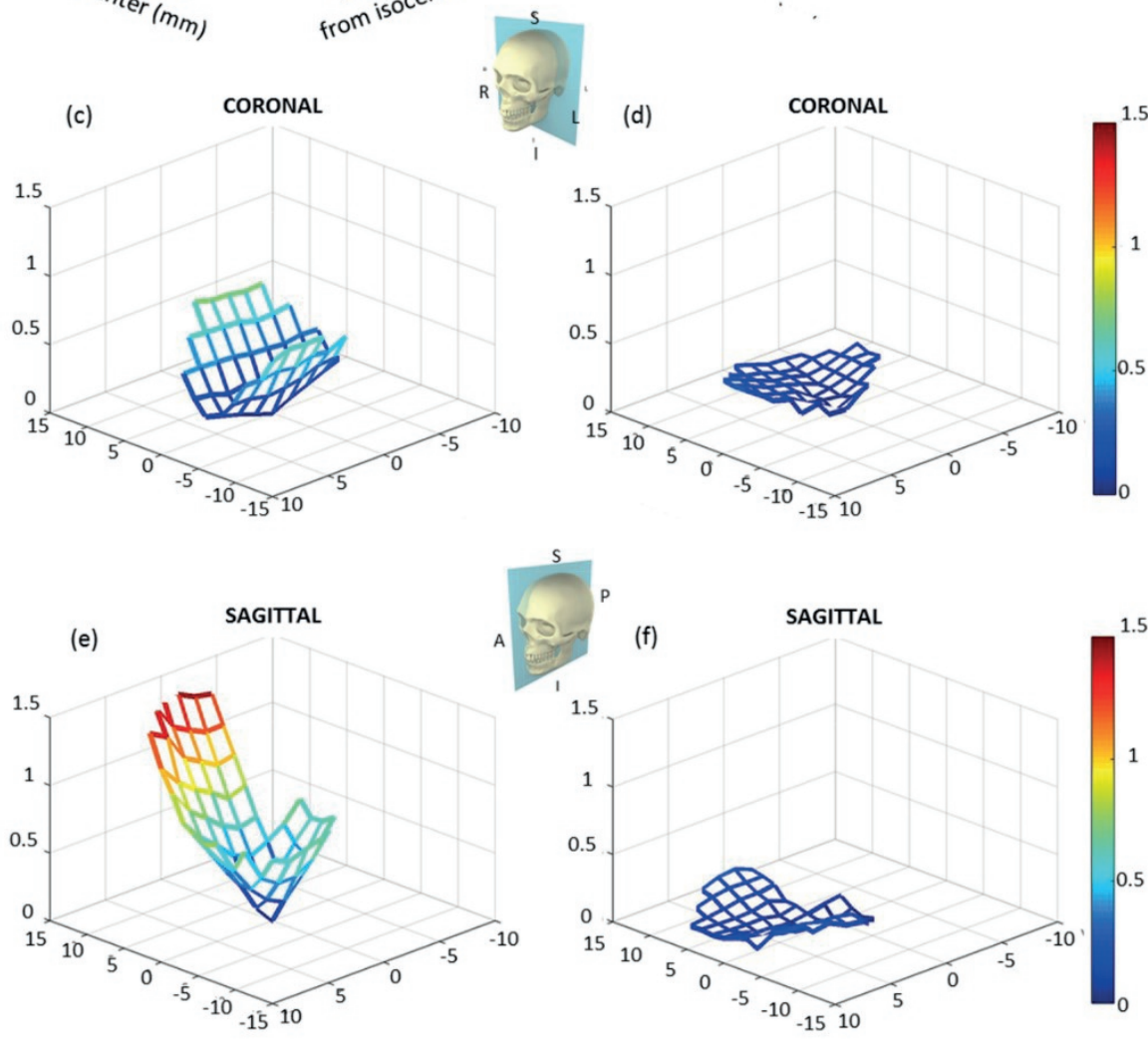

(d)

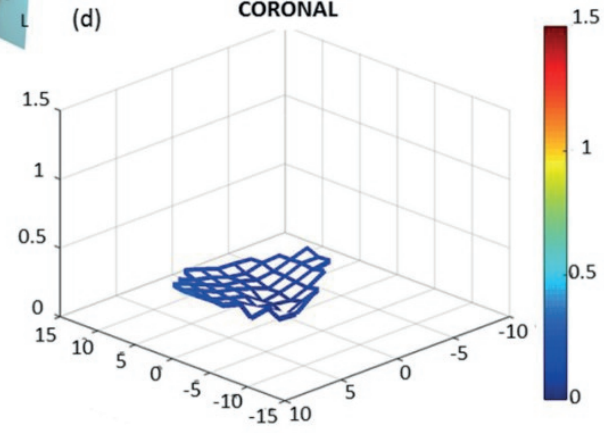

Figure S5.5: Distortion maps of T1-GRE on 7T MRI (a) and T1-FFE on 3T MRI (b) measured in the axial plane nearest the magnetic isocenter $(Y=0)$, in the coronal plane $(Z=0)$, and in the sagittal plane $(X=0)$ 


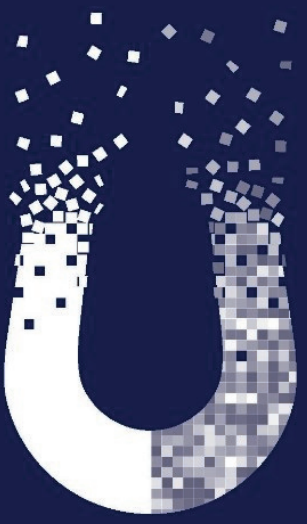




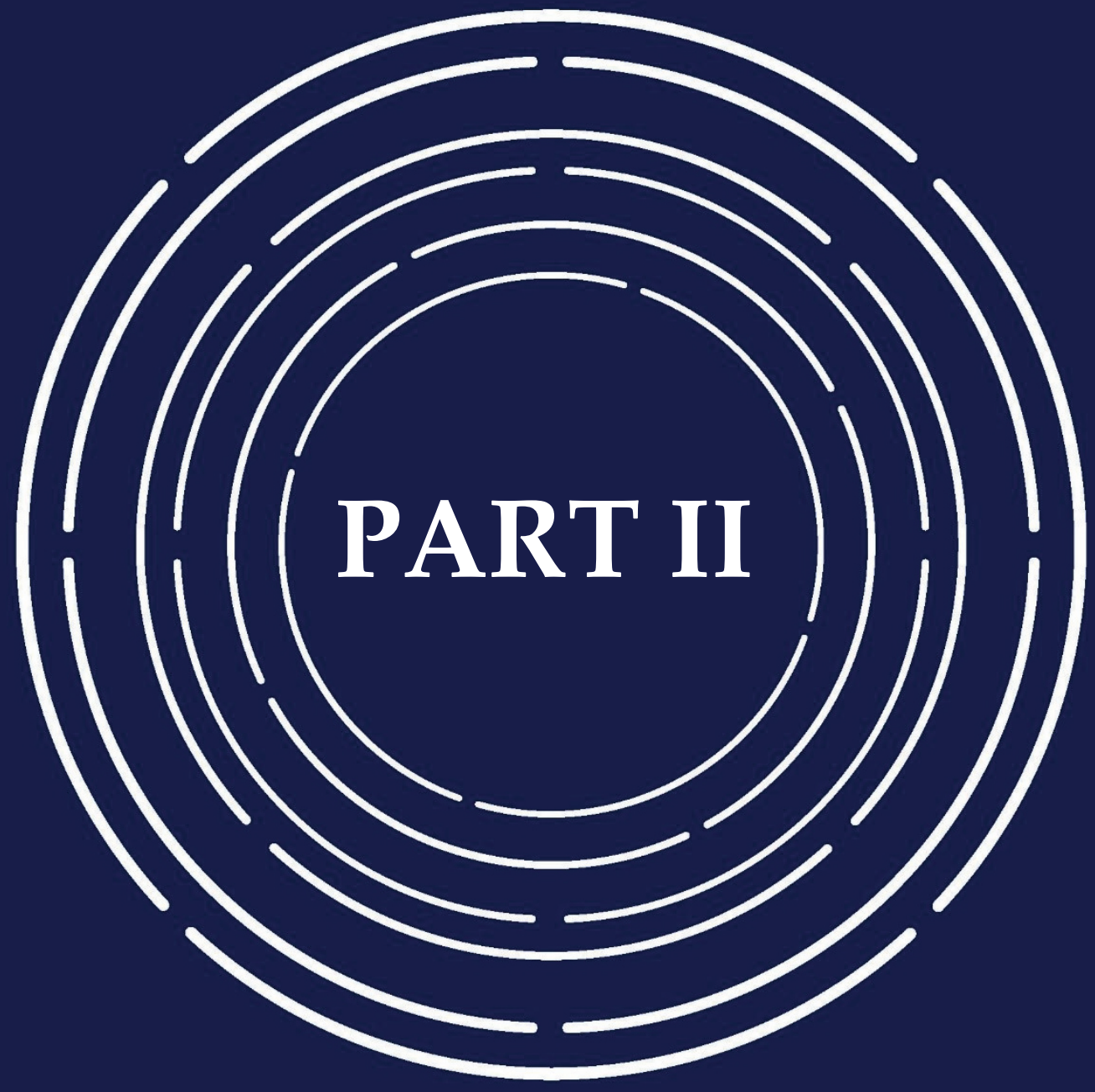

Functional (PET-)MR imaging and quantitative image analysis 


\section{Chapter

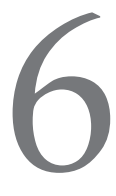

\section{Hypoxia \& hypoxia response-associated molecular markers in oesophageal cancer:} a systematic review

Authors:

J. Peerlings*, L. Van De Voorde*, C. Mitea, R. Larue, A. Yaromina, S. Sanduleanu, L. Spiegelberg, L. Dubois, P. Lambin, F. Mottaghy $\left(^{*}\right)$ equal contribution

Adapted from:

METHODS 2017 Nov 1;130:51-62 DOI: 10.1016/j.ymeth.2017.07.002. Review 


\section{ABSTRACT}

\section{PURPOSE}

In this systematic review, the existing evidence of available hypoxiaassociated molecular response biomarkers in oesophageal cancer (EC) patients is summarized and set into the context of the role of hypoxia in the prediction of oesophageal cancer, treatment response and treatment outcome.

\section{METHODS}

A systematic literature search was performed in Web of Science, MEDLINE, and PubMed databases using the keywords: hypoxia, oesophagus, cancer, treatment outcome and treatment response. Eligible publications were independently evaluated by two reviewers. In total, 22 out of 419 records were included for systematic review. The described search strategy was applied weekly, with the last update being performed on April 3rd, 2017.

\section{RESULTS}

In oesophageal cancer, several (non-)invasive biomarkers for hypoxia could be identified. Independent prognostic factors for treatment response include HIF-1 $\alpha$, CA IX, GLUT-1 overexpression and elevated uptake of the PET-tracer ${ }^{18} \mathrm{~F}$-fluoroerythronitroimidazole $\left({ }^{18} \mathrm{~F}-\mathrm{FETNIM}\right)$. Hypoxia-associated molecular responses represents a clinically relevant phenomenon in oesophageal cancer and detection of elevated levels of hypoxia-associated biomarkers and tends to be associated with poor treatment outcome (i.e., overall survival, disease-free survival, complete response and local control).

\section{CONCLUSION}

Evaluation of tumour micro-environmental conditions, such as intratumoral hypoxia, is important to predict treatment outcome and efficacy. Promising non-invasive imaging-techniques have been suggested to assess tumour hypoxia and hypoxia-associated molecular responses. However, extensive validation in EC is lacking. Hypoxiaassociated markers that are independent prognostic factors could potentially provide targets for novel treatment strategies to improve treatment outcome. For personalized hypoxia-guided treatment, safe and reliable makers for tumour hypoxia are needed to select suitable patients. 


\section{INTRODUCTION}

Hypoxia is one of the hallmarks of cancer and has been associated with a more aggressive tumour phenotype, a higher likelihood of metastatic progression and resistance to (chemo)radiotherapy [1]. Hypoxia occurs when tissue oxygen demand (e.g., increased metabolism) exceeds oxygen supply (e.g., acute and/or chronic vascular changes, anaemia, malfunctioning haemoglobin). In normal tissue, acute hypoxia (i.e., perfusion-limited) is resolved by physiological homeostasis while in cancerous tissue, additional chronic hypoxia (i.e., diffusion-limited) is more likely to manifest. The rapid and uncontrollable tumour growth requires large amounts of nutrients and therefore triggers neo-angiogenesis. However, the resulting tumour neo-vasculature is highly chaotic and inefficient. Oxygenation of tumour regions surrounding perfused blood vessels therefore depends on a diffusion-gradient, relative to the intravascular oxygen partial pressure $\left(\mathrm{pO}_{2}\right)$. Generally, the diffusiongradient is limited to $100-180 \mu \mathrm{m}$, thus inducing chronic hypoxia in remote regions [1].

Clinically, hypoxia is thought to be a key factor contributing to treatment resistance and poor patient prognosis [2]. Although neoadjuvant therapy (i.e., CROSS regimen with weekly carboplatin $\left(2 \mathrm{mg} / \mathrm{ml} / \mathrm{min}\right.$ AUC) and paclitaxel $\left(50 \mathrm{mg} / \mathrm{m}^{2}\right)$ for 5 weeks, concurrent radiotherapy (41.4 Gy in 23 fractions, 5 days per week), followed by surgery) has been proven to be valuable in oesophageal cancer (EC), prognosis remains dismal with approximately $20 \%$ complete responders (5-yr overall survival=20-30\% [3, 4]), making EC the sixth most lethal cancer type in 2012, worldwide [5]. In 2016, over 15.000 patients died from EC in the USA alone [5]. Most EC contain hypoxic areas with a higher percentage in the adenocarcinomas, potentially explaining the poor treatment outcome for these patients [6]. About half of the patients treated with definitive chemoradiation will suffer from a locoregional recurrence. For effective radiation treatment, the presence of molecular oxygen is essential. Under normoxic conditions, ionizing radiation leads to the formation of free radicals and reactive oxygen species (ROS), which can damage DNA. Free radicals produced in the critical target can be fixed in the presence of oxygen, leading to irreversible DNA damage. In hypoxic conditions however, free radicals are reduced and hypoxic regions becomes 2-3 times more radio-resistant, which may explain low rates of complete response (CR) and local control (LC) [1, 7]. Accordingly, patients with hypoxic EC might need a different, personalized treatment approach to reach therapeutic success.

Since tumour hypoxia cannot be predicted based on clinical size, stage, or grade, there is a need for molecular biomarkers that can assess hypoxic status in EC. Such biomarkers could be used to detect hypoxic tumour status at an early stage, evaluate treatment response, predict prognosis in EC patients and select patients for suitable, personalized treatment options. 
In this systematic review, we provide an overview of hypoxia response-associated biomarkers in EC patients and aim to evaluate the prognostic value of elevated expression rate of hypoxia-associated biomarkers with regard to treatment outcome and efficacy [i.e., overall survival (OS), disease-free survival (DFS), CR, and LC]. Markers that are independent prognostic factors could potentially provide targets for novel treatment strategies. In addition, several known methods to improve treatment outcome will be discussed in relationship to these hypoxia-associated biomarkers.

\section{MATERIAL \& METHODS}

\section{SYSTEMATIC SEARCH STRATEGY}

The research question for this systematic review was defined as: "What are the known hypoxia-associated molecular markers in patients with EC and how does elevated expression associate with treatment outcome and response?"

To consider the research question, a comprehensive PRISMA-based literature search was performed to identify relevant studies published in PubMed (National Center for Biotechnology Information, NCBI), MEDLINE (U.S. National Library of Medicine, using NCBI), or Web of Science (Thomson Reuters). The electronic databases were explored using a PICOS-based search string containing a free-text or Medical Subject Headings (MeSH) construction of 5 key search terms: 'hypoxia' AND 'oesophagus' AND 'cancer' AND ('treatment outcome' OR 'treatment efficacy'). For each search term, all known synonyms and associated keywords were included in the search string using Boolean OR-operators. A detailed description of the entire search strings can be found in supplementary material S1 [8]. The complete search strategy was applied weekly, with the last update being performed on April $3^{\text {rd }}, 2017$.

\section{STUDY SELECTION}

Articles were eligible for inclusion when corresponding to the predetermined eligibility criteria: (1) the patient population consisted of human adults diagnosed with oesophageal cancer or clinically acquired EC tissue samples; (2) the index tests were all tests able to assess tumour hypoxia; (3) treatment outcome had to be evaluated and correlated with hypoxia. Only full-text articles written in English were retrieved from the electronic databases. If full-text content was not available to us, the corresponding author was contacted to retrieve the printed publication. Next, duplicate findings were manually discarded to ensure that no data overlap occurred. Further selection was performed by applying several exclusion criteria: (1) reviews, letters, abstracts, case studies, etc.; (2) studies using only oesophageal cell lines or animal-based tumour models; (3) studies aiming to investigate the molecular mechanisms of hypoxia; (4) studies that did not correlate expression rate of hypoxia- 
associated markers with treatment outcome or efficacy (i.e., CR, LC, OS, or DFS). Additional eligible articles were retrieved by manually cross-checking reference lists of relevant articles and reviews (citation tracking). Furthermore, databases were searched to retrieve studies exploring additional methods for non-invasive hypoxiaassessment in cancer patients by performing a secondary search including the MeSH-terms 'Hypoxia' AND ('MRI' OR 'SPECT' OR 'PET' OR 'CT'). This search was not specific for oesophageal cancer and will be reviewed in the second part of this manuscript.

\section{DATA EXTRACTION}

Two investigators (J.P. and L.VDV.) performed each step of this protocol independently (i.e., systematic search, defining eligibility, and data extraction). In cases of disagreement and consensus could not be reached, a third party (L.D.) was consulted to adjudicate. From the included articles, data was extracted concerning study characteristics (i.e., author, publication year), patient characteristics (i.e., number of subjects, country of origin, specimen type, tumour cell type), measurement characteristics (i.e., method of quantifying hypoxia, marker type, definition of hypoxia, percentage of hypoxic elements), and treatment strategy and response outcome characteristics (i.e., OS, DFS, CR, and LC). CR is defined as the total disappearance of a tumour, and LC as the arrest of cancer growth at the site of origin (i.e., stable tumour volume). Survival is assessed by OS, defined as the time interval from end of primary therapy until last known survival data or death, and DSF that is defined as the time interval after primary treatment and the first signs of recurrence, metastasis, or cancer-related disease. Furthermore, statistical outcome was extracted with p-values defining the prognostic/predictive power. P-values $<0.05$ indicated statistically significant differences in treatment outcome between high and low percentages of hypoxia-associated markers based on the reported threshold of hypoxia.

\section{RESULTS}

\section{LITERATURE SEARCH}

As presented in Figure 6.1, a total of 419 records were initially identified in Web of Science $(\mathrm{n}=182)$, PubMed $(\mathrm{n}=215)$ using free-text search strings and in MEDLINE $(\mathrm{n}=22)$ using MeSH-terms. After imposing language-restrictions and removing duplicate findings $(\mathrm{n}=121), 244$ full-text records remained. Further screening of records' title resulted in 85 potentially eligible studies by excluding articles that clearly stated terms did not fit the inclusion criteria (e.g., different tumour-types, reviews, meta-analyses, etc.). Next, abstracts of the remaining 85 articles were screened and based on the exclusion criteria, we excluded reviews $(n=4)$, papers that studied the molecular pathways of hypoxia $(n=7)$, and papers that did not evaluate 
oesophageal cancer $(n=16)$, or treatment outcome $(n=13)$, or studied the effects of hypoxia $(n=14)$. Finally, the full-text content of 31 articles was assessed for eligibility and 11 studies were excluded for following reasons: studies had different study objective than our PICOS-based research question to assess oesophageal tumour hypoxia $(n=6)$, the use of preclinical animal-based tumour models or oesophageal cell lines $(n=4)$, and studies that did not allow the extraction of survival data or clinical effectiveness data $(n=1)$. Through citation tracking, additional papers $(n=2)$ were included that fitted the eligibility criteria. In total, 22 studies were included to be systematically reviewed [9-30].

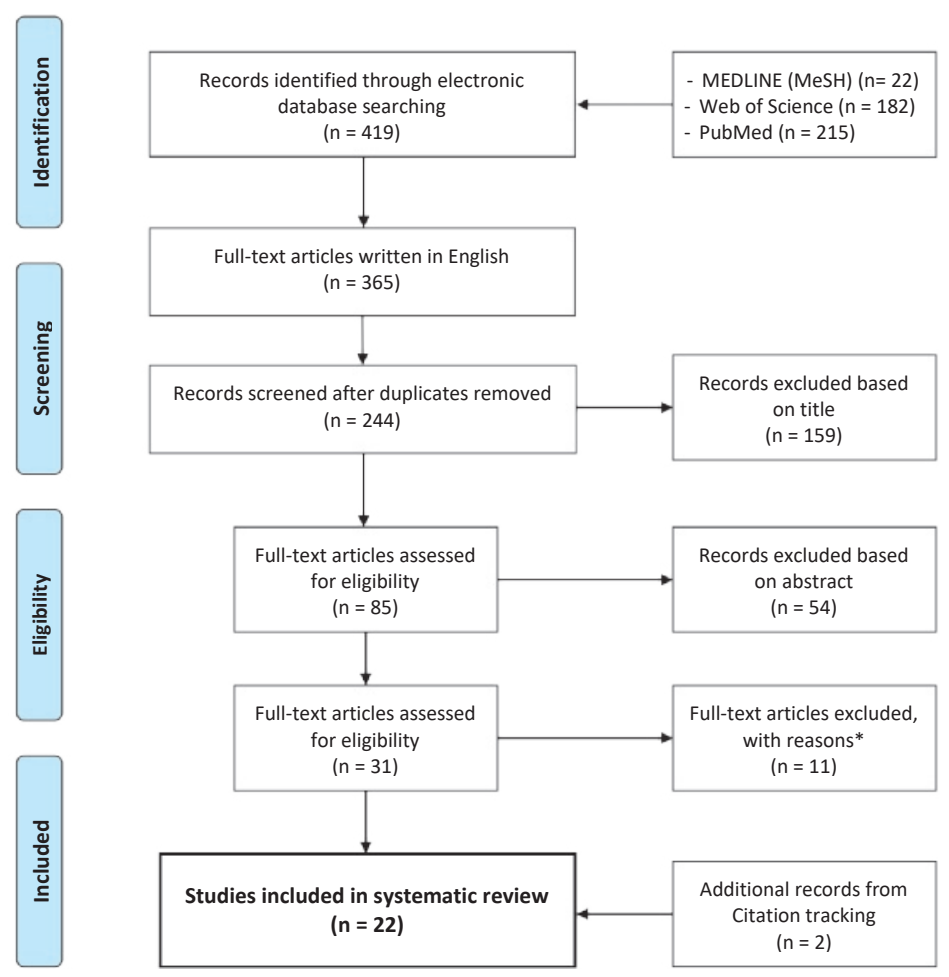

Figure 6.1: Flowchart of information through different phases of systematic review. *Reasons for exclusion on full-text level were: not fitting PICOS-based research question $(n=6)$, use of preclinical tumour models $(n=4)$, or unsuitable to extract survival data $(n=1)$.

\section{DATA EXTRACTION}

Several studies confirm the presence of endogenous hypoxia-associated markers in oesophageal cancer patients. The clinical impact of hypoxia in treatment outcome (i.e., OS, DFS, CR, and LC) is summarized in Table 6.1 and 6.2, respectively. In both tables, clinical impact was defined by Kaplan-Meier analyses (log-rank test) and Cox proportional hazard model (uni- and multi-variate analyses). 
In general, we found mainly endogenous tissue markers (HIF-1 $\alpha$, carbonic anhydrase IX and GLUT-1) with prognostic value and ability to predict treatment response in EC. We found only one study with non-invasive imaging including ${ }^{18}$ F-FETNIM PET which correlated hypoxia to chemoradiotherapy response in EC.

\section{Hypoxia-inducible factor (HIF)}

Hypoxia-inducible factor (HIF) is the master protein in regulating the response of cells to changing oxygen levels and recognizes the hypoxia response element (HRE) on the untranslated region of over 150 genes involved in cell survival, tumour metabolism, proliferation, and angiogenesis [31, 32]. HIF-1 exists as a heterodimer protein composed of constitutively expressed HIF-1 $\beta$ complexed with one of three subunits (HIF- $1 \alpha$, HIF- $2 \alpha$ or HIF- $3 \alpha$ ). Synthesis of HIF- $1 \alpha$ is regulated via $\mathrm{O}_{2-}$ independent mechanisms whereas degradation is primarily $0_{2}$-dependent. Thus, HIF- $1 \alpha$ upregulation could be a promising endogenous marker of hypoxia in EC. Interestingly, strong immunoreactivity for HIF-1 $\alpha$ was presented more often in oesophageal squamous cell carcinomas (ESCC) tumour tissue than in adenocarcinoma (AC) $(\mathrm{p}=0.009)$ [25]. HIF- $1 \alpha$ could be differently upregulated in ESCC than in AC. Together with molecular mutations and epigenetic alterations, the difference in outcome and treatment response of the two histologic subtypes could be explained [4]. Given the scarce data of HIF-1 $\alpha$ in AC, no clear conclusion can be drawn regarding clinical outcome.

In a meta-analysis by Ping et al. (2014), the prognostic significance of HIF-1 $\alpha$ in ESCC has been investigated [3]. They reported that in univariate analyses HIF-1 $\alpha$ overexpression was significantly associated with poor OS ( $\mathrm{p}<0.001,10$ studies), and DFS ( $\mathrm{P}=0.013$, 2 studies). These findings are in accordance with most overlapping studies included in this review. However, Munipalle et al. (2011) showed that HIF$1 \alpha$ overexpression was not correlated with OS in a European population $(\mathrm{p}=0.908)$, contrary to the reported Japanese/Chinese population [20]. Presumably, this information was overlooked in the aforementioned meta-analysis because 11/12 studies included a Japanese or Chinese population (906/942 ESCC patients) and only 1 study was included with 36/942 ESCC patients originating from the UK. In multivariate analyses, opposing results have been presented as some studies indicated that HIF-1 $\alpha$ overexpression is an independent prognostic factor for survival [21, 27], while some studies report the contrary [17, 18, 30]. In the study by Zhang et al. (2014), the prognostic power of HIF-1 $\alpha$ overexpression could be lost by including metastatic/recurrent ESCC in the patient cohort [30]. Therefore, further clarification is needed in a large prospective study that includes both uni- and multivariate analyses to investigate differences in patient cohort, histological subtype, and pathologic origin (primary or metastatic EC). 
In early stage oesophageal cancer, HIF-1 $\alpha$ expression in tumour tissue is associated with lower CR rates to local therapies such as photodynamic therapy (PDT) and concurrent chemoradiotherapy (CCRT) [16, 22, 24, 29]. This suggests that low HIF$1 \alpha$ levels in EC may be a good indicator for early treatment response in otherwise treatment-resistant hypoxic tumours. The significant correlation between HIF-1 $\alpha$ and CR has been confirmed by Ping et al. ( $p=0.001,4$ studies) [3].

\section{Carbonic Anhydrase (CA IX)}

Carbonic Anhydrase IX (CA IX) belongs to the family of zinc metalloenzymes with presence in normal stomach, intestinal and gall bladder tissue. It is involved in maintaining the cells $\mathrm{pH}$-homeostasis by the reversible hydration of carbon dioxide into bicarbonate and hydrogen [33]. CA IX is over-expressed in hypoxic solid tumours through the HIF- $1 \alpha$ activation cascade. Compared to HIF- $1 \alpha$, CA IX is a stable and sustained marker of hypoxia with a half-life of $38 \mathrm{~h}$ [34, 35]. In general, elevated membranous CA IX was mainly found at the tumour centre or at the border of tumours with expression rates being approximately 45-60\% [9, 12, 26]. In 2008, Tanaka et al. reported that although hypoxia-induced CA IX expression correlated with more aggressive clinicopathological parameters and poor outcome, tumour related CA IX expression in ESCC was not an independent prognostic factor in multivariate survival analysis [26]. In contrast, Driessen et al. (2006) showed that CA IX is a significant determinant in AC, and an independent prognostic factor for OS $(p=0.017)$ and DFS $(p=0.041)$ [12]. This was confirmed in a more recent study by Birner et al. (2011) in an evenly-distributed patient cohort of ESCC and AC [9]. In a meta-analysis by van Kuijk et al. (2017), EC-specific subgroup-analyses reported significant association between CA IX expression and both OS and DFS, respectively $(\mathrm{p}<0.001)$ [33]. High CA IX expression was thus regarded as an adverse prognostic marker in EC. Furthermore, the expression of CA IX in tumour-surrounding stroma has also been significantly linked to shorter OS $(p=0.013)$ and DFS $(p=0.007)$ in a large cohort-study ( $n=155$ ESCC, n=206 AC) [13]. It has been postulated that the difference in clinical behaviour between ESCC and AC, could be related to a significant correlation between CA IX and HER-2 and/or a VEGF expression [9, 12]. Nevertheless, these findings indicate the importance of this hypoxia-associated marker in disease progression and treatment resistance. 
Table 6.1: Extracted data concerning Overall survival (OS) and Disease-free survival (DFS) in oesophageal squamous cell carcinoma (SCC) and adenocarcinoma (AC). IHC=immunohistochemistry. ${ }^{*}$ ) P-values $<0.05$ indicate significant differences

\begin{tabular}{|c|c|c|c|c|c|c|c|c|}
\hline Author & Country & Specimen & $\begin{array}{l}\text { Index } \\
\text { test }\end{array}$ & Marker & $\begin{array}{c}\text { Hypoxia criteria } \\
\text { threshold expression rate }\end{array}$ & $\begin{array}{c}\text { Tumour } \\
\text { type }\end{array}$ & $\mathbf{N}$ & $\begin{array}{l}\text { Hypoxic } \\
\text { sample }\end{array}$ \\
\hline Birner (2011) & Austria & Surgical & IHC & CAIX & $\begin{array}{l}>\text { median staining } \\
\text { intensity * staining rate }\end{array}$ & $\begin{array}{l}\text { All } \\
\text { AC } \\
\text { SCC }\end{array}$ & $\begin{array}{l}330 \\
182 \\
148\end{array}$ & $\begin{array}{l}44.5 \% \\
46.7 \% \\
41.9 \%\end{array}$ \\
\hline Chen (2009) & China & Surgical & IHC & $\begin{array}{l}\text { Beclin-2 } \\
\text { HIF-1 } \alpha \\
\text { Both }\end{array}$ & $\begin{array}{l}<10 \% \text { cytoplasm staining } \\
>\text { mean cytoplasm expr } \\
\text { Low Beclin-2, High HIF-1، }\end{array}$ & SCC & 54 & $\begin{array}{l}33.3 \% \\
46.3 \% \\
24.1 \%\end{array}$ \\
\hline Chiba (2010) & Japan & Biopsy & IHC & GLUT-1 & $>30 \%$ membrane expr. & SCC & 25 & $28.1 \%$ \\
\hline Driessen (2006) & $\mathrm{NL} / \mathrm{BE}$ & Surgical & IHC & $\begin{array}{l}\text { CAIX } \\
\text { VEGF }\end{array}$ & $>$ median staining & $\mathrm{AC}$ & 39 & $\begin{array}{l}49.0 \% \\
69.2 \%\end{array}$ \\
\hline Jomrich (2014) & Austria & Surgical & IHC & CAIX & $\geq 10 \%$ stromal staining & $\begin{array}{l}\text { All } \\
\text { AC } \\
\text { SCC }\end{array}$ & $\begin{array}{l}361 \\
206 \\
155\end{array}$ & $\begin{array}{l}11.6 \% \\
11.2 \% \\
12.3 \%\end{array}$ \\
\hline Katsuta (2005) & Japan & Surgical & IHC & $\begin{array}{l}\text { HIF-1 } \alpha \\
\text { VEGF }\end{array}$ & $\begin{array}{c}>10 \% \text { nucl/cytoplasm or } \\
>30 \% \text { cytoplasmic staining }\end{array}$ & SCC & 48 & $\begin{array}{l}70.8 \% \\
60.4 \%\end{array}$ \\
\hline Kimura (2004) & Japan & Surgical & IHC & $\begin{array}{l}\text { HIF-1 } \alpha \\
\text { VEGF }\end{array}$ & $\begin{array}{l}>\text { mean nucl/cytoplasm. } \\
>10 \% \text { cytoplasm. staining }\end{array}$ & SCC & 47 & $\begin{array}{l}36.1 \% \\
53.2 \%\end{array}$ \\
\hline Koukourakis (2001) & ) Greece & Biopsy & IHC & $\begin{array}{l}\text { HIF- } 1 \alpha \\
\text { HIF- } 2 \alpha\end{array}$ & Nuclear staining & SCC & 37 & $\begin{array}{l}51.0 \% \\
13.5 \%\end{array}$ \\
\hline Kurokawa (2003) & Japan & Surgical & IHC & HIF- $1 \alpha$ & $\begin{array}{l}>10 \% \text { nuclear or distinct } \\
\text { cytoplasmic staining }\end{array}$ & SCC & 130 & $30.8 \%$ \\
\hline Ling (2006) & Germany & Biopsy & IHC & prot.HIF-1 $\alpha$ & Nuclear staining & All & 53 & $33.3 \%$ \\
\hline & & Surgical & RNA assay & $y$ HIF- $1 \alpha$ mRNA & $\begin{array}{l}\text { Ratio tumour/normal } \\
\text { epithelium }>1\end{array}$ & $\begin{array}{l}\text { All } \\
\text { AC } \\
\text { SCC }\end{array}$ & $\begin{array}{l}53 \\
22 \\
31\end{array}$ & $\begin{array}{l}53.8 \% \\
15.4 \% \\
38.5 \%\end{array}$ \\
\hline Matsuyama (2004) & Japan & Surgical & IHC & HIF- $1 \alpha$ & $\begin{array}{l}\text { > mean nuclear or } \\
\text { cytoplasmic expr }\end{array}$ & SCC & 215 & $95.0 \%$ \\
\hline Munipalle (2011) & $\begin{array}{l}\text { United } \\
\text { Kingdom }\end{array}$ & Biopsy & IHC & HIF- $1 \alpha$ & $\geq 10 \%$ nuclear staining & SCC & 36 & $52.8 \%$ \\
\hline Ogane (2010) & Japan & Surgical & IHC & HIF-1 $\alpha$ & nuclear staining & SCC & 96 & $67.7 \%$ \\
\hline Ogawa (2011) & Japan & Biopsy & IHC & HIF- $1 \alpha$ & $>10 \%$ staining & Mix & 25 & $18.2 \%$ \\
\hline Schreurs (2014) & NL & Patient & PET-scan & ${ }^{18} \mathrm{~F}-\mathrm{FDG}$ & $\mathrm{SUVmax}(\mathrm{FDG})>3.67$ & Mix & 47 & - \\
\hline Sohda (2004) & Japan & Biopsy & IHC & HIF- $1 \alpha$ & $>10 \%$ cell staining & Mix & 65 & $58.5 \%$ \\
\hline Takala (2011) & Finland & Surgical & IHC & $\begin{array}{l}\text { HIF-1 } \alpha \\
\text { VEGF }\end{array}$ & $\begin{array}{c}>5 \% \text { nuclear staining } \\
>25 \% \text { cytoplasm. staining }\end{array}$ & Mix & $\begin{array}{l}80 \\
84\end{array}$ & $\begin{array}{l}71.3 \% \\
71.4 \%\end{array}$ \\
\hline Tanaka (2008) & Japan & Surgical & $\mathrm{IHC}$ & CAIX & $\begin{array}{l}\text { >median staining rate and } \\
\text { intensity }\end{array}$ & SCC & 127 & $59.8 \%$ \\
\hline Tzao (2008) & Taiwan & Surgical & IHC & $\begin{array}{l}\text { HIF-1 } \alpha \\
\text { VEGF }\end{array}$ & $>10 \%$ positive staining & SCC & 85 & $\begin{array}{l}61.2 \% \\
65.9 \%\end{array}$ \\
\hline Winther (2013) & Denmark & Biopsy & $\begin{array}{c}\text { Gene } \\
\text { Clustering }\end{array}$ & $\begin{array}{l}15 \text { hypoxia- } \\
\text { assoc. genes }\end{array}$ & $\begin{array}{l}\text { weighted hypoxia gene } \\
\text { expression signature }\end{array}$ & Mix & 56 & $31 \%$ \\
\hline Zhang (2014) & China & Surgical & IHC & HIF-1 $\alpha$ & $\begin{array}{c}>\text { median staining } \\
\text { intensity * staining rate }\end{array}$ & $\begin{array}{c}\text { SCC } \\
\text { Locor SCC } \\
\text { Metastatic }\end{array}$ & $\begin{array}{c}136 \\
67 \\
69\end{array}$ & $\begin{array}{l}52.2 \% \\
41.8 \% \\
62.3 \%\end{array}$ \\
\hline
\end{tabular}


Table 6.1: continued. KM=Kaplan-Meier model, Cox=Cox proportional hazard model

\begin{tabular}{|c|c|c|c|c|c|c|c|c|c|c|}
\hline \multirow[b]{2}{*}{ Author } & \multirow[b]{2}{*}{ Marker } & \multicolumn{4}{|c|}{ OS } & \multicolumn{4}{|c|}{ DFS } & \multirow[b]{2}{*}{$\begin{array}{l}\text { Indep } \\
\text { progr } \\
\text { factor }\end{array}$} \\
\hline & & $\begin{array}{l}\text { Hypoxic vs. } \\
\text { non-hypoxic } \\
\text { sample }\end{array}$ & $\begin{array}{c}\mathrm{KM} \\
\mathrm{P}^{*}\end{array}$ & $\begin{array}{c}\text { Cox } \\
\text { Univar } \\
P^{*}\end{array}$ & $\begin{array}{c}\text { Cox } \\
\text { Multivar } \\
\mathrm{P}^{*}\end{array}$ & $\begin{array}{l}\text { Hypoxic vs. } \\
\text { non-hypoxic } \\
\text { sample }\end{array}$ & $\begin{array}{c}\mathrm{KM} \\
\mathbf{P}^{*}\end{array}$ & $\begin{array}{c}\text { Cox } \\
\text { Univar } \\
\mathbf{P}^{*}\end{array}$ & $\begin{array}{c}\text { Cox } \\
\text { Multivar } \\
\mathrm{P}^{*}\end{array}$ & \\
\hline \multirow{3}{*}{ Birner (2011) } & \multirow{3}{*}{ CAIX } & $30 \%$ vs. $54 \%$ & 0.001 & 0.001 & 0.01 & $24 \%$ vs. $52 \%$ & 0.001 & 0.001 & 0.001 & $\mathrm{Y}$ \\
\hline & & $37 \%$ vs. $59 \%$ & 0.001 & 0.01 & 0.03 & $33 \%$ vs. $55 \%$ & 0.001 & 0.01 & 0.02 & Y \\
\hline & & $19 \%$ vs. $48 \%$ & 0.001 & 0.001 & 0.04 & $12 \%$ vs. $45 \%$ & 0.001 & 0.001 & 0.01 & Y \\
\hline \multirow[t]{3}{*}{ Chen (2009) } & Beclin-2 & - & 0.004 & - & - & - & - & - & - & - \\
\hline & HIF- $1 \alpha$ & - & 0.05 & - & - & - & - & - & - & - \\
\hline & Both & - & 0.001 & - & - & - & - & - & - & - \\
\hline Chiba (2010) & GLUT-1 & - & - & - & - & $28 \%$ vs. $30 \%$ & 0.04 & 0.04 & 0.02 & Y \\
\hline \multirow{2}{*}{ Driessen (2006) } & CAIX & $27 \%$ vs. $47 \%$ & 0.01 & - & 0.02 & - & - & - & 0.04 & Y \\
\hline & VEGF & - & - & $>0.05$ & $>0.05$ & - & - & $>0.05$ & $>0.06$ & $\mathrm{~N}$ \\
\hline \multirow[t]{3}{*}{ Jomrich (2014) } & CAIX & $28 \%$ vs. $44 \%$ & 0.01 & - & 0.20 & $25 \%$ vs. $37 \%$ & 0.01 & - & 0.08 & $\mathrm{~N}$ \\
\hline & & $42 \%$ vs. $47 \%$ & 0.14 & - & 0.26 & $40 \%$ vs. $41 \%$ & 0.12 & - & 0.06 & $\mathrm{~N}$ \\
\hline & & $10 \%$ vs. $39 \%$ & 0.04 & - & 0.93 & $8 \%$ vs. $33 \%$ & 0.03 & - & 0.81 & $\mathrm{~N}$ \\
\hline \multirow[t]{2}{*}{ Katsuta (2005) } & HIF- $1 \alpha$ & $71 \%$ vs. $93 \%$ & 0.34 & - & - & $21 \%$ vs. $0 \%$ & 0.09 & - & - & - \\
\hline & VEGF & $66 \%$ vs. $90 \%$ & 0.47 & - & - & $26 \%$ vs. $6 \%$ & 0.22 & - & - & - \\
\hline \multirow[t]{2}{*}{ Kimura (2004) } & HIF- $1 \alpha$ & - & 0.04 & - & - & - & - & - & - & - \\
\hline & VEGF & - & 0.001 & - & - & - & - & - & - & - \\
\hline \multicolumn{2}{|c|}{ Koukourakis (2001)HIF-1 $\alpha$} & - & 0.08 & - & - & - & 0.14 & - & - & - \\
\hline & HIF- $2 \alpha$ & - & 0.03 & - & - & - & 0.10 & - & - & - \\
\hline Kurokawa (2003) & HIF- $1 \alpha$ & $40 \%$ vs. $58 \%$ & 0.001 & 0.001 & 0.17 & - & - & - & - & $\mathrm{N}$ \\
\hline \multirow[t]{4}{*}{ Ling (2006) } & \multirow{4}{*}{$\begin{array}{l}\text { prot.HIF-1o } \\
\text { HIF mRNA }\end{array}$} & - & - & 0.53 & - & - & - & - & - & $\mathrm{N}$ \\
\hline & & - & - & 0.78 & - & - & - & - & - & $\mathrm{N}$ \\
\hline & & - & - & 0.59 & - & - & - & - & - & $\mathrm{N}$ \\
\hline & & - & - & 0.23 & - & - & - & - & - & $\mathrm{N}$ \\
\hline Matsuyama (2004) & HIF- $1 \alpha$ & $43 \%$ vs. $52 \%$ & 0.11 & 0.11 & - & $47 \%$ vs. $55 \%$ & 0.02 & 0.03 & 0.14 & $\mathrm{~N}$ \\
\hline Munipalle (2011) & HIF- $1 \alpha$ & $26 \%$ vs. $18 \%$ & 0.91 & - & 0.001 & - & - & - & - & Y \\
\hline Ogane (2010) & HIF- $1 \alpha$ & - & 0.03 & 0.03 & 0.23 & - & 0.02 & - & - & Y \\
\hline Ogawa (2011) & HIF- $1 \alpha$ & - & $>0.05$ & - & - & $18 \%$ vs. $40 \%$ & 0.001 & 0.001 & 0.001 & Y \\
\hline Schreurs (2014) & ${ }^{18} \mathrm{~F}-\mathrm{FDG}$ & $23 \%$ vs. $57 \%$ & 0.30 & - & - & $17 \%$ vs. $62 \%$ & 0.02 & - & - & - \\
\hline Sohda (2004) & HIF- $1 \alpha$ & - & 0.47 & - & - & - & - & - & - & - \\
\hline \multirow[t]{2}{*}{ Takala (2011) } & HIF- $1 \alpha$ & - & 0.22 & - & - & - & - & - & - & - \\
\hline & VEGF & - & 0.84 & - & - & - & - & - & - & - \\
\hline Tanaka (2008) & CAIX & $33 \%$ vs. $66 \%$ & 0.001 & 0.001 & 0.79 & - & - & - & - & $\mathrm{N}$ \\
\hline \multirow[t]{2}{*}{ Tzao (2008) } & HIF- $1 \alpha$ & - & 0.002 & - & 0.04 & - & - & - & - & Y \\
\hline & VEGF & - & 0.001 & - & 0.03 & - & - & - & - & Y \\
\hline Winther (2013) & $\begin{array}{l}15 \text { hypoxia- } \\
\text { assoc. genes }\end{array}$ & $12 \%$ vs. $49 \%$ & 0.07 & $>0.05$ & 0.11 & $12 \%$ vs. $49 \%$ & 0.13 & 0.02 & 0.22 & $\mathrm{~N}$ \\
\hline \multirow[t]{3}{*}{ Zhang (2014) } & HIF- $1 \alpha$ & $25 \%$ vs. $37 \%$ & 0.03 & - & 0.22 & $22 \%$ vs. $35 \%$ & 0.04 & - & 0.35 & $\mathrm{~N}$ \\
\hline & & $32 \%$ vs. $51 \%$ & 0.04 & - & 0.04 & $32 \%$ vs. $53 \%$ & 0.05 & - & 0.05 & Y \\
\hline & & $11 \%$ vs. $16 \%$ & 0.85 & - & - & - & 0.56 & - & - & $\mathrm{N}$ \\
\hline
\end{tabular}


Table 6.2: Extracted data concerning complete response (CR) and Local control (LC) in oesophageal squamous cell carcinoma (SCC) and adenocarcinoma (AC). CCRT=concurrent chemoradiotherapy, PDT=photodynamic therapy. $\left({ }^{*}\right) \mathrm{P}$-values $<0.05$ indicate significant differences

\begin{tabular}{|c|c|c|c|c|c|c|c|c|}
\hline Author & Country & Specimen & $\begin{array}{c}\text { Index } \\
\text { test }\end{array}$ & Marker & $\begin{array}{c}\text { Hypoxia criteria } \\
\text { threshold expression rate }\end{array}$ & $\begin{array}{l}\text { Tumour } \\
\text { type }\end{array}$ & $\mathbf{N}$ & $\begin{array}{l}\text { Hypoxic } \\
\text { sample }\end{array}$ \\
\hline Chiba (2010) & Japan & Biopsy & IHC & GLUT-1 & $\begin{array}{l}>30 \% \text { membrane } \\
\text { expression }\end{array}$ & SCC & 25 & $28.0 \%$ \\
\hline Koukourakis (2001) & Greece & Biopsy & $\mathrm{IHC}$ & $\begin{array}{l}\text { HIF-1 } \alpha \\
\text { HIF-2 } \alpha\end{array}$ & Epithelial expression & SCC & 37 & $\begin{array}{l}51.0 \% \\
13.5 \%\end{array}$ \\
\hline Ogawa (2011) & Japan & Biopsy & IHC & HIF1 $\alpha$ & $>10 \%$ staining & SCC & 25 & $42.7 \%$ \\
\hline Sohda (2004) & Japan & Biopsy & IHC & HIF-1 $\alpha$ & $>10 \%$ cell staining & Mix & 65 & $58 \%$ \\
\hline Winther (2013) & Denmark & Biopsy & $\begin{array}{c}\text { Gene } \\
\text { Clustering }\end{array}$ & $\begin{array}{l}15 \text { hypoxia- } \\
\text { assoc. genes }\end{array}$ & $\begin{array}{l}\text { weighted hypoxia gene } \\
\text { expression signature }\end{array}$ & SCC & 56 & $31 \%$ \\
\hline Yue (2012) & China & Patient & PET scan & ${ }^{18}$ F-FETNIM & $\begin{array}{c}\text { Ratio SUVmax to } \\
\text { spleen SUVmean }>1,3\end{array}$ & SCC & 28 & $82 \%$ \\
\hline
\end{tabular}

Table 6.2: continued

\begin{tabular}{|c|c|c|c|c|c|c|c|}
\hline \multirow[b]{2}{*}{ Author } & \multirow[b]{2}{*}{ Therapy } & \multicolumn{2}{|c|}{ CR (5yr) } & \multicolumn{3}{|c|}{ LC (5yr) } & \multirow[b]{2}{*}{$\begin{array}{l}\text { Indep. } \\
\text { Predictive } \\
\text { factor }\end{array}$} \\
\hline & & $\begin{array}{l}\text { hypoxic vs. non- } \\
\text { hypoxic group }\end{array}$ & $\begin{array}{c}\text { CR } \\
\text { p-value* }\end{array}$ & $\begin{array}{l}\text { hypoxic vs. non } \\
\text { hypoxic group }\end{array}$ & $\begin{array}{c}\text { Univar. LC } \\
\text { p-value* }\end{array}$ & $\begin{array}{l}\text { Multivar. } \\
\text { LC p-value* }\end{array}$ & \\
\hline Chiba (2010) & CCRT & $14 \%$ vs. $67 \%$ & 0.0185 & $28.5 \%$ vs. $73.4 \%$ & 0.0003 & 0.007 & yes \\
\hline Koukourakis (2001) & $\begin{array}{l}\text { PDT } \\
\text { PDT }\end{array}$ & $\begin{array}{c}20 \% \text { vs. } 56 \%^{*} \\
0 \% \text { vs. } 42 \%\end{array}$ & $\begin{array}{l}0.04 \\
0.13\end{array}$ & - & - & - & $\begin{array}{c}\text { yes } \\
\text { no }\end{array}$ \\
\hline Ogawa (2011) & CCRT & $15.4 \%$ vs. $84.6 \%$ & 0.0027 & $42.7 \%$ vs. $72.5 \%$ & 0.0322 & 0.178 & no \\
\hline Sohda (2004) & CCRT & $7.9 \%$ vs. $44.4 \%$ & 0.009 & - & - & - & yes \\
\hline Winther (2013) & CCRT & $33.3 \%$ vs. $52.4 \%$ & 0.02 & - & - & - & yes \\
\hline Yue (2012) & CCRT & $42 \%$ vs. $100 \%$ & $\begin{array}{c}0.041 \\
\text { (SUVMax) }\end{array}$ & - & - & - & yes \\
\hline
\end{tabular}




\section{Other hypoxia-associated markers}

The expression of glucose-transporter-1 (GLUT-1) is upregulated in hypoxic condition by HIF-1. In immunohistochemistry (IHC) analyses, GLUT-1 expression appeared to be a surrogate marker for hypoxia but also seemed to be prognostic factor for DFS and predictive for initial response to CCRT and LC [11].

Vascular endothelial growth factor (VEGF) is a transcriptional target for HIF and stimulates angiogenesis in EC [25]. Contradicting findings have been reported concerning the prognostic value of VEGF. In AC patients or in a mixed cohort, studies reported no association between VEGF expression and prognosis [12, 25]. In ESCC, however, VEGF expression was regarded as an independent prognostic factor of OS [15, 27].

\section{Non-invasive Imaging techniques}

Non-invasive molecular imaging using positron-emission tomography (PET) has been shown to specifically detect hypoxic cell clusters in individual tumours using several 2-nitroimidazole derivatives [36-42]. Viable hypoxic cells are marked by 2nitroimidazole derivatives through irreversible electron-reduction mechanisms involving nitroreductase enzymes such as cytochrome P450 reductase. Four clinically used, FDA approved hypoxia PET-tracers are presented in Table 6.3 [6, 29, $36,37,43-47]$.

It has been shown that ${ }^{18} \mathrm{~F}$-fluoromisonidazole $\left({ }^{18} \mathrm{~F}-\mathrm{FMISO}\right)$ allows visualization of hypoxic areas in a variety of tumours although data on EC remain scarce. In a study by Brink et al. (2008), 33/38 patients with EC presented noticeable hypoxic volumes [6]. Standard uptake values (SUV) of ${ }^{18} \mathrm{~F}-\mathrm{FMISO}$ were shown to be significantly higher in AC $(n=20$, SUVmean=1.93 \pm 0.43$)$ than in ESCC $(n=18$, SUVmean=1.56 \pm 0.25$)$ $(\mathrm{P}<0.01)$ [6]. However, the ability to visualize hypoxia differs in various cancer-types. For example, a significant correlation between ${ }^{18} \mathrm{~F}-\mathrm{FMISO}$ uptake and tumour markers from IHC (e.g., microvessel density, HIF-1alfa, VEGF, and GLUT-1) have been reported in head-and-neck cancer, whereas no correlation has been published in non-small-cell-lung cancer (NSCLC) [36, 38].

In untreated ESCC, Yue et al. evaluated the spatiotemporal variability of hypoxia and assessed the ability to predict clinical response after CCRT using the PET-marker ${ }^{18} \mathrm{~F}$-fluoroerythronitroimidazole $\left({ }^{18} \mathrm{~F}-\mathrm{FETNIM}\right)$ [29]. In this study, ${ }^{18} \mathrm{~F}$ FETNIM presented pharmacokinetic advantages over ${ }^{18} \mathrm{~F}-\mathrm{FMISO}$ and SUVmax $\left({ }^{18} \mathrm{~F}-\right.$ FETNIM) was found to be predictive for clinical response to CCRT $(\mathrm{P}=0.041)$. A higher baseline SUVmax ( ${ }^{18}$ F-FETNIM) of 5.9 was found in non-responders, while complete or partial responders showed SUVmax (18F-FETNIM) of 3.2 and 4.5, respectively.

Another promising PET-tracer able to visualize tumour hypoxia is ${ }^{18} \mathrm{~F}-3$-Fluoro-2-(4((2-nitro-1H-imidazol-1-yl)methyl)-1H-1,2,3-triazol-1-yl)propan-1-ol ( $\left.{ }^{18} \mathrm{~F}-\mathrm{HX} 4\right)$ [37, 
48, 49]. Klaassen et al. (2015) first studied the feasibility and repeatability of ${ }^{18} \mathrm{~F}-\mathrm{HX} 4$ imaging in oesophageal cancer [45]. Amount and location of elevated ${ }^{18} \mathrm{~F}-\mathrm{HX} 4$ uptake showed good repeatability in 19 EC patients (AD and SCC) suggesting that ${ }^{18} \mathrm{~F}-\mathrm{HX} 4$ PET could be a promising reliable tool to monitor tumour hypoxia in EC patients. Overall maximal tumour-to-background (TBRmax, mean \pm SD) was found to be $1.87 \pm 0.46$ in EC, $4 \mathrm{~h}$ post-injection. ${ }^{18} \mathrm{~F}-\mathrm{HX} 4$ has proven to be clinically useful in the non-invasive detection of tumour hypoxia also in other tumour-types (e.g., head $\&$ neck and lung cancer) (Fig. 6.2) [38, 39, 41, 50].
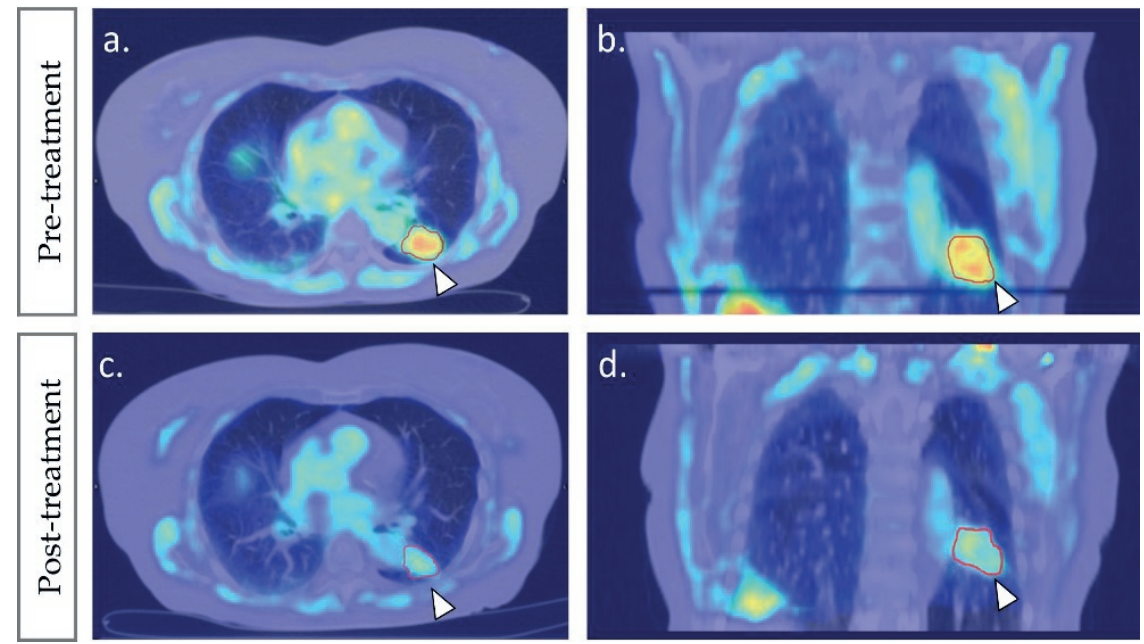

Figure 6.2: Clinical ${ }^{18} \mathrm{~F}-\mathrm{HX} 4$ PET/CT imaging of hypoxic non-small cell lung cancer (4h postinjection). The primary lung tumour (white triangle)is depicted in transversal $(\mathrm{a}, \mathrm{c})$ and coronal plane $(b, d)$, measured before $(a, b)$ and after hypoxia-modified chemoradiotherapy $(c, d)$. SUV $\left({ }^{18}\right.$ F-

HX4) ranged from 0.2-1.8.

Although not yet assessed in EC, several other PET-tracer are known to visualize tumour hypoxia. For example, ${ }^{18} \mathrm{~F}-\mathrm{FAZA}$ has shown promising results in correlating hypoxia in head-and-neck cancer with outcome after CCRT [51]. Less popular clinical PET-tracers include non-nitroimidazole Cu-ATSM [Cu(II)-diacetyl-bis(N4methylthiosemicarbazone)], ${ }^{18} \mathrm{~F}-\mathrm{FETA}$, and ${ }^{18} \mathrm{~F}-\mathrm{EF} 5$. Although several studies have presented $\mathrm{Cu}$-ATSM as a hypoxia marker for radiation treatment outcome in rectal, lung, and head-and-neck cancer, cellular Cu-ATSM retention is affected by multiple mechanisms in addition to hypoxia $[52,53]$. Thus Cu-ATSM would not be a pure marker for hypoxia. Recently, ${ }^{89} \mathrm{Zr}$-labeled cG250 monoclonal antibodies have been show to quantify and map CA IX expression in preclinical models and head-andneck cancer using PET [54]. Directly labelling CA IX for hypoxia-related PET-tracers has also been investigated [55-57].

Ideal PET-tracers for hypoxia should be able to reach hypoxic cells in perfusion-limited microenvironments, have an oxygen-specific retention mechanism, and have a rapid and complete clearance of unbound radioactive tracer 
(i.e., hydrophilic) [43]. These properties ensure safe and optimal PET-imaging of tumour hypoxia. Unfortunately, none of the presented PET-tracers completely meet all these requirements (Table 6.3).

Table 6.3: overview of 2-nitroimidazole PET-tracers able to visualise tumour hypoxia in oesophageal cancer. $\left({ }^{*}\right)$ missing data was substituted with known features in lung cancer.

$\left.{ }^{* *}\right)$ Tumour to background ratio was defined as the ratio of SUVmax(tumour) over SUVmax(spleen).

\begin{tabular}{|c|c|c|c|c|}
\hline & [18F]-FMISO & [18F]-FETNIM & [18F]-FAZA* & [18F]-HX4 \\
\hline Hypoxia PET-tracers & & & & \\
\hline Year of publication ${ }^{44}$ & 1987 & 1995 & 2002 & 2010 \\
\hline Stability & $\begin{array}{c}\text { Metabolites } \\
\text { in blood and urine }\end{array}$ & $\begin{array}{l}\text { Fewer metabolite- } \\
\text { formation than FMISO }\end{array}$ & $\begin{array}{l}\text { Very few metabolite- } \\
\text { formation }(10-15 \%)^{46}\end{array}$ & $\begin{array}{l}\text { Few metabolite- } \\
\text { formation }(18 \%)^{47}\end{array}$ \\
\hline Clearance & $\begin{array}{l}\text { Very slow } \\
\text { Hepatobiliary }\end{array}$ & $\begin{array}{l}\text { Slow } \\
\text { Renal }\end{array}$ & $\begin{array}{l}\text { Fast } \\
\text { Renal }\end{array}$ & $\begin{array}{l}\text { Very fast } \\
\text { Renal }\end{array}$ \\
\hline Optimal scantime & No plateau & 2 h p.i. ${ }^{29}$ & 2 h p.i. ${ }^{37}$ & 3-4h p.i. ${ }^{45}$ \\
\hline TBRmax (mean \pm SD) & Not defined & $2.41 \pm 0.6 * 37$ & $0.98 \pm 0.19^{46}$ & $1.87 \pm 0.46^{45}$ \\
\hline Hypoxic threshold & TBR $>1.2^{6}$ & $\mathrm{TBR}^{* *}>1.3^{29}$ & TBR $>1.4^{83}$ & TBR $>1.0-1.4^{45}$ \\
\hline Hydrophilicity $(\log P)^{43}$ & 0.4 & -0.77 & -0.4 & -0.69 \\
\hline
\end{tabular}

$\left({ }^{*}\right)$ NOT investigated in Esophageal cancer patients. $\left({ }^{* *}\right)$ SUVmax ratio Tumor-to-spleen.

Another non-invasive technique that can detect hypoxia is magnetic resonance imaging (MRI) using exo- or endogenous contrast agents, and MR spectroscopy techniques such as electron paramagnetic resonance and hyperpolarized metabolic MRI [58]. Although MRI has not yet been used to investigate tumour hypoxia in EC, it shows promising results in other tumour types. In cervical cancer, for example, several studies have shown a correlation between tumour hypoxia and dynamic contrast enhanced (DCE-)MRI, using gadolinium bolus-injection, and demonstrated the ability to identify patients with hypoxia-related treatment resistance [59]. However, DCE-MRI estimates tumour perfusion and could therefore not assess the full extent of chronic tumour hypoxia. Similarly, blood-oxygen level dependent (BOLD)-MRI has been reported to be sensitive to tissue oxygenation by indirectly correlating deoxyhaemoglobin-induced changes in $\mathrm{MR}$ signal to $\mathrm{pO}_{2}$. In clinical trials, BOLD-MRI has been reported to map chronic hypoxic regions in prostate cancer by correlation with pimonidazole staining and evaluate hypoxia in breast cancer through correlation with CA IX expression ( $\mathrm{r}=0.616, \mathrm{P}<0.001)[60,61]$. Although these MRI-techniques could be used as surrogate markers for tumour hypoxia, measurements are usually indirect and sensitive to image-related artefacts [62]. 


\section{DISCUSSION}

In this study, we performed a systematic literature search, reviewed the various hypoxia-associated markers used in EC patients, and assessed the clinical impact of hypoxia in treatment outcome (i.e., CR, LC, OS and DFS). Most included studies investigated invasively acquired hypoxia-associated markers (i.e., HIF and CA IX) in IHC analyses. Although all studies confirmed the presence of tumour hypoxia in oesophageal cancer, the prognostic value was not consistent across all studies. Discrepancies might arise from methodology differences for hypoxia detection and quantification. In addition, diverging findings could arise from differences in tumour cell type (AC vs. SCC) or from population differences (Western vs. Far Eastern) in the study cohort. Nevertheless, HIF-1 $\alpha$ overexpression could be regarded as a molecular biomarker for hypoxia-response and could be associated with treatment outcome and clinical response to CCRT and PDT in Asiatic patients with EC. In AC, CA IX levels in both tumour stroma and cell membrane are indicative for hypoxic status and prognostic for OS and DFS. However, we found contradicting results from multivariate survival analyses in studies with HIF-1 $\alpha$ as well as with CA IX. These findings support the need to further elucidate the complex molecular mechanism of tumour hypoxia and construct more reliable prediction models. Because invasively acquired biomarkers report unreliable results and are lacking the ability to capture the full intricacies of tumour hypoxia and its heterogeneity, there is a need for robust and quantitative biomarkers to detect hypoxia or hypoxia-associated responses in EC and determine complete tumour oxygenation. Furthermore, clinical assessment of hypoxic status needs to be performed repeatedly (i.e., before and during(chemo)radiation treatment), since hypoxia is a dynamic process and reoxygenation could occur after irradiation [63, 64]. Non-invasive imaging using radioactive PET-tracers shows great promise in repeatable and quantitative detection of hypoxic sub-regions in the entire tumour, although further validation of the clinical and prognostic value in EC is required.

Combining PET-imaging with MRI in a multimodal hybrid system (i.e., PET/MRI) might be the solution to identify potential new biomarkers and validate hypoxiaassociated biomarkers in EC patients. Recently, Simoncic et al. (2017) demonstrated a high correlation between ${ }^{18} \mathrm{~F}$-FMISO uptake parameters and DCE-MRI kinetic parameters in head-and-neck cancer patients $(n=6)$ [65]. However, the vascular data of dynamic PET and DCE-MRI was not exactly the same and the further development of simultaneous PET/MRI is encouraged to visualize hypoxic status. In addition to DCE- and BOLD-MRI, new techniques to assess tissue oxygenation are under development. Mapping oxygen by imaging lipids relaxation enhancement (MOBILE) detects variations in oxygenation based on MR relaxation rates of tissue lipids, instead of blood-oxygen related signal differences as seen in BOLD-MRI [66]. It has preclinically been confirmed that this novel technique is able to monitor 
changes in tumour oxygenation $(\mathrm{r}=0.51, \mathrm{p}=0.022)$ and changes in lipid relaxation rates show moderate correlation with absolute $\mathrm{pO}_{2}$ values $(\mathrm{r}=0.37, \mathrm{p}=0.027)$ [67]. Another promising novel MR-technique is oxygen-enhanced (OE-)MRI, where tumour oxygenation is detected as oxygen-induced increase in MR signal that is generally larger than signal changes detected using BOLD-MRI or MOBILE. By letting subjects breath $100 \%$ oxygen, $\mathrm{O}_{2}$-saturation in arterial blood plasma $(\mathrm{Hb}$ bound and dissolved) will increase, resulting in an increase in tumour $\mathrm{pO}_{2}$ and tissue oxygenation [68]. In a preliminary study, 10 patients with advanced abdominal or pelvic cancer underwent serial measurement of tumour relaxation rate while breathing medical air ( $21 \%$ oxygen) followed by $100 \%$ oxygen (OE-MRI). The resulting difference in MR signal was significant $(\mathrm{P}<0.005)$, proving the ability of OEMRI to indirectly detect changes in tumour oxygen levels [68, 69]. When combining multiple MRI-techniques with simultaneous PET-based imaging (i.e., PET/MRI), complementary information in tumour perfusion, tissue oxygenation, metabolic activity, and oxygen consumption could be acquired. Similar to PET/CT, this multiparametric method could be used to validate novel hypoxia-associated biomarkers and may help elucidate the complex nature of chronic hypoxia [70]. Moreover, PET/MRI relies on highly sensitive PET-probes and highly specific anatomical and/or functional MR information. However, protocol standardization (i.e., execution and analyses) is needed to allow for reproducible results and validation method of hypoxia-detection in multiple clinics and tumour types.

Non-invasive imaging could be useful to monitor hypoxic status and estimate early clinical response during (chemo)radiation treatment. In non-responsive patients, treatment strategies could be adapted to more hypoxia-guided therapies [71]. In radiotherapy, PET-based dose painting has been proposed to specifically deliver an escalated radiation dose or boost to hypoxic sub-volumes [72]. Such hypoxiatargeted radiotherapy could deliver an optimal dose distribution to radio-resistant regions. Currently, the survival probability of EC patients remains disappointing. Potentially, treatment outcome and patient survival could be improved by targeting hypoxia (i.e., increasing oxygen delivery, normalize tumour vasculature, or reduce oxygen consumption) or by implicating hypoxia-specific treatment strategies. However, pre-treatment hypoxic status must first be assessed since large patientand tumour-variability in oxygenation can exist. By selecting hypoxic patients before the start of treatment, a window-of-opportunity arises wherein attempts to reduce tumour hypoxia could be made. By first applying hypoxia-specific treatment strategies to overcome tumour hypoxia or eradicate hypoxic cells, conventional (chemo-)radiotherapy may become more effective and better treatment outcome can be achieved $[48,71]$. For example, preselected patients with hypoxic laryngeal cancer (i.e., high CA IX-fraction) had better LC and DFS when treated with accelerated radiotherapy with carbogen breathing and nicotinamide (ARCON) compared to accelerated radiotherapy (LC $97 \%$ vs. $71 \%, \mathrm{p}<0.01$ and DFS $92 \%$ vs. $69 \%, \mathrm{p}=0.06$ ) 
[73]. In contrast, a reversed scheme of radiation dose-painting has recently been proposed [74]. Here, hypoxic tumour regions were preclinically assessed using ${ }^{18} \mathrm{~F}-\mathrm{HX} 4-\mathrm{PET} / \mathrm{CT}$ imaging and used for radiation treatment dose planning. Nonhypoxic regions (i.e., low ${ }^{18} \mathrm{~F}-\mathrm{HX}-4$ uptake) received an escalated radiation dose, while hypoxic regions were targeted with hypoxia-activated prodrugs (HAP). Interestingly, this strategy was as effective as conventional radiotherapy plans but was able to reduce the mean overall tumour dose and hereby lowering normal tissue toxicity. Radiation dose was therefore used more efficiently.

It seems that hypoxia represents a 'Janus face' in tumour biology. On the one hand, it is associated with restrained proliferation and oxygen-deprived cell death, but on the other hand, it promotes adaptive processes leading to tumour aggressiveness, progression, and acquired resistance to treatment [75]. The high treatment failure rate seen in EC might therefore be due to a hypoxic microenvironment.

Molecular imaging could help individualizing hypoxia-specific treatment strategies in EC. Several approaches are available that focus on targeting HIF- $1 \alpha$ and VEGF. YC-1 (3-(5'-hydroxymethyl-2'-furyl)-1-benzylindazole) suppresses oesophageal tumour cell growth and inhibit cellular migration activities [76]. Similarly, radiosensitivity could be enhanced by downregulating VEGF and HIF-1 $\alpha$ protein levels. Drugs such as Ginsenoside Rg3, Fenofibrate, and Berberine have been associated with anti-tumour and anti-angiogenesis activities by promoting radiosensitivity of human hypoxic EC cell lines [77-79]. By targeting CA IX expression, therapeutic benefit could be improved when combining conventional treatment with cytotoxic agents such as CA IX-directed ligands or antibodies [80].

Another hypoxia-specific treatment strategy is the use of hypoxia-activated prodrugs (HAP) that become activated by enzymatic reduction under hypoxic conditions to release cytotoxic effectors ("warheads"). For example, evosfosfamide (TH-302) is a HAP that upon activation in severely hypoxic regions induces DNA damage but also diffuses to the surrounding, better oxygenated, cells and creating cytotoxic bystander effects. TH-302 has demonstrated enhanced anti-tumour effects in combination with (chemo)radiotherapy, although levels of toxicity were also elevated [48, 81]. In addition, significant clinical benefit has yet to be reported for treatment strategies involving currently-available HAP (i.e., monotherapy or combined with chemoradiotherapy) [82]. We acknowledge the potential therapeutic effect of additional anti-hypoxia treatment, but also the importance to limit unnecessary toxicity by selecting patients who will benefit from these modifications. Extensive clinical testing of TH-302 in combination with CCRT is therefore advised in pre-selected hypoxic patients using for example HX4-PET imaging [71, 81]. 
Although this systematic review adheres to the PRISMA statement, it holds a few limitations [8]. Several clinicopathological factors such as age, clinical stage, lymph node invasion, and location (i.e., proximal or distal EC) were not investigated, but could explain the different expression rate of hypoxia-associated biomarkers in oesophageal cancer. Furthermore, interactions between these factors and treatment differences (e.g., radiation dose, fractions, and chemo regimens) were not described. Beside hypoxia, reactive oxygen species, genetic alterations and inflammation may also be involved in the stimulation of hypoxia-associated molecular responses. Finally, we did not assess the effect of methodological differences across included studies (e.g., IHC staining procedures, antibody supplier, slice thickness, and threshold for hypoxic status). We assumed that reliable, standardized protocols were applied correctly for optimal detection of hypoxia-associated markers. Although presumed to be inconsequential, the possibility of impure comparison emphasizes the need for protocol standardization.

\section{CONCLUSION}

Evaluation of tumour micro-environmental conditions, such as intra-tumoral hypoxia, is important to predict treatment outcome and efficacy. Until now, the predictive value of hypoxia-associated biomarkers in oesophageal cancer is controversially discussed. Although there is increasing clinical evidence that hypoxia-associated responses can be detected, the perfect biomarker for tumour hypoxia in EC has not yet been established. However, PET-based hypoxia imaging shows great potential in evaluating hypoxic tumour status non-invasively. Knowledge of the presence and dynamics of hypoxia in different oesophageal cancer patients (ESCC vs. AC) is important to exploit and validate novel therapeutic strategies directed against tumour hypoxia. The window-of-opportunity trial concept paves the way for optimal hypoxia diagnosis and individualized hypoxiaguided treatment to improve radiotherapy response in EC patients. For personalized cancer medicine, simple, safe, and efficient methods are needed to determine tumour oxygenation in EC and help select patients with hypoxic tumours. Presumably, the combination of multiple, minimally invasive molecular markers is needed to fully evaluate the hypoxic status in cancer patients. 


\section{REFERENCES}

1. M.R. Horsman, L.S. Mortensen, J.B. Petersen, M. Busk, J. Overgaard, Imaging hypoxia to improve radiotherapy outcome, Nat Rev Clin Oncol, 9 (2012) 674-687.

2. J. Overgaard, Hypoxic modification of radiotherapy in squamous cell carcinoma of the head and neck-a systematic review and meta-analysis, Radiother Oncol, 100 (2011) 22-32.

3. W. Ping, W. Sun, Y. Zu, W. Chen, X. Fu, Clinicopathological and prognostic significance of hypoxiainducible factor-1alpha in esophageal squamous cell carcinoma: a meta-analysis, Tumour biology : the journal of the International Society for Oncodevelopmental Biology and Medicine, 35 (2014) 4401-4409.

4. P. van Hagen, M. Hulshof, J. van Lanschot, E.W. Steyerberg, M.I.V. Henegouwen, B.P.L. Wijnhoven, D.J. Richel, G.a.P. Nieuwenhuijzen, C. group, Preoperative chemoradiotherapy for esophageal or junctional cancer, N Engl J Med, 366 (2012) 2074-2084.

5. R.L. Siegel, K.D. Miller, A. Jemal, Cancer statistics, 2016, CA Cancer J Clin, 66 (2016) 7-30.

6. I. Brink, P. Baier, E. Juttner, T. Paulus, M. Narayanan, U. Podbielski, W. Weber, M. Hentschel, Assessment of hypoxia in esophageal carcinomas using 18F-MISO PET, Journal of nuclear medicine : official publication, Society of Nuclear Medicine, 49 (2008).

7. L.H. Gray, A.D. Conger, M. Ebert, S. Hornsey, O.C. Scott, The concentration of oxygen dissolved in tissues at the time of irradiation as a factor in radiotherapy, The British journal of radiology, 26 (1953) 638-648.

8. D. Moher, A. Liberati, J. Tetzlaff, D.G. Altman, Preferred reporting items for systematic reviews and meta-analyses: the PRISMA statement, Journal of clinical epidemiology, 62 (2009) 1006-1012.

9. P. Birner, B. Jesch, J. Friedrich, M. Riegler, J. Zacherl, M. Hejna, F. Wrba, A. Schultheis, S.F. Schoppmann, Carbonic Anhydrase IX Overexpression is Associated with Diminished Prognosis in Esophageal Cancer and Correlates with Her-2 Expression, Ann. Surg. Oncol., 18 (2011) 3330-3337.

10. Y.S. Chen, Y. Lu, C.L. Lu, L. Zhang, Beclin-1 Expression is a Predictor of Clinical Outcome in Patients with Esophageal Squamous Cell Carcinoma and Correlated to Hypoxia-Inducible Factor (HIF)-1 alpha Expression, Pathol. Oncol. Res., 15 (2009) 487-493.

11. I. Chiba, K. Ogawa, T. Morioka, H. Shimoji, N. Sunagawa, S. Iraha, T. Nishimaki, N. Yoshimi, S. Murayama, Clinical significance of GLUT-1 expression in patients with esophageal cancer treated with concurrent chemoradiotherapy, Oncol. Lett., 2 (2011) 21-28.

12. A. Driessen, W. Landuyt, S. Pastorekova, J. Moons, L. Goethals, K. Haustermans, P. Nafteux, F. Penninckx, K. Geboes, T. Lerut, N. Ectors, Expression of carbonic anhydrase IX (CA IX), a hypoxiarelated protein, rather than vascular-endothelial growth factor (VEGF), a pro-angiogenic factor, correlates with an extremely poor prognosis in esophageal and gastric adenocarcinomas, Ann. Surg., 243 (2006) 334-340.

13. G. Jomrich, B. Jesch, P. Birner, K. Schwameis, M. Paireder, R. Asari, S.F. Schoppmann, Stromal expression of carbonic anhydrase IX in esophageal cancer, Clin. Transl. Oncol., 16 (2014) 966-972.

14. M. Katsuta, M. Miyashita, H. Makino, T. Nomura, S. Shinji, K. Yamashita, T. Tajiri, M. Kudo, T. Ishiwata, Z. Naito, Correlation of hypoxia inducible factor-1 alpha with lymphatic metastasis via vascular endothelial growth factor-C in human esophageal cancer, Exp. Mol. Pathol., 78 (2005) 123-130.

15. S. Kimura, Y. Kitadai, S. Tanaka, T. Kuwai, J. Hihara, K. Yoshida, T. Toge, K. Chayama, Expression of hypoxia-inducible factor (HIF)-1 alpha is associated with vascular endothelial growth factor expression and tumour anglogenesis in human oesophageal squamous cell carcinoma, Eur. J. Cancer, 40 (2004) 1904-1912.

16. M.I. Koukourakis, A. Giatromanolaki, J. Skarlatos, L. Corti, S. Blandamura, M. Piazza, K.C. Gatter, A.L. Harris, Hypoxia inducible factor (HIF-1a and HIF-2a) expression in early esophageal cancer and response to photodynamic therapy and radiotherapy, Cancer research, 61 (2001) 1830-1832.

17. T. Kurokawa, M. Miyamoto, K. Kato, Y. Cho, Y. Kawarada, Y. Hida, T. Shinohara, T. Itoh, S. Okushiba, S. Kondo, H. Katoh, Overexpression of hypoxia-inducible-factor 1 alpha(HIF-1 alpha) in oesophageal squamous cell carcinoma correlates with lymph node metastasis and pathologic stage, Br. J. Cancer, 89 (2003) 1042-1047.

18. F.C. Ling, N. Leimbach, S.E. Baldus, S. Buechel, S. Neiss, J. Brabender, U. Drebber, H.P. Dienes, R.P. Mueller, A.H. Hoelscher, P.M. Schneider, HIF-1alpha mRNA is not associated with histopathological regression following neoadjuvant chemoradiation in esophageal cancer, Anticancer research, 26 (2006) 4505-4509.

19. T. Matsuyama, K. Nakanishi, T. Hayashi, Y. Yoshizumi, S. Aiko, Y. Sugiura, T. Tanimoto, M. Uenoyama, 
Y. Ozeki, T. Maehara, Expression of hypoxia-inducible factor-1 alpha in esophageal squamous cell carcinoma, Cancer Sci., 96 (2005) 176-182.

20. P.C. Munipalle, Y.K.S. Viswanath, P.A. Davis, D. Scoones, Prognostic value of hypoxia inducible factor 1 alpha in esophageal squamous cell carcinoma, Dis. Esophagus, 24 (2011) 177-181.

21. N. Ogane, M. Yasuda, M. Shimizu, M. Miyazawa, S. Kamoshida, A. Ueda, K. Takata, Y. Sakuma, Y. Miyagi, Y. Kameda, Clinicopathological implications of expressions of hypoxia-related molecules in esophageal superficial squamous cell carcinoma, Annals of diagnostic pathology, 14 (2010) 23-29.

22. K. Ogawa, I. Chiba, T. Morioka, H. Shimoji, W. Tamaki, R. Takamatsu, T. Nishimaki, N. Yoshimi, S. Murayama, Clinical Significance of HIF-l alpha Expression in Patients with Esophageal Cancer Treated with Concurrent Chemoradiotherapy, Anticancer Res., 31 (2011) 2351-2359.

23. L.M.A. Schreurs, J.K. Smit, K. Pavlov, B.B. Pultrum, J. Pruim, H. Groen, H. Hollema, J.T.M. Plukker, Prognostic Impact of Clinicopathological Features and Expression of Biomarkers Related to F-18-FDG Uptake in Esophageal Cancer, Ann. Surg. Oncol., 21 (2014) 3751-3757.

24. M. Sohda, H. Ishikawa, N. Masuda, H. Kato, T. Miyazaki, M. Nakajima, M. Fukuchi, R. Manda, Y. Fukai, H. Sakurai, H. Kuwano, Pretreatment evaluation of combined HIF-1alpha, p53 and p21 expression is a useful and sensitive indicator of response to radiation and chemotherapy in esophageal cancer, International journal of cancer, 110 (2004) 838-844.

25. H. Takala, J. Saarnio, H. Wiik, P. Ohtonen, Y. Soini, HIF-1 alpha and VEGF are Associated with Disease Progression in Esophageal Carcinoma, J. Surg. Res., 167 (2011) 41-48.

26. N. Tanaka, H. Kato, T. Inose, H. Kimura, A. Faried, M. Sohda, M. Nakajima, Y. Fukai, T. Miyazaki, N. Masuda, M. Fukuchi, H. Kuwano, Expression of carbonic anhydrase 9, a potential intrinsic marker of hypoxia, is associated with poor prognosis in oesophageal squamous cell carcinoma, British journal of cancer, 99 (2008) 1468-1475.

27. C. Tzao, S.C. Lee, H.J. Tung, H.S. Hsu, W.H. Hsu, G.H. Sun, C.P. Yu, J.S. Jin, Y.L. Cheng, Expression of hypoxia-inducible factor (HIF)-1 alpha and vascular endothelial growth factor (VEGF)-D as outcome predictors in resected esophageal squamous cell carcinoma, Dis. Markers, 25 (2008) 141-148.

28. M. Winther, J. Alsner, T. Tramm, M. Nordsmark, Hypoxia-regulated gene expression and prognosis in loco-regional gastroesophageal cancer., Acta oncologica (Stockholm, Sweden), 52 (2013) 1327-1335.

29. J. Yue, Y. Yang, A.R. Cabrera, X. Sun, S. Zhao, P. Xie, J. Zheng, L. Ma, Z. Fu, J. Yu, Measuring tumor hypoxia with (1)(8)F-FETNIM PET in esophageal squamous cell carcinoma: a pilot clinical study, Diseases of the esophagus : official journal of the International Society for Diseases of the Esophagus, 25 (2012) 54-61.

30. L. Zhang, S.B. Ye, Z.L. Li, G. Ma, S.P. Chen, J. He, W.L. Liu, D. Xie, Y.X. Zeng, J. Li, Increased HIF-1alpha expression in tumor cells and lymphocytes of tumor microenvironments predicts unfavorable survival in esophageal squamous cell carcinoma patients, Int. J. Clin. Exp. Pathol., 7 (2014) 3887-3897.

31. G.L. Semenza, HIF-1 and tumor progression: pathophysiology and therapeutics, Trends Mol Med, 8 (2002) S62-67.

32. B. Krishnamachary, M.F. Penet, S. Nimmagadda, Y. Mironchik, V. Raman, M. Solaiyappan, G.L. Semenza, M.G. Pomper, Z.M. Bhujwalla, Hypoxia regulates CD44 and its variant isoforms through HIF1alpha in triple negative breast cancer, PLoS One, 7 (2012) e44078.

33. S.J. van Kuijk, A. Yaromina, R. Houben, R. Niemans, P. Lambin L.J. Dubois, Prognostic significance of carbonic anhydrase IX expression in cancer patients: a meta-analysis, Front Oncol, 6 (2016) 69 .

34. P. Birner, B. Jesch, J. Friedrich, M. Riegler, J. Zacherl, M. Hejna, F. Wrba, A. Schultheis, S.F. Schoppmann, Carbonic anhydrase IX overexpression is associated with diminished prognosis in esophageal cancer and correlates with Her-2 expression, Ann Surg Oncol, 18 (2011) 3330-3337.

35. U.R. Jewell, I. Kvietikova, A. Scheid, C. Bauer, R.H. Wenger, M. Gassmann, Induction of HIF-1alpha in response to hypoxia is instantaneous, FASEB J, 15 (2001) 1312-1314.

36. S.G. Peeters, C.M. Zegers, A. Yaromina, W. Van Elmpt, L. Dubois, P. Lambin, Current preclinical and clinical applications of hypoxia PET imaging using 2-nitroimidazoles, The quarterly journal of nuclear medicine and molecular imaging : official publication of the Italian Association of Nuclear Medicine (AIMN) [and] the International Association of Radiopharmacology (IAR), [and] Section of the So, 59 (2015) 39-57.

37. S.G. Peeters, C.M. Zegers, N.G. Lieuwes, W. van Elmpt, J. Eriksson, G.A. van Dongen, L. Dubois, P. Lambin, A comparative study of the hypoxia PET tracers [(1)(8)F]HX4, [(1)(8)F]FAZA, and [(1)(8)F]FMISO in a preclinical tumor model, Int J Radiat Oncol Biol Phys, 91 (2015) 351-359. 
38. C.M. Zegers, F.J. Hoebers, W. van Elmpt, J.A. Bons, M.C. Ollers, E.G. Troost, D. Eekers, L. Balmaekers, M. Arts-Pechtold, F.M. Mottaghy, P. Lambin, Evaluation of tumour hypoxia during radiotherapy using [18F]HX4 PET imaging and blood biomarkers in patients with head and neck cancer, Eur J Nucl Med Mol Imaging, 43 (2016) 2139-2146.

39. C.M. Zegers, W. van Elmpt, F.J. Hoebers, E.G. Troost, M.C. Ollers, F.M. Mottaghy, P. Lambin, Imaging of tumour hypoxia and metabolism in patients with head and neck squamous cell carcinoma, Acta oncologica (Stockholm, Sweden), 54 (2015) 1378-1384.

40. C.M. Zegers, W. van Elmpt, B. Reymen, A.J. Even, E.G. Troost, M.C. Ollers, F.J. Hoebers, R.M. Houben, J. Eriksson, A.D. Windhorst, F.M. Mottaghy, D. De Ruysscher, P. Lambin, In vivo quantification of hypoxic and metabolic status of NSCLC tumors using [18F]HX4 and [18F]FDG-PET/CT imaging, Clinical cancer research : an official journal of the American Association for Cancer Research, 20 (2014) 6389-6397.

41. C.M. Zegers, W. van Elmpt, K. Szardenings, H. Kolb, A. Waxman, R.M. Subramaniam, D.H. Moon, J.C. Brunetti, S.M. Srinivas, P. Lambin, D. Chien, Repeatability of hypoxia PET imaging using [(1)(8)F]HX4 in lung and head and neck cancer patients: a prospective multicenter trial, Eur J Nucl Med Mol Imaging, 42 (2015) 1840-1849.

42. C.M. Zegers, W. van Elmpt, R. Wierts, B. Reymen, H. Sharifi, M.C. Ollers, F. Hoebers, E.G. Troost, R. Wanders, A. van Baardwijk, B. Brans, J. Eriksson, B. Windhorst, F.M. Mottaghy, D. De Ruysscher, P. Lambin, Hypoxia imaging with [(1)(8)F]HX4 PET in NSCLC patients: defining optimal imaging parameters, Radiother Oncol, 109 (2013) 58-64.

43. I.N. Fleming, R. Manavaki, P.J. Blower, C. West, K.J. Williams, A.L. Harris, J. Domarkas, S. Lord, C. Baldry, F.J. Gilbert, Imaging tumour hypoxia with positron emission tomography, British journal of cancer, 112 (2015) 238-250.

44. E.M. Hammond, M.C. Asselin, D. Forster, J.P. O'Connor, J.M. Senra, K.J. Williams, The meaning, measurement and modification of hypoxia in the laboratory and the clinic, Clinical oncology (Royal College of Radiologists (Great Britain)), 26 (2014) 277-288.

45. R. Klaassen, R.J. Bennink, G. van Tienhoven, M.F. Bijlsma, M.G.H. Besselink, M.I.V. Henegouwen, J.W. Wilmink, A.J. Nederveen, A.D. Windhorst, M. Hulshof, H.W.M. van Laarhoven, Feasibility and repeatability of PET with the hypoxia tracer F-18 HX4 in oesophageal and pancreatic cancer, Radiother. Oncol., 116 (2015) 94-99.

46. E.E. Verwer, F.H. van Velden, I. Bahce, M. Yaqub, R.C. Schuit, A.D. Windhorst, P. Raijmakers, A.A. Lammertsma, E.F. Smit, R. Boellaard, Pharmacokinetic analysis of 18F]FAZA in non-small cell lung cancer patients, Eur J Nucl Med Mol Imaging, 40 (2013) 1523-1531.

47. E.E. Verwer, C.M. Zegers, W. van Elmpt, R. Wierts, A.D. Windhorst, F.M. Mottaghy, P. Lambin, R. Boellaard, Pharmacokinetic modeling of a novel hypoxia PET tracer [18F]HX4 in patients with nonsmall cell lung cancer, EJNMMI physics, 3 (2016) 30.

48. R. Larue, L. Van de Voorde, M. Berbee, W.J.C. van Elmpt, L.J. Dubois, K.M. Panth, S. Peeters, A. Claessens, W.M.J. Schreurs, M. Nap, F. Warmerdam, F.L.G. Erdkamp, M.N. Sosef, P. Lambin, A phase 1 'window-of-opportunity' trial testing evofosfamide (TH-302), a tumour-selective hypoxia-activated cytotoxic prodrug, with preoperative chemoradiotherapy in oesophageal adenocarcinoma patients, BMC Cancer, 16 (2016) 8.

49. L.J. Dubois, N.G. Lieuwes, M.H. Janssen, W.J. Peeters, A.D. Windhorst, J.C. Walsh, H.C. Kolb, M.C. Ollers, J. Bussink, G.A. van Dongen, A. van der Kogel, P. Lambin, Preclinical evaluation and validation of $[18 \mathrm{~F}] \mathrm{HX} 4$, a promising hypoxia marker for PET imaging, Proceedings of the National Academy of Sciences of the United States of America, 108 (2011) 14620-14625.

50. J. van Loon, M.H. Janssen, M. Ollers, H.J. Aerts, L. Dubois, M. Hochstenbag, A.M. Dingemans, R. Lalisang, B. Brans, B. Windhorst, G.A. van Dongen, H. Kolb, J. Zhang, D. De Ruysscher, P. Lambin, PET imaging of hypoxia using [18F]HX4: a phase I trial, Eur J Nucl Med Mol Imaging, 37 (2010) 1663-1668.

51. E.E. Graves, R.J. Hicks, D. Binns, M. Bressel, Q.T. Le, L. Peters, R.J. Young, D. Rischin, Quantitative and qualitative analysis of [(18)F]FDG and [(18)F]FAZA positron emission tomography of head and neck cancers and associations with HPV status and treatment outcome, Eur J Nucl Med Mol Imaging, 43 (2016) 617-625.

52. H. Yuan, T. Schroeder, J.E. Bowsher, L.W. Hedlund, T. Wong, M.W. Dewhirst, Intertumoral differences in hypoxia selectivity of the PET imaging agent 64Cu(II)-diacetyl-bis(N4-methylthiosemicarbazone), Journal of nuclear medicine : official publication, Society of Nuclear Medicine, 47 (2006) 989-998. 
53. K.S. Chao, W.R. Bosch, S. Mutic, J.S. Lewis, F. Dehdashti, M.A. Mintun, J.F. Dempsey, C.A. Perez, J.A. Purdy, M.J. Welch, A novel approach to overcome hypoxic tumor resistance: Cu-ATSM-guided intensity-modulated radiation therapy, Int J Radiat Oncol Biol Phys, 49 (2001) 1171-1182.

54. B.A. Hoeben, J.H. Kaanders, G.M. Franssen, E.G. Troost, P.F. Rijken, E. Oosterwijk, G.A. van Dongen, W.J. Oyen, O.C. Boerman, J. Bussink, PET of hypoxia with 89Zr-labeled cG250-F(ab')2 in head and neck tumors, Journal of nuclear medicine : official publication, Society of Nuclear Medicine, 51 (2010) 10761083.

55. V. Akurathi, L. Dubois, S. Celen, N.G. Lieuwes, S.K. Chitneni, B.J. Cleynhens, A. Innocenti, C.T. Supuran, A.M. Verbruggen, P. Lambin, G.M. Bormans, Development and biological evaluation of (9)(9)mTc-sulfonamide derivatives for in vivo visualization of CA IX as surrogate tumor hypoxia markers, Eur J Med Chem, 71 (2014) 374-384.

56. L. Dubois, N.G. Lieuwes, A. Maresca, A. Thiry, C.T. Supuran, A. Scozzafava, B.G. Wouters, P. Lambin, Imaging of CA IX with fluorescent labelled sulfonamides distinguishes hypoxic and (re)-oxygenated cells in a xenograft tumour model, Radiother Oncol, 92 (2009) 423-428.

57. D. Sneddon, R. Niemans, M. Bauwens, A. Yaromina, S.J. van Kuijk, N.G. Lieuwes, R. Biemans, I. Pooters, P.A. Pellegrini, N.A. Lengkeek, I. Greguric, K.F. Tonissen, C.T. Supuran, P. Lambin, L. Dubois, S.A. Poulsen, Synthesis and in Vivo Biological Evaluation of (68)Ga-Labeled Carbonic Anhydrase IX Targeting Small Molecules for Positron Emission Tomography, Journal of medicinal chemistry, 59 (2016) 6431-6443.

58. M. Matsuo, S. Matsumoto, J.B. Mitchell, M.C. Krishna, K. Camphausen, Magnetic resonance imaging of the tumor microenvironment in radiotherapy: perfusion, hypoxia, and metabolism, Semin Radiat Oncol, 24 (2014) 210-217.

59. C. Halle, E. Andersen, M. Lando, E.K. Aarnes, G. Hasvold, M. Holden, R.G. Syljuasen, K. Sundfor, G.B. Kristensen, R. Holm, E. Malinen, H. Lyng, Hypoxia-induced gene expression in chemoradioresistant cervical cancer revealed by dynamic contrast-enhanced MRI, Cancer research, 72 (2012) 5285-5295.

60. P.J. Hoskin, D.M. Carnell, N.J. Taylor, R.E. Smith, J.J. Stirling, F.M. Daley, M.I. Saunders, S.M. Bentzen, D.J. Collins, J.A. d'Arcy, A.P. Padhani, Hypoxia in prostate cancer: correlation of BOLD-MRI with pimonidazole immunohistochemistry-initial observations, Int J Radiat Oncol Biol Phys, 68 (2007) 10651071.

61. Y. Wang, M. Liu, M.L. Jin, Blood Oxygenation Level-dependent Magnetic Resonance Imaging of Breast Cancer: Correlation with Carbonic Anhydrase IX and Vascular Endothelial Growth Factor, Chin Med J (Engl), 130 (2017) 71-76.

62. A.R. Padhani, K.A. Krohn, J.S. Lewis, M. Alber, Imaging oxygenation of human tumours, European radiology, 17 (2007) 861-872.

63. A. Yaromina, H. Thames, X. Zhou, S. Hering, W. Eicheler, A. Dorfler, T. Leichtner, D. Zips, M. Baumann, Radiobiological hypoxia, histological parameters of tumour microenvironment and local tumour control after fractionated irradiation, Radiother Oncol, 96 (2010) 116-122.

64. D. Zips, S. Boke, T. Kroeber, A. Meinzer, K. Bruchner, H.D. Thames, M. Baumann, A. Yaromina, Prognostic value of radiobiological hypoxia during fractionated irradiation for local tumor control, Strahlentherapie und Onkologie : Organ der Deutschen Rontgengesellschaft ... [et al], 187 (2011) 306310 .

65. U. Simoncic, S. Leibfarth, S. Welz, N. Schwenzer, H. Schmidt, G. Reischl, C. Pfannenberg, C. Fougere, K. Nikolaou, D. Zips, D. Thorwarth, Comparison of DCE-MRI kinetic parameters and FMISO-PET uptake parameters in head and neck cancer patients, Med Phys, (2017).

66. B.F. Jordan, J. Magat, F. Colliez, E. Ozel, A.C. Fruytier, V. Marchand, L. Mignion, C. Bouzin, P.D. Cani, C. Vandeputte, O. Feron, N. Delzenne, U. Himmelreich, V. Denolin, T. Duprez, B. Gallez, Mapping of oxygen by imaging lipids relaxation enhancement: a potential sensitive endogenous MRI contrast to map variations in tissue oxygenation, Magn Reson Med, 70 (2013) 732-744.

67. F. Colliez, M.A. Neveu, J. Magat, T.T. Cao Pham, B. Gallez, B.F. Jordan, Qualification of a noninvasive magnetic resonance imaging biomarker to assess tumor oxygenation, Clinical cancer research : an official journal of the American Association for Cancer Research, 20 (2014) 5403-5411.

68. J.P. O'Connor, J.H. Naish, G.J. Parker, J.C. Waterton, Y. Watson, G.C. Jayson, G.A. Buonaccorsi, S. Cheung, D.L. Buckley, D.M. McGrath, C.M. West, S.E. Davidson, C. Roberts, S.J. Mills, C.L. Mitchell, L. Hope, N.C. Ton, A. Jackson, Preliminary study of oxygen-enhanced longitudinal relaxation in MRI: a potential novel biomarker of oxygenation changes in solid tumors, Int J Radiat Oncol Biol Phys, 75 (2009) 
1209-1215.

69. J.P. O'Connor, J.K. Boult, Y. Jamin, M. Babur, K.G. Finegan, K.J. Williams, R.A. Little, A. Jackson, G.J. Parker, A.R. Reynolds, J.C. Waterton, S.P. Robinson, Oxygen-Enhanced MRI Accurately Identifies, Quantifies, and Maps Tumor Hypoxia in Preclinical Cancer Models, Cancer research, 76 (2016) 787-795.

70. W. van Elmpt, C.M. Zegers, B. Reymen, A.J. Even, A.M. Dingemans, M. Oellers, J.E. Wildberger, F.M. Mottaghy, M. Das, E.G. Troost, P. Lambin, Multiparametric imaging of patient and tumour heterogeneity in non-small-cell lung cancer: quantification of tumour hypoxia, metabolism and perfusion, Eur J Nucl Med Mol Imaging, 43 (2016) 240-248.

71. L.J. Dubois, R. Niemans, S.J. van Kuijk, K.M. Panth, N.K. Parvathaneni, S.G. Peeters, C.M. Zegers, N.H. Rekers, M.W. van Gisbergen, R. Biemans, N.G. Lieuwes, L. Spiegelberg, A. Yaromina, J.Y. Winum, M. Vooijs, P. Lambin, New ways to image and target tumour hypoxia and its molecular responses, Radiother Oncol, 116 (2015) 352-357.

72. A.J. Even, J. van der Stoep, C.M. Zegers, B. Reymen, E.G. Troost, P. Lambin, W. van Elmpt, PET-based dose painting in non-small cell lung cancer: Comparing uniform dose escalation with boosting hypoxic and metabolically active sub-volumes, Radiother Oncol, 116 (2015) 281-286.

73. Saskia E. Rademakers, Ilse J. Hoogsteen, Paul F. Rijken, Egbert Oosterwijk, Chris H. Terhaard, Patricia A. Doornaert, Johannes A. Langendijk, Piet van den Ende, Robert Takes, Remco De Bree, Albert J. van der Kogel, Johan Bussink, J.H. Kaanders, Pattern of CAIX expression is prognostic for outcome and predicts response to ARCON in patients with laryngeal cancer treated in a phase III randomized trial, Radiat Oncol J, 108 (2013) 517-522.

74. A. Yaromina, M. Granzier, R. Biemans, N.G. Lieuwes, W. Van Elmpt, G. Shakirin, L. Dubois, P. Lambin, A novel concept for tumour targeting with radiation: Inverse dose-painting or targeting the "Low Drug Uptake Volume", Radiother. Oncol., (in press) (2017).

75. J.L. Tatum, G.J. Kelloff, R.J. Gillies, J.M. Arbeit, J.M. Brown, K.S. Chao, J.D. Chapman, W.C. Eckelman, A.W. Fyles, A.J. Giaccia, R.P. Hill, C.J. Koch, M.C. Krishna, K.A. Krohn, J.S. Lewis, R.P. Mason, G. Melillo, A.R. Padhani, G. Powis, J.G. Rajendran, R. Reba, S.P. Robinson, G.L. Semenza, H.M. Swartz, P. Vaupel, D. Yang, B. Croft, J. Hoffman, G. Liu, H. Stone, D. Sullivan, Hypoxia: importance in tumor biology, noninvasive measurement by imaging, and value of its measurement in the management of cancer therapy, Int J Radiat Biol, 82 (2006) 699-757.

76. Y. Feng, H. Zhu, T. Ling, B. Hao, G. Zhang, R. Shi, Effects of YC-1 targeting hypoxia-inducible factor 1 alpha in oesophageal squamous carcinoma cell line Eca109 cells, Cell biology international, 35 (2011) 491-497.

77. X.L. Ge, F.X. Zhen, B.X. Yang, X. Yang, J. Cai, C. Zhang, S. Zhang, Y.D. Cao, J.X. Ma, H.Y. Cheng, X.C. Sun, Ginsenoside Rg3 enhances radiosensitization of hypoxic oesophageal cancer cell lines through vascular endothelial growth factor and hypoxia inducible factor 1 alpha, J. Int. Med. Res., 42 (2014) 628640 .

78. Y.Y. Ge, J. Liu, X. Yang, H.C. Zhu, B.X. Yang, K.L. Zhao, Z.J. Wu, G.J. Cheng, F. Wang, F. Ni, Q. Ge, Y.G. Yang, G.M. Tai, X.C. Sun, J. Cai, Fenofibrate enhances radiosensitivity of esophageal squamous cell carcinoma by suppressing hypoxia-inducible factor-1 alpha expression, Tumor Biol., 35 (2014) 1076510771.

79. X. Yang, B.X. Yang, J. Cai, C. Zhang, Q. Zhang, L.P. Xu, Q. Qin, H.C. Zhu, J.X. Ma, G.Z. Tao, H.Y. Cheng, X.C. Sun, Berberine enhances radiosensitivity of esophageal squamous cancer by targeting HIF-1 alpha in vitro and in vivo, Cancer Biol. Ther., 14 (2013) 1068-1073.

80. P.C. McDonald, S. Dedhar, Carbonic anhydrase IX (CAIX) as a mediator of hypoxia-induced stress response in cancer cells, Sub-cellular biochemistry, 75 (2014) 255-269.

81. S.G. Peeters, C.M. Zegers, R. Biemans, N.G. Lieuwes, R.G. van Stiphout, A. Yaromina, J.D. Sun, C.P. Hart, A.D. Windhorst, W. van Elmpt, L.J. Dubois, P. Lambin, TH-302 in Combination with Radiotherapy Enhances the Therapeutic Outcome and Is Associated with Pretreatment [18F]HX4 Hypoxia PET Imaging, Clinical cancer research : an official journal of the American Association for Cancer Research, 21 (2015) 2984-2992.

82. N. Baran, M. Konopleva, Molecular Pathways: Hypoxia-Activated Prodrugs in Cancer Therapy, Clinical cancer research : an official journal of the American Association for Cancer Research, (2017).

83. D. Di Perri, J.A. Lee, A. Bol, F.X. Hanin, G. Janssens, D. Labar, A. Robert, E. Sterpin, X. Geets, Evolution of [18F]fluorodeoxyglucose and [18F]fluoroazomycin arabinoside PET uptake distributions in lung tumours during radiation therapy, Acta oncologica (Stockholm, Sweden), 56 (2017) 516-524. 


\section{SUPPLEMENTARY MATERIAL}

\section{S1. Systematic search protocol}

\section{Research question:}

HOW DOES HYPOXIA AFFECT TREATMENT EFFICACY AND OUTCOME IN PATIENTS WITH ESOPHAGEAL CANCER?

\section{Search strategy:}

Web of Science: 182 hits

TOPIC: (hypoxia) OR TOPIC: (hypox*) AND TOPIC: TS=(cancer) OR $\mathrm{TS}=$ (tumour) $\mathrm{OR}$ TS=(tumor) $\mathrm{OR}$ TS=(carcinoma) $\mathrm{OR} T S=($ neoplasm $)$ OR TS=(oncology) OR TS=(lymph node) AND TOPIC: (esophag*) OR (oesophag*) $O R$ (esophageal cancer) $O R$ (oesophageal cancer) $O R$ (esophageal carcinoma) $O R$ (oesophageal carcinoma) $O R$ (esophageal tumor) $O R$ (oesophageal tumour) AND TOPIC: TS $=$ (radioresistance) OR TS=(prognosis) OR TS=(treatment outcome) $\mathrm{OR}$ $\mathrm{TS}=($ tumour aggressiveness) $\mathrm{OR}$ TS=(tumor spread) $\mathrm{OR}$ TS=(malignant progression) OR TS $=($ metastasis $)$ OR TS $=($ clinical outcome $)$ OR TS $=($ response prediction) $\mathrm{OR} T S=$ (pathological free survival) $\mathrm{OR} T S=$ (non-responders) $\mathrm{OR}$ $\mathrm{TS}=($ pathological response) $\mathrm{OR} \mathrm{TS}=($ treatment resistance) $\mathrm{OR} \mathrm{TS}=($ therapy resistance) $\mathrm{OR} T S=($ treatment efficacy)

\section{MEDLINE (MeSH): 22 hits}

Search $(((((($ hypoxia[MeSH Terms]) AND $((($ (cancer[MeSH Terms]) OR oncology[MeSH Terms]) OR tumour[MeSH Terms]) OR tumor[MeSH Terms]))

AND ((()((radiation therapy[MeSH Terms]) OR treatment outcome[MeSH Terms]) OR prognosis[MeSH Terms]) OR adjuvant chemotherapy[MeSH Terms]) OR immunotherapy[MeSH Terms]) OR treatment resistant[MeSH Terms])) AND (((()sophageal cancer[MeSH Terms]) OR oesophag*[MeSH Terms]) OR esophag*[MeSH Terms]) OR lymph node[MeSH Terms]) or mediastin* [MeSH Terms]))))

PubMed (Free text): 215 hits

(((((((hypoxia) OR hypox*) OR hypoxia-induced factor)) AND (((()(((((esophageal cancer) OR esophag*) OR oesophageal cancer) OR mediastinum) OR lymphadenopathy) OR oesophageal carcinoma) OR esophageal tumor) OR oesophageal tumour) OR esophageal carcinoma) OR lymph node) AND $(((()(()(()(($ radioresistance $)$ OR prognosis) OR treatment outcome) OR tumour aggressiveness) OR tumor spread) OR malignant progression) OR metastasis) OR clinical outcome) OR response prediction) OR pathological free survival) OR non-responders) OR pathological response) OR treatment resistance) OR therapy resistance) OR treatment efficacy))

\section{Inclusion criteria:}

\section{P= PARTICIPANTS OR PATIENTS}

Species: human

Age: adults (mimimal age $>18 \mathrm{yr}$.)

Sex: no restriction

Condition: esophageal cancer 
Specimen: patients or biopsy-acquired tissue samples or surgical tissue samples Tumour type: Squamous cell carcinoma (SCC) and adenocarcinoma (AC)

Stage: no restriction

\section{I= INDEX TEST}

All clinical tests able to measure hypoxia-related markers

\section{$\mathrm{C}=$ COMPARATIVE TEST}

not relevant

\section{$\mathrm{O}=$ OUTCOME}

Overall survival (OS), Disease-free survival (DSF).

\section{$S=S T U D Y$ DESIGN}

Study design: original diagnostic experiments

Study type: full-text content available, no studies aiming to elucidate molecular mechanisms of hypoxia

Language: English

Publication year: no restriction

\section{Exclusion criteria:}

\section{Based on title \& abstract}

\section{Based on full text}

1.Irrelevant study, not meeting PICOS-characteristics

2. preclinical studies using animal-based tumour models or esophageal cell lines

3.Reviews, letters to the editor, comments, supplements, conference abstracts, reports, essays, symposiums, guidelines

4. Overlapping data-sets

5.Survival analyses were not presented in publication

6. Presented survival analyses did not allow correlation between expression rate of hypoxia-associated markers and treatment outcome analyses

\section{$\underline{\text { Data extraction }}$}

Elements that were extracted comprised of:

Patient characteristics: number of subjects included in the study, country of origin, mean age of the patient population, tumour cell type, specimen type

Index test characteristics: method of quantifying hypoxia, marker type, definition of hypoxia, percentage of hypoxic elements, selected treatment strategy.

Outcome parameters: statistical analysis (uni- or multivariate), overall survival, disease free survival, complete response, local control, p-values indicating statistical difference in treatment outcome between high and low percentages of hypoxia-associated markers.

Study characteristics: First author, publication year

Finally, each article was given a unique identification number 


\section{Chapter}

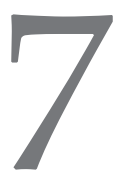

\section{${ }^{18} \mathrm{~F}-\mathrm{HX} 4-\mathrm{PET} / \mathrm{MRI}$ in oesophageal cancer: assessing tumour hypoxia and early treatment response}

Authors:

J. Peerlings, R. T.H.M. Larue, L. Paulis, M. Berbee, J. E. Wildberger, F. M. Mottaghy, P. Lambin

Adapted from:

Combining ${ }^{18} \mathrm{~F}-\mathrm{HX} 4$ tracer and PET/MR imaging for quantifying tumour hypoxia in oesophageal cancer

Clinical Nuclear Medicine - Interesting Images

Submitted 


\section{ABSTRACT}

Chronic hypoxia is associated with poor prognosis in cancer patients since these tumours present a higher likelihood of metastatic progression and resistance to (chemo)radiotherapy. Assessment of hypoxic status could therefore be important for developing personalized treatment strategies to improve therapeutic effectiveness. Potentially, multiparametric ${ }^{18} \mathrm{~F}-\mathrm{HX} 4-\mathrm{PET} / \mathrm{MRI}$ can quantify intratumour heterogeneity and monitor anatomical and cellular changes by combining hypoxia-specific PET-imaging using ${ }^{18} \mathrm{~F}$-flortanidazole $\left({ }^{18} \mathrm{~F}-\mathrm{HX} 4,444 \mathrm{MBq}\right)$ with anatomical MRI (ECG-triggered T1-VIBE and T2-TSE) and background-suppressed diffusion-weighted imaging (DWI, b-value=50, 400, $800 \mathrm{~s} / \mathrm{mm}^{2}$ ).

A patient with oesophageal cancer (male, 56yr) underwent ${ }^{18} \mathrm{~F}-\mathrm{HX} 4-\mathrm{PET} / \mathrm{MR}$ imaging before and mid-chemoradiotherapy. On T1-VIBE and T2-TSE, thickening of oesophageal wall layers indicate an oesophageal tumour near the gastro-oesophageal junction. Partial treatment response was indicated by mid-treatment increase of mean ADC-values and presence of treatment-resistant hypoxic fractions (i.e., tumour-to-blood ratio $>1.4$ ). Post-treatment ${ }^{18} \mathrm{~F}-\mathrm{FDG}-\mathrm{PET} / \mathrm{CT}$ confirmed partial treatment response showing decrease in tumour volume and maximal $\mathrm{SUV}_{\max }\left({ }^{18} \mathrm{~F}-\mathrm{FDG}\right)$ (i.e., 9.6 to $\left.4.3,240 \mathrm{MBq}\right)$. After radical resection, histopathology showed ypT2N1-status. No cancer recurrence was present 1-year post-treatment. In literature, a correlation of SUV $\left({ }^{18} \mathrm{~F}-\mathrm{FDG}\right)$ and ADC has been reported in non-small cell lung cancer, suggesting that ${ }^{18} \mathrm{~F}-\mathrm{HX} 4 \mathrm{might}$ substitute ${ }^{18} \mathrm{~F}-\mathrm{FDG}$ for acquiring synergic data on tumour hypoxia, cellularity, and morphology.

Multimodal and multiparametric ${ }^{18} \mathrm{~F}-\mathrm{HX} 4$-PET/MRI is able to visualize oesophageal cancer with high soft-tissue contrast and detect early treatment response prior to anatomical changes. Consequently, ${ }^{18} \mathrm{~F}-\mathrm{HX} 4-\mathrm{PET} / \mathrm{MRI}$ could pave the way for patient stratification towards individualized hypoxia-adjusted treatment. However, patient-specific treatment strategies are still in an exploratory phase and require further investigation to improve patient outcome. 


\section{INTRODUCTION}

Cancer is a complex disease of heterogenic tumours and micro-environment. Tumour hypoxia is considered as one of the hallmarks of cancers and occurs when there is an imbalance between oxygen supply and consumption, creating oxygendepleted regions. Oxygen supply can be compromised by limited blood-perfusion (e.g., vascular changes, anaemia, or malfunctioning haemoglobin) and could lead to acute hypoxia. In normal tissue, acute hypoxia can be resolved by physiological homeostasis [1]. In addition, tissues can be oxygenated by diffusing $\mathrm{O}_{2}$-molecules according to an intravascular oxygen partial pressure $\left(\mathrm{pO}_{2}\right)$. In cancerous tissue, however, the rapid and uncontrolled cell division causes tumours to outgrow their blood supply and diffusion capabilities of oxygen-molecules (i.e., 100-180 $\mu \mathrm{m}$ ) [2]. To sustain remote regions with oxygen and nutrients, angiogenesis is triggered. However, the newly formed microvasculature in tumours is chaotic and present inefficient, leaky blood vessels (Fig. 7.1). Hence, hypoxic regions could manifest chronically in solid tumours.

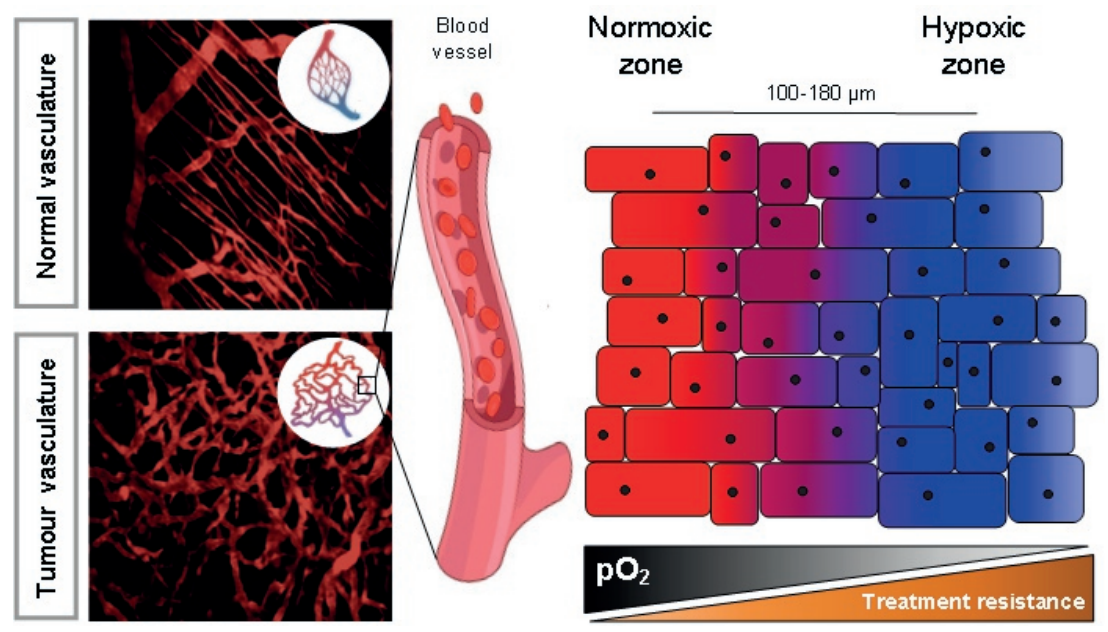

Figure 7.1: the unorganized tumour vasculature is ineffective in oxygenating remote cells. The decreasing pO2 gradient is limited to $100-180 \mu \mathrm{m}$, creating chronically hypoxic tumour regions

The presence of hypoxic regions has a major impact on successful curative treatment as effective radiation treatment requires oxygen to induce irreversible DNAdamage. Consequently, hypoxic cells have a higher likelihood of metastatic progression and express a general resistance to (chemo)radiotherapy, leading to poor patient prognosis [3,4]. Assessment of hypoxic status could therefore be important for developing personalized treatment strategies to improve therapeutic effectiveness and identifying those patients who would benefit the most from hypoxia-adjusted therapy. 
The goal of this study is therefore to assess the potential of multiparametric ${ }^{18} \mathrm{~F}-\mathrm{HX} 4-\mathrm{PET} / \mathrm{MRI}$ in quantifying intra-tumour heterogeneity in patients with oesophageal cancer (EC) by combining hypoxia-specific PET-imaging using ${ }^{18} \mathrm{~F}$-flortanidazole ( $\left.{ }^{18} \mathrm{~F}-\mathrm{HX} 4\right)$ with MRI. In addition, this study aims to assess the effectiveness of chemo-radiotherapy by monitoring changes in hypoxic fraction, tumour volume, and ADC values.

\section{MATERIAL \& METHODS}

\section{STUDY DESIGN}

A patient (male, $56 \mathrm{yr}$ ) with distal EC and possible metastatic lymph nodes was included after informed consent. Standard chemoradiotherapy included neoadjuvant treatment with weekly carboplatin $(2 \mathrm{mg} / \mathrm{ml} / \mathrm{min}$ AUC) and paclitaxel $\left(50 \mathrm{mg} / \mathrm{m}^{2}\right)$ for 5 weeks, concurrent radiotherapy (41.4 Gy in 23 fractions, 5 days per week), followed by surgery (i.e., CROSS regimen).

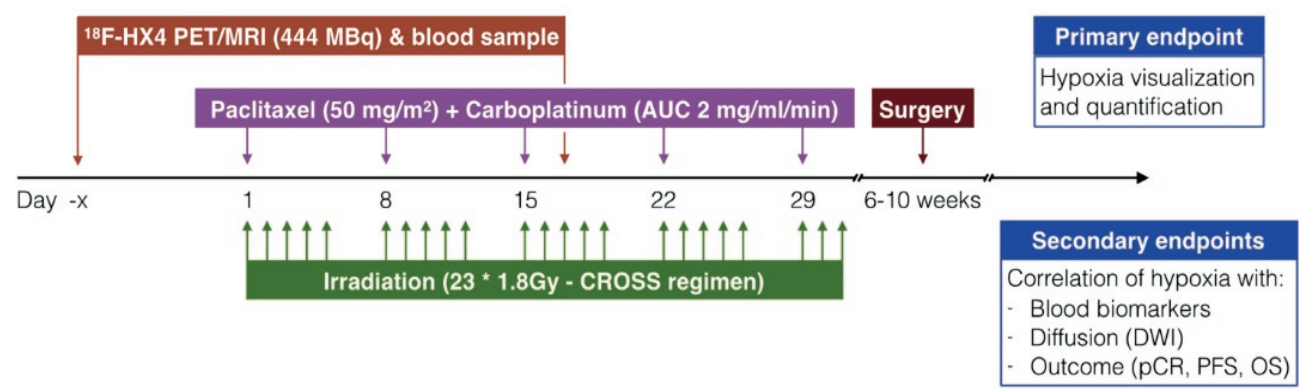

Figure 7.2: overview of study timeline including treatment interventions, imaging, and study endpoints

\section{IMAGING ACQUISITION}

As part of standard patient care, conventional ${ }^{18} \mathrm{~F}-\mathrm{FDG}-\mathrm{PET} / \mathrm{CT}$ (Gemini TF 64, Philips Healthcare, NL) using ${ }^{18} \mathrm{~F}-$ Fluorodeoxyglucose $\left({ }^{18} \mathrm{~F}-\mathrm{FDG}\right)$ was performed before and after treatment at an injected dose of $239 \mathrm{MBq}$ and $245 \mathrm{MBq}$, respectively.

To visualize tumour hypoxia before and mid-chemoradiotherapy, the patient was injected with $444 \mathrm{MBq}$ of ${ }^{18} \mathrm{~F}-\mathrm{HX} 4$ and underwent ${ }^{18} \mathrm{~F}-\mathrm{HX} 4-\mathrm{PET} / \mathrm{MR}$ imaging 4 hours post-injection on a 3-Tesla Magnetom Biograph mMR scanner using two body coils. PET-images were acquired in list-mode for $20 \mathrm{~min}$ at two bed-position and reconstructed using a 3D-iterative ordinary Poisson ordered-subsets expectation maximization (OP-OSEM3D) reconstruction algorithm (3 iterations, 21 subsets, $4 \mathrm{~mm}$ Gaussian smoothing) and fused with MR-images using Syngo.via software (Siemens Healthcare, GER).

Based on the recommendations made in Chapter 3, ${ }^{18} \mathrm{~F}-\mathrm{HX} 4-\mathrm{PET}$ images were acquired over two bed positions (i.e., thorax and upper abdomen) and the PET/MR sequence protocol included ECG-triggered transversal volume interpolated 
breath-hold examination (T1-VIBE) (TR $3.64 \mathrm{~ms}$, TE $1.23 \mathrm{~ms}$, resolution 1.4x1.0x4 mm, acq. matrix 189x384, FOV 262x400, BW $592 \mathrm{~Hz} / \mathrm{px}$, flip 9, GRAPPA 2, TA 0:34). Furthermore, ECG-triggered dark-blood turbo spin-echo (T2-TSE) (TR $750 \mathrm{~ms}$, TE 71

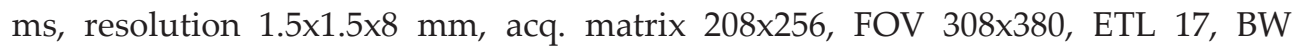
$781 \mathrm{~Hz} / \mathrm{px}$, flip $180^{\circ}$, GRAPPA 2, TA 3:24) and background-suppressed diffusionweighted images (DWI) with b-values of 50, 400, and $800 \mathrm{~s} / \mathrm{mm}^{2}$ (ss-EPI, TR 9200, TE 81, NSA 4, resolution 2.6x2.0x5 mm, acq. matrix 108x192, FOV 284x379, BW 1628 Hz/px, EPI 108, flip 90 ${ }^{\circ}$, GRAPPA 2, TA 5:59) were acquired. T1-VIBE and T2-TSE were acquired over 6 and 8 short breath-hold sessions, respectively.

\section{IMAGE ANALYSES}

Hypoxic fractions were determined by maximum tumour-to-blood ratio (TBR) with a threshold set at $>1.4$, based on previous publications [5,6]. Mean and maximum standardized uptake value (SUV) and apparent diffusion coefficient (ADC) were measured over the gross tumour volume. In addition, SUVMax and SUVMean were measured over the aortic blood pool. SUV was corrected for injected dose and patient weight. ADC maps were constructed by mono-exponential linear fitting of diffusion data. All PET/MR image analyses were performed using OsiriX software (Pixmeo SARL, SUI).

\section{RESULTS}

\section{HYPOXIA IMAGING}

In figure 7.3, ${ }^{18} \mathrm{~F}-\mathrm{HX} 4-\mathrm{PET}$ images indicate hypoxic tumour regions before and mid-treatment. Tumour SUVMean $\left({ }^{18} \mathrm{~F}-\mathrm{HX} 4\right)$ was $0.79 \pm 0.16$ and blood pool $\mathrm{SUV}_{\text {Mean }}\left({ }^{18} \mathrm{~F}-\mathrm{HX} 4\right)$ was $0.48 \pm 0.10$ before treatment and decreased to $0.70 \pm 0.17$ and $0.46 \pm 0.12$ mid-treatment, respectively. Consequently, TBR before treatment was 1.65 (range 1.50-2.35), which decreased to 1.54 (range 1.45-1.72) mid-treatment. On T1-VIBE (Fig. 7.4) and T2-TSE images (Fig. 7.5), thickening of oesophageal wall layers indicate an oesophageal tumour near the gastro-oesophageal junction.
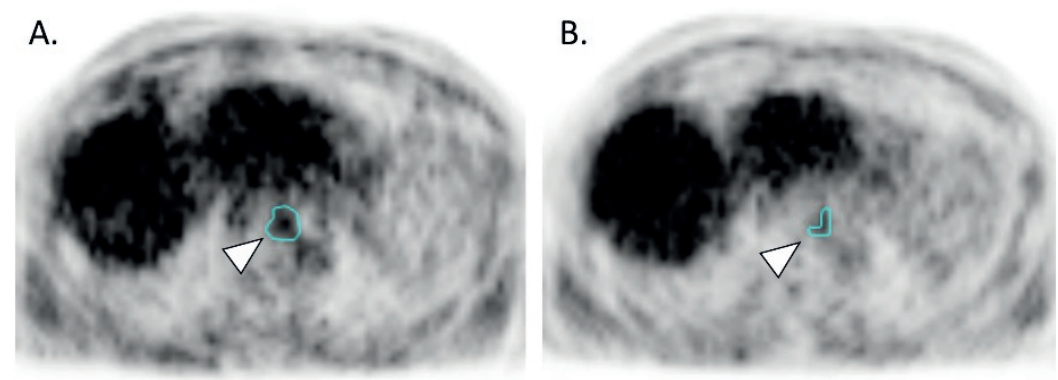

Figure 7.3: ${ }^{18} \mathrm{~F}-\mathrm{HX} 4-\mathrm{PET}$ images of a patient with oesophageal cancer acquired before treatment (A) and mid-treatment (B). Hypoxic fractions are delineated and indicated by the white triangle 

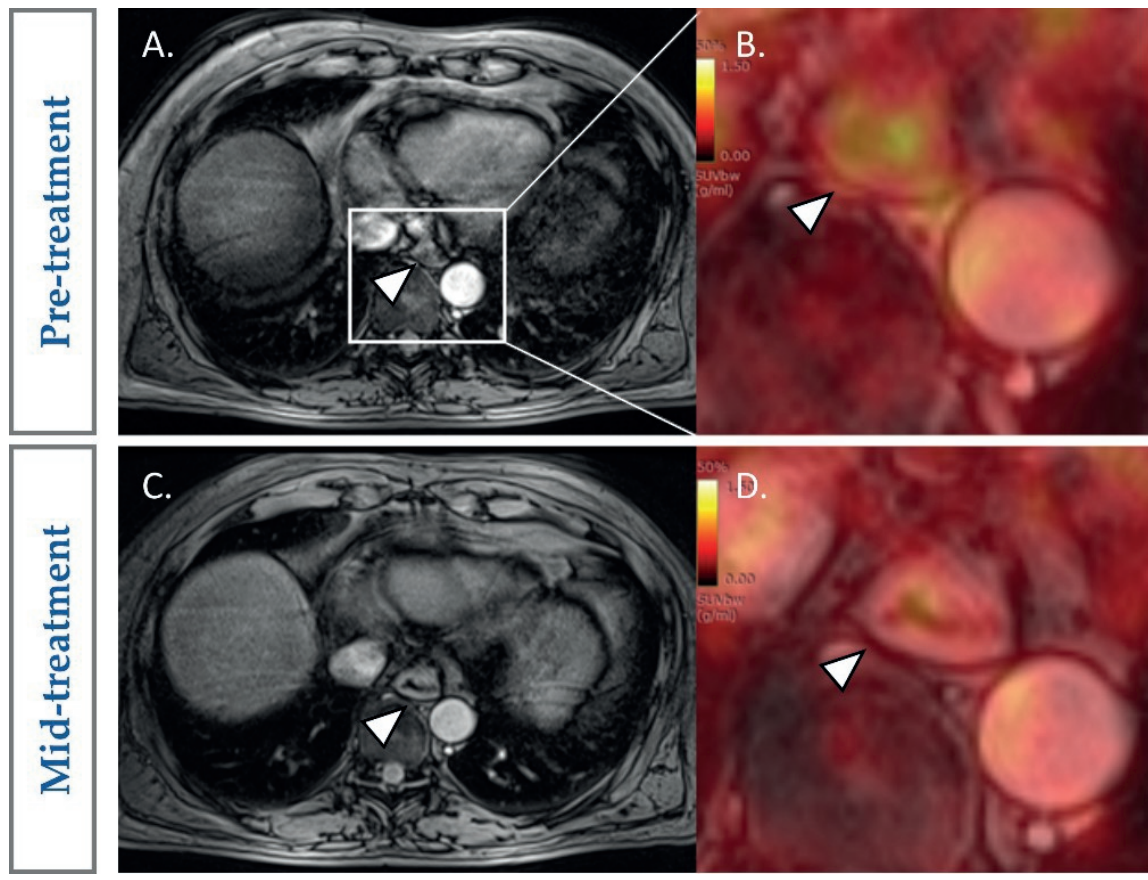

Figure 7.4: ECG-triggered T1-VIBE performed before (A) and mid-treatment (C), fused with ${ }^{18} \mathrm{~F}-\mathrm{HX} 4-\mathrm{PET}(\mathrm{B}, \mathrm{D})$. The white triangles indicate oesophageal carcinoma and hypoxic regions
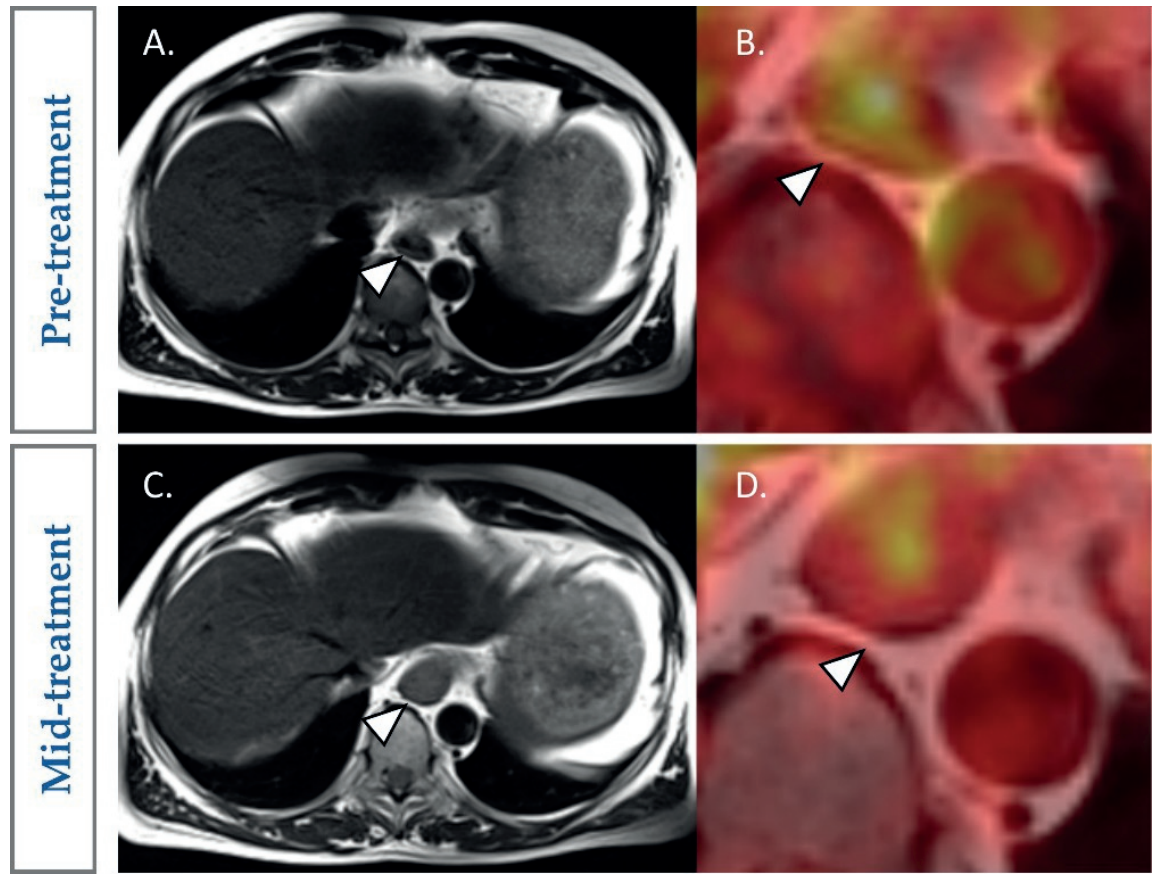

Figure 7.5: ECG-triggered T2-TSE performed before (A) and mid-treatment (C), fused with ${ }^{18} \mathrm{~F}-\mathrm{HX} 4-\mathrm{PET}(\mathrm{B}, \mathrm{D})$. The white triangles indicate oesophageal carcinoma and hypoxic regions 


\section{TREATMENT RESPONSE}

Partial treatment response was indicated by residual tumour hypoxia and midtreatment increase of mean ADC-values from $1287 \pm 574 \times 10^{-6} \mathrm{~mm}^{2} / \mathrm{s}$ to $1488 \pm 455$ $\times 10^{-6} \mathrm{~mm}^{2} / \mathrm{s}$ (Fig. 7.6). Post-treatment ${ }^{18} \mathrm{~F}-\mathrm{FDG}-\mathrm{PET} / \mathrm{CT}$ confirmed partial treatment response showing decrease in tumour volume and $S U V_{\max }\left({ }^{18} \mathrm{~F}-\mathrm{FDG}\right)$ from 9.6 to 4.3 . Mediastinal, hilar, parasternal, and axillary lymph nodes were not enlarged or ${ }^{18} \mathrm{~F}-\mathrm{FDG}$-avid. However, locoregional nodal metastases were detected around the celiac trunk. After radical resection, histopathology revealed ypT2N1-status. No cancer recurrence was present 1-year post-treatment.

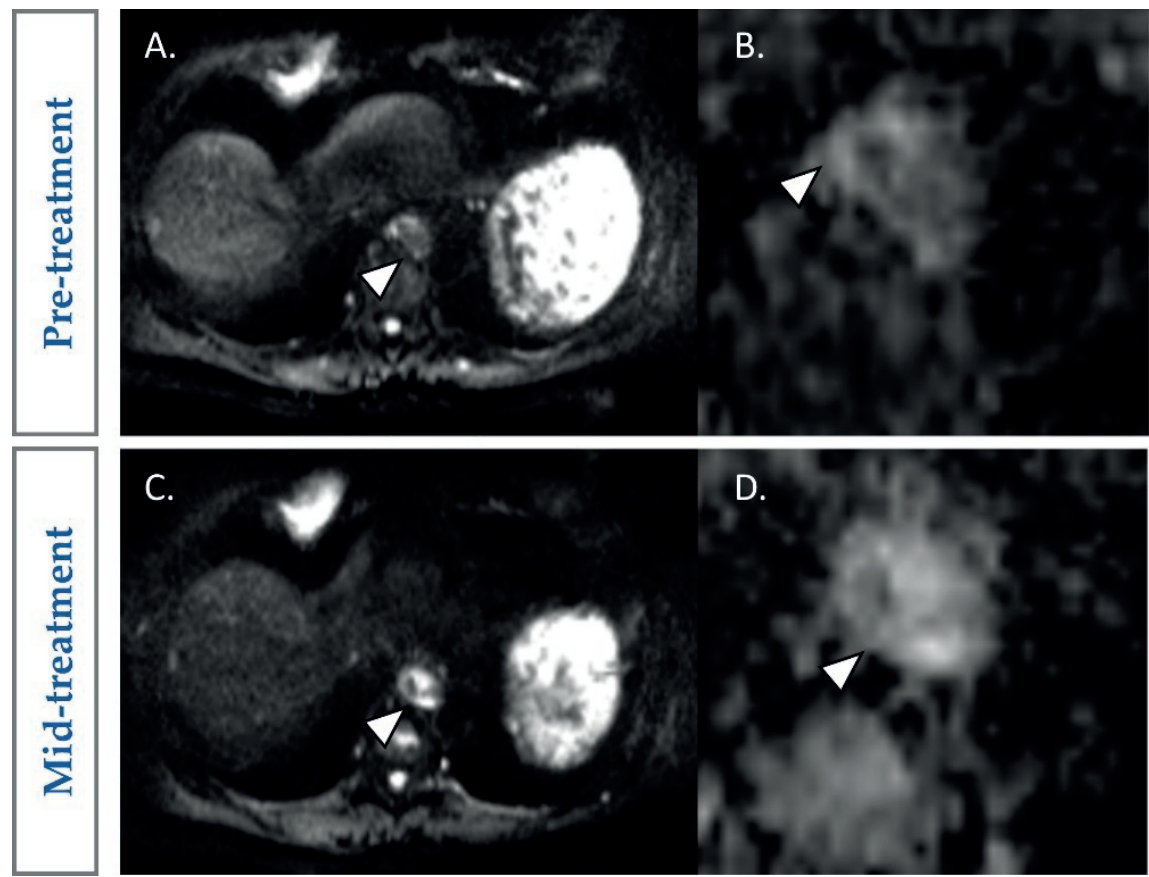

Figure 7.6: DWI (b-value=50, 400, $800 \mathrm{~s} / \mathrm{mm}^{2}$ ) and corresponding ADC map acquired before $(\mathrm{A}, \mathrm{B})$ and mid-treatment (C,D). The white triangles indicate EC and hyper-cellularity
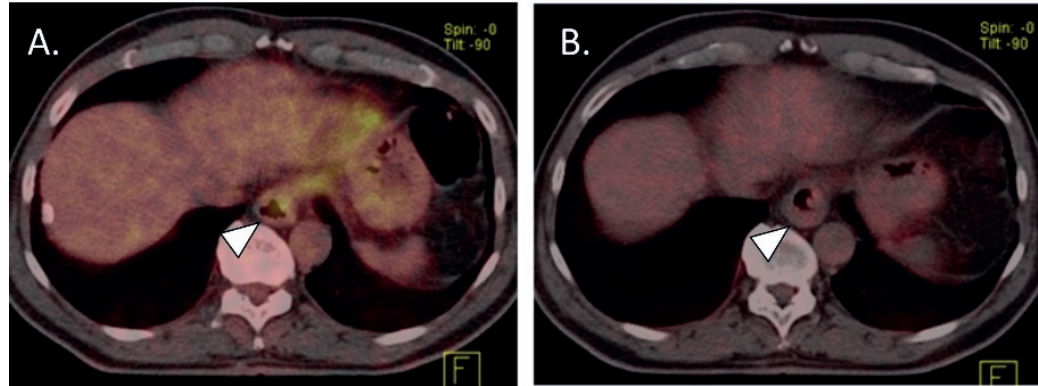

Figure 7.7: ${ }^{18} \mathrm{~F}-\mathrm{FDG}-\mathrm{PET} / \mathrm{CT}$ images of a patient with EC acquired before treatment (A) and after treatment (B). The white triangles indicate EC and metabolically active tumour cells 


\section{DISCUSSION}

By combining highly sensitive ${ }^{18} \mathrm{~F}-\mathrm{HX} 4-\mathrm{PET}$ imaging with anatomical MRI, ${ }^{18} \mathrm{~F}-\mathrm{HX} 4-\mathrm{PET} / \mathrm{MRI}$ is capable to quantify intra-tumour hypoxia and visualize oesophageal cancer with high soft-tissue contrast. Clinically, ${ }^{18} \mathrm{~F}-\mathrm{HX} 4-\mathrm{PET} / \mathrm{MRI}$ can identify patients that could benefit the most from hypoxia-adjusted therapy, define biological target volumes, and personalize treatment strategies $[7,8]$. Moreover, by incorporating DWI into the PET/MR protocol, early clinical response during (chemo)radiation treatment could be evaluated by monitoring changes in tumour volume, hypoxic fraction, and ADC-values.

Partial treatment response was indicated by mid-treatment increase of mean ADC-values and presence of treatment-resistant hypoxic fractions $(T B R>1.4)$. Since functional changes could be detected before anatomical changes manifested, ${ }^{18} \mathrm{~F}-\mathrm{HX} 4-\mathrm{PET} / \mathrm{MRI}$ could offer complementary information for early detection of patient-specific treatment response. Subsequently, treatment strategies can be adjusted to improve therapeutic effectiveness on a case-by-case basis [9-11]. However, hypoxia-adjusted therapy is still in an exploratory phase and requires further investigation to improve patient outcome.

In oncology, qualitative analysis of DWI is used for detecting and characterizing hyper-cellular tumours, cystic and necrotic regions, while quantitative ADC-maps is used for differentiating benign and malignant lesions, and detecting treatment response. Within the first 24 hours after initiating treatment, inflammation and cell swelling could occur and ADC values decrease. However, after this initial phase, treatment-induced cell death will be induced, creating an increase in water mobility and increase in ADC values [12,13]. To assess treatment effect, timing of DWI thus needs to be chosen carefully. It would therefore be more interesting to utilize ADCmaps for predicting treatment response. Generally, tumours with necrotic regions present high baseline ADC values and are more resistant to treatment [13].

In literature, a correlation of SUV $\left({ }^{18} \mathrm{~F}-\mathrm{FDG}\right)$ and $\mathrm{ADC}$ has been reported in non-small cell lung cancer [14,15], suggesting that ${ }^{18} \mathrm{~F}-\mathrm{HX} 4$ might substitute ${ }^{18} \mathrm{~F}-\mathrm{FDG}$ in a dedicated scan protocol to acquire synergic information on tumour hypoxia, cellularity, and anatomical structure. To build on this future perspective, aiming to define key-applications of PET/MR imaging, PET-tracers beyond ${ }^{18}$ F-FDG combined with functional MR imaging could lead to more insight in tumour heterogeneity. However, further research is needed to define the clinical value of ${ }^{18} \mathrm{~F}-\mathrm{HX} 4-\mathrm{PET} / \mathrm{MRI}$ in diagnostic radiology and radiation oncology. 


\section{REFERENCES}

1. Brown JM, Giaccia AJ. The unique physiology of solid tumors: opportunities (and problems) for cancer therapy. Cancer Res 1998;58(7):1408-16.

2. Horsman MR, Mortensen LS, Petersen JB, Busk M, Overgaard J. Imaging hypoxia to improve radiotherapy outcome. Nat Rev Clin Oncol 2012;9(12):674-87.

3. Horsman MR, Overgaard J. The impact of hypoxia and its modification of the outcome of radiotherapy. J Radiat Res 2016;57 Suppl 1:i90-i98.

4. Pettersen EO, Ebbesen P, Gieling RG, Williams KJ, Dubois L, Lambin P, Ward C, Meehan J, Kunkler IH, Langdon SP and others. Targeting tumour hypoxia to prevent cancer metastasis. From biology, biosensing and technology to drug development: the METOXIA consortium. J Enzyme Inhib Med Chem 2015;30(5):689-721.

5. Zegers CM, van Elmpt W, Wierts R, Reymen B, Sharifi H, Ollers MC, Hoebers F, Troost EG, Wanders $\mathrm{R}$, van Baardwijk A and others. Hypoxia imaging with [(1)(8)F]HX4 PET in NSCLC patients: defining optimal imaging parameters. Radiother Oncol 2013;109(1):58-64.

6. Klaassen R, Bennink RJ, van Tienhoven G, Bijlsma MF, Besselink MGH, Henegouwen MIV, Wilmink JW, Nederveen AJ, Windhorst AD, Hulshof M and others. Feasibility and repeatability of PET with the hypoxia tracer F-18 HX4 in oesophageal and pancreatic cancer. Radiotherapy and Oncology 2015;116(1):94-99.

7. Dubois LJ, Niemans R, van Kuijk SJ, Panth KM, Parvathaneni NK, Peeters SG, Zegers CM, Rekers NH, van Gisbergen MW, Biemans R and others. New ways to image and target tumour hypoxia and its molecular responses. Radiother Oncol 2015;116(3):352-7.

8. Ling CC, Humm J, Larson S, Amols H, Fuks Z, Leibel S, Koutcher JA. Towards multidimensional radiotherapy (MD-CRT): biological imaging and biological conformality. Int J Radiat Oncol Biol Phys 2000;47(3):551-60.

9. Thorwarth D, Eschmann SM, Paulsen F, Alber M. Hypoxia dose painting by numbers: a planning study. Int J Radiat Oncol Biol Phys 2007;68(1):291-300.

10. Yaromina A, Granzier M, Biemans R, Lieuwes NG, Van Elmpt W, Shakirin G, Dubois L, Lambin P. A novel concept for tumour targeting with radiation: Inverse dose-painting or targeting the "Low Drug Uptake Volume". Radiotherapy and Oncology 2017;(in press).

11. Zhu T, Das S, Wong TZ. Integration of PET/MR Hybrid Imaging into Radiation Therapy Treatment. Magn Reson Imaging Clin N Am 2017;25(2):377-430.

12. Koh DM, Collins DJ. Diffusion-weighted MRI in the body: applications and challenges in oncology. AJR Am J Roentgenol 2007;188(6):1622-35.

13. Afaq A, Andreou A, Koh DM. Diffusion-weighted magnetic resonance imaging for tumour response assessment: why, when and how? Cancer Imaging 2010;10 Spec no A:S179-88.

14. Schaarschmidt BM, Buchbender C, Nensa F, Grueneisen J, Gomez B, Kohler J, Reis H, Ruhlmann V, Umutlu L, Heusch P. Correlation of the apparent diffusion coefficient (ADC) with the standardized uptake value (SUV) in lymph node metastases of non-small cell lung cancer (NSCLC) patients using hybrid 18F-FDG PET/MRI. PLoS One 2015;10(1):e0116277.

15. Heusch P, Buchbender C, Kohler J, Nensa F, Beiderwellen K, Kuhl H, Lanzman RS, Wittsack HJ, Gomez $\mathrm{B}$, Gauler T and others. Correlation of the apparent diffusion coefficient (ADC) with the standardized uptake value (SUV) in hybrid 18F-FDG PET/MRI in non-small cell lung cancer (NSCLC) lesions: initial results. Rofo 2013;185(11):1056-62. 


\section{Chapter}

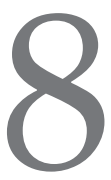

Stability of radiomics features in apparent diffusion coefficient maps from a multi-centre test-retest trial

Authors:

J. Peerlings, H.C. Woodruff, J.M. Winfield, A. Ibrahim, B.E. Van Beers, A. Heerschap, A. Jackson, J.E. Wildberger, F.M. Mottaghy, N.M. deSouza, P. Lambin

Adapted from:

Scientific Reports (Nature Research), 2019 Mar, 18; 9(1):4800

DOI: 10.1038/s41598-019-41344-5 


\section{ABSTRACT}

Quantitative radiomics features, extracted from medical images, characterize tumour-phenotypes and have been shown to provide prognostic value in predicting clinical outcomes. Stability of radiomics features extracted from apparent diffusion coefficient (ADC)-maps is essential for reliable correlation with the underlying pathology and its clinical applications. Within a multicentre, multi-vendor trial we established a method to analyse radiomics features from ADC-maps of ovarian ( $n=12)$, lung $(n=19)$, and colorectal liver metastasis $(n=30)$ cancer patients who underwent repeated ( $<7$ days) diffusion-weighted imaging at $1.5 \mathrm{~T}$ and $3 \mathrm{~T}$. From these ADC-maps, 1322 features describing tumour shape, texture and intensity were retrospectively extracted and stable features were selected using the concordance correlation coefficient $(\mathrm{CCC}>0.85)$. Although some features were tissue- and/or respiratory motion-specific, 122 features were stable for all tumour-entities. A large proportion of features were stable across different vendors and field strengths. By extracting stable phenotypic features, fittingdimensionality is reduced and reliable prognostic models can be created, paving the way for clinical implementation of ADC-based radiomics. 


\section{INTRODUCTION}

Diffusion-weighted magnetic resonance imaging (DWI) is widely used in oncology for identification and characterization of tumours [1], as well as localization [2]. Signal attenuation in DWI arises from Brownian motion of water molecules and reflects their interaction with cellular barriers and tissue macromolecules that restrict their mobility. DWI is used for tumour characterization and as an indirect biomarker of tissue cellularity[3]. By incorporating a number of diffusion-sensitizing gradients with varying strength, duration and time interval (i.e., b-values) into the MR pulse sequence, parametric apparent diffusion coefficients (ADC) maps can be derived. In oncology, ADC maps are used to determine tumour malignancy and assess early treatment response by quantifying the diffusion-related attenuation of MR signal intensity $[3,4]$. There is no consensus regarding the threshold value below which ADC is indicative of tumour, however ADC values around $1000 \times 10^{-6} \mathrm{~mm}^{2} / \mathrm{s}$ are considered normal, while lower values generally reflect restricted diffusion that could relate to hyper-cellularity or hyper-viscosity characteristic of tumour tissue, and higher ADC values represent fluid filled regions where water diffusion is unrestricted (e.g., cystic lesions, necrotic tissue). Unfortunately, an overlap between ADC values characteristic of active and treated tumours often reduces the utility of $\mathrm{ADC}$ in clinical decision-making and variations in estimates of ADC resulting from lack of standardized DWI protocols do not allow the integration of absolute values of ADC as an objective, quantifiable biomarker for personalised healthcare in the clinic [5-7].

Radiomics may provide complementary information from ADC maps by high-throughput extraction of quantitative tumour phenotypic features (i.e., shape, texture, signal intensity, and wavelet features) that have previously been correlated with tissue pathology and treatment response prediction [8, 9]. Please view https://youtu.be/Tq980GEVP0Y for more information. This methodology has shown promising results for CT imaging in oncology and in the development of prediction modelling for treatment response and outcome [10,11]. For DWI, the variability of ADC across different MR systems, vendors and magnetic field-strengths potentially compromises the stability of radiomics features, limiting the clinical implementation of MR radiomics. In addition, there is a realistic risk of overfitting when building a model where the numbers of features extracted greatly outnumber the size of the study cohort. The majority of published radiomics analyses remove features due to redundancy, either because features correlate highly with each other, or because they do not correlate with the endpoint being analysed. Reducing the number of unstable features substantially increases the reliability of radiomics analyses and may not only improve correlation with underlying pathology and tumour biology, but may also allow construction of improved prognostic or predictive models [12- 
14]. Elimination of features with poor clinical reproducibility is critical for achieving a high radiomics quality score [15].

The purpose of this analysis was to evaluate the stability of radiomics features extracted from ADC maps, derived from standardized test-retest DWI acquisitions embedded in prospective multicentre trials. DWI for each subject were acquired twice within 7 days, under similar conditions. We hypothesized that selected ADCbased features are generalizable and unaffected by different sources of data variability (i.e., tumour type, MR system, magnetic field strength) when applying standardized protocols on quality-assured MR scanners across multiple clinical centres. Establishing this methodology and presenting the results derived from the underpinning study would not only stimulate the clinical implementation of MR radiomics using ADC as a biomarker for tumour phenotyping [16] but would also outline a generalizable method for other quantifiable MRI parameters.

\section{MATERIAL \& METHODS}

PATIENT POPUlation

Table 8.1: Main patient cohort characteristics

\begin{tabular}{ccccc}
\hline $\begin{array}{c}\text { Tumour } \\
\text { site }\end{array}$ & Tumour type & Number & Age range & Treatment received \\
\hline Lung & NSCLC/metastases & 19 & $41-86$ & 5 naïve, 14 previously treated \\
Liver & Colorectal metastases & 30 & $44-77$ & No treatment within 6 months \\
Ovary & High grade serous & 12 & $31-77$ & Naïve \\
\hline
\end{tabular}

As part of prospective clinical trials to qualify ADC imaging biomarkers and stability performed by the Quantitative Imaging in Cancer: Connecting Cellular Processes with Therapy (QuICConCePT) consortium (lung and liver) and the Cancer Research UK (ovary), sixty-one patients were included from 6 university hospitals across the UK, Italy, France, and the Netherlands (site A-C, and E-G). Included patients were diagnosed with either lung cancer stage III [17], ovarian cancer (Winfield et al., manuscript in preparation), or colorectal liver metastases [18], and had a minimum of two imaging sessions maximally 7 days apart before start of treatment. Patient cohorts are summarized in Table 8.1. This study was approved by the institutional medical ethics committee of each centre (Medical Ethical Committee VU University Medical Centre, Ethics Committee Humanitas Milan, INSERM Ethics Committee, University Research Ethics Committee at the University of Manchester, Commissie Mensgebonden Onderzoek regio Arnhem - Nijmegen (WMO) at the Radboud University Medical Centre Nijmegen, and the Research Ethics Committee for The Royal Marsden Hospital Sutton). Formal written informed consent was recorded for 
each participant and all data analyses were compliant to the Medical Research Involving Human Subjects Act (WMO).

\section{IMAGE ACQUISITION}

Patients were scanned twice within 7 days before the start of treatment. In total, DWIs were acquired on 4 different MRI systems of 1.5 Tesla (T) and 3 different MRI systems of 3T using a common scan protocol per tumour region (Table 8.2): on 1.5T GE Signa HDxt (site A), Philips Achieva (site B,G) Siemens Avanto (site C,E), and on 3T Siemens Trio Tim (site C), Philips Ingenia (site F), and GE Discovery 750w (site F). The applied MR-protocol was comprised of T1/T2-weighted images for anatomical imaging and diffusion-weighted sequences with three b-values. For DWI of each tumour-type, a common and quality-assured protocol was applied by all centres [19] (Table 8.2). ADC maps were constructed by mono-exponential linear fitting of diffusion data. Images with $\mathrm{b}$-values smaller than $100 \mathrm{~s} / \mathrm{mm}^{2}$ were excluded to minimize components of blood perfusion in parametric ADC maps.

Table 8.2: Diffusion-weighted MR scan protocol. $\left(^{*}\right)$ Philips Ingenia and GE Discovery

\begin{tabular}{|c|c|c|c|c|}
\hline & $\begin{array}{l}\text { Lung Cancer } \\
\text { (site A, B, E, G) }\end{array}$ & $\begin{array}{c}\text { Colorectal liver } \\
\text { metastases }(1.5 T) \\
(\text { site } A, B, C, E)\end{array}$ & $\begin{array}{c}\text { Colorectal liver } \\
\text { metastases }(3 \mathrm{~T}) \\
\left(\text { site } C, \mathrm{~F}^{*}\right)\end{array}$ & $\begin{array}{c}\text { Ovarian } \\
\text { cancer } \\
\text { (site E) }\end{array}$ \\
\hline Sequence & ss-EPI & ss-EPI & ss-EPI & ss-EPI \\
\hline $\mathrm{TR}$ (ms) & $\geq 8000$ & $\geq 8000$ & 5000 & $\geq 8000$ \\
\hline TE (ms) & minimum & minimum & minimum & minimum \\
\hline NSA & 4 & 4 & $2-4$ & 4 \\
\hline $\mathrm{FOV}\left(\mathrm{mm}^{2}\right)$ & $380 \times 273$ & $380 \times 380$ & $380 \times 273$ & $332 \times 380$ \\
\hline Matrix $\left(\mathrm{mm}^{2}\right)$ & $128 \times 112$ & $128 \times 125$ & $128 \times 125$ & $128 \times 112$ \\
\hline Bandwidth (Hz/px) & $1400-1800$ & $1400-1800$ & $1500-2650$ & $1400-1800$ \\
\hline Slice thickness (mm) & 5 & 5 & $5-6$ & 6 \\
\hline Slice gap (mm) & 0 & 0 & 0 & 0 \\
\hline Pixel size $\left(\mathrm{mm}^{2}\right)$ & $3 \times 3$ & $1.5 \times 1.5$ & $1.5 \times 1.5$ & $1.5 \times 1.5$ \\
\hline b-values & $100,500,800$ & $100,500,900$ & $150,400,800$ & $100,500,900$ \\
\hline Fat saturation & yes & yes & yes & yes \\
\hline Parallel imaging & yes & yes & yes & yes \\
\hline
\end{tabular}

\section{SEGMENTATION}

Volumes-of-interest (VOI) were manually delineated over all primary tumours and metastases on DWI images with high b-value. The gross tumour volume (GTV) was determined at central review by experienced radiologists (with a minimum of 2 years experience) using all diagnostic information available, and saved as binary masks. For each patient, the same observer segmented the same lesion in both testretest images, while at the same time making sure that no large anatomical variations occurred. After voxel-wise rigid registration, tumour delineations on DWI were transferred to corresponding ADC maps. If $>2$ tumours were present, the largest 
lesion was delineated while excluding cystic or necrotic regions from segmentations. The same lesion was delineated separately on retest-data while blinded from testdata. In total, 72 lesions were included for analysis. All segmentations were performed using OsiriX (Pixmeo SARL, Bernex, SUI), Mirada RX (Mirada Medical, Oxford, UK), or Adept (in-house software, Institute of Cancer Research, London, UK).

\section{Feature extraction}

Radiomics features were retrospectively extracted from each VOI in the test-retest ADC dataset. ADC maps were pre-processed in two steps: (1) in order to reduce image noise and grey-level matrix (GLM) size, images were rescaled using a bin-size of 25 grey levels; (2) in order to standardise voxel size across all datasets, images were rescaled using a linearly resampled into isotropic voxel-sizes of $3 \times 3 \times 3 \mathrm{~mm}^{3}$ $[3,20]$. A total of 1322 radiomics features were obtained using an in-house developed software-toolbox in MATLAB 2014a (Mathworks, Natick, USA) [21]. These features included signal intensity features $(n=47)$, geometric features $(n=24)$, and texture features $(n=99)$, which respectively described the histogram-distribution of voxel intensity-values (i.e, first-order grey-level statistics, local intensity (Locint), and intensity histogram (IH) features), the 3D shape of delineated volumes, and the spatial distribution of fractal dimensions and voxel intensities using 6 texture matrices (i.e., grey-level co-occurrence (GLCM, 26 features) [22], grey-level distancezone (GLDZM, 16 features) [23], grey-level run-length (GLRLM, 16 features) [5, 24], grey-level size-zone (GLSZM, 16 features) [25], neighbouring grey-level dependence (NGLDM, 17 features) [26], and neighbourhood grey-tone difference matrix (NGTDM, 5 features) [27]. Furthermore, 3D wavelet decompositions of the original image resulted in additional 1152 features focusing on different spatial frequency ranges within tumour values [9].

A mathematical description of all features was previously published in $[9,15$, 28] and were presented as supplemental material with permission of the corresponding authors. Most features used in this study are in compliance with feature definitions as described by the Imaging Biomarker Standardization Initiative (IBSI). Where features differ, a note has been added specifying the difference.

\section{STATISTICAL ANALYSIS}

To select stable radiomics features, the pairwise concordance correlation coefficient (CCC) was calculated between data derived from the test and retest ADC images [29]. CCC-values range from -1 to +1 and describe the negative or positive agreement between 2 datasets. Based on previous work, features with a minimum CCC of 0.85 were regarded as statistically stable and robust $[28,30,31]$. While we Stability is defined as the closeness of agreement between measured quantity values obtained by replicate measurements performed under the same conditions (e.g., patient, scanner, imaging protocol) [17]. Statistical differences in stable features between 
tumour types, and between MR-systems with different magnetic field strength were tested using Kruskal-Wallis 1-way ANOVA with Dunn's correction for multiple testing. Differences between MR-systems from different vendors were tested for statistical significance using a Mann-Whitney test. All statistical analyses were performed using GraphPad Prism version 6.01 (GraphPad, USA). P-values $<0.05$ were considered statistically significant.

\section{FEATURE CORRELATION WITH TUMOUR VOLUME}

Features with a constant value (or near-zero variance) across all images in the test dataset were excluded, and the remainder were examined for correlations with the tumour volume using Spearman's rho statistic to estimate a rank-based measure of association. The Spearman coefficients of all unfiltered features were plotted against the feature stability as measured by the CCC for all tumour types and field strengths.

\section{RESULTS}

\section{FEATURE STABILITY}

Based on previous work, we selected a threshold of 0.85 for the concordance correlation coefficient (CCC), whereby features above this threshold were considered stable between test and retest scans [31].

\section{Tumour-type differences at $1.5 T$}

In 20 ovarian cancer lesions from 12 patients, $29 \%$ of all features (378/1322) were stable in test-retest ADC maps (Table 8.3). Of the unfiltered features, most stable features were related to geometric shape $(22 / 24,92 \%)$ and texture $(37 / 99,37 \%)$. A comprehensive list of all features is presented as supplementary information (online only). After wavelet decomposition, 144 additional radiomics features were calculated for all 8 wavelet-filters, resulting in 27\% (312/1152) of filtered features being stable. In 17 colorectal liver metastases from 17 patients, $25 \%$ of all extracted radiomics features (330/1322) demonstrated CCC-values greater than 0.85 in $1.5 \mathrm{~T}$ ADC-maps. Of these stable features, 36\% (61/170) of unfiltered and 23\% (269/1152) of wavelet-filtered features were stable in colorectal liver metastases. For 22 lung cancer lesions from 19 patients, 25\% of all features (330/1322) showed stability matching our specified threshold. In contrast to colorectal liver metastases, only $16 \%$ (27/170) of unfiltered radiomics signatures were stable, while the percentage of stable wavelet-filtered features were $26 \%$ (303/1152).

122 features ( 23 unfiltered and 99 wavelet-filtered) were regarded as stable in all three tumour entities and 298 features (49 unfiltered and 249 wavelet-filtered) were stable for at least 2 tumour-types on 1.5T ADC maps (Fig. 8.2A, Supplementary Fig. S8.1). Statistically significant differences in CCC from all features (unfiltered and wavelet-filtered) were found between ovarian tumours and colorectal liver 
metastases $(\mathrm{P}<0.0001)$, and between colorectal liver metastases and lung cancer $(\mathrm{P}=0.0051)$ but not between ovarian and lung cancer $(\mathrm{P}=0.56)$.

\section{Magnetic field strength differences}

The effects of magnetic field strength differences were analysed in ADC-maps acquired at $1.5 \mathrm{~T}$ and at $3 \mathrm{~T}$ in 17 and 13 patients with colorectal liver metastases, respectively. On ADC maps acquired at 3T, 32\% (425/1322) of radiomics features were stable over 13 segmented lesions from test-retest scans (Table 8.4). These consisted of 71/170 unfiltered features (i.e., 13/24 (54\%) geometric shape features, $44 / 99$ (44\%) texture features, and 14/47 (30\%) intensity features) and 355/1152 (29\%) of wavelet-filtered features.

No statistically significant differences were found in stability between features extracted from ADC maps acquired at $1.5 \mathrm{~T}$ and $3 \mathrm{~T} \quad(\mathrm{P}=0.51)$. Correspondingly, 245 extracted features (42 unfiltered and 204 filtered) were shown to be stable in both populations, regardless of magnetic field strength (Fig. 8.2B, Supplementary Fig. S8.2). Furthermore, comparable mean and median ADC values were derived from both 1.5T- and 3T-ADC maps of the entire cohort (Table 8.4).

Table 8.3: Stable features in ADC maps acquired at 1.5T over different tumour-entities (i.e., 20 ovarian cancer lesions, 17 colorectal liver metastases, 22 lung cancer lesions)

\begin{tabular}{|c|c|c|c|c|c|c|}
\hline \multirow{3}{*}{$\begin{array}{l}\text { Tumour type } \\
(1.5 \mathrm{~T})\end{array}$} & \multirow{3}{*}{$\begin{array}{l}\text { ADC } \\
\text { Mean } \pm S D \\
\left(10^{-6} \mathrm{~mm}^{2} / \mathrm{s}\right)\end{array}$} & \multicolumn{5}{|c|}{ Stable features $(\mathrm{CCC}>0.85)$} \\
\hline & & \multicolumn{3}{|c|}{ Unfiltered } & \multirow{2}{*}{$\begin{array}{l}\text { Wavelet } \\
\text { filtered }\end{array}$} & \multirow{2}{*}{$\begin{array}{l}\text { ALL } \\
\text { (unfiltered+wavelet) }\end{array}$} \\
\hline & & Intensity & Shape & Texture & & \\
\hline Ovarian & $1086.2 \pm 191.9$ & $7 / 47$ & $22 / 24$ & $37 / 99$ & $312 / 1152$ & $378 / 1322(29 \%)$ \\
\hline Colorectal Liver & $979.2 \pm 420.9$ & $8 / 47$ & $20 / 24$ & $33 / 99$ & $269 / 1152$ & $330 / 1322(25 \%)$ \\
\hline Lung & $1340.2 \pm 412.5$ & $1 / 47$ & $13 / 24$ & $13 / 99$ & $303 / 1152$ & $330 / 1322(25 \%)$ \\
\hline
\end{tabular}

Table 8.4: Test-retest feature stability of colorectal liver metastases measured on $1.5 \mathrm{~T}(\mathrm{n}=17)$ and $3 T$ ADC maps $(n=13)$

\begin{tabular}{|c|c|c|c|c|c|}
\hline \multirow[b]{2}{*}{$\begin{array}{l}\text { Magnetic field } \\
\text { (Colorectal Liver) }\end{array}$} & \multirow[b]{2}{*}{$\begin{array}{c}\text { ADC } \\
\text { Mean } \pm \text { SD } \\
\left(10^{-6} \mathrm{~mm}^{2} / \mathrm{s}\right)\end{array}$} & \multirow[b]{2}{*}{$\begin{array}{c}\text { ADC } \\
\text { Median } \\
\left(10^{-6} \mathrm{~mm}^{2} / \mathrm{s}\right)\end{array}$} & \multicolumn{3}{|c|}{ Stable features $(C C C>0.85)$} \\
\hline & & & Unfiltered & $\begin{array}{l}\text { Wavelet } \\
\text { filtered }\end{array}$ & $\begin{array}{c}\text { ALL } \\
\text { (unfiltered+wavelet) }\end{array}$ \\
\hline $1.5 \mathrm{~T}$ & $979.2 \pm 420.9$ & 953.4 & $61 / 170$ & $269 / 1152$ & $330 / 1322(25 \%)$ \\
\hline $3 \mathrm{~T}$ & $1353.3 \pm 409.8$ & 1202.6 & $71 / 170$ & $355 / 1152$ & $425 / 1322(32 \%)$ \\
\hline
\end{tabular}

\section{Cross-vendor differences}

Vendor-specific subgroups were analysed within the dataset of colorectal liver metastases acquired at 3T (site F). As shown in Table 8.5, feature stability of ADC maps acquired on a Philips Ingenia and a GE Discovery MR-system presented CCC- 
values $>0.85$ for $521 / 1322(39 \%)$ and $506 / 1322$ (38\%) features, respectively. No statistically significant differences were found in the number of features that exceeded the CCC-threshold $(\mathrm{P}=0.49)$. However, 290 features (79 unfiltered and 211 filtered) presented high stability across both vendors' test-retest data (Fig. 8.2C, Supplementary Fig. S8.3). Furthermore, 154 features (34 unfiltered, 120 filtered) were stable in both cross-vendor and in both $1.5 \mathrm{~T}$ and $3 \mathrm{~T}$ datasets.

A detailed description of stable features is described in Supplementary Tables S8.1-4 for each subgroup and listed as supplementary information S8.1-S8.2.

\section{CORRELATIONS BETWEEN FEATURES AND TUMOUR VOLUME}

Across all tissue types and centres, the mean absolute of Spearman's $r(|r|)$ was $0.34 \pm 0.23$. The distribution of Spearman's $r$ values for all features can be seen in supplementary Fig. S8.4. A total of 73 features were found to correlate highly with tumour volume $(|r|>0.8), 10$ of which were shape features such as maximum diameter and surface, and the remaining 63 were made up of 7 texture features and their 56 wavelet filtered equivalents. A further 137 features correlate strongly $(|r|>0.6)$ with volume, of which 11 are texture features, two intensity histogram features, and one is a statistical feature, their associated 112 wavelet features, an additional shape feature, and 10 further wavelets of texture features. For all unfiltered features, supplementary Fig. S8.5 shows the correlation between $|r|$ and CCC for all tumour sites while Supplementary Fig. S8.6 details the strength of the correlations with tumour volume. 

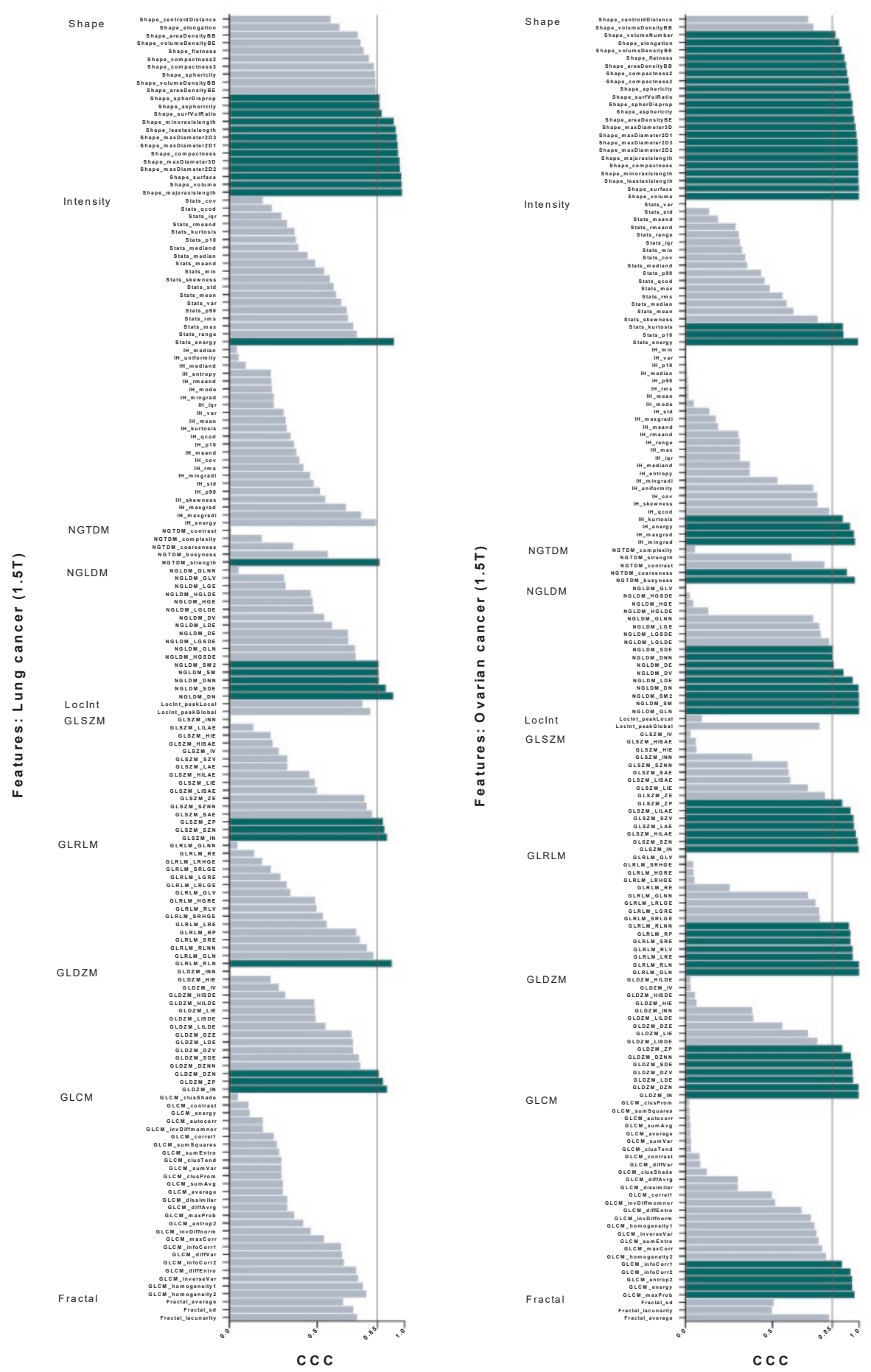

Figure 8.1: Stability of test-retest radiomics features for all patient cohorts. The threshold for stability was set at concordance correlation coefficients (CCC) greater than 0.85 

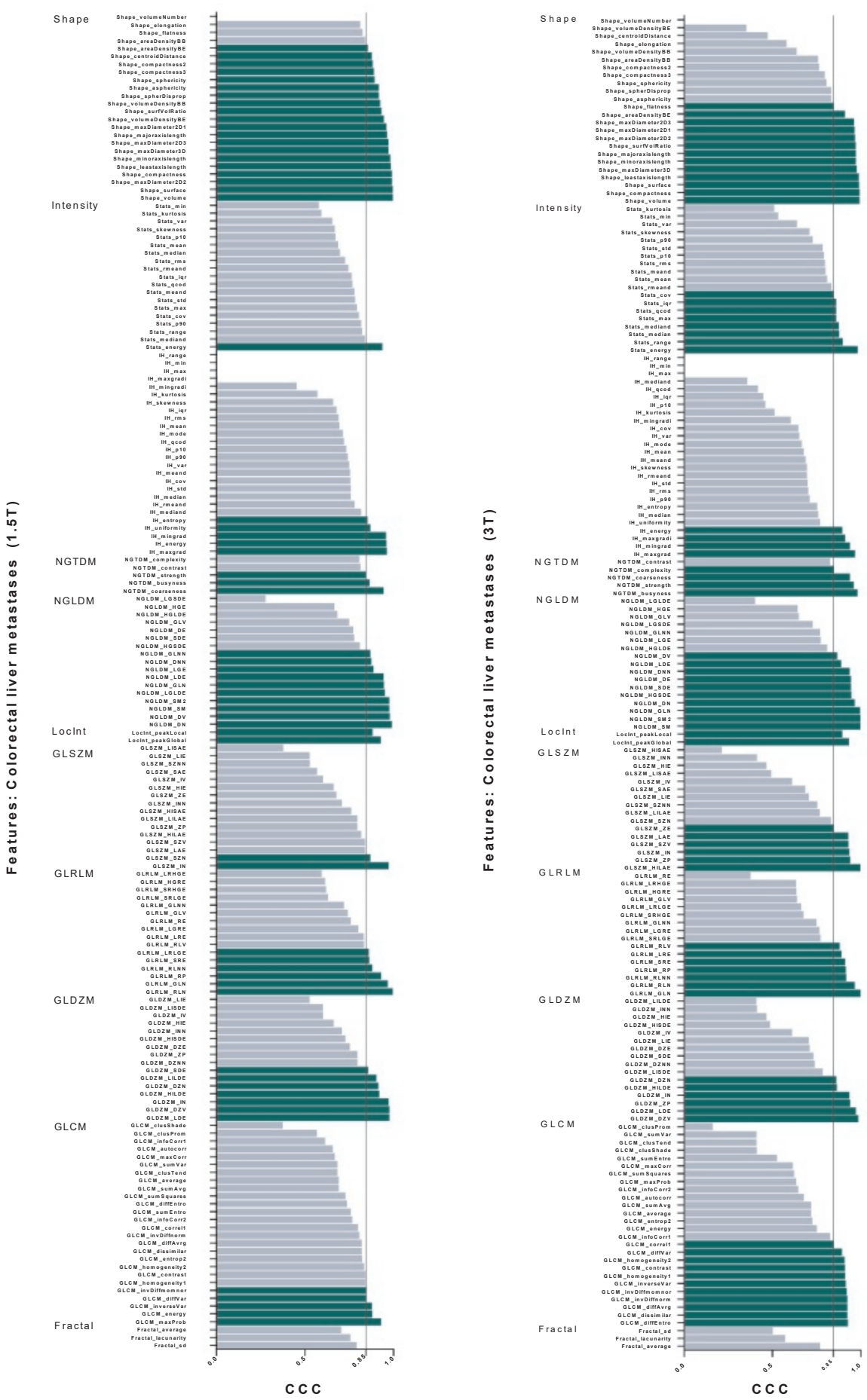

Figure 8.1- Continued: Stability of test-retest radiomics features for all patient cohorts. The threshold for stability was set at concordance correlation coefficients (CCC) greater than 0.85 
Table 8.5: Test-retest feature stability of colorectal liver metastases measured on 3T ADC maps acquired on a Philips Ingenia $(n=10)$ and GE Discovery $(n=8)$ MR systems at the same clinical centre

\begin{tabular}{lccccc}
\hline & & & \multicolumn{2}{c}{ Stable features (CCC $>0.85)$} & \\
\cline { 4 - 6 } $\begin{array}{l}\text { Site F } \\
\text { Colorectal Liver, 3T }\end{array}$ & $\begin{array}{c}\text { ADC } \\
\text { Mean } \pm \text { SD } \\
\left(10^{-6} \mathrm{~mm}^{2} / \mathrm{s}\right)\end{array}$ & $\begin{array}{c}\text { ADC } \\
\text { Median } \\
\left(10^{-6} \mathrm{~mm}^{2} / \mathrm{s}\right)\end{array}$ & Unfiltered & $\begin{array}{c}\text { Wavelet } \\
\text { filtered }\end{array}$ & $\begin{array}{c}\text { ALL } \\
\text { (unfiltered+wavelet) }\end{array}$ \\
\hline Philips & $1237.9 \pm 324.4$ & 1129.8 & $106 / 170$ & $415 / 1152$ & $521 / 1322 \mathbf{( 3 9 \% )}$ \\
GE & $1752.3 \pm 395.6$ & 1882.9 & $100 / 170$ & $406 / 1152$ & $506 / 1322 \mathbf{( 3 8 \% )}$ \\
\hline
\end{tabular}

A) Overlap of all stable unfiltered and wavelet features $(1.5 \mathrm{~T})$

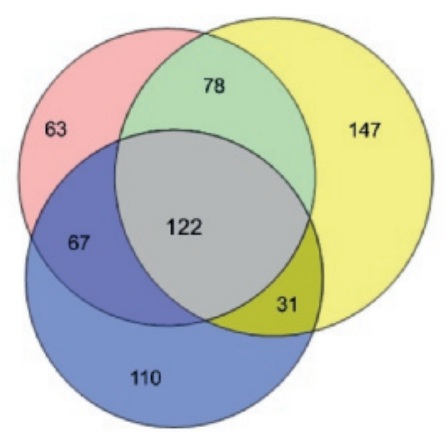

B) Overlap of stable features in colorectal liver metastases (1.5T vs. 3T, Site F)

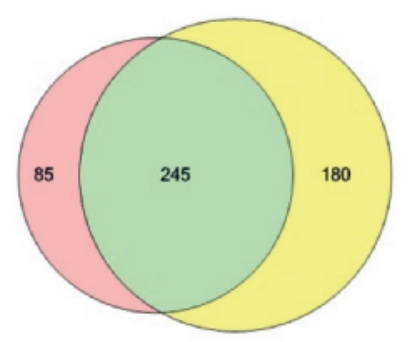

C) Overlap of stable features in colorectal liver metastases (Philips vs. GE, Site F)

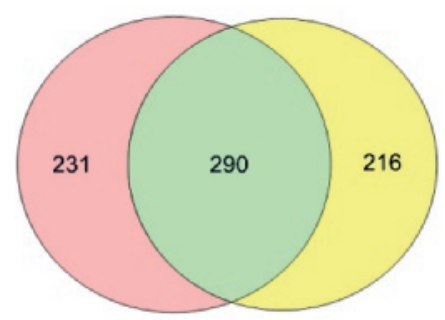

Figure 8.2: Overlapping results in feature stablility extracted from 1.5T-MR images of (A) all tumour-entities (i.e., colorectal liver metastases (red), ovarian cancer (yellow), and lung cancer (blue)); derived from MR images of colorectal liver metastases (B) acquired at 1.5T (red) and 3T (yellow); and obtained from 3T-MR images of colorectal liver metastases (C) acquired on a Philips Ingenia (red) and GE Discovery (yellow)

\section{DISCUSSION}

Within a multicentre trial, we present a method of data analysis to evaluate the stability of radiomics features derived from parametric MRI. This approach has shown that a substantial fraction of ADC-based radiomics features (25-29\%) presented test-retest stability over a variety of tissues, MR-systems, and vendors. In addition, 122 features were stable over all tissues and could be regarded to be independent of tumour origin. These results regarding radiomics feature stability are in line with studies that analysed repeatability of absolute ADC values, a correlation that could be attributed to the low coefficient of variance $(\mathrm{CoV})$ presented in these studies $[5,17,32]$. The methodology for stable feature selection presented in 
this analysis, together with the list of repeatable ADC radiomics features, facilitate the development of reliable MR-based radiomics signatures, and future clinical implementation across multiple centres. We therefore postulate that this method of analysis can be generalized to a larger field of quantifiable MR imaging features.

Shape features describe the volume contained within the segmentation, rendering it volume-dependent. Mismatch in shape features between test and retest scans could thus be attributed to differences in tumour segmentation and inter-observer variability although the same observer outlined the test-retest data in this study [33]. Especially in lung cancer, accurate tumour segmentation is complicated by respiratory motion and motion-related MR artefacts which vary between test-retest studies. Since DWI protocols in the lung were acquired during free-breathing, low stability of shape features is expected. However, these results were also present in radiomics analyses of CT-images where the acquisition is done in breath-hold [31]. Tumorous lesions in the liver and ovaries are less subjected to respiratory motion and as expected produced shape features with higher stability.

Texture features describe the pattern distribution of the voxels and quantify intra-tumour heterogeneity in all three dimensions [21]. As it takes into consideration the spatial relation to nearby voxels, stability across test-retest were similar to those of the shape features. In lung cancer, MR-related susceptibility artefacts are more common in DWI with distortions at the boundaries of tumour and air-filled lung tissue [11]. Hard transitions between tumours and normal lung tissue further complicate tumour segmentation as small delineation differences could have large impact on ADC measurements [33]. Nevertheless, radiomics features derived from lung cancer ADC maps achieved comparable reproducibility after waveletfiltering, which can alleviate boundary inhomogeneity, justifying the use of ADCbased radiomics in multi-centre trials in lung cancer.

Intensity features in lung cancer also showed more variance compared to colorectal liver metastases and ovarian cancer. As the DWI protocol in lung cancer was acquired in free-breathing, variation in partial volume effects during test-retest are very likely to have been responsible for the high variation of the intensity features. There are several other reasons for variability in intensity feature stability in MRI. Unlike CT, variations in signal-intensity result from differences in RF coil sensitivity and coil placement during patient repositioning. Despite attempting to mitigate this by using ADC rather than absolute values of signal-intensity on high b-value images, intensity-features generally showed low stability. Although no treatment was administered to our patients between test-retest scans, eliminating the possibility of treatment-related physiological effects [3, 34], inflammatory processes associated with the tumour (e.g., lung-cancer atelectasis) and small molecular fluctuations of thermal diffusion, may also have affected the stability of the ADC intensity features [35]. 
Stability of radiomics features was unaffected by differences in magnetic field strength, matching the field-independent nature of ADC [3]. In the literature, no significant difference has been reported between 1.5T and 3T ADC values measured in multiple organs [36,37]. However, higher mean CoVs have been reported in $3 \mathrm{~T}$ abdominal ADC [36]. Potentially, this variability is associated with increased difficulty in maintaining homogenous excitation pulses and gradient linearity at higher magnetic fields, and to the presence of artefacts related to magnetic susceptibility and eddy currents [37].

Surprisingly, no statistically significant differences in feature stability were found between vendors, despite potential technical differences (e.g., imaging algorithms, shimming, fat suppression, and $\mathrm{ADC}$ reconstruction) $(\mathrm{P}=0.49)[35,38]$. Previously, studies have shown low longitudinal inter-vendor ADC variability when evaluating an ice/water phantom $(\mathrm{CoV}=1-4 \%)$ and cancer patients $(\mathrm{CoV} \leq 10 \%)$ $[16,19,32]$. High repeatability and low inter-scanner variation of ADC measurements could have a direct effect on radiomics feature stability.

Radiomics features derived from CT-images have been shown to have a prognostic value [9]. For CT, these included tumour intensity ('energy'), texture ('Grey-level non-uniformity'), wavelet (Grey-level non-uniformity $\mathrm{HLH}^{\prime}$ ) and shape ('compactness'). These features were also highly stable in all tumour types in our data with CCC-values ranging from 0.89-0.99 (Fig. 8.1). This opens the possibility that similar radiomics features derived from ADC data might be useful in modelbuilding, or in complementing the currently used method of detecting treatmentrelated changes by analysing absolute ADC metrics (i.e., histogram analysis of centiles, kurtosis and skewness) [17].

Radiomics studies in CT and PET have suggested that some reproducible features could be a surrogate of tumour volume $[39,40]$. This appears to hold true for some but certainly not all the stable features presented in this research, and knowledge of which features correlate highly with volume is important for any radiomics study moving forward. Since radiomics should be purely quantitative imaging, no prognostic or diagnostic features should be excluded a priori, including simple ones such as volume or those that correlate with it.

Our analysis methods and underpinning study set-up had some limitations. Although efforts were made to minimise sources of variability by using a qualityassured standardized protocol and excluding b-values below $100 \mathrm{~mm} / \mathrm{s}^{2}$ from ADC reconstruction to reduce perfusion-related MR-signal, the use of a standardised protocol within a multi-site study does not permit optimization of data from individual MR-systems [38]. Furthermore, DWI protocols did not include respiratory triggering or motion correction. Motion artefacts, predominantly seen in lung cancer patients and patients with colorectal liver metastases (site C, 3T), had an adverse effect on ADC feature stability. However, for the colorectal liver metastases 
data acquired at $3 \mathrm{~T}$, the protocol was adjusted from the one specified at $1.5 \mathrm{~T}$ to avoid specific image-artefacts [36]. For example, a larger bandwidth of 1500-2650 Hz/pixel was set to minimise geometric image distortion. In addition to standardised DWI acquisition, we did not specifically reduce ADC variability through post-processing to further improve the stability of radiomics features [41]. In a recent multi-centre study by Pathak et al (2017), the percentage change in test-retest ADC measurements decreased from $21.1 \%$ to $2.7 \%$ in colorectal liver metastases using a standardizationstrategy to account for measurement uncertainty (i.e., error modelling). This type of approach has the potential to further improve the stability of radiomics features. In addition, radiomics stability could benefit from improvements in tumour segmentation (i.e., reducing inter- and intra-observer variability) and image quality (i.e., increased signal-to-noise ratio and reduced image artefacts) [5, 6, 33, 41]. Although we controlled for observer segmentation (same observer segmented each test-retest), image reconstruction and processing algorithms varied between centres and scanner vendors. Neither the assessment of differences between medical centres nor the number of reproducible features as a function of CCC cut-off (as performed in [31]) were included in this study owing to the small number of patients from each site and should be addressed in future work. However, good agreement in ADCmeasurements between centres previously has been reported [19]. Also, image preprocessing can be regarded as another variable in the feature extraction workflow, and as such is also able to influence feature variability, so minimal pre-processing was performed, using common values from radiomics literature. Performing testretest experiments are crucial to ensure that only stable features are selected for meaningful analysis and inclusion of parametric MR-radiomics as a clinical tool [15].

In conclusion, we have presented the assessment of stability of radiomics features from parametric ADC based on standardized test-retest measurements. This methodology enables selection of stable features that quantitatively represent phenotypic features and enables the exciting use of high-quality radiomics analysis to attain reliable biomarkers complementary to other clinical/imaging data. As extracted ADC-based radiomics features are stable across multiple centres, tumour types, 1.5T-3T systems, and MR-vendors, this analysis can be widely included in multicentre trials. The implementation of such quantitative analysis of tumour phenotype will facilitate the development of diagnostic and theragnostic models that could help detect cancers earlier than the current standard, predict early treatment response and improve treatment decision-making towards personalized healthcare. 


\section{Acknowledgements}

Authors acknowledge the contribution of data from the QuiC-ConCePT partners at VUMC, Amsterdam, Humanitas Milan, INSERM Paris, University of Manchester, Nijmegen Medical Center and The Royal Marsden Hospital, Sutton UK. Particular thanks to Prof S Stroobants, Prof A Chiti for their support and to P G Arteiser, J Wakefield, E Pace, A Weller for their help with patient recruitment and region-of interest selection. Special thanks to JE van Timmeren, RTH Leijenaar, RTHM Larue, A Jochems for their insight in radiomics and statistical analysis.This research received financial support from ERC advanced grant (ERC-ADG-2015, n 694812 - Hypoximmuno) and the QuIC-ConCePT project, which is partly funded by EFPI A companies and the Innovative Medicine Initiative Joint Undertaking (IMI JU) under Grant Agreement No. 115151. This research is also supported by the Dutch technology Foundation STW (grant $\mathrm{n}^{\circ} 10696$ DuCAT \& $\mathrm{n}^{\circ}$ P14-19 Radiomics STRaTegy), which is the applied science division of NWO, and the Technology Programme of the Ministry of Economic Affairs. Authors also acknowledge financial support from the EU 7th framework program (ARTFORCE - $n^{\circ}$ 257144, REQUITE - $n^{\circ}$ 601826), SME Phase 2 (RAIL - ${ }^{\circ} 673780$ ), EUROSTARS (SeDI, CloudAtlas, DART, DECIDE, COMPACT), the European Program H2020-2015-17 (BD2Decide - PHC30-689715, ImmunoSABR - n 733008 and PREDICT - ITN - ${ }^{\circ}$ 766276), Interreg V-A Euregio Meuse-Rhine ("Euradiomics"). We acknowledge funding from Cancer Research UK BIDD grant C1353/A12762 to the Cancer Imaging Centre at the Institute of Cancer Research.We acknowledge funding from Cancer Research UK BIDD grant C8742/A18097 to the Cancer Imaging Centre at Cambridge and Manchester.

\section{Competing interests}

Dr Lambin reports grants/sponsored research from Oncoradiomics, ptTheragnostic, advisor $(\mathrm{SAB}) /$ presenter fee from Oncoradiomics. Dr Lambin is inventor of two patents on radiomics and one non patentable invention (software), licensed to Oncoradiomics and has (minority) shares in the company Oncoradiomics. Dr. Woodruff has (minority) shares in the company Oncoradiomics.

\section{REFERENCES}

1. Peerlings J, Troost EG, Nelemans PJ, Cobben DC, de Boer JC, Hoffmann AL, et al. The Diagnostic Value of MR Imaging in Determining the Lymph Node Status of Patients with Non-Small Cell Lung Cancer: A Meta-Analysis. Radiology. 2016;281:86-98.

2. Pollard JM, Wen Z, Sadagopan R, Wang J, Ibbott GS. The future of image-guided radiotherapy will be MR guided. Br J Radiol. 2017;90:20160667.

3. Koh DM, Collins DJ. Diffusion-weighted MRI in the body: applications and challenges in oncology. AJR American journal of roentgenology. 2007;188:1622-35.

4. Padhani AR, Liu G, Koh DM, Chenevert TL, Thoeny HC, Takahara T, et al. Diffusion-weighted magnetic resonance imaging as a cancer biomarker: consensus and recommendations. Neoplasia (New York, NY). 2009;11:102-25.

5. Pathak R, Ragheb H, Thacker NA, Morris DM, Amiri H, Kuijer J, et al. A data-driven statistical model that estimates measurement uncertainty improves interpretation of ADC reproducibility: a multi-site study of liver metastases. Scientific reports. 2017;7:14084.

6. deSouza NM, Winfield JM, Waterton JC, Weller A, Papoutsaki MV, Doran SJ, et al. Implementing diffusion-weighted MRI for body imaging in prospective multicentre trials: current considerations and future perspectives. European radiology. 2017.

7. Sinkus R, Van Beers BE, Vilgrain V, DeSouza N, Waterton JC. Apparent diffusion coefficient from magnetic resonance imaging as a biomarker in oncology drug development. European journal of cancer (Oxford, England : 1990). 2012;48:425-31.

8. van Timmeren JE, Leijenaar RTH, van Elmpt W, Reymen B, Lambin P. Feature selection methodology for longitudinal cone-beam CT radiomics. Acta Oncol. 2017;56:1537-43.

9. Aerts HJ, Velazquez ER, Leijenaar RT, Parmar C, Grossmann P, Carvalho S, et al. Decoding tumour phenotype by noninvasive imaging using a quantitative radiomics approach. Nat Commun. 2014;5:4006. 
10. van Timmeren JE, Leijenaar RTH, van Elmpt W, Reymen B, Oberije C, Monshouwer R, et al. Survival prediction of non-small cell lung cancer patients using radiomics analyses of cone-beam CT images. Radiotherapy and oncology : journal of the European Society for Therapeutic Radiology and Oncology. 2017;123:363-9.

11. Bowen SR, Yuh WTC, Hippe DS, Wu W, Partridge SC, Elias S, et al. Tumor radiomic heterogeneity: Multiparametric functional imaging to characterize variability and predict response following cervical cancer radiation therapy. Journal of magnetic resonance imaging : JMRI. 2017.

12. Yip SS, Aerts HJ. Applications and limitations of radiomics. Physics in medicine and biology. 2016;61:R150-66.

13. Gillies RJ, Kinahan PE, Hricak H. Radiomics: Images Are More than Pictures, They Are Data. Radiology. 2016;278:563-77.

14. Fave X, Mackin D, Yang J, Zhang J, Fried D, Balter P, et al. Can radiomics features be reproducibly measured from CBCT images for patients with non-small cell lung cancer? Medical physics. 2015;42:678497.

15. Lambin P, Leijenaar RTH, Deist TM, Peerlings J, de Jong EEC, van Timmeren J, et al. Radiomics: the bridge between medical imaging and personalized medicine. Nat Rev Clin Oncol. 2017;14:749-62.

16. Winfield JM, Collins DJ, Priest AN, Quest RA, Glover A, Hunter S, et al. A framework for optimization of diffusion-weighted MRI protocols for large field-of-view abdominal-pelvic imaging in multicenter studies. Medical physics. 2016;43:95.

17. Weller A, Papoutsaki MV, Waterton JC, Chiti A, Stroobants S, Kuijer J, et al. Diffusion-weighted (DW) MRI in lung cancers: ADC test-retest repeatability. European radiology. 2017;27:4552-62.

18. Kumar V, Gu Y, Basu S, Berglund A, Eschrich SA, Schabath MB, et al. Radiomics: the process and the challenges. Magnetic resonance imaging. 2012;30:1234-48.

19. Winfield JM, Papoutsaki MV, Ragheb H, Morris DM, Heerschap A, ter Voert EG, et al. Development of a diffusion-weighted MRI protocol for multicentre abdominal imaging and evaluation of the effects of fasting on measurement of apparent diffusion coefficients (ADCs) in healthy liver. The British journal of radiology. 2015;88:20140717.

20. Shafiq-Ul-Hassan M, Zhang GG, Latifi K, Ullah G, Hunt DC, Balagurunathan Y, et al. Intrinsic dependencies of CT radiomic features on voxel size and number of gray levels. Medical physics. 2017;44:1050-62.

21. Lambin P, Rios-Velazquez E, Leijenaar R, Carvalho S, van Stiphout RG, Granton P, et al. Radiomics: extracting more information from medical images using advanced feature analysis. European journal of cancer (Oxford, England : 1990). 2012;48:441-6

22. Haralick RM, Shanmugam K, Dinstein I. Textural Features for Image Classification. IEEE Transactions on Systems, Man, and Cybernetics. 1973;SMC-3:610-21.

23. Thibault G, Angulo J, Meyer F. Advanced statistical matrices for texture characterization: application to cell classification. IEEE Trans Biomed Eng. 2014;61:630-7.

24. Galloway MM. Texture analysis using gray level run lengths. Computer Graphics and Image Processing. 1975;4:172-9.

25. Thibault G, Fertil B, Navarro C, Pereira S, Cau P, Levy N, et al. Texture indexes and gray level size zone matrix application to cell nuclei classification. Pattern Recognit Inf Process. 2009:140-5.

26. Sun C, Wee WG. Neighboring gray level dependence matrix for texture classification. Computer Vision, Graphics, and Image Processing. 1983;23:341-52.

27. Amadasun M, King R. Textural features corresponding to textural properties. IEEE Transactions on Systems, Man, and Cybernetics. 1989;19:1264-74.

28. van Timmeren JE, Leijenaar RTH, van Elmpt W, Wang JZ, Zhang Z, Dekker A, et al. Test-Retest Data for Radiomics Feature Stability Analysis: Generalizable or Study-Specific? Tomography. 2016;2:361-5.

29. Lin LI. A concordance correlation coefficient to evaluate reproducibility. Biometrics. 1989;45:255-68.

30. Zhao B, Tan Y, Tsai WY, Qi J, Xie C, Lu L, et al. Reproducibility of radiomics for deciphering tumor phenotype with imaging. Scientific reports. 2016;6:23428.

31. Larue R, Van De Voorde L, van Timmeren JE, Leijenaar RTH, Berbee M, Sosef MN, et al. 4DCT imaging to assess radiomics feature stability: An investigation for thoracic cancers. Radiotherapy and oncology : journal of the European Society for Therapeutic Radiology and Oncology. 2017;125:147-53. 
32. Winfield JM, deSouza NM, Priest AN, Wakefield JC, Hodgkin C, Freeman S, et al. Modelling DW-MRI data from primary and metastatic ovarian tumours. European radiology. 2015;25:2033-40.

33. Leijenaar RT, Carvalho S, Velazquez ER, van Elmpt WJ, Parmar C, Hoekstra OS, et al. Stability of FDGPET Radiomics features: an integrated analysis of test-retest and inter-observer variability. Acta Oncol. 2013;52:1391-7.

34. Padhani AR, Koh DM, Collins DJ. Whole-body diffusion-weighted MR imaging in cancer: current status and research directions. Radiology. 2011;261:700-18.

35. Koh DM, Blackledge M, Padhani AR, Takahara T, Kwee TC, Leach MO, et al. Whole-body diffusionweighted MRI: tips, tricks, and pitfalls. AJR American journal of roentgenology. 2012;199:252-62.

36. Rosenkrantz AB, Oei M, Babb JS, Niver BE, Taouli B. Diffusion-weighted imaging of the abdomen at 3.0 Tesla: image quality and apparent diffusion coefficient reproducibility compared with 1.5 Tesla. Journal of magnetic resonance imaging : JMRI. 2011;33:128-35.

37. Donati OF, Chong D, Nanz D, Boss A, Froehlich JM, Andres E, et al. Diffusion-weighted MR imaging of upper abdominal organs: field strength and intervendor variability of apparent diffusion coefficients. Radiology. 2014;270:454-63.

38. Taouli B, Beer AJ, Chenevert T, Collins D, Lehman C, Matos C, et al. Diffusion-weighted imaging outside the brain: Consensus statement from an ISMRM-sponsored workshop. Journal of magnetic resonance imaging : JMRI. 2016;44:521-40.

39. Welch ML, McIntosh C, Haibe-Kains B, Milosevic MF, Wee L, Dekker A, et al. Vulnerabilities of radiomic signature development: The need for safeguards. Radiotherapy and Oncology. 2019;130:2-9.

40. Orlhac F, Soussan M, Maisonobe J-A, Garcia CA, Vanderlinden B, Buvat I. Tumor texture analysis in 18FFDG PET: relationships between texture parameters, histogram indices, standardized uptake values, metabolic volumes, and total lesion glycolysis. Journal of Nuclear Medicine. 2014;55:414-22.

41. Guyader JM, Bernardin L, Douglas NH, Poot DH, Niessen WJ, Klein S. Influence of image registration on apparent diffusion coefficient images computed from free-breathing diffusion MR images of the abdomen. Journal of magnetic resonance imaging : JMRI. 2015;42:315-30. 


\section{SUPPLEMENTAL MATERIAL}

Supplementary Table S8.1: detailed description of stable unfiltered and wavelet radiomics

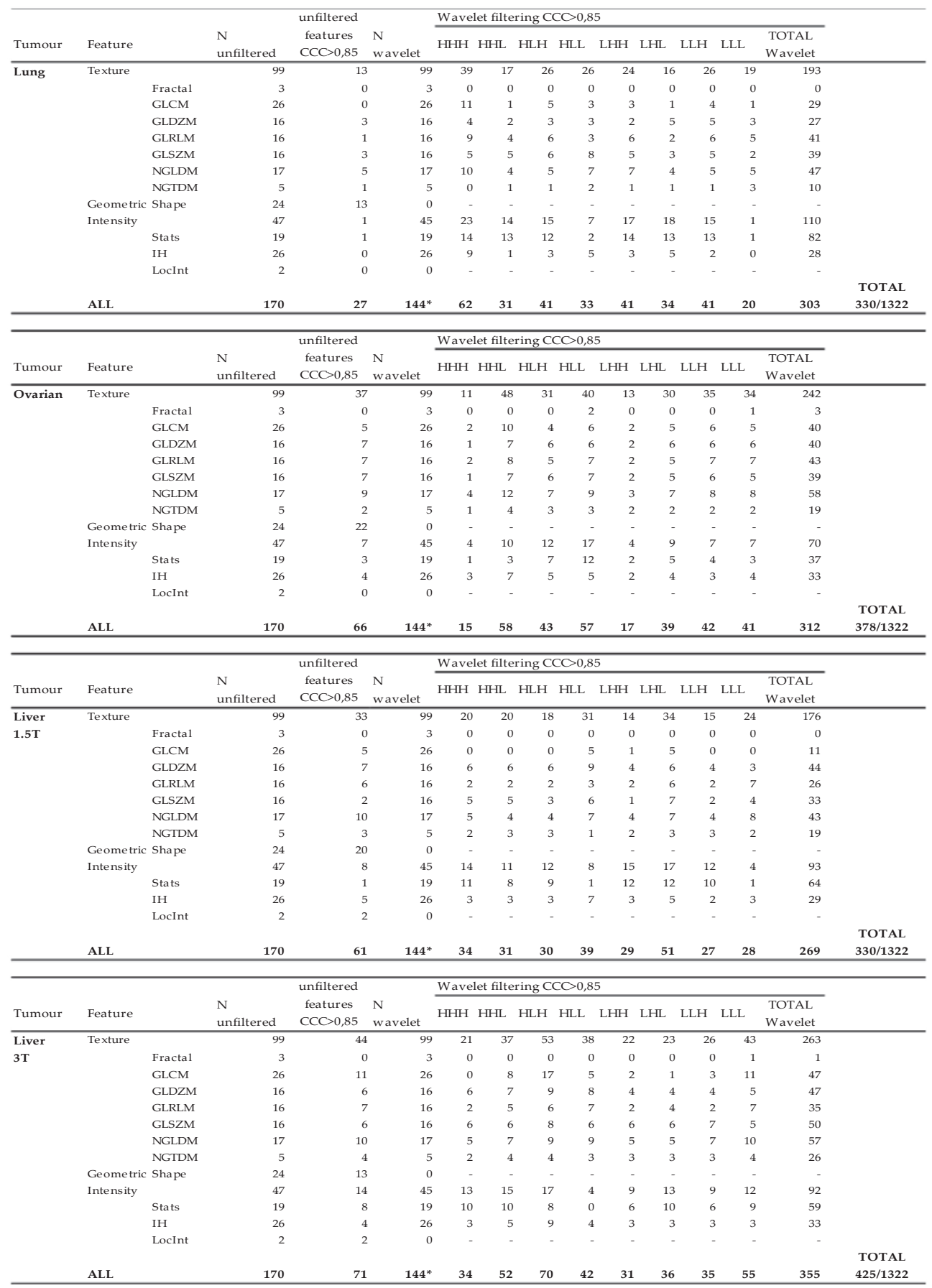


Supplementary Table S8.2: Test-retest feature stability of colorectal liver metastases measured on 3T ADC maps.

\begin{tabular}{|c|c|c|c|c|c|c|c|c|c|c|c|c|c|c|c|}
\hline \multirow{2}{*}{$\begin{array}{l}\text { SITE F1 } \\
\text { Tumour }\end{array}$} & \multirow[b]{2}{*}{ Feature } & & \multicolumn{3}{|c|}{ unfiltered } & \multicolumn{9}{|c|}{ Wavelet filtering $\mathrm{CCO}>0,85$} & \\
\hline & & & $\begin{array}{l}\mathrm{N} \\
\text { unfiltered }\end{array}$ & $\begin{array}{l}\text { features } \\
\mathrm{CCC}>0,85\end{array}$ & $\begin{array}{l}\mathrm{N} \\
\text { wavelet }\end{array}$ & \multicolumn{2}{|c|}{$\mathrm{HHH}$ HHL } & HLH & HLL & LHH & LHL & LLH & LLL & $\begin{array}{c}\text { TOTAL } \\
\text { Wavelet }\end{array}$ & \\
\hline Liver 3T & Texture & & 99 & 61 & 99 & 56 & 51 & 42 & 49 & 17 & 47 & 18 & 48 & 328 & \\
\hline \multirow{13}{*}{ Philips } & & Fractal & 3 & 1 & 3 & 1 & & 0 & 1 & 1 & 0 & 0 & 1 & 3 & \\
\hline & & GLCM & 26 & 18 & 26 & 11 & 17 & 6 & 16 & 6 & 11 & 2 & 13 & 79 & \\
\hline & & GLDZM & 16 & 4 & 16 & 9 & & 5 & 6 & 6 & 4 & 3 & 4 & 44 & \\
\hline & & GLRLM & 16 & 12 & 16 & 11 & & 9 & 8 & 8 & 11 & 2 & 8 & 57 & \\
\hline & & GLSZM & 16 & 7 & 16 & 8 & & 7 & 4 & 4 & 1 & 2 & 4 & 38 & \\
\hline & & NGLDM & 17 & 14 & 17 & 12 & 10 & 12 & 10 & 0 & 10 & 0 & 13 & 77 & \\
\hline & & NGTDM & 5 & 5 & 5 & 4 & & 3 & 4 & 4 & 3 & 3 & 5 & 30 & \\
\hline & Geometric & Shape & 24 & 20 & 0 & - & & - & - & - & - & - & - & - & \\
\hline & Statistics & & 47 & 25 & 45 & 17 & 17 & 7 & 7 & 7 & 15 & & 16 & 87 & \\
\hline & & $\mathrm{IH}$ & 26 & 17 & 26 & 8 & & 7 & 7 & 7 & 10 & & 9 & 53 & \\
\hline & & stats & 19 & 6 & 19 & 9 & & 0 & 0 & 0 & 0 & 5 & 7 & 34 & \\
\hline & & LocInt & 2 & 2 & 0 & - & & - & - & - & - & - & - & - & \\
\hline & ALL & & 170 & 106 & $144^{*}$ & 73 & 68 & 49 & 56 & 18 & 62 & 25 & 64 & 415 & $\begin{array}{r}\text { TOTAL } \\
521 / 1322 \\
\end{array}$ \\
\hline SITE F2 & & & & unfiltered & & Wave & let filte & tering $\mathrm{CC}$ & $\mathrm{CO}>0,8$ & & & & & & \\
\hline Tumour & Feature & & $\begin{array}{l}\mathrm{N} \\
\text { unfiltered }\end{array}$ & $\begin{array}{c}\text { features } \\
\mathrm{CCC}>0,85 \\
\end{array}$ & $\begin{array}{l}\mathrm{N} \\
\text { wavelet }\end{array}$ & $\mathrm{HHH}$ & HHL & HLH & HLL & LHH & I LHL & LLH & LLL & $\begin{array}{c}\text { TOTAL } \\
\text { Wavelet }\end{array}$ & \\
\hline Liver 3T & Texture & & 99 & 53 & 99 & 31 & 27 & 17 & 50 & 49 & 37 & 49 & 41 & 301 & \\
\hline \multirow[t]{13}{*}{ GE } & & Fractal & 3 & 0 & 3 & 0 & & 0 & 0 & 0 & 0 & 2 & 1 & 3 & \\
\hline & & GLCM & 26 & 18 & 26 & 7 & & 0 & 15 & 13 & 13 & 14 & 11 & 69 & \\
\hline & & GLDZM & 16 & 5 & 16 & 5 & & 5 & 6 & 6 & 8 & 5 & 3 & 42 & \\
\hline & & GLRLM & 16 & 9 & 16 & 4 & & 2 & 8 & 8 & 10 & 8 & 10 & 57 & \\
\hline & & GLSZM & 16 & 6 & 16 & 6 & & 4 & 8 & 8 & 8 & 7 & 5 & 50 & \\
\hline & & NGLDM & 17 & 11 & 17 & 9 & & 5 & 11 & 10 & 11 & 11 & 8 & 69 & \\
\hline & & NGTDM & 5 & 4 & 5 & 0 & & 1 & 2 & 2 & 1 & 2 & 3 & 11 & \\
\hline & Geometric & Shape & 24 & 17 & 0 & - & & - & - & - & - & - & - & - & \\
\hline & Statistics & & 47 & 30 & 45 & 10 & & 5 & 14 & 21 & 14 & 7 & 27 & 90 & \\
\hline & & $\mathrm{IH}$ & 26 & 12 & 26 & 6 & & 3 & 11 & 15 & 15 & 5 & 11 & 46 & \\
\hline & & stats & 19 & 17 & 19 & 4 & & 1 & 3 & 3 & 10 & 2 & 16 & 44 & \\
\hline & & LocInt & 2 & 1 & 0 & - & & - & - & - & - & - & - & - & \\
\hline & ALL & & 170 & 100 & $144^{*}$ & 41 & 32 & 20 & 64 & 70 & 51 & 56 & 68 & 402 & $\begin{array}{c}\text { TOTAL } \\
506 / 1322\end{array}$ \\
\hline
\end{tabular}




\section{A) Stable unfiltered features}

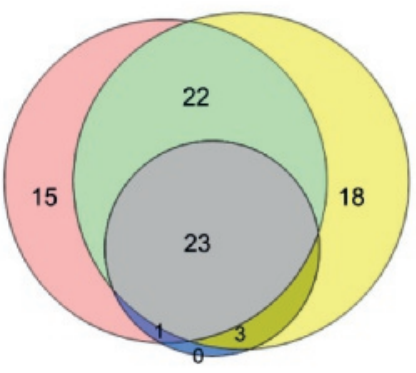

B) Stable Wavelet-filtered features

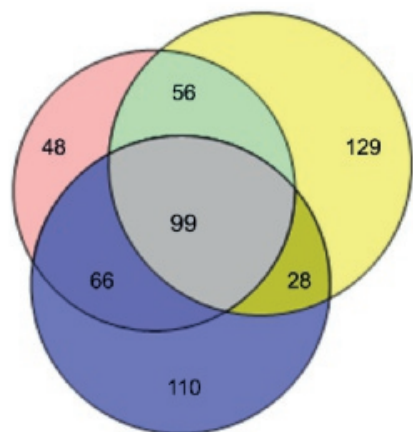

\section{C) All stable features}

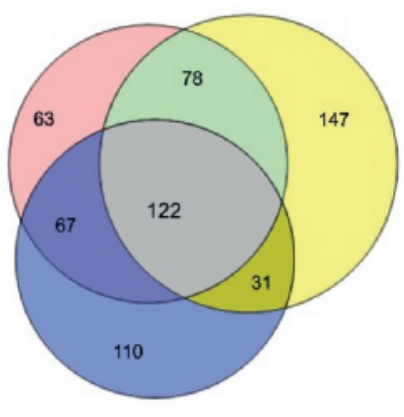

Supplementary Figure S8.1: Overlap in stable unfiltered, wavelet and all features between tumour entities at 1.5T (i.e., colorectal liver metastases (red), ovarian (yellow), and lung (blue) cancer)

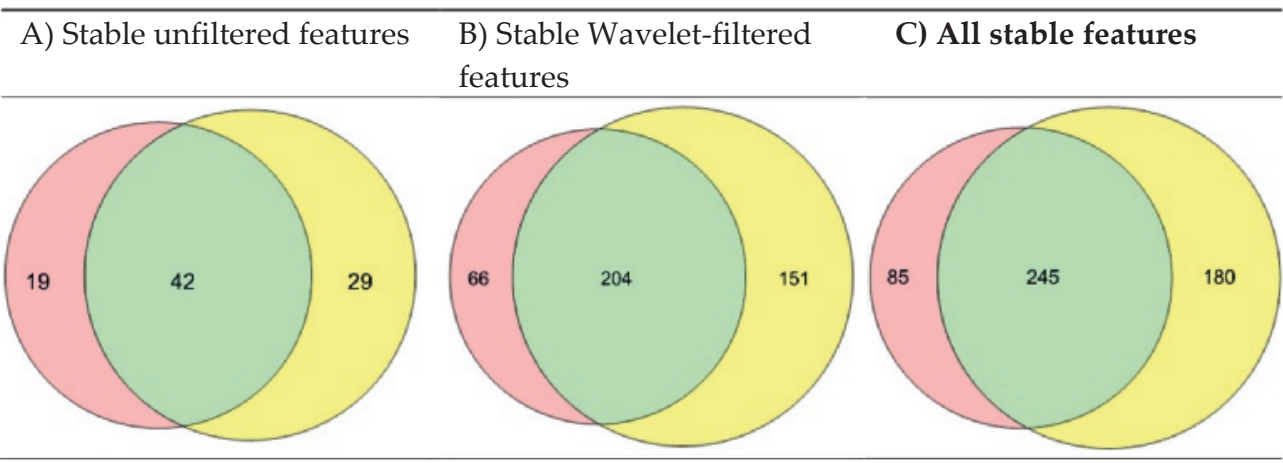

Supplementary Figure S8.2: Overlap in stable features between colorectal liver metastases at 1.5T (red) and 3T (yellow)

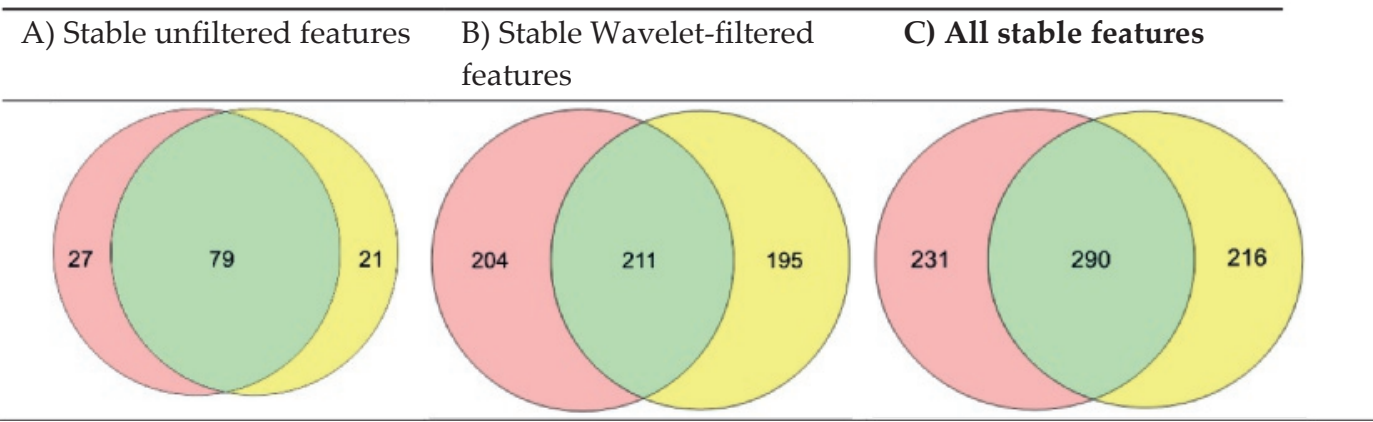

Supplementary Figure S8.3: Overlap in stable features between different MR-systems of Philips (red) and GE (yellow) 


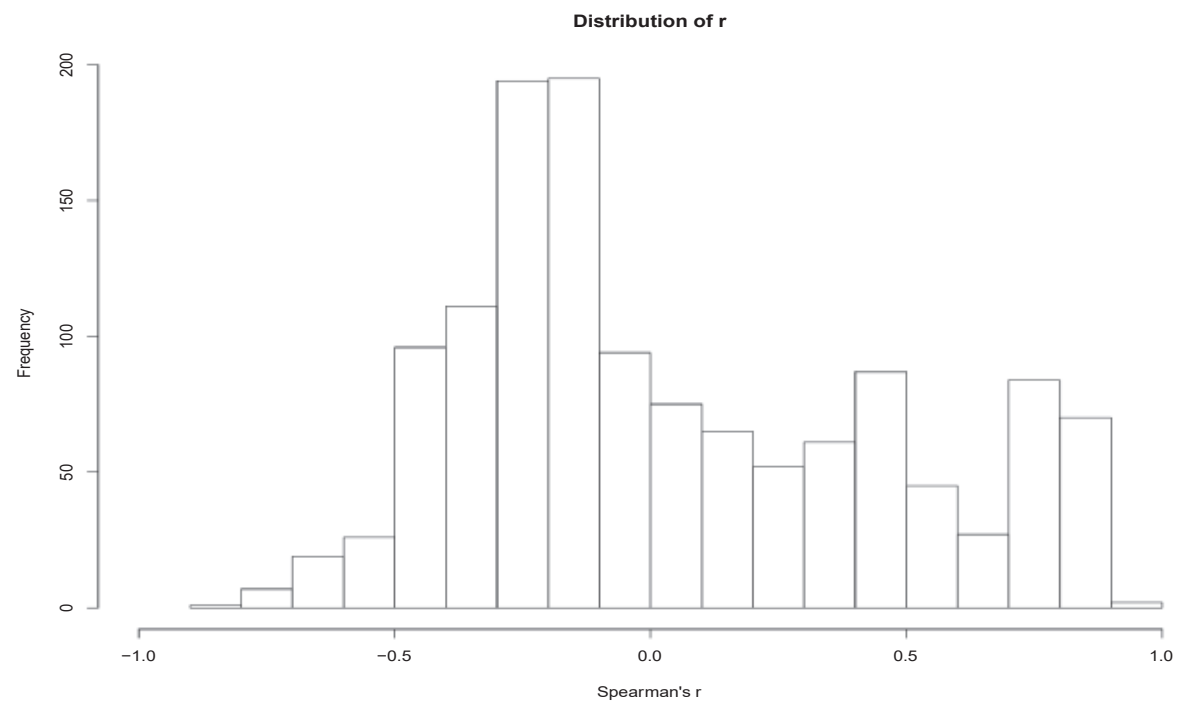

Supplementary Figure S8.4: Histogram of Spearman's r values correlating feature values with tumour volume
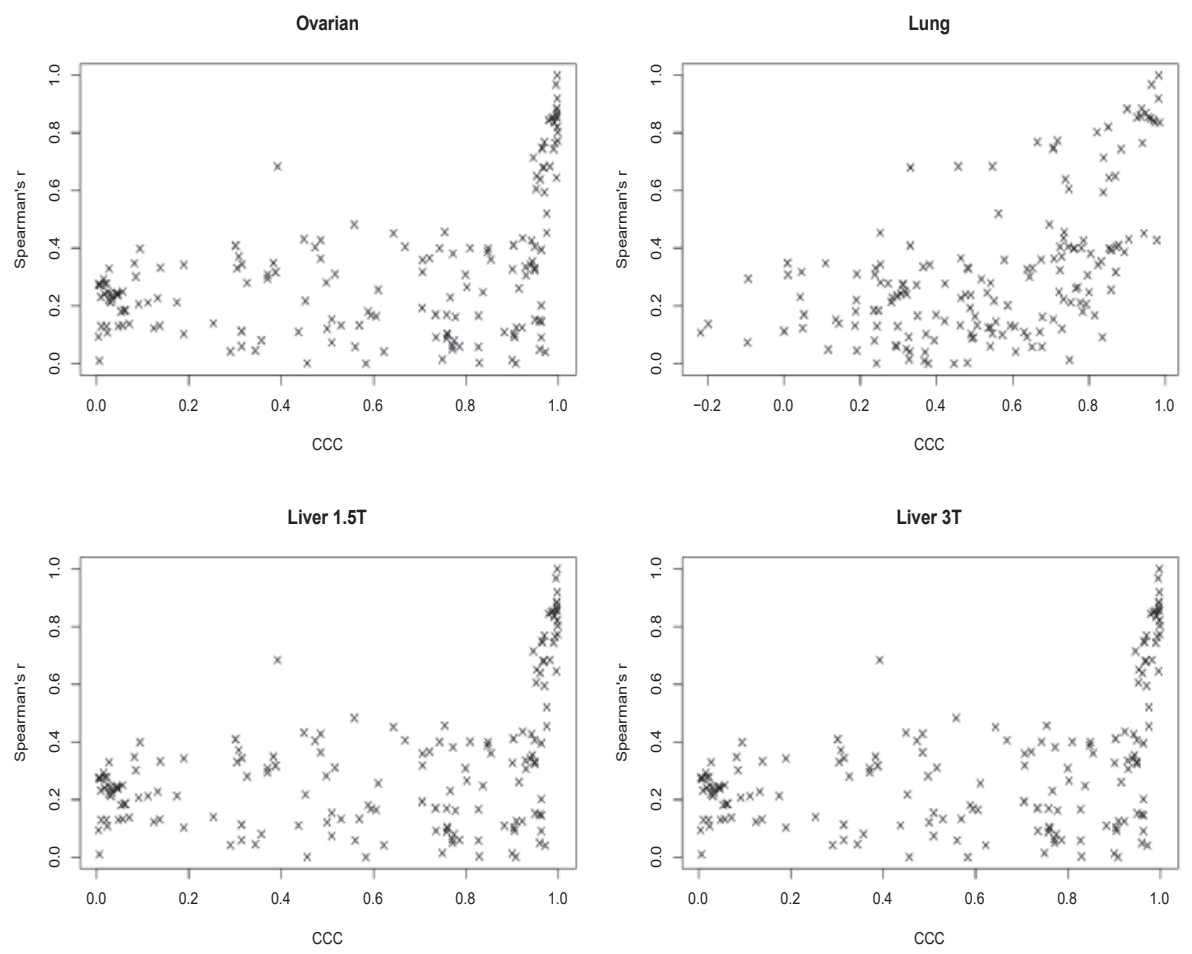

Supplementary Figure S8.5: Absolute value of Spearman's r plotted as a function of CCC 


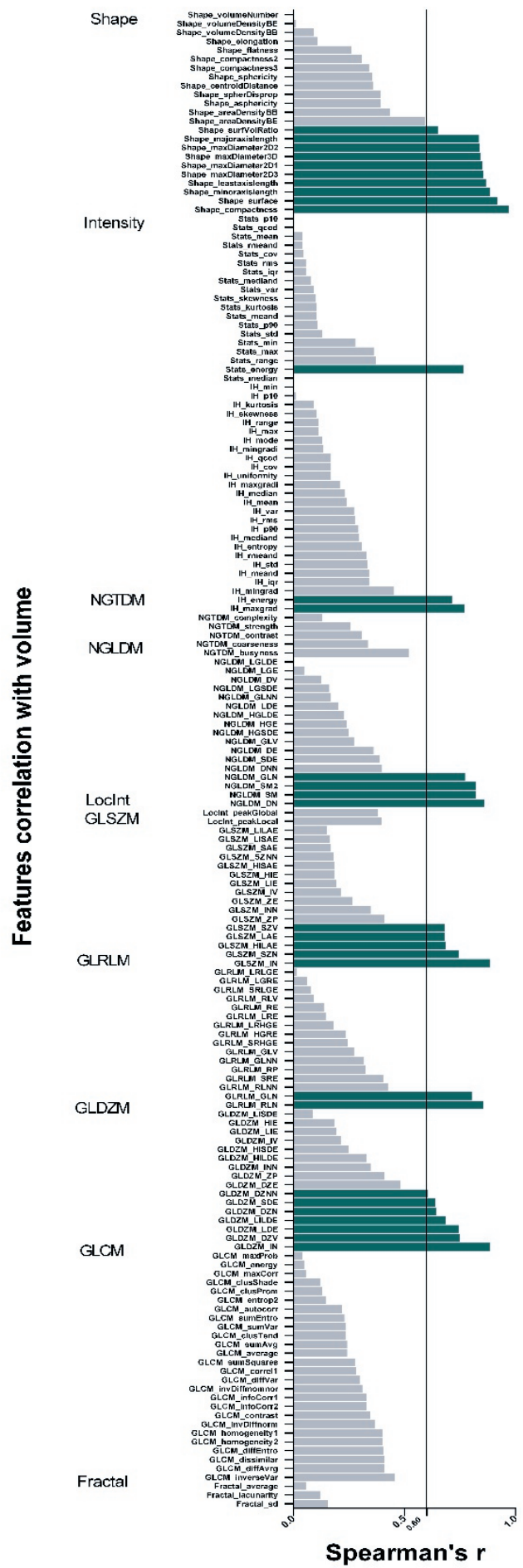

Supplementary Figure S8.6: Spearman correlationss between the radiomics features extracted from the test dataset for all patients. The threshold for strong correlation was set to $r>0.6$ 
Supplementary information S8.1: List of stable features with a concordance correlation coefficients (CCC) greater than 0.85 , for all tumour-entities and subgroups. CLM=colorectal liver metastases

\begin{tabular}{|c|c|c|c|c|}
\hline Feature Name & $\begin{array}{c}\text { CCC } \\
\text { CCrian }\end{array}$ & $\begin{array}{l}\text { Lung } \\
\text { CCC }\end{array}$ & CCC & CCC \\
\hline Fractal_average & 0.83 & 0.65 & 0.7 & 0.77 \\
\hline Fractal_lacunarity & 0.5 & 0.73 & 0.76 & 0.57 \\
\hline Fractal_sd & 0.51 & 0.71 & 0.79 & 0.5 \\
\hline GLCM_autocorr & 0.03 & 0.19 & 0.66 & 0.68 \\
\hline GLCM_average & 0.03 & 0.31 & 0.69 & 0.72 \\
\hline GLCM_clusProm & 0.02 & 0.3 & 0.57 & 0.16 \\
\hline GLCM_clusShade & 0.12 & 0.05 & 0.37 & 0.41 \\
\hline GLCM_clusTend & 0.03 & 0.3 & 0.68 & 0.41 \\
\hline GLCM_contrast & 0.08 & 0.11 & 0.84 & 0.91 \\
\hline GLCM_correl1 & 0.5 & 0.25 & 0.8 & 0.85 \\
\hline GLCM_diffAvrg & 0.3 & 0.33 & 0.82 & 0.93 \\
\hline GLCM_diffEntro & 0.67 & 0.72 & 0.74 & 0.93 \\
\hline GLCM_diffVar & 0.09 & 0.64 & 0.85 & 0.89 \\
\hline GLCM_dissimilar & 0.3 & 0.33 & 0.82 & 0.93 \\
\hline GLCM_energy & 0.96 & 0.12 & 0.88 & 0.75 \\
\hline GLCM_entrop2 & 0.96 & 0.42 & 0.82 & 0.73 \\
\hline GLCM_homogeneity1 & 0.74 & 0.76 & 0.84 & 0.91 \\
\hline GLCM_homogeneity2 & 0.81 & 0.78 & 0.83 & 0.91 \\
\hline GLCM_infoCorr1 & 0.9 & 0.64 & 0.61 & 0.83 \\
\hline GLCM_infoCorr2 & 0.95 & 0.65 & 0.77 & 0.65 \\
\hline GLCM_invDiffmomnor & 0.52 & 0.19 & 0.85 & 0.92 \\
\hline GLCM_invDiffnorm & 0.72 & 0.46 & 0.81 & 0.93 \\
\hline GLCM_inverseVar & 0.75 & 0.74 & 0.88 & 0.91 \\
\hline GLCM_maxCorr & 0.79 & 0.54 & 0.67 & 0.61 \\
\hline GLCM_maxProb & 0.97 & 0.37 & 0.93 & 0.63 \\
\hline GLCM_sumAvg & 0.03 & 0.31 & 0.69 & 0.72 \\
\hline GLCM_sumEntro & 0.77 & 0.28 & 0.76 & 0.52 \\
\hline GLCM_sumSquares & 0.02 & 0.27 & 0.73 & 0.62 \\
\hline GLCM_sumVar & 0.03 & 0.3 & 0.68 & 0.41 \\
\hline GLDZM_DZE & 0.56 & 0.7 & 0.75 & 0.71 \\
\hline GLDZM_DZN & 1 & 0.85 & 0.91 & 0.86 \\
\hline GLDZM_DZNN & 0.95 & 0.75 & 0.8 & 0.74 \\
\hline GLDZM_DZV & 0.96 & 0.71 & 0.98 & 0.99 \\
\hline GLDZM_HIE & 0.06 & 0.24 & 0.66 & 0.47 \\
\hline GLDZM_HILDE & 0.03 & 0.48 & 0.92 & 0.86 \\
\hline GLDZM_HISDE & 0.06 & 0.32 & 0.73 & 0.49 \\
\hline GLDZM_IN & 1 & 0.9 & 0.97 & 0.94 \\
\hline GLDZM_INN & 0.38 & 0.01 & 0.71 & 0.41 \\
\hline GLDZM_IV & 0.03 & 0.28 & 0.6 & 0.61 \\
\hline GLDZM_LDE & 0.97 & 0.71 & 0.98 & 0.97 \\
\hline GLDZM_LIE & 0.7 & 0.49 & 0.53 & 0.71 \\
\hline GLDZM_LILDE & 0.39 & 0.55 & 0.9 & 0.41 \\
\hline GLDZM_LISDE & 0.76 & 0.49 & 0.6 & 0.78 \\
\hline GLDZM_SDE & 0.96 & 0.74 & 0.86 & 0.73 \\
\hline GLDZM_ZP & 0.9 & 0.88 & 0.8 & 0.94 \\
\hline GLRLM_GLN & 1 & 0.82 & 0.97 & 1 \\
\hline GLRLM_GLNN & 0.71 & 0.05 & 0.72 & 0.75 \\
\hline GLRLM_GLV & 0.01 & 0.35 & 0.74 & 0.64 \\
\hline GLRLM_HGRE & 0.05 & 0.49 & 0.61 & 0.64 \\
\hline GLRLM_LGRE & 0.77 & 0.29 & 0.8 & 0.77 \\
\hline GLRLM_LRE & 0.96 & 0.56 & 0.83 & 0.89 \\
\hline GLRLM_LRHGE & 0.05 & 0.19 & 0.6 & 0.63 \\
\hline GLRLM_LRLGE & 0.75 & 0.33 & 0.86 & 0.66 \\
\hline GLRLM_RE & 0.25 & 0.14 & 0.76 & 0.37 \\
\hline GLRLM_RLN & 1 & 0.93 & 1 & 0.97 \\
\hline GLRLM_RLNN & 0.94 & 0.78 & 0.88 & 0.92 \\
\hline GLRLM_RLV & 0.96 & 0.5 & 0.83 & 0.88 \\
\hline GLRLM_RP & 0.95 & 0.72 & 0.93 & 0.92 \\
\hline GLRLM_SRE & 0.95 & 0.74 & 0.86 & 0.91 \\
\hline GLRLM_SRHGE & 0.04 & 0.53 & 0.62 & 0.68 \\
\hline GLRLM_SRLGE & 0.77 & 0.24 & 0.63 & 0.77 \\
\hline GLSZM_HIE & 0.06 & 0.24 & 0.66 & 0.47 \\
\hline GLSZM_HILAE & 0.98 & 0.46 & 0.82 & 1 \\
\hline GLSZM_HISAE & 0.06 & 0.25 & 0.76 & 0.21 \\
\hline GLSZM_IN & 1 & 0.9 & 0.97 & 0.94 \\
\hline GLSZM_INN & 0.38 & 0.01 & 0.71 & 0.41 \\
\hline GLSZM_IV & 0.03 & 0.28 & 0.6 & 0.61 \\
\hline GLSZM_LAE & 0.97 & 0.33 & 0.84 & 0.93 \\
\hline GLSZM_LIE & 0.7 & 0.49 & 0.53 & 0.71 \\
\hline GLSZM_LILAE & 0.95 & 0.14 & 0.8 & 0.77 \\
\hline GLSZM_LISAE & 0.6 & 0.5 & 0.38 & 0.49 \\
\hline GLSZM_SAE & 0.59 & 0.81 & 0.57 & 0.69 \\
\hline GLSZM_SZN & 0.99 & 0.88 & 0.87 & 0.83 \\
\hline GLSZM_SZNN & 0.59 & 0.78 & 0.53 & 0.76 \\
\hline GLSZM_SZV & 0.97 & 0.33 & 0.84 & 0.93 \\
\hline GLSZM_ZE & 0.8 & 0.77 & 0.68 & 0.85 \\
\hline GLSZM_ZP & 0.9 & 0.88 & 0.8 & 0.94 \\
\hline
\end{tabular}


IH_cov

IH_energy

IH_entropy

IH_iqr

IH_kurtosis

IH_max

IH_maxgrad

IH_maxgradi

IH_mean

IH meand

IH median

IH_mediand

IH_min

IH_mingrad

IH_mingradi

IH_mode

IH_p10

IH_p90

IH_qcod

IH_range

IH_rms

IH_skewness

IH_std

IH_uniformity

IH var

LocInt_peakGlobal

LocInt_peakLocal

NGLDM_DE

NGLDM DN

NGLDM_DNN

NGLDM_DV

NGLDM_GLN

NGLDM_GLNN

NGLDM_GLV

NGLDM_HGE

NGLDM HGLDE

NGLDM_HGSDE

NGLDM_LDE

NGLDM LGE

NGLDM LGLDE

NGLDM_LGSDE

NGLDM_SDE

NGLDM_SM

NGLDM_SM2

NGTDM_busyness

NGTDM coarseness

NGTDM complexity

NGTDM_contrast

NGTDM_strength

Shape_areaDensityBB

Shape_areaDensityBE

Shape_asphericity

Shape_centroidDistance

Shape_compactness

Shape compactness2

Shape_compactness3

Shape_elongation

Shape flatness

Shape_leastaxislength

Shape_majoraxislength

Shape maxDiameter2D1

Shape maxDiameter2D2

Shape_maxDiameter2D3

Shape_maxDiameter3D

Shape minoraxislength

Shape_spherDisprop

Shape_sphericity

Shape_surfVolRatio

Shape_surface

Shape_volume

Shape_volumeDensityBB

Shape_volumeDensityBE

Shape volumeNumber

Stats_cov

Stats_energy

Stats_iqr

Stats_kurtosis

Stats_max

Stats_mean

Stats meand

Stats_median

Stats_mediand

\begin{tabular}{|c|c|c|c|}
\hline 0.76 & 0.4 & 0.76 & 0.65 \\
\hline 0.95 & 0.84 & 0.96 & 0.9 \\
\hline 0.37 & 0.24 & 0.85 & 0.75 \\
\hline 0.31 & 0.25 & 0.68 & 0.45 \\
\hline 0.9 & 0.33 & 0.57 & 0.51 \\
\hline 0.31 & - & - & \\
\hline 0.97 & 0.67 & 0.96 & 0.97 \\
\hline 0.17 & 0.75 & 0.01 & 0.91 \\
\hline 0.02 & 0.32 & 0.7 & 0.68 \\
\hline 0.19 & 0.38 & 0.75 & 0.69 \\
\hline 0.01 & 0.04 & 0.76 & 0.76 \\
\hline \multirow[t]{2}{*}{0.37} & -0.09 & 0.82 & 0.36 \\
\hline & - & - & \\
\hline 0.98 & 0.25 & 0.96 & 0.94 \\
\hline 0.53 & 0.46 & 0.45 & 0.61 \\
\hline 0.05 & 0.24 & 0.71 & 0.67 \\
\hline 0.01 & 0.37 & 0.73 & 0.46 \\
\hline 0.01 & 0.52 & 0.74 & 0.71 \\
\hline 0.83 & 0.35 & 0.72 & 0.42 \\
\hline 0.31 & - & - & \\
\hline 0.3 & 0.24 & 0.78 & 0.69 \\
\hline 0.02 & 0.42 & 0.69 & 0.7 \\
\hline 0.76 & 0.55 & 0.66 & 0.69 \\
\hline 0.14 & 0.48 & 0.76 & 0.7 \\
\hline 0.73 & 0.05 & 0.87 & 0.77 \\
\hline 0 & 0.31 & 0.75 & 0.65 \\
\hline 0.77 & 0.8 & 0.93 & 0.93 \\
\hline 0.09 & 0.76 & 0.88 & 0.9 \\
\hline 0.85 & 0.68 & 0.77 & 0.95 \\
\hline 1 & 0.94 & 0.99 & 0.97 \\
\hline 0.85 & 0.85 & 0.87 & 0.94 \\
\hline 0.91 & 0.54 & 0.98 & 0.87 \\
\hline 1 & 0.72 & 0.95 & 1 \\
\hline 0.73 & 0.05 & 0.87 & 0.77 \\
\hline 0.01 & 0.31 & 0.75 & 0.65 \\
\hline 0.04 & 0.48 & 0.67 & 0.64 \\
\hline 0.13 & 0.46 & 0.68 & 0.81 \\
\hline 0.02 & 0.72 & 0.81 & 0.95 \\
\hline 0.96 & 0.59 & 0.94 & 0.89 \\
\hline 0.77 & 0.32 & 0.89 & 0.77 \\
\hline 0.83 & 0.48 & 0.95 & 0.4 \\
\hline 0.78 & 0.68 & 0.28 & 0.73 \\
\hline 0.85 & 0.89 & 0.78 & 0.95 \\
\hline 1 & 0.85 & 0.98 & 1 \\
\hline 1 & 0.85 & 0.98 & 1 \\
\hline 0.97 & 0.56 & 0.87 & 0.98 \\
\hline 0.93 & 0.37 & 0.94 & 0.94 \\
\hline 0.06 & 0.19 & 0.81 & 0.85 \\
\hline 0.8 & 0.01 & 0.81 & 0.83 \\
\hline 0.61 & 0.86 & 0.85 & 0.96 \\
\hline 0.92 & 0.73 & 0.84 & 0.76 \\
\hline 0.97 & 0.84 & 0.85 & 0.91 \\
\hline 0.96 & 0.86 & 0.92 & 0.83 \\
\hline 0.71 & 0.58 & 0.88 & 0.47 \\
\hline 1 & 0.96 & 0.99 & 0.99 \\
\hline 0.93 & 0.8 & 0.88 & 0.76 \\
\hline 0.94 & 0.82 & 0.89 & 0.8 \\
\hline 0.88 & 0.63 & 0.81 & 0.58 \\
\hline 0.91 & 0.77 & 0.83 & 0.85 \\
\hline 1 & 0.95 & 0.99 & 0.99 \\
\hline 0.99 & 0.99 & 0.96 & 0.97 \\
\hline 0.98 & 0.96 & 0.96 & 0.97 \\
\hline 0.99 & 0.97 & 0.99 & 0.97 \\
\hline 0.99 & 0.96 & 0.97 & 0.96 \\
\hline 0.98 & 0.97 & 0.97 & 0.98 \\
\hline 1 & 0.94 & 0.98 & 0.97 \\
\hline 0.96 & 0.86 & 0.92 & 0.83 \\
\hline 0.94 & 0.83 & 0.89 & 0.81 \\
\hline 0.95 & 0.87 & 0.93 & 0.97 \\
\hline 1 & 0.98 & 1 & 0.99 \\
\hline 1 & 0.98 & 1 & 0.99 \\
\hline 0.74 & 0.83 & 0.92 & 0.64 \\
\hline 0.9 & 0.75 & 0.95 & 0.35 \\
\hline 0.86 & - & - & \\
\hline 0.34 & 0.19 & 0.81 & 0.85 \\
\hline 0.99 & 0.94 & 0.94 & 0.98 \\
\hline 0.31 & 0.3 & 0.76 & 0.86 \\
\hline 0.9 & 0.37 & 0.59 & 0.51 \\
\hline 0.48 & 0.71 & 0.79 & 0.86 \\
\hline 0.62 & 0.61 & 0.69 & 0.81 \\
\hline 0.19 & 0.49 & 0.78 & 0.8 \\
\hline 0.58 & 0.45 & 0.7 & 0.88 \\
\hline 0.36 & 0.39 & 0.84 & 0.88 \\
\hline
\end{tabular}


Stats_min

Stats_p10

Stats_p90

Stats_qcod

Stats_range

Stats_rms

Stats_skewnes

Stats_std

Stats var

Wavelet_HHH_Fractal average

Wavelet_HHH_Fractal_lacunarity

Wavelet_HHH_Fractal_sd

Wavelet_HHH_GLCM_autocorr

Wavelet_HHH_GLCM_average

Wavelet_HHH_GLCM_clusProm

Wavelet_HHH_GLCM_clusShade

Wavelet_HHH_GLCM_clusTend

Wavelet_HHH_GLCM_contrast

Wavelet_HHH_GLCM_correl1

Wavelet_HHH_GLCM_diffAvrg

Wavelet_HHH GLCM_diffEntro

Wavelet_HHH_GLCM_diffVar

Wavelet_HHH_GLCM_dissimilar

Wavelet_HHH_GLCM_energy

Wavelet_HHH_GLCM entrop2

Wavelet_HHH_GLCM_homogeneity1

Wavelet_HHH_GLCM_homogeneity2

Wavelet_HHH_GLCM infoCorr1

Wavelet HHH GLCM infoCorr2

Wavelet_HHH_GLCM_invDiffmomnor

Wavelet_HHH_GLCM_invDiffnorm

Wavelet HHH GLCM inverseVar

Wavelet_HHH_GLCM_maxCorr

Wavelet_HHH_GLCM_maxProb

Wavelet_HHH_GLCM_sumAvg

Wavelet_HHH_GLCM_sumEntro

Wavelet_HHH_GLCM_sumSquares

Wavelet_HHH_GLCM_sumVar

Wavelet_HHH_GLDZM DZE

Wavelet_HHH_GLDZM_DZN

Wavelet_HHH_GLDZM_DZNN

Wavelet_HHH_GLDZM_DZV

Wavelet_HHH_GLDZM_HIE

Wavelet_HHH_GLDZM_HILDE

Wavelet_HHH_GLDZM_HISDE

Wavelet_HHH_GLDZM_IN

Wavelet_HHH_GLDZM_INN

Wavelet_HHH_GLDZM_IV

Wavelet_HHH_GLDZM_LDE

Wavelet_HHH_GLDZM_LIE

Wavelet_HHH_GLDZM_LILDE

Wavelet_HHH_GLDZM_LISDE

Wavelet_HHH_GLDZM_SDE

Wavelet_HHH_GLDZM_ZP

Wavelet_HHH_GLRLM_GLN

Wavelet_HHH_GLRLM_GLNN

Wavelet_HHH_GLRLM_GLV

Wavelet_HHH_GLRLM_HGRE

Wavelet_HHH_GLRLM_LGRE

Wavelet_HHH_GLRLM_LRE

Wavelet_HHH_GLRLM_LRHGE

Wavelet HHH GLRLM LRLGE

Wavelet_HHH_GLRLM_RE

Wavelet_HHH_GLRLM_RLN

Wavelet_HHH_GLRLM_RLNN

Wavelet_HHH_GLRLM RLV

Wavelet_HHH_GLRLM_RP

Wavelet_HHH_GLRLM_SRE

Wavelet_HHH_GLRLM_SRHGE

Wavelet_HHH_GLRLM_SRLGE

Wavelet_HHH_GLSZM_HIE

Wavelet_HHH_GLSZM_HILAE

Wavelet_HHH_GLSZM_HISAE

Wavelet_HHH_GLSZM_IN

Wavelet_HHH_GLSZM_INN

Wavelet_HHH_GLSZM_IV

Wavelet_HHH_GLSZM_LAE

Wavelet_HHH_GLSZM_LIE

Wavelet_HHH_GLSZM_LILAE

Wavelet_HHH_GLSZM_LISAE

Wavelet_HHH_GLSZM_SAE

Wavelet_HHH_GLSZM_SZN

\begin{tabular}{|c|c|c|c|}
\hline 0.33 & 0.54 & 0.58 & 0.53 \\
\hline 0.91 & 0.38 & 0.67 & 0.79 \\
\hline 0.44 & 0.67 & 0.82 & 0.73 \\
\hline 0.46 & 0.24 & 0.77 & 0.86 \\
\hline 0.31 & 0.73 & 0.82 & 0.9 \\
\hline 0.29 & 0.33 & 0.75 & 0.84 \\
\hline 0.56 & 0.68 & 0.73 & 0.8 \\
\hline 0.76 & 0.57 & 0.67 & 0.71 \\
\hline 0.14 & 0.59 & 0.78 & 0.78 \\
\hline 0 & 0.64 & 0.66 & 0.64 \\
\hline 0.84 & 0.8 & 0.53 & 0.51 \\
\hline 0.57 & 0.51 & 0.53 & 0.42 \\
\hline 0.45 & 0.55 & 0.52 & 0.49 \\
\hline 0.02 & -0.22 & 0.2 & 0.33 \\
\hline 0.07 & -0.2 & 0.15 & 0.24 \\
\hline 0.01 & 0.6 & 0.35 & 0.34 \\
\hline 0.51 & -0.1 & 0.26 & 0.55 \\
\hline 0.09 & 0.79 & 0.43 & 0.44 \\
\hline 0.11 & 0.78 & 0.42 & 0.43 \\
\hline 0.92 & 0.54 & 0.18 & 0.57 \\
\hline 0.39 & 0.87 & 0.5 & 0.58 \\
\hline 0.45 & 0.9 & 0.65 & 0.65 \\
\hline 0.03 & 0.73 & 0.52 & 0.41 \\
\hline 0.39 & 0.87 & 0.5 & 0.58 \\
\hline 0.48 & 0.98 & 0.59 & 0.75 \\
\hline 0.47 & 0.87 & 0.61 & 0.69 \\
\hline 0.64 & 0.94 & 0.57 & 0.7 \\
\hline 0.64 & 0.94 & 0.57 & 0.71 \\
\hline 0.91 & 0.69 & 0.57 & 0.65 \\
\hline 0.84 & 0.9 & 0.73 & 0.78 \\
\hline 0.73 & 0.81 & 0.43 & 0.46 \\
\hline 0.8 & 0.89 & 0.51 & 0.61 \\
\hline 0.62 & 0.81 & 0.33 & 0.6 \\
\hline 0.47 & 0.53 & 0.3 & 0.7 \\
\hline 0.52 & 0.97 & 0.54 & 0.67 \\
\hline 0.07 & -0.2 & 0.15 & 0.24 \\
\hline 0.46 & 0.91 & 0.64 & 0.66 \\
\hline 0.1 & 0.78 & 0.43 & 0.44 \\
\hline 0.09 & 0.79 & 0.43 & 0.44 \\
\hline 0.15 & 0.62 & 0.7 & 0.12 \\
\hline 0.71 & 0.89 & 0.88 & 0.72 \\
\hline 0.74 & 0.7 & 0.88 & 0.89 \\
\hline 0.8 & 0.58 & 0.97 & 0.98 \\
\hline 0.04 & -0.17 & 0.2 & 0.27 \\
\hline 0.07 & 0.46 & 0.82 & 0.92 \\
\hline 0.05 & -0.23 & 0.42 & 0.38 \\
\hline 0.95 & 0.93 & 0.9 & 1 \\
\hline 0.29 & 0.86 & 0.8 & 0.61 \\
\hline 0.04 & 0.74 & 0.68 & 0.59 \\
\hline 0.72 & 0.57 & 0.98 & 0.98 \\
\hline 0.6 & 0.3 & 0.22 & 0.31 \\
\hline 0.59 & 0.11 & 0.73 & 0.19 \\
\hline 0.59 & 0.44 & 0.44 & 0.32 \\
\hline 0.7 & 0.65 & 0.89 & 0.94 \\
\hline 0.78 & 0.92 & 0.56 & 0.66 \\
\hline 0.95 & 0.9 & 0.9 & 1 \\
\hline 0.56 & 0.91 & 0.71 & 0.77 \\
\hline 0.17 & 0.81 & 0.51 & 0.49 \\
\hline 0.05 & -0.15 & 0.18 & 0.25 \\
\hline 0.71 & 0.26 & 0.19 & 0.39 \\
\hline 0.54 & 0.96 & 0.3 & 0.5 \\
\hline 0.37 & 0.92 & 0.15 & 0.6 \\
\hline 0.59 & 0.73 & 0.47 & 0.19 \\
\hline 0.33 & 0.61 & 0.48 & 0.5 \\
\hline 0.89 & 0.93 & 0.88 & 0.95 \\
\hline 0.7 & 0.96 & 0.69 & 0.66 \\
\hline 0.49 & 0.96 & 0.23 & 0.35 \\
\hline 0.67 & 0.96 & 0.69 & 0.81 \\
\hline 0.67 & 0.96 & 0.73 & 0.66 \\
\hline 0.09 & 0.05 & 0.25 & 0.11 \\
\hline 0.73 & 0.33 & 0.28 & 0.41 \\
\hline 0.04 & -0.17 & 0.2 & 0.27 \\
\hline 0.49 & 0.93 & 0.75 & 0.91 \\
\hline 0.11 & 0.03 & 0.08 & 0.3 \\
\hline 0.95 & 0.93 & 0.9 & 1 \\
\hline 0.29 & 0.86 & 0.8 & 0.61 \\
\hline 0.04 & 0.74 & 0.68 & 0.59 \\
\hline 0.53 & 0.77 & 0.9 & 0.95 \\
\hline 0.6 & 0.3 & 0.22 & 0.31 \\
\hline 0.52 & 0.58 & 0.99 & 0.97 \\
\hline 0.68 & 0.4 & 0.29 & 0.34 \\
\hline 0.73 & 0.79 & 0.27 & 0.02 \\
\hline 0.52 & 0.9 & 0.88 & 0.88 \\
\hline
\end{tabular}


Wavelet_HHH_GLSZM_SZNN Wavelet_HHH_GLSZM_SZV Wavelet_HHH_GLSZM_ZE Wavelet_HHH_GLSZM_ZP Wavelet_HHH_IH_cov Wavelet_HHH_IH_energy Wavelet_HHH_IH_entropy Wavelet_HHH_IH_iqr Wavelet_HHH_IH_kurtosis Wavelet_HHH_IH_max Wavelet_HHH_IH_maxgrad Wavelet_HHH_IH_maxgradi Wavelet_HHH_IH_mean Wavelet_HHH_IH_meand Wavelet_HHH_IH_median Wavelet_HHH_IH_mediand Wavelet_HHH_IH_min Wavelet_HHH_IH_mingrad Wavelet_HHH_IH_mingradi Wavelet_HHH_IH_mode Wavelet_HHH_IH_p10 Wavelet_HHH_IH_p90 Wavelet_HHH_IH_qcod Wavelet_HHH_IH_range Wavelet_HHH_IH_rmeand Wavelet_HHH_IH_rms

Wavelet_HHH_IH_skewness Wavelet_HHH_IH_std

Wavelet_HHH_IH_uniformity Wavelet_HHH_IH_var

Wavelet_HHH_NGLDM_DE Wavelet_HHH_NGLDM_DN Wavelet_HHH_NGLDM_DNN Wavelet_HHH_NGLDM_DV Wavelet_HHH_NGLDM_GLN Wavelet_HHH_NGLDM_GLNN Wavelet_HHH_NGLDM_GLV Wavelet_HHH_NGLDM_HGE Wavelet_HHH_NGLDM_HGLDE Wavelet_HHH_NGLDM_HGSDE Wavelet_HHH_NGLDM_LDE Wavelet_HHH_NGLDM_LGE Wavelet_HHH_NGLDM_LGLDE Wavelet_HHH_NGLDM_LGSDE Wavelet_HHH_NGLDM_SDE Wavelet_HHH_NGLDM_SM Wavelet_HHH_NGLDM_SM2 Wavelet_HHH_NGTDM_busyness Wavelet_HHH_NGTDM_coarsenes Wavelet_HHH_NGTDM_complexity Wavelet_HHH_NGTDM_contrast Wavelet_HHH_NGTDM_strength Wavelet_HHH_Stats_cov Wavelet_HHH_Stats_energy Wavelet_HHH_Stats_iqr

Wavelet_HHH_Stats_kurtosis Wavelet_HHH_Stats_max Wavelet_HHH_Stats_mean

Wavelet_HHH_Stats_meand Wavelet_HHH_Stats_median Wavelet_HHH_Stats_mediand Wavelet_HHH_Stats_min Wavelet_HHH_Stats_p10 Wavelet_HHH_Stats_p90 Wavelet_HHH_Stats_qcod Wavelet_HHH_Stats_range Wavelet_HHH_Stats_rmeand Wavelet_HHH_Stats_rms Wavelet_HHH_Stats_skewness Wavelet_HHH_Stats_std Wavelet_HHH_Stats_var Wavelet_HHL_Fractal_average Wavelet_HHL_Fractal_lacunarity Wavelet_HHL_Fractal_sd Wavelet_HHL_GLCM_autocor Wavelet_HHL_GLCM_average Wavelet_HHL_GLCM_clusProm Wavelet HHL_GLCM clusShade Wavelet_HHL_GLCM_clusTend Wavelet_HHL_GLCM_contrast Wavelet_HHL_GLCM_correl1

Wavelet_HHL_GLCM_diffAvrg Wavelet_HHL_GLCM_diffEntro

\begin{tabular}{|c|c|}
\hline 0.73 & 0.8 \\
\hline 0.53 & 0.77 \\
0.14 & 0.38 \\
\hline 0.78 & 0.92 \\
\hline 0.72 & 0.82 \\
\hline 0.96 & 0.88 \\
\hline 0.53 & 0.93 \\
\hline 0.5 & 0.83 \\
\hline 0.17 & 0.97 \\
\hline 0.31 & - \\
\hline 0.87 & 0.88 \\
\hline 0.02 & 0 \\
0.06 & -0.21 \\
\hline 0.44 & 0.9 \\
\hline 0.08 & -0.12 \\
\hline 0.52 & 0.71 \\
\hline
\end{tabular}

$\begin{array}{cc}0.29 & 0.01 \\ 0.9 & 0.95 \\ 0.67 & 0.5 \\ 0.56 & 0.66 \\ 0.58 & 0.83 \\ 0.93 & 0.99 \\ 0.66 & 0.71 \\ 0.19 & 0.49 \\ 0.45 & 0.54\end{array}$

$0.94 \quad 0.95$

$\begin{array}{ll}0.18 & 0.61 \\ 0.15 & 0.27\end{array}$

$0.51-0.6$

$0.28-0.1$

$\begin{array}{ll}0.16 & 0.78\end{array}$

\begin{tabular}{cccc}
\hline 0.88 & 0.87 & 0.94 & 0.97 \\
0.11 & 0.12 & 0.2 & 0.16 \\
0.08 & -0.12 & 0.28 & 0.12 \\
0.3 & 0.11 & 0.31 & 0.72 \\
0.2 & 0.27 & 0.26 & 0.15
\end{tabular}

0.71

0.

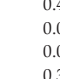

0.38

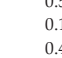

\begin{tabular}{l|c|c|c}
0.47 & 0.82 & 0.48 & 0.5 \\
0.76 & 0.69 & 0.87 & 0.84 \\
0.81 & 0.93 & 0.87 & 0.98 \\
0.62 & 0.92 & 0.42 & 0.77 \\
\hline & 0.9 & 0.5 & 0.56 \\
\hline
\end{tabular}

\begin{tabular}{cccc}
0.62 & 0.9 & 0.5 & 0.56 \\
0.88 & 0.88 & 0.92 & 0.97 \\
0.57 & 0.97 & 0.67 & 0.79 \\
0.18 & 0.82 & 0.48 & 0.5 \\
0.05 & -0.15 & 0.19 & 0.24 \\
\hline .56 & 0.93 & 0.45 & 0.85
\end{tabular}

\begin{tabular}{|c|c|c|c|}
\hline 0.05 & -0.15 & 0.19 & 0.24 \\
\hline 0.56 & 0.93 & 0.45 & 0.85 \\
\hline 0.06 & 0.71 & 0.35 & 0.18 \\
\hline 0.59 & 0.96 & 0.63 & 0.78 \\
\hline 0.72 & 0.25 & 0.19 & 0.4 \\
\hline 0.36 & 0.84 & 0.79 & 0.51 \\
\hline 0.85 & 0.67 & 0.6 & 0.58 \\
\hline 0.78 & 0.93 & 0.58 & 0.64 \\
\hline 0.96 & 0.9 & 0.85 & 0.99 \\
\hline 0.96 & 0.9 & 0.85 & 0.99 \\
\hline 0.84 & 0.81 & 0.38 & 0.68 \\
\hline
\end{tabular}

\begin{tabular}{|c|c|c|c|}
\hline .84 & 0.81 & 0.38 & 0.68 \\
\hline 0.99 & 0.57 & 0.89 & 0.95 \\
\hline 0.07 & 0.64 & 0.42 & 0.03 \\
\hline 0.8 & 0.83 & 0.45 & 0.86 \\
\hline 0.72 & 0.57 & 0.89 & 0.71 \\
\hline
\end{tabular}

\begin{tabular}{|c|c|c|c|}
\hline 0.72 & 0.57 & 0.89 & 0.71 \\
0.14 & 0.18 & 0.08 & 0.32 \\
\hline 0.88 & 0.97 & 0.92 & 0.87 \\
\hline 0.58 & 0.96 & 0.9 & 0.92 \\
\hline 0.16 & 0.96 & 0.52 & 0.52 \\
0.35 & 0.91 & 0.83 & 0.69 \\
\hline
\end{tabular}

0.25

0.52

0.52

0.51

0.6
0.52

0.52

0.54

0.05

0.45

0.59

0.5
0.04

0.36

0.36
0.8

0.68

0.62

0.07

0.22

0.04

0.66

0.35

0.36

0.76

0.63

0.77

\begin{tabular}{|c|c|c|}
\hline 0.62 & 0.83 & 0.69 \\
\hline 0.97 & 0.94 & 0.93 \\
\hline 0.55 & 0.15 & 0.26 \\
\hline 0.96 & 0.9 & 0.92 \\
0.95 & 0.84 & 0.72 \\
0.97 & 0.93 & 0.93 \\
0.96 & 0.93 & 0.95 \\
\hline-0.06 & 0.13 & 0.05 \\
\hline 0.98 & 0.86 & 0.72 \\
0.96 & 0.92 & 0.94 \\
0.98 & 0.93 & 0.87 \\
\hline 0.43 & 0.37 & 0.29 \\
\hline 0.98 & 0.93 & 0.86 \\
0.92 & 0.9 & 0.89 \\
\hline 0.76 & 0.47 & 0.74 \\
0.62 & 0.58 & 0.33 \\
0.67 & 0.58 & 0.25 \\
0.49 & 0.07 & 0.02 \\
0.48 & 0.08 & 0.04 \\
0.37 & 0.49 & 0.92 \\
0.1 & 0.22 & 0.66 \\
\hline 0.62 & 0.58 & 0.87 \\
0.48 & 0.61 & 0.84 \\
-0.02 & 0.65 & 0.59 \\
0.64 & 0.64 & 0.82 \\
0.83 & 0.64 & 0.75 \\
\hline & & \\
\hline
\end{tabular}


Wavelet_HHL_GLCM_diffVar Wavelet_HHL_GLCM_dissimilar Wavelet_HHL_GLCM_energy Wavelet_HHL_GLCM_entrop2 Wavelet_HHL_GLCM_homogeneity1 Wavelet_HHL_GLCM_homogeneity2 Wavelet_HHL_GLCM infoCorr1 Wavelet_HHL_GLCM_infoCorr2 Wavelet_HHL_GLCM_invDiffmomnor Wavelet_HHL_GLCM_invDiffnorm

Wavelet HHL GLCM inverseVar Wavelet_HHL_GLCM_maxCorr Wavelet_HHL_GLCM_maxProb Wavelet_HHL_GLCM_sumAvg Wavelet_HHL_GLCM_sumEntro Wavelet_HHL_GLCM_sumSquares Wavelet_HHL_GLCM_sumVar Wavelet_HHL_GLDZM_DZE Wavelet_HHL_GLDZM_DZN Wavelet_HHL_GLDZM_DZNN Wavelet_HHL_GLDZM_DZV Wavelet_HHL_GLDZM_HIE Wavelet_HHL_GLDZM_HILDE Wavelet_HHL_GLDZM_HISDE Wavelet_HHL_GLDZM_IN Wavelet_HHL_GLDZM_INN Wavelet_HHL_GLDZM_IV Wavelet_HHL_GLDZM_LDE Wavelet_HHL_GLDZM_LIE Wavelet_HHL_GLDZM_LILDE Wavelet_HHL_GLDZM_LISDE Wavelet_HHL_GLDZM_SDE Wavelet_HHL_GLDZM_ZP Wavelet_HHL_GLRLM_GLN Wavelet_HHL_GLRLM_GLNN Wavelet_HHL_GLRLM_GLV Wavelet_HHL_GLRLM_HGRE Wavelet_HHL_GLRLM_LGRE Wavelet_HHL_GLRLM_LRE Wavelet_HHL_GLRLM_LRHGE Wavelet_HHL_GLRLM LRLGE Wavelet_HHL_GLRLM_RE Wavelet_HHL_GLRLM_RLN Wavelet_HHL_GLRLM_RLNN Wavelet_HHL_GLRLM_RLV Wavelet_HHL_GLRLM_RP Wavelet_HHL_GLRLM_SRE Wavelet_HHL_GLRLM_SRHGE Wavelet_HHL_GLRLM_SRLGE Wavelet_HHL_GLSZM_HIE Wavelet_HHL_GLSZM_HILAE Wavelet_HHL_GLSZM_HISAE Wavelet_HHL_GLSZM_IN Wavelet_HHL_GLSZM_INN Wavelet_HHL_GLSZM IV Wavelet_HHL_GLSZM_LAE Wavelet_HHL_GLSZM_LIE Wavelet_HHL_GLSZM_LILAE Wavelet_HHL_GLSZM_LISAE Wavelet_HHL_GLSZM_SAE Wavelet_HHL_GLSZM_SZN Wavelet_HHL_GLSZM_SZNN Wavelet HHL GLSZM SZV Wavelet_HHL_GLSZM_ZE Wavelet_HHL_GLSZM_ZP Wavelet_HHL_IH_cov Wavelet_HHL_IH_energy Wavelet_HHL_IH_entropy Wavelet_HHL_IH_iqr Wavelet_HHL_IH_kurtosis Wavelet_HHL_IH_max Wavelet_HHL_IH_maxgrad Wavelet_HHL_IH_maxgradi Wavelet_HHL_IH mean Wavelet_HHL_IH_meand Wavelet_HHL_IH_median Wavelet_HHL_IH_mediand Wavelet_HHL_IH_min Wavelet_HHL_IH_mingrad Wavelet_HHL_IH_mingradi Wavelet_HHL_IH_mode Wavelet_HHL_IH_p10 Wavelet_HHL_IH_p90

\begin{tabular}{|c|c|c|c|}
\hline 0.2 & 0.51 & 0.58 & 0.87 \\
\hline 0.63 & 0.64 & 0.64 & 0.82 \\
\hline 0.95 & 0.82 & 0.69 & 0.6 \\
\hline 0.91 & 0.79 & 0.62 & 0.68 \\
\hline 0.9 & 0.79 & 0.65 & 0.75 \\
\hline 0.92 & 0.8 & 0.65 & 0.74 \\
\hline 0.96 & 0.58 & 0.56 & 0.93 \\
\hline 0.95 & 0.86 & 0.72 & 0.85 \\
\hline 0.93 & 0.53 & 0.62 & 0.84 \\
\hline 0.93 & 0.69 & 0.64 & 0.81 \\
\hline 0.84 & 0.78 & 0.71 & 0.78 \\
\hline 0.92 & 0.74 & 0.39 & 0.87 \\
\hline 0.96 & 0.75 & 0.71 & 0.6 \\
\hline 0.22 & 0.48 & 0.08 & 0.04 \\
\hline 0.79 & 0.82 & 0.64 & 0.72 \\
\hline 0.36 & 0.55 & 0.6 & 0.86 \\
\hline 0.35 & 0.62 & 0.58 & 0.87 \\
\hline 0.61 & 0.63 & 0.76 & 0.8 \\
\hline 0.95 & 0.92 & 0.81 & 0.98 \\
\hline 0.89 & 0.83 & 0.86 & 0.95 \\
\hline 0.94 & 0.81 & 0.98 & 0.97 \\
\hline 0.05 & 0.35 & 0.09 & 0.19 \\
\hline 0.2 & 0.74 & 0.93 & 0.73 \\
\hline 0.03 & 0.54 & 0.53 & 0.58 \\
\hline 0.99 & 0.95 & 0.98 & 1 \\
\hline 0.68 & 0.63 & 0.76 & 0.67 \\
\hline 0.37 & 0.38 & 0.52 & 0.91 \\
\hline 0.94 & 0.78 & 0.98 & 0.97 \\
\hline 0.53 & 0.53 & 0.3 & 0.78 \\
\hline 0.51 & 0.38 & 0.83 & 0.75 \\
\hline 0.54 & 0.57 & 0.32 & 0.65 \\
\hline 0.92 & 0.81 & 0.89 & 0.97 \\
\hline 0.88 & 0.82 & 0.69 & 0.77 \\
\hline 0.99 & 0.88 & 0.98 & 0.98 \\
\hline 0.91 & 0.84 & 0.69 & 0.74 \\
\hline 0.47 & 0.46 & 0.61 & 0.89 \\
\hline 0.05 & 0.42 & 0.06 & 0.07 \\
\hline 0.5 & 0.41 & 0.12 & 0.87 \\
\hline 0.95 & 0.72 & 0.72 & 0.38 \\
\hline 0.15 & 0.7 & 0.58 & 0.04 \\
\hline 0.46 & 0.48 & 0.54 & 0.52 \\
\hline 0.63 & 0.54 & 0.63 & 0.67 \\
\hline 0.99 & 0.93 & 0.99 & 0.99 \\
\hline 0.95 & 0.88 & 0.74 & 0.74 \\
\hline 0.94 & 0.54 & 0.76 & 0.26 \\
\hline 0.96 & 0.84 & 0.73 & 0.78 \\
\hline 0.96 & 0.89 & 0.73 & 0.74 \\
\hline 0.04 & 0.4 & 0.07 & 0.15 \\
\hline 0.51 & 0.42 & 0.2 & 0.89 \\
\hline 0.05 & 0.35 & 0.09 & 0.19 \\
\hline 0.88 & 0.9 & 0.97 & 0.78 \\
\hline 0 & 0.1 & 0.39 & 0.27 \\
\hline 0.99 & 0.95 & 0.98 & 1 \\
\hline 0.68 & 0.63 & 0.76 & 0.67 \\
\hline 0.37 & 0.38 & 0.52 & 0.91 \\
\hline 0.93 & 0.87 & 0.9 & 0.85 \\
\hline 0.53 & 0.53 & 0.3 & 0.78 \\
\hline 0.92 & 0.83 & 0.81 & 0.92 \\
\hline 0.62 & 0.59 & 0.31 & 0.6 \\
\hline 0.65 & 0.56 & 0.46 & 0.1 \\
\hline 0.9 & 0.93 & 0.91 & 0.97 \\
\hline 0.68 & 0.58 & 0.5 & 0.16 \\
\hline 0.93 & 0.87 & 0.9 & 0.85 \\
\hline 0.83 & 0.71 & 0.78 & 0.28 \\
\hline 0.88 & 0.82 & 0.69 & 0.77 \\
\hline 0.87 & 0.62 & 0.59 & 0.83 \\
\hline 0.9 & 0.88 & 0.98 & 0.97 \\
\hline 0.8 & 0.8 & 0.67 & 0.76 \\
\hline 0.7 & 0.66 & 0.42 & 0.61 \\
\hline 0.93 & 0.77 & 0.54 & 0.18 \\
\hline 0.31 & - & - & \\
\hline 0.98 & 0.81 & 0.98 & 0.9 \\
\hline 0.23 & 0.4 & 0.37 & 0.04 \\
\hline 0.21 & 0.47 & 0.07 & 0.05 \\
\hline 0.67 & 0.61 & 0.64 & 0.83 \\
\hline 0.24 & 0.52 & 0.19 & 0.04 \\
\hline 0.7 & 0.57 & 0.26 & 0.73 \\
\hline- & - & & \\
\hline 0.97 & 0.78 & 0.92 & 0.86 \\
\hline 0.25 & 0.16 & 0.1 & 0.14 \\
\hline 0.32 & 0.44 & 0.02 & 0.12 \\
\hline 0.41 & 0.58 & 0.31 & 0.24 \\
\hline 0.18 & 0.44 & 0.22 & 0.35 \\
\hline
\end{tabular}


Wavelet_HHL_IH_qcod Wavelet_HHL_IH_range Wavelet_HHL_IH_rmeand Wavelet_HHL_IH_rms Wavelet_HHL_IH_skewness Wavelet_HHL_IH_std Wavelet_HHL_IH_uniformity Wavelet_HHL_IH_var Wavelet_HHL_NGLDM_DE Wavelet_HHL_NGLDM_DN Wavelet_HHL_NGLDM_DNN Wavelet_HHL_NGLDM_DV Wavelet_HHL_NGLDM_GLN Wavelet_HHL_NGLDM_GLNN Wavelet_HHL_NGLDM_GLV Wavelet_HHL_NGLDM_HGE Wavelet_HHL_NGLDM_HGLDE Wavelet_HHL_NGLDM_HGSDE Wavelet_HHL_NGLDM_LDE Wavelet_HHL_NGLDM_LGE Wavelet_HHL_NGLDM_LGLDE Wavelet_HHL_NGLDM_LGSDE Wavelet_HHL_NGLDM_SDE Wavelet_HHL_NGLDM_SM Wavelet_HHL_NGLDM_SM2 Wavelet_HHL_NGTDM_busyness Wavelet_HHL_NGTDM_coarseness Wavelet_HHL_NGTDM_complexity Wavelet_HHL_NGTDM_contrast Wavelet_HHL_NGTDM_strength Wavelet_HHL_Stats_cov Wavelet_HHL_Stats_energy Wavelet_HHL_Stats_iqr Wavelet_HHL_Stats_kurtosis Wavelet_HHL_Stats_max Wavelet_HHL_Stats_mean

Wavelet_HHL_Stats_meand Wavelet_HHL_Stats_median Wavelet_HHL_Stats_mediand Wavelet_HHL_Stats_min Wavelet_HHL_Stats_p10 Wavelet_HHL_Stats_p90 Wavelet_HHL_Stats_qcod Wavelet_HHL_Stats_range Wavelet_HHL_Stats_rmeand Wavelet_HHL_Stats_rms Wavelet_HHL_Stats_skewness Wavelet_HHL_Stats_std Wavelet_HHL_Stats_var Wavelet_HLH_Fractal_average Wavelet_HLH_Fractal_lacunarity Wavelet_HLH_Fractal_sd Wavelet_HLH_GLCM_autocorr Wavelet_HLH_GLCM_average Wavelet_HLH_GLCM_clusProm Wavelet_HLH_GLCM_clusShade Wavelet_HLH_GLCM_clusTend Wavelet_HLH_GLCM_contrast Wavelet_HLH_GLCM_correl1 Wavelet_HLH_GLCM_diffAvrg Wavelet_HLH_GLCM_diffEntro Wavelet_HLH_GLCM_diffVar Wavelet_HLH_GLCM_dissimilar Wavelet_HLH_GLCM_energy Wavelet_HLH_GLCM_entrop2 Wavelet_HLH_GLCM_homogeneity 1 Wavelet_HLH_GLCM_homogeneity2 Wavelet_HLH_GLCM_infoCorr1 Wavelet_HLH_GLCM_infoCorr2 Wavelet_HLH_GLCM_invDiffmomnor Wavelet_HLH_GLCM_invDiffnorm Wavelet_HLH_GLCM_inverseVar Wavelet_HLH_GLCM_maxCorr Wavelet HLH GLCM maxProb Wavelet_HLH_GLCM_sumAvg Wavelet_HLH_GLCM_sumEntro Wavelet_HLH_GLCM_sumSquares

Wavelet_HLH_GLCM_sumVar Wavelet_HLH_GLDZM_DZE Wavelet_HLH_GLDZM_DZN Wavelet_HLH_GLDZM_DZNN Wavelet_HLH_GLDZM_DZV Wavelet_HLH_GLDZM_HIE

\begin{tabular}{|c|c|c|c|}
\hline 0.86 & 0.73 & 0.48 & 0.61 \\
\hline 0.31 & - & - & \\
\hline 0.71 & 0.51 & 0.63 & 0.76 \\
\hline 0.19 & 0.44 & 0.08 & 0.09 \\
\hline 0.71 & 0.43 & 0.62 & 0.28 \\
\hline 0.61 & 0.63 & 0.64 & 0.86 \\
\hline 0.94 & 0.84 & 0.67 & 0.72 \\
\hline 0.49 & 0.46 & 0.61 & 0.89 \\
\hline 0.86 & 0.74 & 0.72 & 0.73 \\
\hline 0.95 & 0.95 & 0.99 & 0.99 \\
\hline 0.87 & 0.79 & 0.69 & 0.75 \\
\hline 0.98 & 0.68 & 0.78 & 0.66 \\
\hline 0.98 & 0.85 & 0.97 & 0.9 \\
\hline 0.94 & 0.84 & 0.67 & 0.72 \\
\hline 0.48 & 0.47 & 0.61 & 0.89 \\
\hline 0.05 & 0.42 & 0.06 & 0.06 \\
\hline 0.94 & 0.83 & 0.68 & 0.45 \\
\hline 0.01 & 0.62 & 0.67 & 0.62 \\
\hline 0.97 & 0.81 & 0.75 & 0.72 \\
\hline 0.49 & 0.41 & 0.1 & 0.86 \\
\hline 0.89 & 0.84 & 0.77 & 0.77 \\
\hline 0.67 & 0.53 & 0.4 & 0.94 \\
\hline 0.85 & 0.78 & 0.67 & 0.77 \\
\hline 1 & 0.92 & 0.99 & 0.95 \\
\hline 1 & 0.92 & 0.99 & 0.95 \\
\hline 0.95 & 0.92 & 0.97 & 0.93 \\
\hline 0.99 & 0.74 & 0.92 & 0.9 \\
\hline 0.31 & 0.24 & 0.39 & 0.5 \\
\hline 0.87 & 0.41 & 0.65 & 0.96 \\
\hline 0.9 & 0.59 & 0.94 & 0.95 \\
\hline 0.07 & 0.32 & 0.42 & 0.17 \\
\hline 0.99 & 0.96 & 0.95 & 0.87 \\
\hline 0.84 & 0.97 & 0.84 & 0.96 \\
\hline 0.93 & 0.77 & 0.54 & 0.2 \\
\hline 0.78 & 0.92 & 0.84 & 0.57 \\
\hline 0.83 & -0.23 & 0.51 & 0.13 \\
\hline 0.81 & 0.97 & 0.92 & 0.94 \\
\hline 0.55 & -0.2 & 0.23 & 0.67 \\
\hline 0.84 & 0.97 & 0.84 & 0.96 \\
\hline 0.77 & 0.91 & 0.71 & 0.72 \\
\hline 0.68 & 0.98 & 0.92 & 0.89 \\
\hline 0.83 & 0.97 & 0.91 & 0.96 \\
\hline 0.22 & 0.02 & 0.65 & 0.36 \\
\hline 0.81 & 0.93 & 0.8 & 0.7 \\
\hline 0.86 & 0.97 & 0.86 & 0.95 \\
\hline 0.75 & 0.98 & 0.93 & 0.9 \\
\hline 0.72 & 0.44 & 0.61 & 0.28 \\
\hline 0.74 & 0.98 & 0.93 & 0.9 \\
\hline 0.65 & 0.96 & 0.92 & 0.93 \\
\hline 0.73 & 0.68 & 0.6 & 0.6 \\
\hline 0.79 & 0.69 & 0.6 & 0.36 \\
\hline 0.69 & 0.72 & 0.62 & 0.35 \\
\hline 0.33 & 0.07 & 0.09 & 0.19 \\
\hline 0.46 & 0 & 0.09 & 0.17 \\
\hline 0.08 & 0.58 & 0.21 & 0.92 \\
\hline 0.51 & -0.19 & 0.17 & 0.74 \\
\hline 0.27 & 0.72 & 0.39 & 0.92 \\
\hline 0.19 & 0.73 & 0.33 & 0.94 \\
\hline 0.85 & 0.54 & 0.39 & 0.19 \\
\hline 0.37 & 0.78 & 0.48 & 0.94 \\
\hline 0.53 & 0.9 & 0.6 & 0.88 \\
\hline 0.17 & 0.81 & 0.37 & 0.9 \\
\hline 0.37 & 0.78 & 0.48 & 0.94 \\
\hline 0.87 & 0.87 & 0.72 & 0.69 \\
\hline 0.8 & 0.86 & 0.6 & 0.82 \\
\hline 0.69 & 0.83 & 0.63 & 0.91 \\
\hline 0.73 & 0.84 & 0.64 & 0.91 \\
\hline 0.93 & 0.7 & 0.63 & 0.99 \\
\hline 0.9 & 0.84 & 0.76 & 0.95 \\
\hline 0.68 & 0.75 & 0.36 & 0.94 \\
\hline 0.79 & 0.8 & 0.52 & 0.93 \\
\hline 0.65 & 0.88 & 0.59 & 0.92 \\
\hline 0.84 & 0.74 & 0.17 & 0.89 \\
\hline 0.8 & 0.71 & 0.72 & 0.71 \\
\hline 0.46 & 0 & 0.09 & 0.17 \\
\hline 0.6 & 0.87 & 0.62 & 0.83 \\
\hline 0.23 & 0.73 & 0.36 & 0.94 \\
\hline 0.27 & 0.72 & 0.39 & 0.92 \\
\hline 0.48 & 0.64 & 0.69 & 0.64 \\
\hline 0.97 & 0.9 & 0.78 & 0.96 \\
\hline 0.91 & 0.77 & 0.85 & 0.86 \\
\hline 0.94 & 0.74 & 0.98 & 0.99 \\
\hline 0.31 & -0.05 & 0 & 0.06 \\
\hline
\end{tabular}


Wavelet_HLH_GLDZM_HILDE Wavelet_HLH_GLDZM_HISDE Wavelet_HLH_GLDZM_IN Wavelet_HLH_GLDZM_INN Wavelet_HLH_GLDZM_IV Wavelet_HLH_GLDZM_LDE Wavelet_HLH_GLDZM_LIE Wavelet_HLH_GLDZM_LILDE Wavelet_HLH_GLDZM_LISDE Wavelet_HLH_GLDZM_SDE Wavelet_HLH_GLDZM_ZP Wavelet_HLH_GLRLM_GLN Wavelet_HLH_GLRLM_GLNN Wavelet_HLH_GLRLM_GLV Wavelet_HLH_GLRLM HGRE Wavelet_HLH_GLRLM_LGRE Wavelet_HLH_GLRLM_LRE Wavelet_HLH_GLRLM_LRHGE Wavelet_HLH_GLRLM_LRLGE Wavelet_HLH_GLRLM_RE Wavelet_HLH_GLRLM_RLN Wavelet_HLH_GLRLM_RLNN Wavelet_HLH_GLRLM_RLV Wavelet_HLH_GLRLM_RP Wavelet_HLH_GLRLM_SRE Wavelet_HLH_GLRLM SRHGE Wavelet_HLH_GLRLM_SRLGE Wavelet_HLH_GLSZM_HIE Wavelet_HLH_GLSZM_HILAE Wavelet_HLH_GLSZM_HISAE Wavelet_HLH_GLSZM_IN Wavelet_HLH_GLSZM_INN Wavelet_HLH_GLSZM_IV Wavelet_HLH_GLSZM_LAE Wavelet_HLH_GLSZM_LIE Wavelet_HLH_GLSZM_LILAE Wavelet_HLH_GLSZM_LISAE Wavelet_HLH_GLSZM_SAE Wavelet_HLH_GLSZM_SZN Wavelet_HLH_GLSZM_SZNN Wavelet_HLH_GLSZM_SZV Wavelet_HLH_GLSZM_ZE Wavelet_HLH_GLSZM_ZP Wavelet_HLH_IH_cov Wavelet_HLH_IH_energy Wavelet_HLH_IH_entropy Wavelet_HLH_IH_iqr Wavelet_HLH_IH_kurtosis Wavelet_HLH_IH_max Wavelet_HLH_IH_maxgrad Wavelet_HLH_IH_maxgradi Wavelet_HLH_IH_mean Wavelet_HLH_IH_meand Wavelet_HLH_IH_median Wavelet_HLH_IH_mediand Wavelet_HLH_IH_min Wavelet_HLH_IH_mingrad Wavelet_HLH_IH_mingradi Wavelet_HLH_IH_mode Wavelet_HLH_IH_p10 Wavelet_HLH_IH_p90 Wavelet_HLH_IH_qcod Wavelet_HLH_IH_range Wavelet_HLH_IH_rmeand Wavelet_HLH_IH_rms Wavelet_HLH_IH_skewness Wavelet_HLH_IH_std Wavelet_HLH_IH_uniformity Wavelet_HLH_IH_var Wavelet_HLH_NGLDM_DE Wavelet_HLH_NGLDM_DN Wavelet_HLH_NGLDM_DNN Wavelet_HLH_NGLDM_DV Wavelet_HLH_NGLDM_GLN Wavelet_HLH_NGLDM_GLNN Wavelet_HLH_NGLDM_GLV Wavelet_HLH_NGLDM_HGE Wavelet_HLH_NGLDM_HGLDE Wavelet_HLH_NGLDM_HGSDE Wavelet_HLH_NGLDM_LDE Wavelet_HLH_NGLDM_LGE Wavelet_HLH_NGLDM_LGLDE Wavelet_HLH_NGLDM_LGSDE

\begin{tabular}{|c|c|c|c|}
\hline 0.49 & 0.47 & 0.93 & 0.63 \\
\hline 0.26 & 0.33 & 0.25 & 0.28 \\
\hline 0.99 & 0.93 & 0.97 & 0.99 \\
\hline 0.33 & 0.8 & 0.84 & 0.9 \\
\hline 0.26 & 0.72 & 0.57 & 0.91 \\
\hline 0.94 & 0.72 & 0.99 & 0.99 \\
\hline 0.79 & 0.14 & 0.35 & 0.32 \\
\hline 0.72 & 0.1 & 0.66 & 0.06 \\
\hline 0.75 & 0.19 & 0.46 & 0.61 \\
\hline 0.93 & 0.74 & 0.89 & 0.92 \\
\hline 0.78 & 0.86 & 0.62 & 0.94 \\
\hline 0.99 & 0.89 & 0.98 & 1 \\
\hline 0.68 & 0.92 & 0.69 & 0.82 \\
\hline 0.36 & 0.79 & 0.43 & 0.94 \\
\hline 0.32 & 0.07 & 0.04 & 0.2 \\
\hline 0.74 & 0.15 & 0.34 & 0.77 \\
\hline 0.75 & 0.75 & 0.73 & 0.53 \\
\hline 0.39 & 0.61 & 0.54 & 0.6 \\
\hline 0.64 & 0 & 0.1 & 0.18 \\
\hline 0.38 & 0.69 & 0.49 & 0.69 \\
\hline 0.99 & 0.92 & 0.98 & 0.99 \\
\hline 0.86 & 0.88 & 0.75 & 0.93 \\
\hline 0.67 & 0.59 & 0.74 & 0.37 \\
\hline 0.86 & 0.85 & 0.75 & 0.92 \\
\hline 0.87 & 0.9 & 0.74 & 0.92 \\
\hline 0.3 & -0.07 & 0.03 & 0.05 \\
\hline 0.75 & 0.18 & 0.4 & 0.79 \\
\hline 0.31 & -0.05 & 0 & 0.06 \\
\hline 0.95 & 0.75 & 0.64 & 0.94 \\
\hline 0.24 & -0.07 & 0.11 & 0.14 \\
\hline 0.99 & 0.93 & 0.97 & 0.99 \\
\hline 0.33 & 0.8 & 0.84 & 0.9 \\
\hline 0.26 & 0.72 & 0.57 & 0.91 \\
\hline 0.96 & 0.85 & 0.81 & 0.97 \\
\hline 0.79 & 0.14 & 0.35 & 0.32 \\
\hline 0.97 & 0.91 & 0.95 & 0.71 \\
\hline 0.59 & 0.17 & 0.33 & 0.03 \\
\hline 0.68 & 0.71 & 0.52 & 0.01 \\
\hline 0.93 & 0.89 & 0.86 & 0.98 \\
\hline 0.69 & 0.7 & 0.51 & 0.01 \\
\hline 0.96 & 0.85 & 0.81 & 0.97 \\
\hline 0.76 & 0.66 & 0.63 & 0.6 \\
\hline 0.78 & 0.86 & 0.62 & 0.94 \\
\hline 0.89 & 0.62 & 0.6 & 0.84 \\
\hline 0.92 & 0.77 & 0.92 & 0.75 \\
\hline 0.56 & 0.89 & 0.64 & 0.87 \\
\hline 0.53 & 0.8 & 0.45 & 0.92 \\
\hline 0.32 & 0.83 & 0.43 & 0.57 \\
\hline 0.31 & - & - & \\
\hline 0.98 & 0.82 & 0.92 & 0.97 \\
\hline 0.53 & -0.04 & 0.38 & 0.01 \\
\hline 0.45 & -0.01 & 0.08 & 0.19 \\
\hline 0.51 & 0.82 & 0.52 & 0.92 \\
\hline 0.46 & -0.03 & 0.11 & 0.18 \\
\hline 0.5 & 0.85 & 0.21 & 0.64 \\
\hline- & - & & \\
\hline 0.97 & 0.82 & 0.94 & 0.93 \\
\hline 0.49 & 0.14 & 0.08 & 0.19 \\
\hline 0.57 & -0.03 & 0.16 & 0.18 \\
\hline 0.59 & 0.35 & 0.38 & 0.11 \\
\hline 0.35 & 0.32 & 0.01 & 0.23 \\
\hline 0.9 & 0.65 & 0.44 & 0.9 \\
\hline 0.31 & - & - & \\
\hline 0.55 & 0.8 & 0.51 & 0.93 \\
\hline 0.43 & 0.02 & 0.04 & 0.19 \\
\hline 0.19 & 0.23 & 0.41 & 0.18 \\
\hline 0.45 & 0.84 & 0.55 & 0.93 \\
\hline 0.71 & 0.9 & 0.7 & 0.81 \\
\hline 0.37 & 0.79 & 0.43 & 0.95 \\
\hline 0.8 & 0.47 & 0.68 & 0.68 \\
\hline 0.96 & 0.92 & 0.98 & 0.94 \\
\hline 0.78 & 0.77 & 0.65 & 0.98 \\
\hline 0.92 & 0.6 & 0.76 & 0.79 \\
\hline 0.99 & 0.85 & 0.94 & 0.98 \\
\hline 0.71 & 0.9 & 0.7 & 0.81 \\
\hline 0.37 & 0.79 & 0.43 & 0.95 \\
\hline 0.32 & 0.07 & 0.04 & 0.2 \\
\hline 0.9 & 0.75 & 0.69 & 0.92 \\
\hline 0.17 & 0.83 & 0.44 & 0.76 \\
\hline 0.91 & 0.82 & 0.76 & 0.85 \\
\hline 0.72 & 0.14 & 0.35 & 0.8 \\
\hline 0.29 & 0.81 & 0.44 & 0.51 \\
\hline 0.84 & 0.27 & 0.39 & 0.5 \\
\hline
\end{tabular}


Wavelet_HLH_NGLDM_SDE Wavelet_HLH_NGLDM_SM Wavelet_HLH_NGLDM_SM2 Wavelet_HLH_NGTDM_busyness Wavelet_HLH_NGTDM_coarseness Wavelet_HLH_NGTDM_complexity Wavelet_HLH_NGTDM_contrast Wavelet_HLH_NGTDM_strength Wavelet_HLH_Stats_cov Wavelet_HLH_Stats_energy Wavelet_HLH_Stats_iqr Wavelet_HLH_Stats_kurtosis Wavelet_HLH_Stats_max Wavelet_HLH_Stats_mean Wavelet_HLH_Stats_meand Wavelet_HLH_Stats_median Wavelet_HLH_Stats_mediand Wavelet_HLH_Stats_min Wavelet_HLH_Stats_p10 Wavelet_HLH_Stats_p90 Wavelet_HLH_Stats_qcod Wavelet_HLH_Stats_range Wavelet_HLH_Stats_rmeand Wavelet_HLH_Stats_rms Wavelet_HLH_Stats_skewness Wavelet_HLH_Stats_std Wavelet_HLH_Stats_var Wavelet_HLL_Fractal_average Wavelet_HLL_Fractal_lacunarity Wavelet_HLL_Fractal_sd Wavelet_HLL_GLCM_autocorr Wavelet_HLL_GLCM_average Wavelet_HLL_GLCM_clusProm Wavelet_HLL_GLCM_clusShade Wavelet_HLL_GLCM_clusTend Wavelet_HLL_GLCM_contrast Wavelet_HLL_GLCM_correl1 Wavelet_HLL_GLCM_diffAvrg Wavelet_HLL_GLCM_diffEntro Wavelet_HLL_GLCM_diffVar Wavelet_HLL_GLCM_dissimilar Wavelet_HLL_GLCM_energy Wavelet_HLL_GLCM_entrop2 Wavelet_HLL_GLCM_homogeneity1 Wavelet_HLL_GLCM_homogeneity2 Wavelet_HLL_GLCM_infoCorr1 Wavelet_HLL_GLCM_infoCorr2 Wavelet_HLL_GLCM_invDiffmomnor Wavelet_HLL_GLCM_invDiffnorm Wavelet_HLL_GLCM_inverseVar Wavelet_HLL_GLCM_maxCorr Wavelet_HLL_GLCM_maxProb Wavelet_HLL_GLCM_sumAvg Wavelet_HLL_GLCM_sumEntro Wavelet_HLL_GLCM_sumSquares Wavelet_HLL_GLCM_sumVar Wavelet_HLL_GLDZM_DZE Wavelet_HLL_GLDZM_DZN Wavelet_HLL_GLDZM_DZNN Wavelet_HLL_GLDZM_DZV Wavelet_HLL_GLDZM_HIE Wavelet_HLL_GLDZM_HILDE Wavelet_HLL_GLDZM_HISDE Wavelet_HLL_GLDZM_IN Wavelet_HLL_GLDZM_INN Wavelet_HLL_GLDZM_IV Wavelet_HLL_GLDZM_LDE Wavelet_HLL_GLDZM_LIE Wavelet_HLL_GLDZM_LILDE Wavelet_HLL_GLDZM_LISDE Wavelet_HLL_GLDZM_SDE Wavelet_HLL_GLDZM_ZP Wavelet_HLL_GLRLM_GLN Wavelet_HLL_GLRLM_GLNN Wavelet_HLL_GLRLM_GLV Wavelet_HLL_GLRLM_HGRE Wavelet_HLL_GLRLM_LGRE Wavelet HLL GLRLM LRE Wavelet_HLL_GLRLM_LRHGE Wavelet_HLL_GLRLM_LRLGE Wavelet_HLL_GLRLM_RE Wavelet HLL GLRLM RLN Wavelet_HLL_GLRLM_RLNN

\begin{tabular}{|c|c|c|c|}
\hline 0.77 & 0.83 & 0.62 & 0.95 \\
\hline 1 & 0.92 & 0.97 & 1 \\
\hline 1 & 0.92 & 0.97 & 1 \\
\hline 0.92 & 0.95 & 0.99 & 0.97 \\
\hline 0.99 & 0.76 & 0.93 & 0.94 \\
\hline 0.33 & 0.65 & 0.32 & 0.82 \\
\hline 0.71 & 0.68 & 0.41 & 0.97 \\
\hline 0.88 & 0.71 & 0.93 & 0.93 \\
\hline 0.19 & 0.02 & 0.01 & 0.01 \\
\hline 0.98 & 0.97 & 0.96 & 0.81 \\
\hline 0.9 & 0.94 & 0.83 & 0.92 \\
\hline 0.32 & 0.84 & 0.42 & 0.58 \\
\hline 0.64 & 0.88 & 0.79 & 0.58 \\
\hline 0.45 & 0.09 & 0.2 & 0.2 \\
\hline 0.86 & 0.94 & 0.9 & 0.89 \\
\hline 0.16 & -0.49 & 0.1 & 0.49 \\
\hline 0.9 & 0.93 & 0.83 & 0.92 \\
\hline 0.86 & 0.8 & 0.82 & 0.71 \\
\hline 0.87 & 0.91 & 0.89 & 0.91 \\
\hline 0.71 & 0.95 & 0.88 & 0.85 \\
\hline 0.01 & 0 & 0.13 & 0.01 \\
\hline 0.83 & 0.89 & 0.89 & 0.71 \\
\hline 0.89 & 0.94 & 0.85 & 0.92 \\
\hline 0.82 & 0.94 & 0.91 & 0.85 \\
\hline 0.19 & 0.24 & 0.43 & 0.2 \\
\hline 0.82 & 0.94 & 0.91 & 0.85 \\
\hline 0.7 & 0.94 & 0.9 & 0.83 \\
\hline 0.88 & 0.83 & 0.27 & 0.47 \\
\hline 0.85 & 0.59 & 0.62 & 0.13 \\
\hline 0.7 & 0.58 & 0.68 & 0.13 \\
\hline 0.36 & 0.1 & 0.17 & 0.25 \\
\hline 0.46 & 0.08 & 0.2 & 0.25 \\
\hline 0.02 & 0.77 & 0.75 & 0.27 \\
\hline 0.59 & 0.18 & 0.01 & 0.06 \\
\hline 0.02 & 0.86 & 0.81 & 0.48 \\
\hline 0.38 & 0.82 & 0.85 & 0.63 \\
\hline 0.65 & 0.57 & 0.41 & 0.65 \\
\hline 0.51 & 0.84 & 0.84 & 0.74 \\
\hline 0.53 & 0.71 & 0.85 & 0.83 \\
\hline 0.21 & 0.62 & 0.89 & 0.74 \\
\hline 0.51 & 0.84 & 0.84 & 0.74 \\
\hline 0.94 & 0.76 & 0.82 & 0.9 \\
\hline 0.85 & 0.58 & 0.78 & 0.79 \\
\hline 0.72 & 0.83 & 0.8 & 0.87 \\
\hline 0.76 & 0.82 & 0.8 & 0.86 \\
\hline 0.94 & 0.76 & 0.74 & 0.81 \\
\hline 0.93 & 0.84 & 0.66 & 0.89 \\
\hline 0.79 & 0.83 & 0.85 & 0.65 \\
\hline 0.87 & 0.84 & 0.84 & 0.77 \\
\hline 0.63 & 0.83 & 0.84 & 0.73 \\
\hline 0.75 & 0.73 & 0.56 & 0.77 \\
\hline 0.95 & 0.81 & 0.82 & 0.92 \\
\hline 0.46 & 0.08 & 0.2 & 0.25 \\
\hline 0.6 & 0.64 & 0.8 & 0.65 \\
\hline 0.16 & 0.85 & 0.85 & 0.56 \\
\hline 0.02 & 0.86 & 0.81 & 0.48 \\
\hline 0.5 & 0.4 & 0.87 & 0.72 \\
\hline 0.98 & 0.9 & 0.9 & 0.93 \\
\hline 0.85 & 0.71 & 0.87 & 0.94 \\
\hline 0.91 & 0.7 & 0.98 & 0.97 \\
\hline 0.3 & 0.16 & 0.34 & 0.18 \\
\hline 0.6 & 0.52 & 0.95 & 0.88 \\
\hline 0.16 & 0.44 & 0.31 & 0.3 \\
\hline 1 & 0.94 & 0.99 & 0.99 \\
\hline 0.35 & 0.54 & 0.8 & 0.58 \\
\hline 0.12 & 0.74 & 0.77 & 0.46 \\
\hline 0.91 & 0.67 & 0.99 & 0.98 \\
\hline 0.79 & 0.61 & 0.02 & 0.83 \\
\hline 0.82 & 0.38 & 0.8 & 0.04 \\
\hline 0.78 & 0.66 & 0.15 & 0.75 \\
\hline 0.89 & 0.69 & 0.91 & 0.96 \\
\hline 0.73 & 0.88 & 0.89 & 0.95 \\
\hline 0.99 & 0.89 & 0.99 & 1 \\
\hline 0.8 & 0.74 & 0.81 & 0.84 \\
\hline 0.16 & 0.85 & 0.85 & 0.62 \\
\hline 0.32 & 0.29 & 0.23 & 0.21 \\
\hline 0.72 & 0.71 & 0.1 & 0.57 \\
\hline 0.94 & 0.72 & 0.82 & 0.98 \\
\hline 0.4 & 0.04 & 0.48 & 0.74 \\
\hline 0.67 & 0.68 & 0.13 & 0.1 \\
\hline 0.44 & 0.57 & 0.83 & 0.39 \\
\hline 0.99 & 0.9 & 1 & 0.99 \\
\hline 0.85 & 0.84 & 0.82 & 0.9 \\
\hline
\end{tabular}


Wavelet_HLL_GLRLM_RLV Wavelet_HLL_GLRLM_RP Wavelet_HLL_GLRLM_SRE Wavelet_HLL_GLRLM_SRHGE Wavelet_HLL_GLRLM_SRLGE Wavelet_HLL_GLSZM_HIE Wavelet_HLL_GLSZM_HILAE Wavelet_HLL_GLSZM_HISAE Wavelet_HLL_GLSZM_IN Wavelet_HLL_GLSZM_INN Wavelet_HLL_GLSZM IV Wavelet_HLL_GLSZM_LAE Wavelet_HLL_GLSZM_LIE Wavelet_HLL_GLSZM_LILAE Wavelet_HLL_GLSZM LISAE Wavelet_HLL_GLSZM_SAE Wavelet_HLL_GLSZM_SZN Wavelet_HLL_GLSZM_SZNN Wavelet_HLL_GLSZM_SZV Wavelet_HLL_GLSZM_ZE Wavelet_HLL_GLSZM_ZP Wavelet_HLL_IH_cov Wavelet_HLL_IH_energy Wavelet_HLL_IH_entropy Wavelet_HLL IH iqr Wavelet_HLL_IH_kurtosis Wavelet_HLL_IH_max Wavelet_HLL_IH_maxgrad Wavelet_HLL_IH_maxgradi Wavelet_HLL_IH_mean Wavelet_HLL_IH_meand Wavelet_HLL_IH_median Wavelet_HLL_IH_mediand Wavelet_HLL_IH_min Wavelet_HLL_IH_mingrad Wavelet_HLL_IH_mingradi Wavelet_HLL_IH_mode Wavelet_HLL_IH_p10 Wavelet_HLL_IH_p90 Wavelet_HLL_IH_qcod Wavelet_HLL_IH_range Wavelet_HLL_IH_rmeand Wavelet_HLL_IH_rms Wavelet_HLL_IH_skewness Wavelet_HLL_IH_std Wavelet_HLL_IH_uniformity Wavelet_HLL_IH_var Wavelet_HLL_NGLDM_DE Wavelet_HLL_NGLDM_DN Wavelet_HLL_NGLDM_DNN Wavelet_HLL_NGLDM_DV Wavelet_HLL_NGLDM_GLN Wavelet_HLL_NGLDM_GLNN Wavelet_HLL_NGLDM_GLV Wavelet_HLL_NGLDM_HGE Wavelet_HLL_NGLDM_HGLDE Wavelet_HLL_NGLDM_HGSDE Wavelet_HLL_NGLDM_LDE Wavelet_HLL NGLDM LGE Wavelet_HLL_NGLDM_LGLDE Wavelet_HLL_NGLDM_LGSDE Wavelet_HLL_NGLDM_SDE Wavelet_HLL NGLDM SM Wavelet_HLL_NGLDM_SM2 Wavelet_HLL_NGTDM_busyness Wavelet_HLL_NGTDM coarsenes Wavelet_HLL_NGTDM_complexity Wavelet_HLL_NGTDM_contrast Wavelet_HLL_NGTDM_strength Wavelet_HLL_Stats_cov Wavelet_HLL_Stats_energy Wavelet_HLL_Stats_iqr Wavelet_HLL_Stats_kurtosis Wavelet_HLL_Stats_max Wavelet_HLL_Stats_mean Wavelet_HLL_Stats_meand Wavelet_HLL_Stats_median Wavelet_HLL_Stats_mediand Wavelet_HLL_Stats_min Wavelet_HLL_Stats_p10 Wavelet_HLL_Stats_p90 Wavelet_HLL_Stats_qcod Wavelet_HLL_Stats_range

\begin{tabular}{|c|c|c|c|}
\hline 0.95 & 0.6 & 0.84 & 0.98 \\
\hline 0.9 & 0.83 & 0.81 & 0.94 \\
\hline 0.87 & 0.84 & 0.8 & 0.92 \\
\hline 0.3 & 0.42 & 0.23 & 0.27 \\
\hline 0.73 & 0.71 & 0.17 & 0.63 \\
\hline 0.3 & 0.16 & 0.34 & 0.18 \\
\hline 0.82 & 0.8 & 0.94 & 0.84 \\
\hline 0.19 & 0.61 & 0.13 & 0.03 \\
\hline 1 & 0.94 & 0.99 & 0.99 \\
\hline 0.35 & 0.54 & 0.8 & 0.58 \\
\hline 0.12 & 0.74 & 0.77 & 0.46 \\
\hline 0.99 & 0.9 & 0.86 & 0.96 \\
\hline 0.79 & 0.61 & 0.02 & 0.83 \\
\hline 0.85 & 0.94 & 0.75 & 0.96 \\
\hline 0.86 & 0.24 & 0.3 & 0.78 \\
\hline 0.56 & 0.88 & 0.83 & 0.67 \\
\hline 0.96 & 0.88 & 0.95 & 0.92 \\
\hline 0.58 & 0.88 & 0.84 & 0.68 \\
\hline 0.99 & 0.9 & 0.86 & 0.96 \\
\hline 0.88 & 0.83 & 0.73 & 0.69 \\
\hline 0.73 & 0.88 & 0.89 & 0.95 \\
\hline 0.8 & 0.83 & 0.75 & 0.49 \\
\hline 0.84 & 0.8 & 0.98 & 0.95 \\
\hline 0.58 & 0.76 & 0.85 & 0.8 \\
\hline 0.47 & 0.83 & 0.83 & 0.7 \\
\hline 0.93 & 0.7 & 0.57 & 0.59 \\
\hline 0.31 & - & - & \\
\hline 0.99 & 0.85 & 0.92 & 0.99 \\
\hline 0.4 & -0.38 & 0.04 & 0.25 \\
\hline 0.46 & 0.11 & 0.23 & 0.18 \\
\hline 0.37 & 0.87 & 0.85 & 0.73 \\
\hline 0.5 & 0.11 & 0.23 & 0.34 \\
\hline 0.47 & 0.75 & 0.5 & 0.4 \\
\hline- & - & & \\
\hline 0.98 & 0.82 & 0.92 & 0.99 \\
\hline 0.5 & 0.75 & 0.04 & 0.62 \\
\hline 0.24 & 0.45 & 0.09 & 0.51 \\
\hline 0.69 & 0.62 & 0.11 & 0.25 \\
\hline 0.28 & 0.67 & 0.09 & 0.42 \\
\hline 0.84 & 0.81 & 0.81 & 0.53 \\
\hline 0.31 & - & - & \\
\hline 0.45 & 0.88 & 0.82 & 0.74 \\
\hline 0.42 & 0.3 & 0.24 & 0.23 \\
\hline 0.87 & 0.22 & 0.28 & 0.04 \\
\hline 0.32 & 0.85 & 0.87 & 0.74 \\
\hline 0.85 & 0.78 & 0.75 & 0.89 \\
\hline 0.17 & 0.85 & 0.85 & 0.65 \\
\hline 0.92 & 0.71 & 0.72 & 0.76 \\
\hline 0.97 & 0.91 & 0.99 & 0.98 \\
\hline 0.71 & 0.77 & 0.82 & 0.91 \\
\hline 0.96 & 0.79 & 0.86 & 0.93 \\
\hline 0.99 & 0.88 & 0.97 & 1 \\
\hline 0.85 & 0.78 & 0.75 & 0.89 \\
\hline 0.16 & 0.86 & 0.85 & 0.65 \\
\hline 0.32 & 0.29 & 0.23 & 0.23 \\
\hline 0.84 & 0.61 & 0.76 & 0.75 \\
\hline 0.11 & 0.9 & 0.53 & 0.79 \\
\hline 0.95 & 0.83 & 0.84 & 0.94 \\
\hline 0.71 & 0.71 & 0.11 & 0.57 \\
\hline 0.72 & 0.68 & 0.68 & 0.38 \\
\hline 0.9 & 0.49 & 0.71 & 0.78 \\
\hline 0.7 & 0.88 & 0.87 & 0.95 \\
\hline 1 & 0.93 & 0.99 & 0.99 \\
\hline 1 & 0.93 & 0.99 & 0.99 \\
\hline 0.91 & 0.93 & 0.99 & 0.92 \\
\hline 0.98 & 0.74 & 0.83 & 0.85 \\
\hline 0.25 & 0.89 & 0.52 & 0.41 \\
\hline 0.69 & 0.68 & 0.74 & 0.77 \\
\hline 0.87 & 0.64 & 0.78 & 0.94 \\
\hline 0.85 & 0.14 & 0.24 & 0.07 \\
\hline 0.97 & 0.98 & 0.94 & 0.6 \\
\hline 0.92 & 0.85 & 0.79 & 0.64 \\
\hline 0.93 & 0.7 & 0.57 & 0.59 \\
\hline 0.72 & 0.84 & 0.55 & 0.55 \\
\hline 0.77 & 0.62 & 0.03 & 0.62 \\
\hline 0.93 & 0.84 & 0.81 & 0.59 \\
\hline 0.8 & 0.29 & 0.03 & 0.49 \\
\hline 0.92 & 0.78 & 0.81 & 0.64 \\
\hline 0.8 & 0.74 & 0.53 & 0.74 \\
\hline 0.9 & 0.74 & 0.72 & 0.6 \\
\hline 0.84 & 0.84 & 0.77 & 0.55 \\
\hline 0.49 & -0.02 & 0.1 & 0.06 \\
\hline 0.83 & 0.84 & 0.81 & 0.79 \\
\hline
\end{tabular}


Wavelet_HLL_Stats_rmeand Wavelet_HLL_Stats_rms Wavelet_HLL_Stats_skewness Wavelet_HLL_Stats_std Wavelet_HLL_Stats_var Wavelet_LHH_Fractal_average Wavelet_LHH_Fractal_lacunarity Wavelet_LHH_Fractal_sd Wavelet_LHH_GLCM_autocorr Wavelet_LHH_GLCM_average Wavelet LHH_GLCM_clusProm Wavelet_LHH_GLCM_clusShade Wavelet_LHH_GLCM_clusTend Wavelet_LHH_GLCM_contrast Wavelet_LHH_GLCM_correl1 Wavelet_LHH_GLCM_diffAvrg Wavelet_LHH_GLCM_diffEntro Wavelet_LHH_GLCM_diffVar Wavelet_LHH_GLCM_dissimilar Wavelet_LHH_GLCM_energy Wavelet_LHH_GLCM_entrop2 Wavelet_LHH_GLCM_homogeneity1 Wavelet_LHH_GLCM_homogeneity2 Wavelet_LHH_GLCM_infoCorr1 Wavelet_LHH_GLCM infoCorr2 Wavelet_LHH_GLCM_invDiffmomno Wavelet_LHH_GLCM_invDiffnorm Wavelet_LHH_GLCM_inverseVar Wavelet_LHH_GLCM_maxCorr Wavelet_LHH_GLCM_maxProb Wavelet_LHH_GLCM_sumAvg Wavelet_LHH_GLCM_sumEntro Wavelet_LHH_GLCM_sumSquares Wavelet_LHH_GLCM_sumVar Wavelet_LHH_GLDZM_DZE Wavelet_LHH_GLDZM_DZN Wavelet_LHH_GLDZM_DZNN Wavelet_LHH_GLDZM_DZV Wavelet_LHH_GLDZM_HIE Wavelet_LHH_GLDZM_HILDE Wavelet_LHH_GLDZM_HISDE Wavelet_LHH_GLDZM_IN Wavelet_LHH_GLDZM_INN Wavelet_LHH_GLDZM_IV Wavelet_LHH_GLDZM_LDE Wavelet_LHH_GLDZM_LIE Wavelet_LHH_GLDZM_LILDE Wavelet_LHH_GLDZM_LISDE Wavelet_LHH_GLDZM_SDE Wavelet_LHH_GLDZM_ZP Wavelet_LHH_GLRLM_GLN Wavelet_LHH_GLRLM_GLNN Wavelet_LHH_GLRLM_GLV Wavelet_LHH_GLRLM_HGRE Wavelet_LHH_GLRLM_LGRE Wavelet_LHH_GLRLM_LRE Wavelet_LHH_GLRLM_LRHGE Wavelet_LHH_GLRLM_LRLGE Wavelet_LHH_GLRLM_RE Wavelet_LHH_GLRLM_RLN Wavelet_LHH_GLRLM_RLNN Wavelet_LHH_GLRLM_RLV Wavelet_LHH_GLRLM RP Wavelet_LHH_GLRLM_SRE Wavelet_LHH_GLRLM_SRHGE Wavelet_LHH_GLRLM_SRLGE Wavelet_LHH_GLSZM_HIE Wavelet_LHH_GLSZM_HILAE Wavelet_LHH_GLSZM_HISAE Wavelet_LHH_GLSZM_IN Wavelet_LHH_GLSZM_INN Wavelet_LHH_GLSZM_IV Wavelet_LHH_GLSZM_LAE Wavelet_LHH_GLSZM_LIE Wavelet_LHH_GLSZM_LILAE Wavelet_LHH_GLSZM_LISAE Wavelet_LHH_GLSZM_SAE Wavelet LHH_GLSZM SZN Wavelet_LHH_GLSZM_SZNN Wavelet_LHH_GLSZM_SZV Wavelet_LHH_GLSZM_ZE Wavelet_LHH_GLSZM_ZP Wavelet_LHH_IH_cov

\begin{tabular}{|c|c|c|c|}
\hline 0.92 & 0.84 & 0.8 & 0.61 \\
\hline 0.94 & 0.84 & 0.79 & 0.57 \\
\hline 0.87 & 0.22 & 0.28 & 0.06 \\
\hline 0.92 & 0.83 & 0.81 & 0.57 \\
\hline 0.88 & 0.79 & 0.84 & 0.5 \\
\hline 0.71 & 0.76 & 0.52 & 0.4 \\
\hline 0.18 & 0.76 & 0.35 & 0.25 \\
\hline 0.09 & 0.67 & 0.34 & 0.3 \\
\hline 0.07 & 0.42 & 0.2 & 0.37 \\
\hline 0.22 & 0.38 & 0.19 & 0.36 \\
\hline 0.02 & 0.52 & 0.45 & 0.38 \\
\hline 0.4 & 0.16 & 0.2 & 0.91 \\
\hline 0.41 & 0.6 & 0.52 & 0.51 \\
\hline 0.35 & 0.62 & 0.6 & 0.43 \\
\hline 0.14 & 0.97 & 0.86 & 0.43 \\
\hline 0.57 & 0.71 & 0.59 & 0.55 \\
\hline 0.48 & 0.77 & 0.66 & 0.55 \\
\hline 0.15 & 0.59 & 0.7 & 0.47 \\
\hline 0.57 & 0.71 & 0.59 & 0.55 \\
\hline 0.78 & 0.91 & 0.53 & 0.46 \\
\hline 0.58 & 0.79 & 0.61 & 0.49 \\
\hline 0.66 & 0.82 & 0.57 & 0.6 \\
\hline 0.66 & 0.82 & 0.57 & 0.6 \\
\hline 0.94 & 0.68 & 0.63 & 0.79 \\
\hline 0.89 & 0.75 & 0.79 & 0.95 \\
\hline 0.8 & 0.64 & 0.6 & 0.46 \\
\hline 0.82 & 0.73 & 0.58 & 0.57 \\
\hline 0.62 & 0.67 & 0.45 & 0.6 \\
\hline 0.65 & 0.65 & 0.56 & 0.33 \\
\hline 0.77 & 0.85 & 0.47 & 0.52 \\
\hline 0.22 & 0.38 & 0.19 & 0.36 \\
\hline 0.56 & 0.75 & 0.64 & 0.58 \\
\hline 0.39 & 0.61 & 0.55 & 0.47 \\
\hline 0.41 & 0.6 & 0.52 & 0.51 \\
\hline 0.25 & 0.76 & 0.73 & 0.23 \\
\hline 0.85 & 0.9 & 0.61 & 0.91 \\
\hline 0.8 & 0.84 & 0.8 & 0.73 \\
\hline 0.78 & 0.79 & 0.98 & 0.98 \\
\hline 0.05 & 0.37 & 0.13 & 0.4 \\
\hline 0.16 & 0.76 & 0.96 & 0.75 \\
\hline 0.03 & 0.25 & 0.59 & 0.11 \\
\hline 0.97 & 0.92 & 0.87 & 0.99 \\
\hline 0.49 & 0.5 & 0.73 & 0.65 \\
\hline 0.29 & 0.46 & 0.54 & 0.35 \\
\hline 0.73 & 0.8 & 0.97 & 0.96 \\
\hline 0.52 & 0.57 & 0.3 & 0.72 \\
\hline 0.49 & 0.45 & 0.82 & 0.5 \\
\hline 0.52 & 0.59 & 0.22 & 0.71 \\
\hline 0.76 & 0.84 & 0.83 & 0.78 \\
\hline 0.77 & 0.74 & 0.63 & 0.68 \\
\hline 0.98 & 0.88 & 0.96 & 0.98 \\
\hline 0.65 & 0.84 & 0.64 & 0.59 \\
\hline 0.42 & 0.62 & 0.59 & 0.49 \\
\hline 0.06 & 0.36 & 0.18 & 0.4 \\
\hline 0.5 & 0.51 & 0.25 & 0.82 \\
\hline 0.77 & 0.87 & 0.35 & 0.66 \\
\hline 0.18 & 0.77 & 0.39 & 0.16 \\
\hline 0.47 & 0.54 & 0.24 & 0.63 \\
\hline 0.39 & 0.33 & 0.6 & 0.43 \\
\hline 0.94 & 0.95 & 0.86 & 1 \\
\hline 0.75 & 0.85 & 0.66 & 0.69 \\
\hline 0.77 & 0.81 & 0.22 & 0.67 \\
\hline 0.75 & 0.88 & 0.67 & 0.68 \\
\hline 0.74 & 0.88 & 0.68 & 0.68 \\
\hline 0.04 & 0.28 & 0.16 & 0.27 \\
\hline 0.51 & 0.53 & 0.37 & 0.84 \\
\hline 0.05 & 0.37 & 0.13 & 0.4 \\
\hline 0.85 & 0.83 & 0.81 & 0.92 \\
\hline 0 & 0.28 & 0.1 & 0.28 \\
\hline 0.97 & 0.92 & 0.87 & 0.99 \\
\hline 0.49 & 0.5 & 0.73 & 0.65 \\
\hline 0.29 & 0.46 & 0.54 & 0.35 \\
\hline 0.77 & 0.9 & 0.76 & 0.89 \\
\hline 0.52 & 0.57 & 0.3 & 0.72 \\
\hline 0.64 & 0.92 & 0.7 & 0.86 \\
\hline 0.54 & 0.63 & 0.36 & 0.8 \\
\hline 0.65 & 0.5 & 0.63 & 0.23 \\
\hline 0.67 & 0.92 & 0.79 & 0.85 \\
\hline 0.68 & 0.49 & 0.62 & 0.18 \\
\hline 0.77 & 0.9 & 0.76 & 0.89 \\
\hline 0.63 & 0.58 & 0.55 & 0.63 \\
\hline 0.77 & 0.74 & 0.63 & 0.68 \\
\hline 0.72 & 0.69 & 0.62 & 0.74 \\
\hline
\end{tabular}


Wavelet_LHH_IH_energy Wavelet_LHH_IH_entropy Wavelet_LHH_IH_iqr Wavelet_LHH_IH_kurtosis Wavelet_LHH_IH_max Wavelet_LHH_IH_maxgrad Wavelet_LHH_IH_maxgradi Wavelet_LHH_IH_mean Wavelet_LHH_IH_meand Wavelet_LHH_IH_median Wavelet_LHH_IH_mediand Wavelet_LHH_IH_min Wavelet_LHH_IH_mingrad Wavelet_LHH_IH_mingradi Wavelet_LHH_IH_mode Wavelet_LHH_IH_p10 Wavelet_LHH_IH_p90 Wavelet_LHH_IH_qcod Wavelet_LHH_IH_range Wavelet_LHH_IH_rmeand Wavelet_LHH_IH_rms

Wavelet_LHH_IH_skewness Wavelet_LHH_IH_std Wavelet_LHH_IH_uniformity Wavelet_LHH_IH_var

Wavelet_LHH_NGLDM_DE Wavelet_LHH_NGLDM_DN Wavelet_LHH_NGLDM_DNN Wavelet_LHH_NGLDM_DV Wavelet_LHH_NGLDM_GLN Wavelet_LHH_NGLDM_GLNN Wavelet_LHH_NGLDM_GLV Wavelet_LHH_NGLDM_HGE Wavelet_LHH_NGLDM_HGLDE Wavelet_LHH_NGLDM_HGSDE Wavelet_LHH_NGLDM_LDE

Wavelet_LHH_NGLDM_LGE Wavelet_LHH_NGLDM_LGLDE Wavelet_LHH_NGLDM_LGSDE Wavelet_LHH_NGLDM_SDE Wavelet_LHH_NGLDM_SM Wavelet_LHH_NGLDM_SM2 Wavelet_LHH_NGTDM_busyness Wavelet_LHH_NGTDM_coarseness Wavelet_LHH_NGTDM_complexity Wavelet_LHH_NGTDM_contrast Wavelet_LHH_NGTDM_strength Wavelet_LHH_Stats_cov

Wavelet_LHH_Stats_energy Wavelet_LHH_Stats_iqr Wavelet_LHH_Stats_kurtosis Wavelet_LHH_Stats_max Wavelet_LHH_Stats_mean Wavelet_LHH_Stats_meand Wavelet_LHH_Stats_median Wavelet_LHH_Stats_mediand Wavelet_LHH_Stats_min Wavelet_LHH_Stats_p10 Wavelet_LHH_Stats_p90 Wavelet_LHH_Stats_qcod Wavelet_LHH_Stats_range Wavelet_LHH_Stats_rmeand Wavelet_LHH_Stats_rms Wavelet_LHH_Stats_skewness Wavelet_LHH_Stats_std Wavelet_LHH_Stats_var Wavelet_LHL_Fractal_average Wavelet_LHL_Fractal_lacunarity Wavelet_LHL_Fractal_sd

Wavelet_LHL_GLCM_autocorr Wavelet_LHL_GLCM_average Wavelet_LHL_GLCM_clusProm Wavelet_LHL_GLCM_clusShade Wavelet_LHL_GLCM_clusTend Wavelet_LHL_GLCM_contrast Wavelet_LHL_GLCM_correl1 Wavelet_LHL_GLCM_diffAvrg Wavelet_LHL_GLCM diffEntro Wavelet_LHL_GLCM_diffVar Wavelet_LHL_GLCM_dissimilar Wavelet_LHL_GLCM_energy Wavelet_LHL_GLCM_entrop2 Wavelet_LHL_GLCM_homogeneity1

\begin{tabular}{|c|c|c|c|}
\hline 0.73 & 0.86 & 1 & 0.98 \\
\hline 0.56 & 0.78 & 0.65 & 0.6 \\
\hline 0.71 & 0.68 & 0.49 & 0.28 \\
\hline 0.26 & 0.92 & 0.5 & 0.37 \\
\hline 0.31 & - & - & \\
\hline 0.92 & 0.8 & 0.88 & 0.9 \\
\hline 0.21 & 0.42 & 0.16 & 0.03 \\
\hline 0.2 & 0.39 & 0.19 & 0.4 \\
\hline 0.62 & 0.7 & 0.58 & 0.57 \\
\hline 0.21 & 0.37 & 0.26 & 0.48 \\
\hline 0.64 & 0.8 & 0.4 & 0.43 \\
\hline- & - & & \\
\hline 0.92 & 0.81 & 0.85 & 0.97 \\
\hline 0.26 & 0.48 & 0.12 & 0.5 \\
\hline 0.23 & 0.43 & 0.33 & 0.13 \\
\hline 0.32 & 0.68 & 0.45 & 0.23 \\
\hline 0.17 & 0.19 & 0.2 & 0.12 \\
\hline 0.68 & 0.7 & 0.52 & 0.37 \\
\hline 0.31 & - & - & \\
\hline 0.73 & 0.69 & 0.49 & 0.46 \\
\hline 0.19 & 0.34 & 0.17 & 0.4 \\
\hline 0.48 & 0.59 & 0.54 & 0.02 \\
\hline 0.53 & 0.69 & 0.63 & 0.61 \\
\hline 0.69 & 0.88 & 0.62 & 0.58 \\
\hline 0.44 & 0.64 & 0.57 & 0.5 \\
\hline 0.78 & 0.76 & 0.8 & 0.75 \\
\hline 0.84 & 0.94 & 0.9 & 0.99 \\
\hline 0.8 & 0.71 & 0.53 & 0.68 \\
\hline 0.78 & 0.75 & 0.53 & 0.57 \\
\hline 0.94 & 0.84 & 0.9 & 0.96 \\
\hline 0.69 & 0.88 & 0.62 & 0.58 \\
\hline 0.43 & 0.64 & 0.57 & 0.5 \\
\hline 0.06 & 0.36 & 0.18 & 0.4 \\
\hline 0.38 & 0.85 & 0.63 & 0.51 \\
\hline 0 & 0.42 & 0.57 & 0.31 \\
\hline 0.76 & 0.89 & 0.59 & 0.62 \\
\hline 0.5 & 0.51 & 0.24 & 0.82 \\
\hline 0.73 & 0.85 & 0.53 & 0.56 \\
\hline 0.56 & 0.64 & 0.55 & 0.96 \\
\hline 0.76 & 0.73 & 0.64 & 0.64 \\
\hline 1 & 0.9 & 0.98 & 0.98 \\
\hline 1 & 0.9 & 0.98 & 0.98 \\
\hline 0.85 & 0.93 & 0.91 & 0.97 \\
\hline 0.96 & 0.69 & 0.91 & 0.94 \\
\hline 0.01 & 0.43 & 0.38 & 0.25 \\
\hline 0.84 & 0.74 & 0.6 & 0.5 \\
\hline 0.83 & 0.77 & 0.8 & 0.87 \\
\hline 0.25 & 0.28 & 0.11 & 0 \\
\hline 0.91 & 0.96 & 0.94 & 0.79 \\
\hline 0.49 & 0.98 & 0.91 & 0.9 \\
\hline 0.25 & 0.94 & 0.48 & 0.38 \\
\hline 0.66 & 0.93 & 0.87 & 0.37 \\
\hline 0.89 & 0.81 & 0.43 & 0.56 \\
\hline 0.31 & 0.98 & 0.95 & 0.87 \\
\hline 0.62 & 0.17 & 0.67 & 0.3 \\
\hline 0.49 & 0.98 & 0.91 & 0.89 \\
\hline 0.16 & 0.89 & 0.81 & 0.46 \\
\hline 0.54 & 0.97 & 0.93 & 0.9 \\
\hline 0.17 & 0.98 & 0.94 & 0.89 \\
\hline 0.1 & 0.43 & 0.01 & 0.15 \\
\hline 0.44 & 0.92 & 0.86 & 0.43 \\
\hline 0.46 & 0.98 & 0.93 & 0.91 \\
\hline 0.2 & 0.99 & 0.94 & 0.77 \\
\hline 0.47 & 0.63 & 0.55 & 0.03 \\
\hline 0.19 & 0.99 & 0.94 & 0.76 \\
\hline 0.07 & 0.96 & 0.9 & 0.68 \\
\hline 0.68 & 0.59 & 0.54 & 0.24 \\
\hline 0.06 & 0.52 & 0.56 & 0.19 \\
\hline 0.08 & 0.55 & 0.57 & 0.24 \\
\hline 0.2 & 0.11 & 0.42 & 0.14 \\
\hline 0.25 & 0.13 & 0.37 & 0.13 \\
\hline 0.15 & 0.77 & 0.67 & 0.35 \\
\hline 0.5 & 0.04 & 0.53 & 0 \\
\hline 0.3 & 0.83 & 0.79 & 0.43 \\
\hline 0.13 & 0.83 & 0.8 & 0.31 \\
\hline 0.69 & 0.94 & 0.91 & 0.58 \\
\hline 0.41 & 0.83 & 0.82 & 0.42 \\
\hline 0.6 & 0.78 & 0.86 & 0.5 \\
\hline 0.05 & 0.8 & 0.86 & 0.38 \\
\hline 0.41 & 0.83 & 0.82 & 0.42 \\
\hline 0.87 & 0.57 & 0.82 & 0.67 \\
\hline 0.88 & 0.57 & 0.84 & 0.46 \\
\hline 0.77 & 0.79 & 0.82 & 0.58 \\
\hline
\end{tabular}


Wavelet_LHL_GLCM_homogeneity2 Wavelet_LHL_GLCM_infoCorr1 Wavelet_LHL_GLCM infoCorr2 Wavelet_LHL_GLCM_invDiffmomno Wavelet_LHL_GLCM_invDiffnorm Wavelet_LHL_GLCM_inverseVar Wavelet_LHL_GLCM_maxCorr Wavelet_LHL_GLCM_maxProb Wavelet_LHL_GLCM_sumAvg Wavelet_LHL_GLCM_sumEntro Wavelet_LHL_GLCM_sumSquares Wavelet_LHL_GLCM_sumVar Wavelet_LHL_GLDZM_DZE Wavelet_LHL_GLDZM_DZN Wavelet_LHL_GLDZM_DZNN Wavelet_LHL_GLDZM_DZV Wavelet_LHL_GLDZM_HIE Wavelet_LHL_GLDZM_HILDE Wavelet_LHL_GLDZM_HISDE Wavelet_LHL_GLDZM_IN Wavelet_LHL_GLDZM_INN Wavelet_LHL_GLDZM_IV Wavelet_LHL_GLDZM_LDE Wavelet_LHL_GLDZM_LIE Wavelet_LHL_GLDZM_LILDE Wavelet_LHL_GLDZM_LISDE Wavelet_LHL_GLDZM_SDE Wavelet_LHL_GLDZM_ZP Wavelet_LHL_GLRLM_GLN Wavelet_LHL_GLRLM_GLNN Wavelet_LHL_GLRLM_GLV Wavelet_LHL_GLRLM_HGRE Wavelet_LHL_GLRLM_LGRE Wavelet_LHL_GLRLM_LRE Wavelet_LHL_GLRLM_LRHGE Wavelet_LHL_GLRLM_LRLGE Wavelet_LHL_GLRLM_RE Wavelet_LHL_GLRLM_RLN Wavelet_LHL_GLRLM_RLNN Wavelet_LHL_GLRLM_RLV Wavelet_LHL_GLRLM_RP Wavelet_LHL_GLRLM_SRE Wavelet_LHL_GLRLM_SRHGE Wavelet_LHL_GLRLM_SRLGE Wavelet_LHL_GLSZM_HIE Wavelet_LHL_GLSZM_HILAE Wavelet_LHL_GLSZM_HISAE Wavelet_LHL_GLSZM IN Wavelet_LHL_GLSZM_INN Wavelet_LHL_GLSZM_IV Wavelet_LHL_GLSZM_LAE Wavelet_LHL_GLSZM LIE Wavelet_LHL_GLSZM_LILAE Wavelet_LHL_GLSZM_LISAE Wavelet_LHL_GLSZM_SAE Wavelet_LHL_GLSZM_SZN Wavelet_LHL_GLSZM_SZNN Wavelet_LHL_GLSZM_SZV Wavelet_LHL_GLSZM_ZE Wavelet_LHL_GLSZM_ZP Wavelet_LHL_IH_cov Wavelet_LHL_IH_energy Wavelet_LHL_IH_entropy Wavelet_LHL_IH_iqr Wavelet_LHL_IH_kurtosis Wavelet_LHL IH max Wavelet_LHL_IH_maxgrad Wavelet_LHL_IH_maxgradi Wavelet_LHL_IH_mean Wavelet_LHL_IH_meand Wavelet_LHL_IH_median Wavelet_LHL_IH_mediand Wavelet_LHL_IH_min Wavelet_LHL_IH_mingrad Wavelet_LHL_IH_mingradi Wavelet_LHL_IH_mode Wavelet_LHL_IH_p10 Wavelet_LHL_IH p90 Wavelet_LHL_IH_qcod Wavelet_LHL_IH_range Wavelet_LHL_IH_rmeand Wavelet_LHL_IH_rms Wavelet_LHL_IH_skewness

\begin{tabular}{|c|c|c|c|}
\hline 0.8 & 0.78 & 0.82 & 0.59 \\
\hline 0.95 & 0.69 & 0.66 & 0.8 \\
\hline 0.92 & 0.83 & 0.75 & 0.9 \\
\hline 0.8 & 0.83 & 0.81 & 0.33 \\
\hline 0.86 & 0.83 & 0.82 & 0.45 \\
\hline 0.75 & 0.81 & 0.87 & 0.5 \\
\hline 0.8 & 0.73 & 0.44 & 0.33 \\
\hline 0.81 & 0.63 & 0.83 & 0.65 \\
\hline 0.25 & 0.13 & 0.37 & 0.13 \\
\hline 0.81 & 0.7 & 0.85 & 0.52 \\
\hline 0.23 & 0.83 & 0.78 & 0.38 \\
\hline 0.3 & 0.83 & 0.79 & 0.43 \\
\hline 0.67 & 0.71 & 0.72 & 0.12 \\
\hline 0.93 & 0.94 & 0.89 & 0.94 \\
\hline 0.94 & 0.87 & 0.79 & 0.77 \\
\hline 0.96 & 0.83 & 0.96 & 0.98 \\
\hline 0.21 & 0.31 & 0.38 & 0.12 \\
\hline 0.45 & 0.77 & 0.94 & 0.69 \\
\hline 0.13 & 0.33 & 0.48 & 0.08 \\
\hline 0.97 & 0.96 & 0.99 & 0.99 \\
\hline 0.61 & 0.78 & 0.82 & 0.53 \\
\hline 0.17 & 0.86 & 0.57 & 0.41 \\
\hline 0.96 & 0.83 & 0.97 & 0.96 \\
\hline 0.82 & 0.44 & 0.12 & 0.09 \\
\hline 0.79 & 0.16 & 0.67 & 0.34 \\
\hline 0.83 & 0.5 & 0.52 & 0.23 \\
\hline 0.95 & 0.86 & 0.81 & 0.82 \\
\hline 0.84 & 0.81 & 0.86 & 0.69 \\
\hline 0.97 & 0.84 & 1 & 0.99 \\
\hline 0.81 & 0.75 & 0.89 & 0.61 \\
\hline 0.22 & 0.87 & 0.77 & 0.41 \\
\hline 0.2 & 0.23 & 0.43 & 0.07 \\
\hline 0.83 & 0.49 & 0.19 & 0.12 \\
\hline 0.82 & 0.71 & 0.84 & 0.85 \\
\hline 0.19 & 0.27 & 0.52 & 0.21 \\
\hline 0.76 & 0.37 & 0.74 & 0.18 \\
\hline 0.52 & 0.72 & 0.75 & 0.18 \\
\hline 0.98 & 0.94 & 1 & 0.99 \\
\hline 0.88 & 0.8 & 0.87 & 0.77 \\
\hline 0.8 & 0.67 & 0.84 & 0.85 \\
\hline 0.87 & 0.78 & 0.85 & 0.8 \\
\hline 0.87 & 0.79 & 0.88 & 0.8 \\
\hline 0.2 & 0.34 & 0.57 & 0.07 \\
\hline 0.84 & 0.52 & 0.23 & 0.16 \\
\hline 0.21 & 0.31 & 0.38 & 0.12 \\
\hline 0.28 & 0.59 & 0.99 & 0.93 \\
\hline 0.16 & 0.49 & 0.45 & 0.1 \\
\hline 0.97 & 0.96 & 0.99 & 0.99 \\
\hline 0.61 & 0.78 & 0.82 & 0.53 \\
\hline 0.17 & 0.86 & 0.57 & 0.41 \\
\hline 0.72 & 0.61 & 1 & 0.99 \\
\hline 0.82 & 0.44 & 0.12 & 0.09 \\
\hline 0.96 & 0.63 & 0.98 & 0.96 \\
\hline 0.88 & 0.34 & 0.09 & 0.12 \\
\hline 0.69 & 0.65 & 0.78 & 0.57 \\
\hline 0.86 & 0.93 & 0.95 & 0.97 \\
\hline 0.72 & 0.65 & 0.78 & 0.55 \\
\hline 0.72 & 0.61 & 1 & 0.99 \\
\hline 0.88 & 0.76 & 0.79 & 0.26 \\
\hline 0.84 & 0.81 & 0.86 & 0.69 \\
\hline 0.82 & 0.71 & 0.7 & 0.22 \\
\hline 0.86 & 0.89 & 0.97 & 0.97 \\
\hline 0.65 & 0.79 & 0.86 & 0.55 \\
\hline 0.58 & 0.75 & 0.53 & 0.36 \\
\hline 0.88 & 0.56 & 0.69 & 0.03 \\
\hline 0.31 & - & - & \\
\hline 0.93 & 0.68 & 0.99 & 0.99 \\
\hline 0.01 & 0.27 & 0.3 & 0.22 \\
\hline 0.26 & 0.16 & 0.36 & 0.15 \\
\hline 0.49 & 0.87 & 0.82 & 0.47 \\
\hline 0.27 & 0.23 & 0.34 & 0.14 \\
\hline 0.52 & 0.63 & 0.39 & 0.44 \\
\hline- & - & & \\
\hline 0.88 & 0.65 & 1 & 0.99 \\
\hline 0.23 & 0.24 & 0.49 & 0.06 \\
\hline 0.21 & 0.24 & 0.21 & 0.36 \\
\hline 0.41 & 0.41 & 0.19 & 0.18 \\
\hline 0.24 & 0.46 & 0.65 & 0.53 \\
\hline 0.78 & 0.64 & 0.43 & 0.21 \\
\hline 0.31 & - & - & \\
\hline 0.58 & 0.86 & 0.78 & 0.43 \\
\hline 0.25 & 0.22 & 0.4 & 0.02 \\
\hline 0.77 & -0.12 & 0.45 & 0.42 \\
\hline
\end{tabular}


Wavelet_LHL_IH_std

Wavelet_LHL_IH_uniformity

Wavelet_LHL_IH_var

Wavelet_LHL_NGLDM_DE

Wavelet_LHL_NGLDM_DN

Wavelet_LHL_NGLDM_DNN

Wavelet_LHL_NGLDM_DV

Wavelet_LHL_NGLDM_GLN

Wavelet_LHL_NGLDM_GLNN

Wavelet_LHL_NGLDM_GLV

Wavelet_LHL_NGLDM_HGE

Wavelet_LHL_NGLDM_HGLDE

Wavelet_LHL_NGLDM_HGSDE

Wavelet_LHL_NGLDM_LDE

Wavelet_LHL_NGLDM_LGE

Wavelet_LHL_NGLDM_LGLDE

Wavelet_LHL_NGLDM_LGSDE

Wavelet LHL NGLDM SDE

Wavelet_LHL_NGLDM_SM

Wavelet_LHL_NGLDM_SM2

Wavelet_LHL_NGTDM_busyness

Wavelet_LHL_NGTDM_coarseness

Wavelet_LHL_NGTDM_complexity

Wavelet_LHL_NGTDM_contrast

Wavelet_LHL_NGTDM_strength

Wavelet_LHL_Stats_cov

Wavelet_LHL_Stats_energy

Wavelet_LHL_Stats_iqr

Wavelet_LHL_Stats_kurtosis

Wavelet_LHL_Stats_max

Wavelet_LHL_Stats_mean

Wavelet_LHL_Stats_meand

Wavelet_LHL_Stats_median

Wavelet_LHL_Stats_mediand

Wavelet_LHL_Stats_min

Wavelet_LHL_Stats_p10

Wavelet LHL Stats p90

Wavelet_LHL_Stats_qcod

Wavelet_LHL_Stats_range

Wavelet_LHL_Stats_rmeand

Wavelet_LHL_Stats_rm

Wavelet_LHL_Stats_skewness

Wavelet_LHL_Stats_std

Wavelet_LHL_Stats_va

Wavelet_LLH_Fractal_average

Wavelet_LLH_Fractal_lacunarity

Wavelet_LLH_Fractal_sd

Wavelet_LLH_GLCM autocorr

Wavelet_LLH_GLCM_average

Wavelet_LLH_GLCM_clusProm

Wavelet LLH GLCM clusShade

Wavelet_LLH_GLCM_clusTend

Wavelet_LLH_GLCM_contrast

Wavelet_LLH_GLCM_correll

Wavelet_LLH_GLCM_diffAvrg

Wavelet_LLH_GLCM_diffEntro

Wavelet_LLH_GLCM_diffVar

Wavelet_LLH_GLCM_dissimilar

Wavelet_LLH_GLCM_energy

Wavelet_LLH_GLCM_entrop2

Wavelet_LLH_GLCM_homogeneity1

Wavelet_LLH_GLCM_homogeneity2

Wavelet_LLH_GLCM infoCorr1

Wavelet_LLH_GLCM_infoCorr2

Wavelet_LLH_GLCM_invDiffmomnor

Wavelet_LLH_GLCM invDiffnorm

Wavelet_LLH_GLCM_inverseVar

Wavelet_LLH_GLCM_maxCorr

Wavelet_LLH_GLCM_maxProb

Wavelet_LLH_GLCM_sumAvg

Wavelet_LLH_GLCM_sumEntro

Wavelet_LLH_GLCM_sumSquares

Wavelet_LLH_GLCM_sum Var

Wavelet_LLH_GLDZM_DZE

Wavelet_LLH_GLDZM_DZN

Wavelet_LLH_GLDZM_DZNN

Wavelet_LLH_GLDZM_DZV

Wavelet_LLH_GLDZM_HIE

Wavelet_LLH_GLDZM_HILDE

Wavelet_LLH_GLDZM_HISDE

Wavelet_LLH_GLDZM_IN

Wavelet_LLH_GLDZM_INN

Wavelet_LLH_GLDZM_IV

\begin{tabular}{|c|c|c|c|}
\hline \multirow{2}{*}{0.42} & \multirow[b]{2}{*}{0.86} & \multirow[b]{2}{*}{0.83} & \multirow[b]{2}{*}{0.54} \\
\hline & & & \\
\hline 0.82 & 0.73 & 0.86 & 0.64 \\
\hline 0.23 & 0.87 & 0.77 & 0.41 \\
\hline 0.89 & 0.73 & 0.74 & 0.71 \\
\hline 0.92 & 0.95 & 1 & 0.98 \\
\hline 0.86 & 0.78 & 0.8 & 0.56 \\
\hline 0.83 & 0.64 & 0.84 & 0.77 \\
\hline 0.94 & 0.77 & 0.99 & 0.99 \\
\hline 0.82 & 0.73 & 0.86 & 0.64 \\
\hline 0.23 & 0.87 & 0.77 & 0.41 \\
\hline 0.2 & 0.22 & 0.43 & 0.06 \\
\hline 0.33 & 0.63 & 0.65 & 0.6 \\
\hline 0.13 & 0.78 & 0.75 & 0.86 \\
\hline 0.82 & 0.7 & 0.84 & 0.83 \\
\hline 0.83 & 0.49 & 0.19 & 0.11 \\
\hline 0.54 & 0.6 & 0.9 & 0.76 \\
\hline 0.95 & 0.56 & 0.77 & 0.33 \\
\hline 0.82 & 0.8 & 0.85 & 0.68 \\
\hline 1 & 0.91 & 1 & 0.99 \\
\hline 1 & 0.91 & 1 & 0.99 \\
\hline 0.87 & 0.88 & 0.98 & 0.92 \\
\hline 0.98 & 0.68 & 0.96 & 0.96 \\
\hline 0.15 & 0.81 & 0.58 & 0.06 \\
\hline 0.53 & 0.83 & 0.81 & 0.4 \\
\hline 0.73 & 0.73 & 0.93 & 0.89 \\
\hline 0.07 & 0.39 & 0.06 & 0.12 \\
\hline 0.98 & 0.96 & 0.98 & 0.89 \\
\hline 0.74 & 0.98 & 0.86 & 0.94 \\
\hline 0.86 & 0.56 & 0.7 & 0.02 \\
\hline 0.32 & 0.94 & 0.9 & 0.74 \\
\hline 0.91 & 0.75 & 0.6 & 0.41 \\
\hline 0.64 & 0.99 & 0.92 & 0.93 \\
\hline 0.86 & 0.65 & 0.47 & 0.56 \\
\hline 0.68 & 0.98 & 0.87 & 0.94 \\
\hline 0.51 & 0.92 & 0.81 & 0.58 \\
\hline 0.17 & 0.98 & 0.91 & 0.9 \\
\hline 0.88 & 0.98 & 0.93 & 0.93 \\
\hline 0.15 & 0.04 & 0.05 & 0.79 \\
\hline 0.53 & 0.95 & 0.91 & 0.7 \\
\hline 0.71 & 0.98 & 0.89 & 0.95 \\
\hline 0.72 & 0.98 & 0.93 & 0.89 \\
\hline 0.78 & -0.11 & 0.47 & 0.43 \\
\hline 0.56 & 0.98 & 0.93 & 0.89 \\
\hline 0.44 & 0.98 & 0.94 & 0.88 \\
\hline 0.77 & 0.65 & 0.33 & 0.38 \\
\hline 0.22 & 0.4 & 0.71 & 0 \\
\hline 0.33 & 0.33 & 0.74 & 0.06 \\
\hline 0.3 & 0.03 & 0.31 & 0.28 \\
\hline 0.37 & 0.06 & 0.32 & 0.21 \\
\hline 0 & 0.42 & 0.32 & 0.73 \\
\hline 0.16 & -0.46 & 0.17 & 0.52 \\
\hline 0.09 & 0.58 & 0.46 & 0.74 \\
\hline 0.09 & 0.77 & 0.65 & 0.71 \\
\hline 0.58 & 0.96 & 0.84 & 0.5 \\
\hline 0.34 & 0.82 & 0.63 & 0.72 \\
\hline 0.49 & 0.88 & 0.63 & 0.71 \\
\hline 0.03 & 0.79 & 0.72 & 0.77 \\
\hline 0.34 & 0.82 & 0.63 & 0.72 \\
\hline 0.94 & 0.68 & 0.48 & 0.56 \\
\hline 0.84 & 0.75 & 0.59 & 0.58 \\
\hline 0.73 & 0.85 & 0.58 & 0.71 \\
\hline 0.77 & 0.85 & 0.58 & 0.7 \\
\hline 0.94 & 0.6 & 0.15 & 0.94 \\
\hline 0.93 & 0.81 & 0.55 & 0.95 \\
\hline 0.9 & 0.79 & 0.65 & 0.7 \\
\hline 0.93 & 0.83 & 0.63 & 0.72 \\
\hline 0.72 & 0.83 & 0.64 & 0.69 \\
\hline 0.79 & 0.59 & 0.22 & 0.67 \\
\hline 0.89 & 0.65 & 0.53 & 0.53 \\
\hline 0.37 & 0.06 & 0.32 & 0.21 \\
\hline 0.7 & 0.79 & 0.63 & 0.66 \\
\hline 0.09 & 0.63 & 0.52 & 0.73 \\
\hline 0.09 & 0.58 & 0.46 & 0.74 \\
\hline 0.5 & 0.78 & 0.75 & 0.14 \\
\hline 0.96 & 0.91 & 0.57 & 0.97 \\
\hline 0.9 & 0.88 & 0.84 & 0.33 \\
\hline 0.94 & 0.8 & 0.98 & 0.93 \\
\hline 0.29 & 0.08 & 0.22 & 0.53 \\
\hline 0.52 & 0.56 & 0.66 & 0.77 \\
\hline 0.22 & 0.17 & 0.42 & 0.46 \\
\hline 0.99 & 0.94 & 0.92 & 0.97 \\
\hline 0.44 & 0.75 & 0.73 & 0.68 \\
\hline 0.11 & 0.78 & 0.52 & 0.62 \\
\hline
\end{tabular}


Wavelet_LLH_GLDZM_LDE Wavelet_LLH_GLDZM_LIE Wavelet_LLH_GLDZM_LILDE Wavelet_LLH_GLDZM_LISDE Wavelet_LLH_GLDZM_SDE Wavelet_LLH_GLDZM_ZP Wavelet_LLH_GLRLM_GLN Wavelet_LLH_GLRLM_GLNN Wavelet_LLH_GLRLM_GLV Wavelet_LLH_GLRLM_HGRE Wavelet_LLH GLRLM LGRE Wavelet_LLH_GLRLM_LRE Wavelet_LLH_GLRLM_LRHGE Wavelet_LLH_GLRLM_LRLGE Wavelet_LLH_GLRLM_RE Wavelet_LLH_GLRLM_RLN Wavelet_LLH_GLRLM_RLNN Wavelet_LLH_GLRLM_RLV Wavelet_LLH_GLRLM_RP Wavelet_LLH_GLRLM_SRE Wavelet_LLH_GLRLM_SRHGE Wavelet_LLH_GLRLM_SRLGE Wavelet_LLH_GLSZM_HIE Wavelet_LLH_GLSZM_HILAE Wavelet_LLH_GLSZM_HISAE Wavelet_LLH_GLSZM IN Wavelet_LLH_GLSZM_INN Wavelet_LLH_GLSZM_IV Wavelet_LLH_GLSZM_LAE Wavelet_LLH_GLSZM_LIE Wavelet_LLH_GLSZM_LILAE Wavelet_LLH_GLSZM_LISAE Wavelet_LLH_GLSZM_SAE Wavelet_LLH_GLSZM_SZN Wavelet_LLH_GLSZM_SZNN Wavelet_LLH_GLSZM_SZV Wavelet_LLH_GLSZM_ZF Wavelet_LLH_GLSZM_ZP Wavelet_LLH_IH_cov Wavelet_LLH_IH_energy Wavelet_LLH_IH_entropy Wavelet_LLH_IH_iqr Wavelet_LLH_IH_kurtosis Wavelet_LLH_IH_max Wavelet_LLH_IH_maxgrad Wavelet_LLH_IH_maxgradi Wavelet_LLH_IH_mean

Wavelet_LLH_IH meand Wavelet_LLH_IH_median Wavelet_LLH_IH_mediand Wavelet_LLH_IH_min

Wavelet_LLH_IH_mingrad Wavelet_LLH_IH_mingradi Wavelet_LLH_IH_mode Wavelet_LLH_IH_p10 Wavelet_LLH_IH_p90 Wavelet_LLH_IH_qcod Wavelet_LLH_IH_range Wavelet_LLH_IH_rmeand Wavelet_LLH_IH_rms Wavelet_LLH_IH_skewness Wavelet_LLH_IH_std Wavelet_LLH_IH_uniformity Wavelet_LLH_IH_var Wavelet_LLH_NGLDM_DE Wavelet_LLH_NGLDM_DN Wavelet_LLH_NGLDM_DNN Wavelet_LLH_NGLDM_DV Wavelet_LLH_NGLDM_GLN Wavelet_LLH_NGLDM_GLNN Wavelet_LLH_NGLDM_GLV Wavelet_LLH_NGLDM_HGE Wavelet_LLH_NGLDM_HGLDE Wavelet_LLH_NGLDM_HGSDE Wavelet_LLH_NGLDM_LDE Wavelet_LLH_NGLDM_LGE Wavelet_LLH_NGLDM_LGLDE Wavelet_LLH_NGLDM_LGSDE Wavelet_LLH_NGLDM_SDE Wavelet_LLH_NGLDM_SM Wavelet LLH_NGLDM SM2 Wavelet_LLH_NGTDM_busyness Wavelet_LLH_NGTDM_coarseness

\begin{tabular}{|c|c|c|c|}
\hline 0.94 & 0.82 & 0.98 & 0.83 \\
\hline 0.69 & 0.73 & 0.26 & 0.5 \\
\hline 0.63 & 0.38 & 0.63 & 0.05 \\
\hline 0.69 & 0.76 & 0.54 & 0.67 \\
\hline 0.92 & 0.88 & 0.86 & 0.36 \\
\hline 0.8 & 0.87 & 0.63 & 0.85 \\
\hline 0.99 & 0.86 & 0.98 & 0.98 \\
\hline 0.73 & 0.82 & 0.6 & 0.71 \\
\hline 0.17 & 0.72 & 0.49 & 0.74 \\
\hline 0.29 & 0.01 & 0.3 & 0.32 \\
\hline 0.68 & 0.85 & 0.45 & 0.38 \\
\hline 0.9 & 0.74 & 0.48 & 0.79 \\
\hline 0.35 & 0.52 & 0.09 & 0.58 \\
\hline 0.61 & 0.75 & 0.62 & 0.01 \\
\hline 0.35 & 0.61 & 0.39 & 0.57 \\
\hline 0.99 & 0.94 & 0.93 & 0.99 \\
\hline 0.88 & 0.87 & 0.64 & 0.79 \\
\hline 0.91 & 0.7 & 0.51 & 0.8 \\
\hline 0.89 & 0.84 & 0.63 & 0.79 \\
\hline 0.88 & 0.85 & 0.6 & 0.79 \\
\hline 0.28 & 0 & 0.44 & 0.41 \\
\hline 0.69 & 0.85 & 0.5 & 0.42 \\
\hline 0.29 & 0.08 & 0.22 & 0.53 \\
\hline 0.98 & 0.46 & 0.44 & 0.99 \\
\hline 0.19 & 0.1 & 0.36 & 0.53 \\
\hline 0.99 & 0.94 & 0.92 & 0.97 \\
\hline 0.44 & 0.75 & 0.73 & 0.68 \\
\hline 0.11 & 0.78 & 0.52 & 0.62 \\
\hline 0.9 & 0.69 & 0.77 & 0.98 \\
\hline 0.69 & 0.73 & 0.26 & 0.5 \\
\hline 0.77 & 0.78 & 0.98 & 0.96 \\
\hline 0.78 & 0.65 & 0.29 & 0.59 \\
\hline 0.59 & 0.89 & 0.53 & 0.31 \\
\hline 0.93 & 0.92 & 0.75 & 0.9 \\
\hline 0.64 & 0.86 & 0.54 & 0.34 \\
\hline 0.9 & 0.69 & 0.77 & 0.98 \\
\hline 0.9 & 0.76 & 0.48 & 0.59 \\
\hline 0.8 & 0.87 & 0.63 & 0.85 \\
\hline 0.83 & 0.86 & 0.55 & 0.55 \\
\hline 0.83 & 0.83 & 0.8 & 0.99 \\
\hline 0.53 & 0.85 & 0.62 & 0.72 \\
\hline 0.47 & 0.79 & 0.55 & 0.71 \\
\hline 0.71 & 0.58 & 0.32 & 0.54 \\
\hline 0.31 & - & - & \\
\hline 0.97 & 0.68 & 0.88 & 0.96 \\
\hline 0.28 & 0.18 & 0.35 & 0.36 \\
\hline 0.37 & 0.09 & 0.3 & 0.2 \\
\hline 0.39 & 0.81 & 0.59 & 0.75 \\
\hline 0.38 & -0.04 & 0.35 & 0.15 \\
\hline 0.43 & 0.78 & 0.12 & 0.73 \\
\hline - & - & & \\
\hline 0.96 & 0.73 & 0.91 & 0.98 \\
\hline 0.27 & 0.18 & 0.2 & 0.09 \\
\hline 0.46 & 0.13 & 0.43 & 0.03 \\
\hline 0.53 & 0.57 & 0.18 & 0.41 \\
\hline 0.25 & 0.04 & 0.53 & 0.61 \\
\hline 0.85 & 0.8 & 0.59 & 0.53 \\
\hline 0.31 & - & - & \\
\hline 0.44 & 0.83 & 0.61 & 0.76 \\
\hline 0.34 & 0.04 & 0.33 & 0.26 \\
\hline 0.78 & -0.18 & 0.14 & 0.15 \\
\hline 0.36 & 0.81 & 0.58 & 0.76 \\
\hline 0.77 & 0.8 & 0.59 & 0.7 \\
\hline 0.18 & 0.72 & 0.5 & 0.74 \\
\hline 0.87 & 0.64 & 0.56 & 0.81 \\
\hline 0.97 & 0.93 & 0.95 & 0.99 \\
\hline 0.84 & 0.78 & 0.63 & 0.85 \\
\hline 0.94 & 0.71 & 0.72 & 0.67 \\
\hline 0.98 & 0.77 & 0.93 & 0.98 \\
\hline 0.77 & 0.8 & 0.59 & 0.7 \\
\hline 0.18 & 0.72 & 0.5 & 0.74 \\
\hline 0.29 & 0.01 & 0.3 & 0.31 \\
\hline 0.73 & 0.64 & 0.34 & 0.69 \\
\hline 0.17 & 0.5 & 0.56 & 0.89 \\
\hline 0.92 & 0.77 & 0.58 & 0.75 \\
\hline 0.67 & 0.84 & 0.46 & 0.37 \\
\hline 0.42 & 0.76 & 0.72 & 0.65 \\
\hline 0.87 & 0.88 & 0.75 & 0.69 \\
\hline 0.77 & 0.85 & 0.64 & 0.85 \\
\hline 1 & 0.9 & 0.98 & 0.98 \\
\hline 1 & 0.9 & 0.98 & 0.98 \\
\hline 0.91 & 0.85 & 0.92 & 0.98 \\
\hline 1 & 0.78 & 0.97 & 0.96 \\
\hline
\end{tabular}


Wavelet_LLH_NGTDM_complexity Wavelet_LLH_NGTDM_contrast Wavelet_LLH_NGTDM_strength Wavelet_LLH_Stats_cov

Wavelet_LLH_Stats_energy Wavelet_LLH_Stats_iqr

Wavelet_LLH_Stats_kurtosis Wavelet_LLH_Stats_max Wavelet_LLH_Stats_mean Wavelet_LLH_Stats_meand Wavelet_LLH Stats median Wavelet_LLH_Stats_mediand Wavelet_LLH_Stats_min Wavelet_LLH_Stats_p10 Wavelet_LLH_Stats_p90 Wavelet_LLH_Stats_qcod Wavelet_LLH_Stats_range Wavelet_LLH_Stats_rmeand Wavelet_LLH_Stats_rm Wavelet_LLH_Stats_skewness Wavelet_LLH_Stats_std Wavelet_LLH Stats var

Wavelet_LLL_Fractal_average Wavelet_LLL_Fractal_lacunarity Wavelet_LLL_Fractal_sd

Wavelet_LLL_GLCM autocorr Wavelet_LLL_GLCM_average Wavelet_LLL_GLCM_clusProm Wavelet_LLL_GLCM_clusShade Wavelet_LLL_GLCM_clusTend Wavelet_LLL_GLCM_contrast Wavelet_LLL_GLCM_correl1 Wavelet_LLL_GLCM_diffAvrg Wavelet_LLL_GLCM_diffEntro Wavelet_LLL_GLCM_diffVar Wavelet_LLL_GLCM_dissimilar Wavelet_LLL_GLCM energy Wavelet_LLL_GLCM_entrop2 Wavelet_LLL_GLCM_homogeneity1 Wavelet_LLL_GLCM_homogeneity2 Wavelet_LLL_GLCM infoCorr1 Wavelet_LLL_GLCM_infoCorr2 Wavelet_LLL_GLCM_invDiffmomnor Wavelet_LLL_GLCM_invDiffnorm Wavelet_LLL_GLCM_inverseVar Wavelet_LLL_GLCM_maxCorr Wavelet_LLL_GLCM_maxProb Wavelet_LLL_GLCM_sumAvg Wavelet_LLL_GLCM_sumEntro Wavelet_LLL_GLCM_sumSquares Wavelet_LLL_GLCM_sumVar Wavelet LLL GLDZM_DZE Wavelet_LLL_GLDZM_DZN Wavelet_LLL_GLDZM_DZNN Wavelet_LLL_GLDZM_DZV Wavelet_LLL_GLDZM_HIE Wavelet_LLL_GLDZM_HILDE Wavelet_LLL_GLDZM_HISDE Wavelet_LLL_GLDZM_IN Wavelet_LLL_GLDZM_INN Wavelet_LLL_GLDZM_IV Wavelet_LLL_GLDZM_LDE Wavelet_LLL_GLDZM LIE Wavelet_LLL_GLDZM_LILDE Wavelet_LLL_GLDZM_LISDE Wavelet_LLL_GLDZM_SDE Wavelet_LLL_GLDZM_ZP Wavelet_LLL_GLRLM_GLN Wavelet_LLL_GLRLM_GLNN Wavelet_LLL_GLRLM_GLV Wavelet_LLL_GLRLM_HGRE Wavelet_LLL_GLRLM_LGRE Wavelet_LLL_GLRLM_LRE Wavelet_LLL_GLRLM_LRHGE Wavelet_LLL_GLRLM_LRLGE Wavelet_LLL_GLRLM_RE Wavelet_LLL_GLRLM_RLN Wavelet_LLL_GLRLM RLNN Wavelet_LLL_GLRLM_RLV Wavelet_LLL_GLRLM_RP Wavelet LLL_GLRLM SRE Wavelet LLL_GLRLM SRHGE Wavelet_LLL_GLRLM_SRLGE

\begin{tabular}{|c|c|c|c|}
\hline 0.06 & 0.82 & 0.48 & 0.57 \\
\hline 0.79 & 0.56 & 0.62 & 0.76 \\
\hline 0.69 & 0.84 & 0.96 & 0.94 \\
\hline 0.32 & 0.63 & 0 & 0.47 \\
\hline 0.98 & 0.97 & 0.97 & 0.8 \\
\hline 0.81 & 0.96 & 0.87 & 0.95 \\
\hline 0.71 & 0.58 & 0.3 & 0.54 \\
\hline 0.57 & 0.88 & 0.78 & 0.75 \\
\hline 0.9 & 0.69 & 0.7 & 0.63 \\
\hline 0.75 & 0.98 & 0.91 & 0.92 \\
\hline 0.88 & 0.62 & 0.66 & 0.76 \\
\hline 0.82 & 0.96 & 0.87 & 0.97 \\
\hline 0.55 & 0.95 & 0.71 & 0.42 \\
\hline 0.23 & 0.98 & 0.89 & 0.81 \\
\hline 0.9 & 0.96 & 0.9 & 0.97 \\
\hline 0.04 & 0.51 & 0.06 & 0.02 \\
\hline 0.65 & 0.96 & 0.78 & 0.58 \\
\hline 0.79 & 0.97 & 0.88 & 0.95 \\
\hline 0.84 & 0.98 & 0.9 & 0.85 \\
\hline 0.78 & -0.15 & 0.14 & 0.15 \\
\hline 0.72 & 0.98 & 0.9 & 0.84 \\
\hline 0.64 & 0.98 & 0.86 & 0.69 \\
\hline 0.85 & 0.55 & 0.49 & 0.87 \\
\hline 0.17 & 0.4 & 0.51 & 0.64 \\
\hline 0.17 & 0.48 & 0.53 & 0.53 \\
\hline 0.06 & 0.56 & 0.69 & 0.38 \\
\hline 0.04 & 0.58 & 0.68 & 0.44 \\
\hline 0.02 & 0.09 & 0.53 & 0.19 \\
\hline 0.16 & 0.04 & 0.5 & 0.38 \\
\hline 0.03 & 0.09 & 0.65 & 0.42 \\
\hline 0.21 & 0.49 & 0.78 & 0.94 \\
\hline 0.5 & 0.39 & 0.61 & 0.83 \\
\hline 0.41 & 0.61 & 0.81 & 0.95 \\
\hline 0.66 & 0.85 & 0.81 & 0.92 \\
\hline 0.24 & 0.75 & 0.77 & 0.93 \\
\hline 0.41 & 0.61 & 0.81 & 0.95 \\
\hline 0.98 & 0.47 & 0.77 & 0.62 \\
\hline 0.93 & 0.5 & 0.81 & 0.51 \\
\hline 0.77 & 0.8 & 0.82 & 0.92 \\
\hline 0.82 & 0.82 & 0.82 & 0.92 \\
\hline 0.9 & 0.48 & 0.54 & 0.87 \\
\hline 0.92 & 0.48 & 0.62 & 0.65 \\
\hline 0.6 & 0.51 & 0.78 & 0.95 \\
\hline 0.75 & 0.65 & 0.82 & 0.94 \\
\hline 0.83 & 0.77 & 0.81 & 0.95 \\
\hline 0.73 & 0.39 & 0.6 & 0.31 \\
\hline 0.97 & 0.59 & 0.84 & 0.52 \\
\hline 0.04 & 0.58 & 0.68 & 0.44 \\
\hline 0.75 & 0.36 & 0.74 & 0.32 \\
\hline 0.02 & 0.24 & 0.68 & 0.63 \\
\hline 0.03 & 0.09 & 0.65 & 0.42 \\
\hline 0.5 & 0.49 & 0.7 & 0.51 \\
\hline 0.99 & 0.86 & 0.61 & 0.9 \\
\hline 0.93 & 0.69 & 0.79 & 0.78 \\
\hline 0.95 & 0.69 & 0.95 & 0.94 \\
\hline 0.08 & 0.31 & 0.8 & 0.06 \\
\hline 0.06 & 0.6 & 0.93 & 0.47 \\
\hline 0.08 & 0.34 & 0.82 & 0.28 \\
\hline 1 & 0.92 & 0.77 & 0.92 \\
\hline 0.27 & 0.22 & 0.71 & 0.48 \\
\hline 0.03 & 0.42 & 0.66 & 0.74 \\
\hline 0.95 & 0.7 & 0.94 & 0.93 \\
\hline 0.48 & 0.51 & 0.63 & 0.01 \\
\hline 0.54 & 0.75 & 0.67 & 0.36 \\
\hline 0.48 & 0.46 & 0.68 & 0.09 \\
\hline 0.94 & 0.69 & 0.81 & 0.77 \\
\hline 0.84 & 0.92 & 0.77 & 0.96 \\
\hline 1 & 0.85 & 0.98 & 0.99 \\
\hline 0.58 & 0.64 & 0.86 & 0.62 \\
\hline 0 & 0.36 & 0.76 & 0.63 \\
\hline 0.07 & 0.56 & 0.69 & 0.35 \\
\hline 0.51 & 0.65 & 0.64 & 0.56 \\
\hline 0.93 & 0.66 & 0.82 & 0.92 \\
\hline 0.07 & 0.06 & 0.56 & 0.42 \\
\hline 0.46 & 0.54 & 0.87 & 0.47 \\
\hline 0.26 & 0.26 & 0.73 & 0.35 \\
\hline 1 & 0.93 & 1 & 0.98 \\
\hline 0.91 & 0.87 & 0.85 & 0.92 \\
\hline 0.93 & 0.61 & 0.81 & 0.93 \\
\hline 0.92 & 0.85 & 0.86 & 0.93 \\
\hline 0.92 & 0.85 & 0.86 & 0.92 \\
\hline 0.07 & 0.63 & 0.73 & 0.4 \\
\hline 0.52 & 0.64 & 0.6 & 0.57 \\
\hline
\end{tabular}


Wavelet_LLL_GLSZM_HIE Wavelet_LLL_GLSZM_HILAE Wavelet_LLL_GLSZM_HISAE Wavelet_LLL_GLSZM IN Wavelet_LLL_GLSZM_INN Wavelet_LLL_GLSZM_IV

Wavelet_LLL_GLSZM_LAE Wavelet_LLL_GLSZM_LIE Wavelet_LLL_GLSZM_LILAE Wavelet_LLL_GLSZM_LISAE Wavelet LLL GLSZM SAE Wavelet_LLL_GLSZM_SZN Wavelet_LLL_GLSZM_SZNN Wavelet_LLL_GLSZM_SZV Wavelet_LLL_GLSZM ZE Wavelet_LLL_GLSZM_ZP Wavelet_LLL_IH_cov Wavelet_LLL_IH_energy Wavelet_LLL_IH_entropy Wavelet_LLL_IH_iqr Wavelet_LLL_IH_kurtosis Wavelet_LLL_IH_max Wavelet_LLL_IH_maxgrad Wavelet_LLL_IH_maxgradi Wavelet_LLL_IH_mean

Wavelet_LLL_IH_meand Wavelet_LLL_IH_median Wavelet_LLL_IH_mediand Wavelet_LLL_IH_min

Wavelet_LLL_IH_mingrad Wavelet_LLL_IH_mingradi Wavelet_LLL_IH_mode

Wavelet_LLL_IH_p10

Wavelet_LLL_IH_p90

Wavelet_LLL_IH_qcod Wavelet_LLL_IH_range

Wavelet_LLL_IH_rmeand

Wavelet_LLL_IH_rms

Wavelet_LLL_IH_skewness

Wavelet_LLL_IH_std

Wavelet_LLL_IH_uniformity

Wavelet_LLL_IH_var

Wavelet_LLL_NGLDM_DE

Wavelet_LLL_NGLDM_DN

Wavelet_LLL_NGLDM_DNN

Wavelet_LLL_NGLDM_DV

Wavelet_LLL_NGLDM_GLN

Wavelet_LLL_NGLDM_GLNN

Wavelet_LLL_NGLDM_GLV

Wavelet_LLL_NGLDM_HGE

Wavelet_LLL_NGLDM_HGLDE

Wavelet LLL_NGLDM_HGSDE

Wavelet_LLL_NGLDM_LDE

Wavelet_LLL_NGLDM_LGE

Wavelet_LLL_NGLDM_LGLDE

Wavelet_LLL_NGLDM_LGSDE

Wavelet_LLL_NGLDM_SDE

Wavelet_LLL_NGLDM_SM

Wavelet LLL_NGLDM SM2

Wavelet_LLL_NGTDM_busyness

Wavelet_LLL_NGTDM_coarseness Wavelet_LLL_NGTDM_complexity

Wavelet_LLL NGTDM contrast

Wavelet_LLL_NGTDM_strength

Wavelet_LLL_Stats_cov

Wavelet_LLL_Stats_energy

Wavelet_LLL_Stats_iqr

Wavelet_LLL_Stats_kurtosis

Wavelet_LLL_Stats_max

Wavelet_LLL_Stats_mean

Wavelet_LLL_Stats_meand

Wavelet_LLL_Stats_median

Wavelet_LLL_Stats_mediand

Wavelet_LLL_Stats_min

Wavelet_LLL_Stats_p10

Wavelet_LLL_Stats_p90

Wavelet_LLL_Stats_qcod

Wavelet_LLL_Stats_range

Wavelet_LLL_Stats_rmeand

Wavelet_LLL_Stats_rms

Wavelet_LLL_Stats_skewness

Wavelet_LLL_Stats std

Wavelet_LLL_Stats_var

\begin{tabular}{|c|c|c|c|}
\hline 0.08 & 0.31 & 0.8 & 0.06 \\
\hline 0.78 & 0.36 & 0.98 & 0.81 \\
\hline 0.08 & 0.39 & 0.83 & 0.13 \\
\hline 1 & 0.92 & 0.77 & 0.92 \\
\hline 0.27 & 0.22 & 0.71 & 0.48 \\
\hline 0.03 & 0.42 & 0.66 & 0.74 \\
\hline 0.96 & 0.31 & 0.96 & 0.97 \\
\hline 0.48 & 0.51 & 0.63 & 0.01 \\
\hline 0.92 & 0.2 & 0.9 & 0.8 \\
\hline 0.43 & 0.55 & 0.78 & 0.33 \\
\hline 0.56 & 0.73 & 0.67 & 0.68 \\
\hline 0.97 & 0.82 & 0.68 & 0.76 \\
\hline 0.62 & 0.72 & 0.63 & 0.78 \\
\hline 0.96 & 0.31 & 0.96 & 0.97 \\
\hline 0.84 & 0.79 & 0.64 & 0.91 \\
\hline 0.84 & 0.92 & 0.77 & 0.96 \\
\hline 0.61 & 0.75 & 0.66 & 0.44 \\
\hline 0.89 & 0.78 & 0.91 & 0.8 \\
\hline 0.34 & 0.69 & 0.83 & 0.63 \\
\hline 0.27 & 0.21 & 0.72 & 0.65 \\
\hline 0.86 & 0.26 & 0.49 & 0.52 \\
\hline 0.31 & - & - & \\
\hline 0.98 & 0.65 & 0.96 & 0.88 \\
\hline 0.23 & 0.7 & 0.22 & 0.87 \\
\hline 0.05 & 0.57 & 0.67 & 0.41 \\
\hline 0.18 & 0.4 & 0.79 & 0.7 \\
\hline 0.04 & 0.5 & 0.65 & 0.62 \\
\hline 0.36 & 0.32 & 0.58 & 0.53 \\
\hline - & - & & \\
\hline 0.99 & 0.56 & 0.96 & 0.99 \\
\hline 0.03 & 0.37 & 0.52 & 0.33 \\
\hline 0.16 & 0.26 & 0.49 & 0.54 \\
\hline 0.01 & 0.61 & 0.65 & 0.13 \\
\hline 0 & 0.55 & 0.78 & 0.42 \\
\hline 0.75 & 0.31 & 0.67 & 0.36 \\
\hline 0.31 & - & - & \\
\hline 0.26 & 0.26 & 0.79 & 0.76 \\
\hline 0.04 & 0.54 & 0.69 & 0.45 \\
\hline 0.67 & 0.53 & 0.66 & 0.61 \\
\hline 0.14 & 0.49 & 0.78 & 0.65 \\
\hline 0.63 & 0.64 & 0.8 & 0.62 \\
\hline 0 & 0.34 & 0.75 & 0.66 \\
\hline 0.89 & 0.76 & 0.62 & 0.96 \\
\hline 0.99 & 0.94 & 1 & 0.98 \\
\hline 0.87 & 0.85 & 0.82 & 0.94 \\
\hline 0.96 & 0.76 & 0.87 & 0.87 \\
\hline 1 & 0.76 & 0.96 & 0.99 \\
\hline 0.63 & 0.64 & 0.8 & 0.62 \\
\hline 0 & 0.33 & 0.75 & 0.65 \\
\hline 0.07 & 0.57 & 0.69 & 0.37 \\
\hline 0.03 & 0.15 & 0.56 & 0.71 \\
\hline 0.05 & 0.76 & 0.85 & 0.94 \\
\hline 0.96 & 0.74 & 0.86 & 0.91 \\
\hline 0.51 & 0.63 & 0.71 & 0.59 \\
\hline 0.49 & 0.64 & 0.91 & 0.38 \\
\hline 0.63 & 0.69 & 0.68 & 0.46 \\
\hline 0.81 & 0.9 & 0.76 & 0.97 \\
\hline 1 & 0.89 & 0.98 & 1 \\
\hline 1 & 0.89 & 0.98 & 1 \\
\hline 0.87 & 0.88 & 0.92 & 0.98 \\
\hline 0.95 & 0.86 & 0.89 & 0.93 \\
\hline 0.18 & 0.64 & 0.66 & 0.91 \\
\hline 0.84 & 0.36 & 0.84 & 0.82 \\
\hline 0.48 & 0.88 & 0.81 & 0.92 \\
\hline 0.5 & 0.66 & 0.79 & 0.86 \\
\hline 0.99 & 0.93 & 0.94 & 0.98 \\
\hline 0.29 & 0.19 & 0.75 & 0.91 \\
\hline 0.86 & 0.27 & 0.49 & 0.5 \\
\hline 0.48 & 0.73 & 0.82 & 0.9 \\
\hline 0.75 & 0.62 & 0.69 & 0.82 \\
\hline 0.23 & 0.4 & 0.79 & 0.84 \\
\hline 0.7 & 0.58 & 0.63 & 0.88 \\
\hline 0.38 & 0.22 & 0.68 & 0.91 \\
\hline 0.58 & 0.64 & 0.66 & 0.52 \\
\hline 0.96 & 0.78 & 0.67 & 0.75 \\
\hline 0.57 & 0.56 & 0.82 & 0.75 \\
\hline 0.5 & 0.73 & 0.82 & 0.88 \\
\hline 0.37 & 0.73 & 0.84 & 0.94 \\
\hline 0.29 & 0.25 & 0.77 & 0.87 \\
\hline 0.7 & 0.58 & 0.73 & 0.81 \\
\hline 0.67 & 0.53 & 0.66 & 0.62 \\
\hline 0.2 & 0.49 & 0.8 & 0.82 \\
\hline 0.05 & 0.45 & 0.69 & 0.69 \\
\hline
\end{tabular}


Supplementary information S8.2: Mathematical description of extracted radiomics features not described by the Imaging Biomarker Standardization Initiative (IBSI).

\section{Fractal Dimension features}

The Fractal Dimension (FD) of the image is first computed, as is described by Al-Kadi and Watson [doi: 10.1109/TBME.2008.919735]. Given the FD processed image I, with N elements:

1. Average:

2. Lacunarity:

3. Standard deviation:

$$
\text { average }=\frac{1}{N} \sum I
$$

$$
\text { lacunarity }=\frac{\frac{1}{N} \sum I^{2}}{\frac{1}{N^{2}} \sum I}-1
$$

standard deviation $=\left(\frac{1}{N-1} \sum(I-\bar{I})^{2}\right)^{1 / 2}$

Where $\bar{I}$ is the mean of $I$.

1. Standard deviation

$$
\text { standard deviation }=\left(\frac{1}{N_{v}-1} \sum_{j=1}^{N_{v}}\left(X_{d}(j)-\bar{X}_{d}\right)^{2}\right)^{1 / 2}
$$

\section{Local Intensity features *}

Local Intensity (LocInt) features are defined based on local intensity values around a center voxel.

1. Local intensity peak

Mean intensity level in a $1 \mathrm{~cm}^{3}$ spherical volume, centered on the voxel with the maximum intensity 185 level in the volume of interest. In case multiple voxels contain the maximum intensity level, the highest mean intensity level of all spherical volumes is used.

2. Global Intensity peak

Similar to local intensity peak, but in this case the mean intensity level in a $1 \mathrm{~cm} 3$ spherical volume is calculated for every voxel in the volume of interest. The highest mean intensity 190 level of all spherical volumes is selected as the global intensity peak feature. 


\section{Chapter}

\section{General Discussion \\ \&}

Future Perspectives 


\section{GENERAL DISCUSSION}

The availability and utilization of high-quality images is the cornerstone of modern cancer care, both in diagnostic radiology and therapeutic oncology. Recent innovations in MRI have stimulated improvements in mediastinal and neurologic imaging and have set the scene for clinical implementation in diagnostic radiology and radiation therapy. Firstly, advances in technology have enabled MRI to acquire images with superior spatial resolution and soft-tissue contrast in thoracic regions compared to CT imaging. Secondly, advances in imaging modalities have led to the development of ultra-high field (UHF-)MRI and hybrid PET/MRI. Novel clinical UHF-MRI systems can reach field strengths above 3T, enabling visualization of microvasculature and miniscule structural details. In addition, fully integrated PET/MR systems allow for simultaneous acquisition of PET and MR images, and could be used to gain synergetic insights from anatomical and functional imaging. Lastly, advances in image analyses, such as MR radiomics, enable extraction of stable features describing tumour phenotype and heterogeneity which could be used to construct prognostic models, predict treatment effect and outcome.

The general aim of the research work presented in this thesis was to investigate the potential diagnostic and therapeutic integration of advanced MR techniques and image analysis methods in thoracic and neuro-oncology. As such, the specific aims of this work were to investigate:

1. The diagnostic quality of thoracic MRI and PET/MRI and to compare it against ${ }^{18} \mathrm{~F}-\mathrm{FDG}-\mathrm{PET} / \mathrm{CT}$ imaging.

2. The technical and clinical potential of using 7T MRI for radiation therapy planning of patients with brain tumours.

3. The value of hypoxia PET/MRI and quantitative radiomics analysis of functional MRI in patients with oesophageal carcinoma (EC).

In this chapter, a critical appraisal is made of the work presented in this thesis. The main conclusions of each chapter are summarized and put into clinical perspective. Advances in knowledge and implications for patient care are briefly discussed. Furthermore, a critical reflection of the results and limitations of the work are presented, followed by suggestions for further improvements and future perspectives. 


\section{PART I. Anatomical MR imaging and qualitative image analysis}

\section{THORACIC MRI IN DIAGNOSTIC AND THERAPEUTIC ONCOLOGY}

In Chapter 2, the status quo of the diagnostic value of thoracic MRI has been evaluated in a meta-analysis over 12 studies (1122 patients, 4302 lymph nodes). To test Hypothesis 1, a high diagnostic performance of MRI has been shown in staging hilar/mediastinal lymph nodes in patients with non-small cell lung cancer (NSCLC) with an overall high sensitivity at high specificity. From subgroup analyses, it is apparent that studies relying on anatomical evaluation of thoracic MRI (i.e., visual inspection of lesion size and morphology) presented a lower diagnostic accuracy than studies that relied on functional MR imaging and quantitative analysis (i.e., ADC or lesion-to-phantom ratios). However, our study shows that thoracic MRI could still be of additional value for patient with NSCLC in reliable clinical nodal staging, determining resectability, and associated treatment decision-making (i.e., surgical resection vs. (chemo-)radiotherapy) on a per-patient basis. Compared to four meta-analyses on 18F-FDG-PET/CT, our meta-analysis shows a potential of thoracic MRI to achieve an overall sensitivity that is at least as high as that of ${ }^{18}$ F-FDG-PET/CT at equivalent specificity values on a per-patient basis. Moreover, the overall diagnostic performance of $\mathrm{MR}$ imaging on a per-node basis is substantially higher than that of $18 \mathrm{~F}-\mathrm{FDG}-\mathrm{PET} / \mathrm{CT}$, with superior sensitivity values at comparable specificity values. These advances in knowledge in thoracic MRI may also have clinical implications in radiation oncology by accurately evaluating each lymph node separately, enabling state-of-the-art selective nodal irradiation to be performed [1,2]. For this purpose, however, our meta-analysis is limited as it does not differentiate the $\mathrm{cN}$-stages ( $\mathrm{cN} 1-\mathrm{cN} 3)$, but focusses only on discriminating metastatic and non-metastatic lymph nodes (cN0 vs. cN1-cN3). Further research thus needs to investigate the diagnostic value of thoracic MRI in assessing cN-status of individual lymph nodes beyond malignancy.

In the presented meta-analysis, predominantly studies using MRI at $1.5 \mathrm{~T}$ were evaluated and only a few studies could be retrieved and included that directly compared MRI with ${ }^{18}$ F-FDG-PET/CT in the same population. To be able to state that MRI performs equally well in staging mediastinal and hilar lymph node as ${ }^{18} \mathrm{~F}-\mathrm{FDG}-\mathrm{PET} / \mathrm{CT}$ in NSCLC patients, studies need to be initiated to investigate the diagnostic performance of anatomical and functional MR imaging in a direct comparison to ${ }^{18} \mathrm{~F}-\mathrm{FDG}-\mathrm{PET} / \mathrm{CT}$ and histo-/cytological confirmation. One recent study, that was not included in the meta-analysis, reported no significant difference between PET and DWI in the number of false-positives over 628 hilar lymph nodes [3]. However, DWI presented significantly less false-positive findings than PET over 405 mediastinal lymph nodes larger than $5 \mathrm{~mm}(P=0.011)$. Moreover, DWI correctly diagnosed mediastinal lymph nodes that were false-positive findings on PET (i.e., 
lymphadenitis). These results concurred with our meta-analysis and did not alter our conclusion for implicating thoracic MRI in patient care.

\section{SIMULTANEOUS PET/MR IMAGING: THE BEST OF BOTH WORLDS}

The findings in Chapter 2 suggest that an objective quantitative parameter serves as a better measurement than size and morphology as criteria for detecting metastatic hilar and/or mediastinal lymph nodes. However, a high PET signal or DWI signal alone cannot be regarded as proof of malignancy due to the rate of false-positive findings, and still requires clarification by histo-/cytopathological analyses or anatomical images. With the development of fully-integrated PET/MR systems, the best of both imaging worlds is combined for time-efficient acquisition of images with unprecedented biomolecular information and soft-tissue contrast. However, clinical implications of PET/MR need to meet the demands of clinicians (i.e., high diagnostic accuracy, reliable for target volume definition) and of the patient (i.e., short acquisition time, manageable scanning instructions and tolerable sequences).

Focussing first on anatomical imaging, a mediastinum-specific protocol is tested on clinical performance, workflow efficiency, and patient impact in Chapter 3. This study resulted in a clinically manageable, region-specific PET/MRI protocol (including T1-VIBE and T2-HASTE pulse sequences) with high patient compliance that produces high-quality images within a reasonable acquisition time (Hypothesis 2). Based on these advances in knowledge, ECG-triggered T1-VIBE can be recommended for mediastinal imaging and diagnostic evaluation. However, the diagnostic value and non-inferiority to ${ }^{18} \mathrm{~F}-\mathrm{FDG}-\mathrm{PET} / \mathrm{CT}$ is difficult to prove statistically with only 10 patients included in our study. Large-scale studies are hence needed to determine the clinical impact of the presented protocol on TNMstaging. In literature, no clear (dis)advantage of ${ }^{18} \mathrm{~F}-\mathrm{FDG}-\mathrm{PET} / \mathrm{MR}$ over ${ }^{18} \mathrm{~F}-\mathrm{FDG}-$ PET/CT has been established in TNM-staging or therapeutic decision-making [4-6]. Nevertheless, our study indicated that ECG-triggered T1-VIBE could aid in assessing the local spread of mediastinal tumours (e.g., mediastinal infiltration by NSCLC or infiltration of the trachea by EC). Less over-staging of tumour status was shown on MRI by superior visualisation of pleural infiltration of NSCLC and thickening of oesophageal wall layers than on ${ }^{18} \mathrm{~F}-\mathrm{FDG}$-PET/CT images. These findings are in line with current literature [7-10]. In nodal staging, however, ${ }^{18} \mathrm{~F}-\mathrm{FDG}-\mathrm{PET} / \mathrm{MR}$ presented suboptimal results and was less accurate in differentiating the $\mathrm{cN}$-stages.

As indicated in Chapter 2 (Table 3), the overall diagnostic performance in differentiating nodal malignancy by MRI, and by extension PET/MRI, can profit from the addition of quantitative image analyses (e.g., ADC or lesion-to-phantom ratio). In addition, DWI could be decisive in nodal staging when the PET-positive mediastinal nodes are doubted to be non-metastatic [3]. Dedicated thoracic PET/MRI 
protocols should therefore combine both anatomical and functional MR sequences with PET imaging for accurate TNM-staging and successful integration in clinical oncology.

Although there has been an exponential increase in scientific publications related to PET/MRI since its first clinical application, there is still a lack of evidence demonstrating its cost-effectiveness in clinical oncology [11,12]. In the present study, only standard sequences for the Siemens mMR Biograph PET/MR system were evaluated. Further research is needed to investigate the clinical implications of PET/MRI in large patient cohorts while exploring the versatility of MRI in the mediastinum and other tumour regions (e.g., head-and-neck, abdominal, and pelvic regions). Innovative MR sequences need to be revised for mediastinal imaging, together with advanced methods to compensate for respiratory and cardiac motion.

In radiation oncology, PET/MRI has gained interest for precise target volume delineation on anatomical MR images, for defining biological target (sub-)volumes based on functional imaging information (e.g. tumour burden, tumour proliferation, hypoxia, radiation sensitivity), as well as for monitoring treatment response [13,14]. Hybrid PET/MR images thus have the potential to form a leap towards individualised radiation therapy planning [15]. To this end, T1-VIBE images could be considered for delineating gross tumour volumes as low interobserver variability has been reported [16-18]. However, the full integration of whole-body PET/MRI in radiation oncology requires specific modifications to ensure reproducible patient positioning (e.g., flat table overlay, coil bridges) and the generation of accurate photon attenuation correction templates [17]. In addition, the geometric integrity of MR images is paramount for high-precision radiation treatment planning [19].

\section{UHF-MRI FOR BRAIN TUMOURS: HIGH REWARD - MANAGEABLE RISK?}

In neuro-oncology, 7T MRI could potentially exceed the imaging capabilities of 3T MRI by producing images with higher signal-to-noise ratio (SNR), higher spatial resolution, and novel contrast mechanisms such as quantitative susceptibilityweighted imaging. UHF-MRI could supply radiologists and radiation oncologists with images that depict detailed neurologic malformations that could be the decisive factor for clinical interpretation (i.e., intracerebral tumour spread, tumour angiogenesis, and microbleeds) [20-22]. However, the effect of the high magnetic field strength may induce patient discomfort, compromising the integration into clinical practice. In Chapter 4, an 7T MRI protocol has been optimized for therapeutic image quality and tolerability in patients with brain tumours. Subjects receiving an UHF-MRI scan experienced minimal side effects in our study. In the present work, MP2RAGE, T2-SPACE, and SPACE-FLAIR pulse sequences produced anatomical images with high spatial resolution, SNR, and image contrast. Basal ganglia, cerebellum, and frontal and parietal lobes were excellently depicted, having 
potential clinical implications for interventional radiology (e.g., image-guided biopsy, neurosurgical navigation) and radiation therapy planning (RTP) (Hypothesis 3). However, our study has shown inferior image quality at the frontobasal and temporal lobes due to a decrease in MR signal intensity and image artefacts. In a recent study that tested T2-FLAIR at 7T, similar issues of signal dropout were reported in temporal lobes and near the skull base [23]. These limitations thus need to be further investigated, while respecting the restrictions related to specific absorption rate (i.e. the RF power absorbed per unit mass) and clinically manageable scan duration.

In radiation oncology, advanced visualisation of organs-at-risk, microvasculature, and tumour infiltration along white matter tracts could benefit target volume definition. In a recent study, gross tumour volumes significantly decreased with $7.4 \%$ after reiterating 3T-based RTP on 7T MR images [23]. Furthermore, UHF-MRI has been shown to detect radiation-induced microbleeds in patients with glioblastoma multiforme that consequently required RTP modification $[24,25]$. However, the geometrical fidelity in UHF-MRI might be a major concern for its integration into RTP as geometrical distortion (GD) is directly related to the magnetic field strength. In fact, geometric inaccuracies $>1 \mathrm{~mm}$ in 7T MRI could hold a risk of mistreatment and induce clinically unacceptable dose variations of more than $15 \%$ [26,27].

In Chapter 5, system- and object-related GD in 7T MRI was assessed to test Hypothesis 4 and investigate the clinical applicability of anatomical UHF-MRI in neuro-oncological RTP in respect to standard CT and 3T MR imaging. By evaluating the magnitude and location of GD in an anthropomorphic head phantom, it has been shown that 7T MRI meets the clinical acceptance criteria for RTP of GD $\leq 1 \mathrm{~mm}$ up to $6.9 \mathrm{~cm}$ from the magnetic isocenter for all pulse sequences investigated. Therefore, the risk applying 7T MRI in RTP is clinically manageable in, but limited to, central brain regions that display high image quality and spatial accuracy. In peripheral regions, however, the anatomically-optimised $7 \mathrm{~T}$ sequences did not meet the requirements of geometrical accuracy for RTP. This implies that dedicated 7T MRI scan protocols need to be optimised for the specific clinical usage in diagnostic radiology or in radiation therapy. Potentially, GD could be reduced by appropriate sequence settings (e.g., read-out bandwidth, field of view, and matrix size), creating RTP-specific MR sequences [28-31]. However, sequence settings that would be ideal for minimising GD could also have a negative impact on spatial resolution and SNR which could lead to a loss of diagnostic interpretability of MR images. Future research should therefore comprise a systematic analysis of the effects of the aforementioned sequence parameters on 7T MR image quality.

Differences in GD can have different origins, such as imperfections in hardware (i.e. $\mathrm{B}_{0}$-field inhomogeneity and gradient non-linearities), the temporal structure of the 
applied gradient pulse scheme and sequence parameter settings. The work in this thesis compared GD in anatomically optimised 7T MR sequences with GD in CT and 3T MR sequences, which latter are currently being used for routine RTP. An in-depth assessment of the impact of magnetic field strength on GD was beyond the scope of our study. To resolve this limitation, the GD needs to be investigated systematically on 3T and 7T MR systems using identical gradient systems, distortion correction algorithms, and pulse sequence composition. Furthermore, our study is limited in its ability to separate system-related from object-related GD, and lacks in evaluating tissue-related GD. Although tissue-related GD has been shown to be relatively small in $3 \mathrm{~T}$ sequences, the overall degree of GD is expected to be higher in clinical practice $[29,32,33]$. Therefore, patient-specific correction methods are required for UHF-MRI in addition to gradient correction and shimming.

\section{PART II. Functional (PET-)MR imaging and quantitative image analysis}

\section{HYPOXIA PET/MRI: DEFINING BIOLOGICAL TARGET VOLUMES}

Although PET/MR imaging has promised to deliver the best of both worlds, its clinical value has not shown to be superior over ${ }^{18} \mathrm{~F}-\mathrm{FDG}-\mathrm{PET} / \mathrm{CT}$ and has yet to be established [12]. As previously discussed, functional imaging is needed to fully exploit the diagnostic potential of (PET-)MRI. This thesis argues that tumour-specific protocols combining PET-tracers beyond ${ }^{18} \mathrm{~F}-\mathrm{FDG}$ and dedicated MRI sequences (including DWI) will soon become valuable tools in thoracic oncology. Visualising tumour hypoxia can impact clinical practice by defining biological target volumes (BTV) that can be targeted for hypoxia-adjusted treatment options [34]. Suggested treatment options are still in an exploratory phase and are based on improving therapeutic effectiveness (e.g., targeting radio-resistant regions with boosted RTdose or radio-sensitizers) and on eradicating hypoxic cells prior to conventional (chemo-)radiotherapy (e.g., administrating hypoxia-activated prodrugs) [35-39]. For personalised treatment to be successful, however, it is essential to identify those patients who would benefit the most from hypoxia-adjusted therapy and to monitor early treatment responses.

In Chapter 6 a review is presented of clinically available hypoxia responseassociated biomarkers in patients with oesophageal carcinoma. Overexpression of hypoxia-inducible factor (HIF-1 $\alpha$ ), carbonic anhydrase IX (CA IX), and glucosetransporter 1 (GLUT-1), were regarded as valuable markers to estimate the hypoxiarelated molecular response in patients with oesophageal cancer. However, the presented review also indicates that the current array of invasive biomarkers is insufficient to capture the full intricacies of tumour hypoxia and heterogeneity, and assess the prognostic correlation of hypoxia to treatment outcome. Acquisition of 
invasive biomarkers is tedious and difficult to quantify accurately as other molecular mechanisms, genetic alterations, and inflammation can also affect expression of endogenous biomarkers.

Since hypoxia is a dynamic process and reoxygenation could occur during (chemo)radiation therapy, reliable and repeatable biomarkers are needed to accurately monitor treatment-induced changes in hypoxia [40,41]. Non-invasive imaging using PET-tracers has shown great promise in quantitative detection of hypoxic sub-regions in the entire tumour using several 2-nitroimidazole derivatives, such as ${ }^{18} \mathrm{~F}-$ flortanidazole $\left({ }^{18} \mathrm{~F}-\mathrm{HX} 4\right)$ [42-46]. In 2015, the feasibility and repeatability of ${ }^{18} \mathrm{~F}-\mathrm{HX} 4-\mathrm{PET}$ imaging has been shown in oesophageal and pancreatic cancer patients [45]. However, the prognostic value and implications for patient care have not yet been investigated in mediastinal imaging. In Chapter 7 , advances in knowledge have been made regarding hypoxia imaging using ${ }^{18} \mathrm{~F}-\mathrm{HX} 4-\mathrm{PET} / \mathrm{MRI}$ in patients with EC (Hypothesis 5). By combining highly sensitive ${ }^{18} \mathrm{~F}-\mathrm{HX} 4-\mathrm{PET}$ imaging with anatomical MRI these images can be used to assess intra-tumoural heterogeneity, define BTV, and to stratify patients for hypoxia-adjusted therapy [46,47]. Moreover, by incorporating functional MR sequences (e.g., DWI) into the $\mathrm{PET} / \mathrm{MR}$ protocol, the treatment response could be evaluated by monitoring changes in tumour volume, hypoxic fraction (i.e., tumour-to-blood ratio (TBR)), and ADCvalues. In literature, a correlation of SUV $\left({ }^{18} \mathrm{~F}-\mathrm{FDG}\right)$ and $\mathrm{ADC}$ has been reported in NSCLC $[48,49]$. Therefore, ${ }^{18} \mathrm{~F}-\mathrm{FDG}$ was substituted by ${ }^{18} \mathrm{~F}-\mathrm{HX} 4$ to acquire synergic information on tumour hypoxia, cellularity, and structure in a dedicated scan protocol for mediastinal imaging.

Clinically however the current hypoxia-specific PET-tracers are cumbersome and not very appealing for routine practice. Hypoxia tracers are clinically not widely available, expensive, and time-consuming to implement with tedious tracer-uptake periods. For instance, ${ }^{18} \mathrm{~F}-\mathrm{HX} 4$ imaging is generally performed $4 \mathrm{~h}$ post-injection and analysed using an arbitrary TBR threshold of 1.4. As an alternative to PET imaging, several MRI methods, such as blood-oxygen level dependent imaging (BOLD), oxygen-enhanced MRI or dynamic contrast-enhanced perfusion have been shown to function as a surrogate marker for tumour hypoxia [50,51]. Unfortunately, these measurements are indirect and susceptible to image distortion artefacts. Nevertheless, hypoxia imaging could still be worthwhile to perform, and further improvement of hypoxia-specific PET-tracers needs to be stimulated.

\section{MR RADIOMICS SIGNATURES: IMAGE-BASED PHENOTYPING}

In quantitative MRI, multiparametric quantitative maps of $\mathrm{T} 1, \mathrm{~T} 2, \mathrm{~T} 2 *$ relaxation times and proton density are constructed from anatomical images and used to assess tumour biology [52]. Moreover, ADC maps can be derived from multiple DWIs acquired at different diffusion gradients ( $b$-values) and used to assess water diffusion in tissue and indirectly estimate tissue cellularity. The value of functional 
MRI and the analysis of ADC maps has been evident in the clinic to determine tumour malignancy and assess early treatment response [53,54]. Radiomics analysis might be able to extract complementary information on tumour heterogeneity by compiling quantitative features describing the tumour phenotype (i.e., shape, texture, signal intensity, and wavelet-filtered features). However, unstable features with poor clinical reproducibility need to be excluded to reduce the risk of overfitting and allow for reliable prognostic model-building.

In Chapter 8, it is hypothesised that MR radiomics analysis can extract stable phenotypic features from DWI in a multi-centre study (Hypothesis 6). In the present study, features derived from ADC maps acquired at $1.5 \mathrm{~T}$ demonstrated high testretest stability in lung cancer (330/1322), ovarian cancer (378/1322), and colorectal liver metastases (330/1322) with 122 overlapping stable features in all three tumour entities. However, test-retest stability is highly affected by differences in MRI acquisition, image quality, and tumour segmentation. Respiratory motion and susceptibility differences could instigate image distortion artefacts and complicate repeatable tumour segmentation, affecting the test-retest repeatability [55]. In addition, variation in ADC signal-intensity could result from differences in RF coil sensitivity and coil placement during patient (re)positioning but could also be affected by inflammatory processes and small molecular fluctuations of thermal diffusion between test and retest scans $[56,57]$.

Based on subgroup analyses in the present study, stable radiomics features have shown to be unaffected by different sources of data variability such as MR system, vendor, or magnetic field strength, matching the field-strength independent nature of $\mathrm{ADC}[53,58,59]$. To implement this method of analysis in patient care, however, it is paramount to keep ADC variability at a minimum across multiple clinical centres. In Chapter 8, DWI images were acquired in all centres using a quality-assured protocol, pre-defined by Winfield et al. (2015) [60]. ADC maps were constructed while excluding $b$-values below $100 \mathrm{~mm} / \mathrm{s}^{2}$ to reduce perfusion-related MR signal, and subsequently analysed using the same image reconstruction and processing algorithms. However, these DWI and ADC maps do not necessarily represent the optimal image quality for each individual MR system. Image sequence parameters were fixed and no motion correction or image post-processing was applied [61,62]. In clinical practice, these standardization issues will remain present across centres, MRI systems, and even worsens with the development of vendorspecific innovative sequences. This thesis therefore supports the image biomarker standardisation initiative as proposed by Zwanenburg et al. [63]. Besides improvements in image quality, MR radiomics signatures could benefit from a radiomics-specific DWI protocol, consistent image reconstruction, segmentation tools, and feature extraction algorithms [47,64-66].

Despite these limitations, the advances in knowledge in this thesis suggest that radiomics analyses can be performed on ADC maps and used to complement 
assessment of treatment-related changes in absolute ADC metrics (i.e., histogram analysis of centiles, kurtosis and skewness) [67,68]. Moreover, the tumour phenotype can be quantitatively analysed to develop diagnostic and theragnostic models that could help differentiate between cancers, predict early treatment response and improve treatment decision-making towards personalized healthcare. However, the clinical impact of these implications need to be determined in future work before MR radiomics can be fully implemented in clinical oncology.

\section{FUTURE PERSPECTIVES}

The role of MR imaging in clinical oncology has grown considerably, facilitating improvements in patient healthcare. In this thesis, promising results have been presented for thoracic MRI in depicting tumour infiltrations and differentiating lymph node status. However, ongoing developments in anatomical MR imaging (e.g., tissue-specific contrast agents) could lead to new diagnostic and therapeutic insights [69]. Moreover, the versatility of MRI exceeds anatomical imaging and future scientific breakthroughs are expected from multiparametric and quantitative imaging techniques. MR Fingerprinting, for instance, can simultaneously measure multiple tissue properties in a single time-efficient acquisition and specify MRrelated identification features of the associated tissue. These fingerprints can then be used to detect and analyse early tissue changes [70].

In this thesis, quantitative analysis of body-DWI (i.e., ADC histogram analysis, and MR radiomics) has been investigated. Currently, innovations in wholebody DWI are ongoing and aim to suppress background body signal, image artefacts, and to incorporate diffusion images with high $b$-values beyond $>1000$ $\mathrm{s} / \mathrm{mm}^{2}$ (i.e., diffusion kurtosis imaging (DKI)) [71]. Recently, an interesting quantitative image analysis method (Siemens' Frontier MR Total Tumour Load) has been developed to perform ADC histogram analysis and project this data back onto diffusion images, facilitating response assessment of individual bone metastases $[72,73]$. Potentially, this method may also prove useful in defining a BTV of various tumour types and could be combined with ADC-based radiomics. This thesis therefore supports the motion to approach images as mineable data for advanced quantitative analysis and to build predictive models from that data. To implement such techniques in clinical practice, however, large-scale validation is required in multi-centre studies and on longitudinal datasets.

With high spatial resolution and novel susceptibility-based contrast mechanisms, UHF-MRI is appealing for investigating cardiovascular, musculoskeletal, neurodegenerative diseases (e.g., Parkinson's disease, Multiple sclerosis), acute stroke, and traumatic brain injuries [74-76]. However, the limitation for applying 7T MRI in clinical settings, as for most MR sequences, is the time needed for image acquisition. 
Current research therefore aims to speed up the acquisition of UHF-MRI through faster sequences and parallel imaging [76,77]. Aside from anatomical imaging, as discussed in chapter 5, UHF-MRI could potentially contribute to radiation oncology by elucidating tumour biology through functional MR imaging (e.g., BOLD, chemical exchange saturation transfer, MR spectroscopy, and arterial spin labelling) [75,78-80]. However, the challenge here is to select the appropriate MR sequence for each medical condition and clinical application while considering practical limitations such as time, image quality, and specific absorption rate [81].

Innovations in high-end MR systems are ever growing and evolving to improve cost-effectiveness and patient healthcare. Transcending stand-alone MRI, the future of imaging lies in smartly combining different imaging modalities and parametric imaging techniques to gain comprehensive insights in tumour biology. Moreover, the potential of hybrid MR systems can be explored to help understand cancer and treatment processes through imaging. The recent development of MR-LINAC systems (e.g., ViewRay MRIdian linac and Elekta Unity) allows for on-board MR imaging prior to and during radiation dose delivery. It is expected that this will substantially improve the targeting precision, especially of mobile tumours. In theory, an MR-LINAC can use real-time soft-tissue image feedback to visualise the effect of cardiac and respiratory motion on target volume mobility and on-line adapt the dose delivery accordingly [82].

Future perspectives in PET/MRI foresee an important role for PET-tracers beyond ${ }^{18} \mathrm{~F}-\mathrm{FDG}$ to complement multimodal MR imaging, fully identify BTV, and define key clinical applications. In addition, hybrid imaging modalities have a unique potential as a theragnostic tool by using PET-tracers labelled with radiopharmaceuticals (e.g., ${ }^{68} \mathrm{Ga} / 177 \mathrm{Lu}$-PMSA radioligand in prostate cancer) or radiolabelled antibodies (e.g., ${ }^{64} \mathrm{Cu}$-DOTA-trastuzumab in breast cancer, ${ }^{89} \mathrm{Zr}$ cetuximab in head-and-neck cancer) [83-85]. In the near future, this theragnostic approach could be extrapolated to visualise targeted prodrugs. In prostate cancer, radioactively labelled 5-fluorocytosine (5-FC) is converted to the active anticancer drug 5-fluorouracil (5-FU) under hypoxic conditions that can be used for PET imaging [13]. The clinical use of PET/MRI needs to be explored further throughout different tumour types as this imaging modality has the power to outperform PET/CT due to the superior anatomical and functional imaging capabilities of MRI. 


\section{REFERENCES}

1. De Ruysscher D, Wanders S, van Haren E, Hochstenbag M, Geeraedts W, Utama I, Simons J, Dohmen J, Rhami A, Buell U and others. Selective mediastinal node irradiation based on FDG-PET scan data in patients with non-small-cell lung cancer: a prospective clinical study. Int J Radiat Oncol Biol Phys 2005;62(4):988-94.

2. Van De Voorde L, Larue RT, Pijls M, Buijsen J, Troost EG, Berbee M, Sosef M, van Elmpt W, Schraepen MC, Vanneste B and others. A qualitative synthesis of the evidence behind elective lymph node irradiation in oesophageal cancer. Radiother Oncol 2014;113(2):166-74.

3. Nomori H, Cong Y, Sugimura H, Kato Y. Diffusion-weighted imaging can correctly identify false-positive lymph nodes on positron emission tomography in non-small cell lung cancer. Surg Today 2016;46(10):1146-51.

4. Huellner MW, de Galiza Barbosa F, Husmann L, Pietsch CM, Mader CE, Burger IA, Stolzmann P, Delso G, Frauenfelder T, von Schulthess GK and others. TNM Staging of Non-Small Cell Lung Cancer: Comparison of PET/MR and PET/CT. J Nucl Med 2016;57(1):21-6.

5. Rauscher I, Eiber M, Furst S, Souvatzoglou M, Nekolla SG, Ziegler SI, Rummeny EJ, Schwaiger M, Beer AJ. PET/MR imaging in the detection and characterization of pulmonary lesions: technical and diagnostic evaluation in comparison to PET/CT. J Nucl Med 2014;55(5):724-9.

6. Schaarschmidt BM, Grueneisen J, Metzenmacher M, Gomez B, Gauler T, Roesel C, Heusch P, Ruhlmann V, Umutlu L, Antoch G and others. Thoracic staging with 18F-FDG PET/MR in non-small cell lung cancer - does it change therapeutic decisions in comparison to 18F-FDG PET/CT? Eur Radiol 2017;27(2):681-688.

7. Schwenzer NF, Schraml C, Muller M, Brendle C, Sauter A, Spengler W, Pfannenberg C, Claussen CD, Schmidt H. Pulmonary lesion assessment: comparison of whole-body MR/PET and PET/CT imaging Pilot study. Radiology 2012;264(2):551-58.

8. Sawicki LM, Grueneisen J, Buchbender C, Schaarschmidt BM, Gomez B, Ruhlmann V, Wetter A, Umutlu L, Antoch G, Heusch P. Comparative Performance of 18F-FDG PET/MRI and 18F-FDG PET/CT in Detection and Characterization of Pulmonary Lesions in 121 Oncologic Patients. J Nucl Med 2016;57(4):582-6.

9. Lee G, I H, Kim SJ, Jeong YJ, Kim IJ, Pak K, Park DY, Kim GH. Clinical implication of PET/MR imaging in preoperative esophageal cancer staging: comparison with PET/CT, endoscopic ultrasonography, and CT. J Nucl Med 2014;55(8):1242-7.

10. van Rossum PS, van Lier AL, Lips IM, Meijer GJ, Reerink O, van Vulpen M, Lam MG, van Hillegersberg R, Ruurda JP. Imaging of oesophageal cancer with FDG-PET/CT and MRI. Clin Radiol 2015;70(1):81-95.

11. Miles KA, Voo SA, Groves AM. Additional Clinical Value for PET/MRI in Oncology: Moving Beyond Simple Diagnosis. J Nucl Med 2018;59(7):1028-1032.

12. Bailey DL, Pichler BJ, Guckel B, Barthel H, Beer AJ, Botnar R, Gillies R, Goh V, Gotthardt M, Hicks RJ and others. Combined PET/MRI: from Status Quo to Status Go. Summary Report of the Fifth International Workshop on PET/MR Imaging; February 15-19, 2016; Tubingen, Germany. Mol Imaging Biol 2016;18(5):637-50.

13. Bailey DL, Pichler BJ, Guckel B, Antoch G, Barthel H, Bhujwalla ZM, Biskup S, Biswal S, Bitzer M, Boellaard R and others. Combined PET/MRI: Global Warming-Summary Report of the 6th International Workshop on PET/MRI, March 27-29, 2017, Tubingen, Germany. Mol Imaging Biol 2018;20(1):4-20.

14. Iagaru A, Hope T, Veit-Haibach P. PET/MRI in Oncology: Current Clinical Applications. Springer; 2018.

15. Thorwarth D. Functional imaging for radiotherapy treatment planning: current status and future directions-a review. Br J Radiol 2015;88(1051):20150056.

16. Karki K, Saraiya S, Hugo GD, Mukhopadhyay N, Jan N, Schuster J, Schutzer M, Fahrner L, Groves R, Olsen KM and others. Variabilities of Magnetic Resonance Imaging-, Computed Tomography-, and Positron Emission Tomography-Computed Tomography-Based Tumor and Lymph Node Delineations for Lung Cancer Radiation Therapy Planning. Int J Radiat Oncol Biol Phys 2017;99(1):80-89.

17. Paulus DH, Oehmigen M, Gruneisen J, Umutlu L, Quick HH. Whole-body hybrid imaging concept for the integration of PET/MR into radiation therapy treatment planning. Phys Med Biol 2016;61(9):3504-20.

18. Thorwarth D, Leibfarth S, Mönnich D. Potential role of PET/MRI in radiotherapy treatment planning. 
Clinical and Translational Imaging 2013;1(1):45-51.

19. Owrangi AM, Greer PB, Glide-Hurst CK. MRI-only treatment planning: benefits and challenges. Phys Med Biol 2018;63(5):05TR01.

20. Claes A, Idema AJ, Wesseling P. Diffuse glioma growth: a guerilla war. Acta Neuropathol 2007;114(5):44358.

21. Moenninghoff C, Maderwald S, Theysohn JM, Kraff O, Ladd ME, El Hindy N, van de Nes J, Forsting M, Wanke I. Imaging of adult astrocytic brain tumours with 7 T MRI: preliminary results. Eur Radiol 2010;20(3):704-13.

22. Lupo JM, Chuang CF, Chang SM, Barani IJ, Jimenez B, Hess CP, Nelson SJ. 7-Tesla susceptibility-weighted imaging to assess the effects of radiotherapy on normal-appearing brain in patients with glioma. Int J Radiat Oncol Biol Phys 2012;82(3):e493-500.

23. Regnery S, Knowles BR, Paech D, Behl N, Meissner JE, Windisch P, Ben Harrabi S, Bernhardt D, Schlemmer HP, Ladd ME and others. High-resolution FLAIR MRI at 7 Tesla for treatment planning in glioblastoma patients. Radiother Oncol 2018.

24. Bian W, Hess CP, Chang SM, Nelson SJ, Lupo JM. Susceptibility-weighted MR imaging of radiation therapy-induced cerebral microbleeds in patients with glioma: a comparison between 3T and 7T. Neuroradiology 2014;56(2):91-6.

25. Wahl M, Anwar M, Hess CP, Chang SM, Lupo JM. Relationship between radiation dose and microbleed formation in patients with malignant glioma. Radiat Oncol 2017;12(1):126.

26. Karaiskos P, Moutsatsos A, Pappas E, Georgiou E, Roussakis A, Torrens M, Seimenis I. A simple and efficient methodology to improve geometric accuracy in gamma knife radiation surgery: implementation in multiple brain metastases. Int J Radiat Oncol Biol Phys 2014;90(5):1234-41.

27. Seibert TM, White NS, Kim GY, Moiseenko V, McDonald CR, Farid N, Bartsch H, Kuperman J, Karunamuni R, Marshall D and others. Distortion inherent to magnetic resonance imaging can lead to geometric miss in radiosurgery planning. Pract Radiat Oncol 2016;6(6):e319-e328.

28. Weygand J, Fuller CD, Ibbott GS, Mohamed AS, Ding Y, Yang J, Hwang KP, Wang J. Spatial Precision in Magnetic Resonance Imaging-Guided Radiation Therapy: The Role of Geometric Distortion. Int J Radiat Oncol Biol Phys 2016;95(4):1304-16.

29. Schmidt MA, Wells EJ, Davison K, Riddell AM, Welsh L, Saran F. Stereotactic radiosurgery planning of vestibular schwannomas: Is MRI at 3 Tesla geometrically accurate? Med Phys 2017;44(2):375-381.

30. Walker A, Liney G, Metcalfe P, Holloway L. MRI distortion: considerations for MRI based radiotherapy treatment planning. Australas Phys Eng Sci Med 2014;37(1):103-13.

31. Liney GP, Moerland MA. Magnetic resonance imaging acquisition techniques for radiotherapy planning. Semin Radiat Oncol 2014;24(3):160-8.

32. Stanescu T, Wachowicz K, Jaffray DA. Characterization of tissue magnetic susceptibility-induced distortions for MRIgRT. Med Phys 2012;39(12):7185-93.

33. Wang H, Balter J, Cao Y. Patient-induced susceptibility effect on geometric distortion of clinical brain MRI for radiation treatment planning on a 3T scanner. Phys Med Biol 2013;58(3):465-77.

34. Dewhirst MW, Birer SR. Oxygen-Enhanced MRI Is a Major Advance in Tumor Hypoxia Imaging. Cancer Res 2016;76(4):769-72.

35. Dubois LJ, Niemans R, van Kuijk SJ, Panth KM, Parvathaneni NK, Peeters SG, Zegers CM, Rekers NH, van Gisbergen MW, Biemans R and others. New ways to image and target tumour hypoxia and its molecular responses. Radiother Oncol 2015;116(3):352-7.

36. Overgaard J. Hypoxic modification of radiotherapy in squamous cell carcinoma of the head and neck--a systematic review and meta-analysis. Radiother Oncol 2011;100(1):22-32.

37. Thorwarth D, Eschmann SM, Paulsen F, Alber M. Hypoxia dose painting by numbers: a planning study. Int J Radiat Oncol Biol Phys 2007;68(1):291-300.

38. Thorwarth D, Alber M. Implementation of hypoxia imaging into treatment planning and delivery. Radiother Oncol 2010;97(2):172-5.

39. Wilson WR, Hay MP. Targeting hypoxia in cancer therapy. Nat Rev Cancer 2011;11(6):393-410.

40. A. Yaromina, H. Thames, X. Zhou, S. Hering, W. Eicheler, A. Dorfler, T. Leichtner, D. Zips, M. Baumann, 
Radiobiological hypoxia, histological parameters of tumour microenvironment and local tumour control after fractionated irradiation, Radiother Oncol, 96 (2010) 116-122.

41. D. Zips, S. Boke, T. Kroeber, A. Meinzer, K. Bruchner, H.D. Thames, M. Baumann, A. Yaromina, Prognostic value of radiobiological hypoxia during fractionated irradiation for local tumor control, Strahlentherapie und Onkologie : Organ der Deutschen Rontgengesellschaft ... [et al], 187 (2011) 306-310.

42. Zegers CM, van Elmpt W, Szardenings K, Kolb H, Waxman A, Subramaniam RM, Moon DH, Brunetti JC, Srinivas SM, Lambin P and others. Repeatability of hypoxia PET imaging using [(1)(8)F]HX4 in lung and head and neck cancer patients: a prospective multicenter trial. Eur J Nucl Med Mol Imaging 2015;42(12):1840-9.

43. Peeters SG, Zegers CM, Yaromina A, Van Elmpt W, Dubois L, Lambin P. Current preclinical and clinical applications of hypoxia PET imaging using 2-nitroimidazoles. Q J Nucl Med Mol Imaging 2015;59(1):3957.

44. Zegers CM, van Elmpt W, Reymen B, Even AJ, Troost EG, Ollers MC, Hoebers FJ, Houben RM, Eriksson $\mathrm{J}$, Windhorst $\mathrm{AD}$ and others. In vivo quantification of hypoxic and metabolic status of NSCLC tumors using [18F]HX4 and [18F]FDG-PET/CT imaging. Clin Cancer Res 2014;20(24):6389-97.

45. Klaassen R, Bennink RJ, van Tienhoven G, Bijlsma MF, Besselink MGH, Henegouwen MIV, Wilmink JW, Nederveen AJ, Windhorst AD, Hulshof M and others. Feasibility and repeatability of PET with the hypoxia tracer F-18 HX4 in oesophageal and pancreatic cancer. Radiotherapy and Oncology 2015;116(1):94-99.

46. Ling CC, Humm J, Larson S, Amols H, Fuks Z, Leibel S, Koutcher JA. Towards multidimensional radiotherapy (MD-CRT): biological imaging and biological conformality. Int J Radiat Oncol Biol Phys 2000;47(3):551-60.

47. Jaffray DA, Das S, Jacobs PM, Jeraj R, Lambin P. How Advances in Imaging Will Affect Precision Radiation Oncology. Int J Radiat Oncol Biol Phys 2018;101(2):292-298.

48. Schaarschmidt BM, Buchbender C, Nensa F, Grueneisen J, Gomez B, Kohler J, Reis H, Ruhlmann V, Umutlu L, Heusch P. Correlation of the apparent diffusion coefficient (ADC) with the standardized uptake value (SUV) in lymph node metastases of non-small cell lung cancer (NSCLC) patients using hybrid 18FFDG PET/MRI. PLoS One 2015;10(1):e0116277.

49. Heusch P, Buchbender C, Kohler J, Nensa F, Beiderwellen K, Kuhl H, Lanzman RS, Wittsack HJ, Gomez $\mathrm{B}$, Gauler T and others. Correlation of the apparent diffusion coefficient (ADC) with the standardized uptake value (SUV) in hybrid 18F-FDG PET/MRI in non-small cell lung cancer (NSCLC) lesions: initial results. Rofo 2013;185(11):1056-62.

50. O'Connor J, Robinson S, Waterton J. Imaging tumour hypoxia with oxygen-enhanced MRI and BOLD MRI. Br J Radiol 2018:20180642.

51. Halle C, Andersen E, Lando M, Aarnes EK, Hasvold G, Holden M, Syljuasen RG, Sundfor K, Kristensen GB, Holm R and others. Hypoxia-induced gene expression in chemoradioresistant cervical cancer revealed by dynamic contrast-enhanced MRI. Cancer Res 2012;72(20):5285-95.

52. Yankeelov TE, Pickens D, Price RR. Quantitative MRI in Cancer. Hendee WR, editor. Boca Raton: CRC Press; 2012. 338 p.

53. Koh DM, Collins DJ. Diffusion-weighted MRI in the body: applications and challenges in oncology. AJR Am J Roentgenol 2007;188(6):1622-35.

54. Padhani AR, Liu G, Koh DM, Chenevert TL, Thoeny HC, Takahara T, Dzik-Jurasz A, Ross BD, Van Cauteren M, Collins D and others. Diffusion-weighted magnetic resonance imaging as a cancer biomarker: consensus and recommendations. Neoplasia 2009;11(2):102-25.

55. Larue R, Van De Voorde L, van Timmeren JE, Leijenaar RTH, Berbee M, Sosef MN, Schreurs WMJ, van Elmpt W, Lambin P. 4DCT imaging to assess radiomics feature stability: An investigation for thoracic cancers. Radiother Oncol 2017;125(1):147-153.

56. Scheel M. Diffusion-Based MRI: Imaging Basics and Clinical Applications. 2018:383-393.

57. Koh DM, Lee JM, Bittencourt LK, Blackledge M, Collins DJ. Body Diffusion-weighted MR Imaging in Oncology: Imaging at 3 T. Magn Reson Imaging Clin N Am 2016;24(1):31-44.

58. Donati OF, Chong D, Nanz D, Boss A, Froehlich JM, Andres E, Seifert B, Thoeny HC. Diffusion-weighted MR imaging of upper abdominal organs: field strength and intervendor variability of apparent diffusion 
coefficients. Radiology 2014;270(2):454-63.

59. Rosenkrantz AB, Oei M, Babb JS, Niver BE, Taouli B. Diffusion-weighted imaging of the abdomen at 3.0 Tesla: image quality and apparent diffusion coefficient reproducibility compared with 1.5 Tesla. J Magn Reson Imaging 2011;33(1):128-35.

60. Winfield JM, Papoutsaki MV, Ragheb H, Morris DM, Heerschap A, ter Voert EG, Kuijer JP, Pieters IC, Douglas NH, Orton M and others. Development of a diffusion-weighted MRI protocol for multicentre abdominal imaging and evaluation of the effects of fasting on measurement of apparent diffusion coefficients (ADCs) in healthy liver. Br J Radiol 2015;88(1049):20140717.

61. Taouli B, Beer AJ, Chenevert T, Collins D, Lehman C, Matos C, Padhani AR, Rosenkrantz AB, ShuklaDave A, Sigmund E and others. Diffusion-weighted imaging outside the brain: Consensus statement from an ISMRM-sponsored workshop. J Magn Reson Imaging 2016;44(3):521-40.

62. Pathak R, Ragheb H, Thacker NA, Morris DM, Amiri H, Kuijer J, deSouza NM, Heerschap A, Jackson A. A data-driven statistical model that estimates measurement uncertainty improves interpretation of ADC reproducibility: a multi-site study of liver metastases. Sci Rep 2017;7(1):14084.

63. Zwanenburg A, Leger S, Vallières M, Löck S. Image biomarker standardisation initiative. arXiv preprint arXiv:1612.07003 2016.

64. deSouza NM, Winfield JM, Waterton JC, Weller A, Papoutsaki MV, Doran SJ, Collins DJ, Fournier L, Sullivan D, Chenevert $\mathrm{T}$ and others. Implementing diffusion-weighted MRI for body imaging in prospective multicentre trials: current considerations and future perspectives. Eur Radiol 2017.

65. Guyader JM, Bernardin L, Douglas NH, Poot DH, Niessen WJ, Klein S. Influence of image registration on apparent diffusion coefficient images computed from free-breathing diffusion MR images of the abdomen. J Magn Reson Imaging 2015;42(2):315-30.

66. Kumar V, Gu Y, Basu S, Berglund A, Eschrich SA, Schabath MB, Forster K, Aerts HJ, Dekker A, Fenstermacher D and others. Radiomics: the process and the challenges. Magn Reson Imaging 2012;30(9):1234-48.

67. Weller A, Papoutsaki MV, Waterton JC, Chiti A, Stroobants S, Kuijer J, Blackledge M, Morgan V, deSouza NM. Diffusion-weighted (DW) MRI in lung cancers: ADC test-retest repeatability. Eur Radiol 2017;27(11):4552-4562.

68. Padhani AR, Koh DM, Collins DJ. Whole-body diffusion-weighted MR imaging in cancer: current status and research directions. Radiology 2011;261(3):700-18.

69. Jeong Y, Hwang HS, Na K. Theranostics and contrast agents for magnetic resonance imaging. Biomater Res 2018;22:20.

70. Panda A, Mehta BB, Coppo S, Jiang Y, Ma D, Seiberlich N, Griswold MA, Gulani V. Magnetic Resonance Fingerprinting-An Overview. Curr Opin Biomed Eng 2017;3:56-66.

71. Rosenkrantz AB, Padhani AR, Chenevert TL, Koh DM, De Keyzer F, Taouli B, Le Bihan D. Body diffusion kurtosis imaging: Basic principles, applications, and considerations for clinical practice. J Magn Reson Imaging 2015;42(5):1190-202.

72. Blackledge MD, Collins DJ, Tunariu N, Orton MR, Padhani AR, Leach MO, Koh DM. Assessment of treatment response by total tumor volume and global apparent diffusion coefficient using diffusionweighted MRI in patients with metastatic bone disease: a feasibility study. PLoS One 2014;9(4):e91779.

73. Blackledge MD, Tunariu N, Orton MR, Padhani AR, Collins DJ, Leach MO, Koh DM. Inter- and IntraObserver Repeatability of Quantitative Whole-Body, Diffusion-Weighted Imaging (WBDWI) in Metastatic Bone Disease. PLoS One 2016;11(4):e0153840.

74. Forstmann BU, Isaacs BR, Temel Y. Ultra High Field MRI-Guided Deep Brain Stimulation. Trends in Biotechnology 2017;35(10):904-907.

75. Vargas MI, Martelli P, Xin L, Ipek O, Grouiller F, Pittau F, Trampel R, Gruetter R, Vulliemoz S, Lazeyras F. Clinical Neuroimaging Using 7 T MRI: Challenges and Prospects. J Neuroimaging 2018;28(1):5-13.

76. Kraff O, Quick HH. 7T: Physics, safety, and potential clinical applications. J Magn Reson Imaging 2017;46(6):1573-1589.

77. Wright KL, Hamilton JI, Griswold MA, Gulani V, Seiberlich N. Non-Cartesian parallel imaging reconstruction. J Magn Reson Imaging 2014;40(5):1022-40. 
78. Barrett TF, Sarkiss CA, Dyvorne HA, Lee J, Balchandani P, Shrivastava RK. Application of Ultrahigh Field Magnetic Resonance Imaging in the Treatment of Brain Tumors: A Meta-Analysis. World Neurosurg 2016;86:450-65.

79. van der Zwaag W, Schafer A, Marques JP, Turner R, Trampel R. Recent applications of UHF-MRI in the study of human brain function and structure: a review. NMR Biomed 2016;29(9):1274-88.

80. Zaiss M, Schuppert M, Deshmane A, Herz K, Ehses P, Fullbier L, Lindig T, Bender B, Ernemann U, Scheffler K. Chemical exchange saturation transfer MRI contrast in the human brain at 9.4T. Neuroimage 2018;179:144-155.

81. Marques JP, Norris DG. How to choose the right MR sequence for your research question at $7 \mathrm{~T}$ and above? Neuroimage 2018;168:119-140.

82. Henke LE, Contreras JA, Green OL, Cai B, Kim H, Roach MC, Olsen JR, Fischer-Valuck B, Mullen DF, Kashani R and others. Magnetic Resonance Image-Guided Radiotherapy (MRIgRT): A 4.5-Year Clinical Experience. Clin Oncol (R Coll Radiol) 2018;30(11):720-727.

83. Scarpa L, Buxbaum S, Kendler D, Fink K, Bektic J, Gruber L, Decristoforo C, Uprimny C, Lukas P, Horninger $\mathrm{W}$ and others. The $(68) \mathrm{Ga} /(177) \mathrm{Lu}$ theragnostic concept in PSMA targeting of castrationresistant prostate cancer: correlation of SUVmax values and absorbed dose estimates. Eur J Nucl Med Mol Imaging 2017;44(5):788-800.

84. Sasada S, Kurihara H, Kinoshita T, Yoshida M, Honda N, Shimoi T, Shimomura A, Yunokawa M, Yonemori K, Shimizu C and others. 64Cu-DOTA-trastuzumab PET imaging for HER2-specific primary lesions of breast cancer. Ann Oncol 2017;28(8):2028-2029.

85. Even AJ, Hamming-Vrieze O, van Elmpt W, Winnepenninckx VJ, Heukelom J, Tesselaar ME, Vogel WV, Hoeben A, Zegers CM, Vugts DJ and others. Quantitative assessment of Zirconium-89 labeled cetuximab using PET/CT imaging in patients with advanced head and neck cancer: a theragnostic approach. Oncotarget 2017;8(3):3870-3880. 


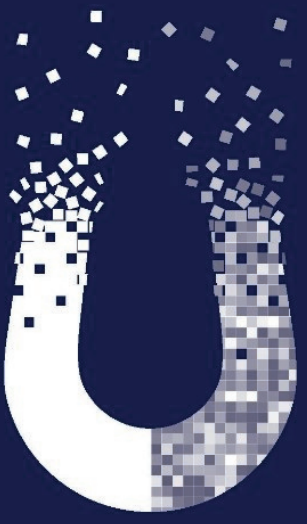




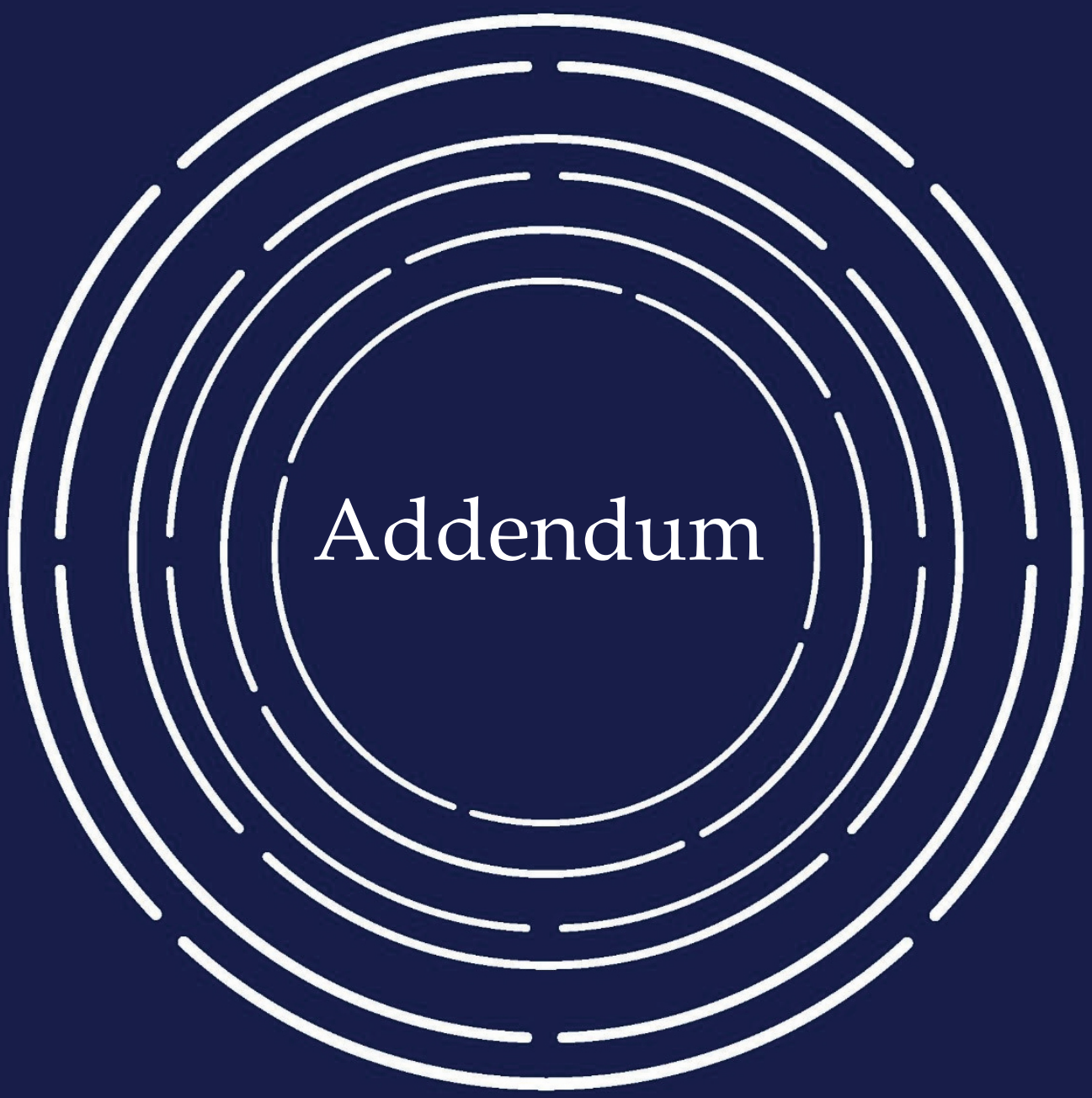


ADDENDUM

Summary 


\section{Towards diagnostic and therapeutic integration of advanced MR imaging in thoracic and neuro-oncology}

\section{BACKGROUND AND AIMS OF THE THESIS}

Cancer is a global health issue that affected 14.1 million people in 2012, leading to 8.2 million deaths worldwide. Despite substantial advances in cancer therapy, successful treatment remains challenging due to the vast diversity and complexity of many cancer types. It is therefore important to detect early development of the disease, differentiate cancer cells from normal cells, and to select the most beneficial treatment-regime for an individual patient and monitor treatment effect. To this end, medical imaging has established a central role in modern cancer management, both in diagnostic radiology and therapeutic oncology. High-resolution anatomical images are clinically used to accurately detect and differentiate cancerous lesions for precise tumour delineation in targeted radiotherapy. Furthermore, functional imaging is clinically valuable in the early detection of cancer development and patient-specific response to treatment. Ultimately, advances in cancer diagnostics and individualised radiation therapy planning (RTP) will result in improved patient outcome.

Over the years, magnetic resonance imaging (MRI) has made incredible technological growth that has resulted in the creation of advanced imaging techniques and image analysis methods (e.g., mediastinal imaging, diffusionweighted imaging, MR radiomics), and innovative imaging modalities such as hybrid PET/MRI and UHF-MRI. These recent innovations in MRI have stimulated improvements in mediastinal and neurologic imaging and have set the scene for clinical implementation in diagnostic radiology and radiation therapy. Nevertheless, practical challenges remain to integrate advanced MRI techniques, image analysis methods, and imaging modalities in thoracic and neuro-oncology. This thesis therefore investigates the potential for diagnostic and therapeutic integration of advanced MR imaging in thoracic and neuro-oncology.

As such, the specific aims of this work were to investigate:

1. The diagnostic quality of thoracic MRI and PET/MRI and to compare it against ${ }^{18} \mathrm{~F}-\mathrm{FDG}-\mathrm{PET} / \mathrm{CT}$ imaging.

2. The technical and clinical potential of using 7T MRI for radiation therapy planning of patients with brain tumours.

3. The value of hypoxia PET/MRI and quantitative radiomics analysis of functional MRI in patients with oesophageal carcinoma (EC). 


\section{PART I. Anatomical MR imaging and qualitative image analysis}

In thoracic oncology, acquiring high-quality MR images of lung and mediastinal tissues is extremely challenging due to the intrinsic properties of thoracic tissues, implementing a low signal-to-noise ratio (SNR), and respiratory and cardiac motion, being susceptible to image-distorting artefacts. However, through technological innovations, modern MRI has the potential to overcome these obstacles and attain clinical advances in thoracic oncology.

In Chapter 2, the diagnostic value of currently available MR imaging methods is summarised in a meta-analysis over 12 studies (1122 patients, 4302 lymph nodes). With a pooled sensitivity of 0.87 [0.78-0.92] on per-patient based and 0.88 [0.78-0.94] on per-nodal based analyses at pooled specificity values of 0.88 [0.77-0.94] and 0.95 [0.87-0.98], respectively, thoracic MRI demonstrated a high diagnostic accuracy in detecting and differentiated (non-)metastatic hilar and mediastinal lymph nodes in NSCLC patients. These findings indicate that MRI can achieve an overall sensitivity that is at least as high as ${ }^{18} \mathrm{~F}$-FDG-PET/CT at equivalent specificity values on a perpatient and per-nodal basis, respectively. Mediastinal MRI could thus be used for reliable nodal staging and treatment decision-making (i.e., surgical resection or (chemo-)radiotherapy). However, subgroup analyses revealed that studies using quantitative diagnostic assessment of metastatic regional lymph nodes showed significantly higher diagnostic performance on a per-nodal basis than studies using qualitative assessment $(\mathrm{rDOR}=7.25$ [1.75-30.09], $P=0.01)$.

To integrate ${ }^{18} \mathrm{~F}-\mathrm{FDG}-\mathrm{PET} / \mathrm{MR}$ into thoracic oncology, clinically manageable imaging protocols need to generate high-quality MR-images within a reasonable acquisition time. In Chapter 3, the clinical value and practical use of mediastinalspecific MR sequences (i.e., T1-VIBE and T2-HASTE) were explored for ${ }^{18} \mathrm{~F}-\mathrm{FDG}$ PET/MR imaging in patients with NSCLC or EC. Making use of ECG-triggering and acquiring images under short multiple breath-holds had a positive effect on the diagnostic MR image quality and patient compliance. Tumour visibility was highest in ECG-triggered T1-VIBE sequences acquired in $\mathrm{BH}(\mathrm{SNR}=62.6$, contrast ratio $=0.86)$ and was qualitatively perceived as high with good reader agreement $(\kappa=0.62)$. Therefore, this sequence could aid in assessing oesophageal wall thickening and evaluating the local spread of mediastinal tumours (e.g., mediastinal infiltration by NSCLC or infiltration of the trachea by EC). However, large-scale studies are recommended to establish the clinical impact of the presented protocol and to validate dedicated, high-quality and patient-friendly PET/MR protocols as an alternative to PET/CT imaging in the thoracic region.

In neuro-oncology, MR images acquired at 1.5 Tesla (T) and 3T are well integrated in clinical practice. Generally, 3T MR images are co-registration with CT images and used for target volume definition and dose calculation, respectively. However, the current clinical MR-techniques are limited in depicting detailed neurologic 
malformations such as intracerebral tumour spread. Through recent technological innovations, however, MR images can be acquired at ultra-high magnetic field (UHF) strengths of 7T or higher. These detailed UHF-MR images have been shown to visualise brain tumours and microscopic neurological malformations with high spatial resolution, SNR, and soft-tissue contrast. To be applicable for interventional radiology (e.g., image-guided biopsy, neurosurgical navigation) and high-precision radiation treatment, however, the spatial accuracy of anatomical images needs to be within $2 \mathrm{~mm}$ for non-stereotactic radiotherapy and within $1 \mathrm{~mm}$ for stereotactic radiotherapy, as is the case for 3T-MRI

In Chapter 4 and 5, the technical feasibility of integrating geometrically reliable UHF-MR images into neuroradiology and high-precision radiotherapy has been demonstrated. After optimization for anatomical imaging, UHF-MR images excellently depicted basal ganglia, cerebellum, frontal lobes, and parietal lobes. In addition, 7T MR images of an anthropomorphic head phantom demonstrated submillimetre levels of system- and object-related geometric distortion (GD) in central brain regions (i.e., distortion $\leq 1 \mathrm{~mm}$ up to $6.9 \mathrm{~cm}$ from the magnetic isocentre) for all pulse sequences investigated. In this region, UHF-MR images could thus be implemented in radiotherapy with clinically-acceptable spatial accuracy equal to the tolerated GD as seen in 3T-MR/CT-based RTP. In peripheral regions, however, the anatomically-optimised 7T sequences did not meet the clinical acceptance criteria for RTP. These studies imply that dedicated 7T MRI protocols need to be optimised for the specific clinical usage in diagnostic radiology or in radiation therapy.

\section{PART II. Functional (PET-)MR imaging and quantitative image analysis}

Functional imaging using dedicated PET and MR imaging biomarkers has the potential to visualise tumour heterogeneity and reveal the radiobiological nature of patient-specific malignancies. Radiosensitive and radioresistant regions can be defined through imaging of tumour sub-volumes and microenvironment before and during treatment. Consequently, functional (PET-)MR imaging paves the way for patient stratification in selecting the most beneficial treatment-regime and adjusting treatment strategies to improve therapeutic effectiveness on a case-by-case basis.

Focussing on tumour hypoxia, a systematic review is presented in Chapter 6 that summarizes the hypoxia-associated molecular response biomarkers that are currently being used clinically in EC patients. Overexpression of hypoxiainducible factor (HIF-1 $\alpha$ ), carbonic anhydrase IX (CA IX), and glucose-transporter 1 (GLUT-1) were regarded as valuable molecular markers that indicate tumour hypoxia. However, diverging findings on the prognostic value for treatment outcome have been reported for different populations (i.e., Western vs. Asian) tumour cell types (i.e., adenocarcinoma vs. squamous cell carcinoma). In addition, 
these invasive biomarkers are insufficient to capture the full intricacies of tumour hypoxia and heterogeneity, and do not allow for multiple patient-friendly measurements.

Since hypoxia is a dynamic process and reoxygenation could occur during (chemo)radiation therapy, reliable and repeatable biomarkers are needed to accurately monitor changes in hypoxia and early treatment effects. In Chapter 7, non-invasive PET/MR imaging using the dedicated PET-tracer ${ }^{18} \mathrm{~F}$-flortanidazole $\left({ }^{18} \mathrm{~F}-\mathrm{HX} 4\right)$ has been shown to quantitatively depict tumour hypoxia in patients with EC. Furthermore, ${ }^{18} \mathrm{~F}-\mathrm{HX} 4-\mathrm{PET} / \mathrm{MRI}$ efficiently assesses tumour heterogeneity by combining hypoxia PET-imaging, anatomical mediastinal MR imaging, and functional diffusion-weighted imaging (DWI) in a single protocol. By correlating changes in hypoxic fraction, tumour volume, and apparent diffusion coefficient (ADC) values, partial treatment response was deduced from mid-treatment increase in mean ADC-values and presence of treatment-resistant hypoxic fractions (TBR $>1.4$ ). Since these molecular changes were detected before anatomical changes manifested, ${ }^{18} \mathrm{~F}-\mathrm{HX} 4-\mathrm{PET} / \mathrm{MRI}$ could therefore be valuable for monitoring therapeutic effectiveness and applying hypoxia-adjusted treatment strategies for personalised patient care.

As an indirect imaging biomarker for tumour hyper-cellularity, DWI and quantitative ADC maps are being used to determine tumour malignancy and assess early treatment response. In Chapter 8, the potential of MR radiomics analyses using $\mathrm{ADC}$ as a biomarker for tumour phenotyping has been investigated. For each patient, standardized DWIs and ADC maps were acquired twice within 7 days, under similar conditions across multiple clinical centres. By analysing the test-retest repeatability, $25-29 \%$ of radiomics features were stable in $1.5 \mathrm{~T}$ ADC maps of various cancers (i.e., lung cancer, ovarian cancer, and colorectal liver metastases). In addition, 122 out of 1322 ADC-based radiomics features were stable over all tissues. By applying standardized protocols, features could be identified that were stable regardless of tumour origin, magnetic field strength, or MR system, stimulating the implementation of ADC-based radiomics across multiple clinical centres. Such stable features have been shown to correlate well with the underlying pathology and tumour biology. By selecting only stable phenotypic features, fitting-dimensionality is reduced and reliable diagnostic and prognostic models can be developed. Quantitative ADC-based radiomics analyses could thus provide complementary information to help detect cancers earlier than the current standard, predict treatment outcome, and improve treatment decision-making towards personalized healthcare. 


\section{FUTURE PERSPECTIVES}

Finally, a critical appraisal has been presented in Chapter 9. This discussion recapitulated the advances in knowledge this work has produced, and reflects on the potential clinical implications. Based on the research presented in this thesis, it is apparent that advanced MRI techniques, MR image analysis methods, and innovative imaging modalities will have a significant role in thoracic and neurooncology in the near future. By further exploring the versatility of MRI, new diagnostic and therapeutic insights could be gained, paving the way towards personalized patientcare in oncology. 
ADDENDUM

Samenvatting

(Dutch summary) 


\section{Een stap richting de diagnostische en therapeutische integratie van geavanceerde MR beeldvorming in thorax en neuro-oncologie}

\section{ACHTERGROND EN DOELEN VAN DE THESIS}

Kanker is een wereldwijde ziekte die in 201214.1 miljoen mensen trof en resulteerde in 8.2 miljoen doden. Ondanks substantiële verbeteringen in de behandeling van kanker blijft het echter moeilijk om succesvolle therapieën te bekomen wegens de grote diversiteit en complexiteit van vele kankersoorten. Het is daarom belangrijk om de ziekte in een vroeg stadium te detecteren, om kankercellen te kunnen onderscheiden van gezonde cellen, om de geschikte behandelingsopties te selecteren en het effect hiervan te controleren. Medische beeldvorming speelt in dit verhaal een centrale rol in zowel diagnostiek als behandeling. Aan de hand van deze anatomische beeldvorming worden tumoren gedetecteerd, diagnoses gesteld en behandelingsplannen voor radiotherapie gecreëerd. Bovendien kan functionele beeldvorming gebruikt worden om kankerontwikkeling en patiënt-specifieke reacties in een vroeg stadium te detecteren. Uiteindelijk zal de vooruitgang in de medische beeldvorming leiden tot verbeterde kankerdiagnose, geïndividualiseerde behandelingsstrategieën, en een verbeterde therapie-uitkomst.

Magnetische resonantie (MR) beeldvorming heeft doorheen de jaren een enorme technologische groei gekend wat enerzijds geleid heeft tot geavanceerde methodes voor beeldacquisitie en beeldanalyse (bv. mediastinale beeldvorming, diffusie-gewogen beeldvorming, MR radiomics), en anderzijds tot de ontwikkeling van innovatieve beeldvormingsmodaliteiten zoals hybride PET/MRI en UHF-MRI. De klinische integratie van mediastinale beeldvorming, geavanceerde beeldanalyse methodes, en innovatieve systemen vormt echter een enorme praktische uitdaging. Deze thesis onderzocht daarom het potentieel van diagnostische en therapeutische integratie van geavanceerde MR beeldvorming in thorax en neuro-oncologie.

Concreet waren de specifieke doelen van deze thesis om:

1. De diagnostische waarde van thorax MRI en PET/MRI te onderzoeken in vergelijking met ${ }^{18} \mathrm{~F}-\mathrm{FDG}$-PET/CT

2. Het technisch en klinisch potentieel van 7T MRI te onderzoeken in radiotherapie planning (RTP) van hersentumoren

3. De klinische waarde van hypoxie PET/MRI en kwantitatieve radiomics beeldanalyse te onderzoeken bij patiënten met slokdarmkanker 


\section{DEEL I. Anatomische MRI en kwalitatieve beeldanalyse}

In thorax oncologie is het een enorme uitdaging om kwaliteitsvolle MR beelden te bekomen van long en mediastinaal weefsel door de intrinsieke eigenschappen van deze weefsels, cardiale pulsatie en ademhalingsbeweging, en gevoeligheid tot beeldverstorende artefacten. Moderne MRI heeft het potentieel om deze beperkingen te overstijgen en een meerwaarde te bieden in klinische oncologie.

In Hoofdstuk 2 werden 12 klinische studies (1122 patiënten, 4302 lymfeklieren) geanalyseerd om de diagnostische waarde van mediastinale MRI samen te vatten. Met een gepoolde sensitiviteit van 0.87 [0.78-0.92] op patiëntenniveau en 0.88 [0.78-0.94] op klierniveau bij een respectievelijke gepoolde specificiteit van 0.88 [0.77-0.94] en 0.95 [0.87-0.98], blijkt MRI een hoge diagnostische accuraatheid in het detecteren en differentiëren van lymfeklieren rond het mediastinum en hilus bij patiënten met longkanker te hebben. Deze resultaten bewijzen dat MRI in staat is om een hogere sensitiviteit dan ${ }^{18} \mathrm{~F}-\mathrm{FDG}-\mathrm{PET} / \mathrm{CT}$ te bekomen bij gelijke specificiteit en dat mediastinale MRI geschikt is voor een betrouwbare klierstadiëring. Subgroep analyses toonde echter aan dat kwantitatieve MR beelden een significant hogere diagnostische precisie hebben, in vergelijk met kwalitatieve beeldanalyse $(\mathrm{rDOR}=7.25$ [1.75-30.09], $P=0.01)$.

Om ook ${ }^{18}$ F-FDG-PET/MR te integreren in thorax oncologie, zijn klinisch haalbare beeldvormingsprotocollen nodig die binnen een aanvaardbare tijd kwaliteitsvolle MR beelden kunnen genereren. In Hoofdstuk 3 werd de klinische waarde en praktisch gebruik onderzocht van specifieke MR sequenties (i.e., T1-VIBE en T2-HASTE) bij patiënten met slokdarm- of longkanker. De diagnostische beeldkwaliteit was het hoogste in sequenties die gebruikmaakten van ECGtriggering en beelden verzamelde terwijl de patiënt zijn/haar adem inhoudt. Tumoren waren immers het beste zichtbaar op ECG-getriggerde T1-VIBE beelden $(\mathrm{SNR}=62.6$, contrast ratio $=0.86)$ die daarenboven het beste beoordeeld werden door twee onafhankelijke artsen $(\kappa=0.62)$. Klinisch kan deze sequentie gebruikt worden om verdikkingen in de slokdarmwand te visualiseren, alsook om de lokale doorgroei van mediastinale tumoren te beoordelen. Desalniettemin zijn er grotere studies nodig om de klinische impact van het gepresenteerde protocol te bepalen en om toegewijde PET/MR protocollen te valideren als een alternatief voor PET/CT beeldvorming in thorax oncologie.

In de neuro-oncologie worden 1.5 Tesla (T) en 3T MR beelden klinisch gebruikt om diagnoses te stellen en om bestralingsplannen voor radiotherapie te creëren. De huidige klinische MRI modaliteiten zijn echter beperkt in het aantonen van bepaalde neurologische aandoeningen zoals intracerebrale tumorspreiding. Met de recente ontwikkeling van klinische ultra-high field (UHF-)MR systemen is het mogelijk om magnetische veldsterkte van 7T of hoger te bekomen en gedetailleerde anatomische beelden te creëren met een hoge signaalsterkte, spatiële resolutie en contrast. Door 
microscopische malformaties te visualiseren, zijn deze beelden uitermate geschikt voor hoogst-nauwkeurig RTP en interventionele radiologie (bv. beeldgestuurde biopsieën en neurochirurgie). Om UHF-MRI klinisch toe te passen in stereotactische radiotherapie, zijn echter anatomische beelden vereist met een geometrische accuraatheid $\leq 1 \mathrm{~mm}$, zoals dit het geval is bij 3T MR beelden.

In Hoofdstuk 4 en 5 werd de technische haalbaarheid beargumenteerd om geometrisch betrouwbare UHF-MR beelden te integreren in neuroradiologie en hoogst-nauwkeurige radiotherapie. $\mathrm{Na}$ optimalisatie van anatomische beeldvorming konden basale ganglia, het cerebellum, en de frontale en pariëtale hersenkwabben goed gevisualiseerd worden zonder belastend te zijn voor de patiënt. Daarnaast werd in een antropomorfisch hoofdfantoom aangetoond dat de systeem- en object-gerelateerde geometrische vervorming in 7T MR beelden kleiner is dan $1 \mathrm{~mm}$ binnen een straal van $6.9 \mathrm{~cm}$ vanaf het magnetisch isocentrum. In centrale hersenregio's voldeden deze UHF-MR beelden aldus aan het klinisch aanvaardbaarheidscriterium voor betrouwbare RTP. In perifere hersenregio's werd er echter een grotere mate van geometrische vervorming vastgesteld. De studies in hoofdstuk 4 en 5 impliceren hiermee dat 7T MRI protocollen geoptimaliseerd moeten worden naargelang het specifiek klinisch doel en gebruik in diagnostische radiologie of radiotherapie.

\section{DEEL II. Functionele (PET-)MRI en kwantitatieve beeldanalyse}

Functionele beeldvorming, door middel van PET en MRI biomarkers, heeft het potentieel om tumorheterogeniteit af te beelden en om de radiobiologische aard van tumoren te onthullen. Door sub-volumes en de micro-omgeving van tumoren te bestuderen, kunnen vóór en tijdens de kankertherapie radio-sensitieve en radioresistente regio's gedefinieerd worden. Deze informatie kan bijgevolg gebruikt worden om patiënten te selecteren die een grotere voorkans hebben op een succesvolle behandeling en op die manier gepersonaliseerde behandelingsstrategieën op te stellen.

Radioresistente sub-volumes kunnen ontstaan door o.a. chronische tumor hypoxie. In Hoofdstuk 6 werd een overzicht gegeven van alle hypoxie-geassocieerde moleculaire response biomarkers die klinisch gebruikt worden in slokdarmkanker. Overexpressie van hypoxia-inducible factor (HIF-1 $\alpha$ ), carbonic anhydrase IX (CA IX), en glucose-transporter 1 (GLUT-1) werden aangeduid als waardevolle moleculaire indicatoren voor tumor hypoxie. De prognostische waarde van deze markers was echter niet eenduidig en tegenstrijdige resultaten werden gerapporteerd in verschillende populaties (Westers vs. Aziatisch) en tumorceltypes (adenocarcinoom vs. plaveiselcelcarcinoom). Bovendien is het onmogelijk om de volledig complexiteit van hypoxische tumoren te bestuderen aan de hand van één 
invasieve staalafname en is het klinisch uitgesloten om meermaals monsters te bekomen doordat deze procedure erg belastend is voor de patiënt.

Doordat tumor hypoxie een dynamisch proces is en re-oxygenatie optreedt ten gevolge van (chemo)radiatie therapie, is er nood aan betrouwbare biomarkers die herhaaldelijk de status van hypoxie kunnen meten en vroegtijdig therapiegebonden veranderingen kunnen detecteren. In Hoofdstuk 7 vertoonde PET/MRI beeldvorming, gebruikmakend van de PET-tracer ${ }^{18} \mathrm{~F}$-flortanidazole $\left({ }^{18} \mathrm{~F}-\mathrm{HX} 4\right)$, het vermogen om hypoxische weefsels te visualiseren in patiënten met slokdarmkanker en vervolgens kwantitatief te analyseren. Door gebruik te maken van de multimodale eigenschappen van PET/MRI, werden ${ }^{18} \mathrm{~F}-\mathrm{H} X 4-\mathrm{PET}$, anatomische MRI, en diffusie-gewogen beeldvorming gecombineerd tot één protocol en kon er een correlatie uitgevoerd worden tussen veranderingen in tumor hypoxie, volume, en 'apparent diffusion coefficient' (ADC) waarden. In een patiënt met slokdarmkanker werd halverwege de behandeling een stijging in gemiddelde ADC gedetecteerd, terwijl tumor hypoxie aanwezig bleef (TBR $>1.4$ ), wat duidde op een partiële response. Aangezien moleculaire veranderingen eerder gedetecteerd kunnen worden dan anatomische veranderingen, kan ${ }^{18} \mathrm{~F}-\mathrm{HX} 4-\mathrm{PET} / \mathrm{MRI}$ klinisch een meerwaarde bieden om vroege behandelingsreacties te observeren en indien nodig de behandelingsstrategie bij te sturen om de therapeutische effectiviteit te verbeteren.

Als een indirecte marker voor tumor hypercellulariteit worden DWI en ADC maps klinisch gebruikt om tumormaligniteit en vroege therapieresponse te bepalen. In Hoofdstuk 8 werd onderzocht of MR radiomics in staat is om ADC maps kwantitatief te analyseren. In deze studie werden voor iedere patiënt twee gestandaardiseerde DWI beelden en ADC maps bekomen onder gelijkaardige omstandigheden in verschillende klinische centra. Door de test-retest reproduceerbaarheid te analyseren, bleek $25-29 \%$ van de radiomics beeldkenmerken stabiel te zijn overheen datasets van verschillende kankersoorten (i.e., longkanker, ovariumkanker, en colorectale levermetastases) waarvan bovendien 122 van de 1322 features stabiel waren bij alle weefsels. Daarnaast konden de beeldkenmerken geïdentificeerd worden die stabiel waren ongeacht de tumorsoort, MR systeem, of magnetische veldsterkte. Deze resultaten moedigen de integratie aan van ADCgebaseerde radiomics analyses in multiple klinische centra. Het is namelijk reeds aangetoond dat stabiele features correleren met de onderliggende pathologie en tumor biologie. Door enkel deze stabiele features te selecteren, kan het risico op overfitting gereduceerd worden en betrouwbare diagnostische en prognostische modellen ontwikkeld worden. Kwantitatieve MR radiomics analyses kan hierdoor complementaire medische informatie opleveren en leiden tot een gepersonaliseerde zorg en een verbeterde behandelingsuitkomst. 


\section{TOEKOMSTPERSPECTIEVEN}

Tot slot werd in Hoofdstuk 9 een kritisch blik geworpen op de studies van deze thesis. Deze discussie vatte de wetenschappelijke van MRI bevindingen samen en reflecteerde op de klinische implicaties hiervan. Gebaseerd op het werk in deze thesis, is het duidelijk dat in de nabije toekomst geavanceerde MRI technieken, analysemethodes, en innovatieve MR systemen een belangrijke rol zal spelen in thorax en neuro-oncologie. Door de vele facetten van MRI verder te onderzoeken, kunnen er mogelijks nieuwe diagnostische en therapeutische inzichten bekomen worden, die vervolgens de aanleiding kunnen geven tot een gepersonaliseerde patiëntenzorg. 
ADDENDUM

Valorisation 


\section{VALORISATION}

\section{SOCIAL RELEVANCE AND TARGET POPULATIONS}

Globally, cancer is considered as the second most common cause of disease-related deaths. In 2012, cancer affected 14.1 million people, leading to 8.2 million deaths worldwide. Despite substantial advances in cancer therapy, successful treatment remains challenging due to the vast diversity and complexity of many cancer types. This thesis focused on patients with thoracic or brain cancer to improve in early diagnostics and cancer treatment, using non-invasive imaging techniques and image analyses. However, the findings of this thesis can be extrapolated to patients with other diseases and other tumour regions (e.g., head-and-neck, abdominal, and pelvic regions). Attributed to the global impact of cancer, improvements in clinical care will economically benefit the whole society as treatment will become more effective and consequently more cost efficient.

Since medical imaging is the cornerstone of modern cancer care, both in diagnostic radiology and therapeutic oncology, implementation of advanced MR imaging techniques, image analysis methods, and imaging modalities can have an enormous impact in clinical healthcare. Primarily, the patient would directly benefit from advances in medical imaging and subsequent increase in diagnostic accuracy (i.e., detecting and differentiating early disease development, tumour infiltration, and tumour metastases) and treatment accuracy (i.e., surgical resection, radiation dose delivery). In addition, MR images can be acquired in a non-invasive, patient-friendly manner without exposing the patient to a harmful, unnecessary ionising radiation dose. By further exploring the versatility of MRI, both high-quality anatomical and functional (PET-)MR images can be acquired to better understanding tumour biology and tumour characteristics related to treatment resistance. Ultimately, multiparametric insights could lead to improvements in current therapies and stimulate the development of individualized treatment strategies.

Selecting the most beneficial treatment-regime for an individual patient does not only aims to obtain the best patient outcome but also aims to maintain a high quality of life. Personalized healthcare therefore also has a social impact, affecting family, friends, and physicians involved in treatment decision-making. Clinical decision support systems based on multiparametric imaging and prediction models can assist physicians and patients in constructing the treatment option that is tailored to the needs and preferences of each individual patient.

To integrate innovative imaging modalities (i.e., UHF-MRI and PET/MRI) into oncology, retraining of radiologists, radiotherapists, and MR technicians is required. 
Acquiring ECG-triggered mediastinal (PET-)MR images and UHF-MR images is not straightforward and often requires hands-on optimisation while acquiring images. Furthermore, the combined representation of nuclear PET-data with MR-data is relatively new to specialised MR technicians and radiologists. For UHF-MRI, expert knowledge is required to acquire and analyse complex anatomical and functional imaging (e.g., susceptibility-weighted imaging and mapping, phase-contrast imaging, CEST, MR spectroscopy, fMRI), making these techniques difficult to translate into daily clinical practice without additional training. Likewise, mediastinal (PET-)MR and UHF-MR images are rarely used in radiation oncology and retraining is needed to learn how to apply dedicated imaging protocols and deal with image-distorting artefacts and geometric integrity.

\section{IMPACT ON CURRENT ACTIVITIES AND PRODUCTS}

Based on the findings presented in Chapter 2, currently-available MRI techniques can be used to clinically assess mediastinal tumours and metastatic lymph nodes with overall high diagnostic accuracy. In radiation oncology, accurate evaluation of each individual lymph node can be used for state-of-the-art selective nodal irradiation. In addition, DWI and ADC-based radiomics analyses can be used to acquire complementary information to specify tumour heterogeneity as indicated in Chapter 7 and 8, respectively. These findings are an important source of information to be used in the development of clinical decision support systems.

In ${ }^{18} \mathrm{~F}-\mathrm{FDG}-\mathrm{PET} / \mathrm{MR}$ imaging, DWI could be decisive in nodal staging when the FDG-avid mediastinal nodes are doubted to be non-metastatic. Practical points of advice have been presented in Chapter 3 for implementing PET/MRI using a dedicated, patient-friendly protocol for thoracic anatomical imaging. By incorporating DWI in the same protocol, ${ }^{18} \mathrm{~F}-\mathrm{HX} 4-\mathrm{PET} / \mathrm{MRI}$ has shown to visualise tumour hypoxia in oesophageal cancer and to detect early treatment responses.

The findings and practical workflow presented in Chapter 4 and 5 stimulate the use of anatomical UHF-MR images in neuro-oncology. UHF-MR images could supply radiologists with images that depict detailed neurologic malformations that could be the decisive factor for clinical interpretation (i.e., intracerebral tumour spread, tumour angiogenesis, and microbleeds). In addition, 7T MR images could be used for high-precision radiation treatment options in the central brain region. However, it is advised to optimise 7T-MR protocols for each specific clinical application and make the trade-off between image-quality and image integrity. 


\section{IMPACT ON FUTURE ACTIVITIES AND PRACTICE}

Although promising results have been presented in this thesis, further academic research is needed to stimulate the integration of (PET-)MRI in thoracic and UHF-MRI neuro-oncology. In future research, innovative MR sequences need to be revised for mediastinal imaging, together with advanced methods to compensate for respiratory and cardiac motion. In addition, the diagnostic value of mediastinal (PET-)MR needs to be investigated and dedicated protocols need to be validated in direct comparison to PET/CT, with histopathological validation. Nevertheless, the presented protocols and practical suggestions of this thesis could have an impact on current radiation treatment. For instance, novel MR-LINAC systems (e.g., ViewRay MRIdian linac and Elekta Unity) could benefit from our imaging protocols as realtime soft-tissue image feedback is used to visualise tumour mobility, related to cardiac and respiratory motion, and improve the precision of radiation dose delivery.

Before PET/MRI can be fully integrated in radiation oncology, however, specific modifications are required to ensure reproducible patient positioning (e.g., flat table overlay, coil bridges) and the generation of accurate photon attenuation correction templates. Moreover, this thesis argues that tumour-specific protocols combining PET-tracers beyond ${ }^{18} \mathrm{~F}-\mathrm{FDG}$ with dedicated MRI sequences (incl. DWI) will soon become valuable tools to fully exploit the diagnostic potential of PET/MRI. However, there is still a lack of evidence defining key clinical applications and demonstrating the cost-effectiveness of PET/MR and need to be investigated further. Nevertheless, hybrid imaging modalities have a unique potential as a theragnostic tool by using PET-tracers labelled with radiopharmaceuticals or radiolabelled antibodies. In addition, the ability to detect hypoxic regions and correlate anatomical and cellular changes by ${ }^{18} \mathrm{~F}-\mathrm{HX} 4-\mathrm{PET} / \mathrm{MRI}$ paves the way for personalized anticancer treatment, as presented in Chapter 6. By selecting patients with hypoxic tumours prior to treatment, a window-of-opportunity arises wherein attempts to reduce tumour hypoxia could be made by enhancing the susceptibility of hypoxic cells to anti-cancer treatment and/or targeting hypoxic tumour cells (e.g., boosted radiation dose painting, hypoxia-activated prodrugs). Consequently, further development of PET/MR tracers and hypoxia targeted agents spikes the interest of health care companies, stimulating further research.

In radiation oncology and neurosurgery, advanced visualisation of organs-at-risk, microvasculature, and tumour infiltration along white matter tracts could benefit target volume definition. the risk applying 7T MRI in RTP is clinically manageable in, but limited to, central brain regions that display high image quality and spatial accuracy. In peripheral regions, however, the anatomically-optimised 7T sequences did not meet the requirements of geometrical accuracy for RTP. This implies that 
dedicated 7T MRI scan protocols need to be optimised for the specific clinical usage in diagnostic radiology or in radiation therapy. To apply UHF-MRI in thoracic regions, new multi-channel excitation coils are being introduced to provide precise control over the RF field distribution to enable high-quality imaging even over large volumes.

Lastly, MR radiomics analyses provide unique insight in tumour heterogeneity by extracting quantitative phenotypic features from ADC maps. Open access has been permitted to the datasets used in Chapter 8 and is publicly available for further research. Based on ADC-based radiomics, prediction models can be developed and are the focus of future research. Some examples from the MAASTRO Clinic research group are already available on www.predictcancer.org. However, MR radiomics is not yet ready to be translated into daily clinical practice and further standardization of quality-assured DWI protocols are needed for multicentre validation. One step towards standardization includes the radiomics quality score (RQS) initiative.

\section{PLANNING AND REALISATION}

Transcending stand-alone MRI, the future of imaging lies in smartly combining different imaging modalities and parametric imaging techniques to gain comprehensive insights in tumour biology that could ultimately lead to individualised anti-cancer treatment. With the increasing amount of data available, analysis methods are required that can manage multiple scans and can combine the knowledge.

In radiation oncology, UHF-MR images might improve delineation of the gross tumour volume (GTV) by visualizing tumour microvascularization. Therefore, a future clinical study (NCT02062372) has been designed to investigate this potential and evaluates the value of 7T-image guided biopsies.

Hybrid PET/MRI has also gained interest for precise target volume delineation on anatomical MR images, for defining biological target (sub-)volumes based on hypoxia imaging, as well as for monitoring treatment response. The study presented in Chapter 7 was part of an ongoing clinical trial (NCT02233387) investigating the potential of ${ }^{18} \mathrm{~F}-\mathrm{HX} 4 \mathrm{PET}$ in patients with oesophageal, cervical, prostate, glioblastoma, rectum cancer or brain metastases. The benefit of boosting specific radioresistant parts of the tumour is still under clinical evaluation. MAASTRO Clinic is participating in a prospective phase II PET-Boost trial in patients with non-small cell lung cancer (NCT01024829). 
ADDENDUM

\author{
Dankwoord \\ (Acknowledgements)
}


Bij het schrijven over het geleverde werk van de voorbije jaren, is het leuk om terug te denken aan het gehele promotietraject. Het was een werk van lange adem maar ik kan ondertussen trots terugblikken op vele mooie momenten. Klinisch onderzoek doe je niet alleen en ik besef nu de omvang hoeveel mensen er op de één of andere manier een impact hebben gehad op mijn promotietraject. Met dit schrijven, grijp ik graag de kans om deze personen even in de bloemetjes te zetten.

Allereerst wil ik graag mijn promotoren Prof. dr. Philippe Lambin en Prof. dr. Felix Mottaghy bedanken voor het faciliteren van mijn onderzoek, maar ook voor al jullie ondersteuning en ongelofelijke wetenschappelijke inzichten. Philippe, het was ongelofelijk leuk om deel uit te maken van een diverse, internationale onderzoeksgroep. Vol bewondering kijk ik op naar uw academische prestaties en erkenningen. Ook al was ik soms de vreemde eend in de bijt, toch heb je me meermaals kunnen inspireren met je vele onderzoeksvoorstellen, wat leidde tot een gevarieerd leertraject en publicaties. Merci! Felix, sinds het vertrek van Regina heb ik het genoegen gehad om nauw met $\mathrm{u}$ te kunnen samenwerken. Bedankt voor je openheid en positieve houding. Het was enorm leuk en motiverend om jouw expertise in nucleaire geneeskunde te kunnen koppelen aan mijn MRI achtergrond.

Uiteraard wil ik ook graag mijn copromotor Dr. ir. Aswin Hoffman bedanken. Vanaf het eerste sollicitatiegesprek tot ons laatste Skypegesprek zaten we op dezelfde golflengte en was het geweldig om met je samen te werken. Je wetenschappelijke inzichten kon je altijd helder overbrengen en je wist me meermaals opnieuw moed in te spreken om er terug volledig in te vliegen. Daarnaast heb je me ook veel bijgebracht op het vlak van project/time management. Je leerde me om 'smart' te werken en om mezelf niet te verliezen in de details. Het was dan ook zeer jammer toen je, samen met Esther, besloten had om Maastricht in te ruilen voor Dresden. In de moderne wereld bestaan er gelukkig voldoende oplossingen om alsnog nauw contact te kunnen houden en dat lukte prima. Vanaf de eerste paper tot het schrijven van het laatste hoofdstuk van deze thesis, kon ik op je rekenen voor uitvoerige iteraties en discussies. Je was een absolute meerwaarde voor mijn promotieteam door een frisse kijk van buitenaf te bieden en wat weerwerk te voorzien. Bovendien heb je ook een heel leuke persoonlijkheid. Bedankt voor alles!

Vervolgens wil ik graag de leden van de beoordelingscommissie, met name voorzitter Prof. dr. ir. Walter Backes, Prof. dr. Anne-Marie Dingemans, Prof. dr. Dirk de Ruysscher, Prof. dr. Daniela Thorwarth, en Dr. Wouter Vogel, bedanken voor het kritisch lezen en beoordelen van mijn thesis.

Dit doctoraat is ontstaan als een gezamenlijk project tussen Maastro Clinic en het MUMC+, en trachtte aan de hand van MR beeldvorming een brug te slaan tussen de wereld van de radiologie en de radiotherapie. Naast mijn promotoren, wil ik daarom graag Prof. dr. Joachim Wildberger bedanken voor het faciliteren van deze 
samenwerking. Doordat dit project ook een logistische en administratieve uitdaging was, bedank ik graag Rianne en Monique voor hun ondersteuning bij het inplannen van meetings en het organiseren van alles rondom mijn promotie.

Dit proefschrift is grotendeels gebaseerd op bevindingen uit klinische studies. Deze klinische studies zijn tot stand gekomen door de multidisciplinaire samenwerking tussen Maastro Clinic en het MUMC+, en de bereidwilligheid van patiënten om vrijwillig deel te nemen. Graag wil ik daarom alle partijen bedanken die dit mogelijk hebben kunnen maken. Om te beginnen bedank ik graag de Trial Poli van Maastro, met name John, Claudia, en Evelyn voor jullie onschatbare waarde in het helpen organiseren en coördineren van de HX4-PET/MR studie. Verder wil ik graag Dr. ir. Wouter van Elmpt bedanken. Jouw deur stond altijd voor me open, ook al had je je handen vol met jouw eigen promovendi.

Vervolgens wil ik graag Dr. Stefan Vöö bedanken voor je medische kennis en expertise in PET-beeldvorming. Naar aanleiding van de wijzigingen in het promotieteam, hebben we samen vele uren gebrainstormd over mogelijke projecten en vervolgens het onderzoeksprotocol geschreven voor de FDG-PET/MR studie. Bij de twee PET/MRI projecten wil ik graag het werk onderstrepen van het gehele PET/MRI-team. Kim, Eslina, Renee, bedankt voor jullie praktische en technische inzichten, en steun tijdens de talloze uren achter de PET/MR console! Het was, naast enorm leerrijk, ook ontzettend leuk en gezellig om samen alle patiënten en gezonde vrijwilligers te scannen. Tijdens de PET/MR workshop in Tübingen heb ik het genoegen gehad om jullie beter te leren kennen. Ook wil ik iedereen van de afdeling Radiologie en Nucleaire geneeskunde bedanken. Roland, bedankt voor het helpen coördineren en inplannen van research tijd. Rik en Roel, bedankt voor al het voorbereidende werk en in te staan voor de kwaliteitscontrole. Graag wil ik tevens het team van Brains Unlimited en Scannexus NV bedanken voor het helpen verwezenlijken van beide UHF-MR studies. Bedankt Dimo, Esther, en Margo voor jullie input tijdens het optimaliseren en creëren van 7T MR beelden.

Bij deze wil ik graag ook alle coauteurs, voor zover nog niet genoemd, bedanken voor al hun inspanningen bij het verzamelen en analyseren van data, en voor de geleverde feedback bij het schrijven. Esther Troost, jouw enthousiasme is zeer aanstekelijk. Je leerde me hierdoor het concept van 'sprintweken' kennen waarin je ongelofelijk veel werk verzette. Regina en Patty, ondanks onze korte samenwerking heb ik veel van jullie geleerd. Regina, je wist in de beginfase van mijn promotie er meteen vaart in te brengen en kon je al snel bijleren hoe een artikel volgens de regels van de kunst op te bouwen. Patty, jij hebt me geleerd hoe een meta-analyse uit te voeren en hoe je moet omgaan met de commentaren van reviewers en deze kan relativeren. Leonie, jij was samen met Kim van onschatbare waarde bij het optimaliseren van de anatomische beeldvorming en het bekomen van de scanprotocollen. Meermaals ben ik bij je komen aankloppen om het te hebben over 
het studieopzet, de eisen van artsen, en manier van data-analyse. Het was ontzettend leuk om met je samen te werken. Bedankt voor alles! Maaike, Frans, Cristina, bedankt voor jullie klinische expertise nodig voor het opstarten van de studie en een beeld te scheppen over wat belangrijk is in MR beelden voor enerzijds radiologen en anderzijds voor radiotherapeuten. Daarnaast wil ik jullie bedanken voor de mogelijkheid om MDO's mee te volgen, jullie hulp bij het verzamelen van patiëntengegevens, en natuurlijk het beoordelen van de anatomische beeldkwaliteit. Lien, Ludwig, Ala, bedankt voor de fijne samenwerking bij het schrijven van de systematische hypoxie review. Fiere, Inge, Christopher, het was een plezier om met jullie samen te werken in een zeer multidisciplinaire setting. Bedankt Fiere voor jouw technische hulp bij de data-analyse en het creëren van de 3D mesh-plots. Christopher, thank you for your extensive expert knowledge in UHF-MR imaging and your insights to rebut the reviewers' comments. De UHF-MR projecten waren echter niet mogelijk zonder de klinische input van jou, Inge. Na een enorme inspanning en een niet-aflatend doorzettingsvermogen is het ons uiteindelijk gelukt om twee mooie publicaties te bekomen. Bedankt. Ik duim voor je eigen studies die hieraan gekoppeld zijn en je eigen promotietraject! Abdalla and Henry, you guys were a key factor in completing the, for me, most difficult paper as it was the furthest outside of my comfort zone. Thanks to your skills in coding we were able to perform radiomics analyses on ADC maps. Henry, thank you for your enthusiasm, lets-go spirit, and role as corresponding author for the final paper.

Over the years, I had the pleasure to encounter many interesting, diverse, and fun people. At Maastro Clinic, all PhDers shared the big research room which lead to a dynamic and fun work atmosphere. I always enjoyed coming to work and your company was a major factor in this, even after moving to Antwerp. We shared so many happy moments during coffee-breaks (often with vlaai or treats from around the world), meeting, conferences, lunch breaks, walks, sports, and other social activities/drinks. Thank you - Abdalla, Adriana, Alberto, Ana, Aniek, Arthur, Bregje, Brent, Cary, Cecile, Celine, Daniela, Davide, Ester, Esther, Evelyn, Frank, Gabriel, Georgi, Henry, Isabel, Inge, Jean, Joana, Johan, José, Karen, Lotte, Lucas, Mark, Marta, Mathieu, Mariana, Murillo, Nicolle, Patrick, Pedro, Raghu, Ralph, Relinde, Ruben, Sara, Scott, Sean, Sebastian, Shane, Simon, Skadi, Stefan, Tessa, Timo, Turkey, and Yvonka. It is nice knowing that many colleagues have become friends.

Sara, you brought an amazing energy to work each day and made it your mission to include everyone and create a tight group. I wish you, Nuno, and Daniel all the best and I look forward to our next visit. Shane, you might as well be the most interesting and colourful person I know. Your ability to befriend everyone is incredible and something I really admire. Hold true to your (many) principles and weirdness. Abdalla, - Porquedee - the word that often described your frustrations. Thank you for not giving up. Timo, you were always in for a laugh - even if the joke was very dark. Turkey, you are one of the most gentle and friendly person I know. 
Gabriel, although you were extremely busy (or at least appeared to be), you were always in a good mood and happy to help or talk about whatever. Mark, you're a really cool person I had the pleasure of meeting. Enjoy your world trip with Aniek. I can't wait to see both again and hear all about your adventures over some beers. Aniek, het was een eer om jouw paranimf te zijn en ik ben trots op de vriendschap die we hebben opgebouwd. Leve de paddenstoelen, leve de gekke hobbies, maar vooral leve de flauwe grappen! Skadi, jij was mijn oorspronkelijke buurvrouw op kantoor en je liet me vanaf dag 1 meteen voelen dat ik bij de groep hoorde. Bedankt voor de gezellige werksfeer en updates van alle roddels en nieuwtjes op de werkvloer. Karen, bij jou kon ik altijd terecht om 'even' gezellig te buurten. Je hebt een aanstekelijke lach en een geweldig gevoel voor (dubbelzinnige) humor. Top! Stefan, telkens wanneer iedereen al iets téveel gedronken had tijdens het stappen, kwam jij aandragen met een plateau Dropshot. 'Bedankt' daarvoor... Brent, eindelijk nog iemand van (Belgisch) niveau op kantoor! Simon, super leuk om je te leren kennen. Je Amsterdams accent en vooral het woord 'penarie' heeft me meermaals op de lachspieren gewerkt. Evelyn, eigenlijk is het jammer dat ik maar twee paranimfen kan kiezen. Je bent een goedlachs en gezellig persoon die de onderzoeksruimte deed opfleuren. Iedereen kon bij jou zijn ei kwijt en je bood altijd een helpende hand aan. Het is dan ook zeer indrukwekkend dat je je promotie versneld hebt kunnen afronden, en meteen aan de slag kon als Klifio. Ik kijk alvast uit naar de volgende BBQ!

Mijn paranimfen, Janita en Ruben, het is een hele eer om jullie achter me te hebben staan op de grote dag en om samen dit hoofdstuk af te sluiten. Het is niet altijd even gemakkelijk om de juiste woorden te vinden maar ik hoop dat jullie weten wat jullie voor me betekenen. Jullie zijn toppers en liggen me nauw aan het hart. Bedankt voor de vele leuke herinneringen. Ik ben ervan overtuigd dat we contact blijven houden en nog meer fantastische momenten gaan beleven! Janita, je academische prestaties en muurklimkunsten zijn zéker iets om naar 'op te kijken'. Bedankt voor alle gezelligheid tijdens en na het werk. Ik hoop dat je nooit zult vergeten dat er ook paarse aardappelen bestaan. Ruben, ons promotietraject verliep haast parallel aan elkaar. Bedankt dat ik mijn frustraties op ieder moment met je kon delen en bedankt voor de diepgaande gesprekken na het sporten en congressen. Je bent mijn maastro-maatje en ik ben zeker dat we elkaar nog zullen zien in de toekomst.

Onder de noemer vrienden wil ik graag Larissa, Marc, Sanne, Stan, Renaat, Ilse, Jorn, Maarten, Kristien, en Jente bedanken voor het pintelieren, het gezever, en de grappen en grollen. Het leven zou maar saai zijn als alles serieus moest verlopen!

Aan mijn nieuwe collega's bij The Clinical Company, bedankt voor het warm welkom en leuke sfeer op kantoor. De afgelopen Super MM zal me altijd bijblijven incl. plateau tequila. 
Tot slot, wil ik graag mijn lieve (schoon)familie bedanken voor hun steun en medeleven. Ik hoop dat dit boekje een beetje duidelijkheid heeft kunnen scheppen over wat ik nu al die jaren precies aan het doen was. Papa en Mama, bedankt voor alles. Jullie stonden altijd voor me klaar en hebben me alles geboden om deze promotie mogelijk te maken. Ik hoop dat jullie evenveel van deze dag genieten als ik. Daar drinken we ene op! Ellen en Teun, bedankt voor de vele keren dat ik bij jullie mocht logeren na een avondje stappen. Thijs, je kan nog niet lezen maar over een paar jaar duw ik je dit boekje in je handen. Eva en Aslan, het is met trots dat ik kan zeggen dat ik je Peter ben.

Mijn liefste Okke, jij mag natuurlijk niet ontbreken in mijn dankwoord. Jij hebt mijn hele promotietraject van dichtbij meegemaakt en misschien wel het meeste ingeleverd van iedereen. Meermaals heb je me van achter mijn laptop moeten halen en weekenden moeten opofferen. Bedankt om de dingen te relativeren en te zorgen voor de nodige ontspanning. Je liefde en aanmoedigingen hebben me door vele moeilijke momenten gebracht. Zolang we samen zijn, weet ik dat het goed komt - Ik hou van je! 
ADDENDUM

Curriculum Vitae 
Jurgen Peerlings was born on the $5^{\text {th }}$ of July 1989 in Bree, Belgium. In 2007, he completed his general secondary education at College Heilig Kruis SintUrsula in Maaseik and started studying Biomedical Life Sciences at the University of Hasselt. Here, he acquired knowledge of human anatomy, cellular processes, and pre-clinical research. During his Bachelor's program, he was also actively involved in the student fraternity Biomedica as pledge master, treasurer, and PR representative.

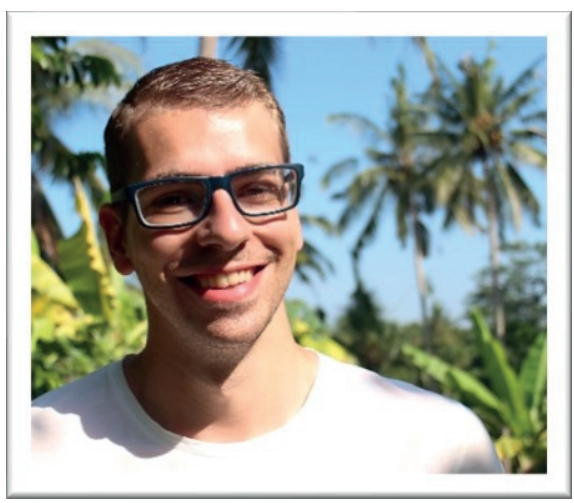
After acquiring his Bachelor's degree, Jurgen continued his academic education at the University of Antwerp to become a Master of Science in Molecular Imaging. Here, he discovered the world of MRI and had the opportunity to attend an European Master program on Molecular Imaging (EMMI) and an Erasmus Intensive Program in the field of Neuro-MRI. During his internships at the Bio-imaging Lab (BIL) and Molecular Imaging Center Antwerp (MICA), he performed pre-clinical research involving small animal imaging using PET and MRI. In 2013, Jurgen graduated Cum laude with a minor in neurology. Later that year, Jurgen made the transition to clinical research and started as a PhD candidate in a joint endeavour at MAASTRO Clinic and Maastricht University Medical Centre. His research focused on bridging the gap between radiology and radiation oncology by exploring advanced molecular imaging techniques such as thoracic (PET-)MRI, ultra-high field MRI, body diffusion-weighted MRI, MR radiomics, and hypoxia PET-imaging for implementation in radiology and radiotherapy. During this time, he was able to present at various (inter)national conferences and publish his work in several academic journals. Since April 2018, Jurgen works as a Clinical Research Associate at The Clinical Company where he is responsible for monitoring and managing multi-centre clinical trials in pharmaceutics and medical devices. 


\section{GRANTS}

Young Fellow workshop stipend UK Tübingen - $4^{\text {th }}$ PET/MRI workshop 2015, Tübingen, DEU

Netherland Respiratory Society - young investigators travel grant, European Congress of Radiology 2015, Vienna, AU

MAASTRO education stipend - ESMRMB course Body Diffusion MRI Theory to Practice 2016, Vienna, AU

\section{CONFERENCES}

International Society for MRI - Benelux (ISMRM):

Oral presentation 2017-18

Poster display 2014, 2016

European Society for MR in Medicine \& Biology (ESMRMB): $\quad$ Oral presentation 2017

Biology-Guided Adaptive Radiotherapy (BiGART): $\quad$ Poster discussion 2017

European Congress of Radiology (ECR): $\quad$ Oral presentation 2015

European Society for Radiotherapy \& Oncology (ESTRO): $\quad$ Poster discussion 2015

Poster display 2016-17

Science day for Oncology \& Developmental Biology GROW: Oral presentation 2013

Poster display 2014, 2016

\section{SCIENTIFIC PUBLICATIONS}

- Peerlings J, Larue RTHM, Paulis L, Berbée M, Wierts R, Wildberger JE, Mottaghy FM, Lambin P, Hoffmann AL. Combining ${ }^{18} \mathrm{~F}-\mathrm{HX} 4$ tracer and PET/MR imaging for quantifying tumour hypoxia imaging in oesophageal cancer. Clin Nucl Med - Interesting images [Submitted]

- Peerlings J, Paulis L, Mitea C, Bakers F, Berbée M, Wierts R, Vöö S, Wildberger JE, Hoffmann AL, Lambin P, Mottaghy FM. Performing clinical ${ }^{18}$ FDG-PET/MR imaging of the mediastinum using a dedicated, patient-friendly protocol. Nucl Med Commun [Article in review]

- Peerlings J, Woodruff HC, Winfield JM, Ibrahim A, Wakefield JC, Larue RTHM, Mottaghy FM, deSouza NM, Lambin P. Repeatability of radiomics features in apparent diffusion coefficient maps from a multi-centre test-retest trial. Sci Rep. 2019 Mar 18;9(1):4800 
- Peerlings J, Compter I, Janssen F, Wiggins CJ, Postma AA, Mottaghy FM, Lambin P, Hoffmann AL. Characterizing geometrical accuracy in clinically optimised $7 T$ and $3 T$ MR images for high-precision radiation treatment of brain tumors. PhiRO. 2019 Jan;(9):35-42.

- Walsh S, de Jong EEC, van Timmeren JE, Ibrahim A, Compter I, Peerlings J, Sanduleanu S, Refaee T, Keek S, Larue RTHM, van Wijk Y, Even AJG, Jochems A, Barakat MS, Leijenaar RTH, Lambin P. Decision Support Systems in Oncology. JCO Clin Cancer Inform. 2019 Feb;(3):1-9.

- Lambin P, Leijenaar RTH, Deist TM, Peerlings J, de Jong EEC, van Timmeren J, Sanduleanu S, Larue RTHM, Even AJG, Jochems A, van Wijk Y, Woodruff H, van Soest J, Lustberg T, Roelofs E, van Elmpt W, Dekker A, Mottaghy FM, Wildberger JE, Walsh S. Radiomics: the bridge between medical imaging and personalized medicine. Nat Rev Clin Oncol. 2017 Dec;14(12):749-762.

- Peerlings $\mathbf{J}^{*}$, Van De Voorde $\mathrm{L}^{*}$, Mitea $\mathrm{C}$, Larue $\mathrm{R}$, Yaromina A, Sanduleanu S, Spiegelberg L, Dubois L, Lambin P, Mottaghy FM. Hypoxia and hypoxia response-associated molecular markers in esophageal cancer: A systematic review. Methods. 2017 Nov 1;130:51-62.

- Lambin P, Zindler J, Vanneste BG, De Voorde LV, Eekers D, Compter I, Panth KM, Peerlings J, Larue RT, Deist TM, Jochems A, Lustberg T, van Soest J, de Jong EE, Even AJ, Reymen B, Rekers N, van Gisbergen M, Roelofs E, Carvalho S, Leijenaar RT, Zegers CM, Jacobs M, van Timmeren J, Brouwers P, Lal JA, Dubois L, Yaromina A, Van Limbergen EJ, Berbee M, van Elmpt W, Oberije C, Ramaekers B, Dekker A, Boersma LJ, Hoebers F, Smits KM, Berlanga AJ, Walsh S. Decision support systems for personalized and participative radiation oncology. Adv Drug Deliv Rev. 2017 Jan 15;109:131-153.

- Guglielmetti C, Le Blon D, Santermans E, Salas-Perdomo A, Daans J, De Vocht N, Shah D, Hoornaert C, Praet J, Peerlings J, Kara F, Bigot C, Mai Z, Goossens H, Hens N, Hendrix S, Verhoye M, Planas AM, Berneman Z, van der Linden A, Ponsaerts P. Interleukin-13 immune gene therapy prevents CNS inflammation and demyelination via alternative activation of microglia and macrophages. Glia. 2016 Dec;64(12):2181-2200. 
- Cobben DC, de Boer HC, Tijssen RH, Rutten EG, van Vulpen M, Peerlings J, Troost EG, Hoffmann AL, van Lier AL. Emerging Role of MRI for Radiation Treatment Planning in Lung Cancer. Technol Cancer Res Treat. 2016 Dec;15(6):NP47-NP60.

- Peerlings J, Troost EG, Nelemans PJ, Cobben DC, de Boer JC, Hoffmann AL, Beets-Tan RG. The Diagnostic Value of MR Imaging in Determining the Lymph Node Status of Patients with Non-Small Cell Lung Cancer: A MetaAnalysis. Radiology 2016 Oct;281(1):86-98.

- Compter $\mathrm{I}^{*}$, Peerlings $\mathrm{J}^{*}$, Eekers DB, Postma AA, Ivanov D, Wiggins CJ, Kubben P, Küsters B, Wesseling P, Ackermans L, Schijns OE, Lambin P, Hoffmann AL. Technical feasibility of integrating 7 T anatomical MRI in imageguided radiotherapy of glioblastoma: a preparatory study. MAGMA. 2016 Jun;29(3):591-603.

- Lambin P, Zindler J, Vanneste B, van de Voorde L, Jacobs M, Eekers D, Peerlings J, Reymen B, Larue RT, Deist TM, de Jong EE, Even AJ, Berlanga AJ, Roelofs E, Cheng Q, Carvalho S, Leijenaar RT, Zegers CM, van Limbergen E, Berbee M, van Elmpt W, Oberije C, Houben R, Dekker A, Boersma L, Verhaegen F, Bosmans G, Hoebers F, Smits K, Walsh S. Modern clinical research: How rapid learning health care and cohort multiple randomised clinical trials complement traditional evidence-based medicine. Acta Oncol. 2015;54(9):1289-300.

\footnotetext{
* indicates equal contribution
} 



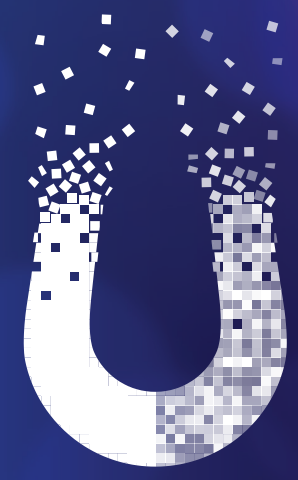

